Florida International University FIU Digital Commons

3-20-2015

\title{
Characterization of the poxAB Operon Encoding a Class D Carbapenemase in Pseudomonas aeruginosa,
}

Diansy Zincke

Florida International University, dzinc001@fiu.edu

DOI: $10.25148 /$ etd.FI15050210

Follow this and additional works at: https:// digitalcommons.fiu.edu/etd

Part of the Bacteriology Commons, Biology Commons, and the Pathogenic Microbiology Commons

\section{Recommended Citation}

Zincke, Diansy, "Characterization of the poxAB Operon Encoding a Class D Carbapenemase in Pseudomonas aeruginosa," (2015). FIU Electronic Theses and Dissertations. 1794.

https://digitalcommons.fiu.edu/etd/1794 


\title{
FLORIDA INTERNATIONAL UNIVERSITY
}

Miami, Florida

\section{CHARACTERIZATION OF THE POXAB OPERON ENCODING A CLASS D CARBAPENEMASE IN PSEUDOMONAS AERUGINOSA}

\author{
A dissertation submitted in partial fulfillment of \\ the requirements for the degree of \\ DOCTOR OF PHILOSOPHY \\ in \\ BIOLOGY \\ by
}

Diansy Zincke 
To: Dean Michael R. Heithaus

College of Arts and Sciences

This dissertation, written by Diansy Zincke, and entitled Characterization of the pox $A B$ Operon Encoding a Class D Carbapenemase in Pseudomonas aeruginosa, having been approved in respect to style and intellectual content, is referred to you for judgment.

We have read this dissertation and recommend that it be approved.

Alejandro Barbieri

Ruben L. Gonzalez

John Makemson

Lynn L. Silver

Kalai Mathee, Major Professor

Date of Defense: March 20, 2015

The dissertation of Diansy Zincke is approved.

\begin{tabular}{r}
\hline $\begin{array}{r}\text { Dean Michael R. Heithaus } \\
\text { College of Arts and Sciences }\end{array}$ \\
\hline Dean Lakshmi N. Reddi \\
University Graduate School
\end{tabular}

Florida International University, 2015 


\section{DEDICATION}

This work is dedicated to my grandmother Marina Diaz Linares and to my parents Maria R. Quintero and Armando Zincke for their constant guidance, support and unconditional love. 


\section{ACKNOWLEDGMENTS}

First and foremost, I would like to thank my PhD advisor Dr. Kalai Mathee for her continued guidance and support throughout my journey with her. I have learned a lot from her and I'm sure her influence and impact on my life will persist for a long time. I would also like to thank my committee members, Alejandro Barbieri, Ruben Gonzalez, John Makemson and Lynn Silver, for their insightful criticism and guidance throughout the years. I have met many wonderful people throughout my time in the Mathee lab and have derived a lot of support from these colleagues. The Mathee crew has become my family and I'm very grateful to have shared this journey with such a diverse and determined group of people. I'm also very grateful to the financial support provided by the FIU MBRS-RISE (NIH/NIGMS R25 GM61347), the FIU Biology TAship, the Student Summer Research Award (RISE Biomedical Research Initiative, 08/09) and the Cystic Fibrosis Foundation (ZINCKE08H0). Last but not least, I would like to thank my family and friends for being supportive and patient. 


\section{ABSTRACT OF THE DISSERTATION \\ CHARACTERIZATION OF THE POXAB OPERON ENCODING A CLASS D \\ CARBAPENEMASE IN PSEUDOMONAS AERUGINOSA}

by

Diansy Zincke

Florida International University, 2015

Miami, Florida

\section{Professor Kalai Mathee, Major Professor}

Pseudomonas aeruginosa is a dreaded opportunistic pathogen that causes severe and often intractable infections in immunocompromised and critically ill patients. This bacterium is also the primary cause of fatal lung infections in patients with cystic fibrosis and a leading nosocomial pathogen responsible for nearly $10 \%$ of all hospital-acquired infections. $P$. aeruginosa is intrinsically recalcitrant to most classes of antibiotics and has the ability to acquire additional resistance during treatment. In particular, resistance to the widely used $\beta$-lactam antibiotics is frequently mediated by the expression of AmpC, a chromosomally encoded $\beta$-lactamase that is ubiquitously found in P. aeruginosa strains. This dissertation delved into the role of a recently reported chromosomal $\beta$-lactamase in P. aeruginosa called PoxB. To date, no detailed studies have addressed the regulation of pox $B$ expression and its contribution to $\beta$-lactam resistance in $P$. aeruginosa. In an effort to better understand the role of this $\beta$-lactamase, pox $B$ was deleted from the chromosome and expressed in trans from an IPTG-inducible promoter. The loss of poxB did not affect susceptibility. However, expression in trans in the absence of $\operatorname{ampC}$ rendered strains 
more resistant to the carbapenem $\beta$-lactams. The carbapenem-hydrolyzing phenotype was enhanced, reaching intermediate and resistant clinical breakpoints, in the absence of the carbapenem-specific outer membrane porin OprD. As observed for most class $\mathrm{D} \beta$ lactamases, PoxB was only weakly inhibited by the currently available $\beta$-lactamase inhibitors. Moreover, poxB was shown to form an operon with the upstream located poxA, whose expression in trans decreased pox promoter $\left(\mathrm{P}_{p o x}\right)$ activity suggesting autoregulation. The transcriptional regulator $\mathrm{AmpR}$ negatively controlled $\mathrm{P}_{\text {pox }}$ activity, however no direct interaction could be demonstrated. A mariner transposon library identified genes involved in the transport of polyamines as potential regulators of pox expression. Unexpectedly, polyamines themselves were able induce resistance to carbapenems. In summary, $P$. aeruginosa carries a chromosomal-encoded $\beta$-lactamase PoxB that can provide resistance against the clinically relevant carbapenems despite its narrow spectrum of hydrolysis and whose activity in vivo may be regulated by polyamines. 


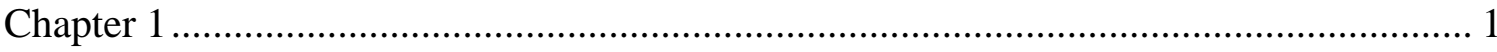

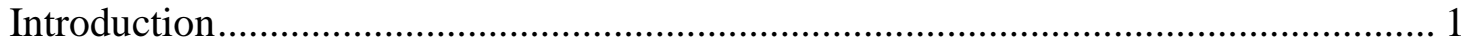

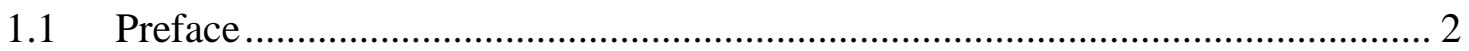

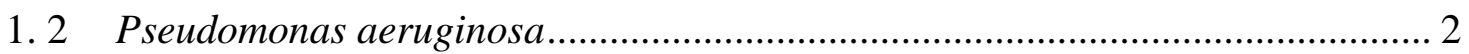

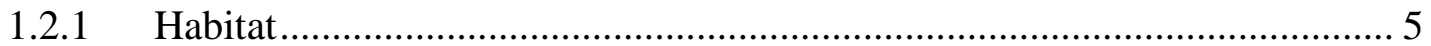

1.2.2 P. aeruginosa infections in healthy individuals..................................... 6

1.2.3 P. aeruginosa in nosocomial or healthcare-associated infections ................ 7

1.2.4 P. aeruginosa in immunocompromised patients ........................................ 8

1.2.5 P. aeruginosa and cystic fibrosis .......................................................... 10

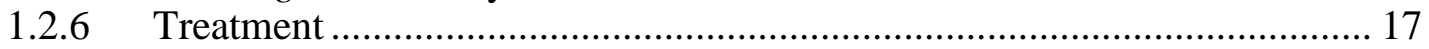

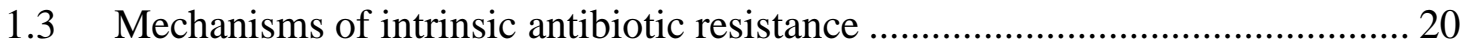

1.3.1 Extrapermeability barrier ..................................................................... 21

1.3.1.1 LPS as a barrier and lipid-mediated uptake ............................................. 23

1.3.1.2 Porin-mediated transport.................................................................. 25

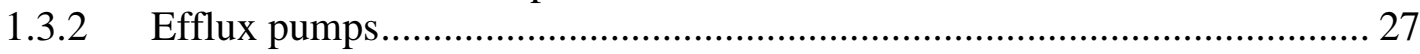

1.3.3 Alteration and modification of penicillin-binding proteins ........................ 32

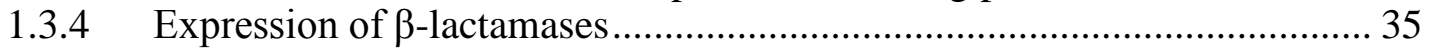

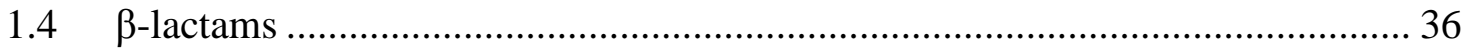

1.4.1 Mechanism of action of $\beta$-lactams.............................................................. 44

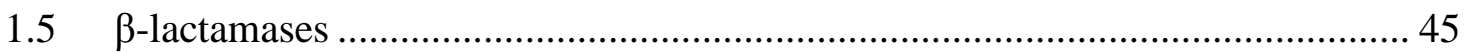

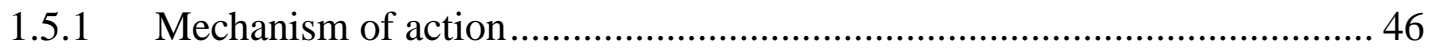

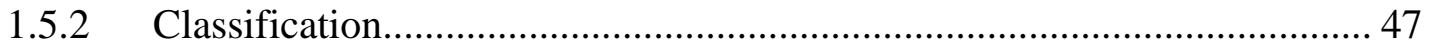

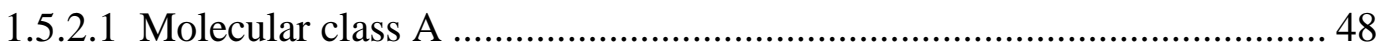

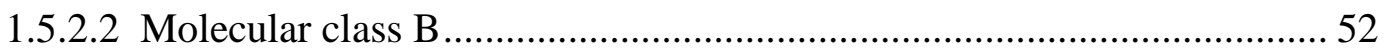

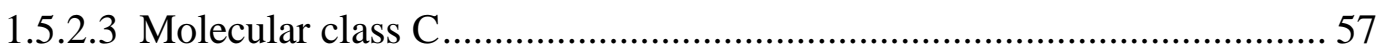

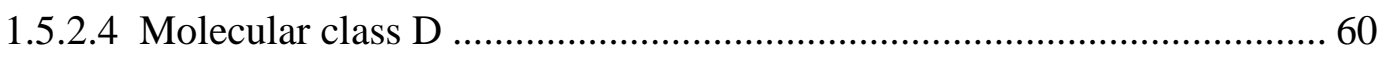

1.5.3 Genetics of inducible class $C \beta$-lactamases ........................................................ 64

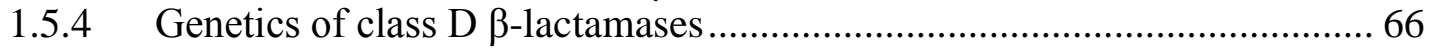

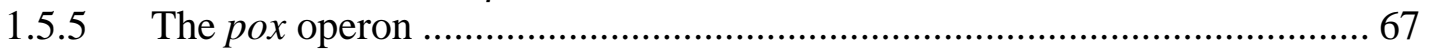

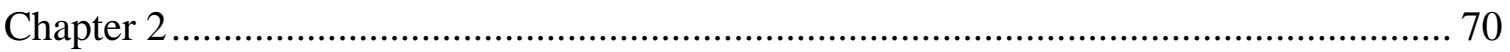

Characterization of a carbapenem-hydrolyzing enzyme, PoxB, in Pseudomonas

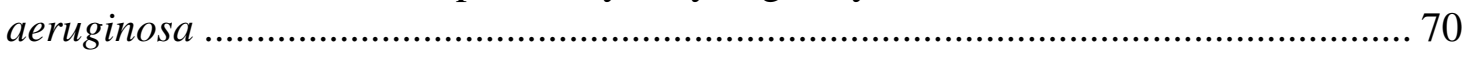

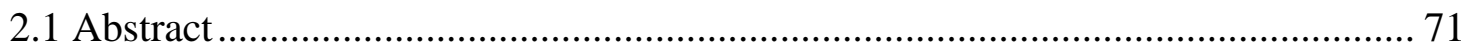

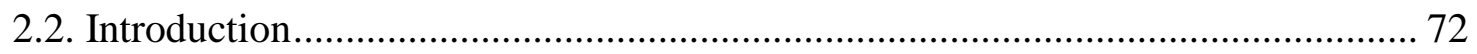

2.3 Materials and Methods................................................................................. 74

2.3.1 Bacterial strains, plasmids and media......................................................... 74

2.3.2 Construction of $\operatorname{pox} A, \operatorname{pox} B$, and $\operatorname{opr} D$ deletion mutants. .............................. 74

2.3.3 PCR amplification and cloning of poxA, poxB and ampC............................. 78

2.3.4 Protein expression and one-dimensional gel electrophoresis. ......................... 79 


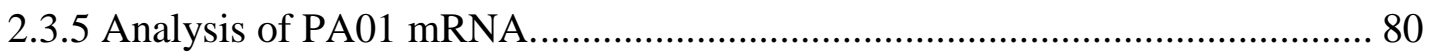

2.3.6 $\beta$-lactamase assay ................................................................................... 80

2.3.7 Minimum inhibitory concentration (MIC) ……………………………….... 81

2.3.8 Colony morphology on Tryptone Agar containing Congo red......................... 81

2.3.9 Pellicle formation and cell-surface interactions.............................................. 82

2.3.10 Congo red binding assay......................................................................... 82

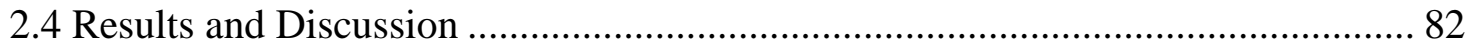

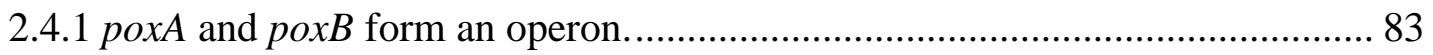

2.4.2 poxA is translated into a 32.4-kDalton protein............................................... 85

2.4.3 poxA and poxB deletions do not alter $\beta$-lactamase activity or $\beta$-lactam

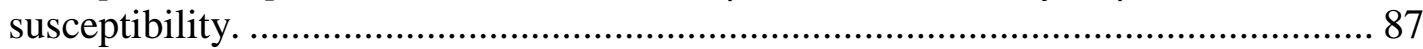

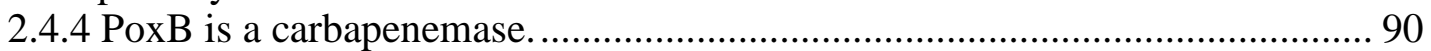

2.4.5 Expression of PoxB does not alter efflux...................................................... 99

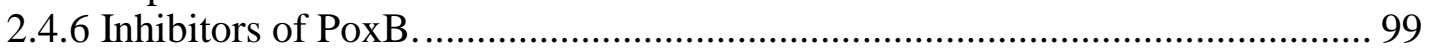

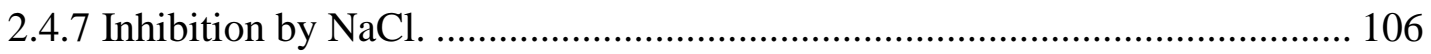

2.4.8 PoxA and Congo red binding phenotype..................................................... 110

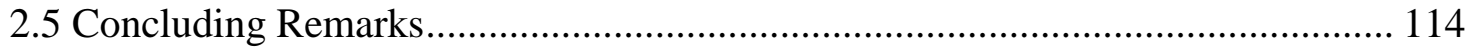

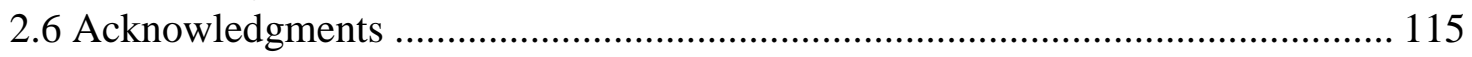

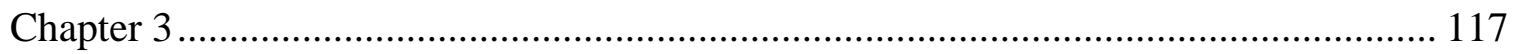

Regulation of the poxAB operon encoding a class D $\beta$-lactamase in Pseudomonas

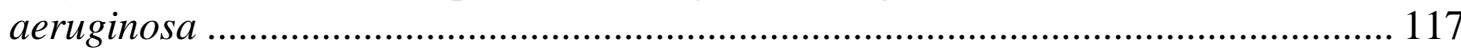

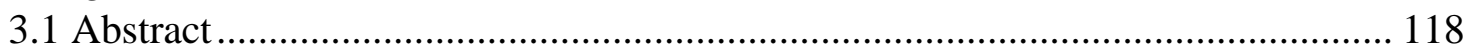

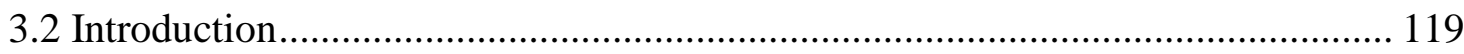

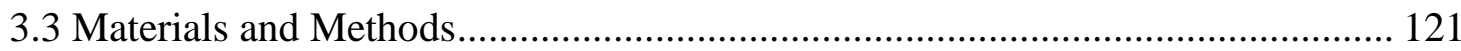

3.3.1 Bacterial strains, plasmids and growth media............................................... 121

3.3.2 Construction of promoter fusions. ............................................................. 123

3.3.3 Quantification of poxB mRNA expression. ................................................ 123

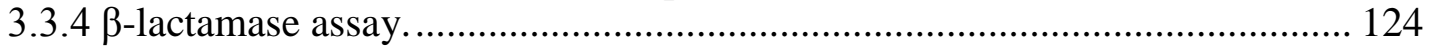

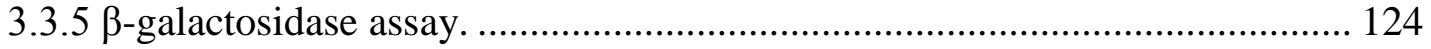

3.3.6 Electrophoretic mobility shift assay (EMSA)............................................ 125

3.3.7 Pilot experiment to construct transposon library. ………………….............. 125

3.3.8 Transposon mutagenesis and screening for regulators of the pox operon. .... 127

3.3.9 Mapping of the transposon $(\mathrm{Tn})$ insertion site.............................................. 131

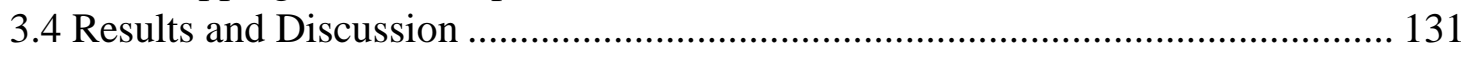

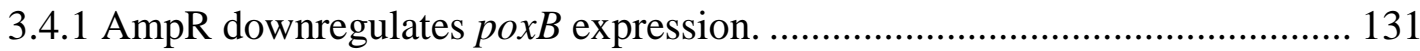

3.4.2 PoxB $\beta$-lactamase activity .......................................................................... 133

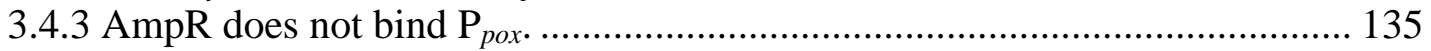

3.4.4 PoxA autoregulates its own promoter........................................................ 137

3.4.5 Approach to identifying regulators of the pox operon.................................. 139

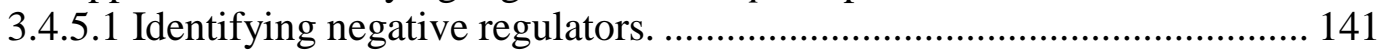

3.4.5.2 Identifying positive regulators............................................................. 142

3.4.6 Construction of mariner Tn library........................................................... 143

3.4.6.1 Generation of the transposon library.................................................... 144

3.4.7 Mapping insertion sites and identification of regulators................................. 145

3.4.7.1 PA0::P $\mathrm{P}_{p o x}$-lacZ library..................................................................... 145 


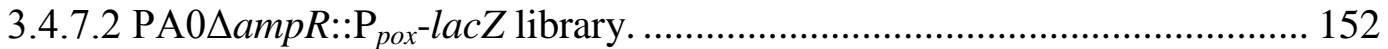

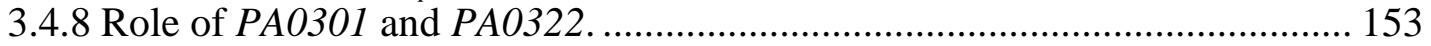

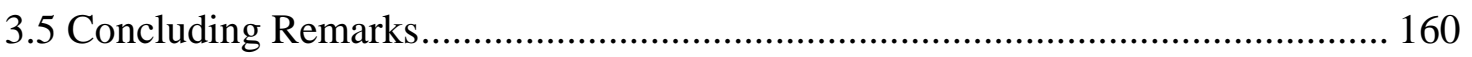

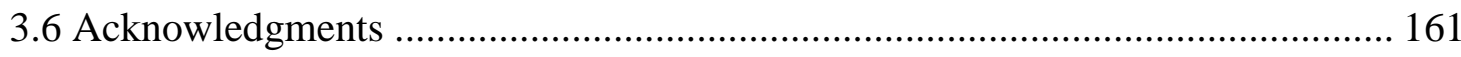

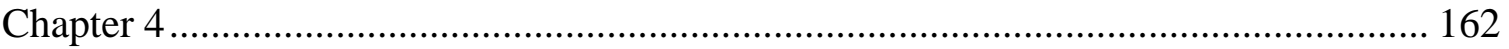

Structural and functional characterization of Pseudomonas aeruginosa global

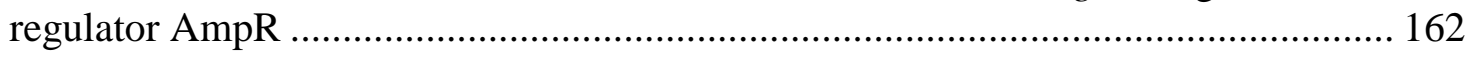

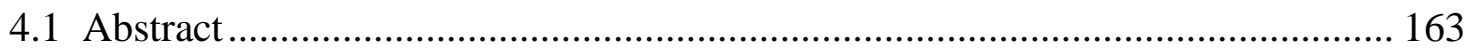

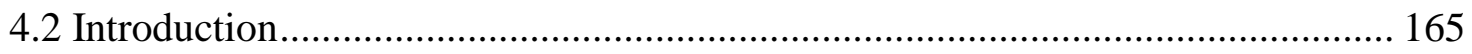

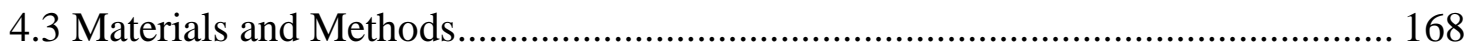

4.3.1 Bacterial strains, plasmids and media......................................................... 168

4.3.2 $\mathrm{P}_{\text {amp }}$ promoter deletions......................................................................... 168

4.3.3 Construction of His-tagged AmpR............................................................ 169

4.3.4 Expression and purification of AmpR-His6............................................... 169

4.3.5 Construction of P. aeruginosa AmpR HTH and point mutants.................... 170

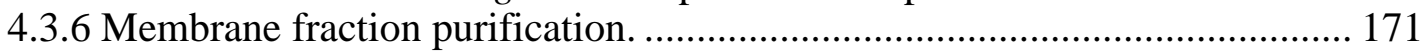

4.3.7 Electrophoretic mobility shift assay (EMSA) ............................................. 171

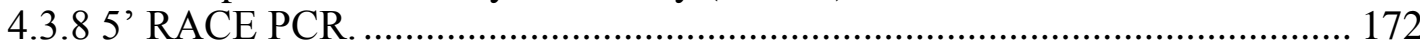

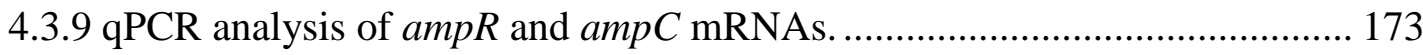

4.3.10 Construction of VSV-G-tagged AmpR.................................................... 174

4.3.11 Chromatin immunoprecipitation quantitative PCR (ChIP-qPCR)............... 174

4.3.12 Protein cross-linking. ............................................................................ 174

4.3.13 Polyclonal anti-AmpR-His6 antibody production. ...................................... 175

4.3.14 Western Blotting and EMSA of HTH mutants.......................................... 175

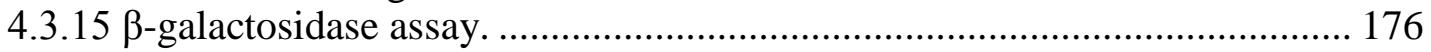

4.3.16 AmpR-LacZ and -PhoA fusion construction and analysis. ......................... 176

4.3.17 Protease protection (shaving) assay. ........................................................... 177

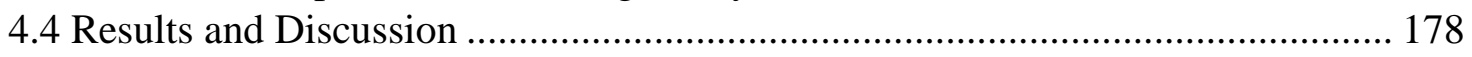

4.4.1 Analysis of the P. aeruginosa ampC-ampR regulatory region..................... 178

4.4.2 Mapping of P. aeruginosa $\mathrm{P}_{\text {amp }}$............................................................... 183

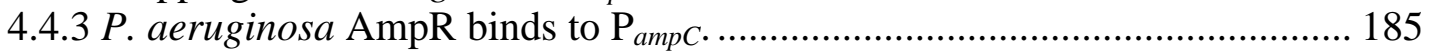

4.4.4 HTH is important for AmpR function........................................................ 187

4.4.5. Gly102 and Asp135 are critical for AmpR function.................................... 193

4.4.6 Cross-linking studies suggest $P$. aeruginosa AmpR dimerizes..................... 195

4.4.7. Localization studies of P. aeruginosa AmpR............................................ 197

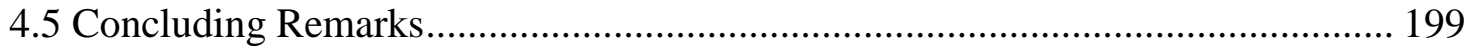

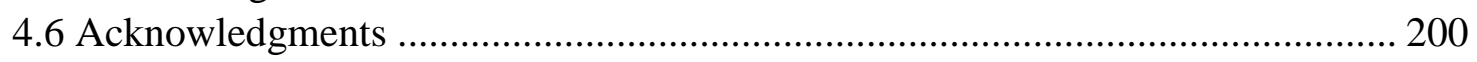

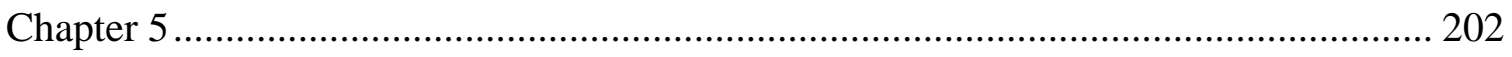

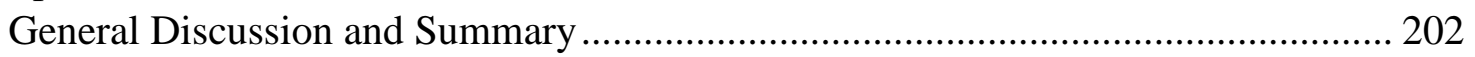

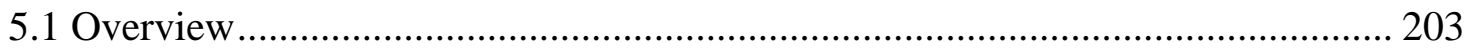

5.2 PoxB is a chromosomal-encoded class D carbapenemase.................................... 204

5.3 Regulation of $p o x B$

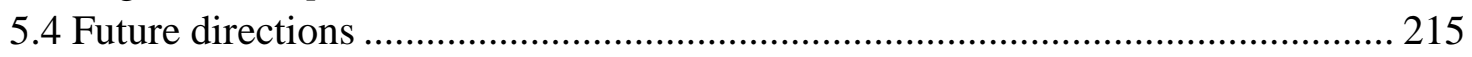




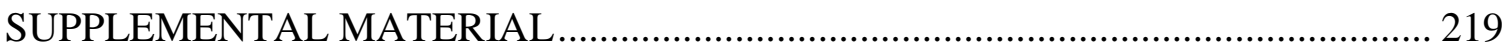

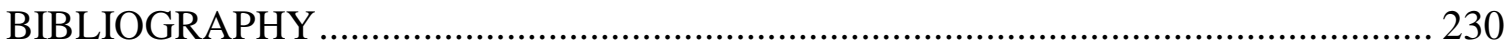

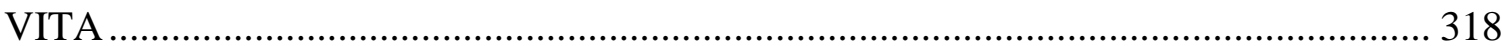


1.1 Substrate profile of RND efflux pumps described in P. aeruginosa ....................30

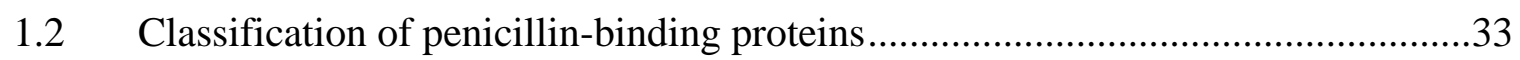

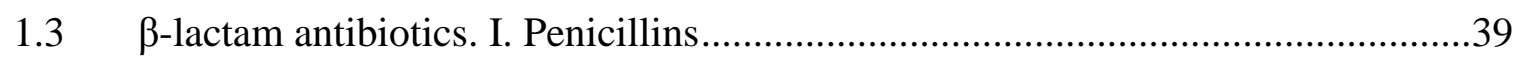

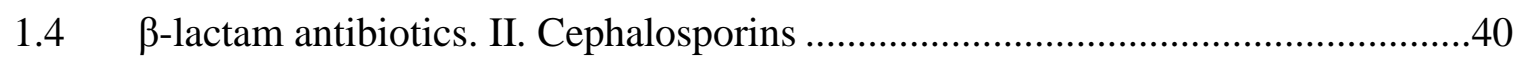

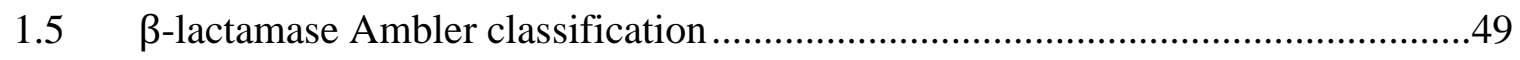

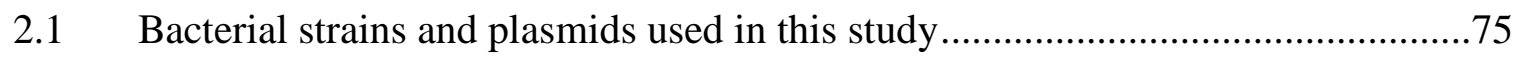

2.2 Susceptibility profiles of $\operatorname{poxA}, \operatorname{poxB}$ and $\operatorname{ampC}$ deletion mutants........................88

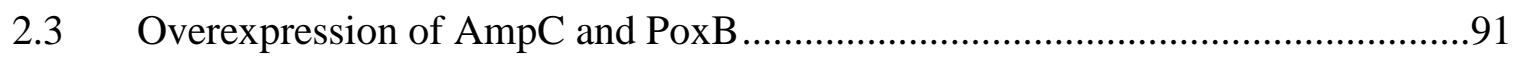

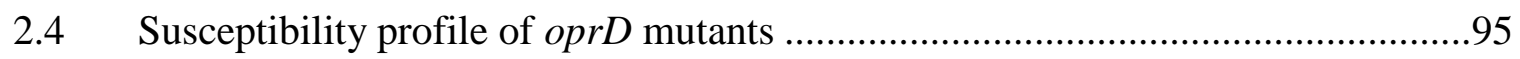

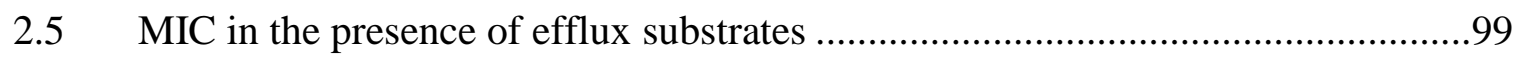

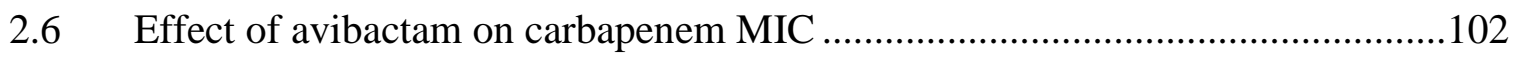

3.1 Bacterial strains and plasmids used in this study ........................................121

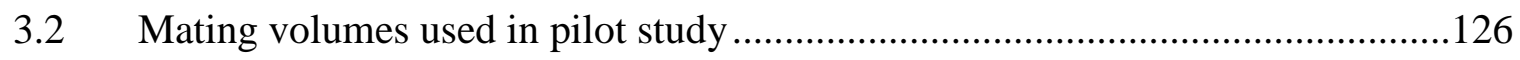

3.3 Approximate colony count obtained for pilot study …...............................128

3.4 Mating ratios used for generation of $P$. aeruginosa library ..............................129

3.5 Approximate colony count after Tn mutagenesis ..........................................144

3.6 Mapped insertions from PA01 library, Plate \# 8 ...........................................146

3.7 $\quad \beta$-lactam MIC in the presence of spermidine ..............................................157

S1 Bacterial strains, plasmids, and primers used in this study ............................220

S2 Complementing assays showing His-tagged AmpR is functional .....................224

S3 Complementing assays showing VSV-G-tagged AmpR is functional ...............225 


\section{LIST OF FIGURES}

PAGE

1.1 Colored scanning electron micrograph of $P$. aeruginosa ...................................

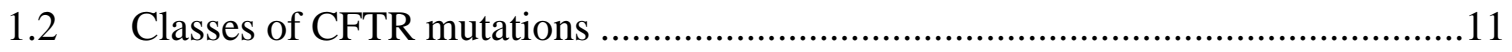

1.3 ASL layer and MCC in the normal and $\mathrm{CF}$ airways ......................................13

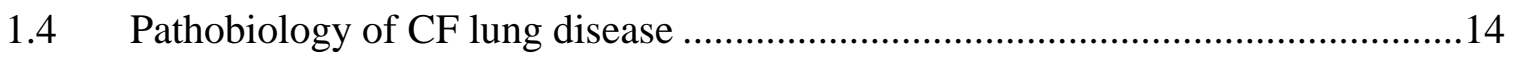

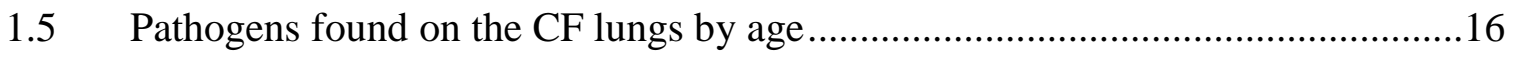

1.6 Molecular structures of two $\beta$-lactams of the penicillin class ...........................17

1.7 Molecular structures of amikacin and tobramycin.........................................18

1.8 Mechanisms of antibiotic resistance in P. aeruginosa ....................................21

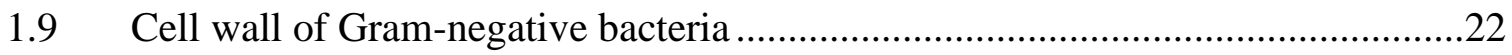

1.10 Molecular structures of the $\beta$-lactam imipenem and of the amino acids

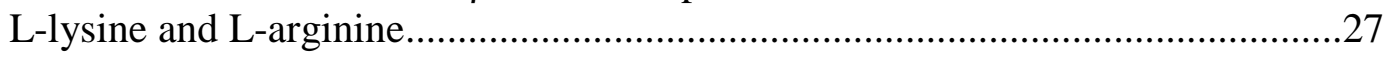

1.11 Family of efflux pumps found in bacteria and their substrates..........................29

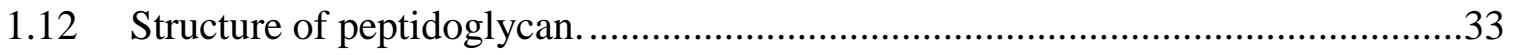

1.13 Molecular structures of penicillin and D-alanyl-D-alanine ...............................34

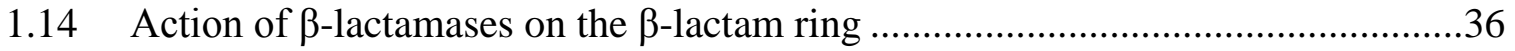

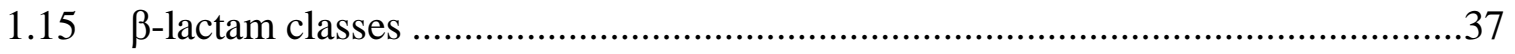

1.16 Molecular structures of 6-aminopenicillanic acid and

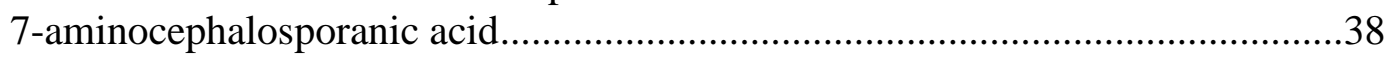

1.17 Structure of antipseudomonal carbapenems approved for used in the US ...........41

1.18 Structure of aztreonam, a monocyclic monobactam......................................42

1.19 Mechanism of action of the serine $\beta$-lactamases .........................................46

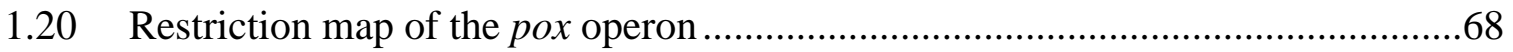

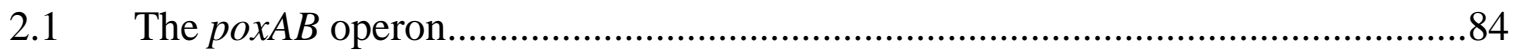


2.2 poxA is translated into a 32.4-kDalton protein....................................................86

$2.3 \quad \beta$-lactamase activity profile of $p o x$ and $\operatorname{ampC}$ deletion mutants............................89

2.4 Determination of meropenem MIC in a PoxB-overexpressing background .........92

2.5 Chemical structures of carbapenems: meropenem, imipenem and doripenem......93

2.6 Determination of carbapenem MICs in P. aeruginosa oprD-deficient strains by the microbroth dilution method

2.7 Effect of tazobactam, sulbactam and clavulanic acid on PoxB activity

2.8 PoxB $\beta$-lactamase activity in the presence of tazobactam, sulbactam, clavulanic acid and avibactam .....

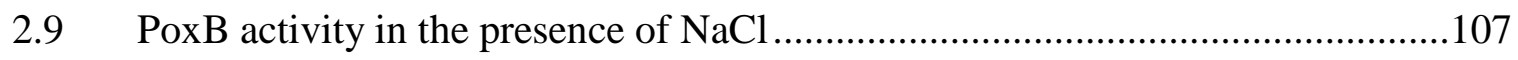

2.10 Determination of $\mathrm{MIC}$ for $\mathrm{NaCl}$.............................................................108

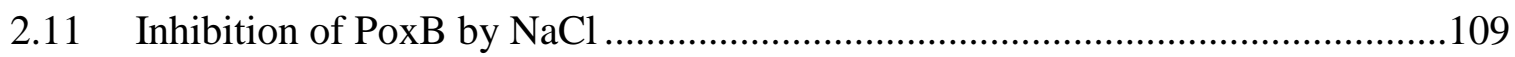

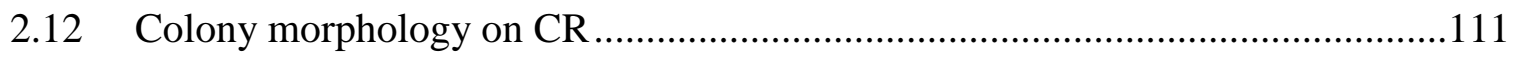

2.13 Pellicle formation in PoxA-expressing cells ....................................................113

2.14 CR-binding assay ………………………............................................114

3.1 Selection and screening of the PA01 library.....................................................130

3.2 Selection and screening of the PA0 $\triangle a m p R$ library..........................................130

3.3 Expression of $\operatorname{pox} B$ in the absence of AmpR.................................................132

$3.4 \quad \beta$-lactamase expression of PA01 and its isogenic mutants .................................134

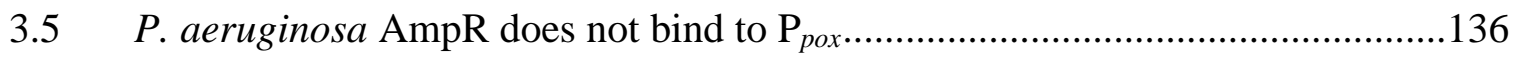

3.6 Activity of the pox promoter in the presence of pPoxA ……..............................138

3.7 Activity of the P. aeruginosa pox promoter .......................................................140

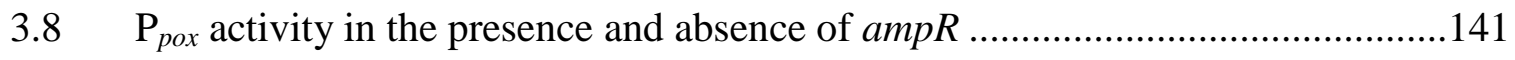

3.9 Predicted color phenotype before and after Tn insertion into putative regulators of the pox operon 
3.10 Plasmid map and features of the mariner transposon vector, pBTK24

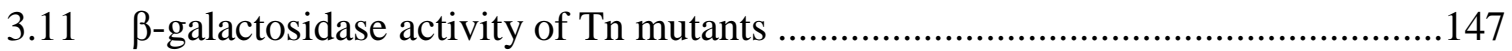

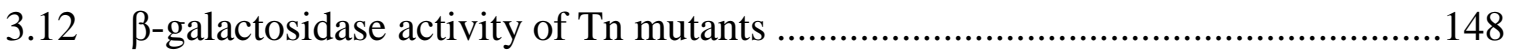

3.13 Qualitative and quantitative screening of PA0:: $\mathrm{P}_{p o x}-l a c Z$ transposon mutants....150

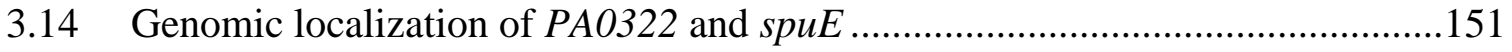

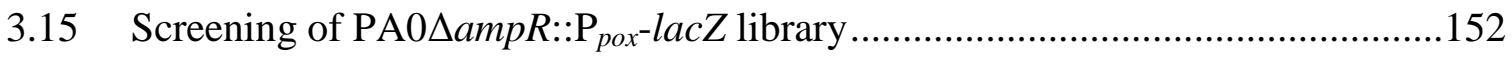

3.16 Structures of some commonly encountered polyamines ................................154

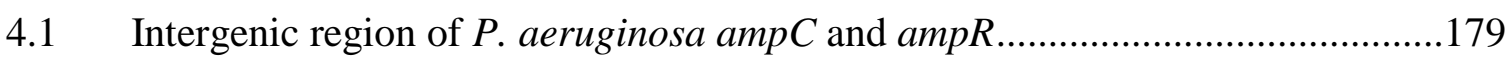

4.2 RpoN downregulates $P$. aeruginosa ampR expression in the presence of

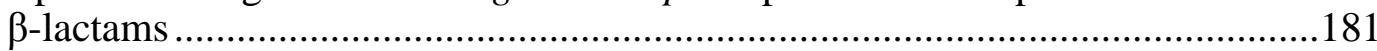

4.3 Mapping of the minimal $P$. aeruginosa ampC promoter .................................184

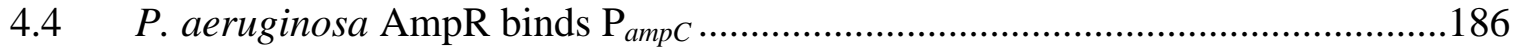

4.5 Analysis of the third helix of the P. aeruginosa AmpR HTH motif....................188

4.6 Functional analysis of the $P$. aeruginosa AmpR HTH motif ...........................189

4.7 Electromobility shift assay of P. aeruginosa AmpR HTH mutants ...................190

4.8 Stability of P. aeruginosa AmpR mutant proteins ........................................192

4.9 Activity of the P. aeruginosa ampC promoter in the presence of AmpRHis6 $_{(\mathrm{Gly} 102 \mathrm{Glu})}$ and AmpR-His6 (Asp135Asn) mutants

4.10 P. aeruginosa AmpR appears to dimerize in vivo .........................................196

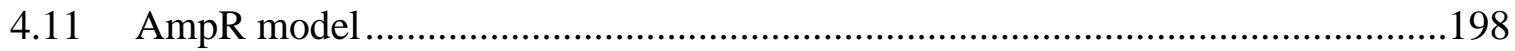

S1 Multiple alignment of the $a m p R-a m p C$ intergenic region ..............................226

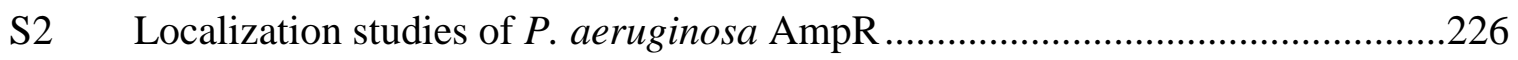

S3 Multiple alignment of the AmpR HTH motif ...........................................228 
LIST OF SYMBOLS AND ABBREVIATIONS

Term/Unit of Measurement

5-bromo-4-chloro-3-indolyl- $\beta$-D-galactopyranoside

6-aminopenicillanic acid

7-aminocephalosporanic acid

Airway surface liquid

Carbapenem-hydrolyzing class D $\beta$-lactamase

Congo Red

Coomassie brilliant blue

Cystic fibrosis

Cystic fibrosis transmembrane conductance regulator

Degrees Celsius

Diaminopimelic acid

Electrophoretic mobility shift assay

et alia

Epsilometer test

Extended-spectrum $\beta$-lactamases

Extended-spectrum oxacillinase

Gram

High molecular mass

High pressure liquid chromatography

Hour
Symbol/Abbreviation

X-gal

6-APA

7-ACA

ASL

CHDL

CR

CB

CF

CFTR

${ }^{\circ} \mathrm{C}$

DAP

EMSA

et al.

E-test

ESBL

ES-OXA

g

HMM

HPLC

$\mathrm{h}$ 
Intensive care unit $\quad$ ICU

Isopropyl $\beta$-D-1-thiogalactopyranoside IPTG

Klebsiella pneumoniae carbapenemase KPC

$\begin{array}{ll}\text { Lipopolysaccharide LPS } & \text { LP }\end{array}$

Liter $\quad$ L

Low molecular mas $\quad$ LMM

$\begin{array}{ll}\text { Luria-Bertani } & \text { LB }\end{array}$

LysR-type transcriptional regulator $\quad$ LTTR

Metallo- $\beta$-lactamase $\quad$ MLB

Microgram $\quad \mu \mathrm{g}$

Microliter $\mu 1$

Micromolar $\quad \mu \mathrm{M}$

Milligram $\mathrm{mg}$

Milliliter $\mathrm{ml}$

Millimolar $\quad \mathrm{mM}$

Minimum inhibitory concentration $\quad$ MIC

Minutes $\quad \min$

Mucociliary clearance $\quad$ MCC

Multicloning site $\quad$ MCS

Multidrug resistant/resistance $\quad$ MDR

Open reading frame ORF

Optical density $\quad$ OD 
Ortho-nitrophenyl- $\beta$-galactoside

ONPG

Outer membrane

$\mathrm{OM}$

Overnight

$\mathrm{O} / \mathrm{N}$

Penicillin-binding protein

PBP

Percent

$\%$

Periciliary liquid

PCL

Peptidoglycan

PG

pox promoter

$\mathrm{P}_{p o x}$

Resistance-Nodulation-Division

RND

Sodium dodecyl sulfate-polyacrylamide gel electrophoresis

SDS-PAGE

Transposon

$\operatorname{Tn}$

Two-component system

TCS

Urinary tract infection

UTI

Ventilator-associated pneumonia

VAP 
Chapter 1

Introduction 


\subsection{Preface}

Pseudomonas aeruginosa is one of the most dreaded pathogens in the clinical setting. It is one of the leading causes of nosocomial infections and one of the most troublesome and intractable organisms colonizing the lungs of patients with cystic fibrosis (CF), a deadly genetic disease. Although there are antibiotics and treatment strategies to combat such infections, the organism's inherent and acquired resistance renders many such antipseudomonal treatments ineffective. One important mechanism of resistance, the expression of $\beta$-lactamases, is the leading cause of $\beta$-lactam resistance in the clinical setting and is the subject of this dissertation. The following introduction is a review of the relevant literature on this important organism and its mechanisms of resistance, with special attention to $\beta$-lactam antibiotics and the expression of $\beta$ lactamases.

\subsection{Pseudomonas aeruginosa}

P. aeruginosa is an aerobic, non-spore forming, monoflagellated, Gram-negative rod ubiquitously found in the environment (Figure 1.1) (1). It is positive for catalase and oxidase, and produces a fruity or grape-like odor due to the production of 2aminoacetophenone (2). Although it is unable to ferment lactose and its metabolism is mostly respiratory, it can also grow under anaerobic conditions by using nitrate as a terminal electron acceptor or by fermenting arginine $(3,4)$.

Like other pseudomonads, renowned for their nutritional versatility, $P$. aeruginosa has very simple nutritional needs and can use a multitude of organic compounds for growth (3). As a chemoorganotroph, it can metabolize a variety of simple 
and complex organic substrates as a sole source of carbon and energy. It is also a very versatile, adaptable microbe that is able to tolerate a variety of physical conditions. It has an optimum growth temperature of $37^{\circ} \mathrm{C}$, but it is also able to grow at $42^{\circ} \mathrm{C}$, unlike some other species of the same genera (3). Most notably, P. aeruginosa is inherently resistant to many commonly used antibiotics and chemotherapeutic agents $(1,5)$.

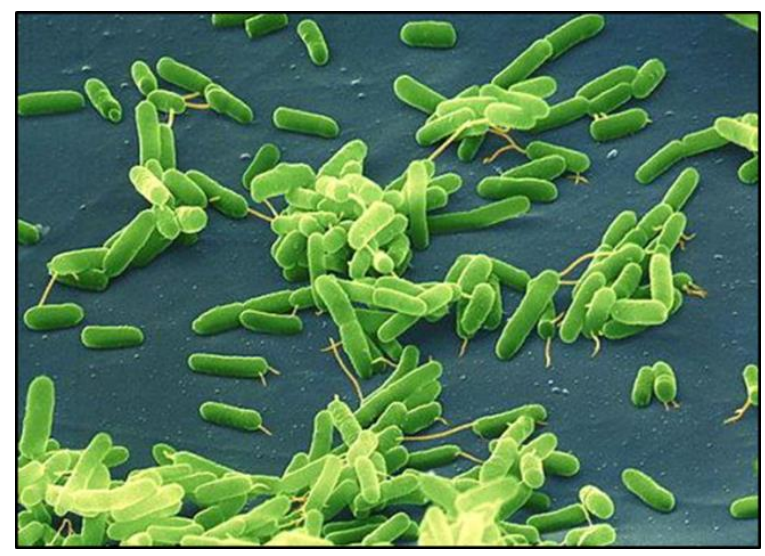

Figure 1.1. Colored scanning electron micrograph of $P$. aeruginosa. (http://www.visualphotos.com/image/1x6040105/coloured_sem_of_pseudomonas_aerugi nosa_bacteria)

Most strains of $P$. aeruginosa produce pyocyanin, a blue-green, water-soluble, non-fluorescent phenazine pigment (3). Phenazine compounds are commonly found in nature and are produced by bacteria such as the Streptomyces and Pseudomonas spp. Pyocyanin (1-hydroxy-5-methyl-phenazine) is derived from chorismate, an intermediate in the biosynthesis of aromatic amino acids in plants and microorganisms (3). Pyocyanin and other phenazine compounds appear to contribute to the virulence and competitive fitness of the producing organisms $(3,6)$. For instance, in $P$. aeruginosa pyocyanin contributes to its ability to colonize the lungs of patients with $\mathrm{CF}$ as it has been shown to 
disrupt the beating of human cilia and inhibit mammalian cell respiration $(7,8)$. Additionally, this pigment inhibits lymphocyte proliferation and epidermal cell growth $(9,10)$. Pyocyanin can also act as an antibiotic agent against other bacterial cells by the generation of reactive oxygen intermediates (6). This antimicrobial activity is oxygendependent and involves the oxidation of NADH to produce superoxide and hydrogen peroxide (6).

Some strains also produce pyoverdin, a water-soluble, fluorescent pigment that gives $P$. aeruginosa a yellowish-green appearance (1). Pyoverdin functions as a siderophore or iron-scavenging compound, and as such it is known to increase the rate of bacterial iron transport. Siderophores, in general, are extracellular compounds that have a high affinity for iron; they are produced under iron-deficient conditions, and have been known to stimulate and increase bacterial growth rate $(1,11)$. Production of siderophores during infection has been implicated with bacterial virulence as a consequence of increased growth rate; it also appears to be a factor that can limit the growth of other bacteria as $P$. aeruginosa can effectively compete for iron with other microorganisms (1, $11)$.

Besides pyocyanin and pyoverdin, $P$. aeruginosa can produce and secrete a myriad of other virulence factors that contribute to its success as a pathogen and allow it to compete against other microorganisms. These include elastase, a zinc protease that destroys immunoglobulins and eukaryotic proteins responsible for maintaining cell structure; exotoxin A, a very toxic protein that inhibits the host cell protein synthesis; exoenzymes $\mathrm{S}$ and $\mathrm{T}$, proteins with cytotoxic activity secreted by the type III secretion 
system; LasA protease, a staphylolytic enzyme that can destroy $\beta$-casein, and rhamnolipids $(1,12)$. Rhamnolipids, in particular, are very powerful virulence factors capable of destroying polymorphonuclear leukocytes, inhibiting phagocytosis by macrophages, and causing disruption of the mucociliary clearance and of epithelial tight junctions leading to paracellular infiltration of $P$. aeruginosa (13-16). Rhamnolipids have also been shown to have antibacterial activity against a number of microbes including

Staphylococcus aureus, Enterobacter aerogenes, Streptococcus faecalis, Serratia marcescens, Klebsiella pneumoniae and Proteus vulgaris to name a few $(17,18)$.

\subsubsection{Habitat}

P. aeruginosa is a ubiquitous microorganism commonly found in water, soil, and sewage as well as on the surfaces of plants, animals, insects, fruits and vegetables $(1,19)$. Natural bodies of water such as lakes and rivers, are also natural sources of the bacterium, especially near sites of urban drainage. It is sometimes part of the human microbial flora infrequently colonizing the skin especially in areas that are moist, and the intestine after ingestion with contaminated foods or fluids (20-23). Its prevalence and colonization in healthy individuals is often low (2.6-25\%) with intestinal loads being reported at $3 \%(24-26)$.

Its ability to thrive in aqueous environments has made it a problem in the hospital setting where it is often isolated from soaps, ointments, disinfectants, irrigation fluids, and eye drops (27). Its very simple nutritional needs allow it to grow even on the negligible impurities contained in aqueous solutions found in hospitals. Specifically, a study found $P$. aeruginosa was able to not only grow but thrive in the distilled water of 
several mist therapy units (28). Tap water is also a major source of nosocomial infections when it is inadvertently used for the preparation of solutions to be used in that setting (29-33). The prevalence of $P$. aeruginosa in tap water likely results from the colonization of showerheads, faucets, and sinks from which it has also been detected (32, 34-38). Additionally, $P$. aeruginosa has also been isolated from holy water, aerators, baby baths, hot tubs, swimming pools, contact lens solutions, cosmetics and even the innersole of sneakers (39-46). In short, aqueous and moist environments seem to be the natural and preferred reservoirs for $P$. aeruginosa and such environments, when contaminated, can be potential sources of infection.

\subsubsection{P. aeruginosa infections in healthy individuals}

$P$. aeruginosa is rarely a cause for concern in healthy individuals with such infections often resulting from contact with contaminated water or solutions or after sustaining some form of external trauma like a puncture wound (19). Folliculitis, for example, is an infection of the hair follicles caused by bacteria such as P. aeruginosa, and can occur after bathing in swimming pools, hot tubs, and whirlpools that are not adequately treated with chlorine $(43,44,47,48)$. Individuals involved in aquatic sports as well as those swimming and bathing in contaminated waters can also develop superficial infections of the ear canal known as external otitis (49-52).

Minor injury to the eye or cornea, often related to the use of contacts lens, especially extended wear lenses, can predispose an individual to eye infections, or keratitis, with $P$. aeruginosa (53-57). Contact lens solutions that are contaminated, or even tap water used for the handling of contact lens, can all serve as potential sources of 
infection (19). Osteomyelitis, or infection of the bone, has also been reported, especially in children after incurring puncture wounds in the feet, with the source of the infection often being the sole or inner pad of the sneaker that was worn at the time of the injury $(46,58)$. Osteomyelitis can also occur in intravenous drug users $(59,60)$.

One of the most severe $P$. aeruginosa infections that can affect an otherwise healthy person is endocarditis, or inflammation of the inner lining of the heart, often requiring replacement of the affected valve (19). The majority of $P$. aeruginosa endocarditis occurs in intravenous drug users, as the drugs are often mixed with contaminated water leading to bacteremia and endocarditis (61-63). P. aeruginosa-related endocarditis, however, can also occur in burn and open heart surgery patients (64-66).

\subsubsection{P. aeruginosa in nosocomial or healthcare-associated infections}

$P$. aeruginosa is one of the leading causes of hospital-acquired infections accounting for as much as $10-13 \%$ of all nosocomial infections with incidences as high as $22 \%$ reported in intensive care units (ICUs) (67-71). P. aeruginosa was the sixth most frequently isolated pathogen accounting for $7.1 \%$ of all healthcare-associated infections in a recent survey of 183 US hospitals from 10 different states (72). Similarly, the latest hospital reporting to the CDC ranks $P$. aeruginosa as the fifth most commonly reported pathogen in nosocomial infections accounting for $7.5 \%$ of all healthcare-associated infections (73).

Pneumonia accounts for the majority of nosocomial diseases caused by $P$. aeruginosa (72, 73). P. aeruginosa can easily colonize endotracheal tubes and mechanical ventilators and as such has been reported as one of the leading causes of 
ventilator-associated pneumonia (VAP), second only to $S$. aureus (73-75). VAP has been associated with high mortality rates that exceed those of other types of pneumonia such as community-acquired, healthcare-associated or hospital-acquired pneumonia $(75,76)$. Mortality rates have been reported as high as or higher than $70 \%$ when $P$. aeruginosa or Acinetobacter spp. are the causative agents $(74,76-78)$. About $11 \%$ of nosocomial urinary tract infections (UTIs) are caused by $P$. aeruginosa $(72,73)$. A higher incidence of infection is associated with the use of urinary catheters, $P$. aeruginosa being the second most commonly identified pathogen in catheter-associated UTIs $(73,79)$.

$P$. aeruginosa is also a leading cause of nosocomial infections in burn units primarily colonizing burn wounds but also responsible for pneumonia, bacteremia and UTIs in that setting (80-84). On admission, generally S. aureus and coagulase-negative staphylococci predominate the wounds of burn patients but the incidence of $P$. aeruginosa quickly increases during the first week of admission and continues to rise with time, often surpassing the incidence of other microorganisms $(82,85,86)$. Burn wound infections with $P$. aeruginosa are especially worrisome since they are correlated with bacteremia, a high rate of sepsis and mortality $(81,82,87,88)$. Other nosocomial infections caused by $P$. aeruginosa include endocarditis, meningitis, bacteremia, gastrointestinal and surgical site infections $(72,73,89)$.

\subsubsection{P. aeruginosa in immunocompromised patients}

P. aeruginosa is an opportunistic human pathogen that readily exploits any deficiency in the host immune system to mount an infection. Since it is often intractable and resistant to a wide range of antibiotics, it represents a very serious problem not only 
for critically ill individuals, such as those in the hospital setting and ICUs, but also for immunocompromised patients. P. aeruginosa has often been isolated as one of the most common pathogens causing septicemia in patients with primary immunodeficiencies $(90$, 91). A number of other conditions can predispose the host to $P$. aeruginosa bacteremia including taking broad spectrum antibiotics, receiving chemotherapy, as well as being an HIV, leukemia, cancer, diabetes, bone marrow or organ transplant patient (92-99).

Although $P$. aeruginosa bacteremia has been reported in patients with AIDS (100, 101), a number of studies agree that pathogens other than $P$. aeruginosa are the most common cause of bacteremia in that population $(95,102)$. P. aeruginosa however, is one of the leading causes of pneumonia in HIV patients (103-106) and a common respiratory pathogen in patients with AIDS, where pulmonary and respiratory disease infections are often chronic and intractable (107-111).

Before 1968, P. aeruginosa bacteremia in cancer patients resulted in $\sim 80-90 \%$ fatalities (112-115). The development of more potent antipseudomonal drugs has greatly improved outcomes and survival rates in these patients, provided that the bacteremia is treated rapidly, with studies from the 1980s and 2000s showing a 60 and $80 \%$ cure rate, respectively $(92,98,116,117)$. Although prognosis has improved, the incidence of $P$. aeruginosa infections in cancer patients for the decade of the ' 90 s was found to range from 5-12\% (118), while in a report from 2008, carbapenem-resistant $P$. aeruginosa infections were documented in $20 \%$ of the cancer patients (119). A recent study found that $P$. aeruginosa was the leading causative agent of bloodstream infections $(42 \%)$ and 
pneumonia in patients with solid tumors, with $22 \%$ incidence of bloodstream infections in patients with haematological malignancies (120).

\subsubsection{P. aeruginosa and cystic fibrosis}

P. aeruginosa is the most common and clinically relevant pathogen found in patients with CF (121-123). It is estimated that over $80 \%$ of CF patients will be infected with this bacterium by the time they reach adulthood (122). Infection of the airways with P. aeruginosa, and the inflammation that follows, represent a major problem for $\mathrm{CF}$ patients as the lungs steadily deteriorate auguring very poor overall prognosis and high mortality rates (124-126). As of 2012, the life expectancy for a patient with CF in the United States was around 37.8 years (122).

CF is an autosomal, recessive genetic disease affecting about 30,000 people in the US and about 70,000 worldwide (122). This disease mainly affects Caucasian populations of European descent and is caused by mutations in the cystic fibrosis transmembrane conductance regulator (CFTR) gene located in the long arm of chromosome VII (127-129). The CFTR protein is a cAMP-regulated chloride channel found on the apical surface of various epithelial cells and responsible for the transport of chloride ions into and out of the cell (130-136). Additionally, the CFTR protein is known to regulate other membrane conductance channels including the outwardly rectifying chloride channel (137-139) and the epithelial sodium channel, ENaC (140-143).

More than 1800 CFTR mutations have been reported so far, the majority being rare and not yet well understood $(122,144)$. Most mutations can be grouped into one of six classes according to the level of disruption or impairment of CFTR function (Figure 
1.2). Classes I, II and III are associated with a more severe form of the disease including pancreatic insufficiency and defective pulmonary function (145-147). Patients with class IV, V and VI mutations, on the other hand, generally exhibit a milder presentation and have a functional pancreas $(146,148,149)$. The most common mutation, a class II mutation present in $86.7 \%$ of the $\mathrm{CF}$ patients worldwide, is a deletion in phenylalanine at position $508(\Delta \mathrm{F} 508)$ resulting in misfolding of the protein and subsequent degradation by proteasomes $(122,147)$.

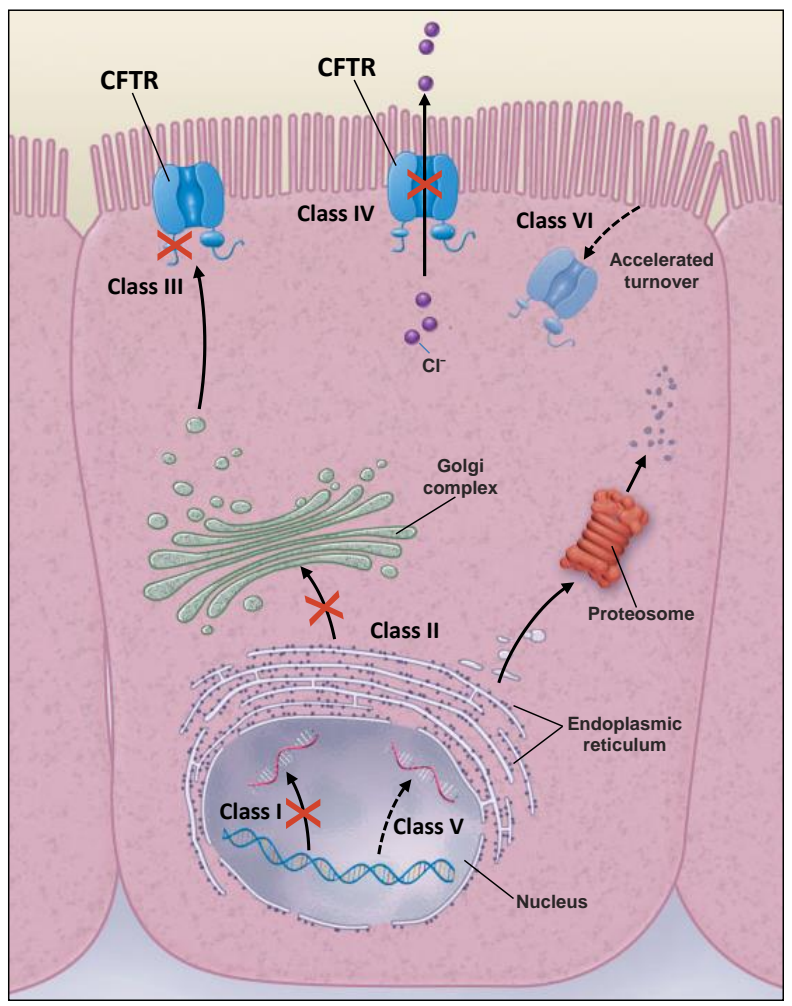

Figure 1.2. Classes of CFTR mutations. CFTR mutations can result in no CFTR protein being expressed (class I), misfolded protein that is degraded by proteasome (class II), a CFTR protein that does not respond to cAMP (class III), defective chloride conductance or channel gating (class IV), a reduced number of CFTR transcripts due to a promoter or splicing abnormality (class V) and accelerated turnover from the cell surface (class VI) (148, 150-153). Reproduced with permission from (153), Copyright Massachusetts Medical Society. 
There is an ongoing debate as to how CFTR mutations cause lung disease in patients with $\mathrm{CF}$ but most proposed models converge on one point: the imbalance of water and ions created by a malfunctioning CFTR protein in the apical surfaces of airway epithelium ultimately leads to abnormal or defective mucociliary clearance (MCC) (154159). MCC is an important defense mechanism against inhaled particles, allergens, bacteria and viruses (160-162). Normal airway epithelium is ciliated and covered with a layer of liquid known as the airway surface liquid (ASL) (163-165). The ASL itself is divided into a fluid layer of low viscosity known as the periciliary liquid (PCL) or sol layer that keeps the cilia hydrated and facilitates its beating, and a more viscous upper gel or mucus layer that is responsible for trapping foreign particles (Figure 1.3A) (164-167). The beating of the cilia continuously propels the mucus layer with its trapped particles upwards towards the nasopharynx and the mouth where foreign substances can be then swallowed or expectorated $(161,166,168,169)$.

The most strongly supported and generally accepted hypothesis of pathobiology in $\mathrm{CF}$ is that abnormal transport of chloride ions across epithelial membranes coupled with the increased absorption of sodium and water through the epithelial sodium channel, $\mathrm{ENaC}$, leads to dehydration of the ASL layer (Figure 1.3B) (170-174). This hypothesis, known as the low-volume hypothesis, states that the depletion of the airway surface liquid reduces the volume or height of the PCL layer and increases the viscosity of the mucus layer leading to compression and impaction of the cilia (170, 174-176). Impaction of the cilia is thought to compromise its proper functioning and to diminish its capacity to work in concert with the mucus layer to remove foreign particles $(154,156)$. The end 
result is impairment of the MCC apparatus setting up the stage for the persistent and chronic bacterial infections that are a hallmark of airway disease in CF patients (Figure 1.4).

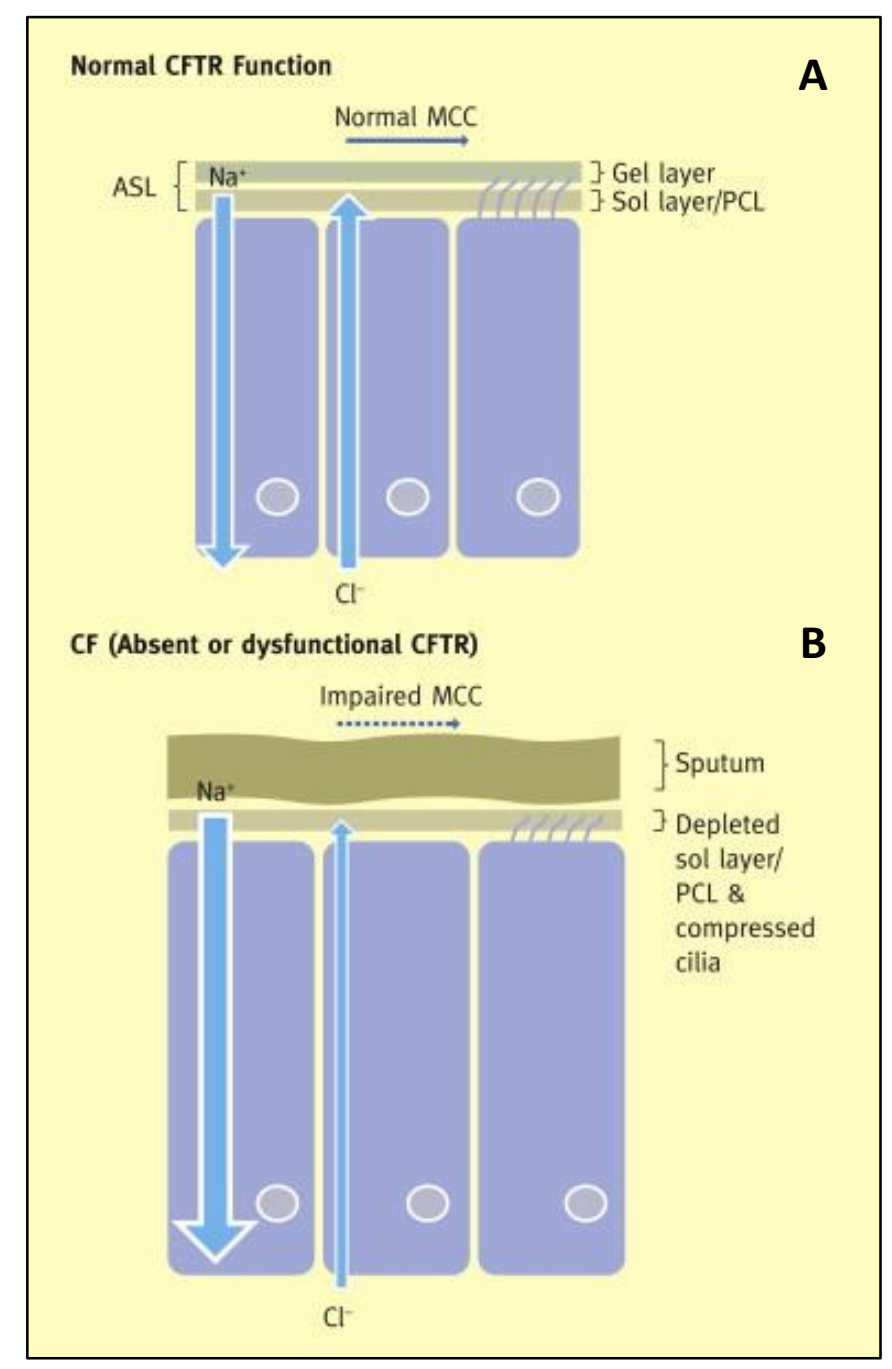

Figure 1.3. ASL layer and MCC in the normal and CF airways. A. Normal CFTR function. Under normal conditions, the PCL layer is hydrated and the cilia beat normally allowing MCC from the mucus layer. B. Abnormal or nonfunctioning CFTR leads to dehydration and reduction in volume of the PCL layer resulting in impacted and improperly beating cilia that hinders MCC (177). Reproduced from (177) with permission from Elsevier. 


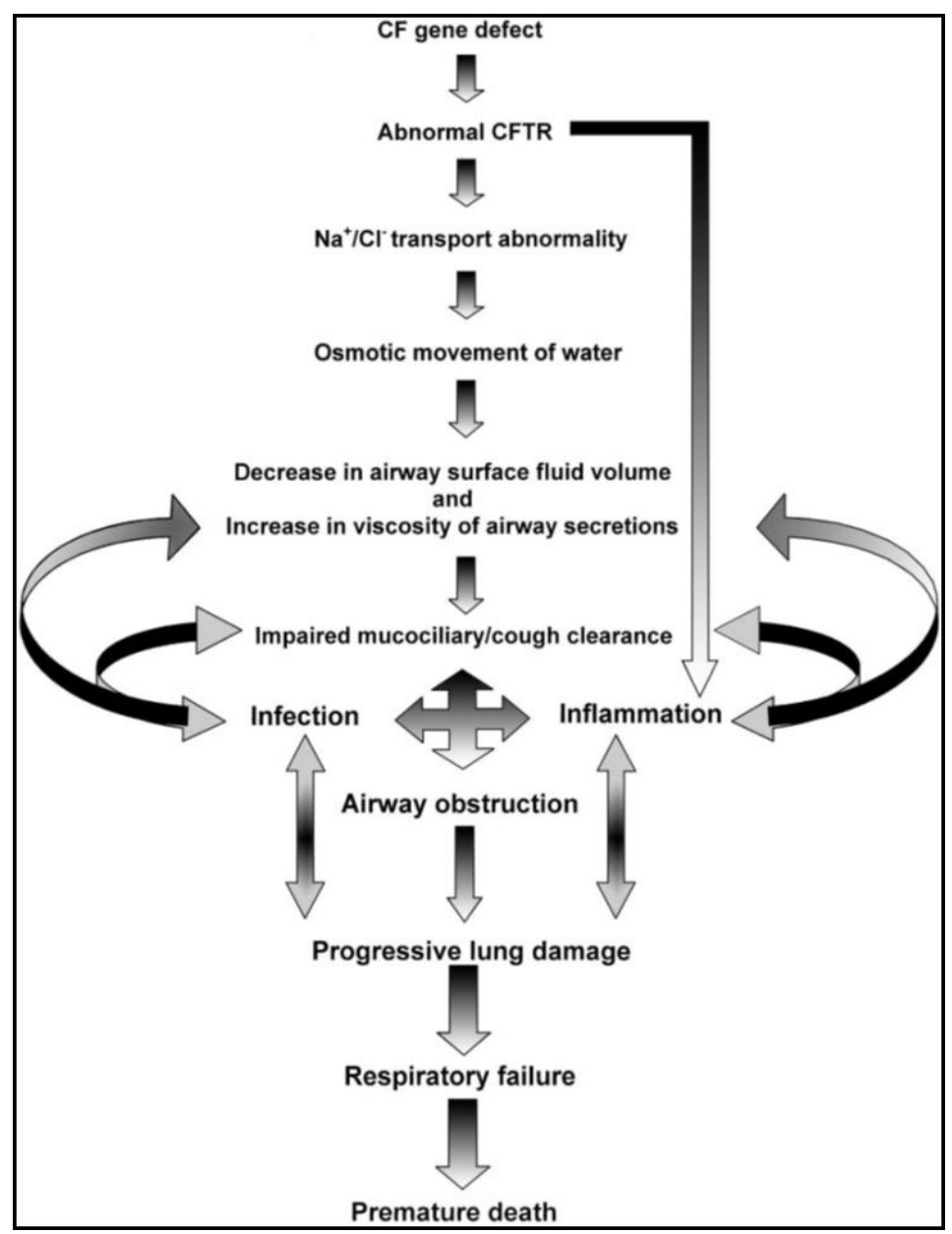

Figure 1.4. Pathobiology of CF lung disease. In the most widely accepted hypothesis of pathobiology of CF lung disease, reduced airway surface liquid results in impairment of MCC leading to successive cycles of infection and inflammation eventually resulting in obstruction of the airways, chronic deterioration of the lungs and death. Reproduced from (154) with permission from John Wiley and Sons.

$\mathrm{CF}$, however, is a multi-organ disease not just limited to the lungs, as the CFTR protein is expressed in the epithelium of several other organs including the pancreas, sweat ducts, intestine, biliary tree and the vas deferens (178-184). For example, in the intestine it can lead to meconium illium, a condition where the first stool of the newborn 
is impacted in the intestine $(182,185)$. Additionally, more than $95 \%$ of men with CF are usually infertile (186-188). Lung disease, however, remains one of the main concerns for patients with $\mathrm{CF}$ as continuous cycles of infection and inflammation herald gradual lung deterioration and eventual pulmonary failure (Figure 1.4).

The conditions present in CF airways, namely dehydrated, thick mucus coupled with impaired mucociliary clearance, provide the ideal environment that is conducive to colonization by a number of pathogens $(123,189)$. S. aureus, for example, often the first to colonize the respiratory tract of CF patients, is common in children less than 10 years old, and responsible for infant morbidity and mortality in the preantibiotic era (Figure 1.5) (122, 123). Haemophilus influenzae is another common pathogen and is predominantly found in young children $(123,189)$. Other less common pathogens such as Stenotrophomonas maltophilia (previously Xanthomonas maltophilia), Alcaligenes xylosoxidans and Burkholderia cepacia complex, have also been isolated from the respiratory tract of CF patients, the latter having the poorest prognosis, although found in less than $10 \%$ of patients (122). A recent study examined the microbiome of sputum from CF patients and found that although the patients were not being treated for fungal infections, many (18/19) did carry pathogenic fugal species of Aspergillus, Candida, Cryptococcus and Exophiala, among others (190). Fungal infections are in fact not uncommon in CF patients and can occur in association with other microorganisms (190193). P. aeruginosa, however, remains the main pathogen associated with morbidity and mortality in CF patients and is more frequently found in adults (Figure 1.5) (122, 124, 126, 194). 


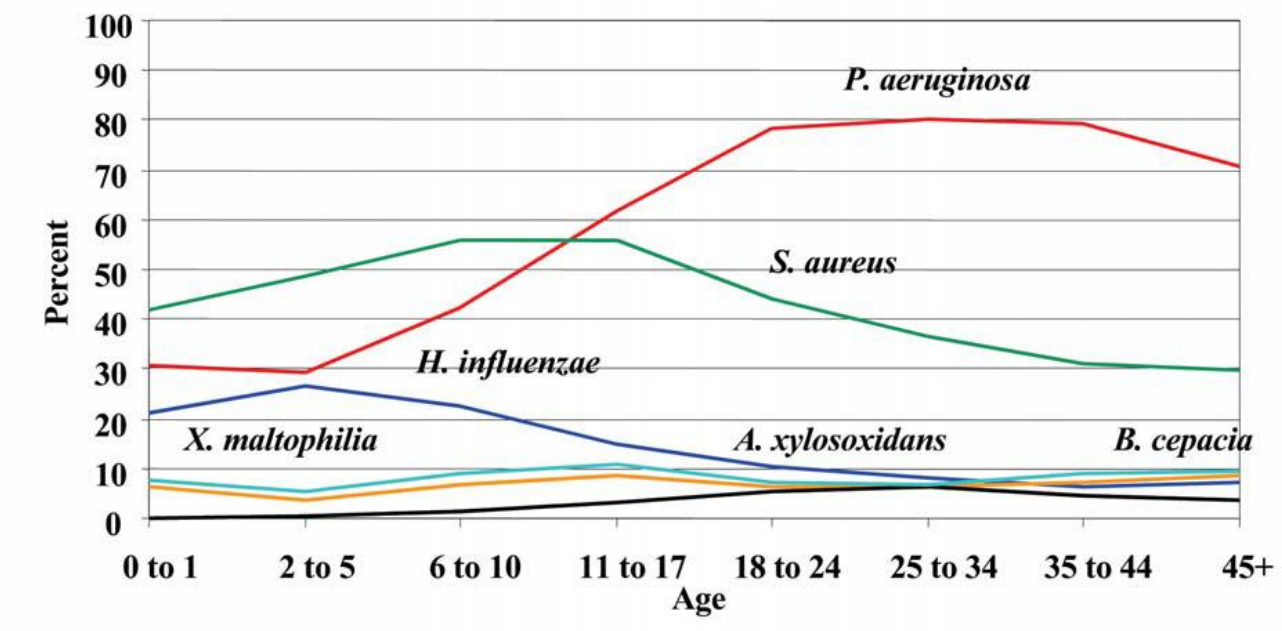

$\begin{array}{cccc}\text { Overall } & - \text { P. aeruginosa } 58.7 \% & - \text { S. aureus } 48.0 \% & - \text { H. influenzae } 15.9 \% \\ & \text { X. maltophilia } 8.4 \% & - \text { B. cepacia } 3.1 \% & - \text { Aylosoxidans } 4.4 \%\end{array}$

Figure 1.5. Pathogens found on the $\mathrm{CF}$ lungs by age. From an early age, $\mathrm{CF}$ patients are infected with a number of different organisms. By the time they reach adulthood, $P$. aeruginosa is the predominant pathogen (122).

An important feature of $P$. aeruginosa infections, is the tendency of this bacterium to convert to a mucoid phenotype in the lungs of $\mathrm{CF}$ patients, an event which signals the beginning of chronic stage of the infection leading to a progressive decline in lung function (195-197). This mucoid phenotype is characterized by the production of large quantities of alginate, a polysaccharide also known as mucoid exopolysaccharide, which plays a very important role in helping the bacterium evade the host immune system $(123,198,199)$. Alginate promotes a biofilm mode of growth, which in turn not only protects the bacteria from antimicrobial agents, but also helps the microorganisms escape phagocytosis by macrophages (200). Alginate overproduction exacerbates the already detrimental conditions of the CF lungs leading to further blocking of the airways and inexorable death. 


\subsubsection{Treatment}

Treatment of $P$. aeruginosa infections varies greatly given severity of infection and resistance profile of the organism. Current strategies include treatment with a single drug, usually a $\beta$-lactam or an aminoglycoside, or combination therapy with at least two different antimicrobial agents $(189,201,202)$. $\beta$-lactams, include a broad class of bactericidal agents, widely used in the clinical setting, and with minimal side effects to eukaryotes (Figure 1.6) (203-205). Several $\beta$-lactams have shown reliable results against $P$. aeruginosa, including the extended-spectrum penicillins ticarcillin and piperacillin that can be combined with the $\beta$-lactamase inhibitors clavulanic acid and tazobactam, respectively, to enhance the antibacterial action of the $\beta$-lactam $(201,202)$. The cephalosporin ceftazidime is stable to most $\beta$-lactamases and is the drug of choice if the isolate is determined to be susceptible $(205,206)$.

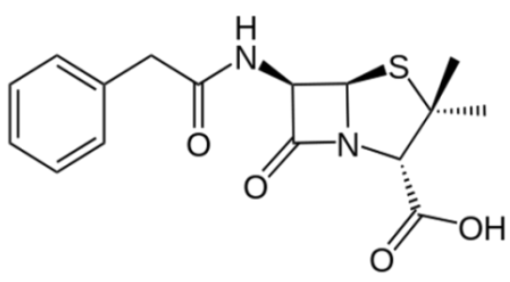

Penicillin G

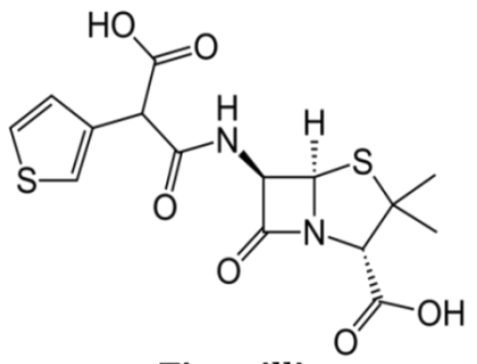

Ticarcillin

Figure 1.6. Molecular structures of two $\beta$-lactams of the penicillin class.

The carbapenem $\beta$-lactams can be used if the organism is resistant to extendedspectrum penicillins and cephalosporins $(207,208)$. Carbapenems have a wide spectrum of activity but can select for multidrug resistant (MDR) strains of $P$. aeruginosa, especially imipenem. The monobactam aztreonam, is the drug of choice if the patient is 
allergic to penicillins and other $\beta$-lactams $(209,210)$. It can be helpful in treating isolates that produce metallo- $\beta$-lactamases as it is resistant to hydrolysis by class B enzymes.

Combination therapy is usually the first line of defense, particularly for MDR pseudomonal infections $(189,201)$. The most commonly used combination includes the administration of a $\beta$-lactam with an aminoglycoside. The aminoglycosides are powerful antimicrobial agents that inhibit bacterial protein synthesis being predominantly active against aerobic, Gram-negative bacteria $(211,212)$. The discovery of gentamicin in 1963 was a breakthrough in the treatment against $P$. aeruginosa infections, and subsequently other more powerful aminoglycosides, such as tobramycin and amikacin, were developed (213). Tobramycin has shown greater activity in vitro than most other aminoglycosides, and thus it is often the drug of choice to treat $P$. aeruginosa infections (211). Amikacin, however, is very effective against bacteria that are resistant to other aminoglycosides such as gentamicin and tobramycin. Its chemical structure makes it less susceptible to the inactivation by the enzymes that normally inactivate gentamicin and tobramycin (Figure 1.7) (211).

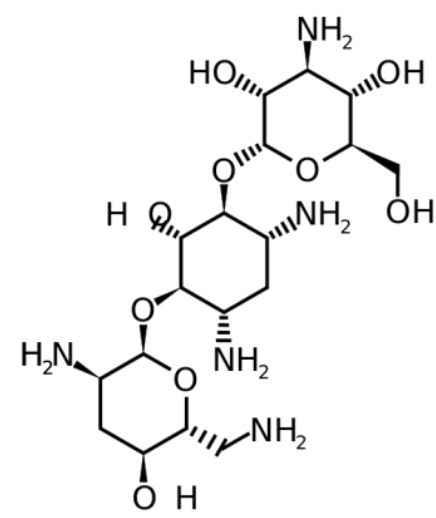

Tobramycin<smiles>NCC[C@H](O)C(=O)NC1C[C@H](N)[C@@H](O[C@@H]2O[C@H](CN)[C@@H](O)[C@H](O)[C@H]2O)[C@H](O)[C@H]1O[C@@H]1O[C@H](CO)[C@@H](O)[C@H](N)[C@H]1O</smiles>

Amikacin

Figure 1.7. Molecular structures of amikacin and tobramycin. 
Aminoglycosides, however, can often have nephrotoxic and ototoxic side effects (214). Nephrotoxicity is usually reversible but ototoxicity, or the toxic effect on the inner ear, is generally irreversible and can lead to hearing loss (215). Thus, aminoglycosides should only be administered when no other antibiotic is deemed as effective, for instance in the treatment of MDR organisms and of critically ill patients, and should be replaced with less toxic agents as soon as the sensitivity profile of the organism is ascertained (211). Combination therapy with aminoglycosides often includes amikacin or tobramycin with either piperacillin, ticarcillin, ceftazidime, imipenem, meropenem or aztreonam $(189,201)$.

Another option when considering therapy for $P$. aeruginosa infections, is the combination of either an aminoglycoside or a $\beta$-lactam with ciprofloxacin $(216,217)$. Ciprofloxacin belongs to a class of antibiotics known as fluoroquinolones (218). Fluoroquinolones have a broad-spectrum of activity and act by preventing DNA replication through the inhibition of the enzyme DNA gyrase (218). Ciprofloxacin has great activity against $P$. aeruginosa and Gram-negatives and unlike the rest of above antibiotics, the majority of which are for parenteral use or aerosolized, it is available for oral administration $(219,220)$. It has been particularly good at treating respiratory infections in CF patients but prolonged monotherapy can lead to resistance $(219,221$ 224). Additionally, serious side effects, such as damage of young cartilage, tendonitis and tendon rupture, hypoglycemia and arrhythmias, have been reported $(225,226)$. It is often used in conjunction with inhaled antibiotics like the aminoglycoside tobramycin or the polymyxin colistin $(123,227)$. 
Colistin was one of the first drugs to show good in vitro activity against $P$. aeruginosa (228-230). It works by disrupting the structure of the outer membrane and increasing cell permeability leading to cell lysis (231). Earlier studies however, reported serious nephrotoxic and neurotoxic effects and thus its use was relegated to the external treatment of eye and skin infections $(232,233)$. The emergence of MDR strains, unresponsive to broad spectrum antibiotics but remaining susceptible to the drug, has brought colistin back into the antimicrobial limelight $(234,235)$. Despite its earlier reported toxicity, recent studies show its usefulness outweighs potential risks, which have also been shown to be lower than originally reported, perhaps as a result of better dosing strategies $(236,237)$. Although reported nephrotoxicity rates for intravenous use are up to $14 \%$, in many cases a lack of better alternatives has redefined colistin as the last line of treatment or salvage therapy $(238,239)$. For the treatment of respiratory infections, in both $\mathrm{CF}$ and non-CF patients, colistin can be administered as an aerosol, which has the advantage of a more direct higher dose delivery to the site of infection while limiting toxicity (240-242). Colistin has also been shown to be effective against the inner structure (core and stalk) of the $P$. aeruginosa biofilm suggesting this antimicrobial peptide can penetrate this complex structure (243).

\subsection{Mechanisms of intrinsic antibiotic resistance}

P. aeruginosa can employ a number of mechanisms to evade, prevent or inhibit the actions of antibiotics, the most common of which include restricted membrane permeability, active efflux of antibiotics, alteration of drug targets, the formation of 
biofilm, and the expression of $\beta$-lactamases (Figure 1.8) (244-247). The following sections will review some of these important mechanisms.

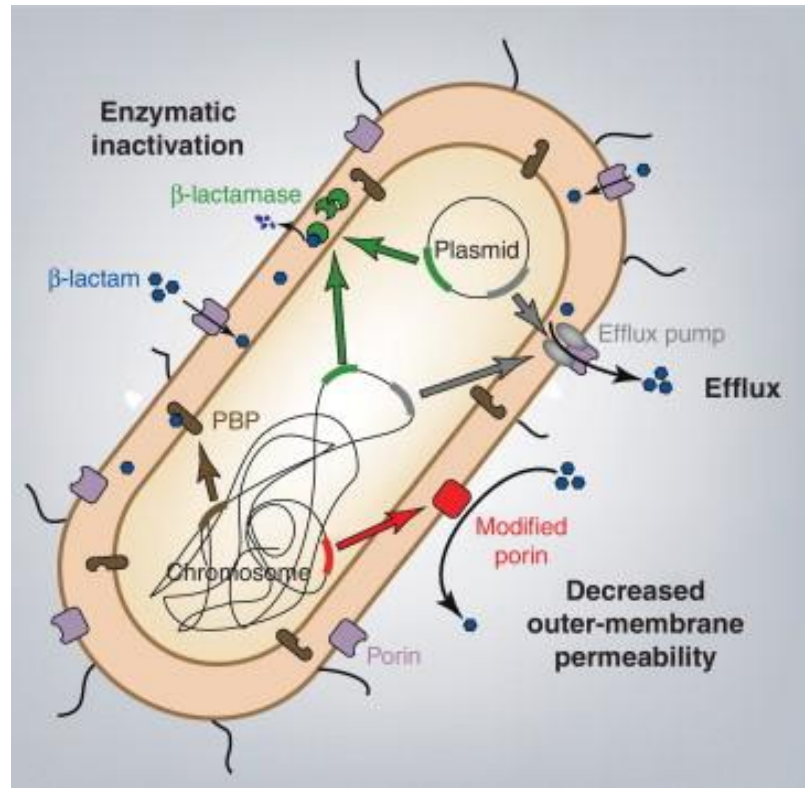

Figure 1.8. Mechanisms of antibiotic resistance in $P$. aeruginosa. $P$. aeruginosa employs multiple mechanisms to inhibit the action of antibiotics including an intrinsically low membrane permeability, expression of efflux pumps, modification of antimicrobial targets and production of $\beta$-lactam-hydrolyzing enzymes. Reproduced from (248) with permission from Elsevier.

\subsubsection{Extrapermeability barrier}

The cell wall of Gram-negative bacteria is composed of an inner membrane, a thin peptidoglycan layer and outer membrane (OM) that serves as a selective permeation barrier by restricting the passage of solutes across the membrane (Figure 1.9) $(249,250)$. The OM is generally an asymmetric lipid bilayer with an inner leaflet of phospholipids and a surface layer rich in lipopolysaccharide (LPS) $(251,252)$. 


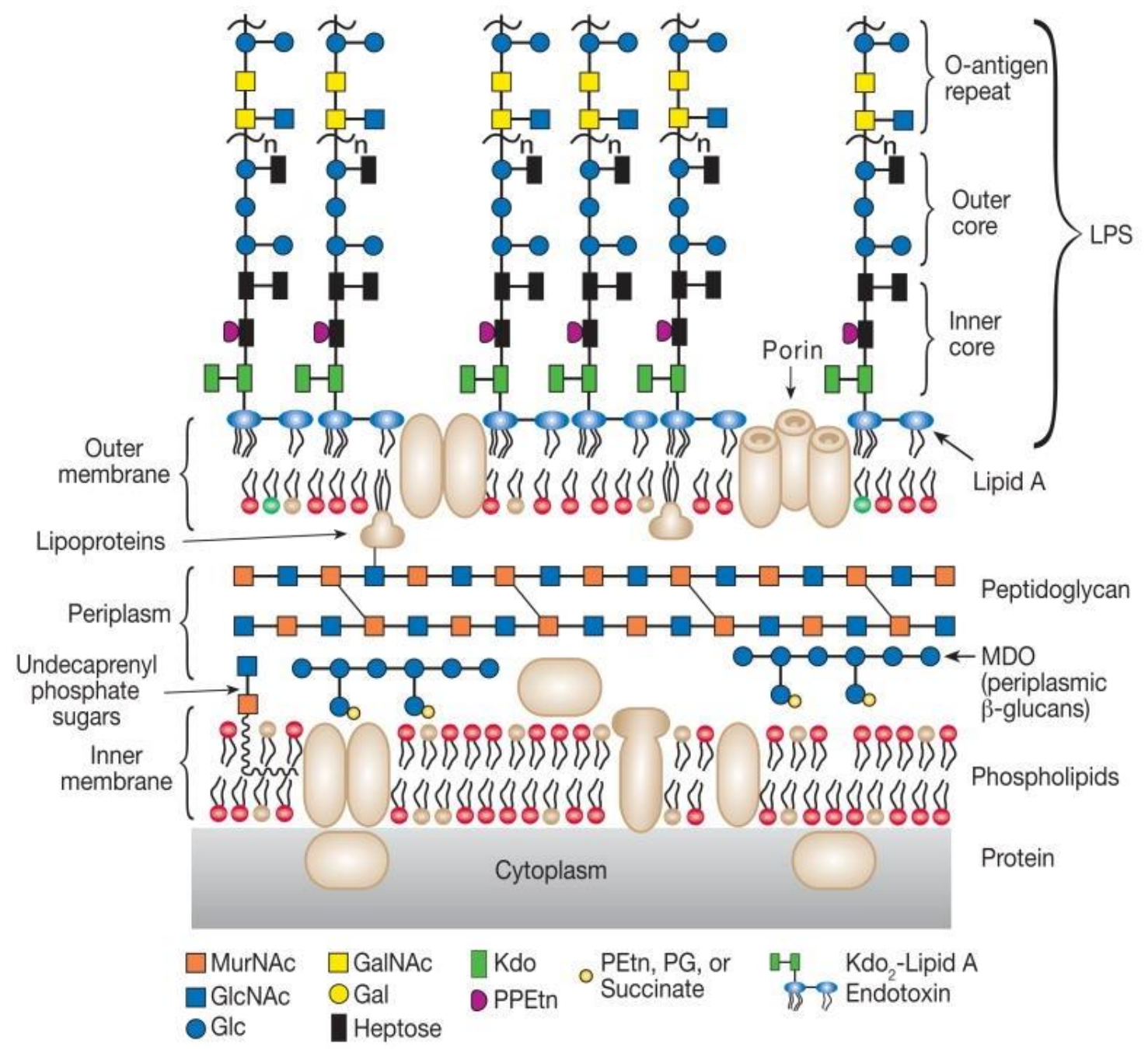

Figure 1.9. Cell wall of Gram-negative bacteria. An inner phospholipid bilayer containing mostly phosphatidylethanolamine (red lipids) with some phosphatidylglycerol (light brown lipids) surrounds the cytoplasm of the bacterial cell. The periplasm, or the space sandwiched between the inner and outer membranes, contains a thin peptidoglycan layer; membrane-derived oligosaccharides (MDO) can also be present. The outer layer of the OM is rich in LPS. Kdo, 3-deoxy-D-manno-octulosonic acid; (n) variable number of O-antigen repeats; (PPEtn), pyrophosphoethanolamine (253).

(https://amit1b.wordpress.com/the-molecules-of-life/10-the-living-cell-gallery/) 
In order to reach their targets inside the cell, antibiotics must first cross the outer membrane. Permeability of the OM is largely governed by the low fluidity structure of the LPS layer and by the presence of channels or pores in the membrane known as porins (250, 254). Generally, small, hydrophilic compounds, like $\beta$-lactams, are taken up by porins, while lipid-mediated pathways are thought to be responsible for the uptake of hydrophobic antibiotics like aminoglycosides and polymyxin (255-257).

\subsubsection{LPS as a barrier and lipid-mediated uptake}

LPS is one of the first barriers that solutes encounter when traversing the cell. It is usually comprised of lipid $\mathrm{A}$, the core sugars and the $\mathrm{O}$ antigen, and it is a very strongly negatively charged structure $(258,259)$. Divalent cations such as calcium $\left(\mathrm{Ca}^{2+}\right)$ and magnesium $\left(\mathrm{Mg}^{2+}\right)$ have a high affinity for LPS and help to neutralize some of these negative charges while at the same time bridging neighboring LPS molecules (260-265). Bridging of adjacent LPS molecules through divalent cations, as well as through hydrogen bonding, promotes strong lateral interactions, stabilizes the structure and creates a gel-like core of low fluidity in the lipid interior restricting the passage of solutes and antibiotics across the membrane (266-268).

The low fluidity of the LPS structure is thought to restrict partitioning by hydrophobic compounds and antibiotics $(255,269)$. Hydrophobic probes have been shown to be excluded from the membranes of P. aeruginosa and Escherichia coli (270, 271). Similarly, P. aeruginosa and other Gram-negative bacteria intrinsically display high MICs (minimum inhibitory concentration) against hydrophobic antibiotics such as macrolides, novobicin, clindamycin and fusidic acid. (255, 272). Defects in efflux, 
however, have been shown to reduce the MICs of hydrophobic antibiotics. The intrinsic resistance to hydrophobic antibiotics thus, is determined not only by the normal structure of LPS but also by the ability of some of these compounds to be effluxed. LPS is generally considered effective at slowing down the passage of hydrophobic antibiotics and permeation rates through the outer membrane have been found to be 50-100 times slower than through a simple lipid bilayer (273).

The structure of LPS, however, can be compromised by the presence of polycationic compounds (274-276). Polycations, having sometimes a higher affinity for LPS than divalent cations, can readily displace them from their regular binding sites in the LPS, leading to disruptions in the membrane barrier and increasing permeability of the outer membrane $(271,275)$. Specifically, the bulkiness of the polycations is thought to create transient cracks in the structure of the LPS, thus allowing uptake of other compounds such as hydrophobic antibiotics and of the polycations themselves in a process known as self-promoted uptake. Examples of antibiotics that promote their own entry into the cell by disrupting the normal structure of LPS via their cationic nature include aminoglycosides, polymyxins, cationic detergents and polycationic peptides, among others (256, 276-281). Permeabilization of the outer membrane with such compounds can additionally sensitize the cells to permeation by other compounds and antibiotics including lysozyme, $\beta$-lactams, and a number of hydrophobic antibiotics (erythromycin, clindamycin, novobiocin, fusidic acid and cloxacillin) (274, 282-284). Membrane permeabilizers, such as the metal chelator EDTA, have a similar effect and 
can enhance the uptake of a number of compounds and antibiotics that are not themselves permeabilizers (285).

\subsubsection{Porin-mediated transport}

A major determinant of permeability is the presence of water-filled channels in the outer membrane called porins. OprF is the major general porin in P. aeruginosa (286, 287). It is a non-specific channel for the passage of large substrates and has an exclusion limit of about 3000 daltons in contrast to 600 daltons for $\mathrm{OmpF}$, the general porin in $E$. coli (287-289). Notwithstanding the large pore size and its abundance in the outer membrane, OprF is considered a slow porin because of its slow rate of diffusion and low permeability $(289,290)$. A study showed that the rate of arabinose influx was 50 times slower through the P. aeruginosa OprF than through the E. coli OmpF channel (291). In general, the rate of solute diffusion across the outer membrane of $P$. aeruginosa has been at least two orders of magnitude lower than that of E. coli $(287,292)$.

The slow rate diffusion has been attributed to the conformation of the OprF channel in $P$. aeruginosa. Various studies suggest that OprF can exist in two different conformations, open or closed, and that only a minority of the channels are ever open, thus accounting for the decreased permeability as compared to E. coli (291, 293-295). While having the majority of channels closed may seem arbitrary, the periplasmic Cterminus of the closed conformer is predicted to interact with the peptidoglycan layer as a way to stabilize the structure of the cell envelope (296-299). Thus, the closed conformer of OprF appears to play a major structural role in $P$. aeruginosa besides that of permeability, its absence leading to abnormal cell morphology and destabilization of the 
outer membrane $(300,301)$. However, even with only about $5 \%$ of the channels open at any one time, OprF is still a major determinant of permeability in P. aeruginosa (293, 302).

Another porin affecting permeability and $\beta$-lactam resistance is the substratespecific porin OprD. OprD is specific for basic amino acids, dipeptides and carbapenems (imipenem, meropenem, doripenem) $(20,21,23,34)$. In the clinical setting, exposure to imipenem often leads to mutations, deletions or insertions in $\operatorname{oprD}$ and loss of the protein resulting in impermeability and clinical resistance to imipenem (29-33, 38). Although loss of OprD also increases the MIC against meropenem, it does not by itself usually lead to clinical resistance (303-305), presumably because meropenem can also get inside the cells via other routes (306-308). Additionally, both meropenem and doripenem are subject to efflux by the MexAB system while imipenem is not (309-312). Thus, clinical resistance to imipenem can happen with just the loss of OprD (313-315), while both absence of OprD and upregulation of the MexAB pump are needed for meropenem and doripenem resistance to occur $(304,309-311,316)$.

As previously mentioned, OprD also mediates the uptake of basic amino acids and peptides that structurally resemble carbapenems (Figure 1.10) (21, 23). The positively charged residues at $\mathrm{C} 2$ of imipenem closely resemble a number of basic amino acids such as histidine, lysine, arginine and ornithine that not surprisingly have been shown inhibit imipenem transport through OprD (317). Small peptides containing lysine have also been shown to inhibit transport, while addition of basic amino acids to different media increased the resistance of different $P$. aeruginosa strains against carbapenems 
$(22,23,36)$. Such finding could be of concern in the clinical setting as the empirically demonstrated MIC might not reflect the real MIC in the blood or serum where the presence of basic amino acids may reduce the efficacy of carbapenem treatment.

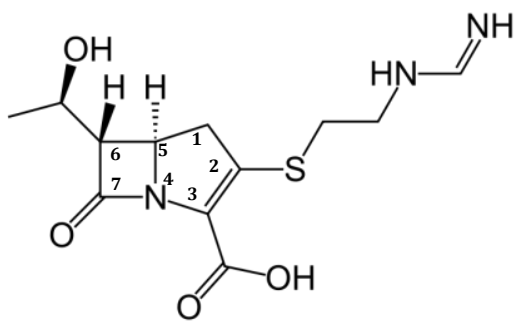

Imipenem

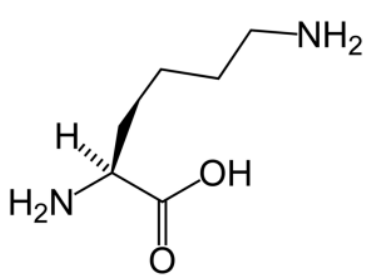

Lysine<smiles>N=C(N)NCCC[C@H](N)C(=O)O</smiles>

Arginine

Figure 1.10. Molecular structures of the $\beta$-lactam imipenem and of the amino acids Llysine and L-arginine.

Many other specific and gated porins, for uptake of a myriad of substrates including iron and carbohydrates, exist in the outer membrane of $P$. aeruginosa (318). As they have not been associated with resistance, they will not be further discussed here. The porins involved in efflux are examined below.

\subsubsection{Efflux pumps}

Efflux pumps are ubiquitous in nature and have been recognized in both prokaryotic and eukaryotic cells (319). They are membrane-bound pumps that transport a variety of solutes such as dyes, antibiotics and toxic compounds to the outside of the cell by using the energy of ATP hydrolysis or the energy derived from transmembrane electrochemical gradients, such as the proton motive force $(320,321)$. Efflux pumps can be substrate specific or they can transport a variety of structurally different compounds, 
including different classes of antibiotics (319). Pumps that are able to export a wide range of antibiotics are often associated with multidrug resistance (319). Effluxdetermined resistance can be caused by an increase in expression of efflux proteins, often as a result of mutations in efflux regulatory genes, or by mutations that enhance the export capabilities of the pump itself (320).

Five different classes of efflux pumps have been described in bacteria: the major facilitator superfamily (MFS), the small multidrug resistance (SMR) family, the multidrug and toxic compound extrusion (MATE) family, the ATP-binding cassette (ABC) family, and the resistance nodulation-division (RND) family (Figure 1.11). RND efflux pumps are of particular interest in Gram-negative bacteria where they play a crucial role in intrinsic and acquired resistance in synergy with the outer membrane barrier $(246,322,323)$. They are responsible for the export of a variety of substrates such as toxic substances, dyes, detergents, antibiotics, as well as substances produced by the host such as bile, hormones and antimicrobial peptides (324-328). RND pumps typically have a tripartite arrangement comprised of an inner membrane transporter, a periplasmic adaptor protein and an outer membrane channel that driven by the proton motive force, work in concert to transport solutes across two membranes towards the extracellular space $(329,330)$. Capture of solutes from the periplasm has also been reported in both $E$. coli and $P$. aeruginosa (331-333).

Twelve RND efflux pumps have now been described in P. aeruginosa: MexABOprM (317, 334, 335), MexCD-OprJ (336, 337), MexEF-OprN (338), MexGHI-OpmD (339, 340), MexJK (341), MexMN (342), MexPQ-OpmE (342), MexVW (343), MexXY 
(344, 345), TriABC-OpmH (346), MuxABC-OpmB (347) and CzrAB-OpmN (348).

Together they form a family of multidrug efflux pumps with an extensive substrate range that includes aminoglycosides, fluoroquinolones, $\beta$-lactams, tetracycline, chloramphenicol and macrolides, among others (Table 1.1).

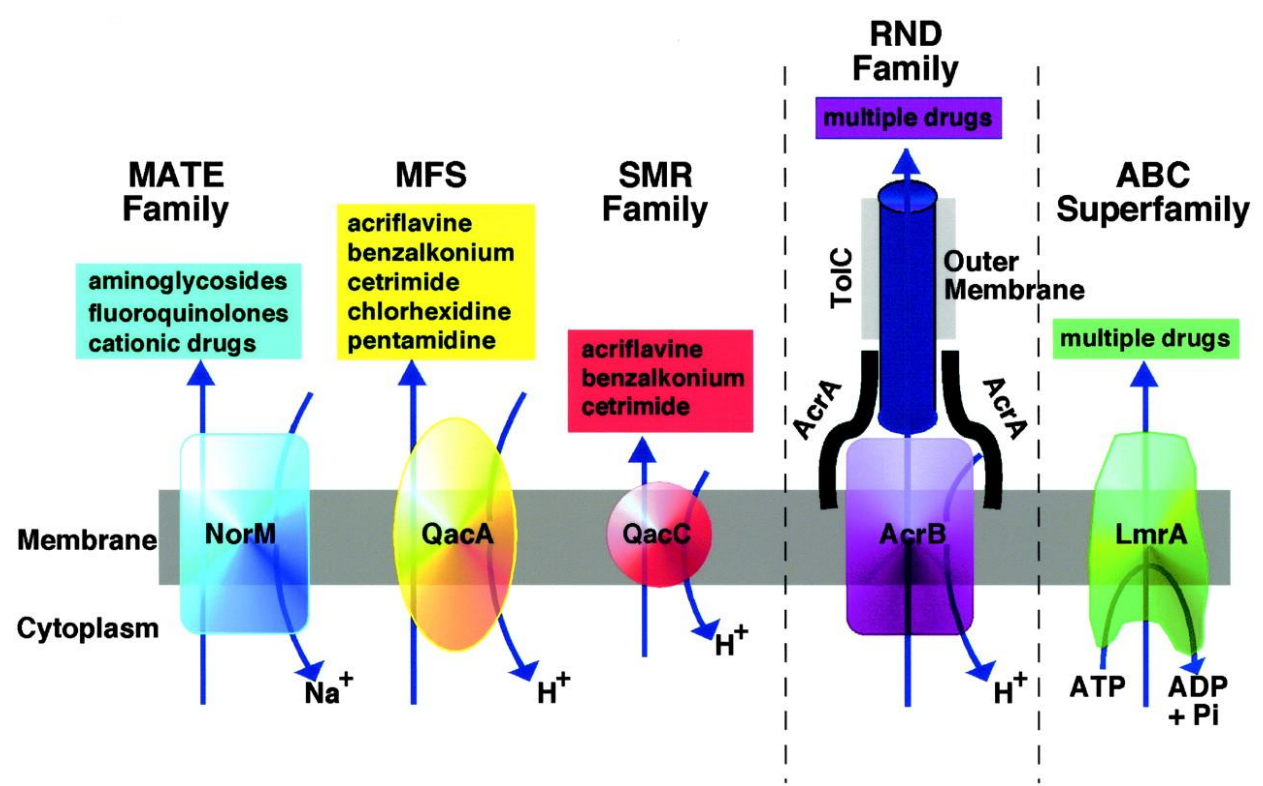

Figure 1.11. Family of efflux pumps found in bacteria and their substrates. Reproduced from (319) with permission by the American Society for Microbiology.

RND efflux-encoding genes are organized into operons frequently in association with an immediately upstream located transcriptional regulatory gene $(320,322,349)$. The first gene of the RND operon (mexA, mex $C$, mexE, and mexX) generally encodes a membrane fusion protein connected to the cytoplasmic membrane and expanding through the periplasm. The second gene $(\operatorname{mex} B, \operatorname{mex} D, \operatorname{mex} F$, and mexY) encodes an inner membrane transporter that moves the substrate across the cytoplasmic membrane into the periplasm. An additional gene may be present in some operons (oprM, oprJ, and oprN). This third gene encodes an OM protein that allows passage of substrates across the outer 
membrane into the extracellular space. Operons that do not code for the outer membrane protein themselves, may instead use the OM protein from another pump. For instance, the

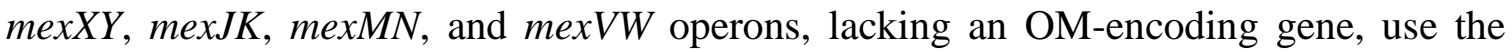
OprM protein of the MexAB system as an outer membrane channel, MexJK can additionally use OpmH (349).

Table 1.1: Substrate profile of RND efflux pumps described in P. aeruginosa $(312,323)$.

\begin{tabular}{|c|c|}
\hline Efflux pump & Substrates \\
\hline MexAB-OprM & $\begin{array}{l}\text { Fluoroquinolones, tetracycline, chloramphenicol, } \beta \text {-lactams } \\
\text { (except imipenem), } \beta \text {-lactamase inhibitors, novobiocin, } \\
\text { macrolides, trimethoprim, tigecycline, triclosan, sulfonamides, } \\
\text { ethidium bromide, SDS, aromatic hydrocarbons, } \\
\text { thiolactomycin, cerulenin, acylated homoserine lactones }\end{array}$ \\
\hline MexCD-OprJ & $\begin{array}{l}\text { Fluoroquinolones, tetracycline, chloramphenicol, } \beta \text {-lactams } \\
\text { (except imipenem), novobiocin, macrolides, trimethoprim, } \\
\text { triclosan, ethidium bromide, SDS, aromatic hydrocarbons, } \\
\text { crystal violet }\end{array}$ \\
\hline MexEF-OprN & $\begin{array}{l}\text { Fluoroquinolones, chloramphenicol, trimethoprim, aromatic } \\
\text { hydrocarbons, triclosan }\end{array}$ \\
\hline MexGHI-OpmD & Norfloxacin, vanadium \\
\hline MexJK & Tetracycline, erythromycin, triclosan \\
\hline MexMN & Chloramphenicol, thiamphenicol \\
\hline MexPQ-OpmE & Fluoroquinolones, tetracycline, chloramphenicol, macrolides \\
\hline MexVW & $\begin{array}{l}\text { Fluoroquinolones, tetracycline, chloramphenicol, } \\
\text { erythromycin, ethidium bromide, acriflavine }\end{array}$ \\
\hline MexXY & $\begin{array}{l}\text { Fluoroquinolones, aminoglycosides, tetracycline, } \\
\text { erythromycin macrolides, chloramphenicol several } \beta \text {-lactams, } \\
\text { not imipenem, }\end{array}$ \\
\hline TriABC-OpmH & Triclosan \\
\hline MuxABC-OpmB & Aztreonam, macrolides, novobiocin, tetracycline \\
\hline CzrAB-OpmN & Cadmium, zinc \\
\hline
\end{tabular}


MexAB-OprM is the major contributor of efflux-mediated intrinsic resistance in P. aeruginosa (350). Although constitutively expressed, the MexAB-OprM pump mediates resistance to varied antimicrobial agents having the widest substrate range of all known RND P. aeruginosa pumps $(317,335)$. Recently, Yang et al. studied the promoter activity of all 12 RND pumps found in P. aeruginosa (351). In agreement with previous reports, mexAB-oprM had the highest activity in all conditions tested. mexJK, mexVW, mexXY and muxABC-opmB were also constitutively expressed, but at much lower levels than mexAB. Still lower, but detectable activity was observed for mexGHI-opmD, mexPQ-opmE and mexMN, with no activity detected for the rest of the pumps.

These promoter expression studies are in agreement with previous reports that show most pumps are either silent or constitutively expressed and that their contribution to innate resistance is null $(322,350)$. In particular, disruptions of mexCD-oprJ and mexEF-oprN were not previously shown to affect susceptibility, suggesting these two pumps do not contribute to intrinsic resistance $(338,352)$. The MexXY system, on the other hand, is thought to contribute to the intrinsic resistance of $P$. aeruginosa against tetracycline, erythromycin, and gentamicin, as deletion of mexXY increases susceptibility against these antibiotics (353). Not surprisingly, these compounds, but not other MexXY substrates, were shown to induce expression of mexXY.

Although most pumps are silent or only weakly expressed, and likely not playing a role in innate resistance, their contribution should not be discounted as it is often mutations in their regulatory genes or regions (known and unknown), that lead to overexpression and multidrug resistance. For instance, MexCD-OprJ is silent in wild-type 
but hyperexpression and variable levels of resistance have been attributed to in vitro and in vivo mutations in its repressor-encoding gene, $n f x B(336,354)$. Such mutations have also been reported in the regulatory genes for mexEF-OprN and mexJK, as well as for the operons already providing intrinsic resistance, mexAB-oprM and mexXY $(322,349)$. Thus collectively, the RND efflux pumps are a major determinant of efflux-mediated resistance in $P$. aeruginosa.

\subsubsection{Alteration and modification of penicillin-binding proteins}

The peptidoglycan layer of Gram-negative bacteria consists of alternating subunits of N-acetylglucosamine (GlcNAc) and N-acetylmuramic acid (MurNAc), where MurNAc bears a stem peptide often consisting of L-alanine, D-glutamic acid, L-lysine or meso-diaminopimelic acid (meso-DAP), and D-alanyl-D-alanine (Figure 1.12) (355-358). Cross-linking of the glycan chains, or transpeptidation, is carried out by essential proteins known as penicillin-binding proteins, PBPs, and generally occurs between the third amino acid (DAP) of a stem peptide and the fourth (D-Ala) of the next peptide chain (Figure 1.12) (359). PBPs additionally catalyze other reactions in the later stages of peptidoglycan synthesis and remodeling including polymerization of the nascent glycan strand (transglycosylation), removal of the last D-alanine of the stem peptide (carboxypeptidation), and hydrolysis of the peptide bond that connects the two strands (endopeptidation) (360).

PBPs are generally classified as high molecular mass (HMM) and low molecular mas (LMM) enzymes and are further subdivided into classes A and B (360-362). A class $\mathrm{C}$ also exists for LMM PBPs. HMM class A PBPs are bifunctional with an N-terminal 
glycosyltransferase domain and a C-terminal transpeptidase domain, while class B enzymes are only transpeptidases (Table 1.2). LMM class A and B PBPs are carboxypeptidases, and class $\mathrm{C}$ can be carboxypeptidases and/or endopeptidases.

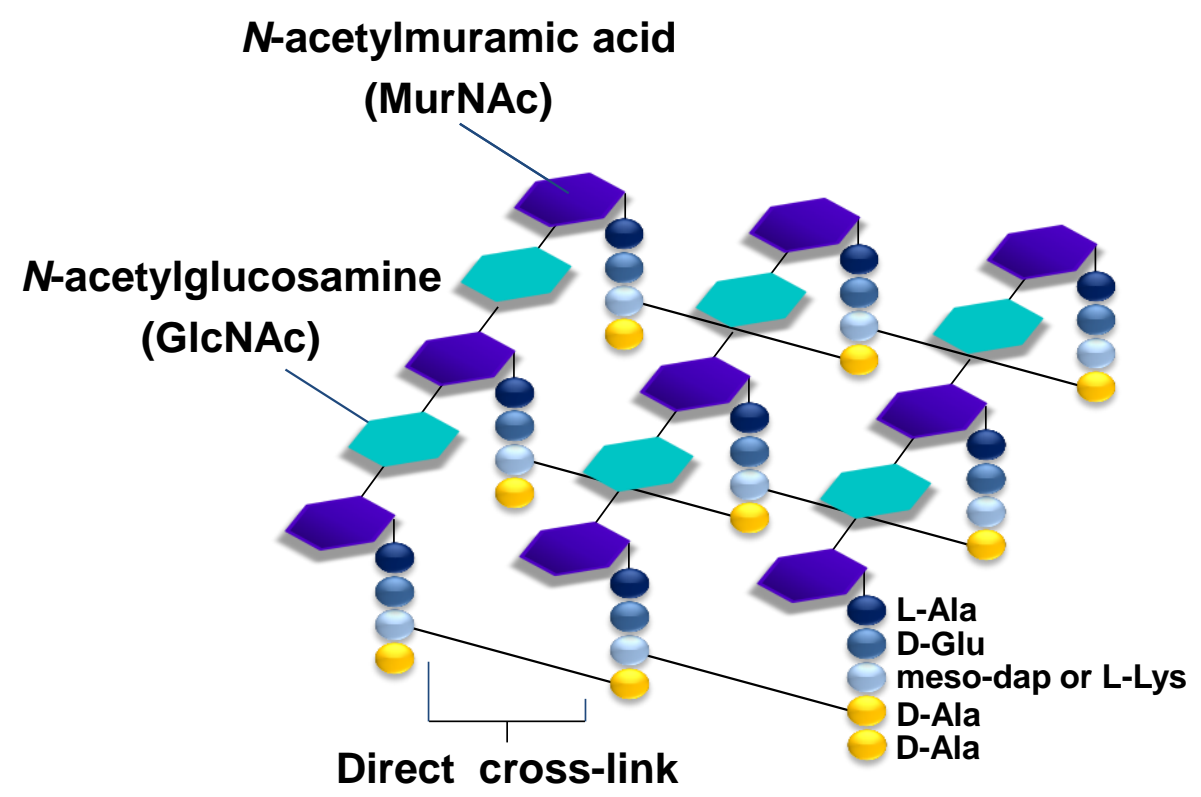

Figure 1.12. Structure of peptidoglycan. In Gram-negative bacteria the peptidoglycan layer is made up of alternating subunits of MurNAc and GlcNAc. A 4-5 amino acid stem peptide is attached to the GlcNAc subunits. Direct cross-linking can occur between the fourth D-Ala of one PG molecule and the third meso-DAP residue of the next.

Table 1.2: Classification of penicillin-binding proteins

\begin{tabular}{|c|c|l|}
\hline $\begin{array}{c}\text { Category } \\
\begin{array}{c}\text { High } \\
\text { Molecular } \\
\text { Mass }\end{array}\end{array}$ & Aubclasses & In vivo function \\
\hline $\begin{array}{c}\text { Low } \\
\text { Molecular } \\
\text { Mass }\end{array}$ & B & glycosyltransferase and transpeptidase \\
\hline transpeptidase \\
\hline B & C & carboxypeptidase \\
\hline
\end{tabular}

Adapted from references (362) and (363). 
HMM PBPs are essential for survival whilst LMM enzymes are not. All PBPs, however, have D-alanyl-D-alanine-peptidase (DD-peptidase) activity in their respective DD-transpeptidase, DD-carboxypeptidase or DD-endopeptidase domains $(360,364)$. Not surprisingly, this DD-peptidase domain is subject to inhibition by $\beta$-lactam antibiotics as $\beta$-lactams resemble the natural substrates of PBPs, namely D-alanyl-D-alanine (Figure 1.13). This is in fact the role of $\beta$-lactam antibiotics, to target and inhibit PBPs.

$\beta$-lactams covalently bind the active site of PBPs and form long-lived acylenzyme complexes effectively acting as suicide inhibitors of the enzymes $(362,365)$. Binding and inhibition of PBPs by $\beta$-lactams prevents the final crosslinking step of the new peptidoglycan layer, thus disrupting cell wall synthesis and leading to cell death. To successfully inhibit the action of PBPs, $\beta$-lactams rely on the specific binding to their PBP target. PBP mutations that reduce the specific binding and lower the affinity with which the $\beta$-lactam and its target bind, can render the drug ineffective and lead to resistance.

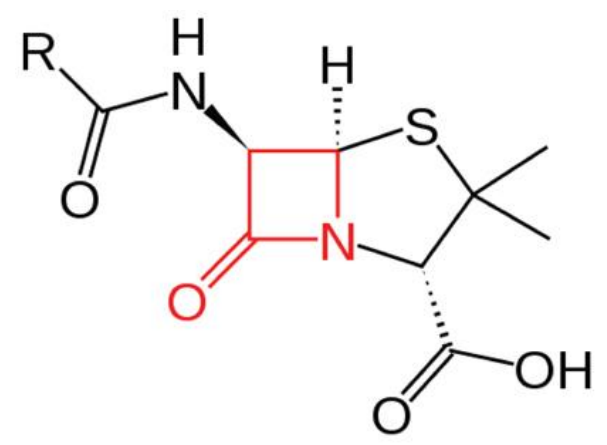

Penicillin

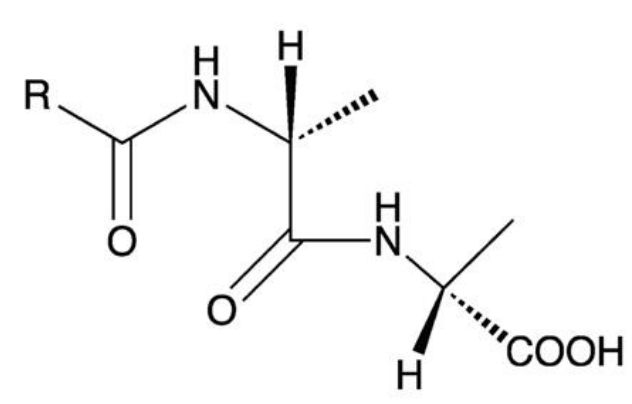

D-Ala-D-Ala

Figure 1.13. Molecular structures of penicillin and D-alanyl-D-alanine (366). 
$\beta$-lactam resistance has been reported in $P$. aeruginosa following treatment of a CF patient with high dosage of tobramycin and piperacillin in association with loss of/or decreased binding to PBP3 (367). Similarly, $\beta$-lactam-resistant strains, selected in vitro following growth on cefsulodin, did not express $\beta$-lactamase but did show reduced affinity of PBP3 towards the two $\beta$-lactams tested, namely cefsulodin and carbenicillin (368). Recently, a new mechanism of PBP-mediated resistance has been elucidated. Specifically, in vitro and in vivo ampC overexpression and $\beta$-lactam resistance were correlated to inactivation of a nonessential PBP4 in an ampD-independent manner (369, 370). It is not known how prevalent this mechanism of resistance is in the clinical setting, but the phenotype was found to be reproducible upon deletion of PBP4.

Although modification of PBPs has also been previously reported in other Gramnegative bacteria, it is not currently considered a major cause of $\beta$-lactam resistance in the clinical setting $(365,371)$. PBP-mediated resistance however, is a prevalent mechanism in Gram-positive bacteria, particularly in Streptococcus pneumoniae, methicillin-resistant S. aureus and the enterococci $(371,372)$.

\subsubsection{Expression of $\beta$-lactamases}

Expression of $\beta$-lactamases is one of the most efficient and common ways of neutralizing the action of the commonly used $\beta$-lactam antibiotics (discussed in section 1.4) $(373,374)$. $\beta$-lactam-hydrolyzing enzymes can be chromosomal- or plasmid-encoded and expressed constitutively or induced upon $\beta$-lactam challenge. Constitutive $\beta$ lactamases are present at basal levels under normal conditions while inducible $\beta$ lactamases are derepressed in the presence of antibiotics but maintained at low levels in 
their absence $(375,376)$. Synthesis of chromosomally encoded $\beta$-lactamases has been found to be inducible in bacterial species having a specific transcriptional regulatory system $(375,377,378)$. In contrast, synthesis of chromosomally encoded $\beta$-lactamases is constitutive in bacterial species lacking such regulatory systems. Derepression of the chromosomally encoded $\beta$-lactamase AmpC is considered the most common mechanism of $\beta$-lactam resistance in $P$. aeruginosa (379-382). The classes, mode of action and genetics of $\beta$-lactamases will be discussed in section 1.5.

\section{$1.4 \quad \beta$-lactams}

$\beta$-lactams are the most widely used class of antibiotics $(204,245,383)$. They contain a signature $\beta$-lactam ring composed of one nitrogen and three carbons that is highly susceptible to hydrolysis by $\beta$-lactamases (Figure 1.14) (206). The major classes of $\beta$-lactam antibiotics include penicillins, cephalosporins, carbapenems, monobactams, and $\beta$-lactamase inhibitors (Figure 1.15).

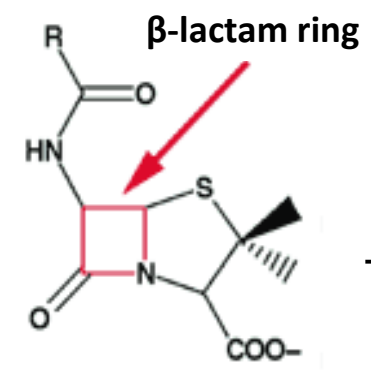

Penicillin

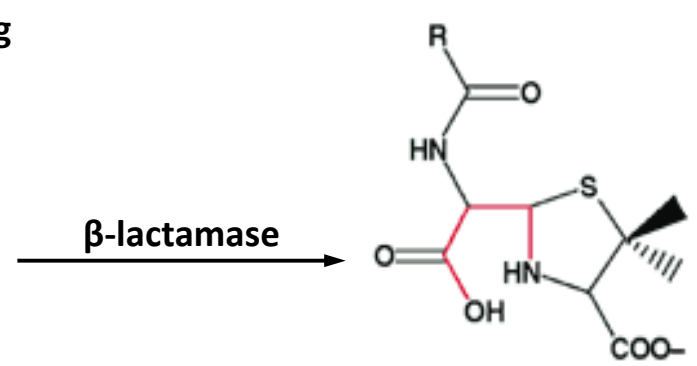

Penicilloic acid

Figure 1.14. Action of $\beta$-lactamases on the $\beta$-lactam ring. $\beta$-lactamases inactivate $\beta$ lactam antibiotics by hydrolyzing the $\mathrm{C}-\mathrm{N}$ bond present in the signature $\beta$-lactam ring. 
$\beta$-lactams
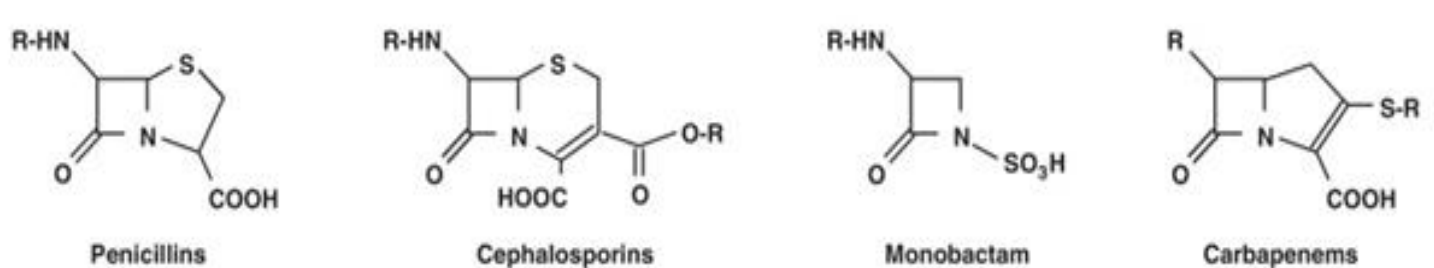

Monobactam

Carbapenems

\section{$\beta$-lactamase inhibitors}

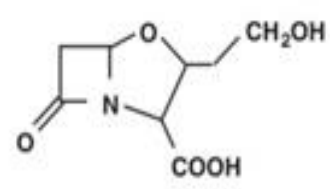

Clavulanic acid

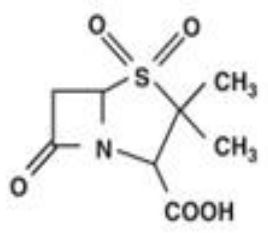

Sulbactam

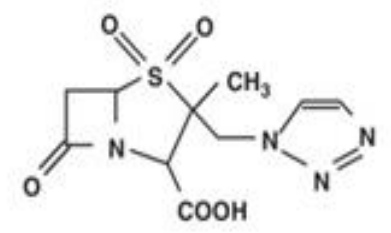

Tazobactam

Figure 1.15. $\beta$-lactam classes. The major classes of $\beta$-lactams, penicillins, cephalosporins, carbapenems, monobactams, and $\beta$-lactamase inhibitors, differ in the ring structure fused to the four-membered signature $\beta$-lactam ring. Derivatives of each class differ in the substituent at the $\mathrm{R}$ groups. Adapted from (248).

Penicillin has the distinction of being the first antibiotic discovered. In 1928 the bacteriologist Alexander Fleming noticed that growth of the mold Penicillium notatum lysed nearby growing Staphylococcus cells (384). Growth of a number of other microorganisms including Pneumococcus, Gonococcus, Streptococcus pyogenes and Streptococcus viridans were also inhibited by what Fleming concluded was an antibacterial agent that was being produced by the fungus. He aptly named this compound penicillin. Subsequent attempts to purify it were relatively unsuccessful, but in 1940 Chain et al. succeeded in purifying penicillin and in using it as a therapeutic agent to treat mice infected with streptococci and staphylococci (385). In 1941, results from the 
first small clinical trial showing low toxicity and high efficacy were published (386). The chemical structure of penicillin was finally elucidated by X-ray crystallography in 1945, although the work was not published until 1949 (387).

A naturally occurring penicillin-like compound, termed cephalosporin C, was later reported by Newton and Abraham (388-390). This compound was isolated from the fungus Cephalosporium, now named Acremonium, and also possessed antibacterial properties but contained a six-membered ring fused to the $\beta$-lactam structure, instead of the five-atom structure normally seen for penicillins (Figure 1.15). The real breakthrough came, however, with the discovery and synthesis of compounds containing only the $\beta$ lactam nucleus without a side chain, namely 6-aminopenicillanic acid (6-APA) and 7aminocephalosporanic acid (7-ACA) (Figure 1.16) (391-393). It was possible to chemically modify these compounds to produce new and improved antibiotics with unusual side chains (394-399). Chemical additions or modifications of the side chains allowed the development of a vast number of semi-synthetic penicillins and cephalosporins with broad and extended-spectrum activity (Tables 1.3 and 1.4) (400403).

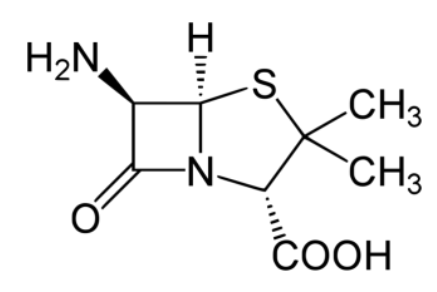

6-aminopenicillanic acid

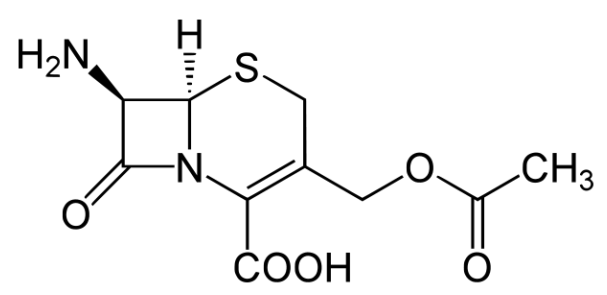

7-aminocephalosporanic acid

Figure 1.16. Molecular structures of 6-aminopenicillanic acid and 7aminocephalosporanic acid. The 6-APA and 7-ACA compounds are useful structures containing only the $\beta$-lactam nucleus without a side chain. The current semi-synthetic penicillins and cephalosporins are derivatives of 6-APA and 7-ACA, where side chains have been added and modified. 
Table 1.3: $\beta$-lactam antibiotics. I. Penicillins

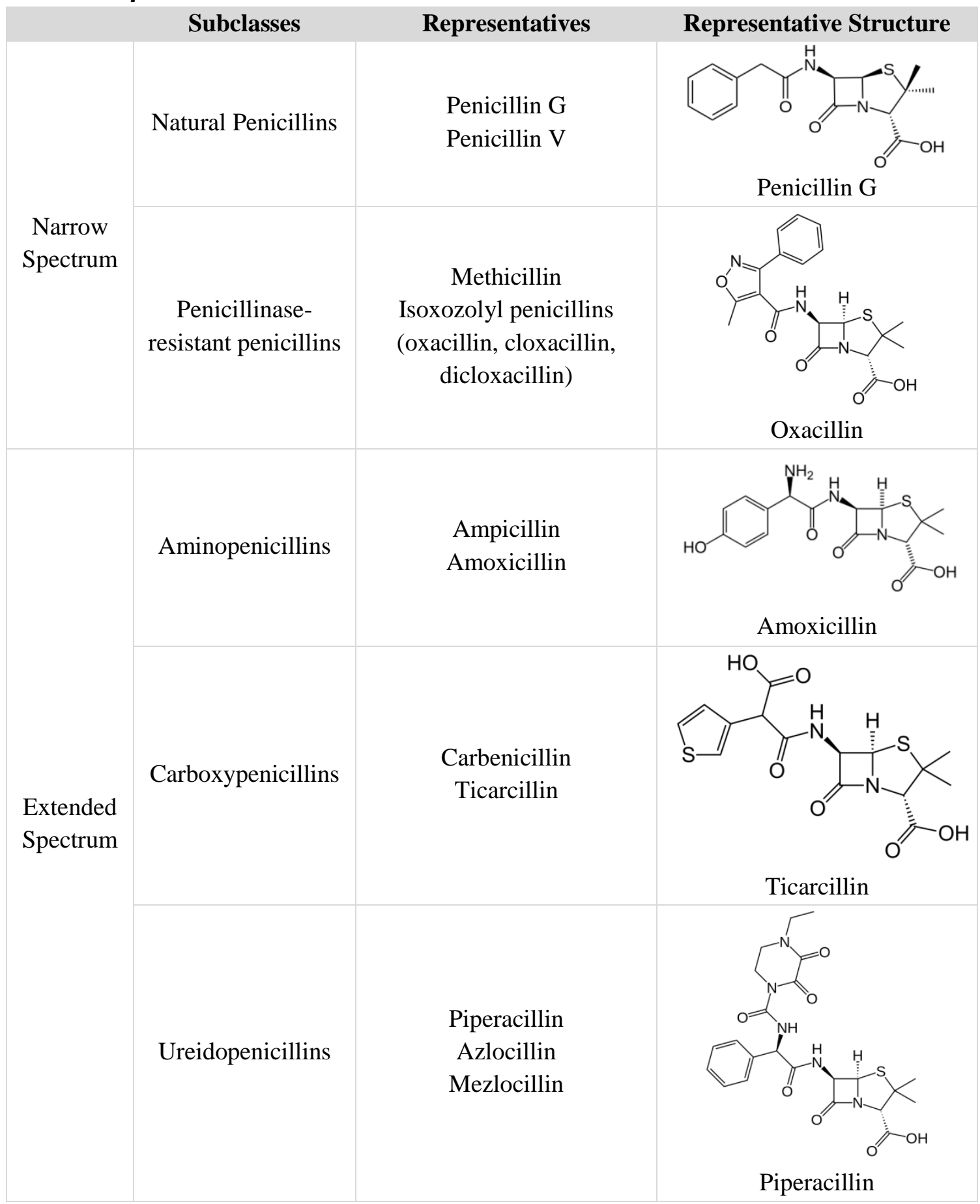


Table 1.4: $\beta$-lactam antibiotics. II. Cephalosporins

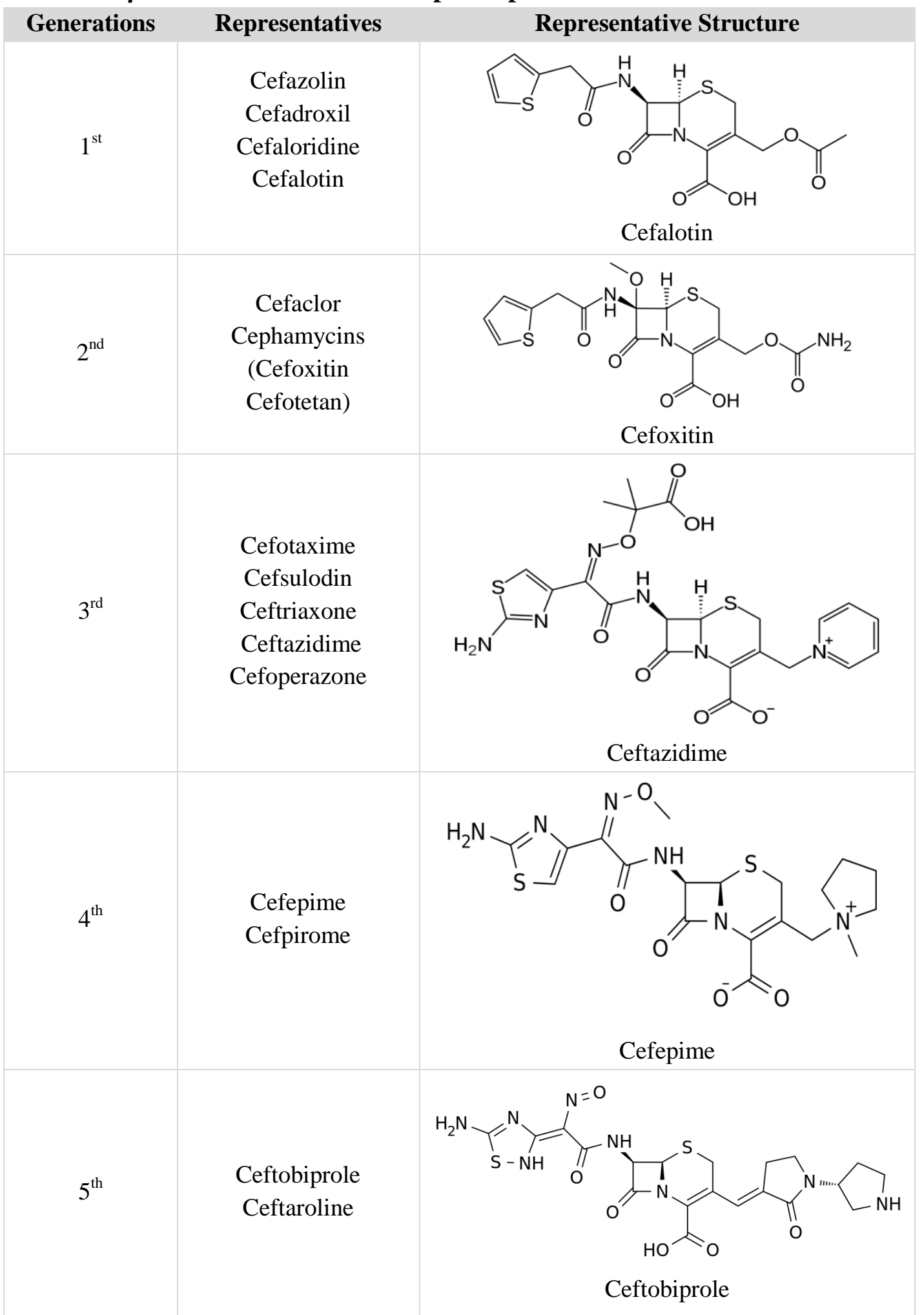


A third class of $\beta$-lactams, termed carbapenems, were first discovered in species of the genus Streptomyces (404). This genus is the source of a plethora of other natural antibacterial compounds including tetracycline, chloramphenicol, aminoglycosides, and cephalosporin C derivatives, among others (405-409). Specifically, thienamycin, the first carbapenem, was discovered in the species Streptomyces cattleya which also produces cephamycin $\mathrm{C}$ and penicillin $\mathrm{N}$ (404). Thienamycin was shown to be a very potent, broad spectrum antimicrobial, generally resistant to degradation by $\beta$-lactamases. However, it was also very unstable. Semisynthetic, stable alternatives of thienamycin have been developed and include those approved for use in the US (imipenem, meropenem, ertapenem, doripenem) (Figure 1.17), and in Japan (panipenem, biapenem, and tebipenem) (207).

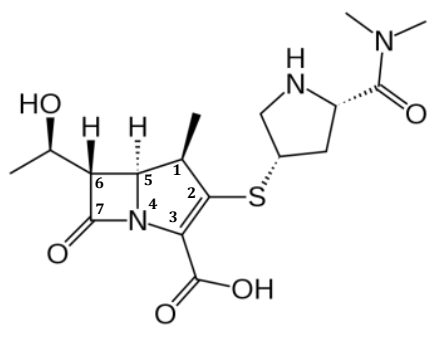

Meropenem

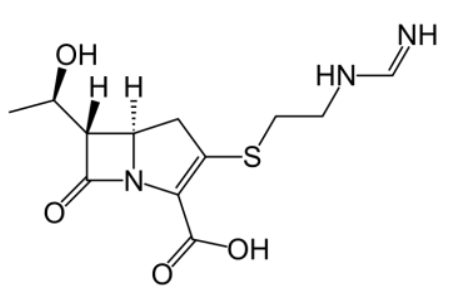

Imipenem

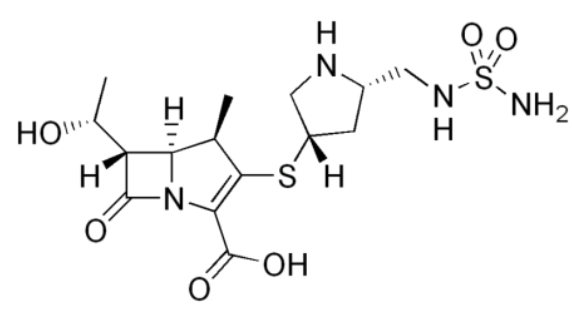

Doripenem

Figure 1.17. Structure of antipseudomonal carbapenems approved for used in the US. Although very similar in structure to penicillins, position 1 of carbapenems has a carbon atom instead of sulfur; the structure is also unsaturated at carbons 2 and 3.

Monobactam antibiotics are also known as monocyclic $\beta$-lactams since they do not possess a ring fused to the $\beta$-lactam nucleus as seen for penicillins, cephalosporins and carbapenems (Figure 1.15). The first monobactam discovered was nocardicin A, produced by the bacterium Nocardia uniformis subsp. tsuyamanensis (410). Several other natural monocyclic $\beta$-lactams have been identified in various other bacterial species 
including Pseudomonas acidophila, Agrobacterium radiobacter, Gluconobacter and Acetobacter (411-414), the simplest of structures being produced by Chromobacterium violaceum (413). Development of semi-synthetic alternatives was undertaken as naturally occurring monobactams were shown to be very poor antimicrobials $(415,416)$. Currently, aztreonam, a synthetic derivative based on the monobactam nucleus, is the only monocyclic $\beta$-lactam approved for clinical use (Figure 1.18) $(209,210)$.

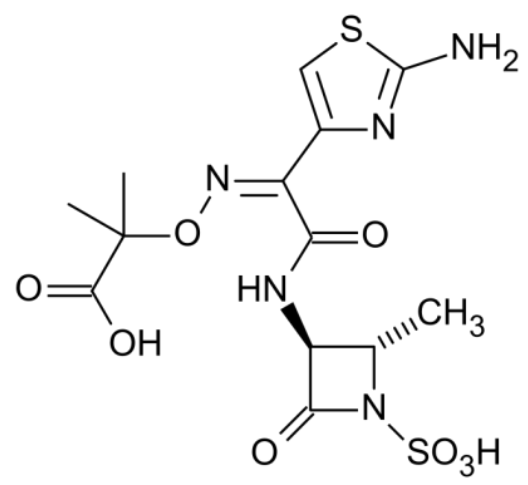

Figure 1.18. Structure of aztreonam, a monocyclic monobactam.

Aztreonam can be used to treat patients with allergies to penicillins and cephalosporins and is considered a safer alternative to aminoglycosides as this $\beta$-lactam does not cause ototoxic or nephrotoxic side effects (210). It is active against aerobic Gram-negative bacteria including $P$. aeruginosa and most of the Enterobacteriaceae, but it does not possess activity against Gram-positives or anaerobic Gram-negatives (417, 418). It is stable to most plasmid- and chromosomal-mediated $\beta$-lactamases of classes A and $\mathrm{D}$, and to all class B enzymes. Aztreonam, however, can be hydrolyzed by the class A Klebsiella pneumoniae carbapenemases (KPCs) $(419,420)$. Additionally, it does not induce expression of the chromosomal cephalosporinases from Citrobacter freundii, 
Enterobacter cloacae, Serratia spp. and P. aeruginosa but can serve as a powerful competitive inhibitor of these enzymes as it is only slowly hydrolyzed by them (421424). Not surprisingly, it has been shown to enhance the antibacterial action of the $\beta$ lactam cefepime by protecting it from degradation by the $P$. aeruginosa cephalosporinase (425).

A final, important category of $\beta$-lactams are the $\beta$-lactamase inhibitors. Three are currently commercially available: clavulanic acid from Streptomyces clavuligerus; and sulbactam and tazobactam, semi-synthetic penicillin derivatives carrying sulfur dioxide at position 1 (Figure 1.15) (426-428). Structurally they are very similar to conventional $\beta$ lactams, having also a five-membered ring fused to the core $\beta$-lactam nucleus. Unlike regular $\beta$-lactams, however, $\beta$-lactamase inhibitors have only weak antibacterial action $(423,428)$. Nonetheless, they are good inhibitors of class A $\beta$-lactamases for which they display high affinity, but generally they are poor inhibitors of the class B, C or D enzymes (429-432).

Clavulanic acid, sulbactam and tazobactam are suicide inhibitors that irreversibly inactivate the $\beta$-lactamases $(423,428)$. Since their main role is inhibitory and not bactericidal, $\beta$-lactamase inhibitors must be co-administered with $\beta$-lactams to achieve the desired antibacterial effect. A good inhibitor thus is one that can competitively bind the enzyme, having a higher affinity for it than that of the co-administered $\beta$-lactam, so as to provide protection to the antibiotic against destruction by the $\beta$-lactamase. The currently approved $\beta$-lactam-inhibitor combinations are amoxicillin-clavulanic acid, ticarcillin-clavulanate, ampicillin-sulbactam and piperacillin-tazobactam (428). Studies 
have shown however, that none of these inhibitors adequately inhibit the chromosomal cephalosporinase of $P$. aeruginosa and are thus deemed ineffective in the treatment of AmpC-expressing isolates (433-435).

\subsubsection{Mechanism of action of $\beta$-lactams}

As described in section 1.3.4, $\beta$-lactams act by inhibiting the synthesis of the peptidoglycan layer, a structure that is important for maintaining cell wall integrity and stability (245). Early studies showed that radio-labeled penicillin covalently binds enzymes that are involved in cell wall synthesis; hence these enzymes were named penicillin-binding proteins (PBPs) (371). PBPs are found in almost all bacteria, but they usually vary amongst species in their affinity for $\beta$-lactam antibiotics, number and size (361).

There are about two to four essential PBPs in any given organism and their inhibition can lead to cell lysis, death or growth arrest $(361,371)$. One of the most important enzymatic activities associated with PBPs is the final transpeptidation step in the synthesis of the peptidoglycan layer. The binding and inhibition of the PBPs by the $\beta$ lactam antibiotics prevents the final crosslinking step of the new peptidoglycan layer, thus disrupting cell wall synthesis $(361,371)$. It has been proposed that the structural and conformational similarities between penicillins and the D-alanyl-D-alanine part of the peptidoglycan (Figure 1.13) facilitates the binding of the $\beta$-lactam antibiotics to the active site of the PBPs. Seven PBPs have been reported in the bacterium P. aeruginosa using the techniques of polyacrylamide gel electrophoresis, but the number could be higher as 
evidenced by the sequencing of the parent strain, PA01, which yielded nine PBPs (361, 436).

\section{$1.5 \quad \beta$-lactamases}

$\beta$-lactamases (EC 3.5.2.6), as defined by the Nomenclature Committee of the International Union of Biochemistry and Molecular Biology, are a diverse group of enzymes that specifically target and hydrolyze the $\mathrm{C}-\mathrm{N}$ bond of the four-atom ring found in $\beta$-lactam antibiotics (Figures 1.14 and 1.15). The first enzyme with penicillinase activity was detected in E. coli by Abraham and Chain in 1940, before the actual introduction of penicillin in the clinical setting (437). The widespread use of penicillin in the 1940s saw the emergence of penicillin-resistant staphylococci and $S$. aureus strains carrying penicillinase (438-441). Staphylococcal penicillinase-mediated resistance became prevalent and of great concern through the 1950s $(439,442-445)$ until the advent of semi-synthetic and more effective $\beta$-lactams in the 1960s led to the emergence and spread of $\beta$-lactamases, especially of the plasmid-encoded types, in Gram-negatives (446-

449). Although $\beta$-lactamases can be found in a number of Gram-positive pathogens, namely the staphylococci and Bacillus spp., these enzymes are more prevalent and widespread in Gram-negative organisms where they remain a major determinant of $\beta$ lactam resistance $(373,374,450,451)$. More than $850 \beta$-lactamases have now been identified. A comprehensive list can be found at the Lahey Clinic (http://www.lahey.org/Studies/). Four main classes, A, B, C and D, are commonly recognized based on amino acid homology $(452,453)$. 


\subsubsection{Mechanism of action}

Class A, C and D $\beta$-lactamases use a serine ester mechanism to disrupt the $\beta$ lactam ring. Specifically, the $\beta$-lactam ring is attacked by the free hydroxyl of the serine at the active site of the $\beta$-lactamase (Figure 1.19) $(454,455)$. The attack opens up the ring producing a covalent acyl enzyme complex. Hydrolysis of the complex regenerates the free, active enzyme and produces a hydrolyzed and inactive $\beta$-lactam. Class B enzymes, on the other hand, usually have one or two zinc ions in the active site, which are absolutely required for enzyme activity, hence they are known as metallo- $\beta$-lactamases (MLBs) $(456,457)$. A zinc ion can recruit and activate a hydroxide to attack the carbonyl carbon of the $\beta$-lactam ring leading to cleavage of the $\mathrm{C}-\mathrm{N}$ bond and sometimes protonation of the nitrogen (456).

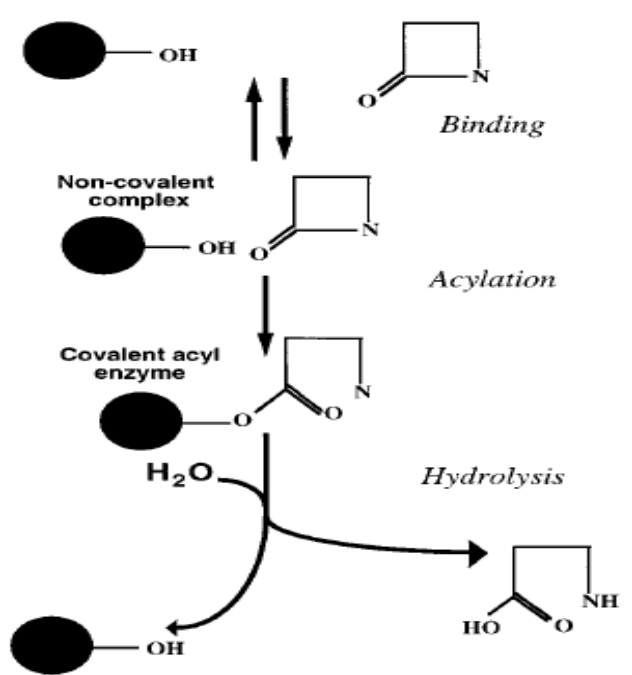

Figure 1.19. Mechanism of action of serine $\beta$-lactamases. The $\beta$-lactamase first associates with the $\beta$-lactam to form a non-covalent complex. A free hydroxyl from the side chain of the active serine residue then attacks the $\beta$-lactam ring forming a covalent acyl ester. The acyl-enzyme complex then undergoes hydrolysis to produce an inactive $\beta$ lactam. Reproduced from (373) with permission by the American Society for Microbiology. 


\subsubsection{Classification}

One of the first classification schemes for $\beta$-lactamases was proposed by Jack and Richmond in 1970 with further updating in 1973 by Richmond and Sykes $(458,459)$. This classification grouped $\beta$-lactamases according to the spectrum of activity, inhibitor susceptibility, and the location of the gene coding for the enzyme (459). A major revision was proposed by Bush in 1989 (460) that was later expanded by Bush and colleagues in 1995 (461). This scheme was based on functional similarities and took into account inhibitor and substrate profiles (461).

The classification proposed by Bush recognized four major groups of $\beta$ lactamases with multiple subgroups $(460,461)$. Group 1 are the cephalosporinases that are poorly inhibited by clavulanic acid (462). Group 2 include the penicillinases, cephalosporinases and broad-spectrum $\beta$-lactamases that are generally inhibited by $\beta$ lactamase inhibitors $(462,463)$. Group 3 comprises the MLBs that can hydrolyze carbapenems, penicillins and cephalosporins (463). Group 4 contains penicillinases not inhibited by clavulanic acid (463).

Phenotypic classifications such as the one proposed by Bush and colleagues can be faulty since point mutations can change the substrate and inhibitor profile for a given enzyme (464). A classification scheme was thus proposed by Ambler based on amino acid similarities rather than phenotypic characteristics (452). The Ambler scheme is widely used today and, as previously mentioned, distinguishes four main classes of $\beta$ lactamases: A, B, C, and D. 


\subsubsection{Molecular class A}

Class A enzymes are among the most common $\beta$-lactamases encountered in the clinical setting and in the Enterobacteriaceae $(373,465)$. They are known as penicillinases since they are mostly active against penicillin and penicillin-derived $\beta$ lactams, but extended-spectrum class A $\beta$-lactamases with activity against cephalosporins and carbapenems have also been reported $(449,461,466)$. Most are well inhibited by the commercially available $\beta$-lactamase inhibitors clavulanic acid, tazobactam, and sulbactam $(461,467)$. The Class A $\beta$-lactamase-encoding genes are widely distributed in bacteria and are generally plasmid-borne but have also been reported in the chromosomes and integrons of Gram-negative bacteria (448, 468-472).

Common representatives include the TEM, SHV, PER and the TEM-, SHV-, and PER-derived $\beta$-lactamases (Table 1.5). TEM-1, the first plasmid-encoded $\beta$-lactamase, was reported in Greece in 1965 (473). It was originally identified in an E. coli isolate from a patient named Temoneira (473). TEM-1 is currently one of the most commonly reported $\beta$-lactamases in Gram-negatives being predominantly found in E. coli and in $K$. pneumoniae but also identified in $H$. influenza and Neisseria gonorrhoeae (373, 446, 474). TEM-2, a less frequently reported point variant of TEM-1, has a more active promoter than its progenitor, a different isoelectric point but the same hydrolytic profile $(475,476)$.

A common group of class A enzymes are the SHV-type $\beta$-lactamases, named after sulfhydryl variable, since it was thought that the inhibition of this enzyme by pchloromercuribenzoate varied according to the substrate used (477). The earliest 
representative of this group, SHV-1 is predominately found in $K$. pneumonia but plasmid dissemination has occurred to other enterobacterial species including E. coli and Proteus mirabilis (477-480). SHV enzymes are very structurally and functionally similar to TEM enzymes, SHV-1 sharing as high as $68 \%$ of its amino acid sequence with TEM-1 (478, 481). TEM and SHV enzymes provide resistance against penicillins such as amoxicillin, ampicillin, tircarcillin and carbenicillin and the narrow-spectrum cephalosporins (478). Most class A extended-spectrum $\beta$-lactamases (ESBLs) are TEM-1, TEM-2- and SHV-1derived enzymes that differ from their precursors in as few as 1-4 amino acids $(466,482$, 483). These point mutations increase the hydrolysis spectrum of the enzymes to include extended-spectrum cephalosporins and monobactams (478).

Table 1.5: $\beta$-lactamase Ambler classification

\section{Molecular class Representatives}

A $\quad$ TEM, SHV, CTX-M, KPC, IMI, SME, GES, PER, VEB

B IMP, VIM, SPM, GIM, SIM, NDM

C AmpC, CMY, MIR, MOX, LAT, FOX, DHA, CFE, ACT

D OXAs (OXA-10, OXA-23, OXA-40, OXA-48, OXA-50)

Another important group of class A $\beta$-lactamases are the CTX-M-types ESBLs. These enzymes were first identified as non-TEM and non-SHV type ESBLs, exhibiting only about $40 \%$ similarity with TEM- and SHV-types, and having distinct hydrolytic activity against cefotaxime (484-487). They can occur in association with other TEM, SHV and OXA enzymes and are frequently carried on transmissible plasmids that can

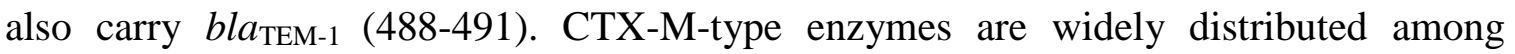


members of the Enterobacteriaceae family including E. coli, P. mirabilis, C. freundii, $S$. marcescens, Salmonella, Klebsiella and Enterobacter spp., but have also been reported in other non-enterobacterial species such as Vibrio spp. and Aeromonas hydrophila (485, 492-503).

Unlike the TEM- and SHV-derived ESBLs that arose by point mutations of their precursors, the CTX-M enzymes appear to be the result of plasmid transfer from naturally occurring chromosomal enzymes in the Kluyvera genus (504-506). Many CTX-M enzymes have been reported to be identical or near identical to the CTX-M-like enzymes KLUA and KLUC of the Kluyvera spp. $(504,505,507)$, with others like KLUG-1 sharing 85-86\% similarity with the CTX-M-1 enzymes (506). Additionally, the genetic regions surrounding the acquired CTX-M enzymes have been shown to be $80-100 \%$ identical to regions surrounding the corresponding $\beta$-lactamase-encoding gene in the Kluyvera chromosome (504, 507-509). ISEcpl or ISEcpl-like insertion sequences have been detected upstream of many CTX-M-plasmid-encoded enzymes (490, 500, 504, 510, 511), while some CTX-M enzyme are encoded in the class I integrons InS21, In35 and In60 (508-510, 512) further strengthening the case for horizontal gene transfer from the Kluyvera chromosome.

A fourth relevant group of class A $\beta$-lactamases are the non-metallocarbapenemases. The chromosomally encoded representatives of this group include the imipenem-hydrolyzing $\beta$-lactamase (IMI) and the non-metallo-carbapenemase (NMC-A) described in E. cloacae (513-515), as well as the SME-types isolated from S. marcescens (516-518). The spectrum of hydrolysis of these enzymes includes not only the 
carbapenems, but also penicillins, narrow-spectrum cephalosporins and aztreonam (519, 520). These chromosomally encoded non-metallo-carbapenemases however, do not generally pose as much a threat as the TEM, SHV and CTX-M ESBLs, as they are only infrequently reported.

Plasmid-borne representatives of class A non-metallo-carbapenemases include the Klebsiella pneumoniae carbapenemase (KPC) and the Guiana extended spectrum (GES) family of enzymes. The first KPC was detected in a $K$. pneumoniae isolate from North Carolina in 1996 (521). KPCs remained relatively rare until the early 2000s when they reemerged in the East Coast of the United States (522-525) causing multiple nosocomial outbreaks in the New York area, sometimes through clonal spread (526-529). Subsequently KPCs have been isolated in several European and South American countries (530-538) as well as in Israel, China and Taiwan (539-541). The bla $_{\mathrm{KPC}}$ genes are usually carried on the plasmid-borne transposon, Tn4401, which undoubtedly contributes to the ease of dissemination observed $(521,524,532,542,543)$. Thus, although most frequently observed in $K$. pneumoniae $(544,545)$, KPCs have also been isolated from P. aeruginosa, E. coli, C. freundii, S. marcescens, as well as from Salmonella and Enterobacter spp. (520, 522, 546, 547).

Similar to the TEM, SHV and CTX-M ESBLs, the KPC enzymes can hydrolyze penicillins and cephalosporins (520). Unlike the ESBLs, however, KPCs can also hydrolyze carbapenems to varying degrees (548). As carbapenems are often the first line of defense against microorganisms that express ESBLs, the emergence of carbapenemresistant organisms, that are already resistant to penicillins and cephalosporins, severely 
limits treatment options. The dissemination of KPCs is thus of great concern. Hydrolysis of carbapenems by KPCs, however, does not always lead to resistance, in some cases it can mean a decrease in susceptibility, with intermediate to high level resistance also being observed $(523,525)$.

GSE-1 was first reported in a K. pneumonia isolate from French Guiana (470). Currently 15 GSE enzymes are recognized, five of which display carbapenemase activity, albeit at low levels $(520,549)$. In every other sense they are typical ESBLs able to hydrolyze penicillins and extended-spectrum cephalosporins (519, 520). The GSE enzymes have been mostly identified in $P$. aeruginosa but have also been reported in some enterobacterial species (469, 550-552). Although GSE $\beta$-lactamases are relatively rare, nosocomial outbreaks have been reported in Korea, Portugal and South Africa (550, $553,554)$.

Most of the class A enzymes, including the extended-spectrum PER- and VEBtypes, have been reported in $P$. aeruginosa $(482,549)$. Class B and D enzymes (discussed below), however, are more prevalent in P. aeruginosa, whereas the class A enzymes are widely distributed in the Enterobacteriaceae (448, 477, 555, 556).

\subsubsection{Molecular class B}

Class B or metallo- $\beta$-lactamases (MLBs) are often described as being a class apart since unlike their serine counterparts they require $\mathrm{Zn}^{+2}$ in their active site in order to hydrolyze $\beta$-lactams $(456,457)$. They have a broad hydrolysis spectrum that includes the carbapenems and most often also penicillins and cephalosporins (456, 461, 463). MLBs are resistant to inhibition by the commercially available serine $\beta$-lactamase inhibitors and 
are unable to hydrolyze the monobactam aztreonam $(456,557)$. The requirement for zinc means they are sensitive to inhibition by EDTA, a known metal chelator $(457,461)$. They are often carried on plasmid-borne transposons and integrons, but can also be chromosomally encoded.

Chromosomally encoded MLBs are mostly found on environmental and sometimes opportunistic pathogenic bacteria that are rarely a cause for concern, with the notable exceptions of Bacillus anthracis and Stenotrophomonas maltophilia (457). Chromosomal MLBs include CphA from A. hydrophila (558), BCII from B. cereus (559), Bla2 from B. anthracis (560), SFH-1 from Serratia fonticola (561), FEZ-1 from Legionella gormanii (562), TUS-1 and MUS-1 from Myroides spp. (563), and L1 from S. maltophilia (564), among others. Frequently the MLBs are coproduced with penicillinases and cephalosporinases and are induced upon $\beta$-lactam challenge. For example, A. hydrophila coordinately expresses two $\beta$-lactamases, CepH (cephalosporinase) and AmpH (oxacillinase) in addition to the MLB CphA, also named $\operatorname{ImiH}(565,566)$. Coproduction of various $\beta$-lactamases is also observed in several other Aeromonas species such as A. veronii, A. jandaei, A. caviae, and A. salmonicida (566568). Similarly, S. maltophilia produces two chromosomally encoded and inducible enzymes, L1, a metallo- $\beta$-lactamase, and L2, a class A cephalosporinase (569-572).

The first occurrence of transferable imipenem resistance was reported in a $P$. aeruginosa isolate from Japan (573). The identified enzyme, IMP-1, was encoded in a conjugative plasmid that could be mobilized into other Pseudomonas strains. IMP-1 was later identified in several $S$. marcescens strains from general hospitals in the nearby cities 
of Anjyo and Okazaki from the Aichi prefecture in Japan (574-576). An IMP-type enzyme was also detected by DNA hybridization in fifteen $P$. aeruginosa clinical isolates from three different geographical areas in Japan (577). DNA fingerprinting showed that, for the most part, the strains were heterogeneous, while the $b l a_{\mathrm{IMP}}$ gene displayed little to no variation, thus illustrating a plasmid-mediated proliferation that was not clonal in nature (577). Further studies have shown that the IMP-1 enzyme and variants have proliferated in Japan in the Enterobacteriaceae, including S. marcescens, E. coli, C. freundii, K. pneumoniae, Enterobacter spp., as well as in non-enterobacterial species such as P. aeruginosa, Pseudomonas putida, Achromobacter xylosoxidans and Alcaligenes spp. (578-583).

PCR analysis of 42 strains carrying the bla $a_{\mathrm{IMP}}$ gene detected class 3 integron sequences in $79 \%$ of them (579). A larger Japanese study involving 357 bla $_{\mathrm{IMP}-1}$-positive strains that included P. aeruginosa, identified class I integron sequences in $99 \%$ of them (580). Association of bla $a_{\mathrm{IMP}}$ with mobile genetic elements has likely facilitated the dissemination of the enzyme in Japan. The IMP family of enzymes has also been reported worldwide in places like Portugal (584), Italy (585), Canada (586), China (587, 588), Australia (589, 590), England (591) and Korea (592, 593), among others. The current data however, suggest that these are local occurrences rather than dissemination from the alleles in Japan. Indeed, phylogenetic trees place IMP-1 in one of the later evolving branches of the dendrogram $(457,594)$.

A second and also prevalent class of MLBs is the integron-borne VIM-type. The first reported representative of this group, VIM-1 (Verona integron-encoded metallo- $\beta$ - 
lactamase) was detected in Italy in 1997 in a $P$. aeruginosa $\beta$-lactam-resistant isolate (595). Expression of the enzyme in a heterologous background conferred high level resistance against penicillins (ampicillin, carbenicillin, piperacillin and mezlocillin) and cephalosporins (cefoxitin, ceftazidime, cefoperazone and cefepime), while decreasing susceptibility to the carbapenems (595). VIM-1 was found to be only distantly related to other MLBs, sharing only $31 \%$ identity with IMP-1 (595). The $b l a_{\mathrm{VIM}-1}$ gene was located in the chromosome and encoded as part of a gene cassette carried in a class 1 integron, which itself carried another gene cassette (aacA4) for aminoglycoside resistance (595). Not surprisingly, a bla $a_{\mathrm{VIM}-1}$ gene was subsequently reported in a plasmid from an $A$. xylosoxidans isolate from the same hospital (596). A VIM-type enzyme, exhibiting a 90\% amino acid similarity with VIM-1, was reported in France in a P. aeruginosa isolate in a nonconjugative plasmid (597). The variant, termed VIM-2, has since been found throughout Europe and the rest of the world, and is often reported as the most detected of acquired MLBs (598-604). A number of other VIM variants have also been reported throughout the world and in a number of bacterial species including E. coli, $K$. pneumoniae, E. cloacae, S. marcescens, C. freundii, Acinetobacter baumannii, P. putida and $P$. aeruginosa; the latter being the most common host for these enzymes $(457,549)$.

Other noteworthy MLBs include SPM-1 (for Sao Paulo MLB), GIM-1 (for German imipenemase) and SIM-1 (for Seoul imipenemase). SPM-1 was first detected in 1997 in a P. aeruginosa clinical isolate from Brazil (605). It was encoded in a plasmid, but unlike the IMP and VIM enzymes that are regularly associated with class 1 integrons, SPM-1 was associated with a novel transposable-like element with probable recombinase 
activity (606). Thereafter, $P$. aeruginosa SPM-1-producing strains have become prevalent in Brazil and isolated from both clinical and environmental sources (607-610). To date, SPM-1 has only been reported outside of Brazil in A. baumannii isolates from various hospitals in Iran (611), and in a P. aeruginosa isolate from a Swiss man who had been previously treated in a Brazilian hospital (612).

GIM-1 was first reported in five multidrug-resistant $P$. aeruginosa isolates from a hospital in Dusseldorf, Germany (613). Subsequent reports identified more GIM-1producing $P$. aeruginosa isolates in hospitals within a $40 \mathrm{~km}$ radius from the location of the original report (614). Although so far GIM-1 has not been reported outside of Germany, it has however, spread to a number of enterobacterial species including Acinetobacter pittii, E. cloacae, Klebsiella oxytoca, S. marcescens, E. coli, and C. freundii (615-618).

SIM-1 was first detected in the chromosome of Acinetobacter clinical isolates from Korea (619). Later, bla $a_{\mathrm{SIM}-1}$ was also detected in two Acinetobacter (genomospecies 10) isolates that additionally carried $b l a_{\mathrm{IMP}-1}$ or $b l a_{\mathrm{VIM}-2}(620)$. To date, SIM-1 has not been detected outside of Korea, except for an Acinetobacter baylyi clinical isolate reported in China that additionally harbored OXA-23 in a large 360-kb plasmid (621). Similar to the IMP and VIM enzymes, both GIM-1 and SIM-1, are carried in class I integrons $(613,619)$. Although both SPM-1 and GIM-1 have been detected in $P$. aeruginosa, the IMP- and VIM-types are the most commonly detected MLBs in this pathogen and in the Acinetobacter spp. $(549,622)$. 


\subsubsection{Molecular class $\mathbf{C}$}

Class C enzymes or AmpC $\beta$-lactamases are generally chromosomally encoded but have also been reported in plasmids $(623,624)$. They can hydrolyze penicillins and cephalosporins including the cephamycins (cefoxitin and cefotetan) and the oxyiminocephalosporins (cefotaxime, ceftriaxone, and ceftazidime), as well as the monobactam aztreonam $(625,626)$. The increased rate of hydrolysis observed against some cephalosporins as compared to penicillins, particularly the first generation cephalosporins cefazolin, cephaloridine, cephalothin and cephalexin, has given rise to their common designation of cephalosporinases (625). Although able to slowly hydrolyze some of the later generation cephalosporins, such as cefotaxime and cefoxitin, these enzymes often display high affinity for these substrates as evidenced by low $K_{m}$ values $(625,627)$. The penicillins cloxacillin, oxacillin and carbenicillin act as transient inhibitors able to efficiently form acyl-enzyme complexes but usually being very poorly hydrolyzed by the class C enzymes (626). Additionally, the class A inhibitors are generally not good inhibitors of the cephalosporinases, although tazobactam and sulbactam have been shown to inhibit some class C enzymes $(429,430,432)$.

Chromosomal class $\mathrm{C}$ enzymes are ubiquitous in Gram-negative bacteria and very frequently encountered in the Proteobacteria including the Aeromonadales (Aeromonas spp.) and the Pseudomonadales (A. baumannii, P. fluorescens and P. aeruginosa) (623). They are particularly well represented in members of the Enterobacteriales, including some commonly encountered species and pathogens such as $C$. freundii, E. aerogenes, $E$. cloacae, E. coli, Morganella morganii, Providencia stuartii, S. marcescens, Shigella spp. 
and Yersinia enterocolitica, among others $(555,623)$. Some well-known members of the Enterobacteria group such as K. pneumoniae, K. oxytoca, P. mirabilis, Salmonella spp. and Yersinia pestis (623), however, do not carry a chromosomal class C enzyme, although this does not preclude the possibility of carrying plasmid-borne AmpC.

In many of the species carrying a chromosomal ampC, including $P$. aeruginosa, C. freundii and E. cloacae, ampC expression is constitutively low but induced upon $\beta$ lactam challenge $(377,628)$. Induction is tightly controlled by the transcriptional regulator AmpR and intricately linked to the recycling of the peptidoglycan layer (375). On the other hand, in E. coli and Shigella spp. ampC does not respond to induction by $\beta$ lactams and is only produced at very low levels $(629,630)$. Consequently the natural AmpC expression in such backgrounds does not lead to resistance. The marginal levels of AmpC $\beta$-lactamase observed in such species stem from the genetic environment surrounding the structural $\beta$-lactamase gene. In species with an inducible system (e.g., AmpC of $P$. aeruginosa), ampR is frequently located upstream of $a m p C$ and divergently transcribed; while species with a non-inducible system lack $a m p R$ and have a very weak promoter $(378,631,632)$. Additionally, an attenuator is located between the promoter and the $\beta$-lactamase-encoding gene leading to the premature termination of the majority of transcripts (633). Derepression and hyperproduction in species having a non-inducible system, i.e., lacking $a m p R$, can occur but often require mutations in the promoter and attenuator regions and/or the combined effect of different mutation types in order to provide any meaningful $\beta$-lactam resistance (634-638). Although such mutations have been reported in both $E$. coli and in Shigella spp. $(634,639,640)$, they are rarer than 
those leading to constitutive hyperexpression in species with an inducible system (376, 630). Mutational derepression or stable derepression of $\operatorname{ampC}$, as it is also known, however, has been reported as one of the most common mechanisms of $\beta$-lactam resistance in species that have an inducible AmpC system such as $P$. aeruginosa, $C$. freundii, E. cloacae, M. morganii, P. stuartii, Enterobacter and Serratia spp. (380, 446, $465,641-645)$. The genetics of inducible class $C \beta$-lactamases will be covered in section

\subsection{3.}

Although chromosomal ampC-types are the predominant class $\mathrm{C}$ enzymes in Gram-negative bacteria, dissemination into plasmids began to emerge in the late 1980s and continued to spread worldwide (646-649). They are predominantly found in species not usually known to possess chromosomal ampC, such as $K$. pneumoniae, K. oxytoca and Salmonella spp., but have also been reported in E. coli $(624,649)$. Representatives include MIR-1, MOX-1, LAT-1, FOX-1, ACT-1 and the CMY-types first reported in $K$. pneumonia, as well as CFE-1 and DHA-1 first identified in E. coli and Salmonella enteritidis, respectively (Table 1.5) $(624,649,650)$. The original chromosomal enzymes of Proteobacteria described above are the likely progenitors of these plasmid-borne enzymes (649). For instance, CMY-2 and the closely related types LAT-1 and CFE-1, appeared to have originated from the AmpC of C. freundii sharing 96, 95 and 99\% amino acid sequence with that enzyme, respectively $(647,651,652)$. CMY-1-types, including MOX-1, were originally thought to have descended from $P$. aeruginosa, but have since been shown to be more closely related to the chromosomal enzymes of the Aeromonas $(646,650,653)$. The genetic support is usually an integron-based plasmid that often 
carries other resistance genes such as those coding for aminoglycoside and quinolone resistance, or even other $\beta$-lactamase-encoding genes such as $b l a_{\mathrm{TEM}-1}, b l a_{\mathrm{CTX}-\mathrm{M}-3}$ and bla $_{\mathrm{VIM}-1}(649,654-658)$. The hydrolysis spectrum remains the same as that of their chromosomal-encoded counterparts $(623,649)$.

Although plasmid-encoded AmpC enzymes have been reported worldwide, they are not as prevalent in the clinical setting as the ESBLs. They are most commonly reported in K. pneumoniae isolates from patients that have had long hospital stays or prolonged care at the ICU, where urinary indwelling catheters and an immunocompromised system are predisposing factors (650, 659-662). Plasmid-encoded AmpC enzymes have not so far been described in P. aeruginosa (549).

\subsubsection{Molecular class D}

Transferable drug resistance among Gram-negative bacteria started being reported and characterized in the early 1960s (663-665). The agents mediating resistance were dubbed resistance or $R$ factors and later shown to be extrachromosomal pieces of DNA, termed plasmids (666). Datta and Kontomichalou recognized two distinct penicillinaseencoding R-factors, $\mathrm{R}_{\mathrm{TEM}}$ and $\mathrm{R} 1818$, on the basis of substrate profile (473). The enzyme from R1818, later renamed R46 (667), was able to hydrolyze methicillin and cloxacillin more efficiently than the penicillinase from $\mathrm{R}_{\mathrm{TEM}}$, which displayed only slight activity against these substrates (473). Additionally the R1818 penicillinase was able to hydrolyze cloxacillin almost as fast as it could hydrolyze penicillin (473). Later work showed that the penicillinase from R1818 also hydrolyzed oxacillin faster than benzylpenicillin (668672). Other studies characterized several more $\mathrm{R}$ factors mediating expression of $\beta$ - 
lactamases that resemble that of R1818 in the rapid hydrolysis of oxacillin and cloxacillin $(670,671,673)$.

Two different classes of oxacillin-hydrolyzing enzymes were then distinguished on the basis of molecular weight and substrate profile (674). One group was characterized by having low molecular weight $(\sim 24,000)$ and high hydrolysis rate against methicillin, while the other had high molecular weight $(\sim 40,000)$ but relatively low hydrolysis of the same compound. The lowest rate of hydrolysis, however, was around 40-fold higher than that previously observed for the original TEM enzyme against methicillin (473). Isoelectric focusing further divided the oxacillinases into three main subclasses that were designated as OXA-1, OXA-2 and OXA-3 to reflect their preference for the isoxazolyl penicillins such as oxacillin and cloxacillin (475).

The first oxacillinases described, particularly OXA-1 (675, 676), OXA-2 (447, $669,677)$ and OXA-3 $(671,678,679)$ from plasmids $\mathrm{R}_{\mathrm{GN} 238}$, $\mathrm{R} 46$, and $\mathrm{R} 57 \mathrm{~b}$, respectively, were characterized by their ability to degrade isoxazolyl penicillins and by their sensitivity to inhibition by $\mathrm{NaCl}$ (674). As more OXAs were reported and characterized, it became apparent that these were a diverse group of enzymes with a heterogeneous substrate profile that in some instances did not include the oxacillin-type $\beta$-lactams (680). More than 350 oxacillinases are now recognized (http://www.lahey.org/studies/webt.asp) and they can be broadly divided into the narrowspectrum, extended-spectrum and the carbapenem-hydrolyzing types (680).

The plasmid-mediated narrow-spectrum OXA enzymes include the originally characterized oxacillinases in E. coli (OXA-1), S. typhimurium (OXA-2), and $K$. 
pneumoniae (OXA-3), as well as a few others also first described in E. coli (OXA-4, OXA-7), K. pneumoniae (OXA-9, OXA-47), A. baumannii (OXA-37) and P. aeruginosa (OXA-5, OXA-6, OXA-10, OXA-20, LCR-1) (680). Generally they can hydrolyze penicillins and sometimes early generation cephalosporins but not extended-spectrum cephalosporins. The hydrolysis spectrum however, can vary greatly from enzyme to enzyme. For instance, OXA-1 can generally hydrolyze amino- and ureidopenicillins, narrow-spectrum cephalosporins (681), as well as the broad-spectrum cephalosporins (675, 681), albeit weakly; whereas the $P$. aeruginosa OXA-5 can only hydrolyze penicillins and cephalothin (682). Similarly the $P$. aeruginosa OXA-10, originally categorized as a $P$. aeruginosa specific enzyme (PSE-2), hydrolyses carbenicillin and oxacillin, as well as aztreonam and some of later generation cephalosporins like cefotaxime and ceftriaxone, albeit slowly (683). The $P$. aeruginosa LCR-1 only hydrolyzes penicillins including oxacillin (684).

The extended-spectrum class D enzymes are mostly point mutation variants of the narrow-spectrum OXA-2 and OXA-10 enzymes (680, 685). These mutations extend the hydrolysis spectrum to include the extended-spectrum cephalosporins. OXA-11, the first reported OXA-10 variant, was identified in a $P$. aeruginosa isolate taken from the bloodstream of a Turkish patient (686). It was carried on a plasmid and had two amino acid substitutions (Asn146Ser and Gly167Asp). Several other OXA-10 variants, including OXA-14 (687), OXA-16 (688), OXA-17 (689), and OXA-19 (690) were also identified in $P$. aeruginosa isolates from both Turkey and France. Up to nine substitutions were observed per variant (OXA-19, OXA-28, OXA-145, OXA-147), with 
OXA-14 having only one point mutation (http://www.lahey.org/Studies/). The Gly167Asp substitution appears to be the common thread among the variants that are very resistant to ceftazidime.

Unlike the OXA-10 ESBLs, the majority of which have more than one point mutation, the seven currently known OXA-2 variants have each only one substitution. OXA-15 (691) and OXA-36 have both a substitution at aspartate 149, to glycine and tyrosine respectively $(685,691)$. Both OXA-10 and OXA-2 ESBLs are carried in plasmid-borne class 1 integrons in association with aminoglycoside resistance cassettes (680). ES-OXAs are not widely distributed and have been mostly reported in $P$. aeruginosa (549).

A very important class of oxacillinases is comprised of the carbapenemhydrolyzing $\beta$-lactamases (CHDLs). The majority has been identified in plasmids from the nosocomial and opportunistic pathogen A. baumannii, not usually in association with integrons $(622,692)$. CHDLs can significantly hydrolyze carbapenems often leading to decreased susceptibility or resistance towards meropenem and imipenem $(685,693)$. They are also able to hydrolyze most penicillins and provide high-level resistance to the aminopenicillins (ampicillin, amoxicillin) and the carboxypenicillins (ticarcillin) with variable hydrolysis against the ureidopenicillins (piperacillin). CHDLs, however, do not generally significantly hydrolyze cephalosporins or provide any meaningful resistance against these compounds $(519,693)$. The notable exception is OXA-146 which hydrolyses ceftazidime (694). 
OXA-23, the first OXA carbapenem-hydrolyzing $\beta$-lactamase identified, was recovered from an A. baumannii isolate taken from a patient in Scotland (695). It was a plasmid-borne enzyme that showed very weak relation to other $\beta$-lactamases. Nineteen different variants have so far been reported for the OXA-23 group, the OXA-23 allele, however, remains the most common of this group with a widespread prevalence (685, 693). The OXA-51 group, currently consisting of 95 variants, is naturally present in the chromosome of $A$. baumannii $(696,697)$. These enzymes are not thought to play a major role in the intrinsic resistance as their expression is unregulated and uninducible. The bla $a_{\text {OXA-51 }}$ gene however, has sometimes been reported in association with the upstreamlocated ISAbal or ISAb9 (698-700). The promoters carried within these insertion sequences have been shown to drive expression of $b l a_{\text {OXA-51 }}$ in carbapenem-resistant isolates (698-700).

Other important CHDLs include the OXA-40, OXA-48, OXA-58 and OXA-143 groups (701-703). Although most display low level of carbapenem hydrolysis, they have often been reported in carbapenem- or multidrug-resistant strains, whereby their transformation into E. coli or susceptible A. baumannii confers decreased susceptibility or carbapenem resistance (703-707). CHDLs are predominant and widespread in $A$. baumannii but relatively rare in $P$. aeruginosa $(549,685,692,693,708,709)$.

\subsubsection{Genetics of inducible class $C \beta$-lactamases}

The inducible expression of AmpC $\beta$-lactamase in the Enterobacteriaceae and in P. aeruginosa is regulated by AmpR, a LysR-type transcriptional regulator (710-712).

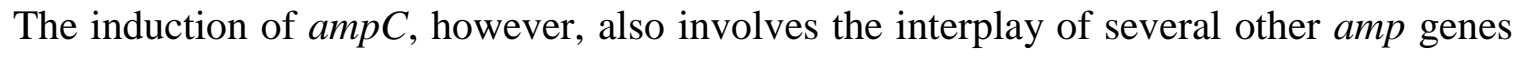


and is coordinately linked to the recycling of the peptidoglycan layer $(375,713,714)$. In the Enterobacteriaceae model, $\operatorname{amp} G$ encodes a transmembrane protein that serves as a permease for passage of the inducer (1,6-anhydromurapeptides) that results from remodeling of the peptidoglycan layer during normal cell growth $(715,716)$. AmpG transports 1,6-anhydromurapeptides into the cytoplasm where the amidase AmpD hydrolyses them for murein recycling (717). It has been proposed that during normal physiological growth, the muramyl peptides processed by AmpD maintain AmpR in an inactive conformation that is unable to induce the expression of $\operatorname{amp} C(716,718,719)$.

In the presence of $\beta$-lactams, however, there is an excessive breakdown of murein leading to an accumulation of 1,6-anhydromurapeptides in the periplasm and in the cytoplasm $(718,720)$. Under these conditions, AmpD cannot keep up with the increased concentration of 1,6-anhydromurapeptides in the cytoplasm. The high number of muramyl peptides can then induce a conformational change in AmpR to promote the high level expression of $\operatorname{amp} C(716,721)$. AmpD can thus function as a negative regulator of ampC expression in the absence of $\beta$-lactams. In fact, mutational inactivation of ampD, in both Enterobacteriaceae and $P$. aeruginosa, leads to constitutive over-expression of AmpC and consequently increased $\beta$-lactam resistance $(722,723)$. Recent work by Juan and colleagues reveals that three ampD homologs in $P$. aeruginosa are responsible for a stepwise ampC up-regulation mechanism that ultimately leads to constitutive hyperexpression of this $\beta$-lactamase (643). Details of this intricate mechanism are further discussed in Chapter 4. 


\subsubsection{Genetics of class D $\beta$-lactamases}

Oxacillinase-encoding genes have been reported in a multitude of bacterial species including $P$. aeruginosa. Most of these and other $\beta$-lactamase-encoding genes appear to be unregulated, with insertion sequences often providing strong promoters for high-level expression of such genes $(471,698,700,724-728)$. To date, the only known regulatory systems of chromosomal-encoded class D $\beta$-lactamases are that of Ralstonia pickettii and Aeromonas spp. (567, 729, 730).

R. pickettii carries two inducible chromosomally encoded $\beta$-lactamases, OXA-22 and OXA-60, exhibiting a narrow-spectrum of hydrolysis that includes the penicillins (731, 732). Induction requires the presence of the divergently transcribed ORF $r p 3$ found 190 bp upstream of the OXA-60-encoding gene $(730,733)$. Imipenem was found to increase the mRNA levels of $b l a_{\text {OXA-22 }}$ and $b l a_{\text {OXA-60 }}$ as well as that of $r p 3$, suggesting regulation by $\mathrm{Rp} 3$ occurs at the transcriptional level (733). Rp3 is thought to function as an activator as a recombinant Rp3 was shown to bind tandem repeats upstream of both OXA-encoding genes and to protect regions of $\sim 30$ bps there (733). RP3 was not similar to any known regulatory proteins. No significant similarities were found with $P$. aeruginosa proteins either.

Aeromonas spp. can produce two-three inducible and unlinked $\beta$-lactamases (552, 696-698). For instance, Aeromonas jandaei AER 14, formerly known as Aeromonas sobria AER 14, produces three chromosomally encoded $\beta$-lactamases, AsbA1 a class C cephalosporinase, AsbB1 (OXA-12) an oxacillinase, and AsbM1, a metallo- $\beta$-lactamase (734-736). Aeromonas veronii bv. sobria similarly produces a penicillinase (AmpS), a 
cephalosporinase (CepS) and metallo- $\beta$-lactamase (ImiS) $(737,738)$, with the homologs, AmpH, CepH, and ImiH, also identified in A. hydrophila $(565,739)$.

The expression of all $\beta$-lactamases in A. hydrophila is coordinately regulated by a two-component system (TCS), encoded by blrA and blrB, and located immediately upstream of $a m p H$, the oxacillinase-encoding gene (729). PCR analysis confirmed the presence of the $b \operatorname{lr} A B$ operon in other species of the Aeromonas genera, including $A$. jandaei and A. veronii bv. sobria, where the TCS-encoding genes are also linked to the bla $a_{\mathrm{OXA}}$ gene (729). Regulation by a TCS thus appears to be a common and effective mechanism of coordinate $\beta$-lactamase expression in Aeromonas spp.

\subsubsection{The pox operon}

Previous work showed that $\operatorname{ampC}$ mutations do not completely abolish $\beta$ lactamase activity in the $P$. aeruginosa PA01 strain (740). Furthermore, the susceptibility profile of an $a m p C$-deficient strain did not show an increase in sensitivity towards the three $\beta$-lactam representatives tested, benzyl-penicillin, meropenem and cefotaxime, as compared to the wild-type PA01 (740). In view of these results, the presence of a second chromosomally encoded $\beta$-lactamase in P. aeruginosa was postulated. In silico analysis revealed that ORF PA5514 encoded a putative $\beta$-lactamase with a signal peptide sequence at the N-terminus. The presence of a signal peptide is consistent with what is known about $\beta$-lactamases since they are localized to the periplasm and require a signal peptide for translocation.

Analysis of the sequences around $P A 5514$, termed poxB, revealed the presence of an upstream ORF annotated PA5513, named poxA, 49 bp upstream. In the absence of 
promoter-like sequences in the intergenic region between $P A 5513$ and PA5514, it was proposed that poxA and poxB form a two-gene operon (Figure 1.20). PoxA displayed high sequence similarity to hypothetical hydrolases and acyltransferases of yet unknown function. The presence of transmembrane spanning domains, as suggested by its hydrophobicity plot, and the absence of any signal sequence, indicate PoxA likely localizes to the inner membrane.

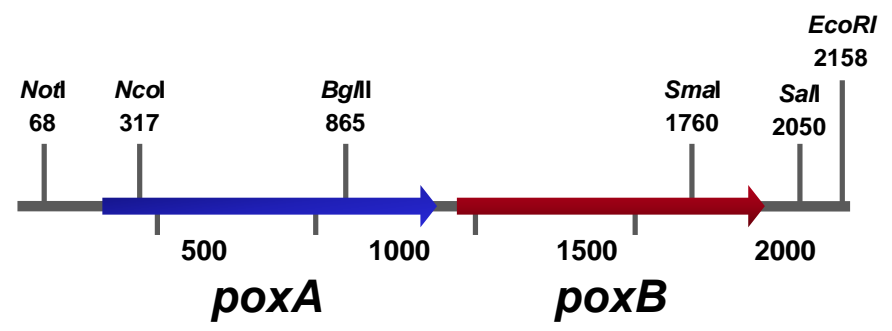

Figure 1.20. Restriction map of the pox operon.

PoxB, on the other hand, shows high sequence identity to class $\mathrm{D} \beta$-lactamases from A. baumannii and Fusobacterium nucleatum (740). Alignment of the PoxB sequence with 26 conserved residues in class D $\beta$-lactamases revealed that 21 of the 26 amino acids were identical. Differences in four of the remaining five amino acids place PoxB in a new branch of a dendogram (740). PoxB, thus appears to be a new kind of oxacillinase, markedly different from other OXA enzymes (740).

Expression studies conducted in E. coli, where there is a naturally low basal level of $\beta$-lactamase expression, confirmed the role of PoxB as a $\beta$-lactamase. Specifically, Girlich et al. showed that introduction of poxB in an E. coli background yields $\beta$ lactamase activity (741). Previous work in our lab also showed that both the poxB gene and the poxAB operon are capable of producing high basal levels of $\beta$-lactamase activity 
in E. coli (740). The goal of this dissertation was to characterize PoxB and the poxAB operon and determine their contribution to $\beta$-lactam resistance in the nosocomial and intractable pathogen $P$. aeruginosa. 


\section{Chapter 2}

Characterization of a carbapenem-hydrolyzing enzyme, PoxB, in

Pseudomonas aeruginosa 


\subsection{Abstract}

Pseudomonas aeruginosa is an opportunistic pathogen often associated with severe and life-threatening infections that are highly impervious to treatment. This microbe readily exhibits intrinsic and acquired resistance to varied antimicrobial drugs. Resistance to penicillin-like compounds is commonplace and provided by the chromosomal AmpC $\beta$-lactamase. In the present work the contribution of a second, recently discovered, chromosomally-encoded $\beta$-lactamase, PoxB, and its relation to AmpC, were investigated. A single deletion of ampC surprisingly did not alter susceptibility against most of the penicillins or cephalosporins tested, however its overexpression provided resistance and/or decreased susceptibility against these $\beta$ lactams. Carbapenem susceptibilities, as evidenced by minimum inhibitory concentrations (MICs), were mostly unaffected by the lack of AmpC. PoxB expression decreased susceptibility against the carbapenems but did not affect susceptibility against the penicillins or cephalosporins. Additionally, PoxB was only poorly inhibited by class A inhibitors, but a novel non- $\beta$-lactam inhibitor, termed avibactam, was a slightly better inhibitor of PoxB activity. Clinical concentrations of avibactam, however, failed to produce a change in MIC against the carbapenems by E-test. In addition, poxB was found to be cotranscribed with the upstream open reading frame, poxA, which itself was shown to encode a 32-kDalton protein of yet unknown function.

Key Words: PoxB, OXA-50, AmpC, Oxacillinase, Class D $\beta$-lactamase

Abbreviations: Congo red, CR; Coomassie brilliant blue, CB; Isopropyl $\beta-\mathrm{D}-1$ thiogalactopyranoside, IPTG; Minimum inhibitory concentration, MIC; Open reading frame, ORF; Sodium dodecyl sulfate-polyacrylamide gel electrophoresis, SDS-PAGE 


\subsection{Introduction}

Pseudomonas aeruginosa is a ubiquitous and versatile opportunistic pathogen commonly affecting immunocompromised individuals such as those with severe burns, AIDS and cancer $(89,92,96,99,109,202)$. In addition, it is a significant source of nosocomial infections and the second most common cause of ventilator-associated pneumonia in the hospital setting $(72,73)$. Most notably, $P$. aeruginosa is the primary pathogen associated with lung deterioration and mortality in patients with cystic fibrosis (CF), a deadly, autosomal, recessive genetic disorder affecting about 70,000 individuals worldwide $(122,742)$. Treatment often proves challenging and ineffective as the bacterium exhibits innate and acquired resistance to a broad range of antibiotics (246, $349,743)$. In particular, resistance to the frequently used $\beta$-lactam-type antibiotics is common and mediated by the expression and derepression of the chromosomally encoded AmpC $\beta$-lactamase (379-381, 722).

The Ambler classification scheme distinguishes four different classes of $\beta$ lactamases (classes A, B, C and D) on the basis of amino acid sequences (452). All four classes have been reported in $P$. aeruginosa and are often transposon or plasmid-borne (601, 744-747). In addition to the acquired $\beta$-lactamases, $P$. aeruginosa strains frequently carry the chromosomal class $C \beta$-lactamase AmpC $(711,748)$. Derepression of ampC has been identified as a common cause of moderate to high-level $\beta$-lactam resistance in $P$. aeruginosa clinical isolates (379, 380, 643-645, 749).

A second chromosomally-encoded $\beta$-lactamase, termed PoxB (PA5514) or OXA50, has been reported in $P$. aeruginosa $(740,741)$. PoxB belongs to the class $\mathrm{D} \beta$ - 
lactamases, also termed oxacillinases, for the ability of some members of this class, particularly, the earlier reported ones, to degrade isoxazolyl penicillins such as oxacillin, methicillin, and cloxacillin $(671,674,682,750-752)$. The DBL numbering system is used to correlate homologous residues and signature sequences across class $\mathrm{D} \beta$-lactamases that may otherwise be located at different amino acid positions (753). Differences in these amino acid signature sequences place PoxB in a new branch of the oxacillinase phylogenetic tree, suggesting it is only weakly related to other oxacillinases $(740,741)$. For instance, class A and D enzymes commonly have a serine-threonine-phenylalaninelysine (STFK) motif at position 70 in the DBL numbering system, where serine, the active site residue, and lysine are conserved in serine $\beta$-lactamases and in penicillinbinding proteins $(753,754)$. To our knowledge, however, PoxB is only one of two oxacillinases where the traditional STFK motif of class D $\beta$-lactamases is replaced by STYK (serine-threonine-tyrosine-lysine) (740, 741), the other being OXA-62 from Pandoraea pnomenusa (755-757). Thus, PoxB appears to be a new kind of oxacillinase, markedly different from other known OXA enzymes. In addition, previous in silico analysis suggested poxB may form a two-gene operon with the upstream open reading frame (ORF), PA5513, termed poxA (740), which is predicted to encode a putative hydrolase or acyltransferase of yet unknown function. The role of poxA and its relation to pox $B$ is yet to be determined.

In the present work we examine the role of PoxB and its contribution to $\beta$-lactam resistance by generating single in-frame deletions of both chromosomal $\beta$-lactamaseencoding genes, namely ampC and $\operatorname{pox} B$. We also study the efficacy of class A enzyme 
inhibitors and of a novel non- $\beta$-lactam inhibitor termed avibactam, against PoxB. Additionally, the hypothesis that poxA and $\operatorname{pox} B$ form an operon and the role of PoxA were investigated.

\subsection{Materials and Methods}

2.3.1 Bacterial strains, plasmids and media. Bacterial strains, plasmids and primers used in this study are shown in Table 2.1. Escherichia coli and P. aeruginosa were cultured routinely in Luria-Bertani medium (LB; $10 \mathrm{~g}$ tryptone, $5 \mathrm{~g}$ yeast extract, and $5 \mathrm{~g}$ $\mathrm{NaCl}$, per liter). Cation-adjusted Mueller Hinton broth (CAMHB) and agar (BBL, BD) were used for susceptibility testing using the broth microdilution method and E-test, respectively. T-broth (10 g Bacto tryptone and $5 \mathrm{~g} \mathrm{NaCl}$, per liter) was used for pellicle formation and Congo red (CR) binding assays. Antibiotics were used at the following concentrations: ampicillin (Ap) at $100 \mu \mathrm{g} / \mathrm{ml}$, and gentamycin (Gm) at $15 \mu \mathrm{g} / \mathrm{ml}$ for $E$. coli; Gm at $75 \mu \mathrm{g} / \mathrm{ml}$ for $P$. aeruginosa.

2.3.2 Construction of $\operatorname{pox} A, \operatorname{pox} B$, and $o p r D$ deletion mutants. A single in-frame deletion of poxA (PA5513) was constructed using overlap extension PCR and homologous recombination as previously described (758). Briefly, sequences upstream (816 bp) and downstream (773 bp) of the target deletion were amplified using primer pairs CApoxAUF1-CApoxAUR1 and CApoxADF2-CApoxADR2, respectively (Table 2.1). The PCR products were then ligated through another round of PCR, cloned into the suicide vector pEXG2 (759) and moved into P. aeruginosa PA01 for homologous 
recombination with the genome. Double-cross-over recombinants were selected for by screening for Gm sensitivity and sucrose resistance. The deletion was confirmed by PCR amplification of deletion product from the genome and sequencing. The strain is referred to as PA0 were generated in a similar manner using primer pairs DBpoxBUF1-DBpoxBUR1 and DBpoxBDF2-DBpoxBDR2; and DZoprDUF1-DZoprDUR1 and DZoprDDF2DZoprDDR2, respectively (Table 2.1). The poxB and oprD deletions were also introduced into a previously generated $a m p C$ deletion strain (PA0 $\Delta a m p C)$ (760) to create PA0 $\Delta a m p C \Delta p o x B, \mathrm{PA} 0 \Delta a m p C \Delta o p r D$ and PA0 $\Delta a m p C \Delta p o x B \Delta o p r D$.

Table 2.1: Bacterial strains and plasmids used in this study

\begin{tabular}{|c|c|c|}
\hline Strains/Plasmids & Relevant phenotype and genotype & $\begin{array}{c}\text { Reference/ } \\
\text { Source } \\
\end{array}$ \\
\hline \multicolumn{3}{|l|}{ Escherichia coli } \\
\hline DH5 $\alpha$ & 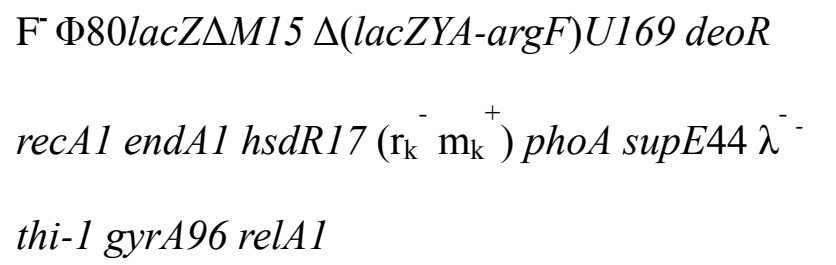 & $\begin{array}{c}\text { New England } \\
\text { Biolabs }\end{array}$ \\
\hline TOP10 & 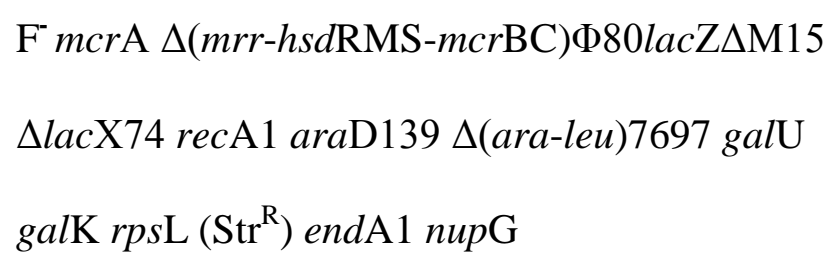 & $\begin{array}{c}\text { Life } \\
\text { Technologies }\end{array}$ \\
\hline BL21(DE3) & $\mathrm{F}^{-}$ompT hsd $S_{\mathrm{B}}\left(\mathrm{r}_{\mathrm{B}^{-}} \mathrm{m}_{\mathrm{B}^{-}}\right)$gal dcm (DE3) & $(761)$ \\
\hline \multicolumn{3}{|c|}{ Pseudomonas aeruginosa } \\
\hline PA01 & Wild-type & $(762)$ \\
\hline
\end{tabular}




\begin{tabular}{|c|c|c|}
\hline PKM119 & $\begin{array}{l}\text { PA0 } \Delta \text { poxA, PA01 containing in-frame deletion } \\
\text { of } p o x A\end{array}$ & This study \\
\hline PKM120 & $\begin{array}{l}\text { PA } 0 \Delta p o x B, \text { PA01 containing in-frame deletion } \\
\text { of poxB }\end{array}$ & This study \\
\hline PKM201 & 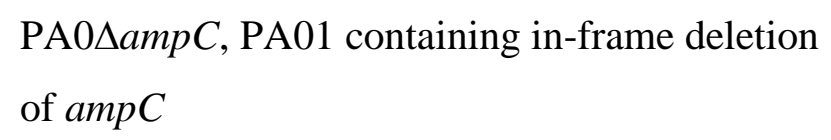 & (760) \\
\hline PKM202 & $\begin{array}{l}\text { PA } 0 \triangle a m p C \triangle p o x B, P A 01 \text { containing in-frame } \\
\text { deletions of } a m p C \text { and } p o x B\end{array}$ & This study \\
\hline PKM121 & $\begin{array}{l}\text { PA0 } \Delta o p r D \text {, PA01 containing in-frame deletion } \\
\text { of } o p r D\end{array}$ & This study \\
\hline PKM203 & $\begin{array}{l}\mathrm{PA} 0 \Delta a m p C \Delta o p r D \text {, PA01 containing in-frame } \\
\text { deletions of } \operatorname{amp} C \text { and } o p r D\end{array}$ & This study \\
\hline PKM204 & $\begin{array}{l}\text { PA0 } 0 \text { amp } C \Delta p o x B \Delta o p r D, \text { PA01 containing in- } \\
\text { frame deletions of } a m p C, \text { poxB and } o p r D\end{array}$ & This study \\
\hline \multicolumn{3}{|l|}{ Plasmids } \\
\hline pEXG2 & $\mathrm{Gm}^{\mathrm{R}}$; pMB1ori sacB & (759) \\
\hline pGEM-T Easy & $\begin{array}{l}\mathrm{Ap}^{\mathrm{R}} \text {; TA cloning vector for PCR amplicons, } \\
\mathrm{pUC} \text { origin, f1 ori }\end{array}$ & Promega \\
\hline pCR2.1-TOPO & $\begin{array}{l}\mathrm{Ap}^{\mathrm{R}}, \mathrm{Km}^{\mathrm{R}} ; \mathrm{TA} \text { cloning vector for } \mathrm{PCR} \\
\text { amplicons, pUC origin, f1 ori lac } Z \alpha\end{array}$ & $\begin{array}{c}\text { Life } \\
\text { Technologies }\end{array}$ \\
\hline pET15bVP & $\begin{array}{l}\mathrm{Ap}^{\mathrm{R}} ; \mathrm{pBR} 322 \text { origin, ori } V \text {, expression vector, } \\
\mathrm{T} 7 \text { promoter, His tag coding sequence }\end{array}$ & (756) \\
\hline pMMB67EH-Gm & $\begin{array}{l}\mathrm{Gm}^{\mathrm{R}} \text {; IncQ, RSF1010, lacl }{ }^{\mathrm{q}} \mathrm{P}_{\text {tac }} \text { expression } \\
\text { vector with ampR::aacC1 insertion at DraI }\end{array}$ & (763) \\
\hline pDZ427 & $\begin{array}{l}\mathrm{Gm}^{\mathrm{R}} ; \mathrm{pET} 15 \mathrm{bVP} \text { carrying an } 891-\mathrm{bp} \text { XhoI and } \\
\text { BamHI poxA fragment }\end{array}$ & This study \\
\hline
\end{tabular}




\begin{tabular}{|c|c|c|}
\hline pAmpC & $\begin{array}{l}\mathrm{Gm}^{\mathrm{R}} ; \mathrm{pMMB} 67 \mathrm{EH}-\mathrm{Gm} \text { carrying a } 1297-\mathrm{bp} \\
\text { fragment containing the } a m p C \mathrm{ORF}\end{array}$ & This study \\
\hline pPoxA & $\begin{array}{l}\mathrm{Gm}^{\mathrm{R}} \text {; pMMB67EH-Gm carrying a 946-bp } \\
\text { fragment containing the poxA ORF }\end{array}$ & This study \\
\hline pPoxB & $\begin{array}{l}\mathrm{Gm}^{\mathrm{R}} ; \mathrm{pMMB} 67 \mathrm{EH}-\mathrm{Gm} \text { carrying an } 835-\mathrm{bp} \\
\text { fragment containing the poxB ORF }\end{array}$ & This study \\
\hline \multicolumn{3}{|l|}{ Primers } \\
\hline $\mathrm{DZampCF}$ & $\begin{array}{l}\text { 5'-GGAATTCAACGACAAAGGACGCCAA- } \\
\text { TCCTC-3' }\end{array}$ & This study \\
\hline $\mathrm{DZampCR}$ & $\begin{array}{l}\text { 5'-CGGGATCCATGGCACCATCATAGCCA- } \\
\text { GGACCGG-3' }\end{array}$ & This study \\
\hline DZpoxAF & $\begin{array}{l}\text { 5'-GGAATTCTTACACTGCGCGCCGTACG- } \\
\text { AGGA-3' }\end{array}$ & This study \\
\hline DZpoxAR & $\begin{array}{l}\text { 5'-CGGGATCCGATTCCCCACGGAAGGAG- } \\
\text { CGTT-3' }\end{array}$ & This study \\
\hline RTpoxARev1 & 5'-AGGAGAAGGGCACTGAAGAGGAGA-3' & This study \\
\hline RTpoxAFord2 & 5'-GACTTTCTCCTGCCCAATCCATGA-3' & This study \\
\hline RTpoxARev2 & 5'-CACCAACCAGAAGTTATCCACA-3' & This study \\
\hline DZpoxAFord4 & $\begin{array}{l}\text { 5'-CTCGAGATGTCGCTATCCCCCTGGTT- } \\
\text { CC-3' }\end{array}$ & This study \\
\hline DZpoxARev4 & $\begin{array}{l}\text { 5'GGATCCTCATGGATTGGGCAGGAGAA- } \\
\text { AGTC-3' }\end{array}$ & This study \\
\hline DZpoxBF & $\begin{array}{l}\text { 5'-GGAATTCAGGATGACCCCACAGGACC- } \\
\text { GAGCCAT-3' }\end{array}$ & This study \\
\hline DZpoxBR & $\begin{array}{l}\text { 5'-CGGGATCCCAGAGCGTCAGGGCAGT- } \\
\text { ATCC-3' }\end{array}$ & This study \\
\hline
\end{tabular}




\begin{tabular}{|c|c|c|}
\hline CApoxAUF1 & $\begin{array}{l}\text { 5'-GGAATTCGATCGTATTGCCGGGTCTC- } \\
\text { 3' }\end{array}$ & This study \\
\hline CApoxAUR1 & $\begin{array}{l}\text { 5'-GAGCATTCTAGCTAGCTAGAACCAGG- } \\
\text { GGATAGCGACAT-3' }\end{array}$ & This study \\
\hline CApoxADF2 & $\begin{array}{l}\text { 5'-CTAGCTAGCTAGAATGCTCCCAATCC- } \\
\text { ATGAACGCTCCTT-3' }\end{array}$ & This study \\
\hline CApoxADR2 & $\begin{array}{l}\text { 5'-CGGGATCCAAGCCGTAGAGCCGCTCG- } \\
\text { TT-3' }\end{array}$ & This study \\
\hline DBрохBUF1 & $\begin{array}{l}\text { 5'-GAATTCGGATTTCGACCTCTGGCTGT- } \\
\text { G-3' }\end{array}$ & This study \\
\hline DBpoxBUR1 & $\begin{array}{l}\text { 5'-GAGCATTCTAGCTAGCTAGCAGGAGA- } \\
\text { AGGGCACTGAAGAG-3' }\end{array}$ & This study \\
\hline DBрохBDF2 & $\begin{array}{l}\text { 5'-CTAGCTAGCTAGAATGCTCTCAAGGC- } \\
\text { TCTC-GGGATACTG-3' }\end{array}$ & This study \\
\hline $\mathrm{DB}$ poxBDR2 & $\begin{array}{l}\text { 5'-CGGGATCCACTGGGTGTGGTTGGAGA- } \\
\text { AT-3' }\end{array}$ & This study \\
\hline DZoprDUF1 & $\begin{array}{l}\text { 5'-GGAATTCCAAAGCGAACATACTGACC- } \\
\text { TCTC-3' }\end{array}$ & This study \\
\hline DZoprDUR1 & $\begin{array}{l}\text { 5'-GAGCATTCTAGCTAGCTAGACTTATA- } \\
\text { GCACGCAGTGTTTCTTG-3' }\end{array}$ & This study \\
\hline DZoprDDF2 & $\begin{array}{l}\text { 5'-CTAGCTAGCTAGAATGCTCCCTATAA- } \\
\text { AGGAAGGGCGTAGGTA-3' }\end{array}$ & This study \\
\hline DZoprDDR2 & $\begin{array}{l}\text { 5'-CGGGATCCCGGTGGAAAGCAGATACT- } \\
\text { GACTGGTA-3' }\end{array}$ & This study \\
\hline
\end{tabular}

2.3.3 PCR amplification and cloning of poxA, poxB and ampC. The $P$. aeruginosa PA01 genome was used as the template for PCR amplification of the poxA (PA5513), poxB (PA5514) and ampC (PA4110) ORFs with primer pairs DZpoxAF-DZpoxAR, 
DZpoxBF-DZpoxBR, and DZampCF-DZampCR, respectively (Table 2.1). The amplicons were independently cloned into pCR2.1-TOPO and sequenced. Each insert was then moved into the EcoRI-BamHI sites of the broad-host range expression vector pMMB67EH-Gm (763) and propagated in E. coli TOP10 or DH5a. The plasmid carrying ampC, (pAmpC), was subsequently introduced by electroporation into PA0 $\triangle a m p C \triangle p o x B$ while pPoxA was introduced into PA0 $\triangle a m p C$, PA0 $\Delta$ poxA, and PA0 $\Delta p o x B$. The pPoxB plasmid was introduced into PA0 $\Delta$ poxA, PA0 $\Delta p o x B, \mathrm{PA} 0 \Delta a m p C$, PA0 $\Delta a m p C \Delta p o x B$ and PA0 $\triangle a m p C \triangle \operatorname{poxB} \Delta o p r D$.

For protein expression in E. coli BL21(DE3), the poxA ORF was amplified using primers DZpoxAFord4 and DZpoxARev4, cloned into the pGEM-T Easy vector (Promega, Madison, WI) and sequenced. The 891-bp fragment was then subcloned into the XhoI and BamHI sites of the broad host range shuttle vector pET15bVP (756) and propagated in E. coli TOP10 (Life Technologies, Grand Island, NY). The resultant plasmid was then introduced into E. coli BL21(DE3) (761) for expression studies.

2.3.4 Protein expression and one-dimensional gel electrophoresis. To determine if poxA is translated, the ORF was cloned into the expression vector pET15bVP and introduced into E. coli BL21(DE3) as described in section 2.3.3. Stationary-phase cultures were diluted to an $\mathrm{OD}_{600}$ of 0.02 in $25 \mathrm{ml}$ of $\mathrm{LB}$ broth and incubated with shaking at $37^{\circ} \mathrm{C}$ until the culture density reached an $\mathrm{OD}_{600}$ of 0.5 . Cells were then induced with $1 \mathrm{mM}$ of isopropyl $\beta$-D-1-thiogalactopyranoside (IPTG) and reincubated at $37^{\circ} \mathrm{C}$. One-ml samples were taken at the time of induction and every hour for a total of 
four hours for both IPTG-induced and non-induced samples. The cells were recovered by centrifugation, resuspended in $100 \mu \mathrm{l}$ of $4 \mathrm{X}$ SDS protein sample buffer and boiled for 10 minutes. Proteins were separated on a $12 \%$ SDS-PAGE gel and stained with Coomassie brilliant blue $(\mathrm{CB})$.

2.3.5 Analysis of PA01 mRNA. To determine if poxA and poxB are cotranscribed, RNA was extracted from wild-type $P$. aeruginosa PA01, using the RNeasy mini kit (Qiagen). cDNA was synthesized with Superscript III (Invitrogen) and an (NS) $)_{5}$ random primer using standard methods (764). Two sets of primers were used to amplify the intergenic between poxA and poxB plus 24 bp corresponding to the 3' end of poxA and 53 or $486 \mathrm{bp}$ from the 5' end of poxB. RTpoxAFord2 and RTpoxARev1 were designed to amplify 126bp product, while amplification with RTpoxAFord2 and with RTpoxARev2 would yield a 559-bp product. RNA samples not treated with Superscript were also tested to ensure no carryover DNA contamination resulted from the RNA isolation procedure.

2.3.6 $\beta$-lactamase assay. The $\beta$-lactamase assay was modified from a previously published protocol (765). Briefly, stationary-phase cultures were diluted in $25 \mathrm{ml}$ of LB broth to an $\mathrm{OD}_{600}$ of 0.02 and incubated with shaking at $37^{\circ} \mathrm{C}$. At an $\mathrm{OD}_{600}$ of 0.6 , cells were induced with $50 \mu \mathrm{g} / \mathrm{ml}$ of benzylpenicillin, while cells containing the expression plasmid (pPoxB) were induced with $1 \mathrm{mM}$ IPTG. In both cases induction was carried out for an hour. Ten milliliter of cells were harvested by centrifugation and resuspended in $1000 \mu 1$ of 1x BugBuster solution (Novagen, EMD Millipore, Billerica, MA) containing 1 
$\mu \mathrm{l}$ of benzonase (Novagen) and $1 \mathrm{KU}$ of rLysozyme (Novagen). Lysate was rotated for 20 min at room temperature before centrifuging at $4^{\circ} \mathrm{C}$ for 15 minutes to collect the $\beta$ lactamase-containing supernatant. A $2-\mu 1$ aliquot of cell lysate was added to nitrocefin (final concentration, $100 \mu \mathrm{M}$ ) in $250 \mu \mathrm{l}$ of assay buffer. The reaction was incubated at $37^{\circ} \mathrm{C}$ for 20 minutes and the hydrolysis of nitrocefin was measured spectrophotometrically at $482 \mathrm{~nm}$. Total protein concentration was determined with Bradford on the same supernatant. The activity was expressed as nmol of nitrocefin degraded per minute per microgram of total protein.

2.3.7 Minimum inhibitory concentration (MIC). MICs were determined by the E-test system according to the manufacturer's instructions (bioMérieux, Marcy l'Etoile) or by the broth microdilution method according to standard protocols $(766,767)$. E-test values were often reported as a range or as two independent values to illustrate variation seen in biological and technical replicates.

2.3.8 Colony morphology on Tryptone Agar containing Congo red. Plates containing $1 \%$ agar, $1 \%$ tryptone, $40 \mu \mathrm{g} / \mathrm{ml}$ of $\mathrm{CR}$ and $20 \mu \mathrm{g} / \mathrm{ml}$ of CB were spotted with $10 \mu \mathrm{l}$ of $0.025 \mathrm{OD}_{600}$ cells. Plates were also supplemented with $1 \mathrm{mM}$ IPTG and 10 or $20 \mathrm{mM}$ $\mathrm{NaNO}_{3}$, when appropriate. Plates were incubated at room temperature or in an anoxic chamber for 5-7 days and pictures were taken with the Epson Perfection V550 scanner (Long Beach, CA). 
2.3.9 Pellicle formation and cell-surface interactions. For pellicle formation, the strains were grown in $6 \mathrm{ml}$ of T-broth at room temperature without shaking for 7 days as previously described (768). The tubes were inspected visually for formation of mats at the liquid-air interphase. To assess cell-cell and cell-surface interactions, cells were grown in borosilicate glass tubes overnight $(\mathrm{O} / \mathrm{N})$ with shaking at $37^{\circ} \mathrm{C}$ in $\mathrm{T}$-broth in the presence and absence of $1 \mathrm{mM}$ IPTG and inspected visually the next day for aggregates on the walls of the tubes (769).

2.3.10 Congo red binding assay. The CR-binding assay was performed as previously described with minor modifications (770). Briefly, strains were grown in $2 \mathrm{ml}$ of T-broth at $37^{\circ} \mathrm{C}$ without shaking for 48 hours. Cells were harvested by centrifugation, and washed with T-broth. The pellet was resuspended in $1 \mathrm{ml}$ of T-broth containing $40 \mu \mathrm{g} / \mathrm{ml}$ of CR and incubated with shaking at $37^{\circ} \mathrm{C}$. After 90 minutes, the cells and the bound $\mathrm{CR}$ were pelleted by centrifugation. The optical density of the supernatant was determined at 495 $\mathrm{nm}$ and the percentage of CR left in the supernatant was calculated.

\subsection{Results and Discussion}

P. aeruginosa infections are commonly treated with a combination of $\beta$-lactams and aminoglycosides. Resistance to the $\beta$-lactam antibiotics is frequently observed in the clinical setting and is commonly due to the expression of enzymes that can hydrolyze these compounds, namely $\beta$-lactamases $(645,743,771-773)$. P. aeruginosa clinical isolates often harbor acquired $\beta$-lactamases particularly of the oxacillinase- (class D) and 
metallo- $\beta$-lactamase IMP- and VIM-types (class B) $(549,608,610,614,747,748,774$, 775). Additionally, P. aeruginosa expresses a chromosomal cephalosporinase, AmpC, that provides intrinsic resistance and that can be expressed at high levels in the presence of $\beta$-lactams $(711,712,776)$. A second chromosomally encoded oxacillinase-type $\beta$ lactamase, PoxB, has also been reported $(740,741)$. The aim of this study was to examine the role of the class D $\beta$-lactamase PoxB in P. aeruginosa PA01.

2.4.1 $\operatorname{pox} A$ and $\operatorname{pox} B$ form an operon. Previous in silico analysis revealed the oxacillinase-encoding gene (PA5514) to be located 49-bp downstream of a putative ORF annotated PA5513 (Figure 2.1A). Given the close proximity between PA5513 and PA5514, the absence of promoter like sequences in the intergenic region, and the presence of a potential $\rho$-independent terminator downstream of PA5514, we previously hypothesized that poxA and poxB form a two-gene operon (740). To determine if in fact these two genes are cotranscribed, RNA was extracted from the prototypic $P$. aeruginosa PA01, cDNA was synthesized and two sets of primers (RTpoxAFord2-RTpoxARev1 and RTpoxAFord2-RTpoxARev2) were used to amplify the intergenic region plus small portions of the 3 ' of poxA and the 5' of poxB (Figure 2.1A). As expected, the first set of primers yielded a 126-bp amplicon in both genomic DNA and cDNA templates (Figure 2.1B, lanes 1 and 2), with the second set yielding a 559-bp product in the same templates (Lanes 5 and 6). In the absence of template DNA or reverse transcriptase, no amplification was detected for both primer sets (Lanes 3, 4, 7 and 8). This work confirms that these two genes are cotranscribed and that they form an operon. 


\section{A}

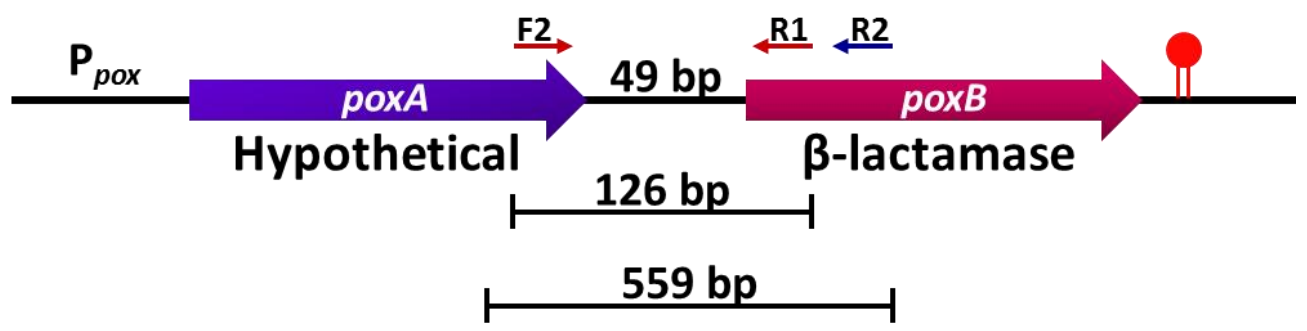

B

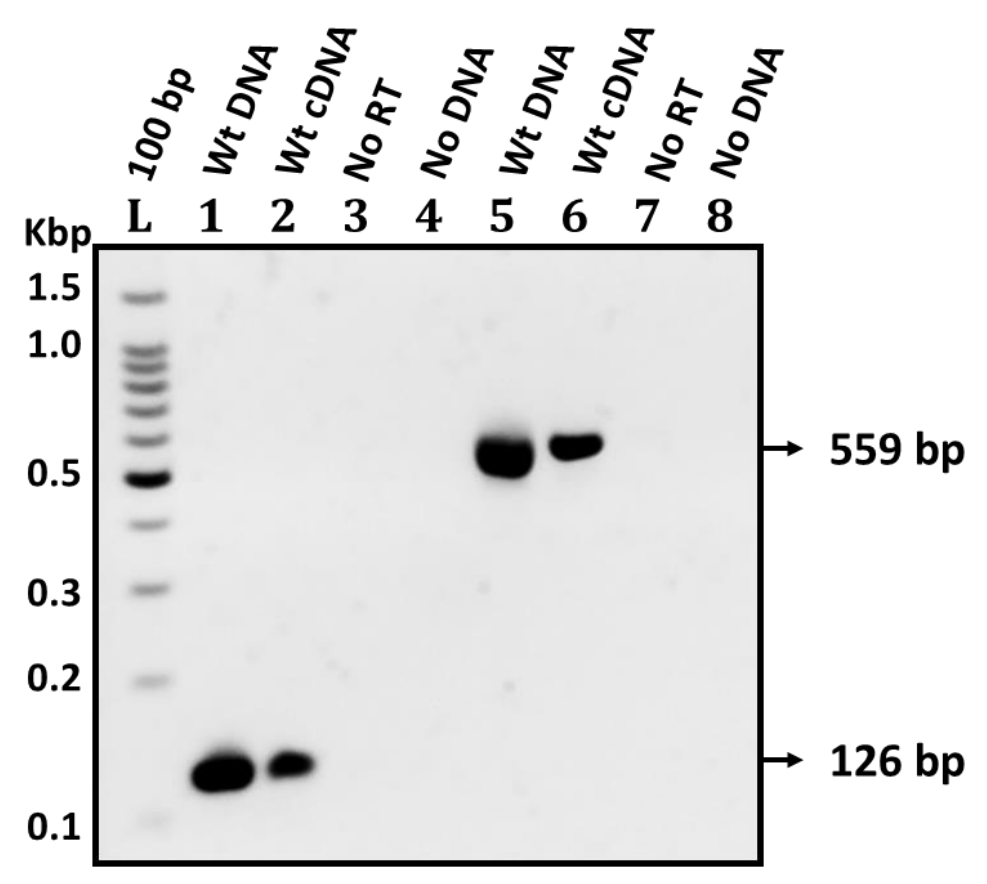

Figure 2.1. The pox $A B$ operon. (A) The pox genes and the approximate location of primers used to show PA5513 and PA5514 form an operon. Two sets of primers were used to amplify the intergenic region as well as the 3' end of poxA and the 5' start of poxB from wild-type cDNA. Primer pair RTpoxAFord2 and RTpoxARev1, denoted as F2 and R1 respectively, were used for amplification of a 126-bp product, while RTpoxAFord2 and RTpoxARev2, denoted F2 and R2, were used to amplify a 559-bp region. (B) A 126-bp amplicon was detected in wild-type genomic and cDNA samples (Lanes 1 and 2) upon amplification with F2 and R1. Similarly, a 559-bp product was observed upon amplification with F2 and R2 in wild-type genomic and cDNA samples (Lanes 5 and 6). No amplification could be detected with either primer set in the absence of reverse transcriptase or template DNA (Lanes 3, 4, 7 and 8). 
2.4.2 poxA is translated into a 32.4-kDalton protein. Since our present work indicates poxA and poxB are cotranscribed, it is also expected that poxA mRNA is translated into a protein with a function potentially related to that of PoxB. To confirm our prediction, the poxA ORF was amplified and cloned into the expression vector pET15bVP (756) and expressed in E. coli BL21(DE3) (761). Whole cell extracts were obtained from cells carrying the poxA-overexpressing plasmid in the presence and absence of IPTG. Proteins were visualized in an SDS-PAGE gel. A protein band corresponding to PoxA was detected at around $32 \mathrm{kDalton}$ an hour after induction with IPTG and thereafter (Figure 2.2, Lanes 3-6). The same band was also present but reduced in whole cell extracts from uninduced cells that had been grown for 4 hours after induction (Lane 7). PoxA was absent from the uninduced and induced conditions at the 0 time point (Lanes 1 and 2), as well as from cells containing only empty vector (Figure 2.2, lanes 8-9). Thus the poxA ORF codes for a protein.

Since both poxA and poxB are part of a single operon, they could potentially have related functions. In silico analysis reveals that the nucleotide sequences composing this operon are not found anywhere else but in P. aeruginosa. Sequences showing high similarity to both the poxA ORF (73-82\%) and the PoxA protein (66-78\%) were also identified in other Pseudomonas species such as P. denitrificans, P. entomophila, $P$. monteilii, and $P$. putida, but were unlinked to any $\beta$-lactamase-encoding gene. Similar to PoxA, these hypothetical proteins are classified as putative hydrolases or acyltransferases of the $\alpha / \beta$ hydrolase superfamily. The $\alpha / \beta$ hydrolase fold is present in a varied number of proteins that share little in terms of function or sequence homology but show structural 


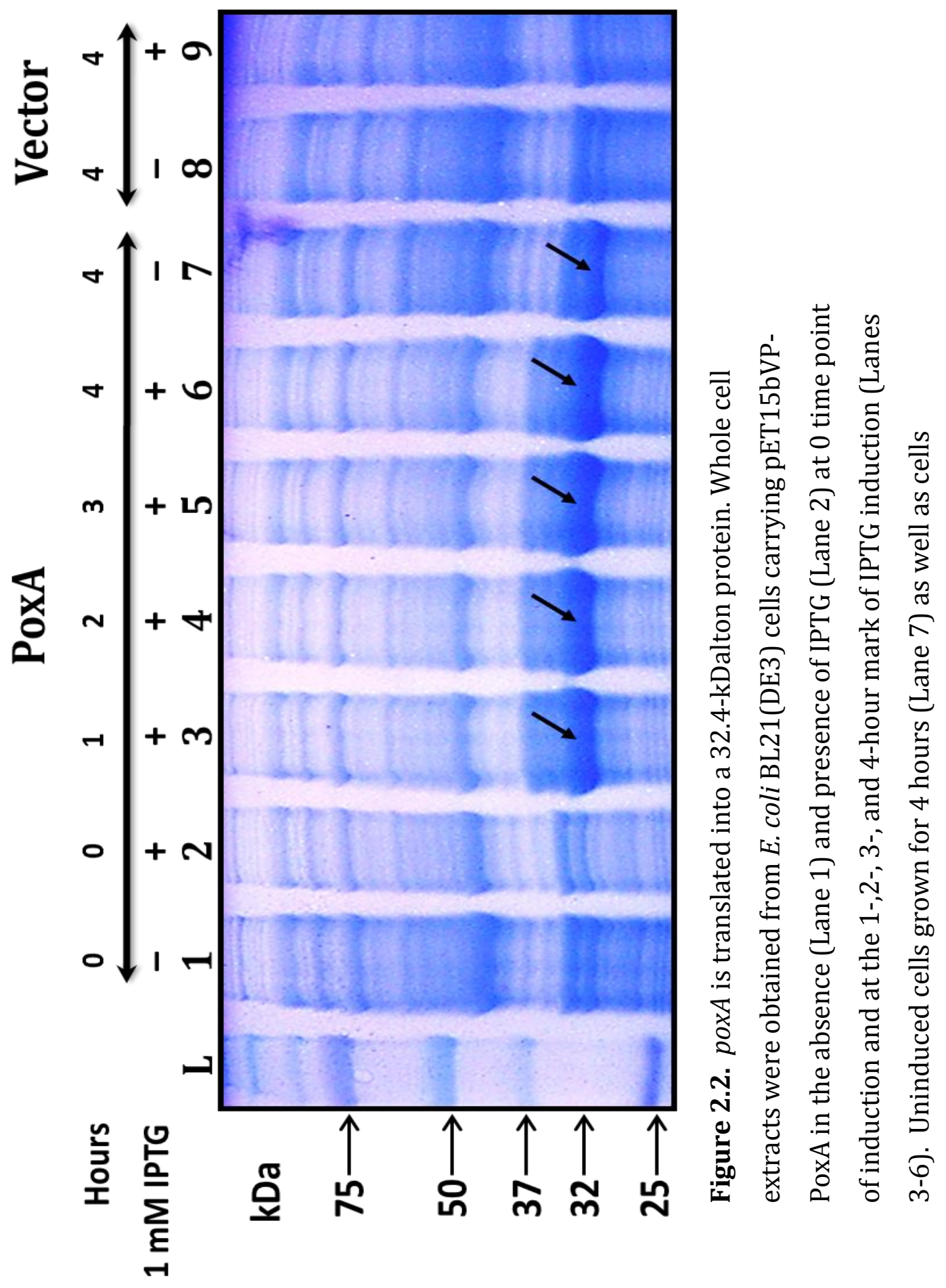


similarity $(777,778)$. Proteins carrying the canonical $\alpha / \beta$ fold include lipases, esterases, proteases, peroxidases as well as transporters and hormone precursors. The role of PoxA and its relation to PoxB are yet to be determined.

\subsection{3 poxA and poxB deletions do not alter $\beta$-lactamase activity or $\beta$-lactam} susceptibility. To investigate the role of poxA and poxB in $P$. aeruginosa, single inframe deletions of each of the genes were generated. The $\beta$-lactamase and $\beta$-lactam susceptibility profiles were compared with that of the parent strain PA01. An in-frame deletion of $\operatorname{poxB}$ was also introduced into a previously generated PA0 $\triangle a m p C$ strain (760). Single deletions of poxA or poxB did not alter the $\beta$-lactamase or susceptibility profiles of the strains as compared to the wild-type PA01 (Table 2.2 and Figure 2.3). Since the AmpC $\beta$-lactamase was present in these backgrounds and potentially obscuring the role of PoxB, the susceptibility and $\beta$-lactamase activity were also examined in strains lacking ampC.

Little to no $\beta$-lactamase was detected in both PA0 0 ampC and PA0 $\triangle a m p C \Delta p o x B$ suggesting AmpC is responsible for the activity observed in wild-type and in deletion $\operatorname{pox} A$ and $\operatorname{pox} B$ strains (Figure 2.3). Interestingly, a deletion of $\operatorname{ampC}$ alone significantly increased susceptibility against the aminopenicillins (ampicillin and amoxicillin), but produced only minor changes against the carbapenems (imipenem and doripenem), with no effect observed against the cephalosporins and the rest of the penicillins (Table 2.2). When overexpressed, however, AmpC was capable of restoring the phenotype and/or increasing resistance against all penicillins and cephalosporins tested (Table 2.3), in most 
cases past clinical breakpoints, while having little to no effect on the carbapenems. These results are in agreement with the known hydrolysis spectrum of AmpC that includes penicillins and cephalosporins but rarely carbapenems $(625,626,779,780)$. Resistance against penicillins and cephalosporins has been observed in the clinical setting and correlates with different levels of AmpC derepression (379-381, 722, 781-783). Lastly, deleting $р о x B$ from an already $a m p C$-deficient background did not further alter susceptibility suggesting either that PoxB plays no role in $\beta$-lactam resistance or that it is not expressed under the tested experimental conditions (Table 2.2).

Table 2.2: Susceptibility profiles of $\operatorname{poxA}$, $\operatorname{pox} B$ and $\operatorname{amp} C$ deletion mutants

\begin{tabular}{|c|c|c|c|c|c|}
\hline \multirow[b]{2}{*}{$\beta$-lactam } & \multicolumn{5}{|c|}{ Minimum Inhibitory Concentration $(\mu \mathrm{g} / \mathrm{ml})$} \\
\hline & PA01 & $\Delta p o x A$ & $\Delta p o x B$ & $\triangle a m p C$ & $\triangle a m p C \Delta p o x B$ \\
\hline Ampicillin & $>256$ & $>256$ & $>256$ & $16-24$ & 24 \\
\hline Ampicillin/sulbactam & $>256$ & $>256$ & $>256$ & $16-32$ & $16-32$ \\
\hline Amoxicillin & $>256$ & $>256$ & $>256$ & $8-12$ & $16-24$ \\
\hline Amoxicillin/clavulanate & $>256$ & $>256$ & $>256$ & 8 & 8 \\
\hline Ticarcillin/clavulanate & $8-12$ & 12 & $8-12$ & 8 & 12 \\
\hline Piperacillin/tazobactam & $3-4$ & 3 & 3 & 3 & $3-4$ \\
\hline Piperacillin & $4-6$ & 4 & 4 & & \\
\hline $4-6$ & & & & & \\
\hline Aztreonam & $1.5-3$ & 2 & 2 & $1.5-3$ & $2-3$ \\
\hline Cefepime & $1-1.5$ & $1-1.5$ & 1 & $0.75-2$ & 1 \\
\hline Cefotaxime & $4-6$ & 6 & 6 & $4-6$ & 4 \\
\hline Ceftazidime & $1-1.5$ & 1.5 & 1.5 & 1.5 & 1 \\
\hline Imipenem & $1-1.5$ & 1.5 & 1.5 & $0.25-0.5$ & $0.38-0.5$ \\
\hline N $\mathrm{K} \sim \ldots \ldots$ & nof $n E$ & $\cap 20 \cap$ ก & n 20 & ก DE ก TE & O DE $\cap 20$ \\
\hline
\end{tabular}




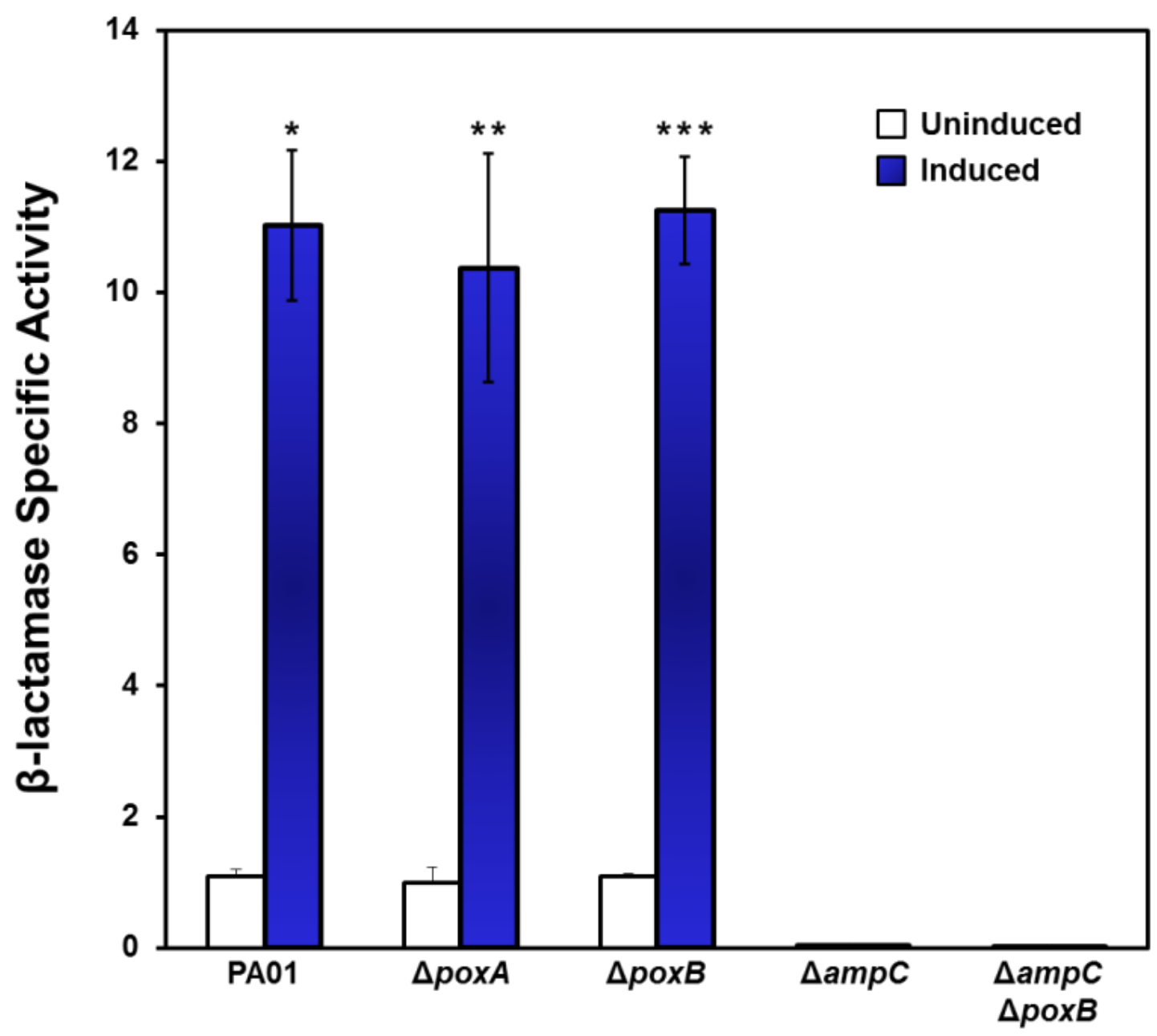

Figure 2.3. $\quad \beta$-lactamase activity profile of pox and ampC deletion mutants. The $\beta$ lactamase activities of PA0 0 poxA, PA0 $\Delta p o x B, \mathrm{PA} 0 \Delta a m p C$ and PA0 $\Delta a m p C \Delta p o x B$ were quantified in the presence (blue bars) and absence (white bars) of the inducer (benzylpenicillin at $50 \mu \mathrm{g} / \mathrm{ml}$ for $1 \mathrm{~h}$ ). Assays were carried out on cell lysate with $100 \mu \mathrm{M}$ of nitrocefin as chromogenic substrate. One milliunit of $\beta$-lactamase was defined as 1 nanomole of nitrocefin hydrolyzed per minute per microgram of protein. * $p$-value< 0.005 for $\beta$-lactamase activity in PA01 induced versus activity in PA01 uninduced; ** $p$ value $<0.05$ for $\beta$-lactamase activity in PA0 poxA induced versus activity in PA0 0 poxA uninduced; *** $p$-value $<0.005$ for $\beta$-lactamase activity in PA0 $\Delta$ poxB induced versus activity in PA0 0 poxB uninduced, as determined by paired $t$-test. 
2.4.4 PoxB is a carbapenemase. To determine the role of PoxB in P. aeruginosa, we overexpressed poxB in a low-copy plasmid under the control of an IPTG-inducible promoter in both PA0 $\triangle a m p C$ and PA0 $\triangle a m p C \triangle p o x B$. Overexpression of PoxB provided little to no resistance against the penicillins and the cephalosporins (Table 2.3). There was, however, a significant decrease in susceptibility (increase in MIC) against the carbapenems, meropenem and doripenem, as determined by E-test (Table 2.3). A fourfold increase in MIC was also observed against meropenem (not tested against doripenem) using the broth microdilution method (Figure 2.4). PoxB, however, did not

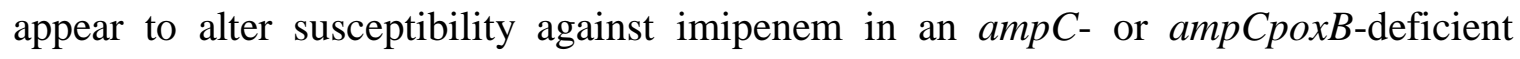
background suggesting PoxB may not be able to hydrolyze this carbapenem.

In carbapenems, the signature $\beta$-lactam ring, composed of one nitrogen and three carbons, is fused to an unsaturated five-atom ring that binds sulfur at position C2 (Figure 2.5). The basic structural differences between the carbapenems (imipenem, doripenem, and meropenem) are imparted by the varying side chains extending from the sulfur at $\mathrm{C} 2$. Meropenem and doripenem have bulkier side groups than imipenem. Additionally, there is a methyl group at $\mathrm{C} 1$, absent from imipenem that could be the basis for the lack of activity of PoxB against this compound. However, beyond structural differences, resistance/susceptibility to imipenem in $P$. aeruginosa must also take into account the role of the outer membrane porin OprD. 


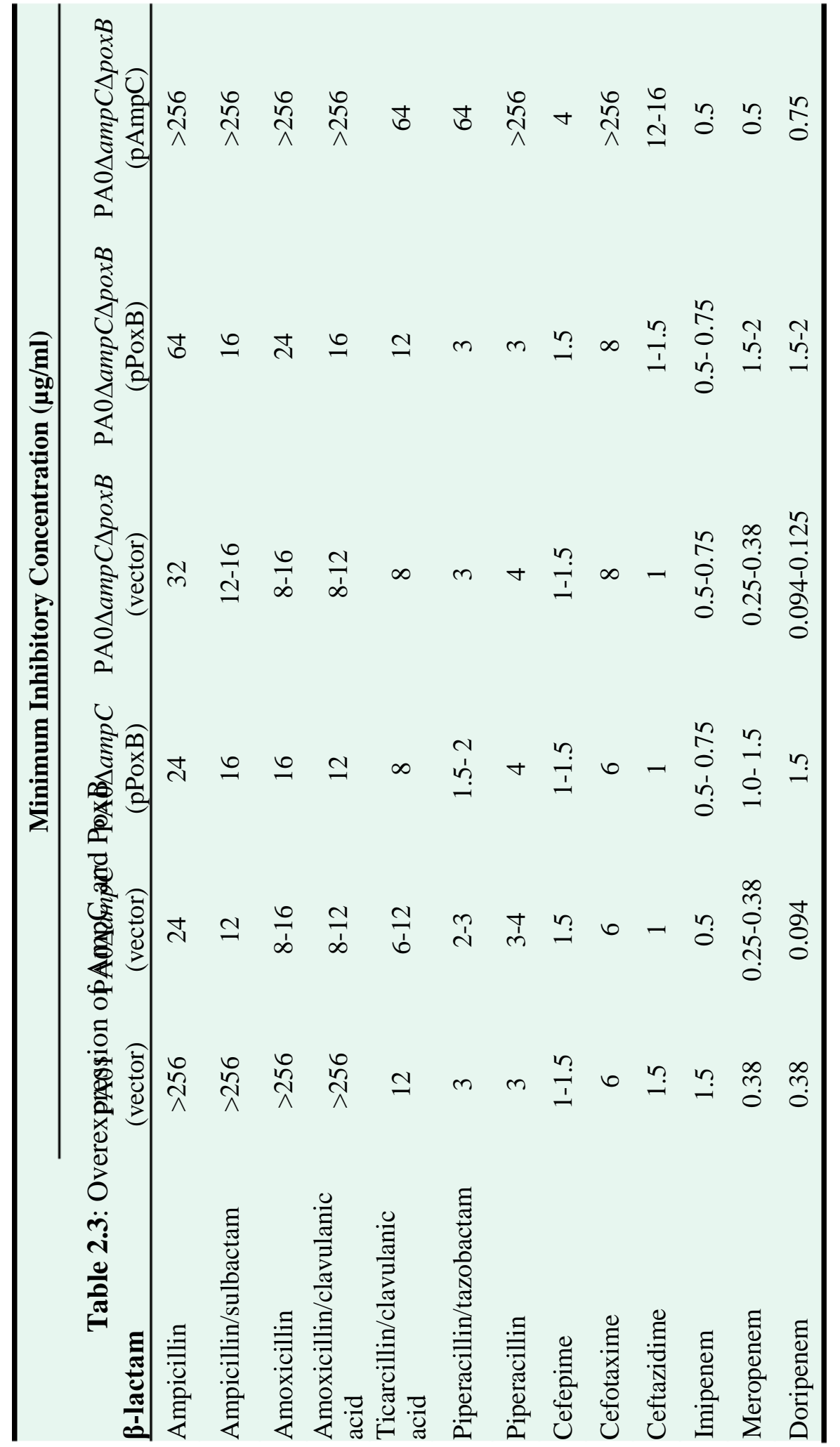




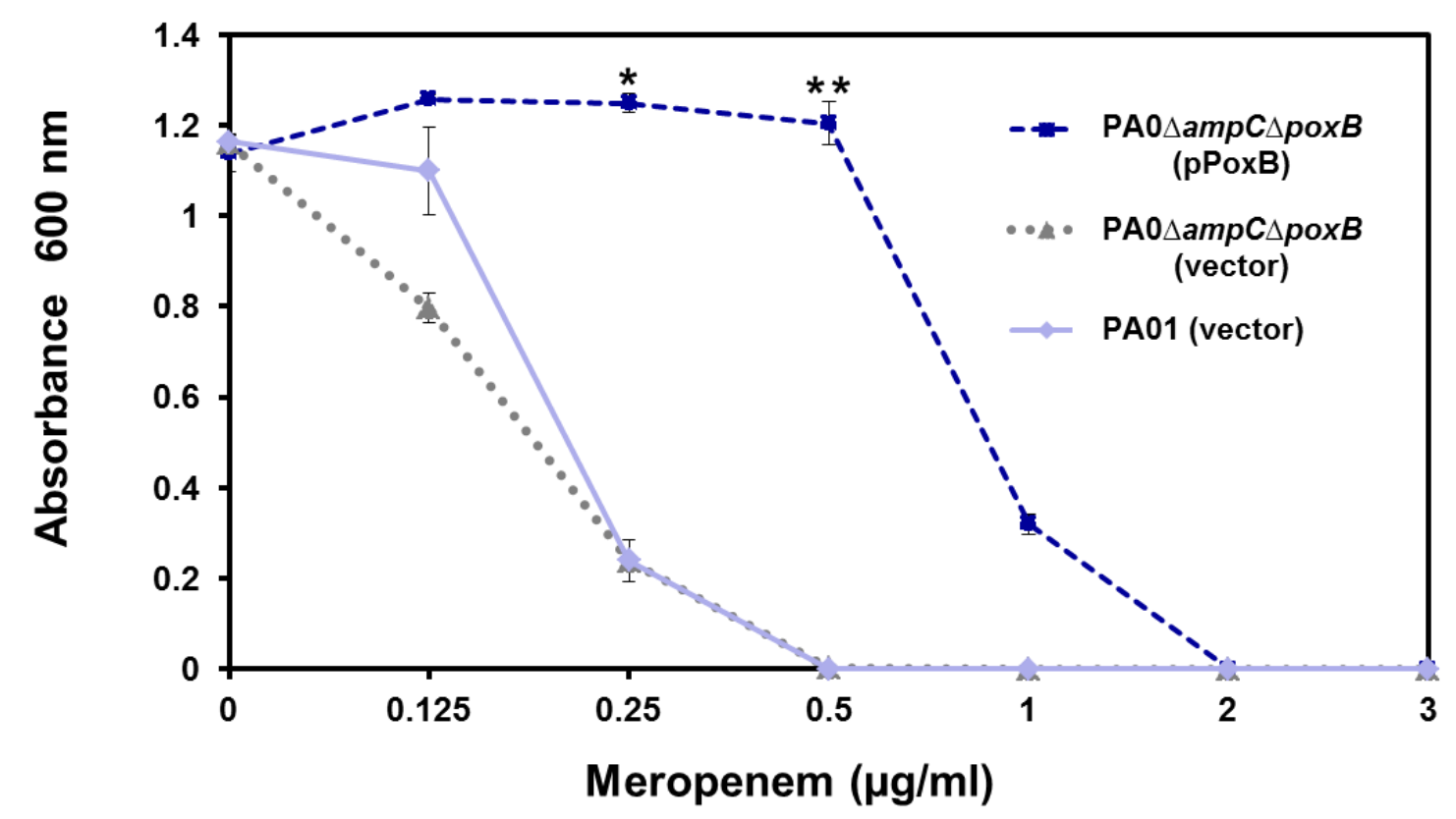

Figure 2.4. Determination of meropenem MIC in a PoxB-overexpressing background. The MIC of meropenem was determined by the microbroth dilution method in PA0 $\triangle a m p C \triangle p o x B$ (pPoxB) (blue square), PA0 $\triangle a m p C \Delta p o x B$ (vector) (grey triangles) and in PA01 (vector) (light blue diamond). Cells were grown $\mathrm{O} / \mathrm{N}$ in the presence of 1 $\mathrm{mM}$ IPTG with increasing concentrations of meropenem $(0-3 \mu \mathrm{g} / \mathrm{ml})$. The final cell density measured at $\mathrm{OD}_{600}$ after 18 hours of growth is reported. * $p$-value $<0.0001$ for $\mathrm{OD}_{600}$ of PA0 $\Delta a m p C \Delta p o x B$ (pPoxB) versus optical density for PA0 $\Delta a m p C \Delta p o x B$ (vector) with $0.25 \mu \mathrm{g} / \mathrm{ml}$ meropenem; ** $p$-value $=0.0005$ for $\mathrm{OD}_{600}$ of PA0 $\triangle a m p C \Delta p o x B$ (pPoxB) versus optical density for PA0 $\triangle a m p C \Delta p o x B$ (vector) with 1 $\mu \mathrm{g} / \mathrm{ml}$ meropenem, as determined by unpaired $t$-test. 


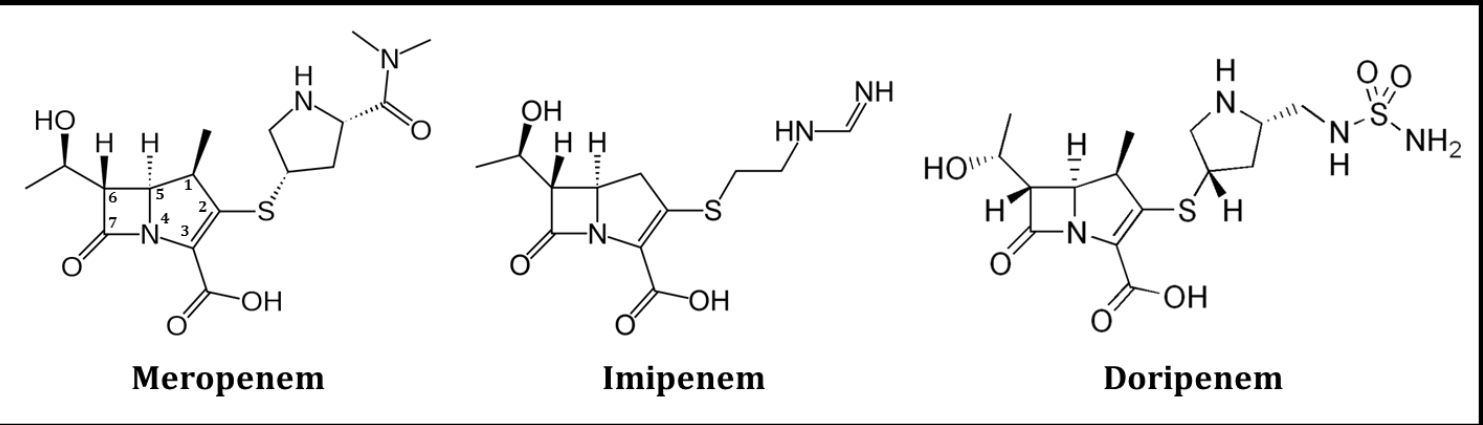

Figure 2.5. Chemical structures of carbapenems: meropenem, imipenem and doripenem. The positions of atoms in the core $\beta$-lactam ring and the carbapenem nucleus structure are numbered and depicted in meropenem.

OprD is a substrate-specific porin that mediates the diffusion of basic amino acids and imipenem into the cell $(757,784,785)$. Imipenem remains a viable and powerful treatment option against $P$. aeruginosa, however, mutations in or loss of $\operatorname{opr} D$ and subsequent resistance to this $\beta$-lactam, often arise after imipenem treatment $(313,315$, 786). In the absence of $\operatorname{oprD}$, the MIC for imipenem increases to $\sim 8-16 \mu \mathrm{g} / \mathrm{ml}$ as the antibiotic cannot penetrate the cell as fast as it could in the wild-type $(305,314,315)$. Imipenem will still enter the cell, most likely through other pores and porins, albeit at a much lower rate (L. Silver, Personal communication). This slower rate of diffusion makes imipenem a better substrate for the AmpC $\beta$-lactamase, which is active in the periplasm of the cell. Thus, in an oprD-deficient background, the activity of imipenem is determined by the expression of $\operatorname{ampC}$ and potentially poxB, but not by the mex efflux system, as imipenem, unlike meropenem and doripenem, is not subject to efflux (312,

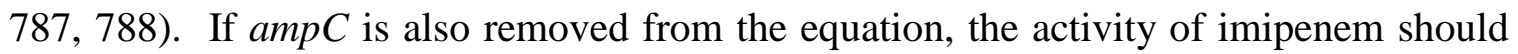
be determined by PoxB. Previous studies have shown PoxB has some affinity against 
imipenem (741), however, PoxB activity against imipenem has not been examined in an oprD-deficient background. We thus set out to test the ability of PoxB to provide resistance against imipenem in the absence of $o p r D$.

As expected, deletion of $\operatorname{oprD}$ alone significantly decreased susceptibility of the strains against the carbapenems, with the greatest increase in MIC observed for meropenem followed by imipenem (Table 2.4). In such a background, the MIC is presumably dictated by the action of $\mathrm{AmpC}$ and $\mathrm{PoxB}$, along with efflux for meropenem and doripenem. AmpC plays a major role in providing resistance here, since in its absence, the strain becomes very susceptible to imipenem, but less so to meropenem and doripenem (Table 2.4). This work corroborates previous studies where imipenem resistance was found to be dependent upon both the loss of $\operatorname{oprD}$ and the expression of AmpC $\beta$-lactamase (305). Similarly, in our study AmpC afforded greater protection against imipenem in a less permeable background, e.g., loss of oprD (Table 2.4). Specifically the loss of $\operatorname{orD}$ raised the MIC from 1 to $8 \mu \mathrm{g} / \mathrm{ml}$, with a further deletion of ampC restoring susceptibility to that of wild-type levels (MIC $=1 \mu \mathrm{g} / \mathrm{ml}$ ). Resistance against meropenem and doripenem in our study, was found to be affected mainly by the loss of $\operatorname{opr} D$, with only minor changes observed upon deletion of $\operatorname{ampC}$, suggesting these two carbapenems are not good substrates for AmpC and/or they are efficiently effluxed. In agreement with these results, our single $\operatorname{ampC}$ deletion mutant also showed a slight increase in susceptibility against imipenem but no change against meropenem (Table 2.2). 


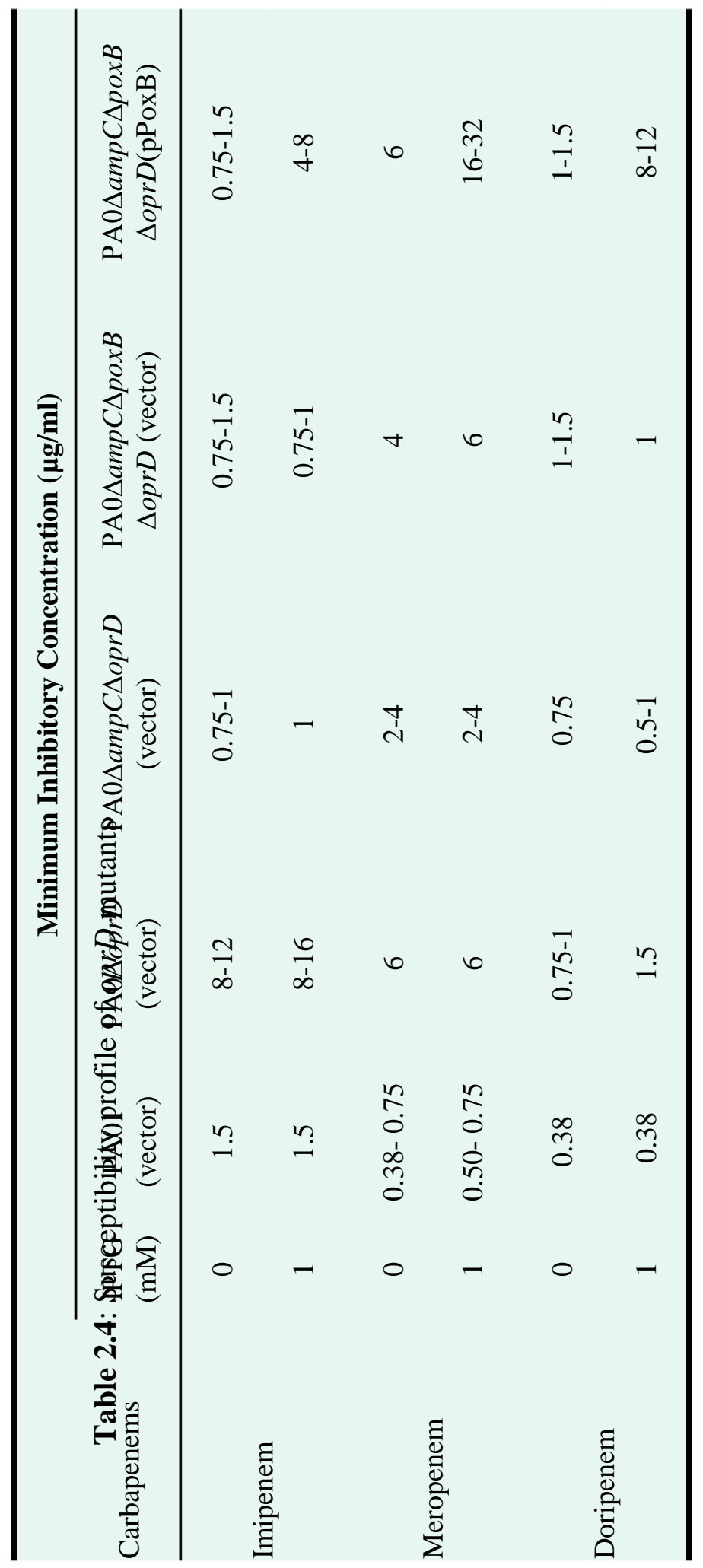


A further deletion of poxB from an ampCoprD-deletion mutant did not alter susceptibility (Table 2.4). However, overexpression of PoxB in an oprDampCpoxBdeficient background significantly increased the MICs of all the carbapenems, including imipenem (Table 2.4), showing that in fact PoxB can hydrolyze all of these $\beta$-lactams. In particular, the increase in MIC observed for meropenem reached the CLSI clinical resistant breakpoint for this carbapenem ( $\geq 8 \mu \mathrm{g} / \mathrm{ml}$ ) (767). Determination of MIC by the microbroth dilution method corroborated the E-test results (Figure 2.6 A-D).

Previous biochemical studies showed that the supposed preferred substrates of oxacillinases, namely oxacillin and cloxacillin, were hydrolyzed very poorly, if at all by PoxB (741). Additionally, PoxB exhibited very low affinity towards most of the substrates tested, which included benzylpenicillin, ampicillin, piperacillin and cephalothin. PoxB, however, did exhibit the highest affinity towards imipenem, although its ability to hydrolyze it was low (741). Against meropenem, PoxB exhibited low affinity and weak hydrolysis, while overexpression in trans did afford the cells a decreased in susceptibility (increase in MIC) against meropenem but not imipenem (741). Similarly, our work shows that PoxB does not significantly hydrolyze penicillin and cephalosporin $\beta$-lactams. In contrast to previous work, however, we show PoxB is capable of hydrolyzing not only meropenem but also imipenem and doripenem producing a decrease in susceptibility (higher MIC) towards these $\beta$-lactams when overexpressed. Although PoxB biochemically may not have the greatest affinity towards some of these carbapenems, its expression does afford the cell greater protection against these $\beta$ lactams. PoxB thus is a carbapenemase with a narrow spectrum of hydrolysis. 
A

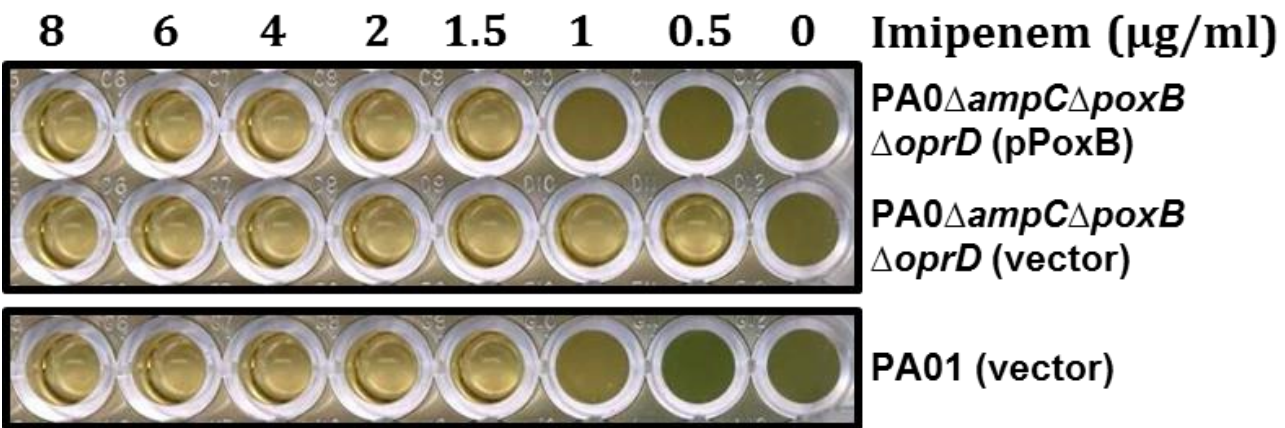

B

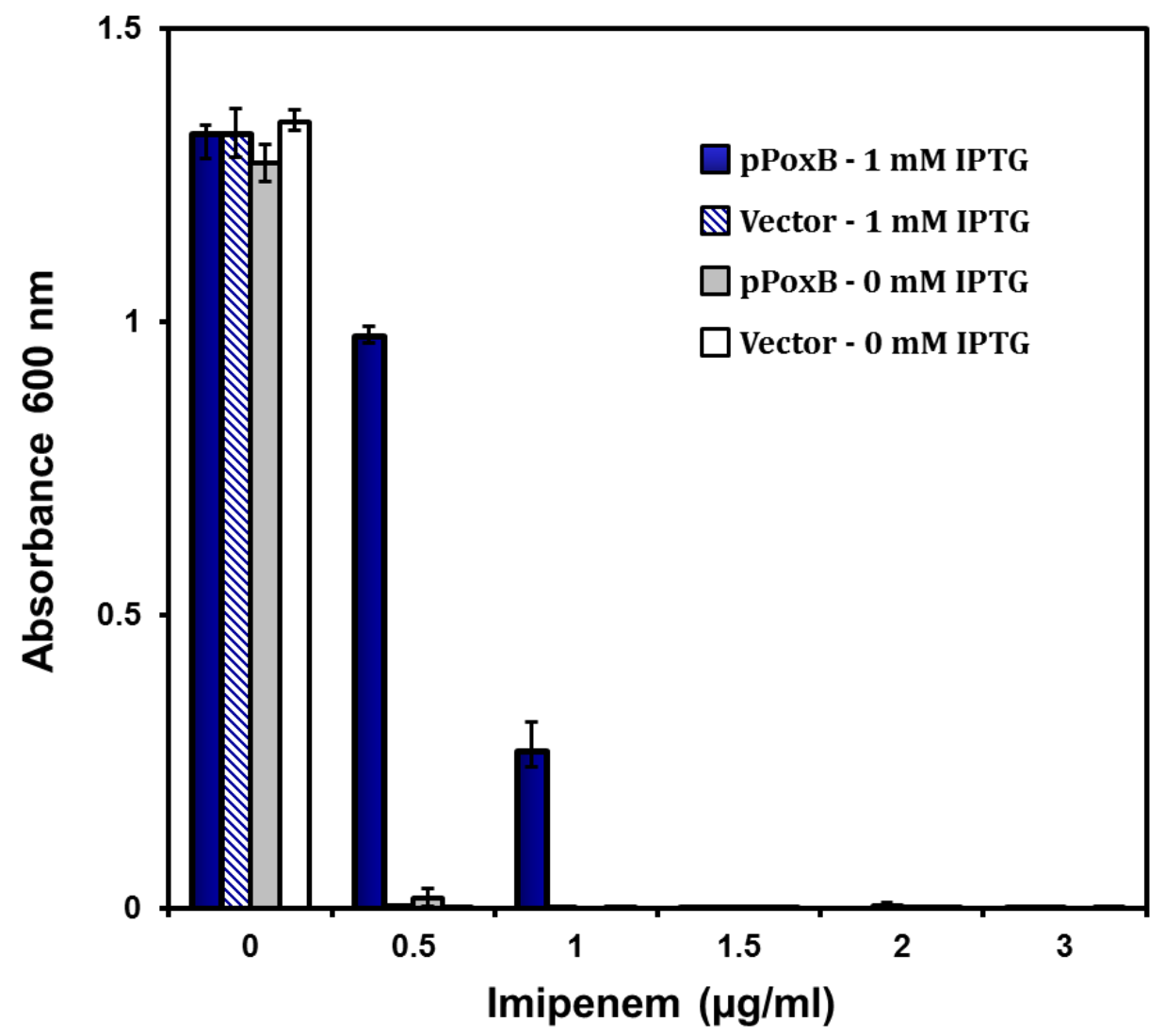


C

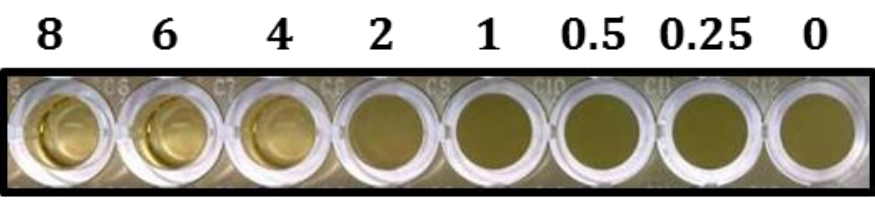

Meropenem $(\mu \mathrm{g} / \mathrm{ml})$

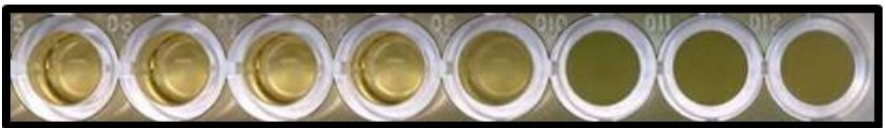

$\mathrm{PAO} \triangle \mathrm{ampC} \triangle \mathrm{poxB}$

$\triangle o p r D(p P o x B)$

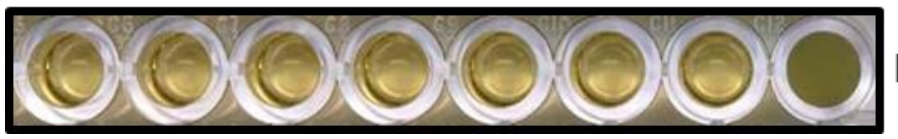

$\mathrm{PAO} \triangle \mathrm{ampC} \triangle \mathrm{pox} B$

$\triangle o p r D$ (vector)

PA01 (vector)

D

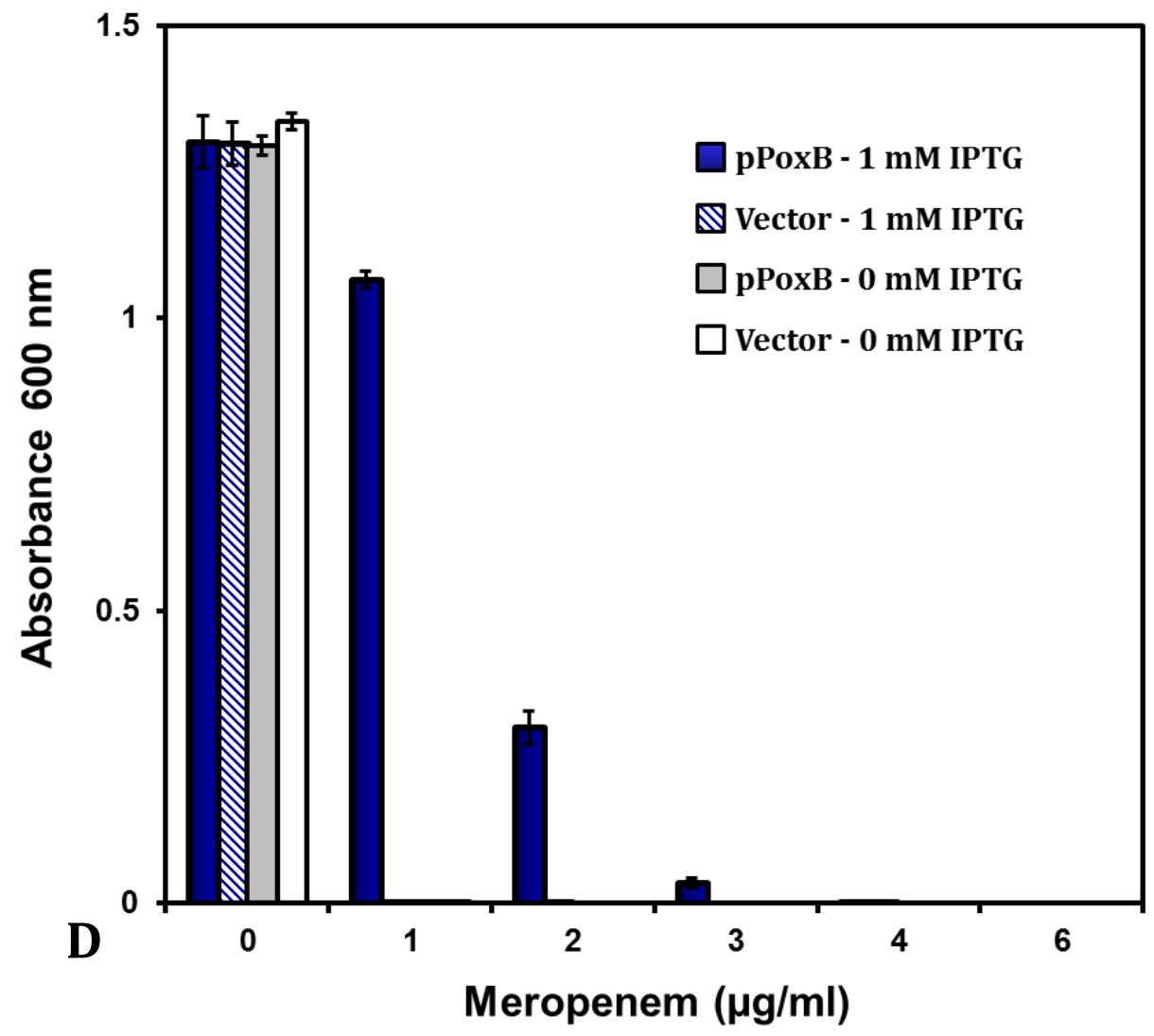

Figure 2.6. Determination of carbapenem MICs in $P$. aeruginosa oprD-deficient strains by the microbroth dilution method. (A) MIC of imipenem in the presence of $1 \mathrm{mM}$ IPTG. (B) Optical density of cells grown $\mathrm{O} / \mathrm{N}$ with increasing concentrations of imipenem (0-3 $\mu \mathrm{g} / \mathrm{ml})$ in the presence and absence of IPTG $(1 \mathrm{mM})$. (C) MIC of meropenem in the presence of $1 \mathrm{mM}$ IPTG. (D) Optical density of cells grown $\mathrm{O} / \mathrm{N}$ with increasing concentrations of meropenem $(0-3 \mu \mathrm{g} / \mathrm{ml})$ in the presence and absence of IPTG $(1 \mathrm{mM})$. 
2.4.5 Expression of PoxB does not alter efflux. It is expected that the significant decrease in susceptibility against carbapenems observed above is caused by the expression of PoxB and not by an increase in the activity of efflux pumps, especially as imipenem is not the subject of efflux. Nonetheless, the activity of efflux pumps was checked by examining the susceptibility of PoxB-overexpressing strains against known efflux substrates. As expected, the MIC values did not significantly differ between wildtype and PoxB-overexpressing strains (Table 2.5), suggesting efflux systems were neither enhanced nor compromised by the increased expression of PoxB. Thus, these results clearly argue that the increase in carbapenem MIC (Tables 2.3 and 2.4) is provided by the expression of PoxB.

Table 2.5: MIC in the presence of efflux substrates

\begin{tabular}{|c|c|c|c|}
\hline \multirow[b]{2}{*}{ Antibiotic } & \multicolumn{3}{|c|}{ Range $(\mu \mathrm{g} / \mathrm{ml})$} \\
\hline & PA01 vector & $\begin{array}{c}\text { PA0 } 0 \text { amp } C \Delta p o x B \\
\text { (vector) }\end{array}$ & $\begin{array}{c}\mathrm{PA} 0 \Delta a m p C \Delta p o x B \\
(\mathrm{pPoxB})\end{array}$ \\
\hline Chloramphenicol & 32 & 32 & 32 \\
\hline Trimethoprim & 150 & 150 & 100 \\
\hline Novobiocin & 512 & 512 & 512 \\
\hline Tetracycline & 8 & 8 & 8 \\
\hline Ofloxacin & 1 & 1.5 & 1 \\
\hline Norfloxacin & 0.75 & 0.75 & 0.75 \\
\hline Ciprofloxacin & 0.19 & 0.19 & 0.19 \\
\hline Levofloxacin & 0.38 & 0.38 & 0.38 \\
\hline
\end{tabular}

2.4.6 Inhibitors of PoxB. $\beta$-lactam antibiotics are often co-administered with $\beta$ lactamase inhibitors to improve the efficacy of treatment against $\beta$-lactamase-expressing pathogens $(423,428,557) . \beta$-lactamase inhibitors are $\beta$-lactams themselves with only 
minor antibacterial activity (789-792) that can either reversibly or irreversibly inhibit the $\beta$-lactamase thus preventing it from hydrolyzing the co-administered antibiotic $(423,793$ 796). Currently, three irreversible $\beta$-lactamase inhibitors, clavulanic acid $(797,798)$, sulbactam $(426,799,800)$ and tazobactam $(801,802)$ are approved for clinical use. Their activity is limited to most class A enzymes, being generally ineffective against classes B, $\mathrm{C}$ and $\mathrm{D} \beta$-lactamases $(428,467)$. PoxB has been shown to be only poorly inhibited by these (741). A new $\beta$-lactamase inhibitor, termed avibactam, with a broader spectrum of activity has been developed and is currently undergoing phase III clinical trials in combination with ceftazidime (803-805). Unlike the aforementioned compounds, avibactam is not a $\beta$-lactam, although its structure closely resembles one (804). The ability of avibactam to inhibit PoxB activity was examined and compared to that of weak inhibitors of oxacillinases, namely tazobactam, sulbactam and clavulanic acid.

Subinhibitory concentrations of imipenem $(0.5 \mu \mathrm{g} / \mathrm{ml})$ and meropenem $(1 \mu \mathrm{g} / \mathrm{ml})$ that only allowed PA0 $\operatorname{ampC} \triangle \operatorname{poxB} \Delta$ oprD to grow when PoxB was overexpressed (Figure 2.6A and 2.6C) were used to examine the inhibitory action of tazobactam, sulbactam and clavulanic acid against PoxB. A slight inhibition of growth was apparent with tazobactam in the presence of imipenem, and to a smaller extent with sulbactam (Figure 2.7). In the presence of clavulanic acid, growth either remained unaltered or was slightly enhanced. Overall, as expected, the ability of PoxB-overexpressing strains to grow on imipenem or meropenem was not significantly affected by the presence of these inhibitors at the tested clinical concentrations, indicating these compounds did not inhibit PoxB activity (Figure 2.7). 


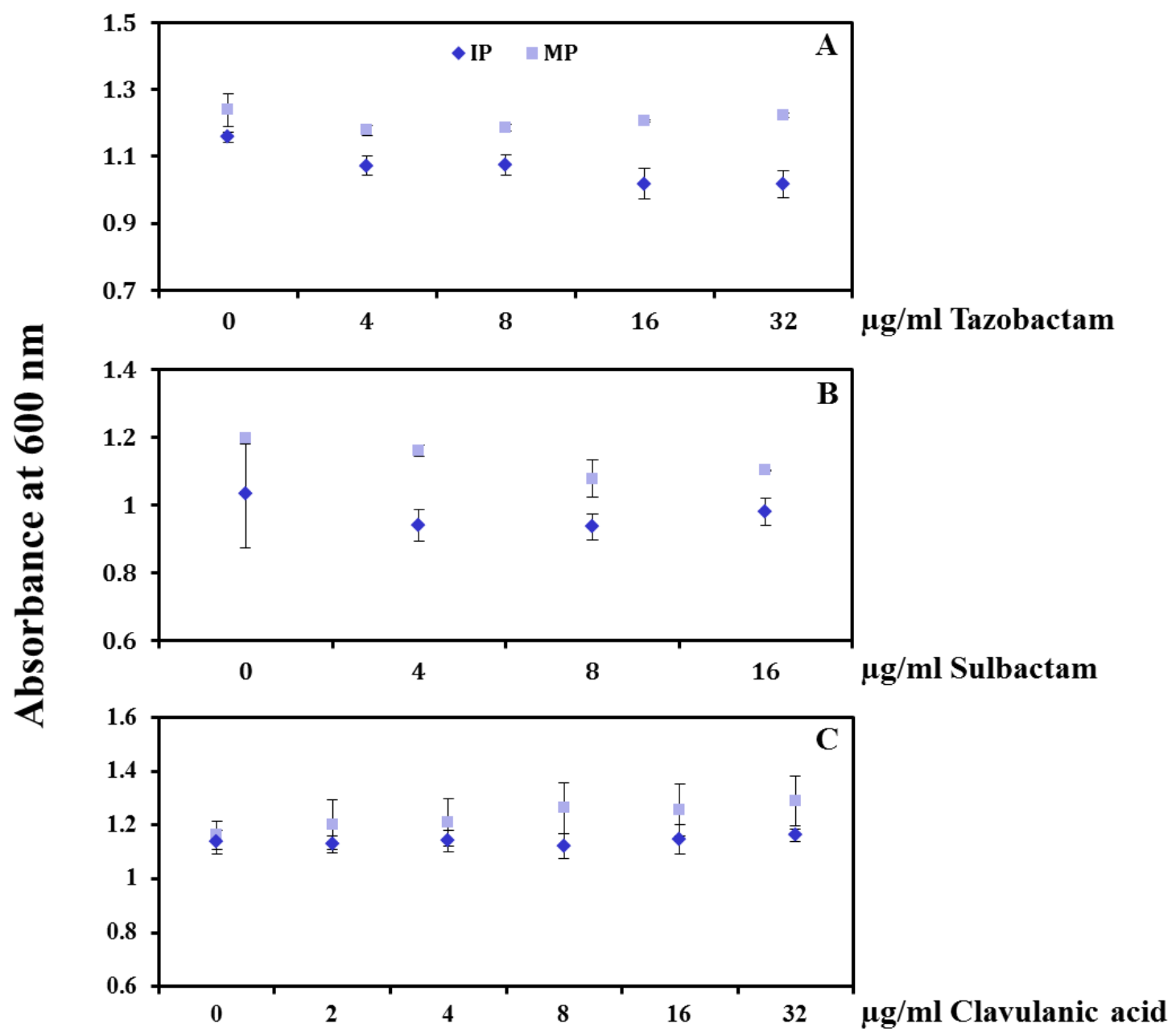

Figure 2.7. Effect of tazobactam, sulbactam and clavulanic acid on PoxB activity. The ability of PA0 $\operatorname{PampC} \triangle \operatorname{poxB} \Delta o p r D(\mathrm{pPoxB})$ to grow in the presence of subinhibitory concentrations of imipenem $(0.5 \mu \mathrm{g} / \mathrm{ml})$ or meropenem $(1 \mu \mathrm{g} / \mathrm{ml})$ was examined in the presence of the class A enzyme inhibitors tazobactam (A), sulbactam (B) and clavulanic acid $(\mathbf{C}) . \mathrm{OD}_{600}$ readings after $\mathrm{O} / \mathrm{N}$ growth with aeration at $37^{\circ} \mathrm{C}$ are reported. 
Similarly, the ability of avibactam to inhibit PoxB activity was tested by growing PoxB-expressing strains in CAMH agar with IPTG in the presence and absence of avibactam at a clinically used concentration $(4 \mu \mathrm{g} / \mathrm{ml})$. The presence of PoxB increases the MIC against imipenem, meropenem and doripenem (as detailed in Section 2.4.4, Table 2.4) since PoxB can hydrolyze these carbapenems. Inhibition of PoxB by avibactam, should then produce a decrease in MIC against the carbapenems. Our results show, however, that the MIC of PoxB-expressing cells was not altered in the presence of this inhibitor (Table 2.6), suggesting avibactam does not significantly inhibit PoxB activity.

Table 2.6: Effect of avibactam on carbapenem MIC

\begin{tabular}{|c|c|c|c|}
\hline \multirow[b]{2}{*}{ Carbapenems } & \multicolumn{3}{|c|}{ Minimum Inhibitory Concentration $(\mu \mathrm{g} / \mathrm{ml})$} \\
\hline & CAMH & $\begin{array}{c}\text { PA0 } \Delta a m p C \Delta p o x B \\
\Delta o p r D \text { (vector) }\end{array}$ & $\begin{array}{c}\text { PA0 } \Delta a m p C \Delta p o x B \\
\Delta o p r D(\text { pPoxB })\end{array}$ \\
\hline \multirow{3}{*}{ Imipenem } & Alone & 0.25 & 0.75 \\
\hline & IPTG & 1.5 & 6 \\
\hline & $\mathrm{IPTG}+\mathrm{AV}$ & 1.5 & 4,6 \\
\hline \multirow{3}{*}{ Meropenem } & Alone & 6 & 4 \\
\hline & IPTG & 4,6 & 32 \\
\hline & $\mathrm{IPTG}+\mathrm{AV}$ & 6,4 & 32 \\
\hline \multirow{3}{*}{ Doripenem } & Alone & $0.75-1$ & $0.75-1$ \\
\hline & IPTG & $0.75-1$ & 8 \\
\hline & $\mathrm{IPTG}+\mathrm{AV}$ & $1-1.5$ & 8 \\
\hline
\end{tabular}

AV- Avibactam at $4 \mu \mathrm{g} / \mathrm{ml}$ 
The inhibitory action of these compounds was also tested directly on cell lysate from PoxB-overexpressing cells by quantification of the $\beta$-lactamase activity. Concentrations as high as $700 \mu \mathrm{M}$ of tazobactam failed to reduce the $\beta$-lactamase activity in cell lysates from PA0 $\triangle а т р C \triangle \operatorname{poxB}(\mathrm{pPoxB})$ cells, while only a minor reduction was observed with $4000 \mu \mathrm{M}$ of sulbactam (Figure 2.8A and 2.8B). A general downward trend of inhibition was observed with clavulanic acid starting at $50 \mu \mathrm{M}$, but a $50 \%$ inhibition was not reached even at high levels of the inhibitor $(4000 \mu \mathrm{M})$. Avibactam decreased PoxB activity by more than half (Figure 2.8C) at a concentration of $2000 \mu \mathrm{M}(530.488$ $\mu \mathrm{g} / \mathrm{ml}$ ), which is 75 -fold higher than that of the highest concentration of avibactam used in clinical studies $(0.5-7 \mu \mathrm{g} / \mathrm{ml})(806-808)$. Notwithstanding that avibactam produced greater inhibition than the other tested inhibitors, the large quantities of the compound needed to inhibit PoxB activity, makes avibactam a weak inhibitor.

Avibactam at a concentration of $4 \mu \mathrm{g} / \mathrm{ml}$ has proven sufficient and efficient at increasing susceptibility and/or eliminating resistance against a number of $\beta$-lactams in a large number of clinical isolates (809-811). For instance, in a study of 1466 Gramnegative isolates collected from patients hospitalized with pneumonia, bloodstream, intraabdominal and urinary tract infections, avibactam in combination with ceftazidime effectively reduced MICs from 16 or 32 to $0.25 \mu \mathrm{g} / \mathrm{ml}$ in $90 \%$ of Enterobacterial isolates (809). About $99.8 \%$ of such isolates exhibited an MIC of $\leq 4 \mu \mathrm{g} / \mathrm{ml}$, the CLSI clinical susceptible breakpoint for ceftazidime (809). Similarly, avibactam reduced the MIC anywhere from 16 or 32 to $4-8$ in $90 \%$ of P. aeruginosa isolates, with an average of 


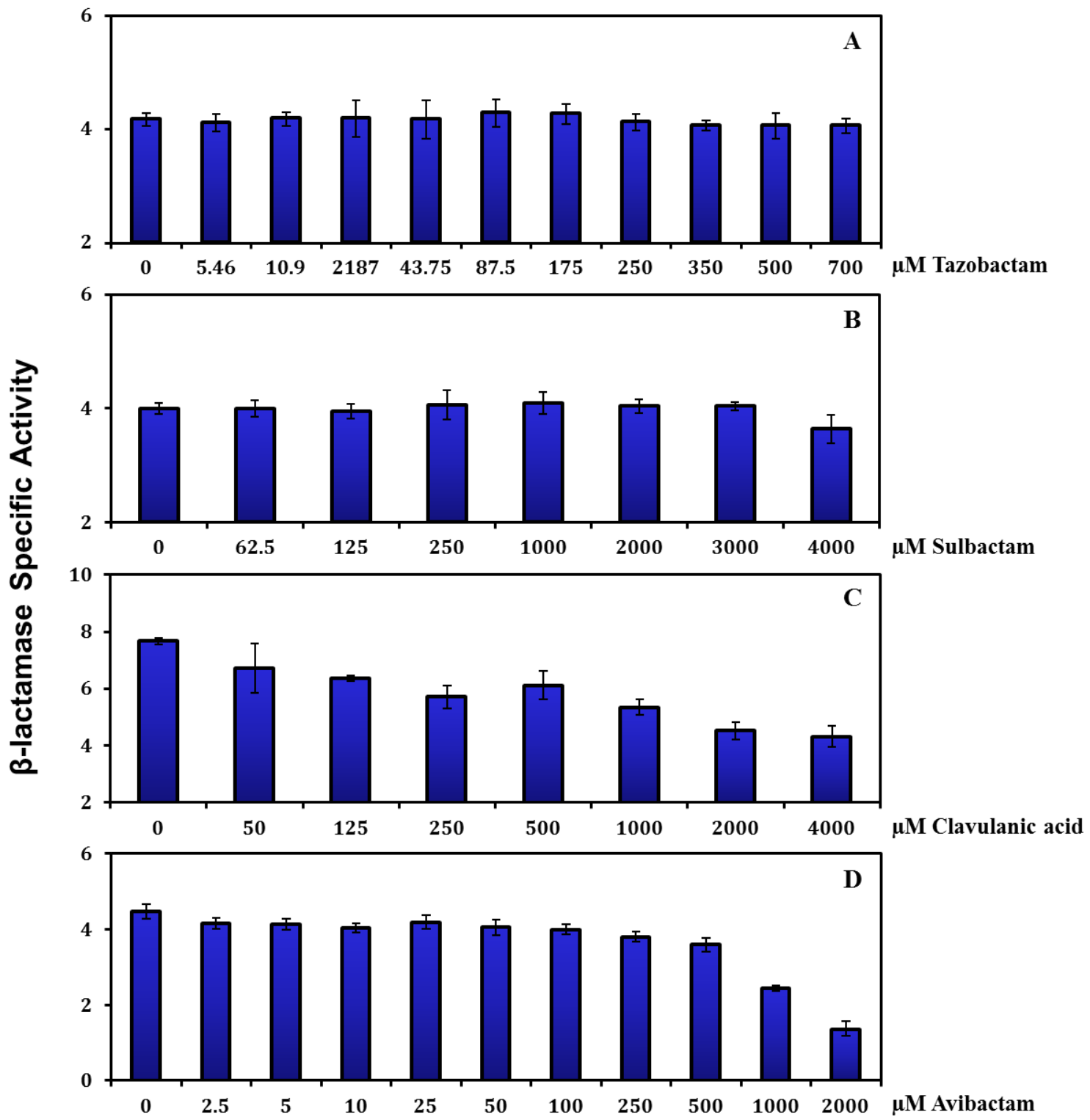

Figure 2.8. PoxB $\beta$-lactamase activity in the presence of tazobactam, sulbactam, clavulanic acid and avibactam. The $\beta$-lactamase assay was performed on lysate from IPTG-induced (1 mM) PA0 $\Delta a m p C \Delta p o x B$ (pPoxB) cells. Lysate was incubated for $20 \mathrm{~min}$ with increasing concentrations of tazobactam (A), sulbactam (B), clavulanic acid (C), and avibactam (D). Chromacef was used as a chromogenic substrate. One milliunit of $\beta$ lactamase was defined as 1 nanomole of chromacef hydrolyzed per minute per microgram of protein. 
$96.8 \%$ of all isolates showing a ceftazidime/avibactam MIC of $\leq 8 \mu \mathrm{g} / \mathrm{ml}$, the CLSI susceptible breakpoint for ceftazidime in this organism (809).

Although avibactam has shown variable activity against class D enzymes, it remains a more potent and effective inhibitor of class A $(805-808,812)$ and $\mathrm{C}(805,807$, 812, 813) enzymes, including the problematic class A Klebsiella pneumoniae carbapenemases or KPCs $(814,815)$. A study found that avibactam potentiated the activity of both ceftazidime and aztreonam against meropenem-nonsusceptible Gramnegative bacilli expressing multiple $\beta$-lactamases of the class $\mathrm{A}$ and $\mathrm{C}$ types (811). Avibactam, however, was less successful at reducing the MIC of Acinetobacter baumannii isolates carrying OXA-23-like genes $\left(\mathrm{MIC}_{90}\right.$ fold reduction of 2-4 vs fold reduction of 32-256 against class A, B and C enzymes in that particular study) (811). Similarly, avibactam did not significantly improve the activity of cefepime, ceftazidime or imipenem in A. baumannii isolates carrying PER-1, OXA-51 and OXA-58, with only a minor increase in susceptibility ( $\sim 2$-fold decrease in resistance) observed (816). The efficacy of ceftazidime against an OXA-5-expressing $P$. aeruginosa clinical isolate was also not improved by the presence of avibactam (817).

OXA-48 appears to be just one of a few class D enzymes against which avibactam is active. A study showed that $K$. pneumoniae and E. coli isolates harboring the carbapenemase OXA-48 exhibited significant reduction in the MIC of imipenem, cefepime and ceftazidime when combined with avibactam, leading to susceptibility in $90 \%$ of isolates (816). In another study, the number of $K$. pneumoniae OXA-48producers showing resistance was eliminated and/or susceptibility was enhanced by the 
addition of avibactam (818). However, a kinetics study also found the rates of reaction of avibactam with OXA-48 and OXA-10 to be much slower than that of the other tested class A and C enzymes (804). The $P$. aeruginosa OXA-10 was actually found to be poorly inhibited by avibactam (804). Although avibactam has not been tested against very many OXA enzymes and while the structure and hydrolytic activities of oxacillinases is wide and varied, the emerging trend suggests that avibactam is not a very good inhibitor of class D enzymes. It is thus not surprising avibactam is only a weak inhibitor of PoxB (OXA-50).

2.4.7 Inhibition by $\mathrm{NaCl}$. Oxacillinases are known to be inhibited by $\mathrm{NaCl}$ in vitro $(669,674,682)$. This characteristic is commonly attributed to the presence of a Tyr residue at position 144, with replacement by Phe leading to resistance to inhibition (703, 819). Inhibition is often showed directly by measuring enzyme activity ( $\beta$-lactamase assay), or indirectly by determining MIC changes (820). Previous work showed inhibition of purified PoxB by $\mathrm{NaCl}\left(\mathrm{IC}_{50}, 50 \mathrm{mM}\right)$, which not surprisingly carries $\mathrm{Tyr}$ at position 144 (741). We here further test if the previously reported $\mathrm{NaCl}$ inhibition of PoxB can translate into quantifiable changes that can affect the ability of PoxB to provide resistance. To that end, PA0 $\triangle a m p C \Delta \operatorname{poxB} \Delta \operatorname{oprD}(\mathrm{pPoxB})$ was grown in the presence of subinhibitory concentrations of imipenem $(0.5 \mu \mathrm{g} / \mathrm{ml})$ or meropenem $(1 \mu \mathrm{g} / \mathrm{ml})$ that only allowed growth if PoxB was expressed. Growth was monitored $\mathrm{O} / \mathrm{N}$ and inhibition of growth was used as an indirect indicator of PoxB activity. 
$\mathrm{NaCl}$ produced a small, but steady increase in growth up to a concentration of 50 and $100 \mathrm{mM}$ in the presence of meropenem or imipenem, respectively (Figure 2.9). A slight growth decrease was detected in the presence of meropenem at $\mathrm{NaCl}$ concentrations of $100 \mathrm{mM}$ or higher. Concentrations of $\geq 200 \mathrm{mM}$ decreased growth below the level of untreated cells, suggesting $\mathrm{NaCl}$ was compromising PoxB-dependent growth (Figure 2.9). In the presence of imipenem, growth never fell below that of untreated cells, but the enhancement of growth seen with up to $100 \mathrm{mM}$ was lost at $\mathrm{NaCl}$ concentrations of $200 \mathrm{mM}$ or higher, also suggesting that this higher range of solute was affecting the ability of PoxB to hydrolyze the $\beta$-lactam.

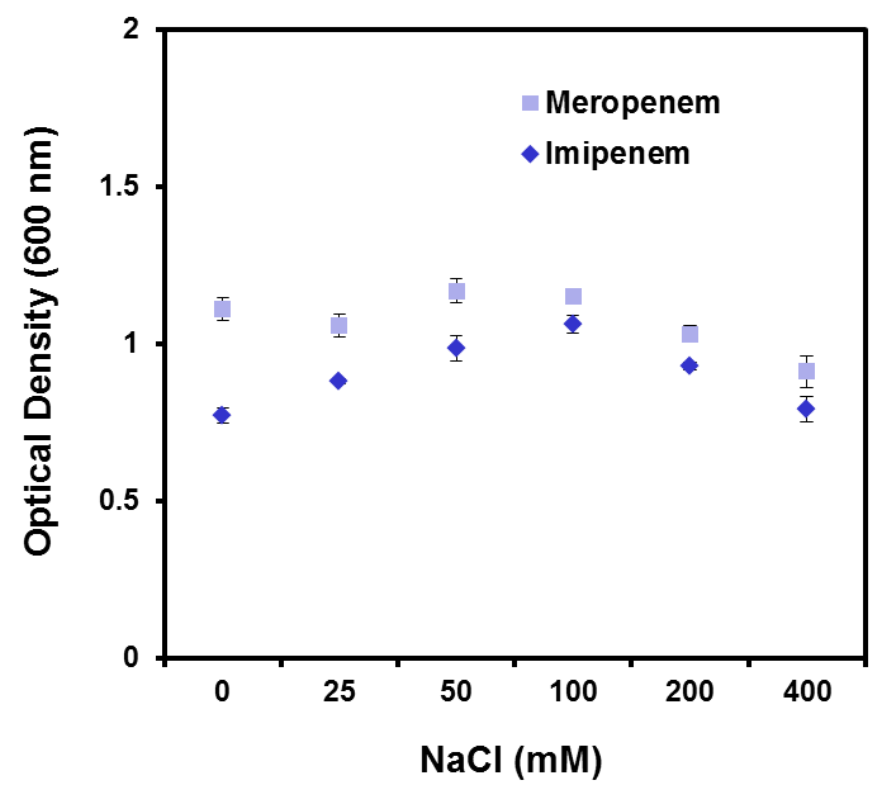

Figure 2.9. PoxB activity in the presence of $\mathrm{NaCl}$. Inhibition of $\mathrm{PoxB}$ by $\mathrm{NaCl}$ was examined by growing PA0 $\Delta a m p C \Delta \operatorname{poxB} \Delta o p r D$ (pPoxB) $\mathrm{O} / \mathrm{N}$ in the presence of subinhibitory concentrations of meropenem or imipenem with IPTG $(1 \mathrm{mM})$ and with increasing concentrations of $\mathrm{NaCl}$. The subinhibitory concentrations of meropenem and imipenem selected were those previously shown to allow growth of only PoxBoverexpressing cells. 
Although small inhibition of growth is detected with $\mathrm{NaCl}$, particularly at higher concentrations, $\mathrm{NaCl}$ did not completely abolish growth of PA0 $\Delta a m p C \Delta$ poxB $\Delta$ oprD $(\mathrm{pPoxB})$ in LB or CAMH broth containing meropenem or imipenem (Figure 2.9). In short, PoxB activity did not appear to be inhibited by $\mathrm{NaCl}$ to levels significant enough to prevent hydrolysis of the carbapenems and subsequently growth in media containing these $\beta$-lactams. The slight decrease in growth observed, however, was determined to be mostly unrelated to osmolarity, since cells grown in the presence of $\mathrm{NaCl}$ only lysed at a concentration of $800 \mathrm{mM}$ of solute (Figure 2.10). A concentration of $400 \mathrm{mM}$ does appear to slightly affect osmolarity leading to a <2-fold reduction in absorbance as compared to growth in $200 \mathrm{mM}$ of $\mathrm{NaCl}$.

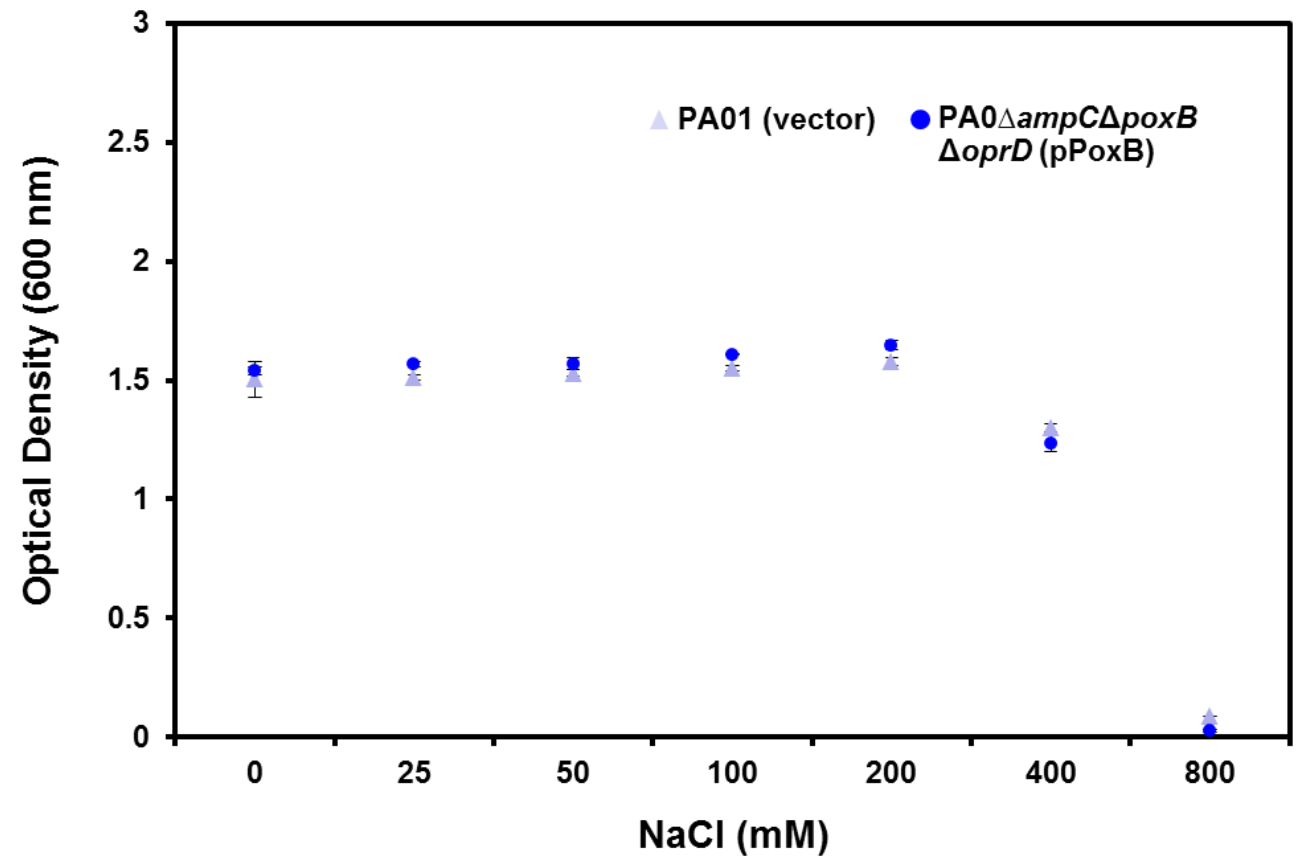

Figure 2.10. Determination of $\mathrm{MIC}$ for $\mathrm{NaCl}$. The concentration of $\mathrm{NaCl}$ needed to lyse cells was determined by growing PA01 (vector) and PA0 $\Delta a m p C \Delta p o x B \Delta o p r D$ (pPoxB) $\mathrm{O} / \mathrm{N}$ with increasing concentrations of $\mathrm{NaCl}$ in $\mathrm{CAMHB}$ media. 
Inhibition by $\mathrm{NaCl}$, however, was apparent on cell lysate from PA0 $\Delta a m p C \Delta p o x B$ (pPoxB) cells (Figure 2.11). Specifically, $\beta$-lactamase activity decreased with increasing concentrations of $\mathrm{NaCl}$. The phenotype was readily observed in the absence of IPTG. However, in the presence of IPTG, where the levels of PoxB are significantly higher, $\mathrm{NaCl}$ inhibition could not be appreciated (Data not shown).

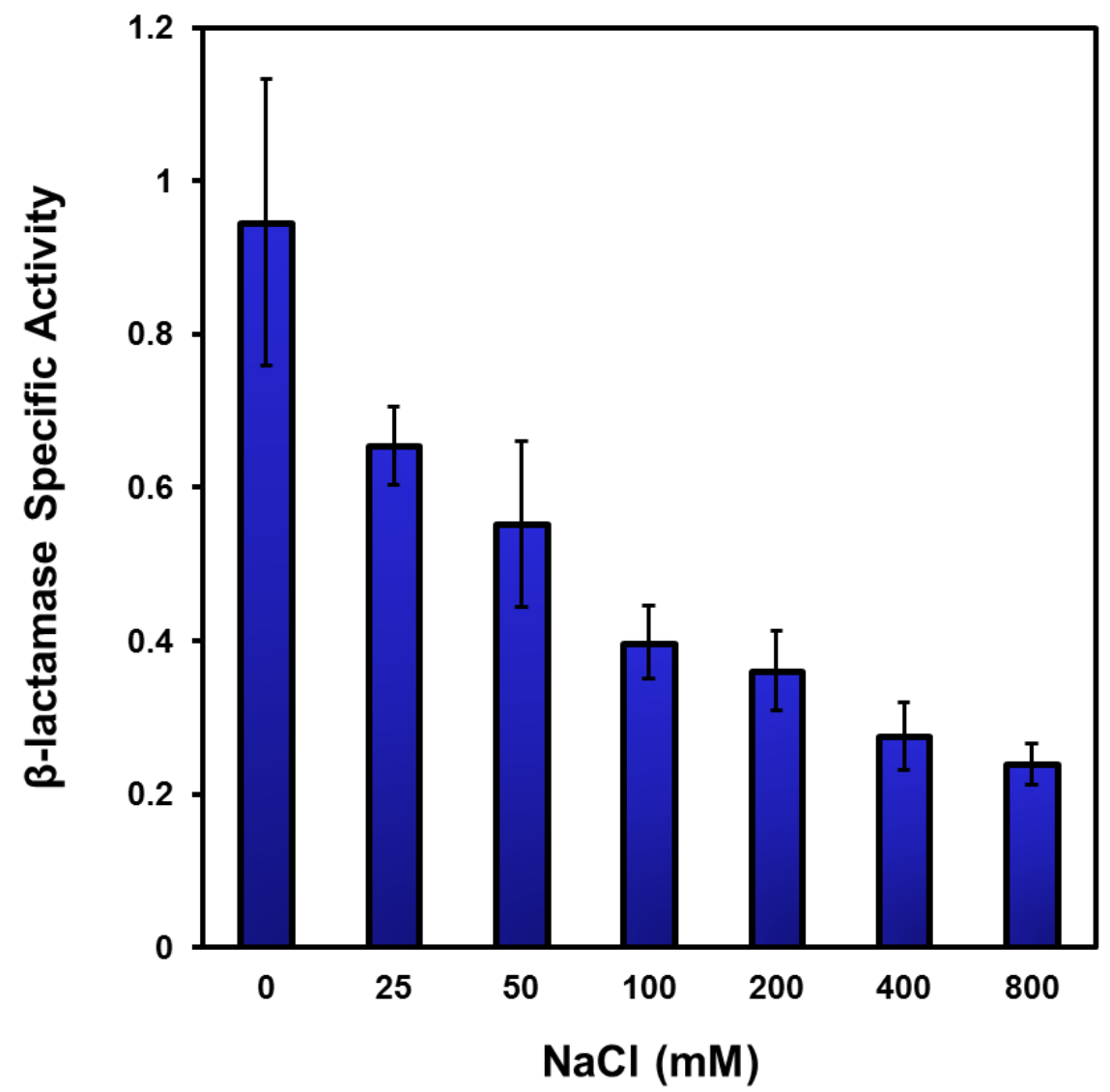

Figure 2.11. Inhibition of $\mathrm{PoxB}$ by $\mathrm{NaCl}$. PoxB inhibition was measured in vitro as a function of $\beta$-lactamase activity. The $\beta$-lactamase assay was performed on lysate from IPTG-induced and uninduced $(1 \mathrm{mM})$ PA0 $\triangle a m p C \triangle p o x B$ (pPoxB) cells. Lysate was incubated for 20 min with increasing concentrations of $\mathrm{NaCl}(0-800 \mathrm{mM})$. Only data for uninduced lysate is shown. Nitrocefin was used as a chromogenic substrate. One milliunit of $\beta$-lactamase was defined as 1 nanomole of nitrocefin hydrolyzed per minute per microgram of protein. 
The CF lung is notoriously susceptible to bacterial infections and in particular to colonization by $P$. aeruginosa $(122,123)$. The most widely accepted hypothesis of CF pathobiology, the low volume hypothesis, states that mutations in the CFTR gene cause an excessive intake of $\mathrm{NaCl}$ in the lung epithelium leading to surface airway dehydration and impaction of mucus thus setting up the stage for the chronic and persistent bacterial infections that are the hallmarks of CF lung disease $(155,177,189)$. The CF lung, with its imbalance of $\mathrm{Na}^{+}$and $\mathrm{Cl}^{-}$ions transport, may confer an advantage to OXA-producing organisms susceptible to inhibition by $\mathrm{NaCl}$ than otherwise provided by healthy lung. By the same token, OXA-enzymes susceptible to $\mathrm{NaCl}$ inhibition may exhibit decreased activity in the normal lung. However, it has been difficult to measure the salt content on surface airway epithelium. Mice and epithelial culture studies, although substantiating the low volume hypothesis, have not shown a difference in ion content between healthy and CF models $(174,176,821-823)$. Thus, it is difficult to ascertain the ramification of the imbalance of $\mathrm{Na}^{+}$and $\mathrm{Cl}^{-}$transport caused by $C F T R$ mutations on the activity of OXAtype $\beta$-lactamases, if at all.

2.4.8 PoxA and Congo red binding phenotype. A Biolog microarray phenotypic assay pointed to a growth defect for the PA0 $\Delta$ poxA mutant in the presence of $10 \mathrm{mM} \mathrm{NaNO}_{3}$. Since $P$. aeruginosa is a facultative anaerobe capable of using $\mathrm{NO}_{3}$ as a terminal electron acceptor (824), we investigated the colony morphology of poxA mutants on $\mathrm{CR}$ and $\mathrm{CB}$ plates with $\mathrm{NaNO}_{3}$ in aerobic and anaerobic conditions. Interestingly, a phenotype was observed in the presence of oxygen and in the absence of $\mathrm{NaNO}_{3}$ (Figure 2.12). 
Specifically, overexpression of PoxA produced colonies with an outer dark ring and a darker inner ring, while the center of the colony remained white (Figure 2.12). The observed red phenotype here is indicative of hyperbinding to CR and suggests enhanced production of extracellular polysaccharides such as Pel or Psl $(768,770)$.

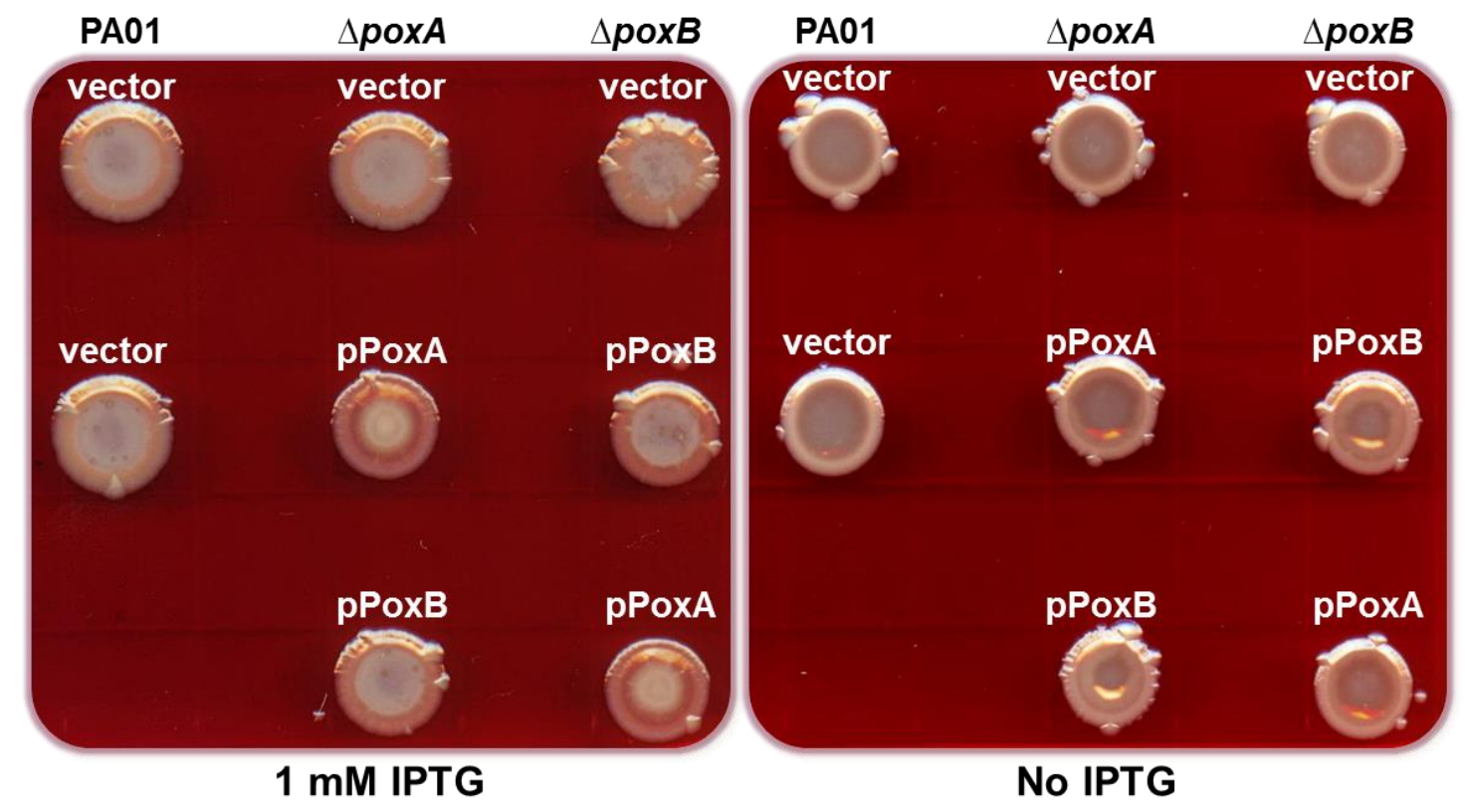

Figure 2.12. Colony morphology on $\mathrm{CR}$. Fresh cultures were diluted to an $\mathrm{OD}_{600}$ of 0.05 and $10 \mu \mathrm{l}$ of each were spotted on agar plates containing 1\% tryptone and $1 \%$ agar supplemented with $40 \mu \mathrm{g} / \mathrm{ml} \mathrm{CR}$ and $20 \mu \mathrm{g} / \mathrm{ml} \mathrm{CB}$ in the presence of gentamycin (75 $\mu \mathrm{g} / \mathrm{ml})$ and IPTG $(1 \mathrm{mM})$. Plates were incubated at $25^{\circ} \mathrm{C}$ in the dark for five days. The colonies were imaged every 24 hours using an Epson scanner. Fifth-day colonies are shown.

Non-mucoid $P$. aeruginosa strains can secrete two extracellular polymeric substances, Pel $(768,825,826)$ and Psl $(769,827)$ polysaccharides, that are known to contribute to biofilm formation. Although the P. aeruginosa strain PA01 has all the genes necessary for production of both Pel and Psl, the latter has been shown to play a bigger 
role in biofilm formation and maturation in this background $(768,769,826,827)$. The PA14 strain, on the other hand, can only produce Pel due to a three-gene deletion in the psl locus $(768,825,826)$. Nonetheless, a recent study showed that both Pel and Psl polysaccharides were involved in micro- and macrocolony formation and in subpopulation interactions in the PA01 biofilms (828), while a PA01 psl mutant was able to form biofilm after acquiring mutations that allowed it to overexpress Pel (829).

$\mathrm{CR}$ is known to bind polysaccharides containing $\beta$-1,3- or $\beta$-1,4-glycosidic linkages (830), and thus has been used to detect the carbohydrates-rich components in the extracellular matrices of a number of bacteria (831-833). CR is in fact routinely used to detect the presence of the Pel and Psl polysaccharides in P. aeruginosa (768-770, 825). In order to determine if hyperbinding to $\mathrm{CR}$ observed upon expression of PoxA (Figure 2.12), is the result of increased production of either Pel or Psl polysaccharides, pellicle formation, autoaggregative properties and binding to CR were examined in liquid media. All strains were able to form pellicle at the liquid-air interphase and there was no difference by visual inspection in the amount of pellicle being formed (Figure 2.13). Similarly, not much difference was observed in the ability of the pellicles to bind CR (Figure 2.14) or in the ability of the strains to form aggregates on the walls of the tubes (Data not shown).

Expression of PoxA thus, does not appear to contribute to an increased production of Pel or Psl in liquid media. Thus, at first glance, the CR-binding phenotype observed in Figure 2.12, does not appear to be the result of an increased production of Pel or Psl polysaccharides. Clearly the surface attached biofilm growing on plates are able to bind 
CR there, but the basis for binding remains unknown. Given that $\mathrm{CR}$ is known to bind polysaccharides, it is likely that the CR phenotype observed here is due to increased production of a carbohydrate or polysaccharide. Alternatively, the surface attached biofilms that grow on plates may be conducive to the production of Pel or Psl in plates but not in liquid media. CR, however, has also been shown to bind non-cellulose material of the extracellular matrix, such as the proteinaceous thin aggregative fimbriae and curli polymers produced by Salmonella spp. and E. coli isolates, respectively (834-836).

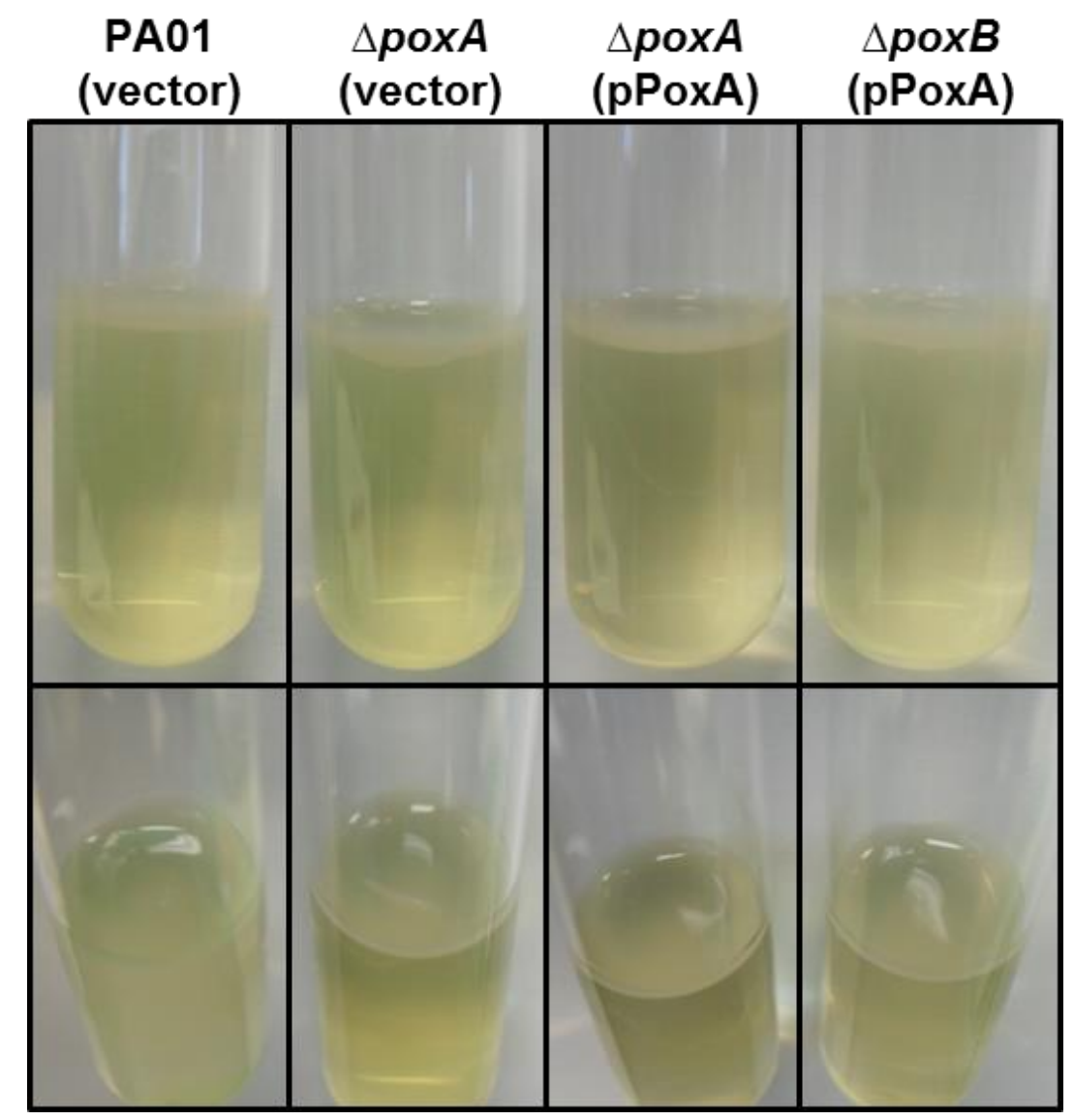

Figure 2.13. Pellicle formation in PoxA-expressing cells. Stationary phase cultures were diluted to an $\mathrm{OD}_{600}$ of 0.025 in $\mathrm{T}$-broth and pellicle formation was monitored at room temperature without shaking for seven days. Seventh-day cultures are shown. 


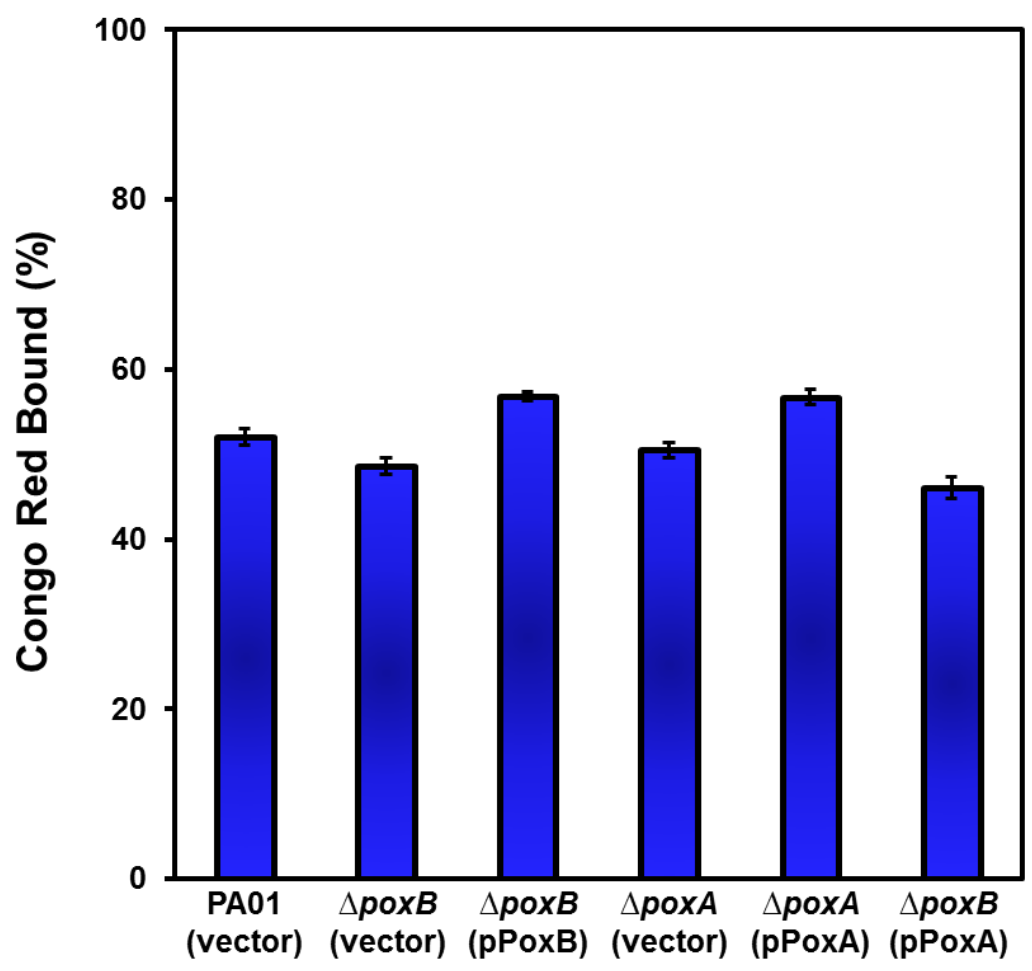

Figure 2.14. CR-binding assay. Two-day old cells were pelleted, resuspended in Tbroth containing $\mathrm{CR}(40 \mu \mathrm{g} / \mathrm{ml})$ and grown with aeration at $37^{\circ} \mathrm{C}$ for 90 minutes. The cells along with bound CR were pelleted by centrifugation and the $\mathrm{OD}_{495}$ of the supernatant was used to calculate the percentage of bound CR.

\subsection{Concluding Remarks}

P. aeruginosa carries two chromosomal $\beta$-lactamases, AmpC, a penicillinase and cephalosporinase; and PoxB, a carbapenemase. The role of $\mathrm{AmpC}$ and its contribution to $\beta$-lactam resistance are well-established and evident here. In our study, AmpC expression readily afforded protection against the cephalosporins and penicillins but not against the carbapenems. PoxB, on the other hand, had a narrow spectrum of hydrolysis that mostly included the carbapenems. Similar to other class D enzymes, PoxB was only poorly 
inhibited by the currently available inhibitors: clavulanic acid, tazobactam and sulbactam. A novel inhibitor, avibactam, was slightly more effective at reducing PoxB activity but clinical concentrations failed to decrease MIC of carbapenems by E-test. Additionally, poxB was shown to form an operon with an upstream ORF of yet unknown function, termed poxA. Expression of PoxA in trans enhanced CR-binding abilities suggesting the production of a CR-binding substance likely rich in carbohydrates.

PoxB appears to be constitutively expressed, but its relevance in the clinical setting is still unknown. Mutational derepression of $\mathrm{AmpC}$ is a common mechanism of resistance. Thus, it is plausible that mutations in $\operatorname{pox} B$ regulatory genes may lead to increased expression and carbapenem resistance in the clinical setting. Expression and regulation of $p o x B$ are examined in Chapter 3.

\subsection{Acknowledgments}

We would like to thank Lars Dietrich and Hassan Sakhtah (Columbia University for insightful discussions on Congo Red and Pel polysaccharides and Dr. Lynn Silver for suggestions on inhibitor studies. This study was supported in part by the National Institutes of Health - Minority Biomedical Research Support SCORE (SC1AI081376; KM), Research Initiative for Scientific Enhancement graduate student fellowship (NIH/NIGMS R25 GM61347; DZ), NIH/NIAID R37 AI021451 (SL), National Science Foundation IIP-1237818 [PFI-AIR: CREST-I/UCRC-Industry Ecosystem to Pipeline Research] (KM), Florida International University Teaching Assistantship (DZ) and Florida International University Dissertation Year Fellowship (DB). We are additionally 
thankful for the funds provided by the Biomedical Research Initiative student summer research award in biomedical sciences (DZ) and to the Cystic Fibrosis Foundation student traineeship grant $(\mathrm{DZ})$. 


\section{Chapter 3}

Regulation of the pox $A B$ operon encoding a class $D \beta$-lactamase in

Pseudomonas aeruginosa 


\subsection{Abstract}

Regulation of AmpC $\beta$-lactamase in Pseudomonas aeruginosa is a tightly controlled process that involves the global transcriptional regulator AmpR and the recycling of muramyl peptides of the peptidoglycan layer. AmpC is normally produced at low basal levels, but $\beta$-lactams such as benzylpenicillin, cefoxitin and imipenem, can induce its high level expression. A second chromosomally encoded carbapenemase, PoxB, has been characterized in $P$. aeruginosa, but its regulation has not yet been examined. Although expression of $\operatorname{pox} B$ appears to be constitutive, studies in a heterologous host suggest AmpR could also be involved in its regulation. In the present work regulation of $p \circ x B$ by AmpR was detected at the transcriptional level by RT-PCR. Quantification of $\beta$-lactamase activity, however, did not show regulation at the protein level. Further, AmpR failed to bind the pox promoter suggesting indirect regulation. A global genetic screen for transposon insertions into pox regulators identified a possible link between polyamine transport and pox promoter activity.

Key Words: PoxB, AmpR, $\beta$-lactamase regulation

Abbreviations: Electrophoretic mobility shift assay, EMSA; Open reading frame, ORF; pox promoter, $\mathrm{P}_{\text {pox }}$; RT-PCR, Real-time polymerase chain reaction; Transposon, Tn 


\subsection{Introduction}

Pseudomonas aeruginosa PoxB is a class D $\beta$-lactamase with a narrow spectrum of hydrolysis that mostly includes the carbapenems $(741,837)$. It is poorly inhibited by the clinically used class A enzyme inhibitors and by avibactam, a new non- $\beta$-lactam inhibitor that is currently undergoing clinical trials (Chapter 2) (741). Additionally, PoxB forms a two-gene operon with the upstream open reading frame (ORF), poxA, whose function is still unknown. The pox operon appears to be constitutively expressed but its regulation has not been investigated.

It is not uncommon for regulatory proteins to be encoded in the general vicinity of the genes they regulate $(730,739)$. For instance, the transcriptional regulator AmpR is encoded immediately upstream of, and divergently transcribed from, the ampC structural gene that it regulates $(711,776)$. It was our previous hypothesis that a two-component regulatory system encoded by mifSR, located immediately upstream of the pox operon and divergently transcribed, could be involved in its regulation. The response regulator MifR has recently been implicated in the formation of biofilm (838). Deletion of either or both mifS and mifR, however, did not affect $\beta$-lactam and carbapenem susceptibility or poxB expression (G. Tatke, Personal communication).

Previous work from our lab, however, seems to implicate AmpR as possible regulator of pox expression (710). Specifically, expression of $a m p R$ in an Escherichia coli heterologous host resulted in decreased PoxB $\beta$-lactamase activity (710). AmpR is an interesting candidate for regulator of pox expression. As a LysR-type transcriptional regulator, AmpR regulates $\operatorname{ampC}$ expression by binding to the promoter region in the 
presence and absence of $\beta$-lactam inducers in both the Enterobateriaceae and $P$. aeruginosa $(839,840)$. In this manner, AmpR can accomplish either repression or induction given the presence/absence of activating inducer. Recently, AmpR has been redefined as a global regulator, differentially controlling expression of various other genes involved in virulence, quorum sensing, biofilm formation as well as various transcriptional regulators (841-844). The AmpR regulon is quite extensive and possibly includes both direct and indirect interactions. It would thus not be surprising if AmpR also regulates expression of the pox operon.

In the present work we analyze the role of $A m p R$ as potential regulator of poxB expression. Our findings suggest AmpR regulates poxB but does not directly interact with the pox promoter $\left(\mathrm{P}_{p o x}\right)$ which indicates indirect regulation. A $P$. aeruginosa transposon library was constructed and screened for insertions into regulators of pox expression. Two transposon mutants exhibiting increased $\mathrm{P}_{\text {pox }}$ activity carried insertions in genes involved in the transport and uptake of polyamines, essential molecules implicated in a wide range of cellular functions (845-847). Polyamines reduced the susceptibility of $P$. aeruginosa towards various $\beta$-lactams but provided resistance only against the carbapenems. The mechanism by which polyamines are able to induce carbapenem resistance is not well understood but could be related to a decrease in cell permeability or to the increased expression of the carbapenem-hydrolyzing $\beta$-lactamase PoxB. Polyamines have, in fact, been shown to modulate gene expression through binding of both DNA and RNA (848-851). Thus, polyamine-dependent regulation, although unprecedented for $\beta$-lactamases, is plausible. 


\subsection{Materials and Methods}

3.3.1 Bacterial strains, plasmids and growth media. Bacterial strains and plasmids used in this study are listed in Table 3.1. E. coli and P. aeruginosa were cultured regularly in Luria-Bertani medium (10 $\mathrm{g}$ tryptone, $5 \mathrm{~g}$ yeast extract, and $5 \mathrm{~g} \mathrm{NaCl}$, per liter) at $37^{\circ} \mathrm{C}$. Antibiotics were used at the following concentrations: ampicillin (Ap) at 100, gentamicin $(\mathrm{Gm})$ at 15 and tetracycline (Tc) at $20 \mu \mathrm{g} / \mathrm{ml}$ for E. coli; Gm at 75 and Tc at $60 \mu \mathrm{g} / \mathrm{ml}$ for $P$. aeruginosa. For construction of PA0 $\Delta a m p R \Delta p o x B$, a previously generated in-frame deletion of $\operatorname{poxB}$ was introduced into PA0 $\triangle a m p R$ (843) using overlap extension PCR and homologous recombination as previously described (758). The presence of the deletion was checked by gene-specific PCR.

Table 3.1: Bacterial strains and plasmids used in this study

\begin{tabular}{|c|c|c|}
\hline Strains/Plasmids & Relevant phenotype and genotype & $\begin{array}{c}\text { Reference/ } \\
\text { Source }\end{array}$ \\
\hline \multicolumn{3}{|l|}{ Escherichia coli } \\
\hline DH5 $\alpha$ & 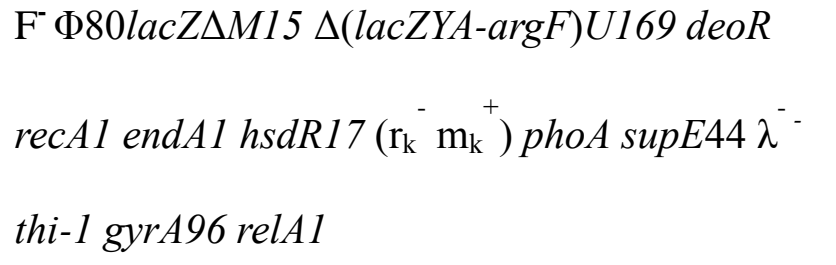 & $\begin{array}{c}\text { New England } \\
\text { Biolabs }\end{array}$ \\
\hline TOP10 & $\begin{array}{l}\mathrm{F}^{-} m c r \mathrm{~A} \Delta(m r r-h s d \mathrm{RMS}-m c r \mathrm{BC}) \Phi 80 l a c \mathrm{Z} \Delta \mathrm{M} 15 \\
\Delta l a c \mathrm{X} 74 \operatorname{rec} \mathrm{A} 1 \text { araD139 } \Delta(\text { ara-leu }) 7697 \text { galU } \\
\text { galK } r p s \mathrm{~L}\left(\mathrm{Str}^{\mathrm{R}}\right) \text { end } \mathrm{A} 1 \text { nup } \mathrm{G}\end{array}$ & $\begin{array}{l}\text { Life } \\
\text { Technologies }\end{array}$ \\
\hline SM10גpir & $\begin{array}{l}\mathrm{Km}^{\mathrm{R}}, \text { thi-1, thr, leu, tonA, lacY, supE, } \\
\text { recA::RP4-2-Tc::Mu, pir }\end{array}$ & Stephen Lory \\
\hline
\end{tabular}


Pseudomonas aeruginosa

PA01 Wild-type

PKM201 PA0 $\triangle a m p C$, PA01 containing in-frame deletion of $\operatorname{ampC}$

PKM315 PA0 $\triangle a m p R$, PA01 containing in-frame deletion of $a m p R$

PKM332 PA0 $\triangle a m p R \Delta p o x B$, PA01 containing in-frame deletions of ampR and poxB

PKM106 PA0attB::mini-CTX-lacZ; $\mathrm{Tc}^{\mathrm{R}}$

PKM117

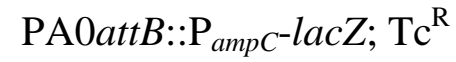

This study

PKM118

PA0attB::P $\mathrm{P}_{p o x}-l a c Z ; \mathrm{Tc}^{\mathrm{R}}$

This study

PKM319

PA0 AampRattB::mini-CTX-lacZ; Tc ${ }^{\mathrm{R}}$

This study

PKM335

PA0 $\Delta a m p R a t t B:: \mathrm{P}_{p o x}-l a c Z ; \mathrm{Tc}^{\mathrm{R}}$

This study

Plasmids

\begin{tabular}{|c|c|c|}
\hline mini-CTX-lacZ & $\mathrm{Tc}^{\mathrm{R}}$; Integration-proficient vector for single- & $(853)$ \\
\hline pMMB67EH-Gm & $\begin{array}{l}\text { copy chromosomal } l a c Z \text { fusion at the } a t t B \text { site } \\
\mathrm{Gm}^{\mathrm{R}} \text {; IncQ, RSF1010, } l a c I^{\mathrm{q}} \mathrm{P}_{t a c} \text { expression } \\
\text { vector with } a m p R:: a a c C 1 \text { insertion at DraI }\end{array}$ & (763) \\
\hline pBTK24 & $\mathrm{Ap}^{\mathrm{R}}, \mathrm{Gm}^{\mathrm{R}}$; transposon delivery vector, ori $\mathrm{R} 6 \mathrm{~K}$ & Stephen Lory \\
\hline pPoxA & $\begin{array}{l}\mathrm{Gm}^{\mathrm{R}} \text {; pMMB67EH-Gm carrying a 946-bp } \\
\text { fragment containing poxA ORF }\end{array}$ & Chapter 2 \\
\hline pOC19 & $\begin{array}{l}\mathrm{Gm}^{\mathrm{R}} ; \mathrm{pMMB} 67 \mathrm{EH}-\mathrm{Gm} \text { containing His-tagged } \\
\text { AmpR at C-terminus }\end{array}$ & $(852)$ \\
\hline pDZ29 & $\begin{array}{l}\mathrm{Ap}^{\mathrm{R}} ; \mathrm{pCR} 2.1-\mathrm{TOPO} \text { derivative containing a } \\
\text { 217-bp EcoRI-BamHI pox promoter fragment }\end{array}$ & This study \\
\hline pDZ197 & $\mathrm{Tc}^{\mathrm{R}} ; \mathrm{P}_{p o x}$ fused to the $l a c Z$ of mini-CTX-lac $Z$ & This study \\
\hline
\end{tabular}


3.3.2 Construction of promoter fusions. A ФCТX-based system was used to construct single-copy promoter fusions, where the expression of the promoterless reporter gene lac $\mathrm{Z}$ is under the control of $\mathrm{P}_{p o x}$. Briefly, $\mathrm{P}_{p o x}$ was amplified using primers KM_PpoxAFov (5'-GAATTCTCACAGGCCGCCGAGCAGCGGCAG-3') and KM_PpoxARev (5'-GGATCCCTCACCAGCCGCGCAGGGTGAAACC-3'). The amplicon was cloned into the TA cloning vector pCR2.1-TOPO, sequenced and subcloned into the EcoRI-BamHI sites of the integrative vector mini-CTX-lacZ and resequenced for fidelity. The transcriptional fusion was subsequently introduced into $P$. aeruginosa PA01 and the isogenic mutant PA0 $\triangle a m p R$ as a single copy at the attB site. A previously generated PA0::P $\mathrm{P}_{a m p c}-l a c Z$ was used as positive control for promoter-driven expression of $l a c Z$ (710).

3.3.3 Quantification of poxB mRNA expression. Total RNA was extracted from $P$. aeruginosa PA01 and a previously generated PA0 $\Delta a m p R$ (843) in the presence and absence imipenem $(0.15 \mu \mathrm{g} / \mathrm{ml})$ using the RNeasy mini kit (Qiagen). cDNA synthesis was performed with Superscript III (Invitrogen) and the (NS) 5 random primer as previously described (764). qPCR was performed with the ABI 7500 cycler (Applied Biosystems) using the Power SYBR Green PCR Master Mix with ROX (Applied Biosystems). $c l p X$ (PA1802) was used as endogenous control and amplified with DBS_qRT_clpXF (5'-TGCGATTACGATGTGGAGA-3') and DBS_qRT_clpXR (5'-CCCTCGATGAGCTTCAGCA-3') (843). DZRTpoxBFord1 (5'-AATCGGCCAGGTTGTGGATAA-3') and DZRTpoxBRev1 (5'-GGAGCAGAAAGCGGGTCTGT-3') were 
used for the real time amplification of poxB. Gene expression was normalized to that of the PA01 uninduced value.

3.3.4 $\beta$-lactamase assay. The $\beta$-lactamase assay was performed as previously described with some modifications (765). Briefly, stationary-phase cultures of PA01, PA0 $\Delta a m p R$ and PA0 $\triangle a m p R \Delta$ poxB were diluted in $25 \mathrm{ml}$ of $\mathrm{LB}$ broth to an $\mathrm{OD}_{600}$ of 0.01 and incubated with shaking at $37^{\circ} \mathrm{C}$. At $\mathrm{OD}_{600}$ of $0.6-0.8$, cells were induced with $0.15 \mu \mathrm{g} / \mathrm{ml}$ of imipenem and reincubated for 1 hour at $37^{\circ} \mathrm{C}$. Ten milliliters of cells were harvested by centrifugation and resuspended in $800 \mu \mathrm{l}$ of $1 \mathrm{x}$ BugBuster solution (Novagen). Benzonase (1 $\mu \mathrm{l} / \mathrm{ml}$ of BugBuster used) (Novagen) and rLysozyme (1 KU (Novagen) were then added and the mixture was rotated for $20 \mathrm{~min}$ at room temperature. The lysate was centrifuged at $4^{\circ} \mathrm{C}$ for 15 minutes to collect the $\beta$-lactamase-containing supernatant. A $2-\mu 1$ aliquot of cell lysate was added to nitrocefin (final concentration, $100 \mu \mathrm{M}$ ) in 250 $\mu \mathrm{l}$ of $50 \mathrm{mM}$ sodium phosphate buffer. The reaction mixture was incubated at $37^{\circ} \mathrm{C}$ for 20 minutes and the hydrolysis of nitrocefin was measured spectrophotometrically at 482 $\mathrm{nm}$. The Bradford assay was used to determine total protein concentration of each sample. $\beta$-lactamase activity was expressed as nmol of nitrocefin degraded per minute per microgram of total protein.

3.3.5 $\beta$-galactosidase assay. The $\beta$-galactosidase activity was quantified as a measure of $\mathrm{P}_{p o x}$ activity as previously described (854). Briefly, fresh cultures of PA01 and PA0 $\triangle a m p R$ harboring $\mathrm{P}_{p o x}-l a c Z$ were grown at $37^{\circ} \mathrm{C}$ and induced with benzylpenicillin 
$(50 \mu \mathrm{g} / \mathrm{ml})$ at an $\mathrm{OD}_{600}$ of 0.5 for 1 hour. The assay was performed on $1-\mathrm{ml}$ cell lysate using ortho-nitrophenyl- $\beta$-galactoside (ONPG) as a chromogenic substrate and the activity was expressed in Miller units. Assays performed as part of transposon screening were routinely done in the absence of induction.

3.3.6 Electrophoretic mobility shift assay (EMSA). The pox promoter region was amplified using $P$. aeruginosa PA01 genome as template with primers EM-PpoxAFor (5'-CGATCAGGGTCTGCAGCGAGC-3') and EM-PpoxARev (5'-GGCTTGCCGCTCGGTTCGC-3') and the 335-bp amplicon was used to perform EMSA. Briefly, the promoter fragment was radiolabelled at the 5 ' end by incubation with T4 polynucleotide kinase (NEB) and $\left[\gamma_{-}{ }^{32} \mathrm{P}\right]$ ATP $\left(3,000 \mathrm{Ci} \mathrm{mmol}^{-1}\right.$; Perkin Elmer). The labeled fragment was diluted to a final concentration $100 \mathrm{nM}$ and unincorporated nucleotides were removed by sephadex G-25 (Biorad, Hercules, CA) spin chromatography. DNA binding reactions containing $50 \mathrm{fmol}$ of ${ }^{32} \mathrm{P}$-labeled DNA probe and varying amounts of total protein membrane fractions were incubated for 20 minutes and loaded thereafter in a nondenaturating 5\% PAGE. Protein membrane fractions were used as AmpR was shown to be membrane-associated (852). Radioactive signals were detected by scanning a phosphostorage cassette with the GE Healthcare Typhoon 9400 scanner.

3.3.7 Pilot experiment to construct transposon library. Before attempting to generate a transposon library it was necessary to determine the conditions needed to obtain good coverage over the PA01 genome. About 20,000 to 40,000 mutagenized colonies, which is 
roughly 5-7 times the number of genes $(\sim 5500)$ in PA01, are necessary. A pilot study was

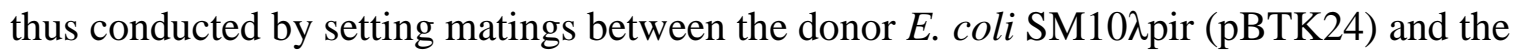
recipient PA0 $\Delta a m p R:: \mathrm{P}_{\text {pox }}-l a c Z$. PA0 $\Delta a m p R:: \mathrm{P}_{\text {pox }}-l a c Z$ was grown overnight $(\mathrm{O} / \mathrm{N})$ at

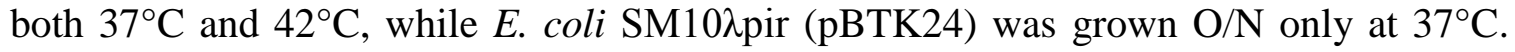
The donor and recipient were then diluted (1/5) and grown at $37^{\circ} \mathrm{C}$ in $\mathrm{LB}$ for about 4 hours. The recipient strain that was grown $\mathrm{O} / \mathrm{N}$ at $42{ }^{\circ} \mathrm{C}$ was also diluted and incubated in a dry bath at $42^{\circ} \mathrm{C}$ for 4 hours. When the cultures reached an $\mathrm{OD}_{600}$ of 2.5 or higher, after approximately $4 \frac{1}{2}$ hours, $6 \mathrm{mls}$ of both the donor and recipient were centrifuged at 13,000 rpm for a minute. After discarding the supernatant, the cells were washed in $1 \mathrm{ml}$ of LB broth, repelleted and resuspended again in $1 \mathrm{ml}$ of LB broth. One set of matings was set up using the recipient that was grown at $42^{\circ} \mathrm{C}$ (Matings $\mathrm{A}-\mathrm{C}$ ), while the others were done with the recipient that had grown at $37^{\circ} \mathrm{C}$ (Matings D-E) (Table 3.2).

Table 3.2: Mating volumes used in pilot study

\begin{tabular}{|c|c|c|c|c|c|c|c|c|}
\hline \multirow[b]{2}{*}{ Strains } & \multicolumn{5}{|c|}{ Pilot Matings $(\mu \mathrm{l})$} & \multicolumn{3}{|c|}{ Controls $(\mu \mathrm{l})$} \\
\hline & $\mathbf{A}$ & B & C & D & $\mathbf{E}$ & $\begin{array}{c}\text { Recipient } \\
\left(37^{\circ} \mathrm{C}\right)\end{array}$ & $\begin{array}{c}\text { Recipient } \\
\left(42^{\circ} \mathrm{C}\right)\end{array}$ & Donor \\
\hline $\begin{array}{l}\mathrm{PA} 0 \Delta a m p R:: \mathrm{P}_{p o x^{-}} \\
\quad l a c Z \text { at } 37^{\circ} \mathrm{C}\end{array}$ & - & - & - & 200 & 400 & 400 & - & - \\
\hline $\begin{array}{c}\mathrm{PA} 0 \Delta a m p R:: \mathrm{P}_{p o x^{-}} \\
\text {lacZ at } 42^{\circ} \mathrm{C}\end{array}$ & 200 & 600 & 400 & - & - & - & 600 & - \\
\hline $\begin{array}{l}\text { E. coli SM10 } \lambda \text { pir } \\
\text { (pBTK24) }\end{array}$ & 1000 & 600 & 800 & 1000 & 800 & - & - & 1000 \\
\hline
\end{tabular}


All mating mixtures were centrifuged, the supernatant discarded and the pellet resuspended in $30 \mu \mathrm{l}$ of $\mathrm{LB}$. The suspensions were then spotted on LB plates and incubated at $37^{\circ} \mathrm{C}$ for 4 hours. All spots were resuspended in $200 \mu 1$ of LB. Fifty and 150 $\mu \mathrm{l}$ of each were plated on selective media (LB-PIA-Gm ${ }^{75}$ ). For the controls, only $100 \mu 1$ of each strain were plated (Table 3.3). The most efficient matings had the recipient grown at $42^{\circ} \mathrm{C}$ at a $1: 1$ ratio of donor to recipient (Table 3.2 and 3.3).

\subsubsection{Transposon mutagenesis and screening for regulators of the pox operon. The} reporter strains, $\mathrm{PA} 0:: \mathrm{P}_{p o x}-l a c Z$ and $\mathrm{PA} 0 \Delta a m p R:: \mathrm{P}_{p o x}-l a c Z$, displaying distinctly different levels of pox promoter activity, were each mutagenized on four different occasions. Twenty matings were set-up each time with the objective of obtaining enough transposon-mutagenized colonies to cover the entire PA01 genome. Matings were set-up as described in the pilot study. Briefly, $\mathrm{O} / \mathrm{N}$ cultures of recipients and donor grown at 42 and $37^{\circ} \mathrm{C}$, respectively, were freshly diluted $1 / 5$ in LB broth and grown for four hours at 42 and $37^{\circ} \mathrm{C}$, respectively. At the four-hour time point, enough culture of both donor and recipient strains were centrifuged at $13,000 \mathrm{rpm}$ for one minute $(1 \mathrm{ml}$ culture/eppendorf tube). The suppernatants were discarded and the pellets resuspended in $1 \mathrm{ml}$ of LB. Matings were set up in donor to recipient ratios of 5:1, 2:1, and 1:1 (Table 3.4). All mating mixtures were centrifuged, the supernatant discarded and the pellet resuspended in $30 \mu \mathrm{l}$ of $\mathrm{LB}$, which were then spotted on $\mathrm{LB}$ plates and incubated at $37^{\circ} \mathrm{C}$ for an additional four hours. All spots were individually resuspended in $200 \mu$ of LB and 50 and $100 \mu 1$ were plated on selective media (LB-PIA-Gm ${ }^{75}$ or LB-PIA-Gm ${ }^{75}-\mathrm{X}_{- \text {gal }^{100}}$ ). 


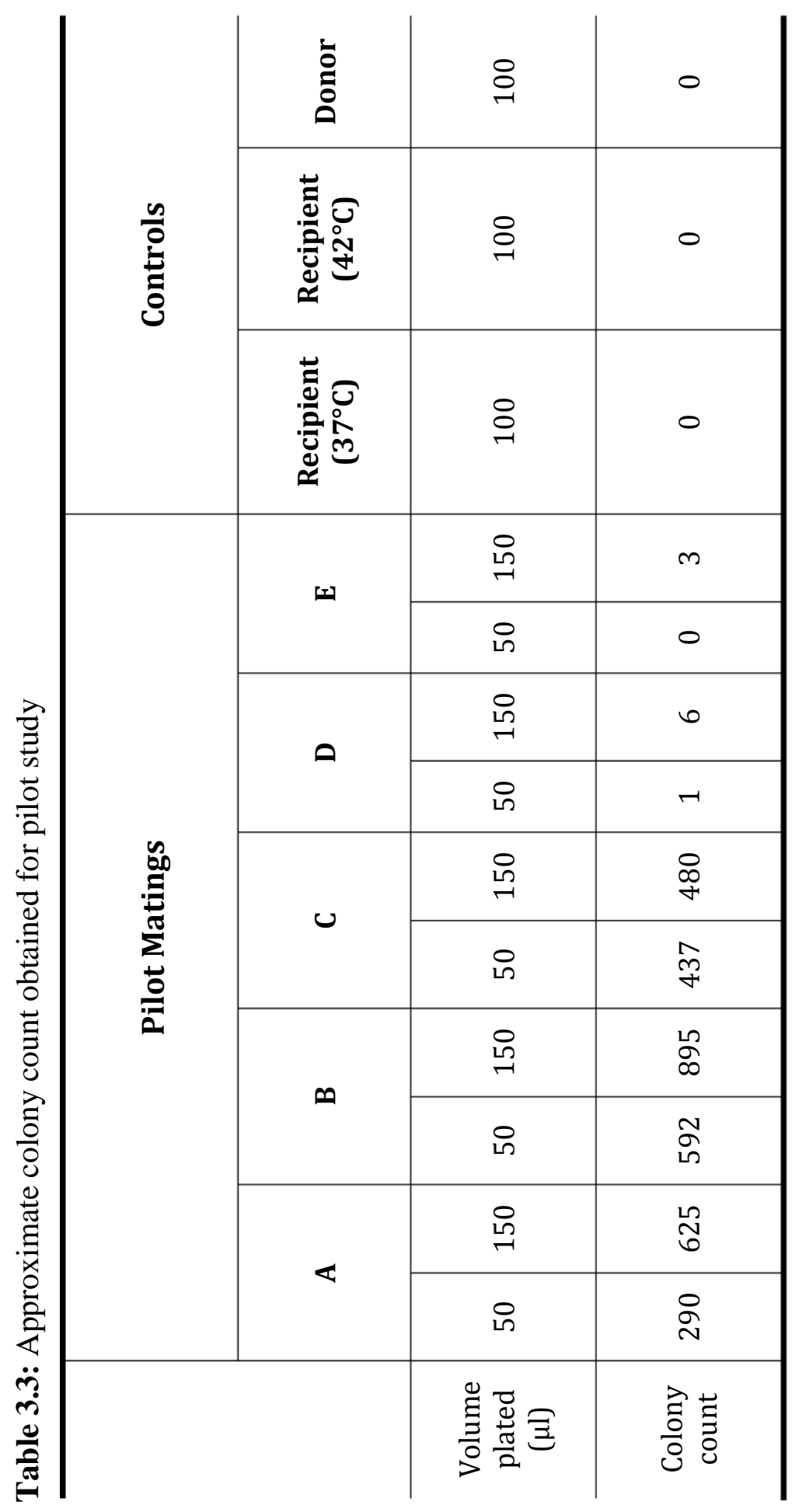


Table 3.4: Mating ratios used for generation of $P$. aeruginosa library

\begin{tabular}{|c|c|c|c|c|c|}
\hline \multirow{2}{*}{ Strains } & \multicolumn{3}{|c|}{ Matings } & \multicolumn{2}{|c|}{ Controls } \\
\hline & $1-7$ & 8-14 & $15-20$ & Recipient & Donor \\
\hline $\begin{array}{c}\text { PA01:: } \mathrm{P}_{p o x}-\text { lacZ or } \\
\text { PA0 } \Delta a m p R:: \mathrm{P}_{p o x}-l a c Z(\mu \mathrm{l})\end{array}$ & 200 & 600 & 400 & 600 & - \\
\hline E. coli SM10 $\lambda$ pir (pBTK24) ( $\mu \mathrm{l})$ & 1000 & 600 & 800 & - & 1000 \\
\hline
\end{tabular}

For the PA0:: $\mathrm{P}_{\text {pox }}$-lacZ library, colonies obtained with the $1^{\text {st }}$ and $2^{\text {nd }}$ mating events, were all pooled and frozen into five 96-well plates since selection was done only in LB-PIA-Gm ${ }^{75}$. Screening of these pools for dark blue colonies remains to be done on LB-PIA-Gm ${ }^{75}-\mathrm{X}-$ gal $^{100}$ (Figure 3.1). Colonies for the $3^{\text {rd }}$ and $4^{\text {th }}$ mating events were directly selected on LB-PIA-Gm ${ }^{75}-\mathrm{X}-\mathrm{gal}^{100}$. The blue colonies resulting from this screening were individually frozen in 4 x 96-well plates and further analyzed as described in later sections.

For the PA0 $\triangle a m p R:: \mathrm{P}_{p o x}-l a c Z$ library, all colonies obtained were pooled and frozen with $10 \%$ skim milk in 57 x 2-ml-vials (mating events 1-3) and in two 96-well plates $\left(4^{\text {th }}\right.$ mating event) (Figure 3.2). Screening for white colonies has so far been done on 34 wells (A1-C10, Plate \#1, mating event 4). The screening was done by inoculating a scraping from a well into $10 \mathrm{ml}$ of $\mathrm{LB}$ broth and growing at $37^{\circ} \mathrm{C}$ for 45 minutes. Each culture was then serially diluted and the $10^{-5}$ and $10^{-6}$ dilutions were plated onto LB-PIA$\mathrm{Gm}^{75}$-X-gal ${ }^{100}$. White to light blue colonies were selected for further analysis and individually frozen on $10 \%$ skim milk. 


\section{PA0:: $P_{\text {pox }}-l a c Z$ \\ (White to light blue phenotype)}

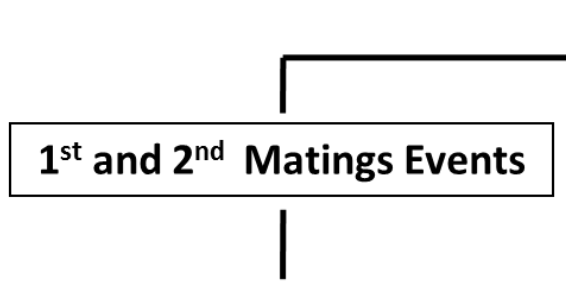

Selection on LB-PIA-Gm ${ }^{75}$

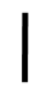

All colonies pooled and frozen

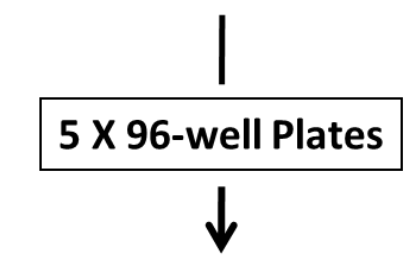

Screen for blue colonies

\section{L phenotypel}

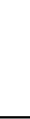

$3^{\text {rd }}$ and $4^{\text {th }}$ Matings Events

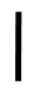

Selection on LB-PIA-Gm ${ }^{75}$-X-gal ${ }^{100}$

Blue colonies individually frozen

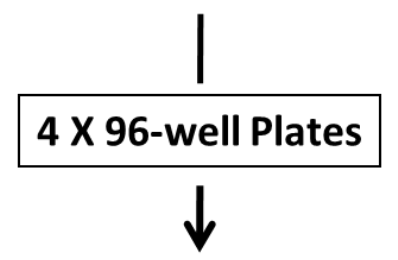

Identify transposon insertion site

Figure 3.1. Selection and screening of the PA01 library

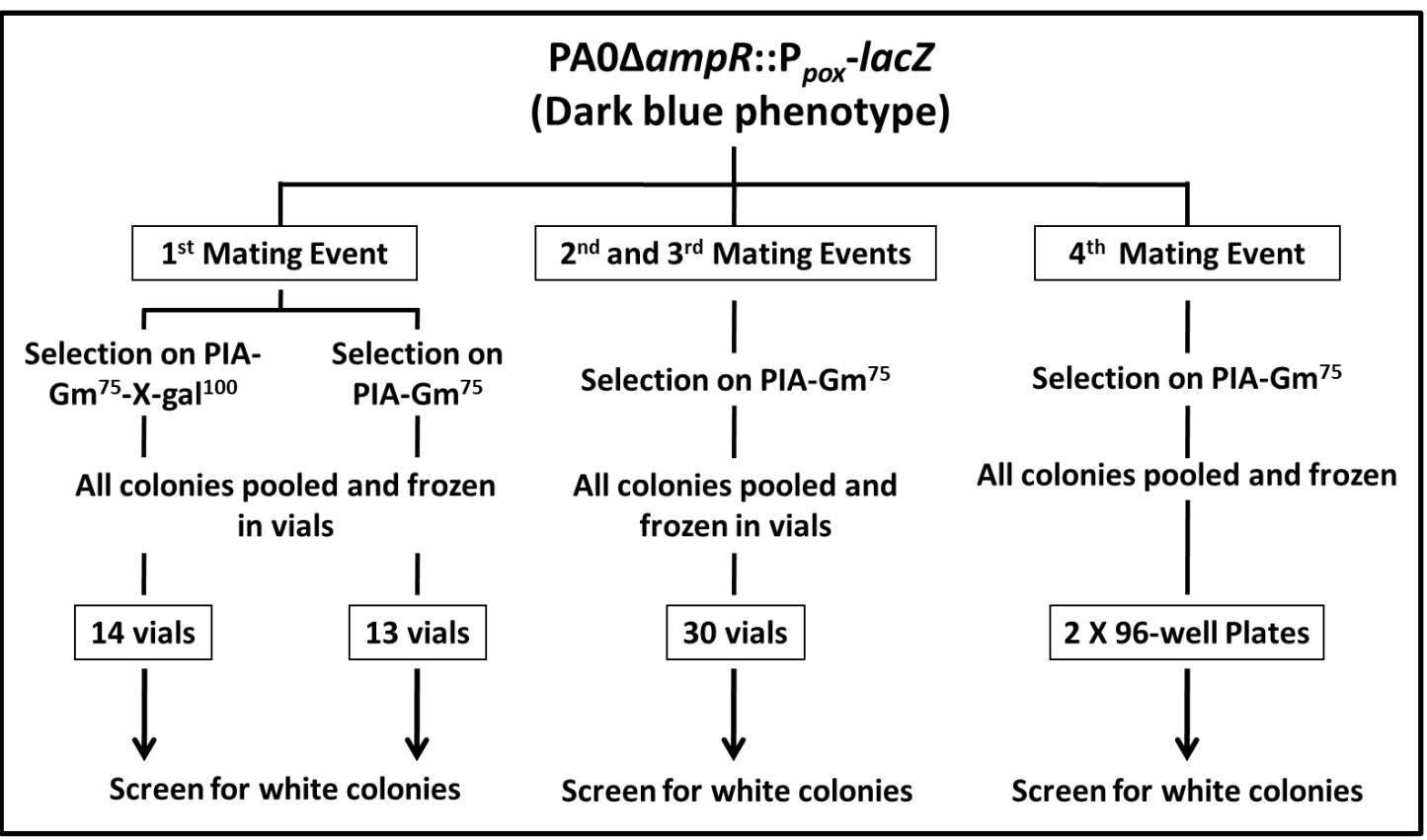

Figure 3.2. Selection and screening of the PA0 $\triangle a m p R$ library 
3.3.9 Mapping of the transposon (Tn) insertion site. Insertion sites were mapped by a two-round semi-random PCR (825) using either pure colony or purified DNA as template. The first round of PCR was performed using the Tn-specific primer Round1BTK24 (5'-GTCGTAAATCACTGCATAATTCG-3') and the random primer Round1Pa(1) (5'-GGCCACGCGTCGACTAGTACNNNNNNNNNNGATAT-3') (855), that can anneal anywhere on the genome and has a nonrandom tail. One microliter of purified product from the first round of PCR was used as template for a nested PCR with primers Round2-BTK24 (5'-GCACTCCCGTTCTGGATAAT-3') and Round2-Pa (5'-GGCCACGCGTCGACTAGTAC-3') (855). The products were gel-purified and sequenced with another nested Tn-specific, outward-facing primer BTK24-seq (5'-GCGGATAACAATATAATGTGTGGA-3), that will read through the junction and into the genome. Mapped insertions were subsequently confirmed by gene-specific PCR.

\subsection{Results and Discussion}

3.4.1 AmpR downregulates poxB expression. Previous work in an E. coli heterologous background showed increased PoxB activity in the absence of AmpR suggesting the transcriptional regulator normally acts to downregulate poxB expression (710). Regulation of the pox operon, however, has not yet been examined in P. aeruginosa. The expression of $\operatorname{pox} B$ was thus quantified in the presence and absence of $\beta$-lactams in an $a m p R$ deletion strain (PA0 $\triangle a m p R)$ by RT-PCR. In the absence of the inducer, equivalent amounts of poxB mRNA were observed in both wild-type and PA0 $\triangle a m p R$ (Figure 3.3). In the presence of the inducer, however, significantly higher amount of poxB mRNA was 
detected in an $a m p R$-deficient background. This finding confirms previous work done in a heterologous host (710) and suggests AmpR negatively regulates pox expression in the presence of $\beta$-lactams.

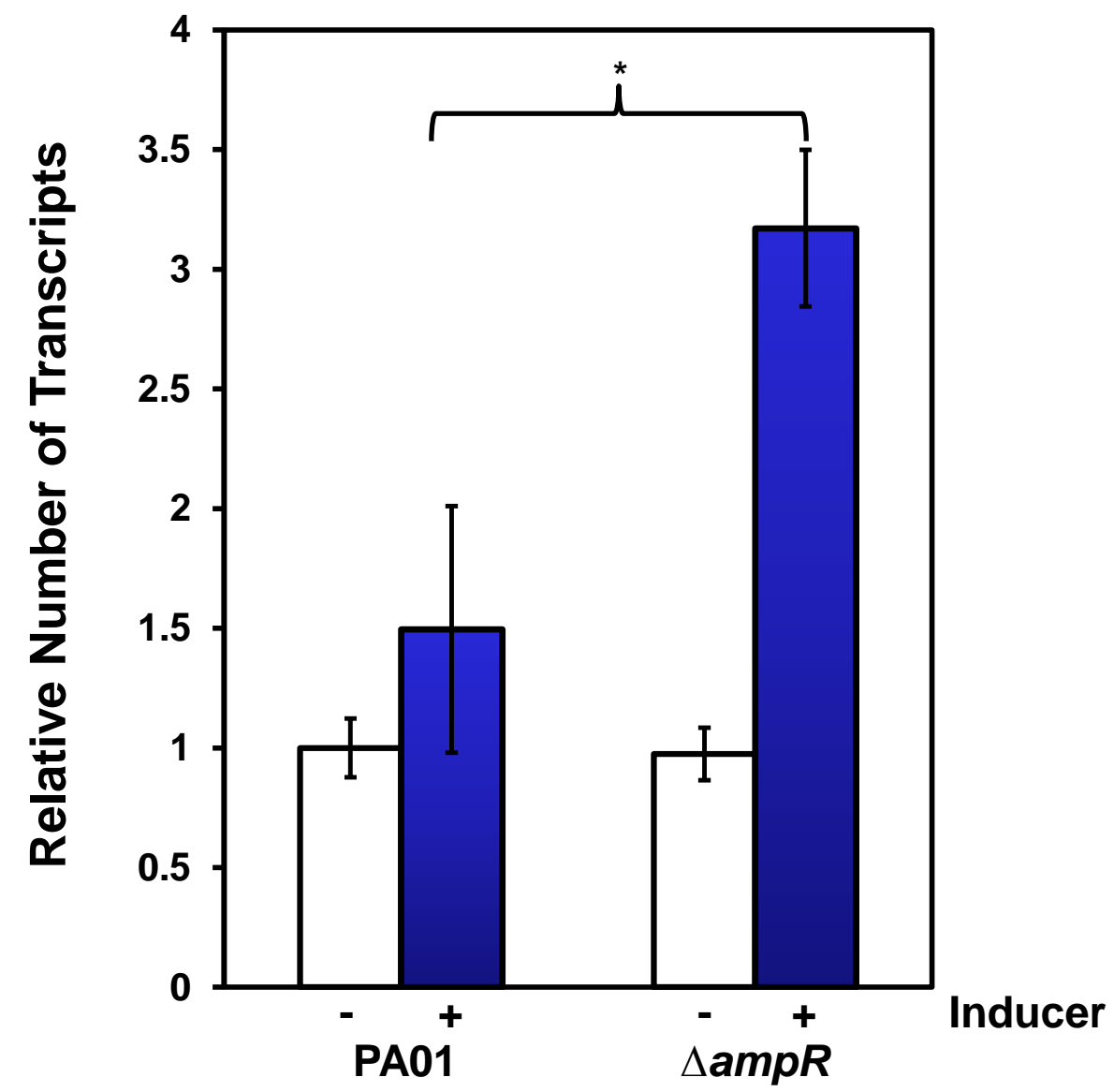

Figure 3.3. Expression of poxB in the absence of AmpR. RNA was isolated from PA01 and PA0 $\triangle a m p R$ in the presence and absence of imipenem $(0.15 \mu \mathrm{g} / \mathrm{ml})$, reversed transcribed to cDNA and tested by qPCR with poxB specific primers, as described in the Materials and Methods. Values were normalized to the expression of the wild-type uninduced sample and represent the mean $( \pm \mathrm{SD})$ of two experiments conducted in triplicates. * $p$-value $<0.01$ for poxB expression in PA0 $\Delta a m p R$ induced as compared to PA01 induced, as determined by unpaired $t$-test. 
3.4.2 PoxB $\beta$-lactamase activity. To determine if the differential RNA expression observed in the absence of $a m p R$ actually translates into quantifiable changes at the protein level, the $\beta$-lactamase activity of PA0 $\Delta a m p R$ and PA0 $0 a m p R \Delta p o x B$ strains was measured in the presence and absence of the inducer. As expected, in the wild-type $\beta$ lactamase levels are detectable and induced upon $\beta$-lactam challenge (Figure 3.4). This activity could be due to either AmpC alone or AmpC and PoxB combined.

The $\beta$-lactamase activity did not significantly increase in the absence of induction in PA0 $\triangle a m p R$, the levels being equivalent to that of wild-type uninduced. In the presence of the inducer, however, deletion of $a m p R$ resulted in the loss of induction, suggesting the

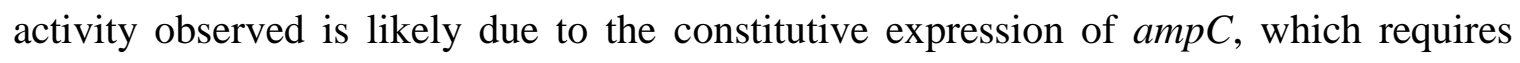
AmpR for induction. A further deletion of $p o x B$ from an already ampR-deficient strain yielded $\beta$-lactamase levels similar to that of PA0 $\triangle a m p R$, with no inducibility being detected upon exposure to $\beta$-lactams. A PA0 $\triangle a m p C \triangle a m p R$ strain behaved similarly to an ampC deletion strain characterized in Chapter 2, in that it produced negligible amounts of $\beta$-lactamase. The activity of PoxB thus, appears to be very low and masked by the expression of ampC. The inducibility of poxB observed at the mRNA level (Figure 3.3) does not translate into a detectable difference in terms of $\beta$-lactamase activity in the conditions tested. 


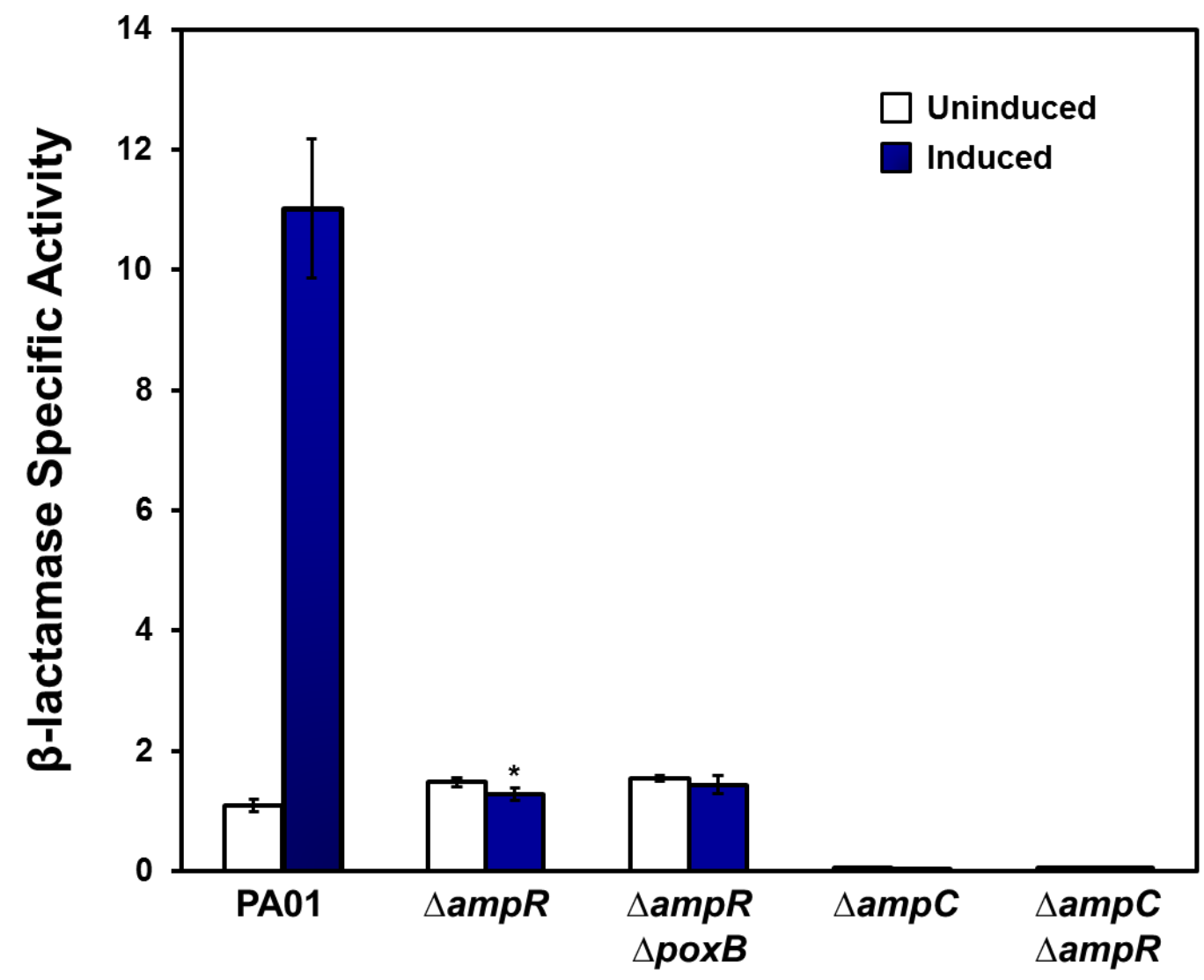

Figure 3.4. $\beta$-lactamase expression of PA01 and its isogenic mutants. The $\beta$-lactamase activity was quantified for the wild-type strain PA01 and the isogenic mutants $\mathrm{PA} 0 \triangle a m p R, \mathrm{PA} 0 \Delta a m p R \triangle p o x B, \mathrm{PA} 0 \Delta a m p C$ and $\mathrm{PA} 0 \Delta a m p C \triangle a m p R$, in the presence and absence of the inducer. Logarithmic-phase cultures were induced with $0.15 \mu \mathrm{g} / \mathrm{ml}$ of imipenem for 1 hour before harvesting. Assays were performed in triplicates on BugBuster and rLysozyme-treated lysate using nitrocefin as chromogenic substrate. One milliunit of enzyme is defined as $1 \mathrm{nmol}$ of nitrocefin hydrolyzed per minute per microgram of total protein. $* p$-value $=0.0001$ for $\beta$-lactamase activity in PA0 $\Delta a m p R$ induced versus activity in PA01 induced, as determined by unpaired $t$-test. 
3.4.3 AmpR does not bind $\mathbf{P}_{p o x}$. As a LysR-type transcriptional regulator, AmpR regulates gene expression by binding to DNA with its canonical N-terminal HTH motif (856). Binding to the ampC promoter has been found to occur in both the presence and absence of $\beta$-lactams $(839,840,852)$. An EMSA was thus performed to determine if AmpR directly binds the pox promoter. A $\left[\gamma-{ }^{32} \mathrm{P}\right]$ ATP radiolabeled $\mathrm{P}_{p o x}$ fragment was incubated with increasing concentrations of total membrane fractions extracted from cells overexpressing a C-terminal His-tagged AmpR that was previously shown to be functional (852). Cell extracts were recovered in the presence and absence of benzylpenicillin. A 193-bp fragment corresponding to the ampC promoter region was used as positive control as it was been previously shown to bind AmpR (852).

As expected and previously shown, AmpR was able to shift $\mathrm{P}_{\text {amp }}$ in the presence and absence of the inducer (Figure 3.5, Lanes 16 and 18), indicating EMSA conditions were working properly. AmpR, however, did not shift a 335-bp $\mathrm{P}_{p o x}$ fragment in the presence (Lanes 8-12) or absence (Lanes 2-6) of benzylpenicillin, indicating AmpR does not bind the pox promoter. If $A m p R$ is in fact regulating expression of poxB, as suggested by previous work and by qPCR here, then this regulation is indirect. This hypothesis is further substantiated by the absence of an AmpR-binding site in the pox promoter region.

Previous work from our lab has shown AmpR is global regulator controlling

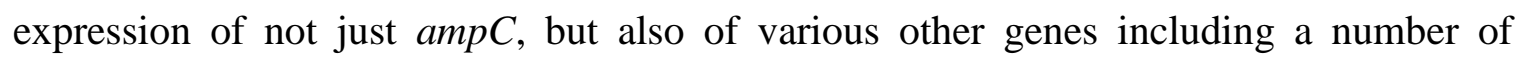
putative and characterized transcriptional regulators and sigma factors such as creB, $\operatorname{ppr} B, \operatorname{alg} B, \operatorname{alg} T / U$ and $\operatorname{rpoS}(710,842,843)$. Thus it is possible that AmpR regulates pox $B$ through these or other, as of yet, unidentified regulators. 


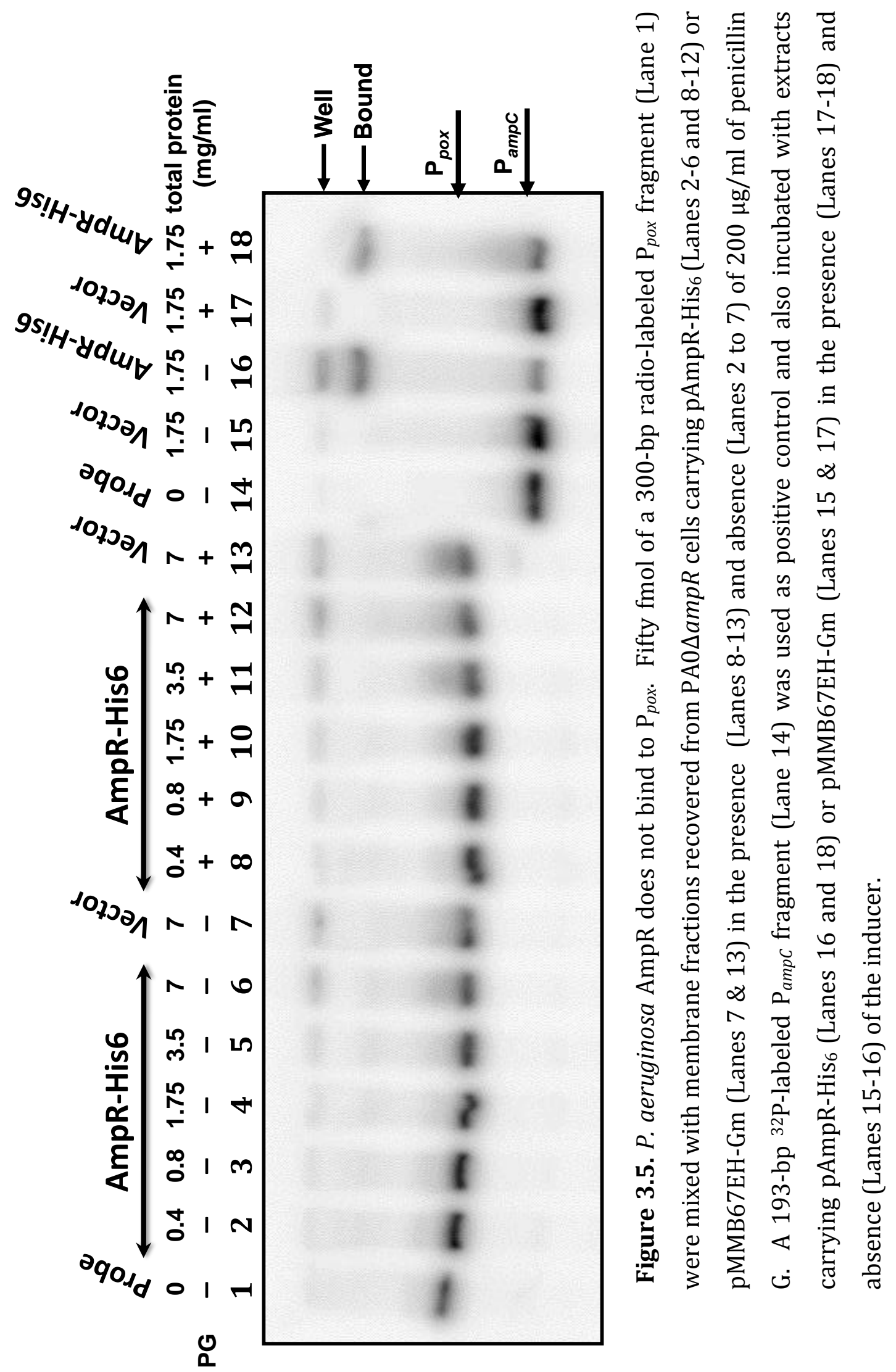


3.4.4 PoxA autoregulates its own promoter. Autogenous regulation is a common regulatory mechanism in which a gene product regulates expression of the very gene that encodes it (857). Autoregulation, especially negative autoregulation, is a common theme among transcriptional factors and regulators, with over $40 \%$ of E. coli transcriptional factors regulating their own synthesis (857-859). Autoregulation however, also occurs in non-regulatory structural genes including those coding for enzymes involved in a myriad of metabolic processes, often with the first gene regulating the expression of the rest of the genes in that operon $(857,860)$.

Since the role of PoxA was unclear, we postulated that PoxA may autoregulate the pox operon. In order to determine if this was the case, poxA was expressed in a lowcopy plasmid under an IPTG inducible promoter and introduced into PA01 carrying a chromosomal $\mathrm{P}_{\text {pox }}$-lac $Z$ fusion. In the absence of induction, the pox promoter exhibited high basal, constitutive levels in both the presence and absence of poxA in trans (Figure 3.6). After one hour induction with IPTG, a small but significant decrease in $\mathrm{P}_{p o x}$ activity was observed in the presence of poxA, suggesting PoxA at high levels can negatively regulate its own transcription. The trend, although no longer statistically significant, was also observed 2 hours after IPTG induction. In wild-type, PoxA may contribute to maintaining low levels of PoxB. Indeed poxB mRNA levels are relatively low and appear for the most part to be uninducible in PA01 (Figure 3.3). PoxA is a putative hydrolase of yet uncharacterized function. 


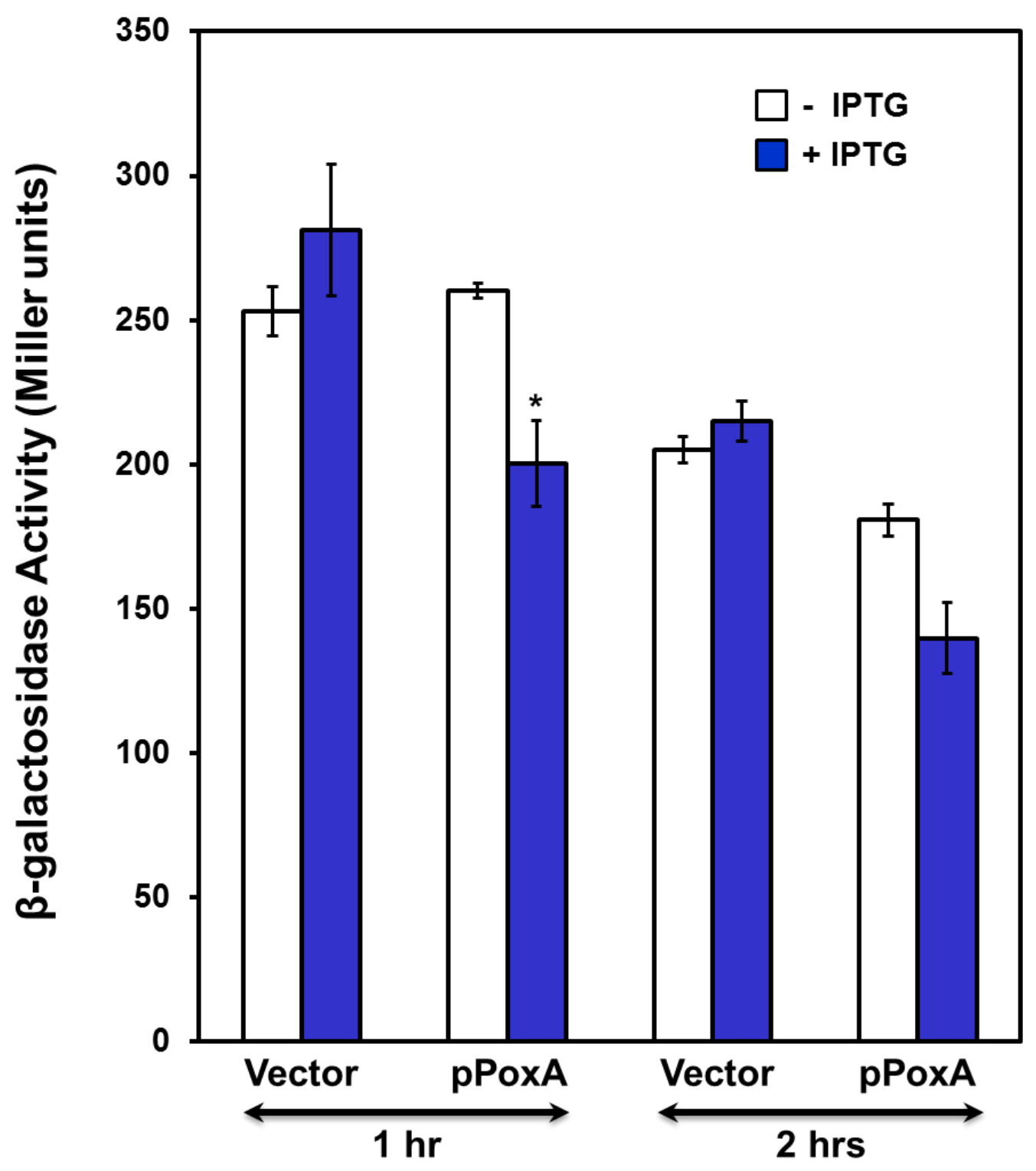

Figure 3.6. Activity of the pox promoter in the presence of pPoxA. The activity of the pox promoter activity was quantified in PA01 carrying a chromosomal lac $Z$ fusion in the presence and absence of PoxA in trans. Exponentially growing cultures were induced with $1 \mathrm{mM}$ IPTG for 1 or 2 hours before harvesting for quantification of $\beta$-galactosidase activity. * $p$-value $<0.05$ for promoter activity of PA0:: $\mathrm{P}_{p o x}-l a c Z$ (pPoxA) in the presence of IPTG vs $\mathrm{P}_{p o x}$ activity in the same strain in the absence of IPTG, as determined by paired $t$-test. 
3.4.5 Approach to identifying regulators of the pox operon. To identify other possible regulators of the pox operon we have taken a global approach that involves construction of a $P$. aeruginosa mutant library with potential transposon insertions into regulators of pox expression. In order to screen for desired insertions, two reporter strains, PA0:: $\mathrm{P}_{p o x}-$ lacZ and PA0 $\triangle a m p R:: \mathrm{P}_{p o x}-l a c Z$, were constructed and the conditions needed for a successful genetic screening were investigated by examining promoter activity in the presence of X-gal or ONPG.

As expected from previous work, the pox promoter was found to be more active in the absence of $a m p R$ as evidenced by the distinctly dark blue phenotype displayed by PA0 $\triangle a m p R:: \mathrm{P}_{p o x}-l a c Z$ on plates containing X-gal (Figure $3.7 \mathrm{~B}$ and D). PA0::P $\mathrm{P}_{p o x}-l a c Z$, on the other hand, having an intact $\mathrm{AmpR}$ that can repress $\mathrm{P}_{\text {pox }}$, displayed a greenish to light blue colony phenotype (Figure $3.7 \mathrm{~A}$ and C). For subsequent selection and screening of the mutant library, $100 \mu \mathrm{g} / \mathrm{ml}$ of X-gal was used, however, lower concentrations of 40 and $60 \mu \mathrm{g} / \mathrm{ml}$ of X-gal were also effective at showing color differences.

The promoter activity was also investigated by the $\beta$-galactosidase assay. In the positive control $\mathrm{P}_{a m p C}$ activity was detected in the absence of induction and induced 3fold upon $\beta$-lactam challenge (Figure 3.8). On the other hand, $\mathrm{P}_{p o x}$ activity was detected in both PA0::P $\mathrm{P}_{p o x}-l a c Z$ and $\mathrm{PA} 0 \Delta a m p R:: \mathrm{P}_{p o x}-l a c Z$, with higher activity observed in the absence of ampR upon induction with $\beta$-lactams (Figure 3.8). These findings are in agreement with the mRNA study (Figure 3.3) and the visual analysis of $\mathrm{P}_{p o x}$ activity (Figure 3.7). 


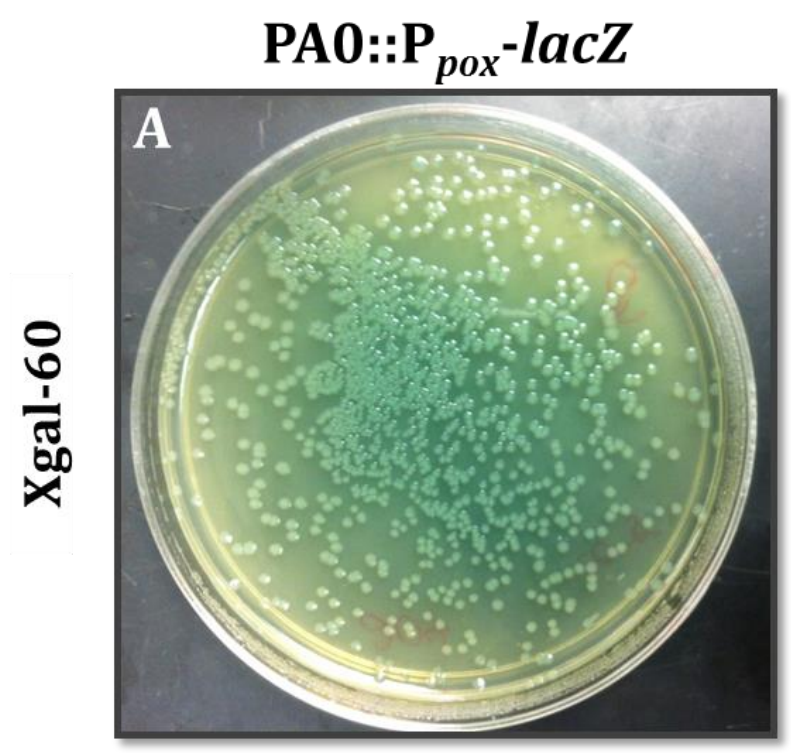

\section{PA0DampR:: $\mathrm{P}_{\text {pox }}-$ IacZ}
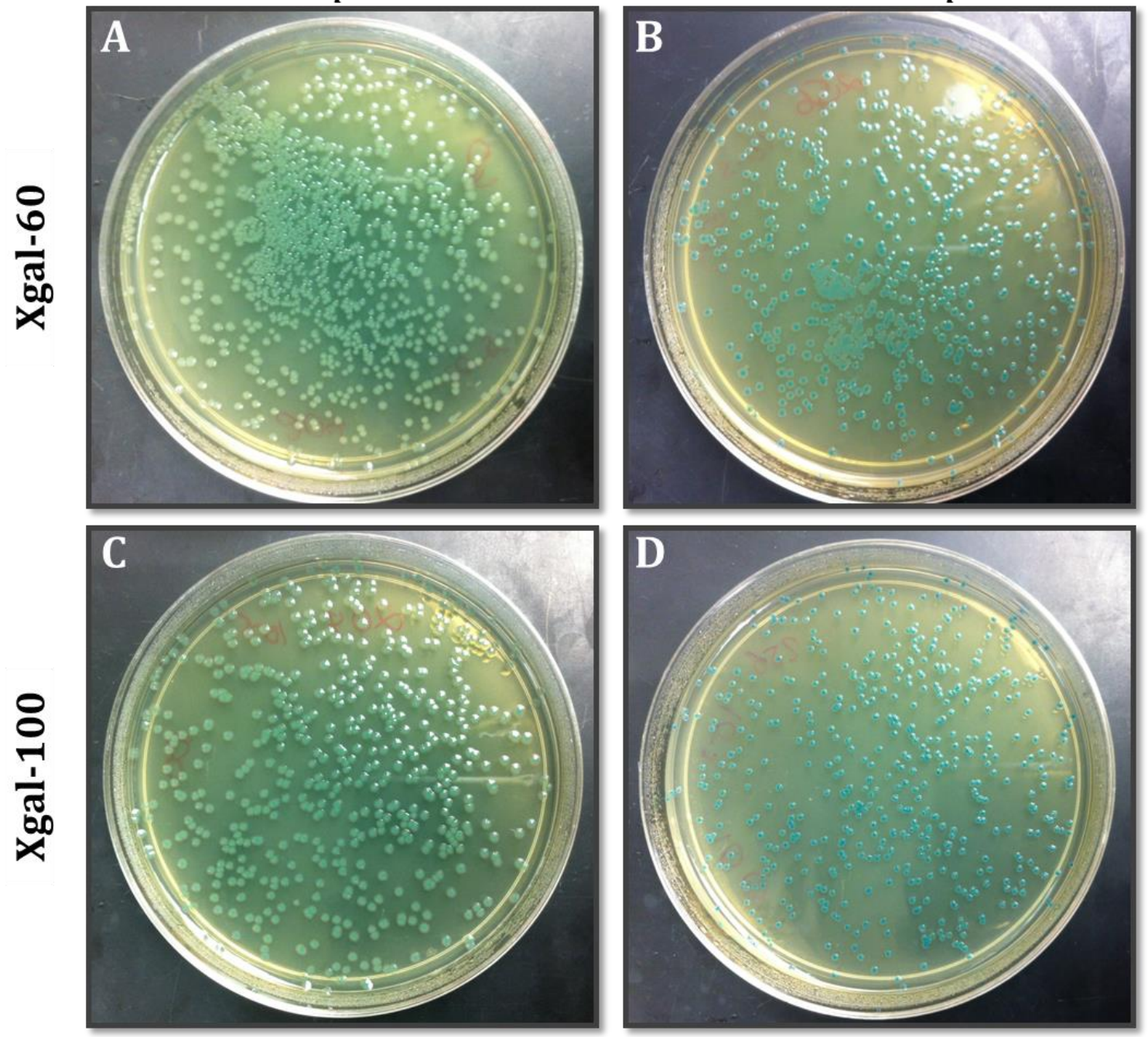

Figure 3.7. Activity of the $P$. aeruginosa pox promoter. The $10^{-5}$ and $10^{-6}$ dilutions of freshly grown PA01 (Panels A and C) and PA0 $\mathrm{P}_{\text {pox }}$-lac $Z$ were plated on LB agar plates containing 40 (data not shown), 60 or $100 \mu \mathrm{g} / \mathrm{ml}$ of X-gal. The promoter activity was ascertained by visually inspecting colony color phenotype after an $\mathrm{O} / \mathrm{N}$ incubation at $37^{\circ} \mathrm{C}$. 


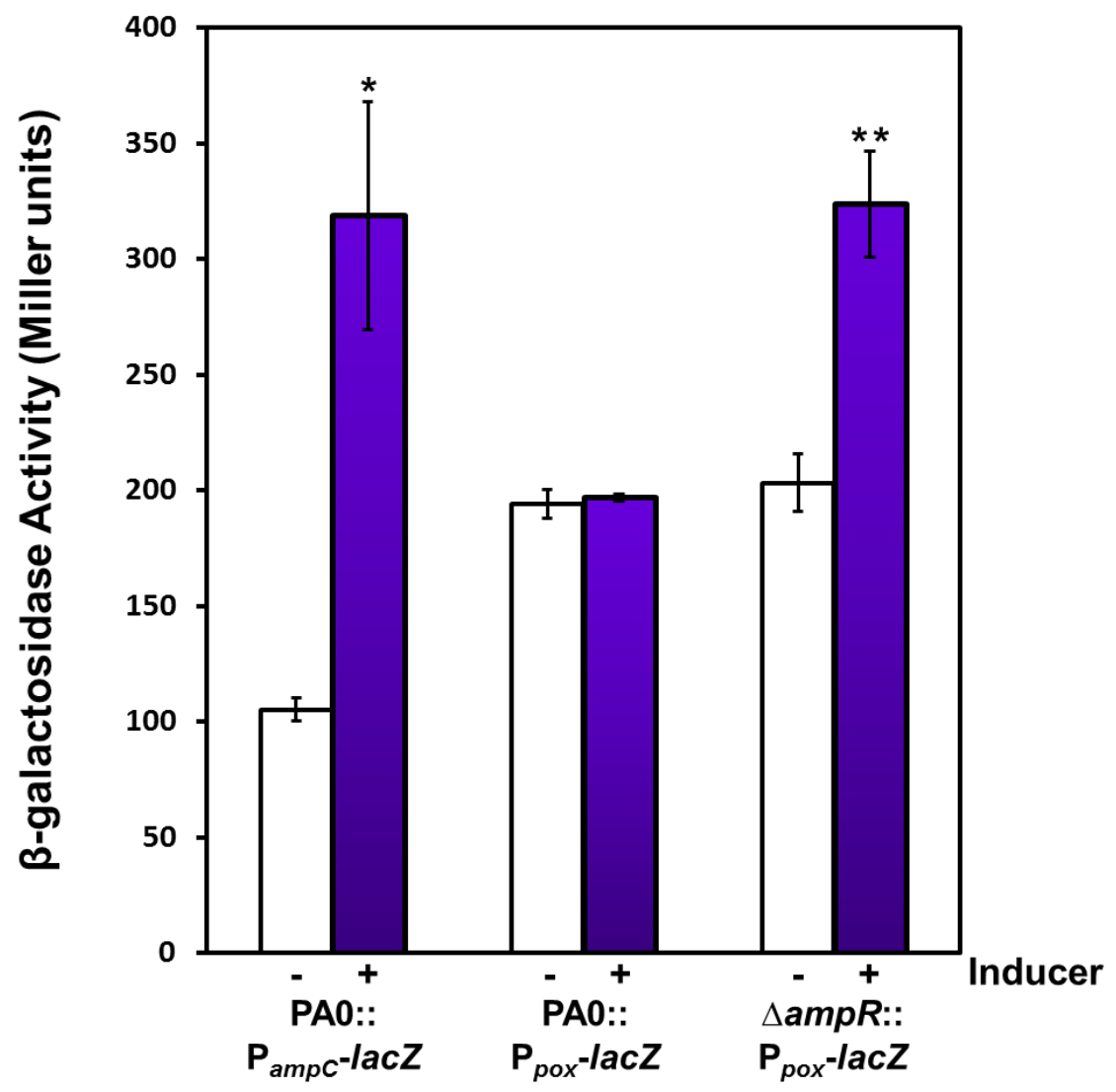

Figure 3.8. $\mathrm{P}_{p o x}$ activity in the presence and absence of $a m p R$. PA01 and PA0 $\triangle a m p R$ cells carrying $\mathrm{P}_{\text {pox }}$-lacZ were grown at $37^{\circ} \mathrm{C}$ and induced with benzylpenicillin at an $\mathrm{OD}_{600}$ of 0.5 for 1 hour. The assay was performed on cell lysate using ONPG as a chromogenic substrate. $\beta$-galactosidase activity was quantified in Miller units in the presence and absence of $\beta$-lactams. * $p$-value $<0.05$ versus uninduced PA0:: $\mathrm{P}_{\text {amp }}$-lacZ; ** $p$-value $<0.02$ versus uninduced PA0 $0 a m p R:: \mathrm{P}_{p o x}-l a c Z$ as determined by paired $t$-test.

3.4.5.1 Identifying negative regulators. Having an intact $a m p R, \mathrm{PA} 0:: \mathrm{P}_{p o x}-l a c Z$ displays a light blue phenotype on X-gal (Figure 3.8). Thus we postulate that the dark blue colonies resulting from the transposon mutagenesis of this strain are likely to have insertions into negative regulators of pox expression (Figure 3.9). Alternatively, a falsenegative dark blue colony could result from insertions immediately upstream of lac $Z$ such 
that transcription from the Tn drives expression of the $\beta$-galactosidase gene. Only mapping can determine the exact Tn insertion site.

Strain $\begin{gathered}\begin{array}{c}\text { Colony } \\ \text { color on } \\ \text { X-gal }\end{array} \\ \begin{array}{c}\text { WA0:: } \\ P_{p o x} \text {-lacZ }\end{array} \text { light blue }\end{gathered}$

Figure 3.9. Predicted color phenotype before and after Tn insertion into putative regulators of the pox operon. Blue triangles indicate transposon insertion sites; yellow arrows denote any positive or negative regulator-encoding genes.

\subsubsection{Identifying positive regulators. PA0 $\Delta a m p R:: \mathrm{P}_{p o x}-l a c Z$ displays a} distinctly dark blue phenotype on X-gal (Figure 3.8). Upon Tn mutagenesis of this strain, colonies that display a white to light blue color, indicative of a decrease in $\mathrm{P}_{\text {pox }}$ activity, potentially have insertions in genes encoding positive regulators of pox expression (Figure 3.9). There are, however, two other possible Tn insertions that can lead to repression of $l a c Z$ resulting in a white phenotype: (a) Tn insertion into $\mathrm{P}_{p o x}$ that controls the transcription of $l a c Z$, or (b) Tn insertion into $l a c Z$ itself. To determine where in the genome the insertion occurred, Tn-specific primers will be used to sequence through the transposon junctions and into the genome. 
3.4.6 Construction of mariner Tn library. A P. aeruginosa PA01 mutant library was constructed by mutagenesis of PA0:: $\mathrm{P}_{p o x}-l a c Z$ and PA0 $0 a m p R:: \mathrm{P}_{p o x}-l a c Z$ with the Tn delivery vector pBTK24 (Figure 3.10). This vector is derived from the pBT20 minitransposon (861) and like its predecessor, carries the Himarl mariner transposase element isolated from the horn fly Haematobia irritans $(855,862,863)$.

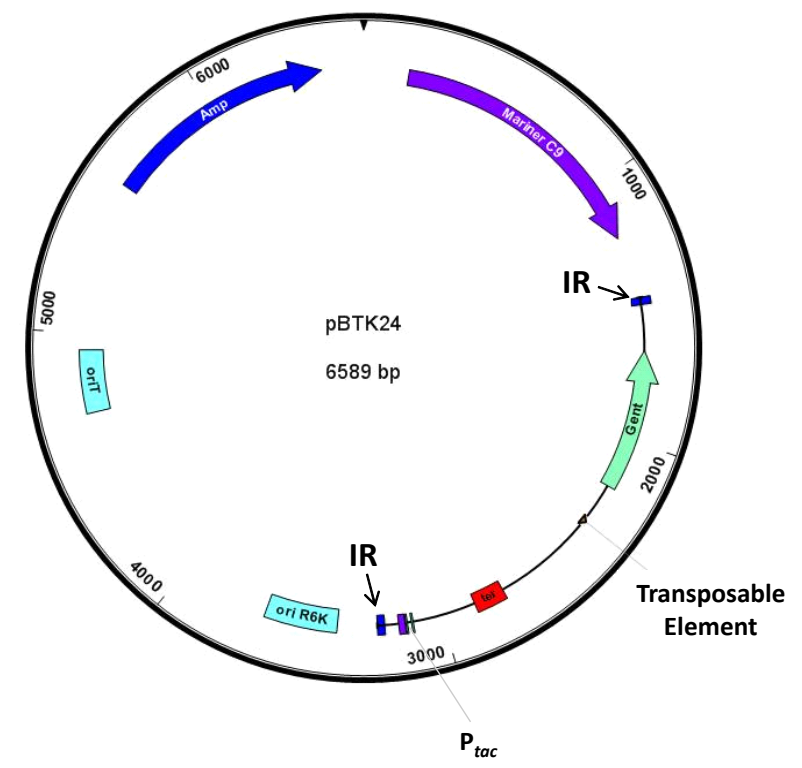

Figure 3.10. Plasmid map and features of the mariner transposon vector, pBTK24. pBTK24 has an ori R6K origin for replication in E. coli and an ampicillin marker for plasmid propagation. The transposase-encoding gene, mariner $\mathrm{C} 9$, is responsible for excision and recombination of the transposable element. Inverted repeats depicted in blue flank the transposon, which carries a gentamicin cassette for selection and an outwardfacing $\mathrm{P}_{\text {tac }}$ promoter for transcription of downstream genes.

The pBTK24 vector also carries an oriT origin for transfer into Pseudomonas, but having only an E. coli origin of replication (ori R6K), is a suicide delivery vector that cannot be propagated in a $P$. aeruginosa background. The mariner $\mathrm{C} 9$ transposase is encoded on the plasmid backbone to promote transposition and to prevent transposase integration into the genome. The transposable element itself is flanked by 29-bp inverted 
terminal repeats (ITRs), which are recognized by the transposase for transposition by a cut-and-paste mechanism (861). Additionally, the transposable element contains a gentamicin cassette for selection and an outward-facing $\mathrm{P}_{t a c}$ promoter for transcription of downstream genes to prevent polarity.

3.4.6.1 Generation of the transposon library. Transposon libraries of $\mathrm{PA} 0:: \mathrm{P}_{p o x}-l a c Z$ and PA0 $\mathrm{PampR}: \mathrm{P}_{\text {pox }}-l a c Z$ were obtained by mutagenesis with E. coli SM10 $\lambda$ pir (pBTK24). Each recipient strain was mutagenized on four different occasions (mating events), with a total of 20 matings per event, in order to generate a library with coverage over the entire PA01 genome. A total of 163,266 colonies were obtained for the PA0 $\Delta a m p R:: \mathrm{P}_{p o x}-l a c Z$ library and more than 503,988 colonies were obtained for PA0:: $\mathrm{P}_{p o x}$-lacZ (Table 3.5).

Table 3.5. Approximate colony count after Tn mutagenesis

\begin{tabular}{|c|c|c|}
\hline Strains & Mating Events & Colony Count \\
\hline \multirow{5}{*}{ PA0::P ${ }_{p o x}-l a c Z$} & 1 & 387,625 \\
\hline & 2 & 86,427 \\
\hline & 3 & 299,36 \\
\hline & 4 & ND \\
\hline & Total & $>503,988$ \\
\hline \multirow{5}{*}{ PA0 $\Delta a m p R:: \mathrm{P}_{p o x}-l a c Z$} & 1 & 973 \\
\hline & 2 & 3,329 \\
\hline & 3 & 19,766 \\
\hline & 4 & 139,198 \\
\hline & Total & 163,266 \\
\hline
\end{tabular}

ND-Not determined 


\subsubsection{Mapping insertion sites and identification of regulators}

3.4.7.1 PA0:: $\mathbf{P}_{\text {pox }}$-lacZ library. Mapping was first attempted on 44 blue colonies obtained from the PA0:: $\mathrm{P}_{\text {pox }}$-lacZ library $\left(3^{\mathrm{rd}}\right.$ and $4^{\text {th }}$ mating events, Plate \# 8 , Figure 3. 1). Out of these, 15 insertions could be confirmed by gene-specific PCR and were selected for further analysis. The majority of the Tn insertions were mapped to genes and operons involved in the biosynthesis of lipopolysaccharide (LPS) (Table 3.6). Often insertions occurred twice in the same gene but at different positions within the ORF (e.g., $w b p J, w z t, w b p Y$, and $r m l A$ ). Two clones had a Tn insertion that mapped to the same location within $w z t$, suggesting these two clones were siblings. Three different insertions were found within the PA3488 ORF, coding for a hypothetical, unclassified protein.

Although these 15 colonies displayed a dark-blue phenotype on X-gal indicative of increased lac $Z$ expression, the promoter activity as determined by $\beta$-galactosidase assay was equivalent to that of wild-type (Figure 3.11 A and B). Thus, determination of promoter activity by blue/white screening alone was deemed to be insufficient for identification of regulators.

It is likely that disturbances in LPS created by Tn insertions in such genes, led to porosity of the membrane and leakage of the enzyme where it could readily cleave X-gal. Subsequently, all blue PA01 colonies frozen in the 4 x 96-well plates (Plates 8-11) from the $3^{\text {rd }}$ and $4^{\text {th }}$ mating events (Figure 3.1) were further screened by assaying the level of $\beta$-galactosidase activity before selecting for mapping. Colonies producing significantly different levels of enzyme as compared to the control strain were selected for mapping. 
Table 3.6. Mapped insertions from PA01 library, Plate \# 8

\begin{tabular}{|c|c|c|c|c|}
\hline $\begin{array}{l}\text { Well } \\
\text { ID }\end{array}$ & $\begin{array}{l}\text { Insertion } \\
\text { Site }\end{array}$ & $\begin{array}{l}\text { Insertion } \\
\text { Coordinate }\end{array}$ & Gene & Function \\
\hline A4 & PA3147 & 3530530 & wbpJ & Glycosyltransferase \\
\hline B4 & PA3147 & 3530649 & wbpJ & Glycosyltransferase \\
\hline E1 & PA5450 & 6140010 & $w z t$ & $\begin{array}{l}\text { ABC subunit of A-band LPS } \\
\text { efflux transporter }\end{array}$ \\
\hline F1 & PA5450 & 6140164 & $w z t$ & $\begin{array}{l}\text { ABC subunit of A-band LPS } \\
\text { efflux transporter }\end{array}$ \\
\hline E9 & PA5450 & 6140164 & $w z t$ & $\begin{array}{l}\text { ABC subunit of A-band LPS } \\
\text { efflux transporter }\end{array}$ \\
\hline E11 & PA5448 & 6137971 & $w b p Y$ & Glycosyltransferase \\
\hline $\mathrm{H} 1$ & PA5448 & 6137221 & $w b p Y$ & Glycosyltransferase \\
\hline $\mathrm{C} 1$ & PA5451 & 6141320 & $w z m$ & $\begin{array}{l}\text { Membrane subunit of A- } \\
\text { band LPS efflux transporter }\end{array}$ \\
\hline A1 & PA5163 & 5812349 & $r m l A$ & $\begin{array}{l}\text { dTDP-glucose } \\
\text { pyrophosphorylase }\end{array}$ \\
\hline E6 & PA5163 & 5812475 & $r m l A$ & $\begin{array}{l}\text { dTDP-glucose } \\
\text { pyrophosphorylase }\end{array}$ \\
\hline D3 & PA4999 & 5616945 & waal & 0 -antigen ligase \\
\hline F7 & PA0795 & 874014 & $\operatorname{prpC}$ & Citrate synthase \\
\hline B5 & PA3488 & 3906941 & - & Hypothetical protein \\
\hline E4 & PA3488 & 3906772 & - & Hypothetical protein \\
\hline F5 & PA3488 & 3907035 & - & Hypothetical protein \\
\hline
\end{tabular}

Screening did not reveal any noteworthy colonies in Plates 9 and 11, however, in Plate 8, two colonies were identified, B6 and G7, as having 8-fold higher promoter activity than the control (Figure 3. 12). These, however, turned out to be false negative as mapping revealed the Tn inserted immediately upstream of the lacZ ORF and mapping 

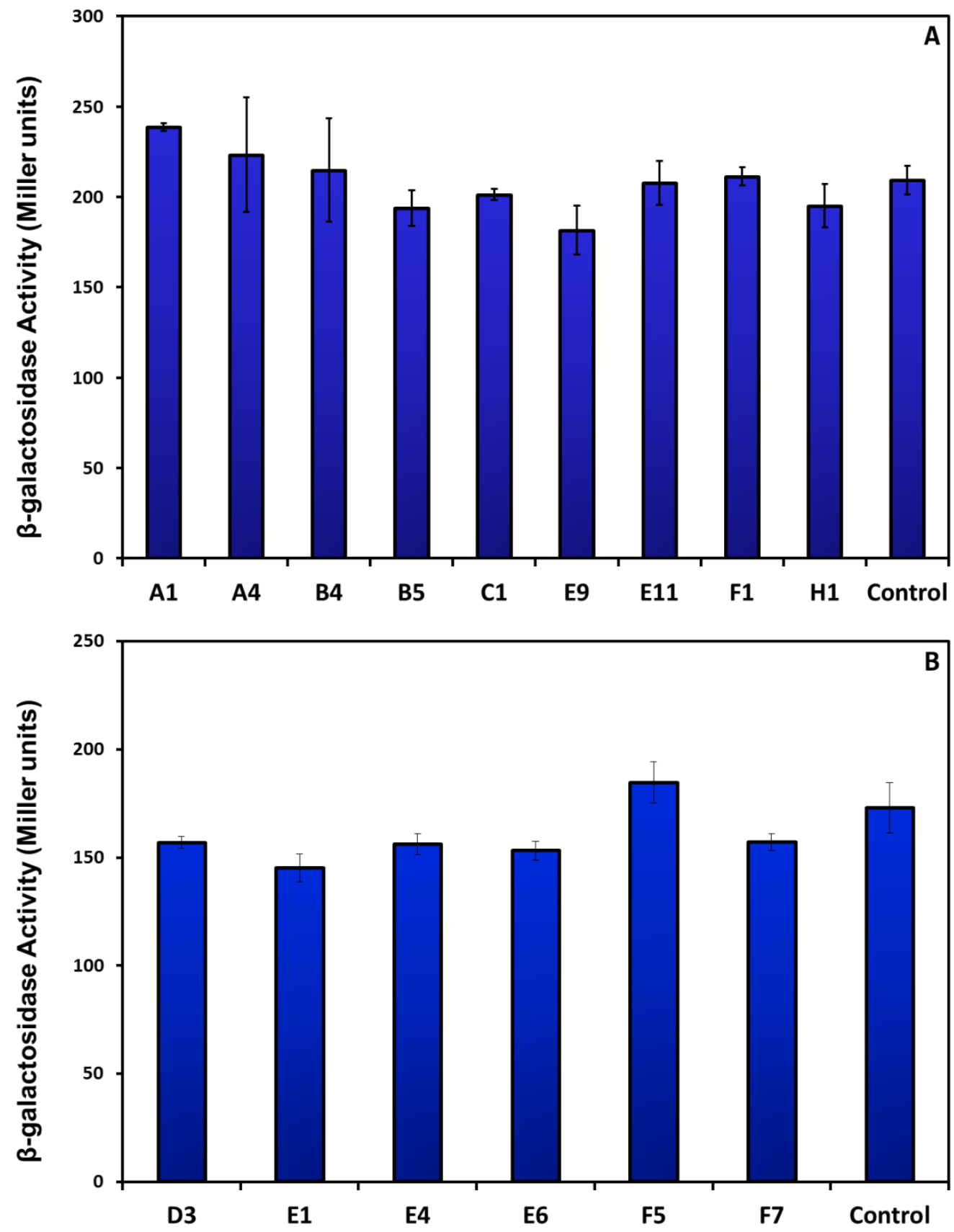

Figure 3.11. $\beta$-galactosidase activity of Tn mutants. The $\beta$-galactosidase activities of 15 Tn mutants showing a dark-blue phenotype on X-gal were quantified in the absence of induction as described in Materials and Methods. The pox promoter activity of the mutants (panel A and B) was found to be similar to the unmutagenized parent strain PA0:: Pox $_{\text {placZ }}$ (Control). 
revealed the Tn inserted immediately upstream of the lacZ ORF and downstream of the cloned pox promoter, with the -35 and -10 sequences of the Tn inserted in the direction of lacZ. Thus, the outward-facing promoter of the transposable element appears to be driving the high level expression of lacZ observed in these clones.

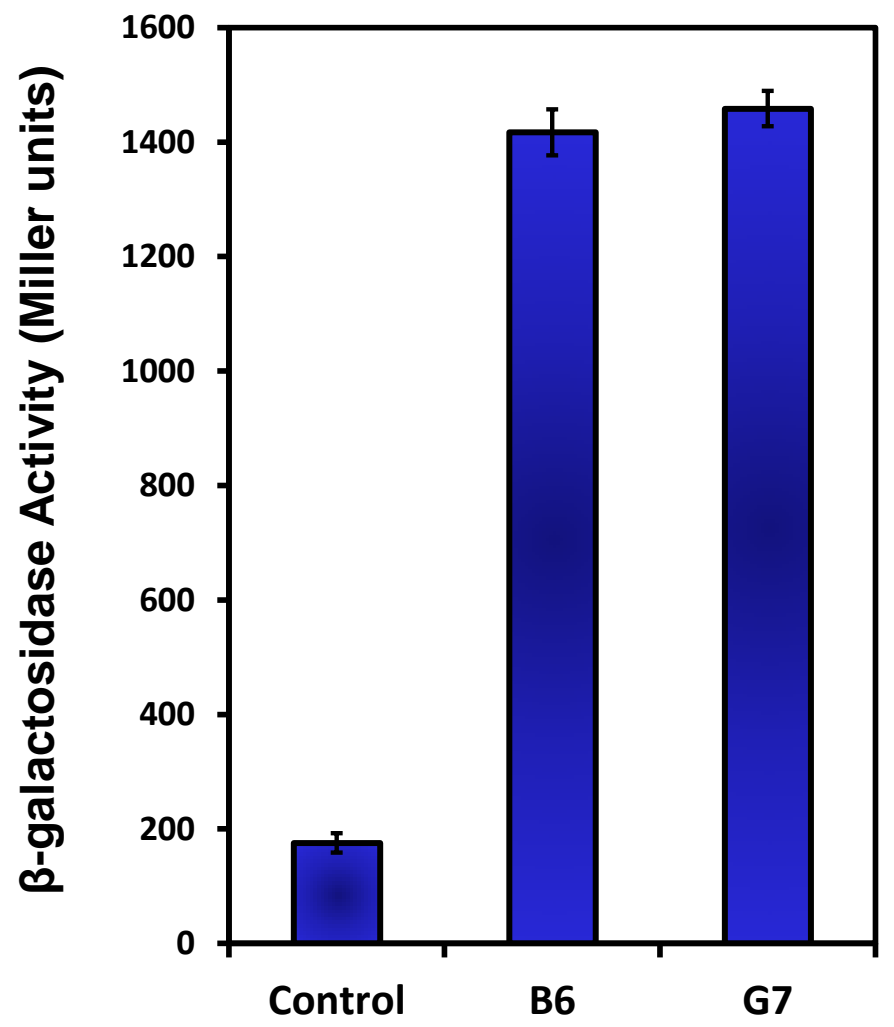

Figure 3.12. $\beta$-galactosidase activity of Tn mutants. The $\beta$-galactosidase activities of 96 blue colonies from the PA01 library (Plate 8) were quantified in the absence of induction as described in Materials and Methods. Only two colonies from plate 8, B6 and G7, were identified as having significantly higher lac $Z$ expression than the unmutagenized control, PA01:: $\mathrm{P}_{\text {pox }}-$ lacZ. 
Screening of Plate 10 revealed that colonies A3, A4, A5, A6, B8 and B11 had both a darker blue and higher $\beta$-galactosidase activity than the control (Figure $3.13 \mathrm{~K}$ and L). Similar to clones B6 and G7 above, colonies A3, A4 hat the Tn inserted immediately upstream of the lacZ ORF and downstream of the cloned pox promoter. Colonies A6 and A12 had insertions in the LPS genes $w b p Y$ and $w b p L$. As these two colonies only had slightly higher $\beta$-galactosidase activity than control, they were not pursued further. Clones A5 (DZ478) and B8 (DZ481) had insertions in PA0322 and in PA0301 (spuE), respectively (Figure 3.14). The role of these two genes will be explored in a later section. Although the data from Plate 8 failed to identify any regulators, these data serve to validate the study and screening methods. In general, colonies displaying high lacZ activity were also dark in color. On the other hand, having a dark blue phenotype does not always correlate with increased lacZ expression. Specifically, disturbances in the LPS layer can allow for the color phenotype to be enhanced as a leakier, more porous membrane presumably allows escape of LacZ or uptake of X-gal (J. Goldberg, personal communication). Lastly, the outward-facing promoter of the transposable element was found to be very efficient at transcribing downstream genes, with expression of lacZ increasing up to 8 -fold when such promoter was located upstream (Figure 3.12). Thus evaluation of potential clones must take into account not only the contribution of the Tndisrupted gene but also the transposon-driven expression of nearby genes. 


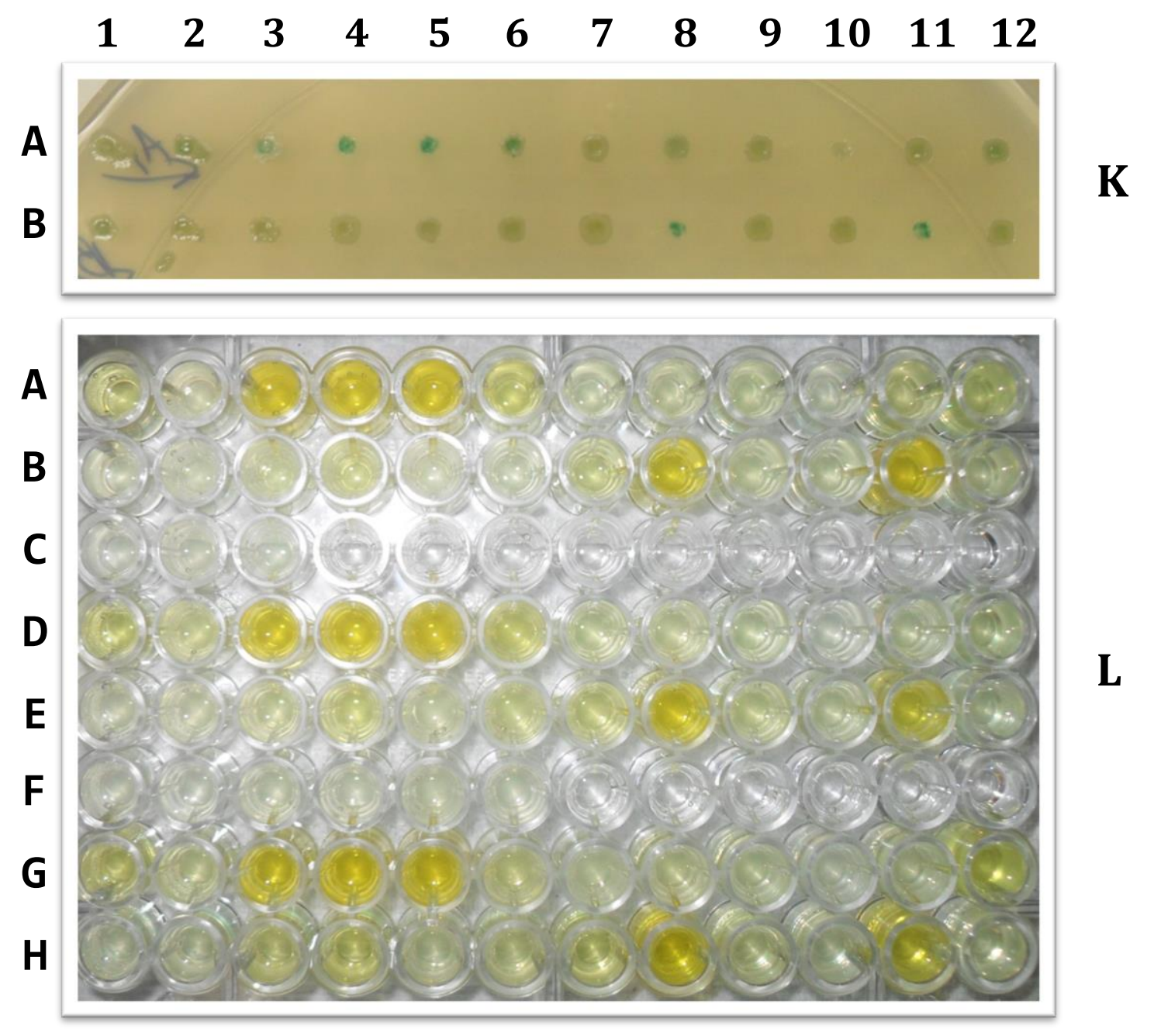

Figure 3.13. Qualitative and quantitative screening of $\mathrm{PA} 0:: \mathrm{P}_{p o x}-l a c Z$ transposon mutants. Twenty-four dark blue colonies from Plate 10, wells A1 through B12, were patched onto an LB plate containing imipenem $(0.15 \mu \mathrm{g} / \mathrm{ml}), \mathrm{Gm}(75 \mu \mathrm{g} / \mathrm{ml})$ and X-gal $(100 \mu \mathrm{g} / \mathrm{ml})$ and incubated $\mathrm{O} / \mathrm{N}$ at $37^{\circ} \mathrm{C}$ for qualitative determination of promoter activity (Panel K). The $\beta$-galactosidase activities were also quantified in triplicates in the absence of induction as described in Materials and Methods (Panel L). Rows D-E and G-H are technical replicates of rows A-B. Wells C1-C3 and F1-F6 represent activity of the control unmutagenized strain PA0:: $\mathrm{P}_{p o x}$ lacZ. The promoter activity, as measured by $\beta$ galactosidase assay (Panel L), is consistent with the activity observed in X-gal plates (Panel K). Colonies A3, A4, A5, A6, B8 and B11 have both a dark blue phenotype and increased hydrolysis of ONPG as evidenced by the darker yellow color. Although colonies A1 and A12 appear to have slightly more $\beta$-gal activity than then control (Panel L), only data from A3, A4, A5, A6, B8 and B11 could be subsequently reproduced. 


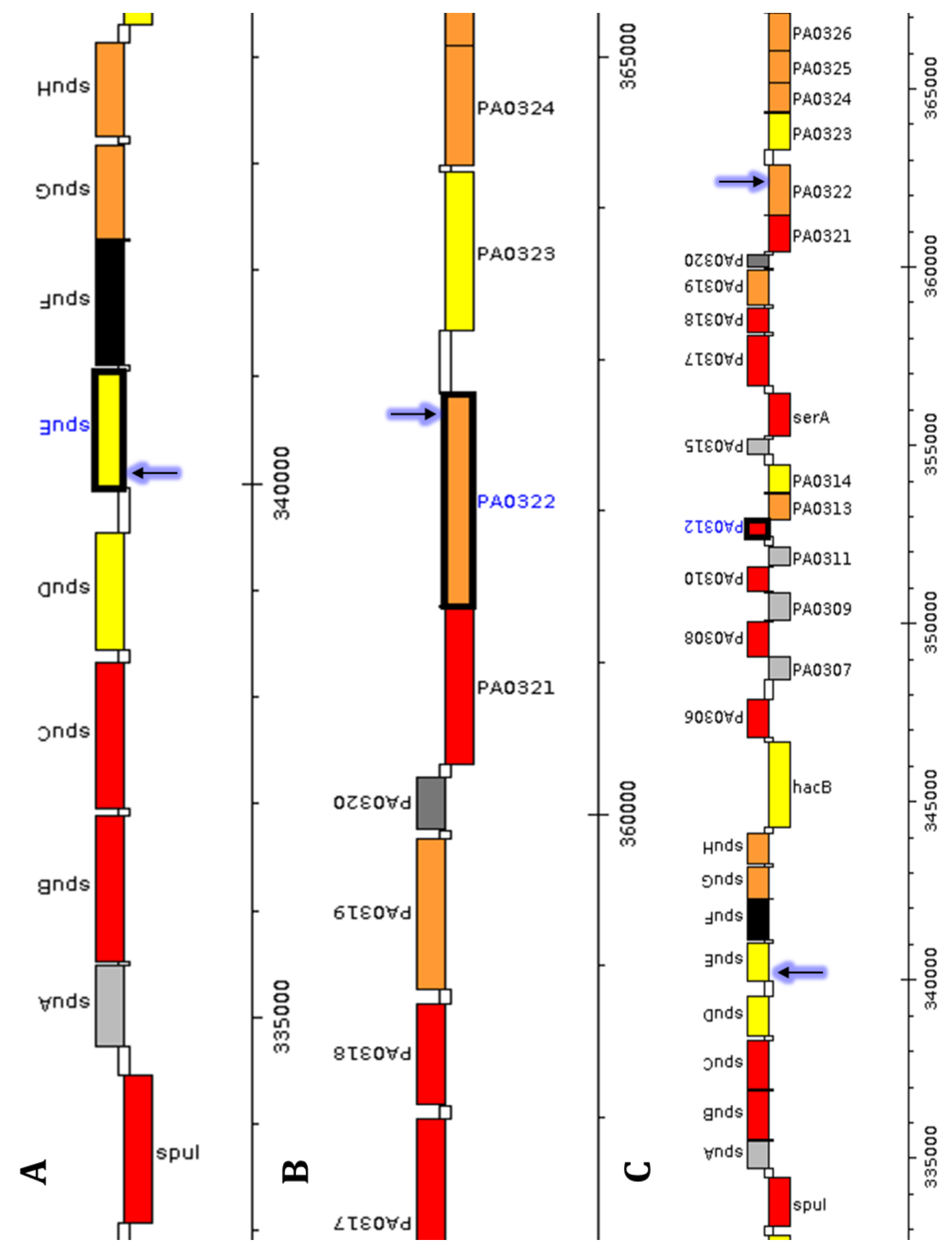


Figure 3.14. Genomic localization of PAO322 and spuE. PA0322 and SpuE were identified as possible regulators of the pox operon using genome-wide genetic screen. spuE encodes a polyamine transport protein and is part of a transcriptional unit containing two operons involved in the biosynthesis and transport of polyamines (Pannel A). PA0322, is a probable amino acid transporter with homology to the E. coli amine transport protein YcjJ, also implicated in a polyamine-utilizing pathway (Pannel B). These two genes are about 25, $000 \mathrm{bp}$ and 21 ORFs away from each other (Pannel C) and localized in a clusters of genes mostly involved with polyamine transport, utilization and metabolism.

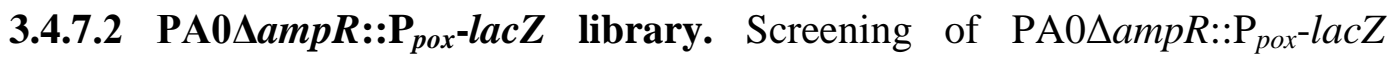
library has thus far identified 77 white to light blue colonies (Figure 3.15A), 14 of which showed varying degrees of mucoidy. Additionally, 21 colonies displaying a dark blue phenotype were selected for further analysis as they appeared to be darker than the unmutagenized parent strain, PA0 $\Delta a m p R:: \mathrm{P}_{p o x}$-lacZ (Figure 3.15B).
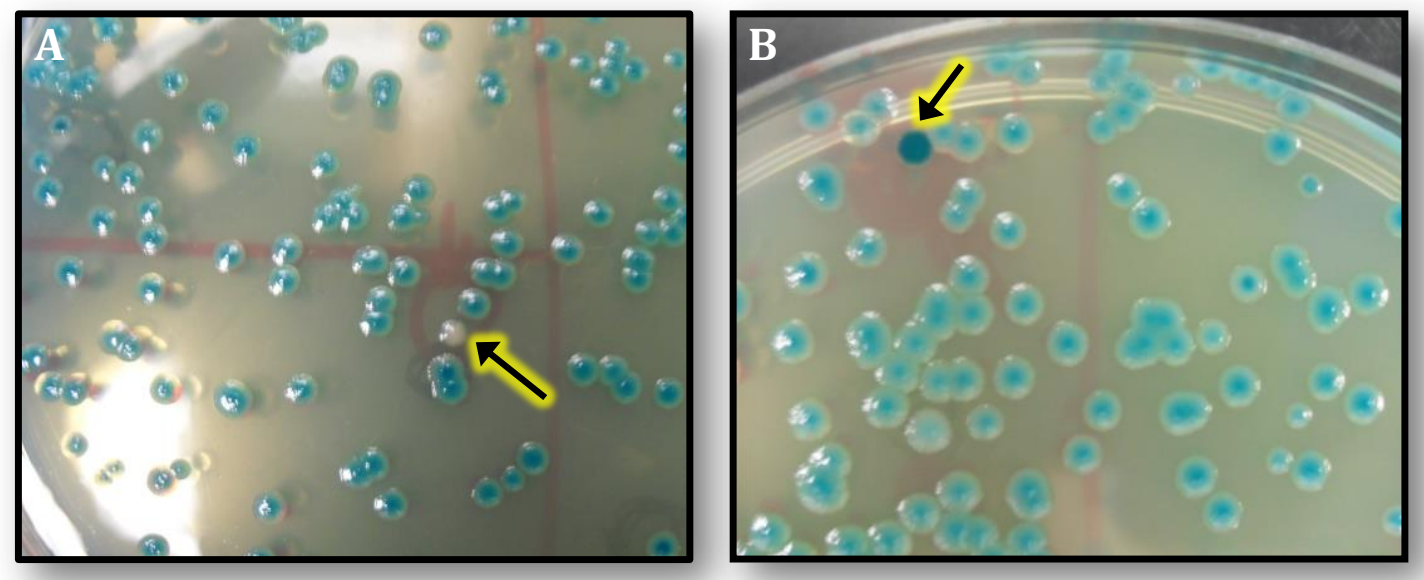

Figure 3.15. Screening of PA0 $\triangle a m p R:: \mathrm{P}_{\text {pox }}$-lacZ library. Colonies obtained after $\mathrm{Tn}$ mutagenesis of PA0 $\triangle a m p R$ were all pooled and frozen in $10 \%$ skim milk in vials and 96well plates. Screening was performed on a number of wells (A1-C10) from Plate \#1 by growing scrapings in $\mathrm{LB}$ at $37^{\circ} \mathrm{C}$ for 45 minutes. The $10^{-5}$ and $10^{-6}$ dilutions were plated on LB-PIA-Gm ${ }^{75}$-X-gal ${ }^{100}$. Seventy-seven white (Panel A) and 21 darker blue (Panel B) colonies were selected for further analysis. 
The 77 white to light blue colonies were further screened by patching them onto LB-Gm ${ }^{75}-\mathrm{X}-$ gal $^{100}$ plates. Ten of these colonies displayed a clear white phenotype, and not surprisingly carried insertions within various positions of lacZ. The rest of the 77 colonies, ranging in color from light blue to the typical light green of $P$. aeruginosa, were further analyzed by assaying $\beta$-galactosidase activity. Only two colonies, CR88 and 90, were found to produce low $\beta$-galactosidase activity but similarly carried insertions in lacZ.

The 21 dark blue colonies showing a darker phenotype than that of the unmutagenized parental strain, were suspected of having the insertion immediately upstream of $l a c Z$, with the promoter from Tn likely responsible for intense blue color observed. Alternatively, the intense blue color could be due to increased leakage from the cell, as previously hypothesized. A region immediately upstream of lac $Z$ was thus PCR amplified to check for transposon insertion. All but six of these colonies had insertions in the MCS of mini-ctx-lacZ, thus explaining the intense dark color observed. The remaining six colonies were assayed for promoter activity, however all colonies produced $\beta$-gal similar to that of the isogenic strain. Thus far, the PA0 $\Delta a m p R$ library has not yielded any potential leads, however, screening has not yet been completed for this library.

3.4.8 Role of PA0301 and PA0322. Since colonies B8 and A5 (plate 8, PA01 library) had increased lacZ activity, a darker blue phenotype and carried Tn insertions in spuE and PA0322, respectively, we decided to further explore the role of these two genes and 
their possible link to $\beta$-lactam resistance. SpuE (PA0301) forms part of the spuABCDEFGH gene cluster (Figure 3.14A) involved in the utilization and transport of polyamines (putrescine, cadaverine, spermidine, spermine) (864). Polyamines are small polycationic organic compounds having more than one primary amine ( $-\mathrm{NH}_{2}$ group) and ubiquitously found in prokaryotes and eukaryotes (Figure 3.16) $(845,865)$. They are essential for growth and due to their cationic nature they are able to interact with polyanionic molecules such as nucleic acids $(848,866-868)$. They have also been shown to stabilize and condense DNA (869-871). Their ability to bind DNA and RNA translates into a range of pleiotropic effects such as modulation of protein-DNA interaction, transcription and translation among many others $(846,849-851,872-874)$. They have also been reported to affect the activity of a number of ion channels such as the inwardly rectifying potassium channel and to decrease outer membrane permeability in E. coli (875-877).

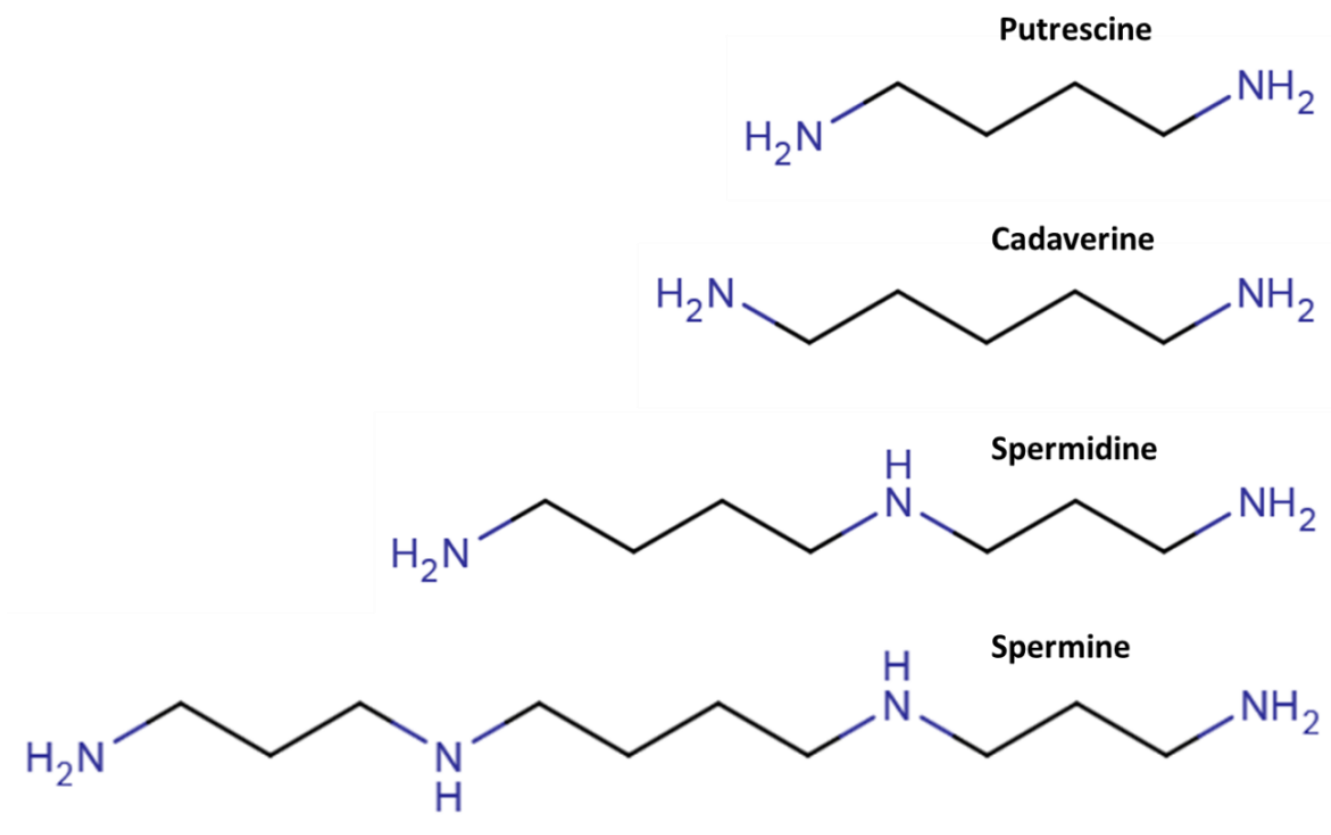

Figure 3.16. Structures of some commonly encountered polyamines 
Besides their synthesis and catabolism, bacteria are able take up polyamines from the environment $(847,878)$. P. aeruginosa can use polyamines as sole source of carbon and nitrogen $(864,879)$. Two main ABC-transporter-type polyamine uptake systems have been reported in E. coli: the PotA/B/C/D or the spermidine-preferential pathway, and the PotF/G/H/I or the putrescine-specific system (880-882). A homologous system has been found in $P$. aeruginosa encoded by the $s p u A B C D E F G H$ gene cluster, where $s p u D$ and spuE encode the periplasmic polyamine binding proteins for putrescine-preferential and spermidine-specific binding, respectively $(864,883)$. SpuG and SpuH are the poreforming permeases in the inner membrane and SpuF is the ATPase for energy-driven transport. SpuA, B and C encode putative enzymes for the utilization of polyamines.

Previous work showed that polyamines decreased expression of T3SS secretions and virulence genes in PA01 (884). Polyamines were also shown to increase resistance in P. aeruginosa against aminoglycosides, with resistance against quinolones and cationic peptides being mediated by the expression of the oprH-phoPQ operon (885). In contrast, polyamines significantly increased susceptibility of $P$. aeruginosa against a number of $\beta$ lactams including the penicillins ampicillin, carbenicillin, piperacillin and ticarcillin, and the third generations cephalosporins ceftazidime, cefoperazone and ceftriaxone (886). This reduction in resistance was not linked to a decrease in expression of $\operatorname{ampC}$ or to disruption of the outer membrane as expected of polycationic ions which are known to be outer membrane disorganizers (886). Similarly, A. baumannii clinical and lab strains were shown to be more susceptible to the $\beta$-lactams aztreonam, carbenicillin, piperacillin 
and ticarcillin when treated with spermine and spermidine, but only a slight to moderate effect was observed against ceftazidime or meropenem (887).

PA0322 also encodes a probable amino acid transporter sharing $61 \%$ homology with the E. coli YcjJ (PuuP) enzyme, which coincidently is a putrescine importer (888890). The Puu system is one of several putrescine utilization pathways reported in E. coli besides the Pot system. Not much is yet known about the role of PA0322 as it still remains uncharacterized. PA0322 does appear to form a putative operon with the upstream ORF PA0321, which displays $67 \%$ similarity to an acetylpolyamine aminohydrolase from Mycoplana ramosa involved in putrescine catabolism (891).

While identifying two targets that involve polyamine transport/utilization is promising, the range of pleiotropic effects these molecules have can obscure the real connection to the pox promoter. The observed phenotype may simple be an indirect effect with no actual connection to $\mathrm{P}_{\text {pox }}$. Additionally, membrane perturbations cannot be ruled out as the main cause of the observed phenotype.

Increased poxB expression has been shown to decrease susceptibility against the carbapenems (Chapter 2). In order to see if carbapenem susceptibility was altered in these clones and if polyamines can affect susceptibility towards these $\beta$-lactams, the MIC against carbapenems was determined in the presence and absence of the polyamine spermidine. Addition of $1 \mathrm{mM}$ of spermidine produced little to no change in susceptibility across strains and $\beta$-lactams, while a concentration of $20 \mathrm{mM}$ spermidine was bactericidal to all strains tested (Table 3.7). 
Table 3.7. $\beta$-lactam MIC in the presence of spermidine

\begin{tabular}{|c|c|c|c|c|}
\hline$\beta$-lactam & $\begin{array}{c}\text { Polyamine } \\
(\mathrm{mM})\end{array}$ & $\begin{array}{l}\text { PA0:: } P_{\text {pox }^{-}} \\
\text {lacZ }\end{array}$ & $\begin{array}{c}\text { PA0::P }{ }_{p o x}-\text { lacZ } \\
\text { PA0322::Tn }\end{array}$ & $\begin{array}{c}\text { PA0::P }{ }_{\text {pox }}-\text { lacZ } \\
\text { spuE::Tn }\end{array}$ \\
\hline \multirow{4}{*}{ Meropenem } & 0 & 1.5 & 1.5 & 1 \\
\hline & 1 & 1.5 & 2 & 1.5 \\
\hline & 5 & $>32$ & $>32$ & $>32$ \\
\hline & 10 & $>32$ & $>32$ & $>32$ \\
\hline \multirow{4}{*}{ Doripenem } & 0 & 0.19 & 0.19 & 0.19 \\
\hline & 1 & 0.25 & 0.25 & 0.50 \\
\hline & 5 & 0.75 & 1 & 1 \\
\hline & 10 & 2 & 2 & 2 \\
\hline \multirow{4}{*}{ Imipenem } & 0 & 0.75 & 1 & 1 \\
\hline & 1 & 1.5 & 1.5 & 1.5 \\
\hline & 5 & 6 & 8 & 12 \\
\hline & 10 & $>32$ & $>32$ & $>32$ \\
\hline \multirow{4}{*}{ Ceftazidime } & 0 & 1.5 & 1.5 & $1.5-2$ \\
\hline & 1 & 1.5 & 1.5 & 1.5 \\
\hline & 5 & 2 & 1.5 & 2 \\
\hline & 10 & 8 & 8 & 8 \\
\hline \multirow{4}{*}{ Piperacillin } & 0 & 4 & 3 & 4 \\
\hline & 1 & 4 & 3 & 3 \\
\hline & 5 & 4 & 4 & 4 \\
\hline & 10 & 12 & 12 & 12 \\
\hline \multirow{4}{*}{$\begin{array}{l}\text { Ticarcillin/ } \\
\text { Clavulanate }\end{array}$} & 0 & 12 & 12 & 12 \\
\hline & 1 & 12 & 12 & 8 \\
\hline & 5 & 6 & 8 & 8 \\
\hline & 10 & 8 & 8 & 8 \\
\hline \multirow{4}{*}{ Aztreonam } & 0 & 2 & 2 & 1.5 \\
\hline & 1 & 2 & 1.5 & 1.5 \\
\hline & 5 & 1 & 1 & 1 \\
\hline & 10 & 1.5 & 1.5 & 1.5 \\
\hline
\end{tabular}


The presence of 5 or $10 \mathrm{mM}$ of spermidine rendered all strains very resistant towards meropenem (MIC $>32 \mu \mathrm{g} / \mathrm{ml}$ ). This change however, occurred in both the unmutagenized and mutagenized strains and thus is likely unrelated to altered $\mathrm{P}_{p o x}$ activity in Tn mutants. Similarly, spermidine at $5 \mathrm{mM}$ produced a significant increase in MIC against imipenem, with $10 \mathrm{mM}$ leading to resistance against this $\beta$-lactam in a strain-independent manner (MIC $>32 \mu \mathrm{g} / \mathrm{ml}$ ). A smaller but significant decrease in susceptibility was also produced against doripenem that, although not leading to resistance, raised MIC values 4-5-fold and 10-fold in the presence of 5 and $10 \mathrm{mM}$ of spermidine, respectively. Spermidine also produced a 2-3-fold increase in MIC against ceftazidime and piperacillin, while having no effect on aztreonam. Overall, although spermidine was able to alter the MIC values against various $\beta$-lactams tested, the most dramatic increase in MICs were produced against the carbapenems, leading to resistance against meropenem and imipenem. This experiment does not reveal if poxB expression is altered or enhanced by the presence of polyamines, merely that polyamines significantly raise the MIC values against the carbapenems and other $\beta$-lactams.

The mechanism by which carbapenem MIC is raised is not clear but it could be related to a decrease in cell permeability. Previous studies in E. coli have shown that polyamines decrease outer membrane permeability to the $\beta$-lactam cephaloridine by inhibiting permeation through the porin channels $\mathrm{OmpF}$ and $\mathrm{OmpC}(875,877)$. Similarly, the polyamine cadaverine induced closing of liposome-reconstituted porins in E. coli and was proposed to be a modulator of porin activity in vivo (876). Under conditions of oxidative stress, polyamines also increased resistance in E. coli against 
fluoroquinolones, antibiotics that are known to traverse the cell via porins (892). Thus a decrease in outer membrane permeability could account for the decrease in susceptibility to carbapenems that is observed in the presence of polyamines in the current study. However, other studies show that polyamines can sensitize E. coli, S. aureus, Salmonella and $P$. aeruginosa to most $\beta$-lactams effectively increasing susceptibility against these and other drugs $(886,893)$. The reduction in MIC suggested that any possible decrease in permeability, caused by polyamine inhibition of porin transport, did not play a major role or was not enough of a factor to generate resistance against most $\beta$-lactams in those studies $(886,893)$.

Porins blockage by polyamines in $P$. aeruginosa does appear to be a factor contributing to resistance at least against imipenem (886). Kwon and Lu showed that while polyamines increased susceptibility against most $\beta$-lactams (decreased MIC), the MIC against imipenem is increased in the presence of polyamines $(886,893)$. This increase appeared to occur by blockage of the OprD porin. Inhibition of OprD activity may explain the results of the present work showing that the MIC against all carbapenems tested is increased in the presence of polyamines. However, MICs of various other non-carbapenem $\beta$-lactams such as ceftazidime and piperacillin, were also slightly altered in this study. These $\beta$-lactams, however, are not known to use the OprD porin for passage into the cell. Thus, if polyamines affect susceptibility by blocking of porins, they may be able to block more than one type of porin besides OprD. In the present study, spermidine however, appears to be more effective at blocking OprD than other porins, since it is the carbapenem MIC that is mostly altered. Alternatively, 
although permeability may be an issue contributing to resistance, other polyamine intracellular effects may be responsible for the increase in MIC observed. Future studies may elucidate on the expression of poxB in the presence of polyamines and the mechanism by which polyamines are able to induce resistance against the carbapenems.

\subsection{Concluding Remarks}

Regulation of class D chromosomal $\beta$-lactamases has only been previously described in Ralstonia pickettii and Aeromonas spp. (567, 730). In R. pickettii, the induction of two chromosomally encoded $\beta$-lactamases, OXA-22 and OXA-60, requires the regulatory element $\mathrm{Rp} 3$ encoded upstream of $b l a_{\text {OXA-60 }}$ and divergently transcribed (730). Aeromonas spp. can produce two-three inducible and unlinked $\beta$-lactamases, one of which is an oxacillinase $(566,734-736)$. Expression of all is coordinately regulated by a two-component system, BlrAB, encoded immediately upstream of the $b l a_{\text {OXA }}$ gene (567, 739). Unpublished studies from our lab showed a two-component system, MifSR, encoded upstream of poxAB and divergently transcribed, played no role in poxB expression or carbapenem resistance.

Previous work suggested the transcriptional regulator AmpR may be involved in the regulation of the pox operon (710). Our transcription studies reveal poxB mRNA levels increase in the absence of AmpR. The increase in pox mRNA levels, however, do not translate into quantifiable changes at the protein levels as measured by PoxB $\beta$ lactamase activity. Additionally, binding of AmpR to the pox promoter was not detected indicating a possible indirect regulation by AmpR. As genes can often regulate their own 
synthesis and that of the operon they are encoded in, the activity of $\mathrm{P}_{p o x}$ was investigated in the presence of PoxA. PoxA was found to decrease $\mathrm{P}_{\text {pox }}$ was activity when expressed in trans from an IPTG inducible promoter. This finding suggests PoxA is capable of regulating the expression of the pox operon. Autoregulation by PoxA may account for the low PoxB activity that is normally observed.

Interestingly, this work has unexpectedly connected the role of the small, essential molecules polyamines with carbapenem resistance. Specifically, a genome search for pox regulators identified Tn insertions in two polyamine transport genes leading to increased $\mathrm{P}_{\text {pox }}$ activity. Although the link between polyamine transport and pox expression is not yet clear, polyamines were found to drastically raise MICs against carbapenems leading to clinical resistant phenotypes. Whether or not the polyamine-induced carbapenem resistance is connected to enhanced pox expression, polyamine research may be a new and worthwhile area of study in the lab.

\subsection{Acknowledgments}

We would like to thank Dr. Stephen Lory for kindly providing mini-transposon strains for mutagenesis and Camille de Rimonteil for the invaluable help with the transposon mutagenesis project. This study was supported in part by the National Institutes of Health -Minority Biomedical Research Support SCORE (SC1AI081376; KM), Research Initiative for Scientific Enhancement graduate student fellowship (NIH/NIGMS R25 GM61347; DZ), and Florida International University Teaching Assistantship (DZ). 


\title{
Chapter 4
}

\section{Structural and functional characterization of Pseudomonas aeruginosa global regulator AmpR}

\author{
As published in the Journal of Bacteriology \\ November 2014 \\ Volume 196, Pages 3890-3902 \\ Copyright (C) The American Society for Microbiology
}

Olivier Caille, Diansy Zincke, Massimo Merighi, Deepak Balasubramanian, Hansi Kumari, Kok-Fai Kong, Eugenia Silva-Herzog, Giri Narasimhan, Lisa Schneper, Stephen Lory, Kalai Mathee

Author contributions:

O.C. and D.Z. contributed equally to the paper. O.C., D.Z., M.M., D.B., H.K., K-F.K., E.S-H., G.N., L.S., S.L., and K.M. designed research; O.C., D.Z., M.M., D.B., H.K., KF.K., E.S-H., N.A.N., and L.S. performed research; O.C., D.Z., M.M., D.B., H.K., KF.K., E.S-H., G.N., L.S., S.L., and K.M. analyzed data; and O.C., D.Z., and K.M. wrote the paper. 


\subsection{Abstract}

Pseudomonas aeruginosa is a dreaded pathogen in many clinical settings. Its inherent and acquired antibiotic resistance thwarts therapy. In particular, derepression of the AmpC $\beta$-lactamase is a common mechanism of $\beta$-lactam resistance among clinical isolates. The inducible expression of $\operatorname{ampC}$ is controlled by the global LysR-type transcriptional regulator (LTTR) AmpR. In the present study we investigated the genetic and structural elements that are important for $a m p C$ induction. Specifically, the $a m p C$ $\left(\mathrm{P}_{a m p C}\right)$ and $\operatorname{ampR}\left(\mathrm{P}_{a m p R}\right)$ promoters and the $\mathrm{AmpR}$ protein were characterized. The transcription start sites (TSSs) of the divergent transcripts were mapped using 5' rapid amplification of cDNA ends-PCR (RACE-PCR), and strong $\sigma^{54}$ and $\sigma^{70}$ consensus sequences were identified at $\mathrm{P}_{a m p R}$ and $\mathrm{P}_{a m p C}$, respectively. Sigma factor RpoN was found to negatively regulate $a m p R$ expression possibly through promoter blocking. Deletion mapping revealed the minimal $\mathrm{P}_{a m p C}$ extends 98-bp upstream of the TSS. Gel shifts using membrane fractions showed $\mathrm{AmpR}$ binds to $\mathrm{P}_{a m p C}$ in vitro whereas in vivo binding was demonstrated using ChIP-qPCR. Additionally, site-directed mutagenesis of the AmpR helix-turn-helix (HTH) motif identified residues critical for binding and function (Ser38, and Lys42), and critical for function but not binding (His39). Amino acids Gly102 and Asp135, previously implicated in the repression state of AmpR in the enterobacteria, were also shown to play a structural role in P. aeruginosa AmpR. Alkaline phosphatase fusion and shaving experiments suggest that AmpR is likely to be membrane associated. Lastly, in vivo cross-linking study shows that AmpR dimerizes. In conclusion, a potential membrane-associated AmpR dimer regulates ampC expression by direct binding. 
Key Words: $\beta$-Lactam resistance, LysR-type transcriptional regulator, AmpC $\beta$ lactamase

Abbreviations: EMSA, electrophoretic mobility shift assay; HTH, helix-turn-helix; LTTR, LysR-type transcriptional regulator; PG, peptidoglycan; RACE-PCR, rapid amplification of cDNA ends-PCR; TSS, transcriptional start site 


\subsection{Introduction}

Pseudomonas aeruginosa, a Gram-negative opportunistic pathogen, causes severe and life-threatening infections in susceptible individuals. This pathogen is primarily associated with morbidity and mortality in patients with cystic fibrosis, a deadly genetic disease (742). The bacterium's innate ability to counteract the action of antibiotics often complicates treatment strategies. Intrinsic resistance is conferred by its low membrane permeability, the expression of efflux pumps and hydrolyzing enzymes, the alteration of antimicrobial targets and the ability to form biofilm $(246,436,781)$. In particular, resistance to $\beta$-lactam antibiotics is mediated by the expression and overproduction of a chromosomally-encoded class C $\beta$-lactamase AmpC (379-381).

Ambler class C $\beta$-lactamases were first described in members of the Enterobacteriaceae family where expression is either constitutively low or inducible (375, 376, 630, 894). In species where expression is inducible, such as Citrobacter freundii and Enterobacter cloacae, the induction requires $\beta$-lactam challenge and the presence of a transcriptional regulator $\operatorname{AmpR}(375,712,895,896)$. AmpR is a member of the LysR family of transcriptional regulators and as such is a DNA-binding protein with a predicted helix-turn-helix $(\mathrm{HTH})$ motif at the N-terminus and an inducer-binding domain at the C-terminus $(856,897)$. Comprehensive studies in the Enterobacteriaceae have established the critical role of AmpR as the regulator of $a m p C$ expression and the paradigm of $\beta$-lactamase induction.

In the Enterobacteriaceae, $\operatorname{amp} C$ inducibility is intimately linked to the recycling of the peptidoglycan $(\mathrm{PG})$ of the murein sacculus $(713,716,718,721)$. During normal 
physiological growth, N-actylglucosaminyl-1,6-anhydromuropeptides (GlcNAc-1,6anhydro-MurNAc tri, tetra, and pentapeptides) are continuously being released from the murein sacculus due to remodeling $(716,718)$. The permease, AmpG, transports the metabolites into the cytoplasm where the glycosidase NagZ removes the GlcNAc moiety and the amidase AmpD removes the stem peptides from either the incoming GlcNAc-1,6anhydro-MurNAc-peptides or from the NagZ-processed product $(716,717,719,898$ 901). The resulting muramyl peptides are recycled back into the PG biosynthetic pathway to form the PG precursor UDP-MurNAc-pentapeptide (902). It has been proposed that during normal cell growth, the cytosolic concentrations of UDP-MurNAc-pentapeptide maintain AmpR in an inactive conformation that represses the expression of $\operatorname{ampC}$ (716, 718). In the presence of $\beta$-lactams, however, there is an excessive breakdown of murein leading to accumulation of 1,6-anhydromuropeptides in the cytoplasm, which in turn overwhelm the hydrolytic activity of $\operatorname{AmpD}(714,716,718,903)$. The increased number of AmpD-unprocessed muramyl peptides presumably displaces the repressor UDPMurNAc-pentapeptide from AmpR and induce a conformational change in the protein to promote expression of $\operatorname{amp} C(714,718,903)$.

All amp gene homologs $(\operatorname{ampC}, \operatorname{ampR}, a m p D$ and $a m p G)$ have been identified and studied in $P$. aeruginosa $(643,710,711,776,782,904-906)$. Whether a similar induction mechanism is employed by $P$. aeruginosa is not yet known, however, recent work illustrates significant departures from the classical enterobacterial induction system. In particular, there are three $a m p D$ homologs in $P$. aeruginosa that are responsible for a stepwise up-regulation mechanism leading to constitutive $\beta$-lactamase hyperexpression 
(246, 643, 904). Additionally, P. aeruginosa harbors two AmpG homologs, PA4218 (AmpP) and PA4393 (AmpG) that appear to be required for induction of ampC (837, 906). Further, our lab has shown that $P$. aeruginosa AmpR is a global transcriptional regulator involved in the control of amp and various other genes (710, 842-844).

AmpR exhibits high sequence identity with its counterparts in $C$. freundii and $E$. cloacae, and as in the Enterobacteriaceae, $a m p R$ is located immediately upstream of ampC and divergently transcribed $(711,776)$. Such similarities suggest a common regulatory mechanism among the species, however, the $P$. aeruginosa ampR-ampC intercistronic region bears little resemblance to that of the enterobacteria. In vitro studies using crude extracts have shown $P$. aeruginosa AmpR binds to this region, but the exact binding site and the identity of the amino acids involved in the interaction have not yet been determined (776). In essence, not much is known about the structural elements that are critical to the functioning of $P$. aeruginosa AmpR as regulator of $\beta$-lactamase expression.

In the present work, we define some of the genetic elements in the ampR-ampC intergenic region including the $a m p R$ and $\operatorname{ampC}$ transcriptional start sites, as well as, the minimal length of the $a m p C$ promoter needed for induction of the $a m p C$ system. We further show AmpR specifically binds to the 193-bp $\mathrm{P}_{a m p C}$ fragment identified by promoter mapping as being required for induction. We also identify amino acids in the AmpR HTH motif that are critical for the interaction with the promoter. Additionally, we examine the role of two amino acids, Gly102 and Asp135, previously implicated in the repression state of AmpR from the Enterobacteriaceae. Lastly, we show that $P$. 
aeruginosa AmpR likely functions as dimers as previously seen for $C$. freundii and is potentially a membrane protein.

\subsection{Materials and Methods}

4.3.1 Bacterial strains, plasmids and media. Bacterial strains, plasmids and primers employed in this study are shown in Table S1 of the Supplemental Material. E. coli and P. aeruginosa were routinely cultured in Luria-Bertani medium (10 g tryptone, $5 \mathrm{~g}$ yeast extract, and $5 \mathrm{~g} \mathrm{NaCl}$, per liter). Pseudomonas Isolation Agar (Difco) was used with LB at a 1:1 ratio in triparental mating experiments. Antibiotics were used at the following concentrations (per milliliter): ampicillin (Ap) at $100 \mu \mathrm{g}$, tetracycline (Tc) at $15 \mu \mathrm{g}$, and gentamycin $(\mathrm{Gm})$ at $15 \mu \mathrm{g}$ for E. coli; $\mathrm{Gm}$ and Tc each at $75 \mu \mathrm{g}$ for P. aeruginosa. PA0 $\triangle a m p C$ and PA0 $\triangle a m p R$ strains used in this work were previously constructed using overlap extension PCR and homologous recombination $(760,843)$.

4.3.2 $P_{\text {ampC }}$ promoter deletions. To characterize the minimal promoter necessary for full activity, 5'-end deletions of $\mathrm{P}_{a m p C}$ were constructed and transcriptionally fused to a promoterless lacZ. Briefly, 352-, 193-, 171-, 151-, 130-, 111-, 90-, 70-, and 51-bp fragments were generated by PCR with the following primer pairs respectively: SBJ03ampCRFor-OCP ${ }_{a m p}$ RevBc, $\quad \mathrm{OCP}_{a m p}$ For 193-OCP $_{a m p C}$ RevBc,$\quad \mathrm{OCP}_{a m p}$ For173-

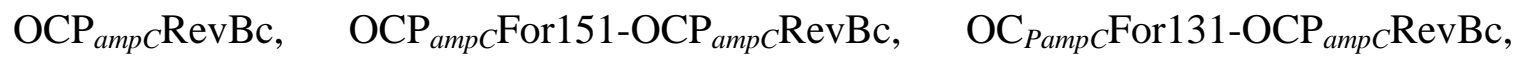
$\mathrm{OCP}_{a m p} C$ For111-OCP ${ }_{a m p} \mathrm{RevBc}, \quad \mathrm{OCP}_{a m p}$ For91-OCP $\mathrm{Omp}_{\mathrm{C}} \mathrm{RevBc}, \quad \mathrm{OCP}_{a m p c}$ For71$\mathrm{OCP}_{a m p} \mathrm{RevBc}$, and $\mathrm{OCP}_{a m p} \mathrm{~F}_{\mathrm{F}}$ 51-OCP ${ }_{a m p} \mathrm{RevBc}$ (Table S1, Supplemental material). 
The fragments were sequenced, then cloned into the EcoRI-BamHI sites of the integrative vector mini-CTX-lacZ and integrated into PA01.

4.3.3 Construction of His-tagged AmpR. Primers OCAmpR-His-For and OCAmpRHis-Rev (Table S1) were used to amplify PA01 genomic ampR. The 933-bp amplicon, carrying a $\mathrm{His}_{6}$ sequence at the 3 '-end was cloned into pBluescriptSK(+) and sequenced (Table S1). The fragment was subsequently subcloned into the EcoRI-BamHI sites of pMMB67EH-Gm, a broad-host range expression vector (763). His-tagged AmpR was shown to be functional by $\beta$-lactamase assay and E-test (Text S1 and Table S2, Supplemental material).

4.3.4 Expression and purification of AmpR-His6. AmpR-His 6 was purified according to standard protocols. Briefly, stationary-phase cultures of PA0 $\Delta a m p R\left(\mathrm{pAmpR}^{\left.-\mathrm{His}_{6}\right)}\right.$ were diluted to an $\mathrm{OD}_{600}$ of 0.02 in 2 liters of LB broth and incubated with shaking at $37^{\circ} \mathrm{C}$ until the culture density reached an $\mathrm{OD}_{600}$ of 0.2 . Cells were then induced with 1 $\mathrm{mM}$ of isopropyl $\beta$-D-1-thiogalactopyranoside (IPTG) and incubated for an additional six hours before harvesting. The cells were recovered by centrifugation at $6,000 \mathrm{Xg}$ for 10 minutes at $4^{\circ} \mathrm{C}$ and resuspended in $25 \mathrm{ml}$ of lysis buffer $(20 \mathrm{mM}$ HEPES pH8, $0.5 \mathrm{M}$ $\mathrm{NaCl}, 10 \%$ glycerol, $1 \mathrm{mM}$ PMSF, 2 pellets of EDTA-free protease inhibitor cocktail tablets complete, $100 \mu \mathrm{l}$ of $0.1 \mathrm{mg} \mathrm{ml}^{-1}$ of lysozyme and $5 \mu 1$ of DNase1). Following disruption of the cells on ice with sonication (20-s pulse ON and 20-s pulse OFF for 20 minutes, amplitude 50\%), the cell lysate was centrifuged at 10,000 X g for 10 minutes at 
$4^{\circ} \mathrm{C}$. The supernatant was further ultracentrifuged at $36,000 \mathrm{rpm}$ for $1 \mathrm{~h}$ at $4^{\circ} \mathrm{C}$. Membranes pellets were resuspended in $20 \mathrm{ml}$ of solubilization buffer (20 mM HEPES pH8, $0.5 \mathrm{M} \mathrm{NaCl}, 10 \%$ glycerol, $5 \mathrm{mM}$ imidazole, $1 \mathrm{mM}$ PMSF, 2 pellets of EDTA-free Protease Inhibitor cocktail tablets complete, and $2 \%$ of CHAPS) and loaded onto a HisTrap FF $1 \mathrm{ml}$ column. AmpR-His 6 was eluted with buffer B (20 mM HEPES pH8, $0.5 \mathrm{M} \mathrm{NaCl}, 10 \%$ glycerol, $500 \mathrm{mM}$ imidazole and $0.6 \%$ of CHAPS) by using an FPLC chromatograph (Akta, Amersham Biosciences). About $20 \mathrm{ml}$ were recovered and dialyzed to remove imidazole. This AmpR preparation was used to make AmpR-specific antibodies.

4.3.5 Construction of $P$. aeruginosa AmpR HTH and point mutants. Site-directed mutagenesis was used to replace various amino acid residues in AmpR. Briefly, Ser38, His39, Lys42, Ser43, and Glu46 were replaced with Ala; Gly102 and Asp135 were replaced with Glu and Asn, respectively. Substitutions were constructed by PCR using the following primer pairs: AmpRSer38AlaFor-AmpRSer38AlaRev, AmpRHis39AlaForAmpRHis39AlaRev, AmpRLys42AlaFor-AmpRLys42AlaRev, AmpRSer43AlaForAmpRSer43AlaRev, AmpRGlu46AlaFor-AmpRGlu46AlaRev, AmpRGly102GluForAmpRGly102GluRev, and AmpRAsp135AsnFor-AmpRAsp135AsnRev (Table S1, Supplemental material). 
4.3.6 Membrane fraction purification. Preliminary studies showed pAmpR-His 6 expression and $\beta$-lactamase induction were achieved with a 2 -hour incubation at a $1 \mathrm{mM}$ IPTG concentration. Thus, PA0 $\Delta a m p R$ (pAmpR-His 6 ) cells at $\mathrm{OD}_{600}$ of 0.2 were induced with $1 \mathrm{mM}$ of IPTG and incubated for two hours before harvesting for membrane fractionation. For $\beta$-lactamase induction, cells were further treated with $200 \mu \mathrm{g} \mathrm{ml}^{-1}$ of penicillin $\mathrm{G}$ an hour after IPTG addition. Cells were recovered by centrifugation at 6,000 $\mathrm{X} \mathrm{g}$ for 10 minutes at $4^{\circ} \mathrm{C}$ and resuspended in $50 \mathrm{ml}$ of lysis buffer $(20 \mathrm{mM}$ HEPES pH8, 0.1 M NaCl, $1 \mathrm{mM}$ EDTA, $1 \mathrm{mM}$ PMSF, and $50 \mu$ of DNase1). Following disruption of the cells on ice with sonication (15 cycles of 10 second pulse $\mathrm{ON}$ and $30 \mathrm{~S}$ pulse OFF, amplitude $40 \%$ ), the cell lysate was centrifuged at 5,000 rpm for 10 minutes at $4^{\circ} \mathrm{C}$. The supernatant was ultracentrifuged at 36,000 rpm (Rotor Ti70) for one hour at $4^{\circ} \mathrm{C}$ and the pellets were resuspended with $2 \mathrm{ml}$ of membrane buffer (25\% sucrose, $20 \mathrm{mM}$ Tris $\mathrm{pH} 8$, and 0.5 mM PMSF). Two-hundred milliliters of membrane fractions were aliquoted and stored at $-80^{\circ} \mathrm{C}$.

\subsubsection{Electrophoretic mobility shift assay (EMSA). The 193-bp PCR fragment} containing the $a m p R$ - $a m p C$ intergenic region plus a small part of $a m p R$ ORF was used to perform EMSA. Ten pmol of this fragment were radiolabelled at the 5' end by incubation with T4 polynucleotide kinase (NEB) and $\left[\gamma^{32} \mathrm{P}\right] \mathrm{ATP} \quad\left(3,000 \mathrm{Ci} \mathrm{mmol}{ }^{-1}\right.$; Perkin Elmer). The labeled fragment was diluted to a final concentration $100 \mathrm{nM}$ and unincorporated nucleotides were removed by sephadex G-25 (Biorad) spin chromatography. DNA binding reactions containing $50 \mathrm{fmol}$ of ${ }^{32} \mathrm{P}$-labeled DNA probe 
and varying amounts of total protein membrane fractions were incubated for 20 minutes and loaded thereafter in a non-denaturating 5\% PAGE. Radioactive signals were detected by scanning a phosphostorage cassette with the GE Healthcare Typhoon 9400 scanner.

For competition assays, $50 \mathrm{fmol}$ of the $193-\mathrm{bp}{ }^{32} \mathrm{P}$-labeled probe were mixed with the unlabeled 193-bp fragment in 10-, 100- and 500-fold molar excess in the EMSA assay. For nonspecific assays, $50 \mathrm{fmol}$ of the $193-\mathrm{bp}{ }^{32} \mathrm{P}$-labeled probe were mixed with a PCR-amplified 233-bp fragment (alg44) in 10-, 100- and 500-fold molar excess in the EMSA assay.

4.3.8 5' RACE PCR. The $a m p C$ and $a m p R$ TSSs were mapped using a classical 5' RACE-PCR on total mRNAs extracted from PA01, PA0 $\Delta a m p R$ and PA0 $a m p C$ (907). Stationary-phase cultures of PA01, PA0 $\triangle a m p R$ and PA0 $\triangle a m p C$ were diluted to an $\mathrm{OD}_{600}$ of 0.02 and incubated with shaking at $37^{\circ} \mathrm{C}$ until the cultures reached an $\mathrm{OD}_{600}$ of 0.6 . The cultures were then induced with $200 \mu \mathrm{g} \mathrm{ml}^{-1}$ of penicillin $\mathrm{G}$ for one hour and subsequently blocked on ice for 15 minutes with $1 / 5^{\text {th }}$ of the final culture volume in $5 \%$ acidic phenol-95\% ethanol, $\mathrm{pH}$ 4. One milliliter of the cells were recovered by centrifugation and resuspended with $3 \mathrm{mg} \mathrm{ml}^{-1}$ of lysozyme (Tris EDTA, pH 8). RNA was then extracted according to the RNeasy mini kit protocol (Qiagen), treated with $10 \mathrm{U}$ of RQ1 DNase (Promega) for one hour, extracted with phenol/chloroform/isoamyl alcohol (25:24:1) and chloroform, precipitated and dried. Superscript III (Invitrogen) was then used to reverse transcribe $10 \mu \mathrm{g}$ of RNA as previously described (907) using primers 
5RA- $\mathrm{P}_{a m p c} 233$ and 5RA-P $\mathrm{P}_{a m p R} 229$ for determination of the ampC and ampR TSS, respectively. In the first round of PCR amplification (Pfu, Stratagen), primers $\mathrm{Q}_{t}, \mathrm{Q}_{0}$, and 5RA- $\mathrm{P}_{a m p} 154$ were used for $\operatorname{ampC}$ TSS determination, while $\mathrm{Q}_{\mathrm{t}}, \mathrm{Q}_{0}$, and 5RA-P $\mathrm{P}_{a m p R} 169$ were used for determination of the ampR TSS. In the second round of PCR amplification (Pfu, Stratagen), primer pairs $\mathrm{Q}_{\mathrm{i}}-5 \mathrm{RA}-\mathrm{P}_{a m p C} 113$, and $\mathrm{Q}_{\mathrm{i}}-5 \mathrm{RA}-\mathrm{P}_{a m p R} 99$ were used for ampC and $\operatorname{ampR}$ TSS determination, respectively (Table S1). PCR products were cloned into TOPO (Invitrogen), blue colonies were selected for screening and clones were sequenced.

4.3.9 qPCR analysis of $a m p R$ and $a m p C$ mRNAs. Total RNA was extracted from PA01, PA0 $\Delta r p o N$ and PA0 $\Delta r p o N(\mathrm{pRpoN})$ in the presence and absence of the inducer $\left(0.2 \mu \mathrm{g} \mathrm{ml}^{-1}\right.$ imipenem) using the RNeasy mini kit (Qiagen). RNA was reverse transcribed into cDNA with Superscript III (Invitrogen) and an (NS) $)_{5}$ random primer using standard methods (764). For qPCR, the ABI 7500 (Applied Biosystems) cycler was used with Power SYBR Green PCR Master Mix with ROX (Applied Biosystems). The reading was normalized to $\operatorname{clpX}$ (PA1802), whose expression remains constant in all the samples and conditions tested. Melting curves were generated to ensure primer specificity. Gene expression of each sample was normalized to PA01 uninduced value, to see the effect of induction and mutation at the same time. Primer pairs DBS_QRTAmpRFwd-DBS_QRTAmpRRev and qRT_ampCF-qRT_ampCR were used for the real time amplification of $a m p R$ and $\operatorname{ampC}$, respectively. 
4.3.10 Construction of VSV-G-tagged AmpR. A 540-bp fragment corresponding to the 3' end of $a m p R$, minus the stop codon, was amplified using primers DB_ampR3'_F and DB_ampR3'_R. The amplicon was cloned into pP30 4 FRT-MvaT-V (908), a replicationincompetent vector in $P$. aeruginosa, such that the 3 ' end of $a m p R$ was fused in frame with three alanines and the VSV-G tag (YTDIEMNRLGK). The construct was then was introduced into PA01 as single copy by mating and clones were selected for gentamycin resistance.

\subsubsection{Chromatin immunoprecipitation quantitative PCR (ChIP-qPCR). Cells} harboring the VSV-G-tagged AmpR were harvested after sub-MIC $\beta$-lactam exposure (843) and treated with formaldehyde to cross-link proteins with DNA as previously described (908). DNA was sheared by sonication to an average length of $0.5-1.0 \mathrm{~kb}$ and AmpR was immunoprecipitated with anti-VSV-G agarose beads (BETHYL Laboratories, Inc.). ChIP-qPCR was performed using Power SYBR Green PCR Master Mix (Applied Biosystems) with primers DBS_ChIP_ampCF and DBS_ChIP_ampCR. Fold enrichment was normalized to $\operatorname{clpX}(P A 1802)$.

4.3.12 Protein cross-linking. PA0 $\triangle a m p R\left(\mathrm{pAmpR}^{-H i s_{6}}\right)$ was grown to an $\mathrm{OD}_{600}$ of 0.3 and induced with $1 \mathrm{mM}$ IPTG for 2 hours. Cells were grown for two additional hours in the presence and absence of $\beta$-lactam antibiotics $\left(0.1 \mu \mathrm{g} \mathrm{ml}^{-1}\right.$ of imipenem). Cultures were then treated with $0.1 \%$ formaldehyde for 20 and 40 minutes at room temperature. Crude extracts containing $10 \mu \mathrm{g}$ of total protein were separated on an SDS- 
polyacrylamide gel and AmpR was visualized using anti-His antibody. The blot was subsequently stripped and reprobed using anti- $\sigma^{70}$ antibody (NeoClone).

4.3.13 Polyclonal anti-AmpR-His6 antibody production. Purified AmpR-His 6 was used as antigen to raise anti-AmpR-His 6 rabbit polyclonal antibodies (Covance, Princeton, NJ). AmpR-His ${ }_{6}$ antibody was affinity-purified as previously described (909).

4.3.14 Western Blotting and EMSA of HTH mutants. Concentration of purified AmpR-His 6 was determined by the bicinchoninic acid (BCA) method (910). A calibration Western blot was generated using FujiFilm LAS-3000 imager to correlate intensities with the concentration of purified AmpR-His 6 . Membrane fractions were purified from PA0 $\triangle a m p R$ pAmpR-HTH mutants and their concentrations were determined using the BCA method, whereas the exact quantity of AmpR was deduced from Western blotting. Preliminary gel shifts with increasing concentrations of membrane fractions of AmpR-HTH mutants showed that a $0.4 \mathrm{mg} \mathrm{ml}^{-1}$ is sufficient to shift the 193-bp $\mathrm{P}_{a m p C}$ PCR fragment (Data not shown). For a second gel shift, a $0.4 \mathrm{mg}$ $\mathrm{ml}^{-1}$ of total membrane fraction (8.44 $\mathrm{ng}$ of AmpR), recovered from PA0 $\triangle a m p R$ overexpressing AmpR HTH mutants in the presence and absence of $200 \mu \mathrm{g} \mathrm{ml}{ }^{-1}$ penicillin G, was hybridized with the 193-bp PCR fragment spanning the ampC-ampR intergenic region (Fig. 7). As this concentration was not enough to visualize AmpR, a higher quantity (33 ng) was used for Western blotting of the HTH mutants to show that the amount of AmpR-His 6 is equivalent under all conditions and thus in the EMSA 
experiment (Data not shown). Further, the stability of AmpR-His ${ }_{6}$ mutants was verified by Western using equal amounts of total protein with AmpR-specific antibodies (Figure 4.8). Sigma $^{70}$ (NeoClone) was used as control to show the same amount of total protein was loaded per sample. All Western blots were developed according to standard protocols. Briefly, proteins were transferred to a PVDF membrane (Bio-Rad) and blocked with TBST (TBS $0.1 \%$ Tween) and $5 \%$ non-fat dry milk at $4{ }^{\circ} \mathrm{C}$ overnight or for four hours, followed by rinsing with the same solution and probing with rabbit antiAmpR antibody (1:3000). Membranes were subsequently washed with TBST, incubated with goat anti-rabbit $\operatorname{IgG}(\mathrm{H}+\mathrm{L})$-horseradish peroxidase-conjugated antibody (1:5000) (Bio-Rad), rinsed and developed using Enhanced Chemiluminescence Western Blotting Substrate (Pierce).

4.3.15 $\beta$-galactosidase assay. $\beta$-galactosidase assays were performed as previously described (854).

4.3.16 AmpR-LacZ and -PhoA fusion construction and analysis. The topology of AmpR was investigated using phoA and lacZ fusions. The plasmid pSJ01 (710) carrying a 1220-bp fragment containing ampR was digested at HindIII, HincII and PstI, corresponding to the amino acid positions, Gln15, Val134 and Gln186, respectively (Fig. S2A). The resultant fragments were ligated in-frame upstream of phoA and lacZcontaining plasmids, pTrcphoA and pTrclacZ (Table S1, Supplemental material), (911). 
The phoA and lacZ activities were qualitatively determined in E. coli according to standard protocols (854).

4.3.17 Protease protection (shaving) assay. A stationary-phase culture of PA0 $\Delta a m p R$ (pAmpR-His ${ }_{6}$ ) was diluted to an $\mathrm{OD}_{600}$ of 0.02 and incubated with shaking at $37^{\circ} \mathrm{C}$ until the culture reached an $\mathrm{OD}_{600}$ of 0.4 . The cells were then induced with $1 \mathrm{mM}$ IPTG for four hours and chloramphenicol $\left(500 \mu \mathrm{g} \mathrm{ml}^{-1}\right)$ was added 30 minutes prior to harvesting to stop protein synthesis. The cells were harvested by centrifugation and resuspended in $40 \mathrm{mM}$ Tris- $\mathrm{Cl} \mathrm{pH} 8.0,0.5 \mathrm{M}$ sucrose. Spheroplasts were obtained by adding $1 \mathrm{mg} \mathrm{ml}^{-1}$ of lysozyme and $4 \mathrm{mM}$ EDTA for 10 minutes in a $30^{\circ} \mathrm{C}$ water bath followed by the addition of $20 \mathrm{mM} \mathrm{MgCl}$. The formation of spheroplasts was monitored by light microscopy. Spheroplasts were harvested by centrifugation at $4,000 \mathrm{X} \mathrm{g}$ for 10 minutes and resuspended in $40 \mathrm{mM}$ Tris- $\mathrm{Cl} \mathrm{pH} 8.0,0.5 \mathrm{M}$ sucrose. Proteinase $\mathrm{K}\left(10 \mu \mathrm{g} \mathrm{ml}^{-1}\right)$ was added to $1 \mathrm{ml}$ aliquot of the spheroplasts and incubated in a $30^{\circ} \mathrm{C}$ water bath. Samples were taken at different time points and added to $2 \mathrm{mM}$ PMSF and 4X SDS PAGE Sample Buffer. Samples were then boiled for 5 minutes and ran in a 4-20\% SDS-PAGE (Criterion Biorad). Proteins were transferred to a nitrocellulose membrane (Biorad) and identified using AmpR (Covance), $\sigma^{70}$ (NeoClone) and His-tag (Qiagen) antibodies. The immunoblot was developed using SuperSignal West Pico Chemiluminescent Substrate (Thermo Scientific). 


\subsection{Results and Discussion}

\subsubsection{Analysis of the $P$. aeruginosa ampC-ampR regulatory region. $P$. aeruginosa}

AmpR shares high identity with its homologs in C. freundii and E. cloacae (58.3 and $62.5 \%$, respectively), as well as, the same gene organization, with $a m p R$ located upstream of $\operatorname{ampC}$ and divergently transcribed $(711,776)$. The $P$. aeruginosa ampR-ampC intercistronic region, however, shares no significant similarities with that of $C$. freundii and $E$. cloacae, with the exception of an inverted 38-bp sequence that in $C$. freundii is protected by AmpR $(711,840)$. The lack of conservation in the promoter region could point to different sigma factor requirements and/or different regulatory mechanisms. To elucidate the transcriptional regulatory elements of $P$. aeruginosa $\operatorname{ampC}$ and $\operatorname{ampR}$, their 148-bp intergenic region was characterized.

The transcriptional start sites (TSS) were determined using 5' RACE-PCR on total mRNAs isolated from PA01, PA0 $\triangle a m p R$ and PA0 $\triangle a m p C$ (Figure 4.1). A strong similarity to RpoN $\left(\sigma^{54}\right)$ sigma factor sequences was detected at -12 and -24 positions of the $a m p R$ TSS, whereas strong $\sigma^{70}$ promoter sequences were observed at the -10 and -35 positions of ampC. Alignment of the C. freundii, E. cloacae and P. aeruginosa intergenic region reveals a fair conservation for the $\operatorname{ampC}-10$ and -35 . However, there is a downstream shift in the $P$. aeruginosa ampR TSS that could contribute to the change in sigma factor control observed here (Figure S1, Supplemental material).

The presence of putative -12 and -24 recognition sequences in the ampR promoter

$\left(\mathrm{P}_{a m p R}\right)$ suggested that its expression might be RpoN or $\sigma^{54}$-dependent. Quantitative realtime PCR was used to validate this hypothesis. Total RNA was extracted from $\beta$-lactam 


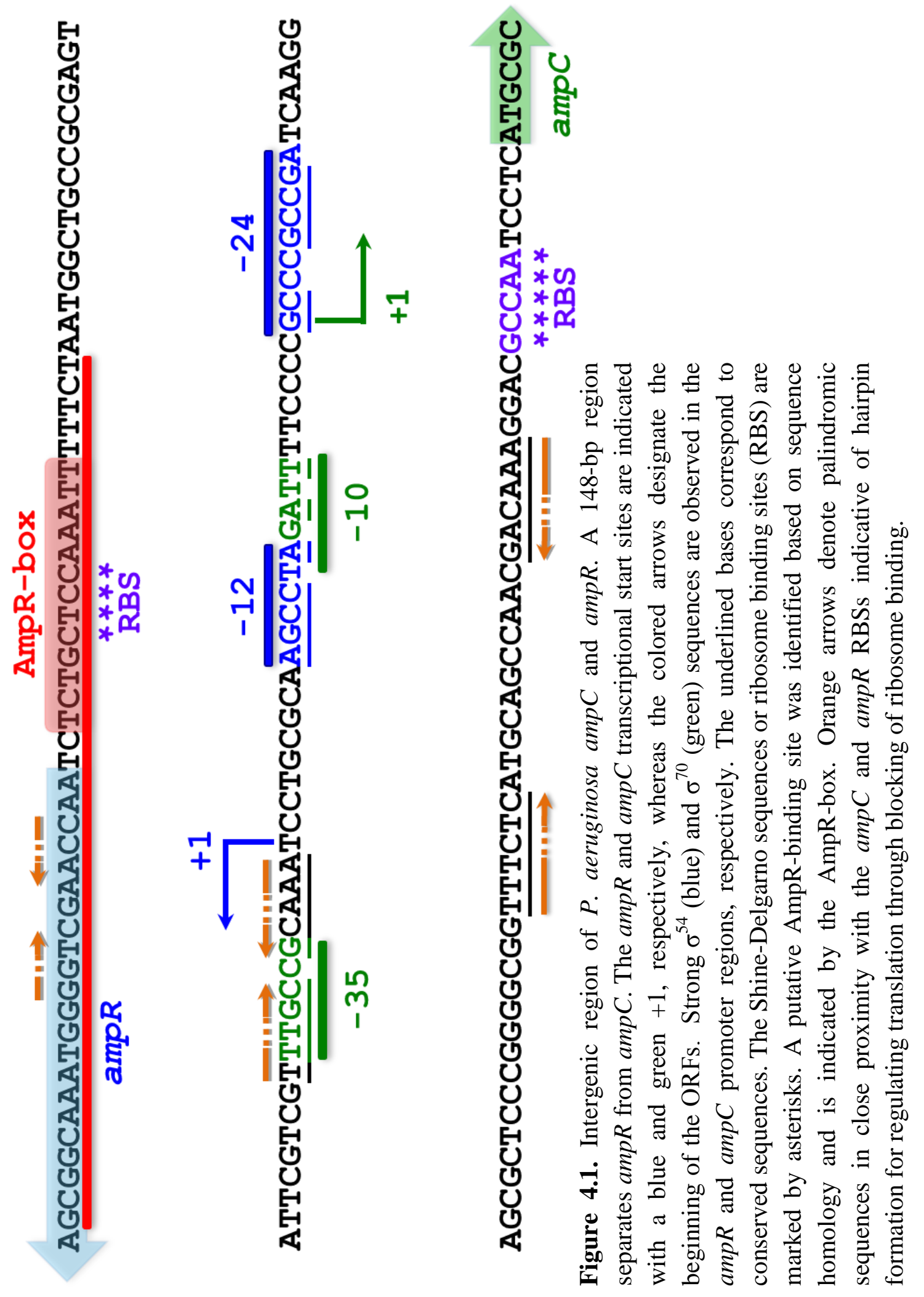


induced and uninduced PA01, PA0 $\Delta r p o N$ and PA0 $\Delta r p o N(\mathrm{pRpoN})$ strains and reverse transcribed into cDNA using standard methods (764). In the wild-type, exposure to $\beta$ lactams increased $\operatorname{ampR}$ and $\operatorname{ampC}$ expression approximately 100-fold over the uninduced samples (normalized to 1) (Figure 4.2). A further, significant increase in expression of both genes was observed in PA0 0 rpoN upon $\beta$-lactam challenge that could be rescued by pRpoN. In the absence of $\beta$-lactams, a small, but significant reduction in expression was observed in the PA0 $\Delta r p o N$ that could not be complemented.

In spite of the presence of a probable binding site on $\mathrm{P}_{a m p R}, \sigma^{54}$ is not required for ampR expression. The significant increase in the absence of $r p o N$ and in the presence of the $\beta$-lactams suggests $\sigma^{54}$ negatively impacts $\operatorname{ampR}$ expression. RpoN may exert this negative effect indirectly by enabling transcription of a negative regulator or directly by competing for the RNA polymerase holoenzyme with another sigma factor. Alternatively, $P$. aeruginosa RpoN can directly repress transcription by blocking promoter access to a different sigma factor in a phenomenon referred to as $\sigma$-factor antagonism as previously reported (912). Specifically, $\sigma^{54}$ has been shown to repress $\sigma^{22}$ dependent transcription from $\mathrm{P}_{a l g D}$ by directly binding to the overlapping promoter sequences in the absence of an external stimulus (912). Similarly, $\sigma$-factor antagonism has also been reported in E. coli, where mutagenesis of a $\sigma^{54}$-dependent promoter created a new TSS with $\sigma^{70}$ requirement that exhibited decreased transcriptional activity in the presence of $\sigma^{54}(913)$. 


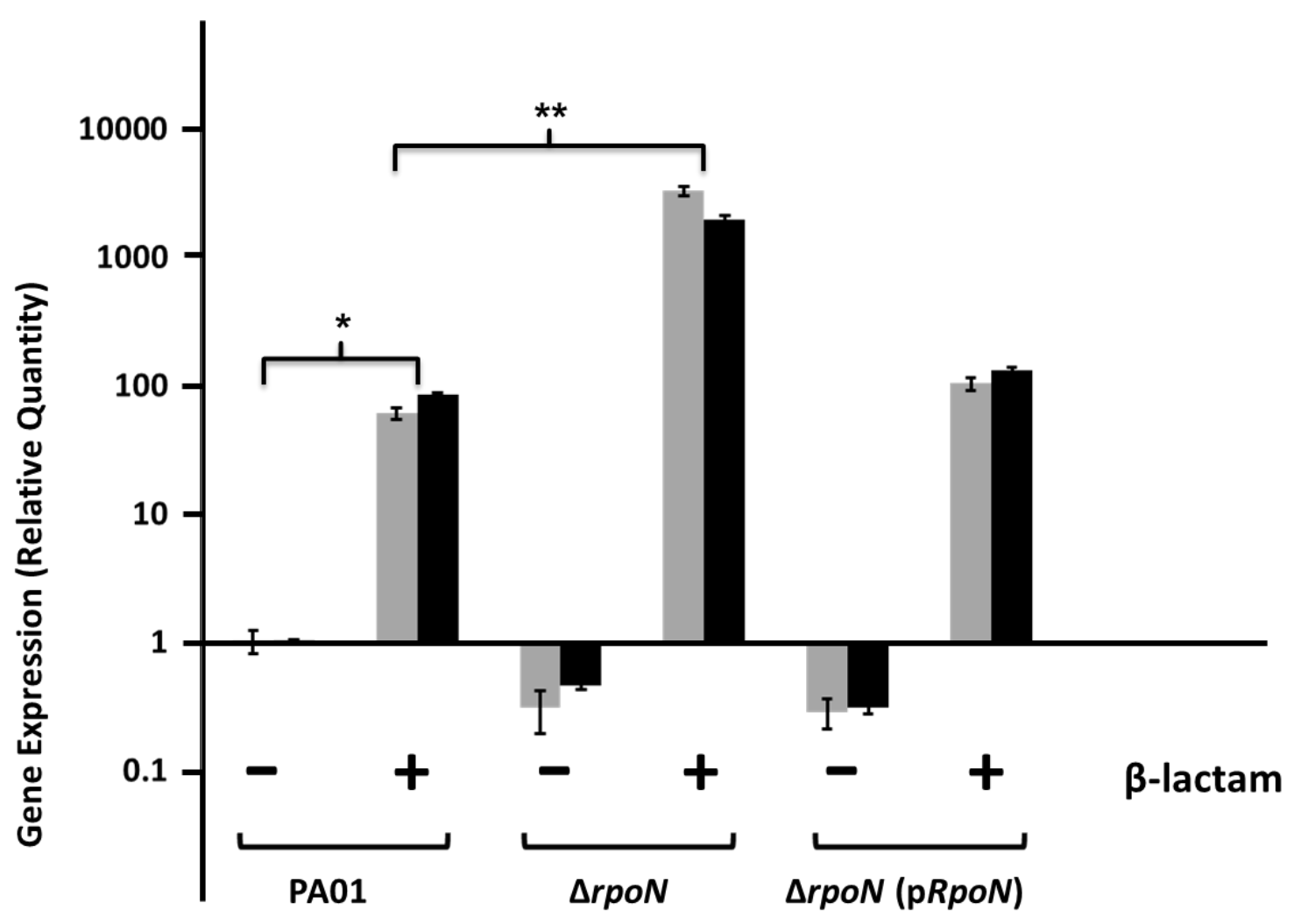

Figure 4.2. RpoN downregulates $P$. aeruginosa ampR expression in the presence of $\beta$ lactams. RNA was isolated from PA01, PA0 $\Delta r p o N$ and PA0 $\Delta r p o N(\mathrm{pRpoN})$ in the presence and absence of $\beta$-lactams, reversed transcribed to cDNA and tested by $\mathrm{qPCR}$ with $\operatorname{ampC}$ (grey bars) and $\operatorname{ampR}$ (black bars) specific primers, as described in the Experimental Procedures. Values were normalized to the expression of the wild-type uninduced sample and represent the mean $( \pm \mathrm{SD})$ of two experiments conducted in triplicates. * ${ }^{*}$-value $<0.001$ for both $a m p R$ and $a m p C$ expression in induced PA01 versus expression in uninduced PA01, ** p-value $<0.005$ for $a m p R$ and $a m p C$ expression in induced PA0 4 rpoN versus expression in PA01 induced as determined by unpaired $t$ test. 
A model of repression by $\sigma^{54}$ through promoter blocking is conceivable, where in the absence of some external stimuli, in this case $\beta$-lactams, $\sigma^{54}$ binds $\mathrm{P}_{a m p R}$ and prevents access and thus transcription by the sigma factor. In the presence of the inducer, however, $\sigma^{54}$ may be partially or completely displaced by the sigma-RNA polymerase complex to promote $\operatorname{ampR}$ transcription. Thus, the loss of $r p o N$ leads to complete derepression of ampR expression in the induced condition.

Lastly and very interestingly, the expression of $\operatorname{ampC}$ followed a pattern similar to that of $\operatorname{ampR}$ in all backgrounds. Previously, ampR expression in $P$. aeruginosa was shown to be low and not significantly induced upon exposure to the $\beta$-lactam benzylpenicillin (710). Similarly, expression from the $C$. freundii ampR promoter in $E$. coli was found to be constitutive in the presence and absence of the inducer (6aminopenicillanic acid) (840). In E. cloacae, induction with cefoxitin significantly increased transcription of $a m p C$ but had no effect on $a m p R$ expression (895). Recent work from our lab however, showed that in the presence of very powerful known inducers, namely imipenem and meropenem, expression of both $a m p C$ and $a m p R$ is equally and very significantly induced in wild-type $P$. aeruginosa (760). In light of such results, it is not surprising that our current data shows that wild-type $P$. aeruginosa has similar $a m p C$ and $a m p R$ mRNA levels in the presence of imipenem. Similar induction profiles in the absence of $r p o N$ suggest that the $a m p C$ and $a m p R$ promoters can reach their full induction potential upon removal of the restricting negative effect imposed by RpoN. It is not clear how exactly RpoN, or its absence, can accomplish this, but it is worth noting that in the $a m p C$ and $a m p R$ TSSs, the -12 sequence of $a m p R$ overlaps the - 
10 sequence of $a m p C$. Thus, if $\sigma^{54}$ is in fact blocking promoter access to RNA polymerase, it could be blocking access to both the $\operatorname{ampR}$ and $\operatorname{ampC}$ promoters until such time as inducers lead to its partial or complete displacement from the intergenic region. Further studies are needed to elucidate the mechanism of ampC and $a m p R$ downregulation by RpoN.

4.4.2 Mapping of $\boldsymbol{P}$. aeruginosa $\mathbf{P}_{\text {ampc }}$. To map the minimal promoter needed for AmpRdependent activity, a series of 5'-end deletions of $\mathrm{P}_{a m p C}$ were transcriptionally fused to the promoterless lacZ gene of the integrative vector mini-CTX-lacZ (853). The activity of each promoter deletion was then analyzed in PA01 by assaying $\beta$-galactosidase activity in the presence and absence of $200 \mu \mathrm{g} \mathrm{ml}^{-1}$ of penicillin $\mathrm{G}$. The minimum length of the promoter needed for full $\mathrm{P}_{\text {ampc }}$ activity is 193-bp (Figure 4.3). This fragment consists of the full ampR-ampC intergenic region plus small parts of the $\operatorname{ampR}(22 \mathrm{bp})$ and $\operatorname{amp} C$ (23 bp) ORFs. The high activity seen with the 193-bp fragment in the absence of $\beta$-lactams as compared to the wild-type may be the result of the partial or complete removal of the repressor-binding site. Subsequent loss of a 22-bp ampR fragment from the 5 ' end of the 193-bp segment resulted in a 2-fold decrease in induction likely indicating partial removal of the activator-binding site. This 22-bp fragment corresponding to the beginning of the $a m p R$ ORF seems to be necessary for full induction

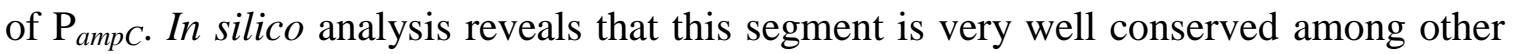
species but has no real identifiable features. Induction was abolished with a further 20-bp deletion (151-bp fragment). Thus, the 42-bp region (denoted red in Figures 4.1 and 4.3), 


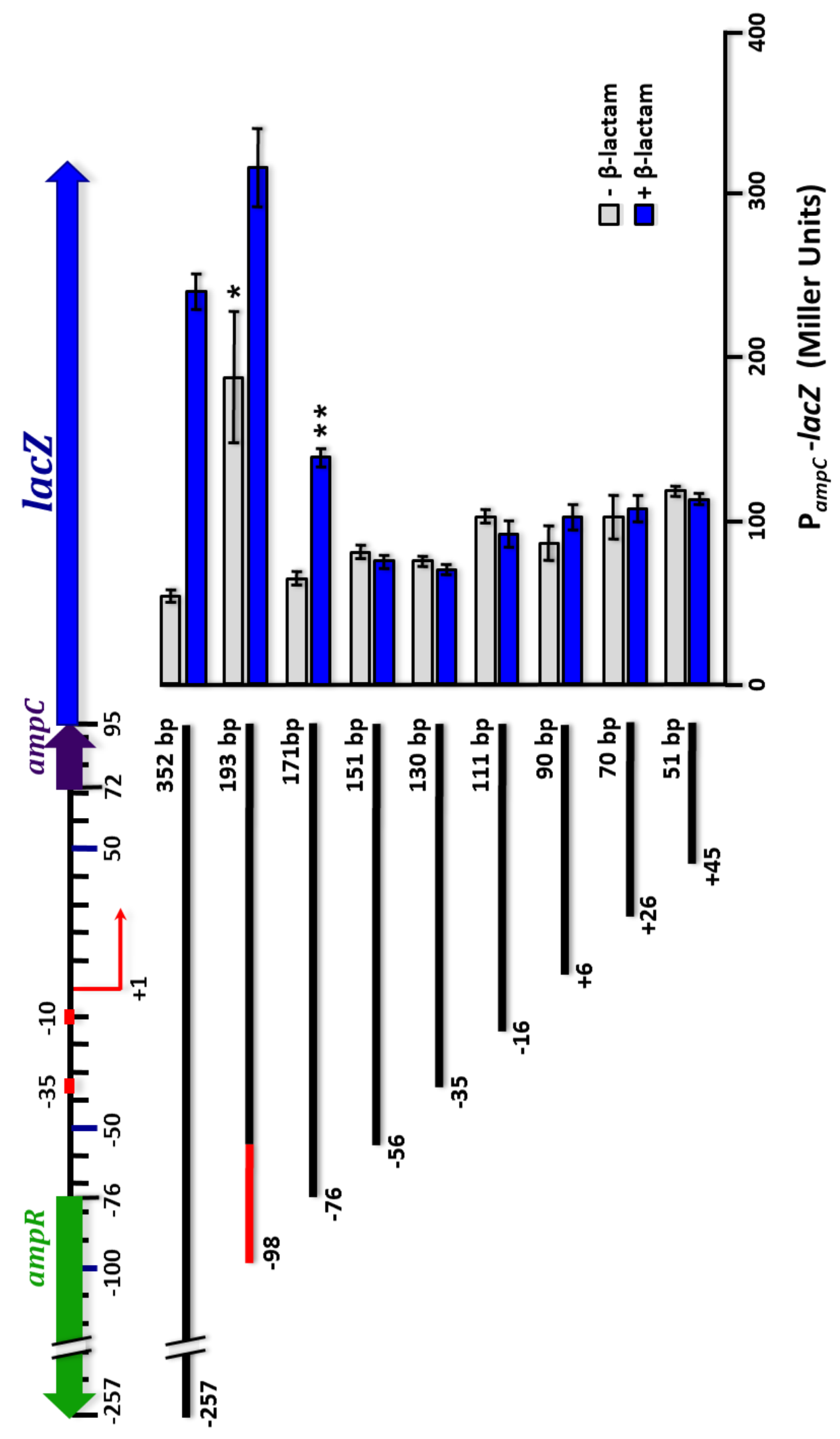


Figure 4.3. Mapping of the minimal $P$. aeruginosa ampC promoter. 5 '-end deletions of $\mathrm{P}_{\text {ampC }}$ were constructed and transcriptionally fused to a promoterless lac $Z$ gene in the integrative vector mini-CTX-lacZ as described in the Materials and Methods. Constructs were introduced into PA01 for integration at the attB site to generate single-copy promoter fusions. Promoter activities are expressed in Miller units. The +1 denotes the ampC transcriptional start site. The 42-bp segment, missing from the 151-bp construct, that appears to be necessary for activator binding, is depicted in red at the 5 ' end of the 193-bp fragment. * $p$-value $<0.05$ as compared to uninduced PA0attB:: $\mathrm{P}_{\text {ampC352-lacZ; } * *}$ $p$-value $<0.05$ versus uninduced PA0attB::PampC171-lacZ as determined by unpaired $t$-test using ANOVA.

present at the 5' end of the 193-bp fragment but deleted from the 151-bp construct, demarcates the outer bounds of the functional promoter needed for activation of $\mathrm{P}_{a m p C}$. Since this 42-bp fragment includes the AmpR box, an in silico derived putative AmpRbinding site (Figure 4.1), this region could be critical for activator binding.

4.4.3 P. aeruginosa AmpR binds to $\mathbf{P}_{\text {ampc }}$. Previously, $P$. aeruginosa AmpR has been shown to bind $\mathrm{P}_{a m p C}$ using AmpR-overexpressing E. coli whole cell extracts (776). Similarly, crude preparations of $C$. freundii AmpR were also shown to retard a radiolabeled ampR-ampC intergenic region (840). Since preliminary work from our lab suggested that $P$. aeruginosa AmpR is likely to be a membrane-associated protein (See Localization studies of $P$. aeruginosa AmpR section below and Figure. S2), we tested the ability of PA01 membrane fractions to bind $\mathrm{P}_{a m p c}$. EMSA was performed using AmpRHis $_{6}$ enriched membrane fractions and a $\left[\gamma_{-}{ }^{32} \mathrm{P}\right]$ ATP radiolabeled $\mathrm{P}_{a m p C}$ fragment. Shift was observed with increasing concentrations of total membrane protein up to $0.4 \mathrm{mg} \mathrm{ml}^{-1}$ (Figure 4.4). The binding was competed-out by mixing labeled DNA with unlabeled promoter DNA in 100-fold molar excess confirming AmpR binding to $\mathrm{P}_{a m p C}$ (Figure 4.4). 
Additionally, competition with a nonspecific, unlabeled fragment (233-bp alg44 PCR fragment) mixed in 10-, 100-, and 500-fold molar excess with the labeled $\mathrm{P}_{\text {amp }}$ fragment, failed to displace AmpR-His 6 from $\mathrm{P}_{\text {ampc }}$ illustrating the binding specificity.

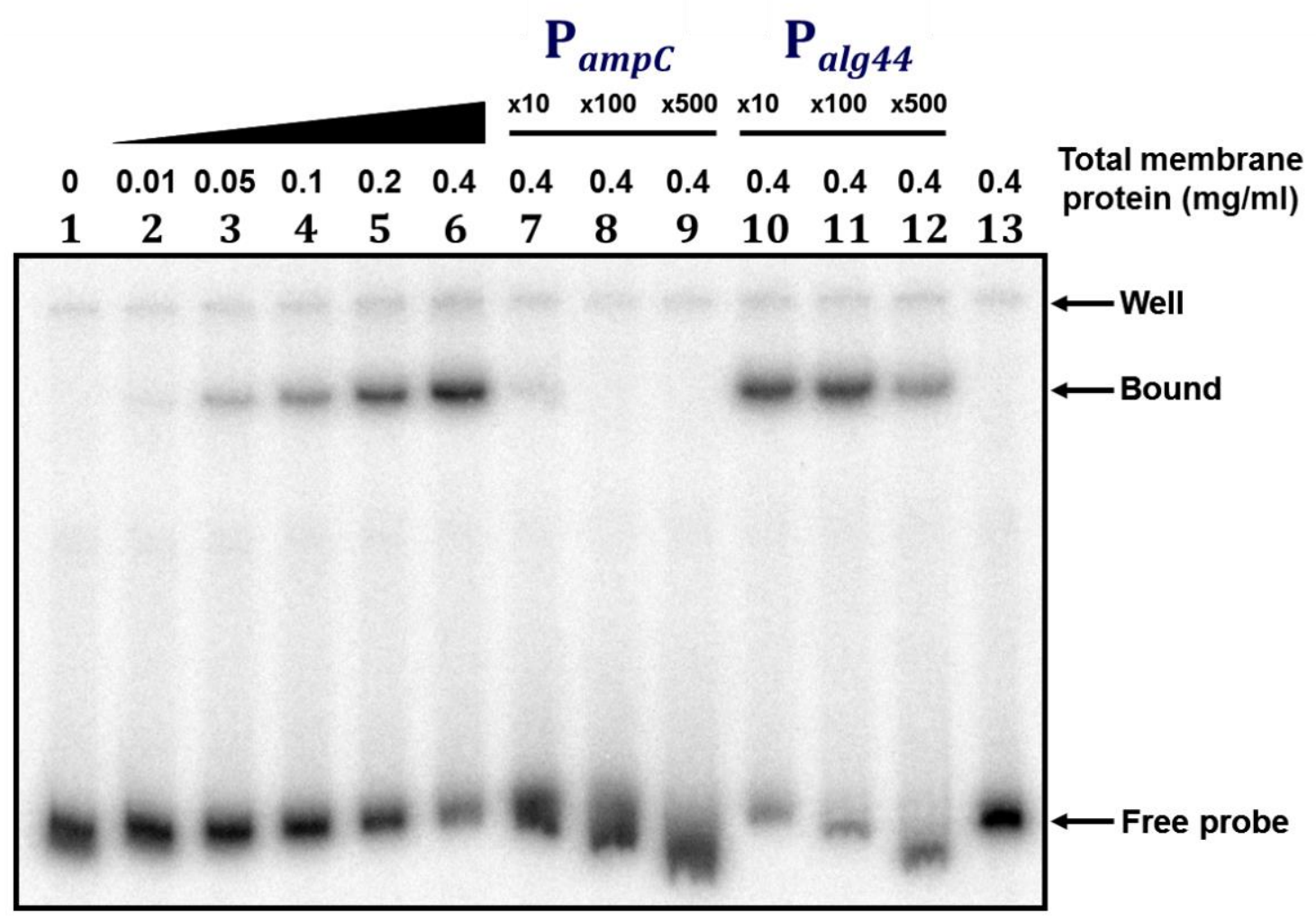

Figure 4.4. P. aeruginosa AmpR binds $\mathrm{P}_{\text {ampc }}$. Fifty fmol of a 193-bp radio-labeled $\mathrm{P}_{\text {ampC }}$ fragment (Lane 1) spanning the ampC-ampR promoter region were mixed with increasing concentrations of $\mathrm{AmpR}-\mathrm{His}_{6}$ enriched membrane fractions extracted from PA0 $\triangle a m p R$ (pAmpR-His 6 ) in the presence of $200 \mu \mathrm{g} \mathrm{ml}^{-1}$ of penicillin $\mathrm{G}$ (Lanes 2 to 6). Competition assays were carried out with the cold $\mathrm{P}_{\text {ampC }}$ fragment in 10- (Lane 7), 100(Lane 8) and 500-fold (Lane 9) molar excess. To characterize the binding specificity, a nonspecific fragment (233-bp alg44 PCR fragment) was mixed in 10- (Lane 10), 100(Lane 11) and 500-fold (Lane 12) molar excess with the radio-labeled reaction mix. Lane 13 is the control showing the 193-bp radio-labeled $\mathrm{P}_{\mathrm{ampC}}$ fragment in the presence of membrane fractions extracted from PA0 $\triangle a m p R$ containing the plasmid backbone alone. 
To determine if AmpR interacts with $\mathrm{P}_{\text {ampc }}$ in vivo, ChIP-qPCR was employed (908). A functional VSV-G-tagged AmpR (Table S3, Supplemental material) was introduced into PA01 as single copy and then immunoprecipitated with anti-VSV-G antibody. Sequence-specific primers were used to detect the presence of $\mathrm{P}_{\text {amp }}$ DNA with qPCR. Promoter occupancy was detected in the presence and absence of $\beta$-lactams as expected of LTTRs (856) (Fold enrichment over $c l p X$ control- Uninduced: 10.6 \pm 1.73 , Induced: 13.3 \pm 4.63 ; Fold enrichment for negative control target $\operatorname{aprX}$ - Uninduced: $1.30 \pm 0.02$, Induced: $1.34 \pm 0.22$ ). AmpR thus binds $\mathrm{P}_{\text {amp }}$ in vivo in the presence and absence of the inducer.

4.4.4 HTH is important for AmpR function. The majority of prokaryotic DNA-binding proteins, including LTTRs, use the HTH motif to interact with DNA $(897,914)$. In LTTRs, this domain is often found at the $\mathrm{N}$-terminus $(856,897)$. The canonical HTH motif is comprised of three helical bundles where the second and third helices interact with the DNA, and the third makes the essential contacts with the major groove to provide recognition $(897,914,915)$. A multiple alignment of the AmpR family HTH motif shows the highest degree of conservation is found in the first two helices, with the most variation in the third helix that provides specificity (Figure S3, Supplemental material). Although $P$. aeruginosa AmpR has been shown to bind the ampR-ampC intercistronic region (776), the amino acids involved in the interaction have not been identified. 
An amphipathic wheel of the third helix (residues Gln34 to Leu48), generated using DNASTAR Protean, identified polar and charged amino acids potentially facing the major groove of the DNA (Figure 4.5). Point mutations corresponding to these residues were generated by site-directed mutagenesis of AmpR-His 6 . Residues Ser38, His39, Lys42, Ser43, and Glu46 were thus replaced with alanine and the mutants were overexpressed in PA01 and PA0 $\triangle a m p R$ strains carrying the chromosomal $\mathrm{P}_{\text {ampC }}$-lacZ fusion (Figure 4.6). The $\mathrm{P}_{a m p C}$ activity observed in PA01 is the result of both ampR alleles, from the chromosome $\left(a m p R_{\text {chr }}\right)$ and from the plasmid $\left(a m p R_{p l s}\right)$, whereas in PA0 $\Delta a m p R$ only $a m p R_{p l s}$ contributes.

\section{$\frac{\text { Helix } 1}{{ }^{8} \text { LNALRAFEASARHLSFTRAAIELCVTQAAV } \underline{\text { H }} \text { QVVISLEEERLG }}{ }^{49}$}

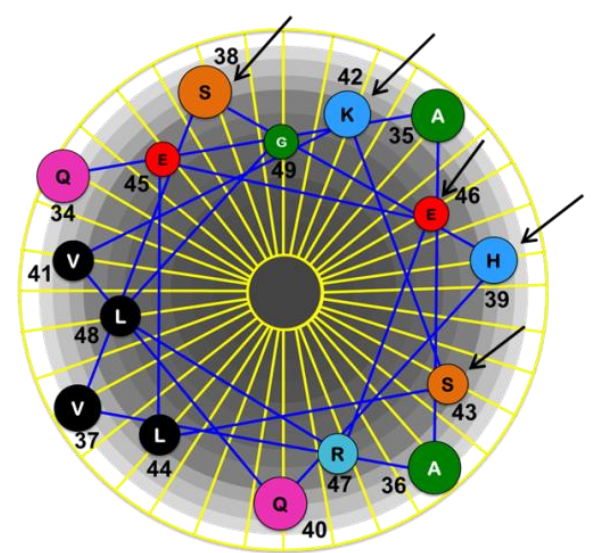

Figure 4.5. Analysis of the third helix of the P. aeruginosa AmpR HTH motif. An amphipathic wheel of the third helix (residues Q34 to L48) was generated using DNASTAR Protean in order to identify polar and charged amino acids likely facing the major groove of the DNA. The AmpR residues Ser38, His39, Lys42, Ser43, and Glu46, were identified as amino acids likely to interact with the DNA and were thus targeted for mutagenesis. They are indicated by the black arrows and denoted as S5, H6, K9, S10 and E13 in the helical wheel, respectively. The N-terminal sequence of $P$. aeruginosa AmpR illustrates the HTH motif and the location of the above amino acids (in red) in the third helix of AmpR. 


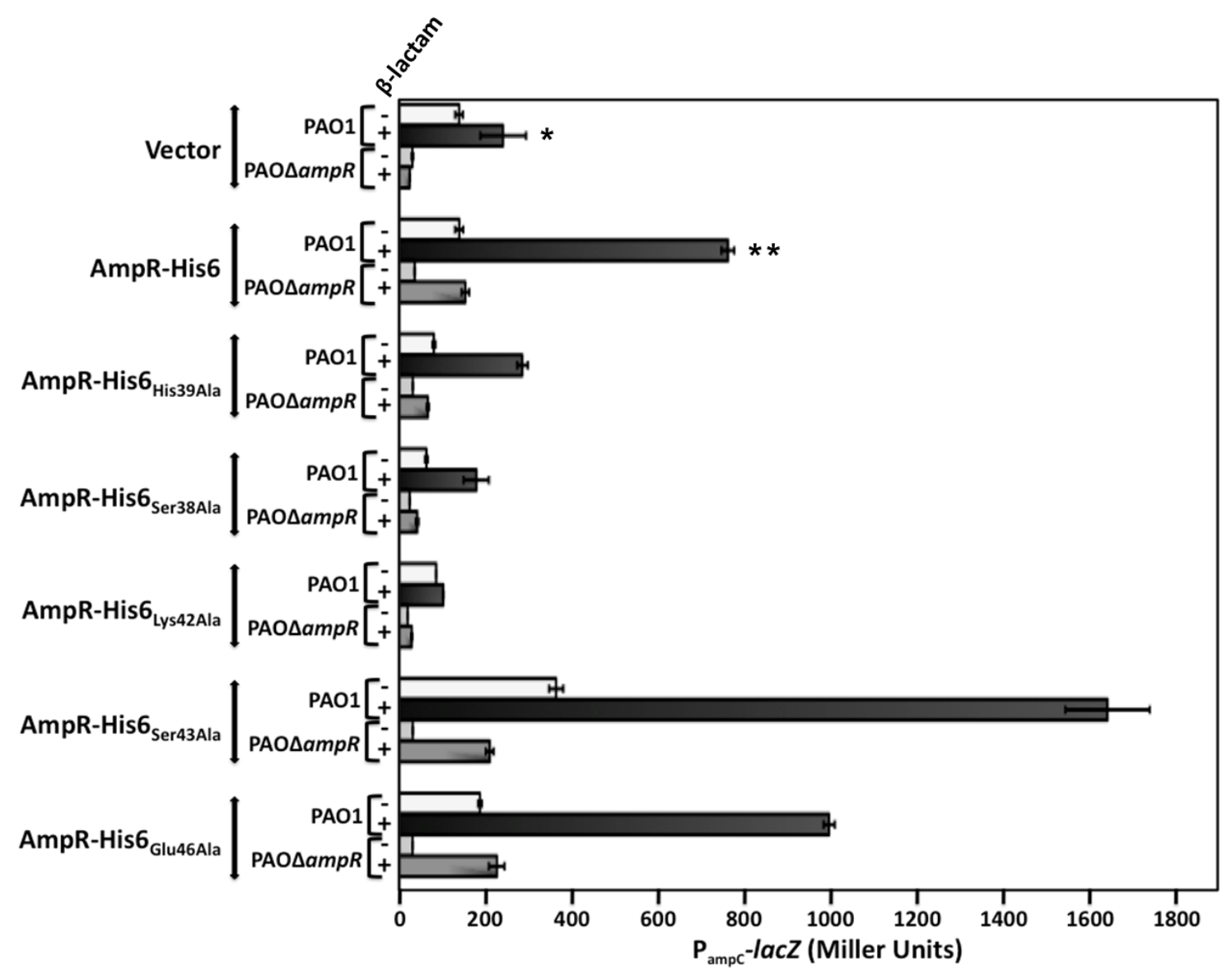

Figure 4.6. Functional analysis of the $P$. aeruginosa AmpR HTH motif. Site-directed mutagenesis was used to replace Ser38, His39, Lys42, Ser43, and Glu46 with Ala in AmpR-His 6 . The mutant proteins were overexpressed in PA01 and PA0 $\Delta a m p R$ strains carrying a single copy of the $\mathrm{P}_{\text {amp }}$-lac $Z$ fusion integrated at the attB site. $\beta$-galactosidase activity was quantified in the presence and absence of $\beta$-lactams. * $p$-value $<0.05$ versus uninduced PA01 vector control; ** $p$-value $<0.005$ versus induced PA01 vector control as determined by unpaired $t$-test using ANOVA.

Alanine substitutions at Ser43 and Glu46 did not affect the ability of AmpR to activate $\mathrm{P}_{a m p C}$ in PA0 $\mathrm{PampR}$ (Figure 4.6). In addition, both $\mathrm{AmpR}_{\text {Ser43Ala }}$ and AmpR $\mathrm{Alu}_{\text {46Ala }}$ were able to bind $\mathrm{P}_{\text {ampC }}$ in the presence and absence of $\beta$-lactams (Figure 4.7). These two findings suggest Ser43 and Glu46 are not critical for AmpR function. However, expression of $A m p R_{\text {Ser43Ala }}$ and $A m p R_{\text {Glu46Ala }}$ in PA01 that carries $A m p R_{\text {chr }}$, 
significantly increased $\mathrm{P}_{\text {ampC }}$ activity by more than 2-fold in the presence of inducers suggesting a possible interaction between chromosomal encoded $A m p R_{c h r}$ and the variants (Figure 4.6). In particular, the Ser43Ala substitution increased basal levels in the absence of inducers, while leading to hyperinduction in the presence of $\beta$-lactams. Similarly, significant activation of $\mathrm{P}_{\text {ampC }}$ in PA01 in the presence of AmpR-His ${ }_{6}$ further strengthens the idea that AmpR functions as a multimer.

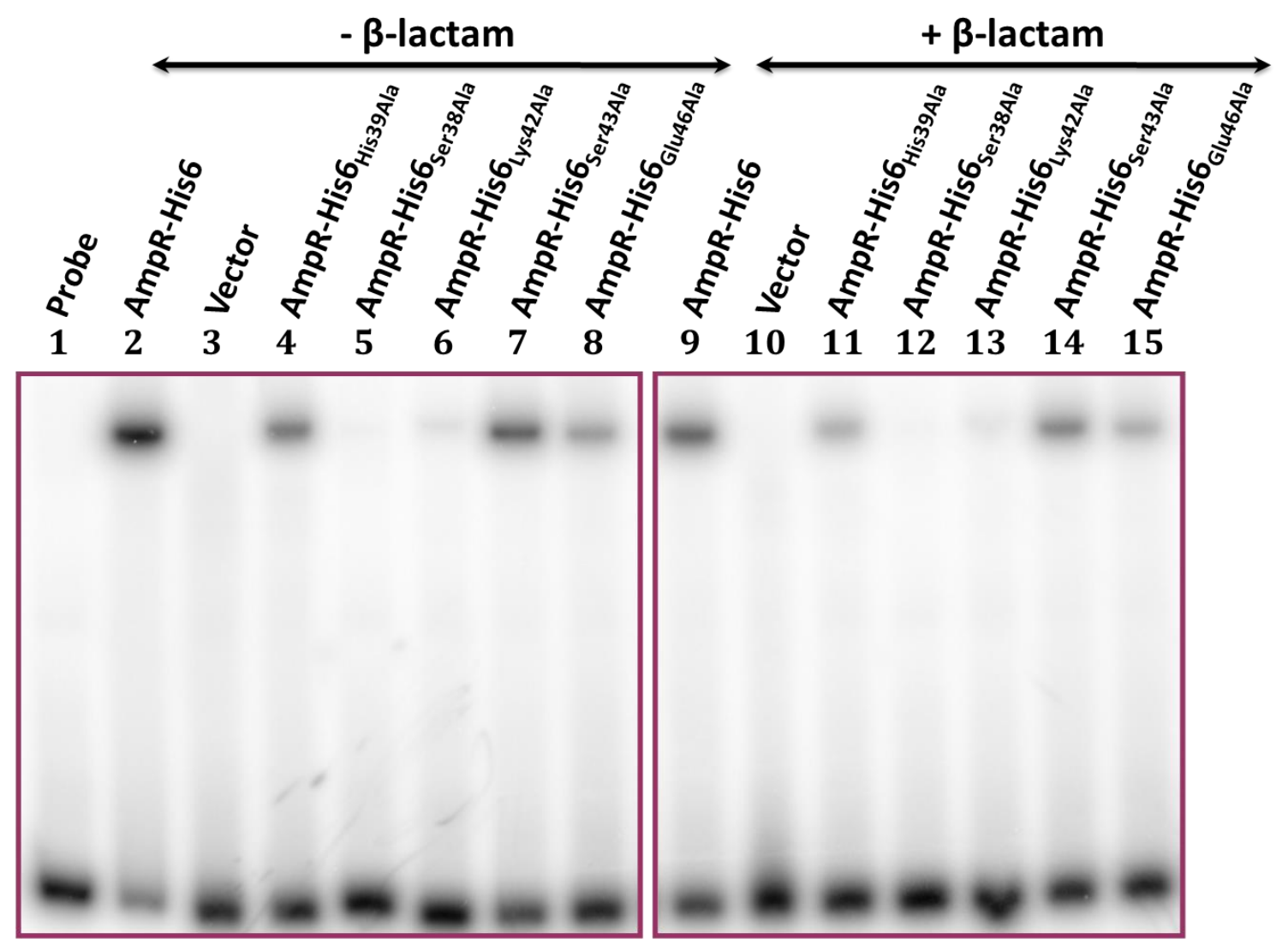

Figure 4.7. Electromobility shift assay of $P$. aeruginosa AmpR HTH mutants. A 50 fmol of the 193-bp radio-labeled $\mathrm{P}_{\mathrm{amp}}$ fragment (Lane 1) were mixed with membrane fractions recovered from PA0 $\triangle a m p R$ in the absence (Lanes 2 to 8 ) and presence of $\beta$ lactams (Lanes 9 to 15) and carrying pMMB67EH-Gm (Lanes 3 \& 10), pAmpR-His6

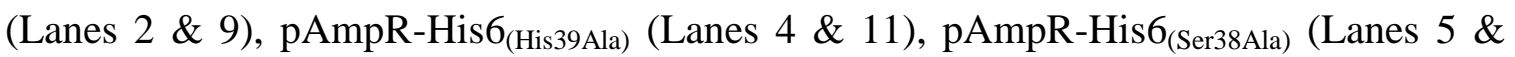
12), pAmpR-His6 (Lys42Ala) $($ Lanes $6 \& 13)$, pAmpR-His6 $6_{\text {(Ser43Ala) }}$ (Lanes $\left.7 \& 14\right)$, and pAmpR-His6 (Glu46Ala) $($ Lanes $8 \& 15)$. 
AmpR mutant proteins failed to activate $\mathrm{P}_{a m p c}$ when Ser38, His39, or Lys42 were substituted with alanine. These three residues are thus essential for AmpR activity and are presumably involved in the binding to $\mathrm{P}_{\text {ampc }}$. A multiple alignment reveals Ser38 and Lys 42 are well-conserved in members of the AmpR family as expected of amino acids that play a critical role in the functionality of a protein (Figure S3, Supplemental material).

The loss of $\mathrm{P}_{\text {ampC }}$ activity in $A m p R_{\text {Ser38Ala }}, \mathrm{AmpR}_{\text {His39Ala }}$ and $\mathrm{AmpR}_{\mathrm{Lys} 42 \mathrm{Ala}}$ could be attributed to the destabilization of the proteins. Their expression was thus analyzed using Western blotting with anti-AmpR antibody (Figure 4.8). Interestingly, not only are these three mutant AmpR proteins made, it appears that they, and in particular $A m p R_{\text {Ser38Ala }}$ and $A m p R_{\text {Lys42Ala, }}$ are made in large quantities. These amino acid substitutions, therefore, appear to stabilize rather than destabilize the proteins. Thus, we argued that the loss of $\mathrm{P}_{\text {ampC }}$ activity may be due to their inability to bind DNA. Gelshifts revealed that $A m p R_{\text {Ser38Ala }}$ failed to bind to $\mathrm{P}_{a m p C}$ while $A m p R_{\text {Lys42Ala }}$ bound very poorly correlating well with the loss of $\mathrm{P}_{a m p C}$ transcriptional activity (Figure 4.7). Surprisingly, the His39Ala substitution did not prevent AmpR from binding to $\mathrm{P}_{\text {ampC }}$ in the presence or absence of $\beta$-lactams, although it clearly prevented it from activating transcription from $\mathrm{P}_{\text {ampC }}$ (Figures 4.6 and 4.7). $\mathrm{AmpR}_{\mathrm{His} 39 \mathrm{Ala}}$ is thus a positive control mutant that can bind DNA but cannot activate transcription from the promoter to which it binds. 


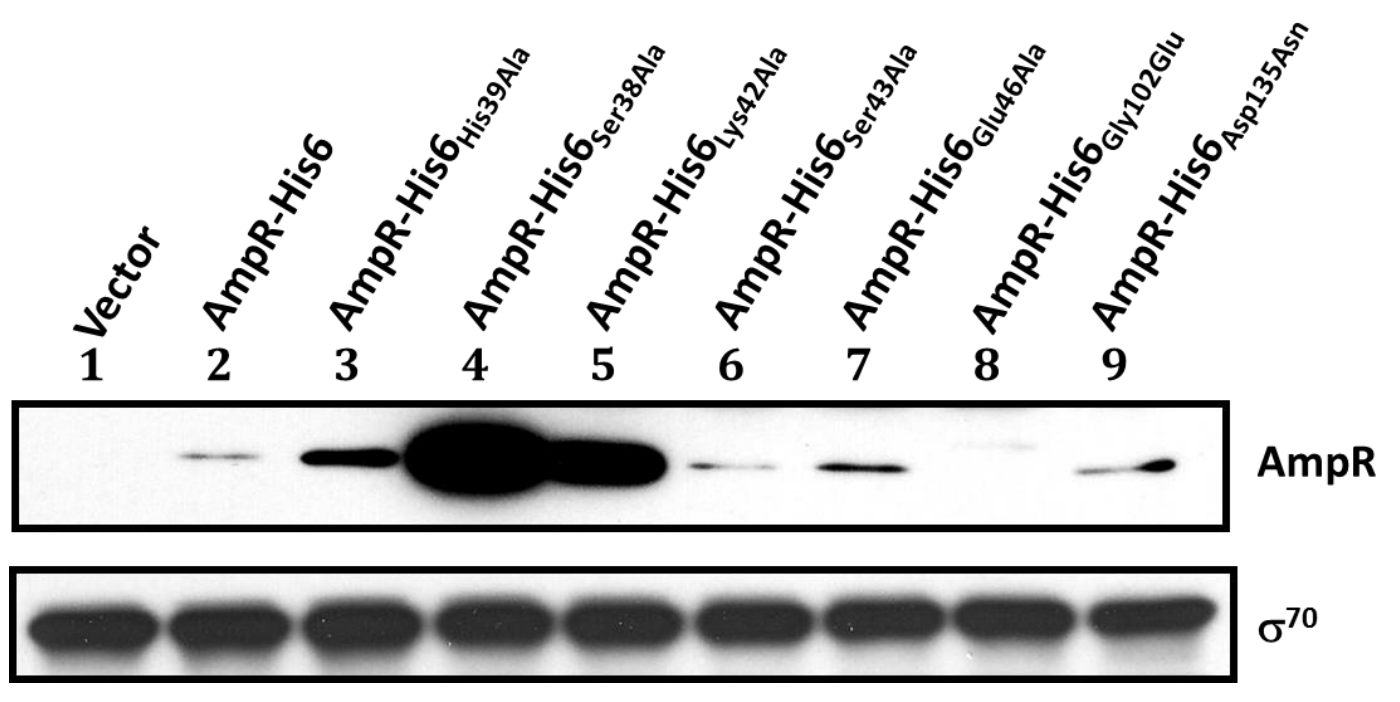

Figure 4.8. Stability of $P$. aeruginosa AmpR mutant proteins. Total protein extracts were recovered from AmpR HTH and point mutants after a 11/2-hour incubation with 1 $\mathrm{mM}$ of IPTG. The stability of each mutant was verified by Western blotting using AmpR-specific antibodies. Equal amounts of total membrane protein were loaded per well; $\sigma^{70}$ was used as a loading control and detected with anti- $\sigma^{70}$ antibody.

Positive control $(p c)$ mutants are proteins that are defective in transcriptional activation but retain the ability to bind DNA. The $p c$ phenotype is caused by the disruption of favorable protein-protein interactions between the activator protein and the RNA polymerase (916-918). Several $p c$ mutants of other proteins have been characterized with mutations in or near the DNA-binding domain (916-921). Mutations away from this region have also been reported (922). In particular, mutations in the DNA-binding region have been mapped to the second helix of the HTH motif and to the junction between the second and third helix of the same domain in the activator proteins $\lambda$ cI, 434 cI, and P22 c2 (916-919). AmpR $\mathrm{His}_{\text {HaAla }}$ is different from previously reported $p c$ mutants in that its mutation is found in the helix of the DNA-binding domain that is 
thought to directly interact with the major groove of the DNA (helix 3), and not in the helix which usually lies across the major groove (helix 2), and makes contacts with the DNA backbone. Although it is not clear whether this third helix can contact the RNA polymerase, it may interact with other sites in the nearby helix to indirectly affect transcription. Our work here does not reveal how the disruption of His39 affects activation of transcription, but merely that it is required for it.

In the present work we show that the highly conserved residues Ser38 and Lys42 (not conserved in Klebsiella pneumoniae), in the HTH motif are critical for binding and function of AmpR. The less conserved His39 is also necessary for promoter activation but not for binding to the DNA.

4.4.5. Gly102 and Asp135 are critical for AmpR function. Previous work identified $C$. freundii ampR mutants that constitutively express $\beta$-lactamase $(839,923)$. Specifically, a change in Gly102 to Glu (Gly102Glu) resulted in high constitutive $\beta$-lactamase expression in an inducer-independent manner, while a Gly102Asp substitution yielded a similar but less pronounced phenotype $(839,923)$. In addition, Asp135 was also found to play a role in the function of $C$. freundii and E. cloacae AmpR (923, 924). The expression of $C$. freundii $\mathrm{AmpR}_{\mathrm{Asp} 135 \mathrm{Tyr}}$ led to constitutive $\beta$-lactamase hyperexpression in an ampG mutant background. In E. cloacae AmpR Asp135 substitutions to Val or Asn resulted in higher $\beta$-lactamase activity in the presence and absence of inducers and contributed to increased $\beta$-lactam resistance in two different E. coli backgrounds (923, 
924). Gly102 and Asp135, thus appear to play important roles in the activation/repression state of AmpR in, at least, the Enterobacteriaceae.

To investigate the role of these amino acids, P. aeruginosa AmpR Gly102 and Asp135 were replaced with Glu and Asn, respectively. The two mutant AmpR proteins were overexpressed in PA01 and PA0 $\triangle a m p R$ strains carrying $\mathrm{P}_{a m p C^{-}}$lacZ (Figure 4.9). Unlike in C. freundii, in $P$. aeruginosa the Gly102Glu substitution resulted in the loss of $\mathrm{P}_{\text {ampC }}$ activity in the presence and absence of $\beta$-lactams. The loss of activity is due to destabilization of the protein (Figure 4.8), suggesting a structural role for Gly102 in $P$. aeruginosa AmpR.

On the other hand, the Asp135Asn substitution led to an inducer-independent increase in $\mathrm{P}_{a m p C}$ transcriptional activity in both $\mathrm{PA} 01$ and $\mathrm{PA} 0 \Delta a m p R$ with no concomitant increase in the amount of protein being made (Figures 4.8 and 4.9). Thus, we postulate that the Asp135Asn substitution in the effector binding domain appears to stabilize the active conformation effectively turning AmpR into a constitutive activator of ampC transcription. The Asp135Asn substitution has also been reported in AmpR from a P. aeruginosa clinical variant that exhibited hyper-constitutive $\beta$-lactamase expression and high resistance to $\beta$-lactams (722). The importance of Asp135 corroborates the previous work in E. cloacae and C. freundii (923, 924). Gly102, on the other hand, clearly plays different roles in the $P$. aeruginosa and $C$. freundii AmpR proteins. 


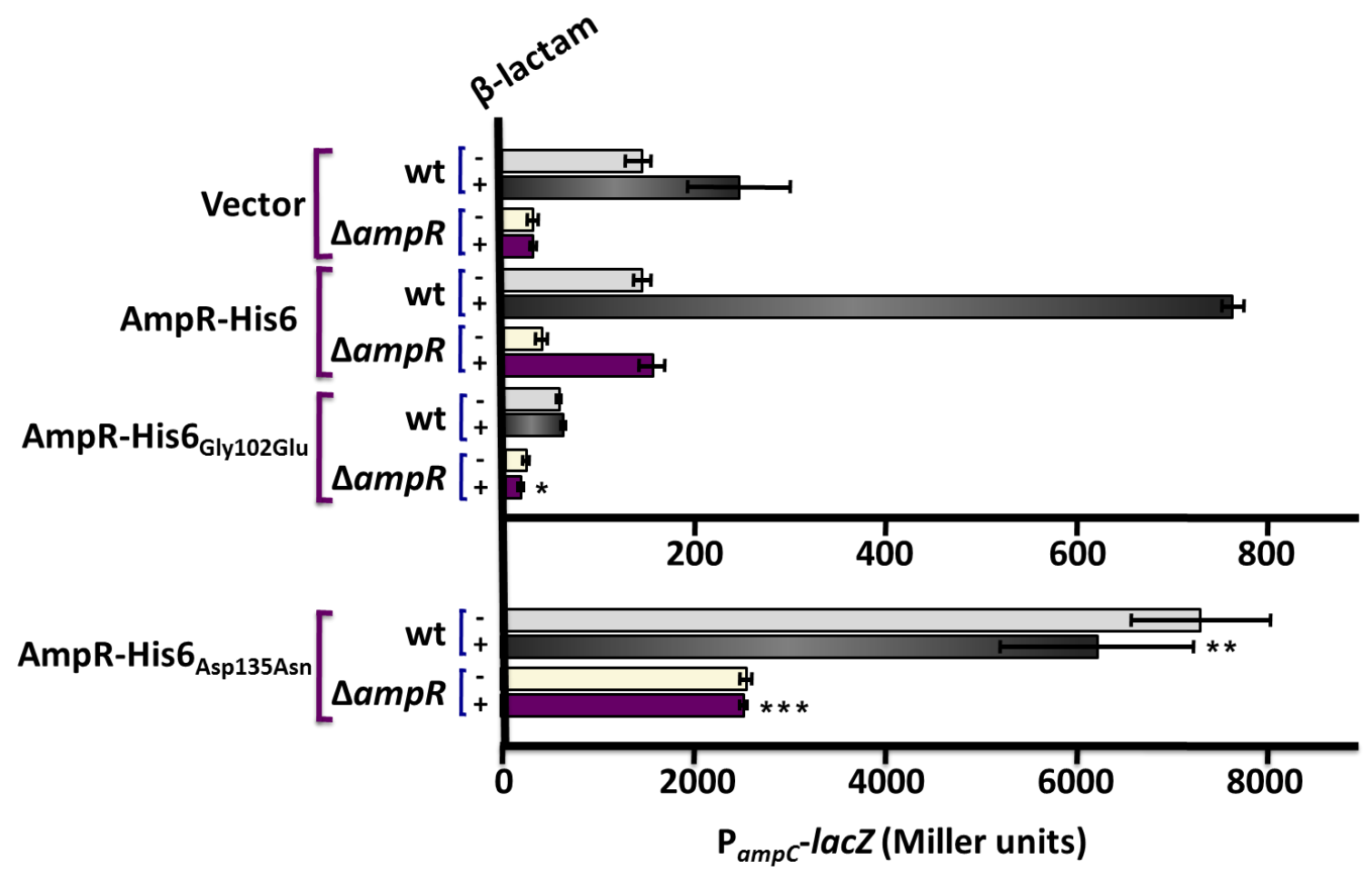

Figure 4.9. Activity of the $P$. aeruginosa ampC promoter in the presence of AmpRHis6 $_{(\mathrm{Gly} 102 \mathrm{Glu})}$ and AmpR-His6 (Asp135Asn) mutants. Site-directed mutagenesis was used to replace Gly102 and Asp135 of $P$. aeruginosa AmpR with Glu and Asn, respectively. Mutant AmpRs were expressed in wild-type PA01 and PA0 $\triangle a m p R$ strains carrying

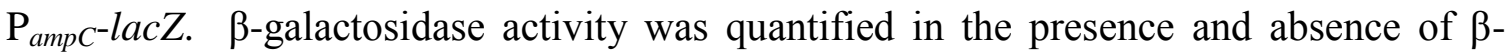
lactams and is represented in Miller units. ${ }^{*} p$-value $<0.01$ versus induced PA0 $\Delta a m p R$ pAmpR-His 6 ; ** $p$-value<0.01 versus induced PA0attB::mini-CTX-lacZ; *** $p$ value $<0.001$ versus induced PA0 $\triangle a m p R$ pAmpR-His ${ }_{6}$ as determined by unpaired $t$-test using ANOVA.

4.4.6 Cross-linking studies suggest $P$. aeruginosa AmpR dimerizes. The $\mathrm{P}_{\text {amp } C}$ activity in the presence of both $a m p R_{\mathrm{chr}}$ and $a m p R_{p l s}$ is always considerably higher than that in PA01 and PA0 $\Delta a m p R\left(a m p R_{p l s}\right)$ (Figure 4.6). These findings suggest a possible interaction between the wild-type and His-tagged AmpR. This is not surprising as LTTRs exist and/or function as dimers or tetramers $(856,897,925-929)$. To determine if in fact 


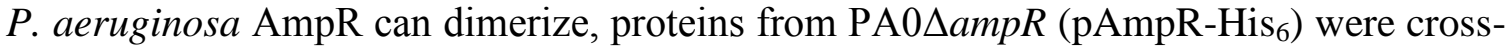
linked and AmpR was visualized with anti-His antibody. The detection of a 64-kD and a $32 \mathrm{kD}$ species in cross-linked and non-cross-linked samples, respectively, suggests that AmpR dimerizes in vivo (Figure 4.10). Only the monomeric form of $\sigma^{70}$ was detected after stripping and reprobing the blot. Our findings corroborate previous work in $C$. freundii where both AmpR and its effector binding domain were shown to dimerize in solution and in the crystallized form, respectively $(930,931)$.

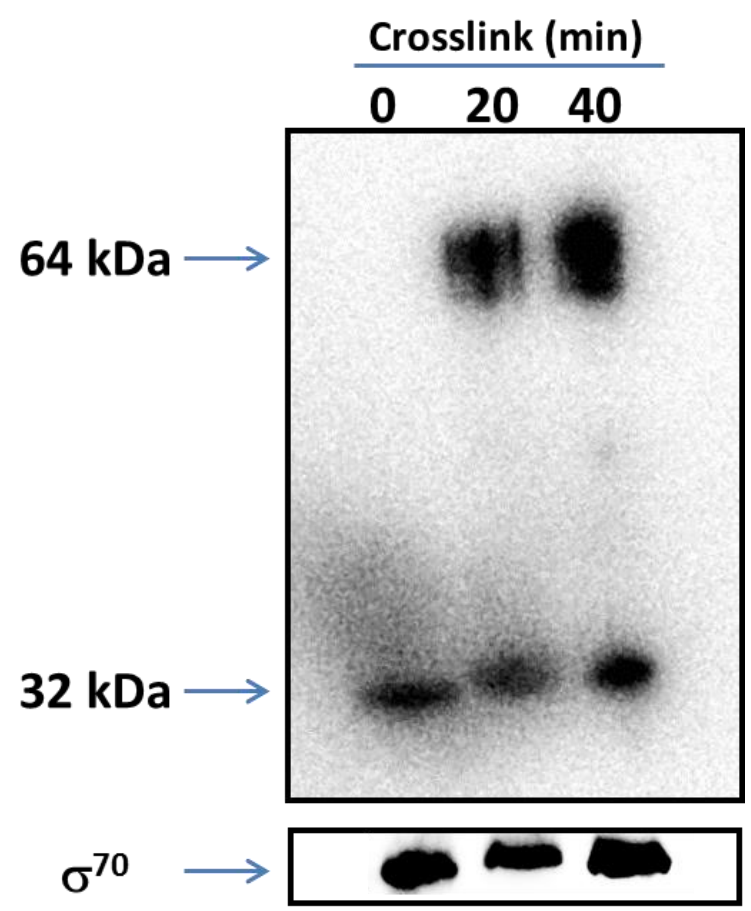

Figure 4.10. $P$. aeruginosa AmpR appears to dimerize in vivo. A fresh culture of PA0 $\triangle a m p R$ strain harboring $\mathrm{pAmpR}^{-\mathrm{His}_{6}}$ was treated with $0.1 \%$ formaldehyde for 20 and 40 minutes at room temperature to achieve protein cross-linking. Crude extracts containing $10 \mu \mathrm{g}$ of total protein were separated on an SDS-polyacrylamide gel and AmpR was visualized using anti-His antibody. The blot was later stripped and reprobed using anti- $\sigma^{70}$ antibody. Monomeric AmpR is detected in non-cross-linked samples at zero time point, while AmpR dimeric entities (64KDa) are detected 20 and 40 minutes after protein cross-linking. 
4.4.7. Localization studies of $\boldsymbol{P}$. aeruginosa AmpR. Although it is generally accepted that AmpR is a cytoplasmic protein, our bioinformatics analyses suggested that AmpR may be membrane associated. More specifically, a Kyte-and-Doolittle hydrophobicity plot (932) and the topology prediction softwares TopPred2 (933), DAS (934), MEMSAT (935), TMpred (936), and SCAMPI (937) suggested the presence of a transmembrane domain somewhere between amino acids 92 and 114 of P. aeruginosa AmpR. The crystal structure of $C$. freundii AmpR, however, reveals this segment is near the proteinprotein interface of the dimer and thus unlikely to traverse the membrane (930).

In order to localize AmpR, phoA and $l a c Z$ were fused in-frame at amino acid positions Glu15, Val134 and Gln185 (Figure S2A, Supplemental material). Fusions at Glu15 were LacZ-positive and PhoA-negative, whereas fusions at Val134 and Gln185 were PhoA-positive and LacZ-negative suggesting AmpR may traverse the inner membrane with the $\mathrm{N}$ and $\mathrm{C}$ termini in the cytoplasm and periplasm, respectively (Figure 4.11). Since this data is only qualitative, localization of $P$. aeruginosa AmpR was further investigated with a protease protection assay using a C-terminal His-tagged AmpR that was shown to be functional (Table S2, Supplemental material). Full length AmpR (32 $\mathrm{kDa})$ was detected in whole cell extracts, as well as, in spheroplasts preparations treated with Proteinase K (0-min incubation) that were immediately processed for immunoblotting (Figure S2B, Supplemental material). Incubations with Proteinase K of 5 minutes or longer resulted in the visible reduction of full length AmpR and the appearance of the degradation product (10-kDa fragment). However, slight degradation of $\sigma^{70}$ was observed. The evidence provided here is suggestive of AmpR being an inner 
membrane-associated protein. If confirmed, this would be an important finding and could have major implications regarding the regulation of two $\operatorname{ampD}$ amidase homologs that have now been localized to the periplasm $(938,939)$. The identity of the muramylpeptides that are important for regulating AmpR will further confirm its localization and is the subject of ongoing work in the lab.

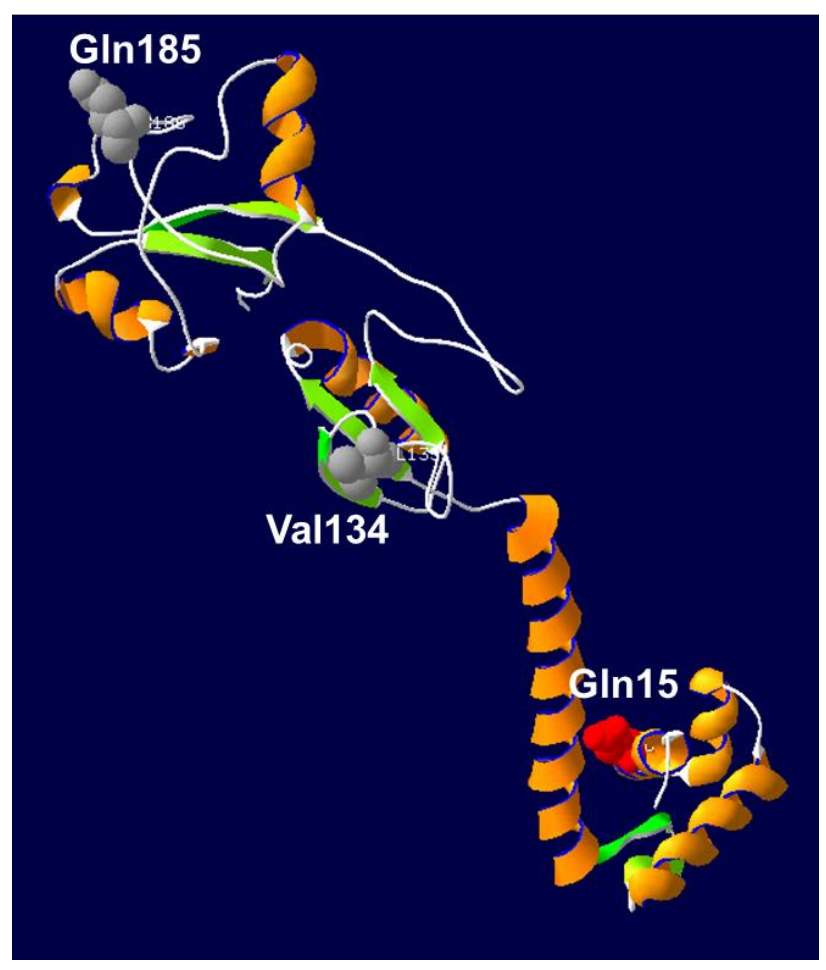

Figure 4.11. AmpR model. A three-dimensional structure of $A m p R$ was generated using RasMol (http://www.umass.edu/microbio/rasmol/). The topology of AmpR was investigated by introducing $p h o A$ and lac Z fusions at amino acid positions Glu15, Val134, and Gln185. Fusions at Glu15 were LacZ positive but PhoA negative, whereas fusions at Val134 and Gln185 were PhoA positive and LacZ negative.

Although the majority of purified LTTRs appear to be soluble cytoplasmic proteins, the nodulation factor, NodD, from Rhizobium species appears to be a peripheral membrane protein associated with the inner leaflet of the cytoplasmic membrane (940). A 
few membrane-bound non-LTTRs have also been reported, such as the Salmonella enterica serovar Typhimurium acid-sensing CadC, the streptococcal CpsA involved in regulation of capsule production, and the Vibrio cholera toxin activator, ToxR (941-943). Since these proteins act as both signal sensor and response regulator, they form a simple but sophisticated form of transmembrane signaling system.

\subsection{Concluding Remarks}

The role of AmpR as regulator of ampC expression has been clearly established in both the Enterobacteriaceae and $P$. aeruginosa. Our recent work has further redefined AmpR as a major global regulator, playing an important role in acute infections through its regulation of virulence, biofilm formation, quorum sensing and non- $\beta$-lactam resistance $(710,841-844)$. Regulation of $\operatorname{amp} C$, however, remains one of its most critical roles, as AmpC derepression is a prevalent mechanism of $\beta$-lactam resistance in $P$. aeruginosa.

In the present work we characterize some of the genetic and structural elements necessary for induction of $a m p C$ and important for the functioning of AmpR as regulator of AmpC $\beta$-lactamase expression. The presence of strong $\sigma^{54}$ consensus sequences in the $a m p R$ promoter led us investigate its possible involvement in the regulation of ampR. However, contrary to what was expected, RpoN was not required for $a m p R$ expression in the conditions tested. Instead, RpoN was found to downregulate expression of both ampR and $\operatorname{ampC}$, although the exact mechanism is yet unknown. 
Like other LTTRs, AmpR has two important regions critical for its functioning as activator/repressor of $a m p C$ expression: the HTH motif for binding to DNA and the effector binding domain for ligand interaction. Analysis of polar and charged amino acids on the AmpR HTH revealed two residues, Ser38 and Lys42, important for binding of AmpR to the promoter region and consequently for $\operatorname{ampC}$ promoter activation. A third residue, His39, was shown to be important for function but not for binding to $\mathrm{P}_{a m p C}$. In the effector binding domain, we examined the role of two amino acids, Gly102 and Asp135, previously shown to be important for maintaining AmpR in an inactive conformation in the enterobacteria. In P. aeruginosa, Gly102 appears to be responsible for maintaining a stable structural conformation, while Asp135 is responsible for keeping AmpR in an inactive state that represses $\operatorname{ampC}$ expression. Additionally, our work suggests that AmpR dimerizes and that it is likely to be membrane associated. This is the first comprehensive look at the $P$. aeruginosa AmpR and the promoter elements that it regulates.

\subsection{Acknowledgments}

This study was supported in part by the National Institutes of Health - Minority Biomedical Research Support SCORE (SC1AI081376; KM), Research Initiative for Scientific Enhancement graduate student fellowship (NIH/NIGMS R25 GM61347; DZ), NIH/NIAID R37 AI021451 (SL), National Science Foundation IIP-1237818 [PFI-AIR: CREST-I/UCRC-Industry Ecosystem to Pipeline Research] (KM), and Florida International University Dissertation Year Fellowship (DB). The content is solely the 
responsibility of the authors and does not necessarily represent the official views of the funding agencies.

We thank Dr. D. Haas from UNIL, Switzerland for kindly providing PA0 $\Delta r p o N$ and PA0 0 rpoN (pRpoN), Dr. G. Plano from University of Miami, Miami, FL for sharing reagents for Western blotting and Nikolay Atanasov Nachev from Florida International University, Miami, FL for bioinformatics analysis of P. aeruginosa AmpR. 


\section{Chapter 5}

\section{General Discussion and Summary}




\subsection{Overview}

The Infectious Diseases Society of America classified Pseudomonas aeruginosa as one of the troublesome ESKAPE ${ }^{1}$ pathogens that, along with Enterococcus faecium, Staphylococcus aureus, Klebsiella pneumoniae, Acinetobacter baumannii, and Enterobacter spp., causes the majority of nosocomial infections and effectively evades the action of antibiotics $(944,945)$. The intractability of $P$. aeruginosa is owned to its intrinsic low membrane permeability, the presence of multidrug efflux pumps that can export more than one type of antimicrobial, and the expression of $\beta$-lactamases, enzymes which can hydrolyze and inactivate the commonly used $\beta$-lactam antibiotics (246, 270, $317,549,946)$. In addition, $P$. aeruginosa can evolve resistance after treatment with antibiotics through mutations in resistance genetic determinants or their regulatorencoding genes (247, 313, 336, 749, 947-949).

P. aeruginosa also has an innate ability to acquire resistance genes from the environment and is often a natural reservoir of multiple plasmid-encoded $\beta$-lactamases (549, 950). Chromosomally, P. aeruginosa carries AmpC, a class C $\beta$-lactamase that plays a major role in intrinsic resistance against penicillin- and cephalosporin-type $\beta$ lactams (711). Mutational derepression of this enzyme, whereby AmpC is produced at high levels irrespective of the presence or absence of $\beta$-lactams, is currently one of the most common mechanisms of $\beta$-lactam resistance in the clinical setting $(379,380,722$, 749).

\footnotetext{
${ }^{1}$ ESKAPE is an acronym for the six pathogens, Enterococcus faecium, Staphylococcus aureus, Klebsiella pneumoniae, Acinetobacter baumannii, Pseudomonas aeruginosa and Enterobacter spp., which are currently a major problem in the hospital setting.
} 
This dissertation delved into the role of a second chromosomally encoded $\beta$ lactamase in P. aeruginosa termed PoxB. This work established PoxB is an oxacillinasetype $\beta$-lactamase with a narrow spectrum of hydrolysis that includes the carbapenems imipenem, meropenem and doripenem. The currently available $\beta$-lactamase inhibitors did not significantly affect PoxB activity as commonly seen for class D enzymes. In addition, PoxB was shown to form a two-gene operon with the upstream open reading frame poxA, whose expression exerted negative control on the pox promoter. The transcriptional regulator AmpR negatively impacted pox expression but this regulation did not occur by binding to the promoter. A mutant transposon library, constructed to screen for regulators of pox expression, identified two clones with insertions in genes involved in polyamine transport. Polyamines coincidently were shown to provide resistance against imipenem and meropenem. In summary, two chromosomal-encoded $\beta$-lactamases with complementing hydrolytic spectrums have the potential to provide $P$. aeruginosa with resistance against most classes of $\beta$-lactams including the penicillins, cephalosporins and carbapenems.

\subsection{PoxB is a chromosomal-encoded class $D$ carbapenemase. Class $D \beta$-lactamases are} predominantly found in P. aeruginosa and in A. baumannii, but have also been reported in Escherichia coli, K. pneumoniae, and Proteus mirabilis, among others (606, 685). Three distinct oxacillinase categories are now recognized: the narrow-spectrum, the extended-spectrum and the carbapenem-hydrolyzing oxacillinases (680). Carbapenemhydrolyzing class D $\beta$-lactamases (CHDLs), are widespread in the nosocomial pathogen 
A. baumannii but have also been occasionally reported in $K$. pneumoniae and Acinetobacter spp., as well as and in a few of the Enterobacteriaceae (680, 685). CHDLs are less common in $P$. aeruginosa and to date only three CHDLs have been reported there: OXA-40, OXA-198 and, of course, OXA-50 or PoxB $(549,740,741,951,952)$.

Specifically, a plasmidic carbapenemase was detected in two unrelated imipenemresistant $P$. aeruginosa isolates from northern Spain where OXA-40-producing $A$. baumannii were found to be common $(951,953,954)$. The nucleotide sequence in fact showed $100 \%$ identity to the previously described bla $a_{\text {OXA-40 }}$ from an A. baumannii isolate endemic to this area (951). Thus, one of the first OXA-type carbapenemases detected in P. aeruginosa was termed OXA-40 (954). Similarly, OXA-198 was identified in the imipenem-resistant clinical isolate PA41437 (952). OXA-198 was encoded in a plasmidborne integron but was not closely related to other OXA enzymes. Biochemically, OXA198 behaved similarly to other CHDLs, exhibiting a strong affinity for carbapenems but low rate of hydrolysis (952). Its expression in a heterologous host provided resistance against ticarcillin and decreased susceptibility against piperacillin, cefepime, and the carbapenems and but not against aztreonam and extended-spectrum cephalosporins (952). Although the clinical strain PA41437 was resistant to imipenem and displayed a high minimum inhibitory concentration (MIC) against meropenem $(12 \mu \mathrm{g} / \mathrm{ml})$, OXA-198 expression in trans only decreased susceptibility against these drugs, suggesting other mechanisms of resistance were operating in the PA41437. Sequencing of oprD revealed a number of mutations and an 88-bp deletion in this strain (952). 
To our knowledge PoxB (OXA-50), is the first carbapenem-hydrolyzing class D $\beta$-lactamase reported in P. aeruginosa $(740,741)$. Similar to OXA-198, OXA-50 is only weakly related to other class D enzymes, displaying 43-47\% homology to CHDLs from the OXA-23 and OXA-229 group found in A. baumannii and Acinetobacter spp. Unlike the other two OXA enzymes reported in $P$. aeruginosa, OXA-50 is carried on the chromosome and ubiquitously found in environmental and clinical $P$. aeruginosa isolates (740). The goal of this dissertation was to characterize the naturally occurring class $\mathrm{D} \beta$ lactamase PoxB and determine its contribution to intrinsic resistance in P. aeruginosa.

Since the class $\mathrm{C}$, chromosomal encoded AmpC $\beta$-lactamase is known to play a major a role in both the intrinsic and acquired resistance against $\beta$-lactams in $P$. aeruginosa, the role of PoxB was investigated in strains carrying clean deletions of the $\beta$ lactamase-encoding genes (PA0 $\triangle a m p C, \mathrm{PA} 0 \Delta p o x B$ and $\mathrm{PA} 0 \Delta a m p C \Delta p o x B)$. This work clearly showed AmpC confers protection against penicillins and cephalosporins. Specifically, an ampC deletion rendered strains susceptible to the aminopenicillins (ampicillin and amoxicillin) but produced only a small decrease in MIC for imipenem and doripenem (Chapter 2, Table 2.2). Overexpression of AmpC in PA0 $A a m p C$ restored resistance against the aminopenicillins and additionally provided resistance or significantly increased MICs (decreased susceptibility) against the cephalosporins and the rest of the penicillins (Chapter 2, Table 2.3). A slight decrease in susceptibility was also observed against doripenem but not against imipenem or meropenem suggesting the carbapenems are not good substrates of the AmpC $\beta$-lactamase. 
The role of PoxB, on the other hand, was not readily observed in the natural background. For instance, a poxB deletion failed to alter susceptibility in the presence or absence of $\operatorname{ampC}$ (Chapter 2, Table 2.2). Similarly, the amount $\beta$-lactamase activity produced by a $p o x B$ deletion strain $(\mathrm{PA} 0 \Delta p o x B)$ was comparable to that of wild-type PA01 (Chapter 2, Figure 2.3). PA0 $\triangle a m p C$, on the other hand, failed to produce any detectable $\beta$-lactamase activity in the presence or absence of chromosomal poxB. This suggests that the activity detected in wild-type and poxB deletion strains was due to the presence of AmpC. PoxB thus appears to be expressed at very low, marginal levels in the lab strain PA01 under the tested experimental conditions.

Expression of $p o x B$ from an IPTG-inducible promoter in an $a m p C$-deficient strain

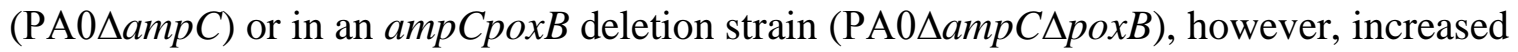
the MIC, thereby decreasing susceptibility, against the carbapenems doripenem and meropenem, but produced little to no change against the penicillins and cephalosporins (Chapter 2, Table 2.3). It is well-documented that clinical strains can develop resistance to imipenem after treatment with said carbapenem $(304,313,314,955)$. Mutations, deletions and insertions usually occur in $\operatorname{opr} D$, encoding an outer membrane porin specific for the uptake of imipenem, and result in impaired/lost function of the porin $(313,315,947,955)$. Similarly, in our studies loss of the outer membrane porin, OprD, specific for the uptake of imipenem, extended the hydrolysis spectrum of PoxB to include imipenem and enhanced the carbapenem-hydrolyzing phenotype leading to clinical intermediate and resistant phenotypes (Chapter 2, Table 2.4). Thus, PoxB is a carbapenem-hydrolyzing class D $\beta$-lactamase. 
In agreement with our studies, previous work by Girlich et. al examining the biochemical properties of PoxB, failed to detect hydrolysis of oxacillin, cloxacillin, cefepime, cefotaxime, ceftazidime and aztreonam, among others (741). Hydrolysis of various other penicillins and cephalosporins, however, was detected, as well as of nitrocefin, meropenem and imipenem. PoxB, however, displayed low affinity, high $\mathrm{K}_{m}$, for most $\beta$-lactams tested, with the exception of imipenem and nitrocefin for which $\mathrm{K}_{m}$ values of 20 and $200 \mu \mathrm{M}$ were obtained, respectively (741). Hydrolysis of both imipenem and meropenem, nonetheless, was very low, with meropenem exhibiting even weaker hydrolysis than that of imipenem. PoxB expression did afford the cell decreased susceptibility against meropenem in the previous and present study (741). In the current work, PoxB expression additionally increased MIC against doripenem, and in the absence of OprD against imipenem. Protection against imipenem was not previously detected in the work of Girlich et. al despite PoxB exhibiting high affinity for this $\beta$-lactam, as experiments were conducted in $\operatorname{opr} D^{+}$strains.

The current study further tested the effect of class A $\beta$-lactamase inhibitors, tazobactam, sulbactam and clavulanic acid, on the activity of PoxB (Chapter 2, Figures 2.7 and 2.8). These compounds did not significantly affect PoxB activity and thus were considered poor inhibitors and likely also poor substrates of the enzyme. That OXA-50 is resistant to inhibition is not surprising as in general, class D enzymes are not significantly inhibited by clavulanic acid, sulbactam, and tazobactam $(428,467)$. Exceptions include OXA-53 (956), OXA-18 (957) and OXA-45 (958) all inhibited by clavulanic acid, OXA- 
12 (735) inhibited by both clavulanic acid and tazobactam, and OXA-2 and its point mutant OXA-32 that are susceptible to inhibition by tazobactam (959).

Avibactam, a new non- $\beta$-lactam-type inhibitor, was also a poor inhibitor of PoxB (Chapter 2, Table 2.6). Avibactam has been shown to successfully inhibit class A and C enzymes, but has generally lacked activity against $A$. baumannii isolates expressing OXA-type $\beta$-lactamases $(804,805)$. Significant activity has been observed against the CHDL OXA-48, but a kinetics study showed that the rate of inhibition was lower than that for class $\mathrm{A}$ and $\mathrm{C}$ enzymes $(805,816)$. Thus inhibition of class $\mathrm{D}$ enzymes still remains a problem in the clinical setting where the commercially available inhibitors are not generally active against OXA enzymes. In this respect, OXA-50 is a typical class D $\beta$-lactamase. In vitro inhibition by $\mathrm{NaCl}$, often used as a defining characteristic of most oxacillinases, was also observed for PoxB. This inhibition, although readily detected in crude extracts from PoxB-expressing cells, was only slightly noticeable by the broth microdilution method (Chapter 2, Figures 2.9 and 2.11).

In summary, although exhibiting relatively low homology to other class D enzymes, in most respects, PoxB is a typical class D carbapenemase. In general, CHDLs do not significantly hydrolyze extended-spectrum cephalosporins but do exhibit some level of hydrolysis against the penicillins, mainly benzylpenicillin, ampicillin, piperacillin and ticarcillin $(680,693,960)$. Additionally, CHDLs display low $\mathrm{K}_{m}$ values for imipenem, lower than for other $\beta$-lactams, suggesting very high affinity for this substrate (680, 685, 960). Hydrolysis rates for both imipenem and meropenem, however, remain very low. 
In its natural host and under the conditions tested, poxB seems to be expressed at very low levels which do not contribute to the intrinsic resistance of PA01. This raises the question of the natural role of this operon in $P$. aeruginosa. Does the pox $A B$ operon play a yet unidentified role besides that of resistance? Further, we know that increasing the expression of PoxB can lead to decreased susceptibility or resistance against the carbapenems, but the relevance of OXA-50 in the clinical setting is not yet known.

Although poxB expression appears to be constitutive and unregulated, it is possible that mutations in the promoter region or insertion elements could lead to its expression in a way that contributes to resistance as seen in other bacteria. For example, A. baumannii often carries the chromosomal ampC but lacking ampR, expression is normally non-inducible $(961,962)$. Ceftazidime resistance in this pathogen however, has been linked to increased expression of $\operatorname{ampC}$ due to the presence of the upstream insertion element ISAbal with strong promoter sequences $(963,964)$. ISAbal was absent from ceftazidime-susceptible strains $(724,965)$. This insertion sequence seems to be prevalent in carbapenem-resistant A. baumannii isolates where it has been reported in association with $b l a_{\text {OXA-51-like }}$ and bla $a_{\text {OXA-23-like }}$ genes $(700,725,727)$. Although ISAbal has not been reported in P. aeruginosa, promoter sequences in ISPa12 have been found to drive expression of the extended-spectrum $\beta$-lactamase-encoding gene $b l a_{\mathrm{PER}-1}$ in $P$. aeruginosa, Providencia stuartii, A. baumannii and Salmonella enterica serovar Typhimurium (728). Similarly, expression of the extended-spectrum $\beta$-lactamaseencoding gene, $b l a_{\mathrm{VEB}-1}$, in ceftazidime-resistant $P$. aeruginosa isolates was found to be driven by promoter sequences within the upstream-located IS1999 (966). Thus, 
upregulation of gene expression by insertion sequences is a plausible mechanism of PoxB overexpression.

As a sole mechanism of resistance, poxB expression may be easy to overlook as the changes in MIC against carbapenems may not be substantial. Moreover, carbapenem resistance is often linked to mutations or insertions in $\operatorname{oprD}$, and screening of the poxB ORF and its promoter are not routinely done. This work showed that, when combined, the concerted expression of $\operatorname{poxB}$ and the loss of $\operatorname{oprD}$ can lead to resistance and/or significant MIC changes. Thus, although constitutively expressed in PA01, the role of PoxB in $P$. aeruginosa in the clinical setting may currently be overlooked.

5.3 Regulation of poxB. After establishing the role of PoxB as a carbapenemhydrolyzing class D $\beta$-lactamase, its regulation was investigated (Chapter 3 ). As previous work pointed to the involvement of the transcriptional regulator AmpR (710), expression of $p o x B$ was examined by RT-PCR in the presence and absence of $a m p R$ (Figure 3.3). A small but significant increase in expression was observed in the absence of ampR and in the presence of $\beta$-lactams suggesting AmpR normally acts to downregulate poxB expression. Similarly, promoter studies using reporter strains carrying $\mathrm{P}_{p o x}-$ lac $Z$ fusions were in agreement with the mRNA data (Figure 3.8). Specifically, induction of poxB could be achieved only in the absence of $\operatorname{ampR}$ and in the presence of $\beta$-lactams (Chapter 3). Interestingly these changes could not be observed at the protein level in terms of $\beta$ lactamase activity. 
AmpR is an interesting candidate for regulator of pox expression. As a LysR-type transcriptional regulator, AmpR carries an N-terminal HTH motif to interact with DNA and an effector binding domain in the C-terminus for ligand interaction $(852,856,897)$. As detailed in Chapter 4 of this dissertation, AmpR activates transcription of ampC in the presence of $\beta$-lactams but maintains expression at low basal levels in the absence of such compounds, thus acting as both a negative and positive regulator of ampC expression (710, 711, 776). AmpR has additionally been shown to have an extensive regulon that includes a number of characterized and putative transcriptional and sigma factors, as well as genes involved in quorum sensing and virulence $(710,841,843)$. That the regulon of AmpR includes transcriptional regulatory proteins suggests AmpR-dependent expression may occur by indirect regulation. Our EMSA studies in fact, failed to show binding of AmpR to the pox promoter (Figure 3.5), suggesting regulation by AmpR is indirect.

In an effort to identify possible pox regulators, a genome-wide approach was undertaken using the reporter strains $\mathrm{PA} 0:: \mathrm{P}_{p o x}-l a c Z$ and $\mathrm{PA} 0 \Delta a m p R:: \mathrm{P}_{p o x}-l a c Z$ (Chapter 3, Section 3.4.5). The strains were mutagenized with a random mariner transposon ( $\mathrm{Tn})$ in order to identify clones with increased or decreased lac $Z$ expression indicative of altered pox promoter expression. This study generated a wealth of clones that are still being screened and evaluated. Two noteworthy clones however, identified based on their increased lacZ expression, had insertions in spuE (PA0301) and PA0322, genes involved in a characterized and a putative polyamine uptake system, respectively.

Polyamines (putrescine, cadaverine, spermidine, spermine) are essential molecules found in all domains of life $(845,847)$. Bacteria can synthesize, catabolize and 
uptake these molecules for a number of diverse processes as described in Chapter 3 (845, $847,878)$. Interestingly, polyamines have been found to sensitize a number of bacterial species to multiple $\beta$-lactams including penicillins, cephalosporins and monobactams $(886,893)$. This sensitization did not appear to occur by an increase in outer membrane permeability as expected of polyamines given their cationic nature. On the other hand, polyamines provided resistance against the $\beta$-lactam imipenem in $P$. aeruginosa seemingly through blockage of $\mathrm{OprD}$, the major route of entry for imipenem into the cell (893).

In order to determine if the increased lacZ expression in mutants containing $\mathrm{Tn}$ insertions in spuE and PA0322, was due to an increase in pox expression, the susceptibility of the mutants to carbapenems was investigated in the presence and absence of polyamines (Chapter 3, Table 3.7). Our studies show that polyamines significantly increased MICs, or decreased susceptibility, against ceftazidime, piperacillin and the carbapenems, but not against aztreonam, in both unmutagenized and Tnmutagenized strains. This increased in MIC is likely the result of blockage of OprD as well as other porins, as ceftazidime and piperacillin are not known to use the OprD porin for passage into the cell. If resistance is occurring by blocking of porins, then the polyamines do not necessarily need to come into the cell to exert their action. This would explain why polyamines could raise MICs in what appear to be a strain-independent manner. The resistance provided was independent of polyamine uptake pathways and as such the spuE and PA0322 Tn insertions did not affect susceptibility. That the polyamine spermidine $(10 \mathrm{mM})$ could induce resistance against both imipenem and meropenem 
(>32 $\mu \mathrm{g} / \mathrm{ml}$ ) likely suggests that polyamines more efficiently block OprD than other porins and that meropenem and imipenem preferentially enter the cell via this route.

Alternatively, although the function of these genes may be impaired, since there are other polyamine transport systems, both putative and characterized, the disruptions imposed by the Tn insertions could potentially be overcome by other such systems. Thus, polyamines could still enter the cell to exert their action, e.g., modulation of gene expression. For instance, SpuE is a periplasmic binding protein that specifically binds spermidine, but the spuABCDEFGH gene cluster that encodes it also encodes SpuD, another periplasmic binding protein that although preferentially binds to putrescine, can also bind and transport spermidine $(864,883)$. Thus, this system is also likely involved in the uptake and transport of both spermidine and putrescine. The presence of spuABCDEFGH probably also compensates for the loss of $P A 0322$ a putative, not yet proven putrescine transporter. Other putative polyamine transport and utilization systems are also encoded in the general vicinity of the spu gene cluster. The redundancy of such system may illustrate how essential these molecules are for survival.

It is worth noting that the Tn insertion into $s p u E$ likely also led to disruption of transcription for the rest of the operon, spuFGH, encoding the ATPase for transport and the two permeases, respectively. Since the Tn contained an outwardly-directed promoter and the insertion occurred with the promoter in the direction of transcription of these genes, it is likely that transcription of these downstream ORFs did occur. As experiments revealed in Chapter 3, the outward-facing promoter of this mariner transposon was very strong. 
An alternative explanation for the polyamine-dependent MIC increase could be the induction of genes that themselves confer resistance, such as those involved in efflux. Although polyamines have not been shown to induce expression of efflux pumps, spermidine did provide resistance against cationic peptides and quinolones by inducing expression of $o p r H-p h o P Q$ and the LPS modification operon (885). It is likely we don't yet know all the current targets of polyamines.

The $\beta$-lactamase profiles of spuE (PA0301) and PA0322 Tn mutants were not altered as compared to wild-type as would be expected if such insertions affected pox expression. Although the increased lacZ expression phenotype displayed by these mutants appears to be unconnected to $\mathrm{P}_{\text {pox }}$ expression, that we have stumbled upon yet another condition that contributes to carbapenem resistance is very interesting. To conclusively prove pox involvement, the transcript levels would have to be quantified in spuE and PA0322 mutants.

5.4 Future directions. Previous studies showed poxB is ubiquitous in $P$. aeruginosa clinical and environmental isolates (741), yet its expression and contribution to $\beta$-lactam resistance has not been analyzed in such backgrounds. Traditionally, carbapenem resistance in $P$. aeruginosa has been provided by the loss of OprD and the expression of class B $\beta$-lactamases, especially the VIM and IMP-types (549, 947, 955, 967-969). OXA50 , however, is not routinely screened for and thus its true contribution in the clinical setting is unknown. 
Future studies should elucidate on the role of PoxB in clinical isolates, especially for those that are shown to be carbapenem non-susceptible (intermediate and resistant phenotypes). Using PCR-based methods, the gold standard for identifying $\beta$-lactamases, the presence of metallo- $\beta$-lactamases and OXA-type enzymes, including OXA-50, could be detected. Since the mere presence of PoxB does not imply its expression, its mRNA levels should be quantified. Alternatively, the actual protein could be quantified by doing Western blot. Sequencing of the oprD ORF should also be done in carbapenem nonsusceptible isolates to determine contribution of this porin to resistance.

Another area of this research to expand could involve identification of other potential roles for PoxB and the pox operon besides that of resistance. Phenotypic microarrays (Biolog, Hayward, CA) could help identify other such functions. Such arrays typically measure the rate of respiration over a period of time under a number of environmental conditions including ranges of salinity, $\mathrm{pH}$, nitrogen, carbon, phosphorus and sulfur sources, as well as many antimicrobial drugs and chemical compounds. In this respect two strains will be critical: poxB and $\operatorname{pox} A B$ deletion mutants.

A phenotypic microarray has been carried out in PA0ApoxA and pointed to a growth defect in the presence of $\mathrm{NaNO}_{3}$, leading us to investigate morphology in anoxic and oxic environments. While the $\mathrm{NaNO}_{3}$ phenotype could not be reproduced, we did observed PoxA-expressing strains were able to bind the dye Congo red (CR) better presumably because of increased production of an extracellular polymeric substance. The CR-binding phenotype was not reproducible or readily observed in liquid media as is often seen with strains that overproduce Pel and Psl polysaccharides. Thus, it is likely 
that the CR-binding phenotype is due to production of a polysaccharide or a CR-binding substance other than Pel or Psl. In order to rule out the involvement of Pel or Psl polysaccharides, expression of some of the genes of pel an psl operons could be quantified by RT-PCR. Additionally, the carbohydrate composition of the CR-binding substance being produced in PoxA-expressing cells could be investigated by gas chromatography/mass spectrometry as previously described $(768,825)$.

Although screening and confirmation of the Tn mutant library was well underway, more than $50 \%$ of the library still remains to be screened and/or evaluated. Thus, a continuing project could involve further screening with the clones that still remain to be investigated. Although the relationship between pox expression and polyamine transport identified by the transposon screening is not yet clear, polyamineinduced resistance and susceptibility in $P$. aeruginosa is by itself an interesting and worthy area of study. A microarray could elucidate on the targets of these essential molecules and the link to carbapenem resistance.

Carbapenem resistance has become an increasing problem in the nosocomial pathogen A. baumannii with the predominant mechanism of resistance being the expression of OXA enzymes $(622,692,708,709,726,820)$. Carbapenems are often the drug of choice for treating organisms expressing extended-spectrum $\beta$-lactamases (ESBLs) which can hydrolyze penicillins and cephalosporins. There is a real concern that treatment with such compounds can lead to ESBLs that additionally can hydrolyze carbapenems. Thus, there is a need to develop effective class D $\beta$-lactamase inhibitors. Although not yet prevalent in $P$. aeruginosa, CHDLs have the potential to become 
widespread, as they have in the closely related Pseudomonadales species A. baumannii, given its genomic plasticity and ability to acquire resistance genes from the environment. P. aeruginosa already carries PoxB, a chromosomal carbapenem-hydrolyzing $\beta$ lactamase, ubiquitous in clinical and environmental isolates, and with the potential to become yet another tool in the arsenal of this intractable pathogen against the $\beta$-lactam antibiotics. 
SUPPLEMENTAL MATERIAL 
Table S1: Bacterial strains, plasmids, and primers used in this study

\begin{tabular}{|c|c|c|}
\hline Strains/ Plasmids & Relevant phenotype and genotype & Source \\
\hline \multicolumn{3}{|l|}{ Escherichia coli } \\
\hline E. coli $\mathrm{DH} 5 \alpha$ & $\begin{array}{l}\mathrm{F}^{-} \Phi 80 \text { lacZDM15 } \Delta(\text { lacZYA-argF }) U 169 \text { deoR recA1 } \\
\text { endA1 hsdR17 }\left(\mathrm{r}_{\mathrm{k}}^{-} \mathrm{m}_{\mathrm{k}}^{+}\right) \text {phoA supE44 } \lambda^{-} \text {thi-1 gyrA96 } \\
\text { relA1 }\end{array}$ & $\begin{array}{l}\text { New } \\
\text { England } \\
\text { Biolabs }\end{array}$ \\
\hline \multicolumn{3}{|c|}{ Pseudomonas aeruginosa } \\
\hline PA01 & Wild-type & $(762)$ \\
\hline PA06358 & $\begin{array}{l}\text { PA0 } 0 \text { rpoN }, \text { PA01 containing in-frame deletion of a } \\
0.9 \text {-kb fragment from the rpoN gene (PA4462) }\end{array}$ & $(970)$ \\
\hline PKM106 & PA0attB::mini-CTX-lacZ; $\mathrm{Tc}^{\mathrm{R}}$ & This study \\
\hline PKM107 & $\mathrm{PA0attB:: \textrm {P } _ { a m p c 3 5 2 } - l a c Z ; \mathrm { Tc } ^ { \mathrm { R } }}$ & This study \\
\hline PKM108 & PA0attB::P $\mathrm{P}_{a m p c 193-l a c Z ;} \mathrm{Tc}^{\mathrm{R}}$ & This study \\
\hline PKM109 & $\mathrm{PA0attB:: \textrm {P } _ { a m p c 1 7 1 } - l a c Z ; \mathrm { Tc } ^ { \mathrm { R } }}$ & This study \\
\hline PKM110 & PA0attB::Pampc151-lacZ; Tc ${ }^{\mathrm{R}}$ & This study \\
\hline PKM111 & 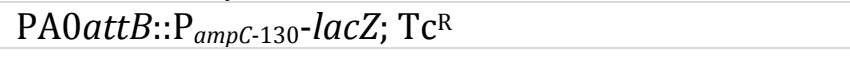 & This study \\
\hline PKM112 & PA0attB:: $\mathrm{P}_{a m p c-111}-l a c Z ; \mathrm{Tc}^{\mathrm{R}}$ & This study \\
\hline PKM113 & PA0attB:: $\mathrm{P}_{a m p c 90}-l a c Z ; \mathrm{Tc}^{\mathrm{R}}$ & This study \\
\hline PKM114 & 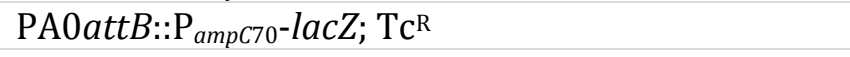 & This study \\
\hline PKM115 & PA0attB:: $\mathrm{P}_{a m p c 51}-l a c Z ; \mathrm{Tc}^{\mathrm{R}}$ & This study \\
\hline PKM116 & PA0attB::PampR193-lacZ; $\mathrm{Tc}^{\mathrm{R}}$ & This study \\
\hline PKM201 & $\begin{array}{l}\text { PA0 } \triangle a m p C, \text { PA01 containing in-frame deletion of } \\
a m p C\end{array}$ & $(760)$ \\
\hline PKM315 & $\begin{array}{l}\text { PA0 } \Delta a m p R, \text { PA01 containing in-frame deletion of } \\
\text { ampR }\end{array}$ & $(843)$ \\
\hline PKM318 & PA0ampR $R_{V S V-G} ; V S V-G$ tag added in-frame to AmpR & This study \\
\hline \multicolumn{3}{|l|}{ Plasmids } \\
\hline pBSK(+) & $\mathrm{Ap}^{\mathrm{R}}$; ColE1ori lacZ $\alpha$ & Stratagene \\
\hline pEXG2 & $\mathrm{Gm}^{\mathrm{R}}$; pMB1ori sacB & $(759)$ \\
\hline pP30 $\Delta$ FRT-MvaT-V & $\begin{array}{l}\mathrm{Gm}^{\mathrm{R}} \text {; ColE1ori RP4 (mob) aacC1, integration- } \\
\text { proficient vector containing VSV-G epitope tag }\end{array}$ & (908) \\
\hline pSJ01 & $\begin{array}{l}\text { Ap }^{\mathrm{R}}, \mathrm{pGEMEX}-1 \text { with a } 1220 \text {-bp EcoRI-BamHI- } \\
\text { flanked fragment containing ampR }\end{array}$ & $(710)$ \\
\hline pTrcphoA & $\begin{array}{l}A p^{\mathrm{R}} \text {, low-copy trc promoter expression vector } \\
\text { carrying } l a c I^{\mathrm{q}} \text { and } p h o A\end{array}$ & $(911)$ \\
\hline pTrclac $Z$ & $\begin{array}{l}A p^{\mathrm{R}} \text {, low-copy } \operatorname{trc} \text { promoter expression vector } \\
\text { carrying } l a c I^{\mathrm{q}} \text { and } l a c Z\end{array}$ & $(911)$ \\
\hline Mini-CTX-lacZ & $\begin{array}{l}\mathrm{Tc}^{\mathrm{R}} \text {; Integration-proficient vector for single-copy } \\
\text { chromosomal lacZ fusion at the } a t t B \text { site }\end{array}$ & $(853)$ \\
\hline pMMB67EH-Gm & $\begin{array}{l}\mathrm{Gm}^{\mathrm{R}} \text {; IncQ, RSF1010, } l a c I^{\mathrm{q}} \mathrm{P}_{t a c} \text { expression vector } \\
\text { with } \operatorname{ampR}:: a a c C 1 \text { insertion at } \text { DraI }\end{array}$ & $(763)$ \\
\hline
\end{tabular}




\begin{tabular}{|c|c|c|}
\hline pP30 $\Delta$ FRT-AmpR-V & $\begin{array}{l}\text { pP30 } 3 \text { FRT-MvaT-V digested with HindIII and NotI } \\
\text { to release mvaT and carrying 540-bp 3' fragment of } \\
\text { ampR fused in-frame with VSV-G tag }\end{array}$ & This study \\
\hline p0C1 & $\mathrm{Tc}^{\mathrm{R}} ; \mathrm{P}_{\text {ampc352 }}$ fused to the lacZ of mini-CTX-lacZ & This study \\
\hline p0C2 & $\mathrm{Tc}^{\mathrm{R}} ; \mathrm{P}_{a m p c 193}$ fused to the $l a c Z$ of mini-CTX-lacZ & This study \\
\hline pOC3 & $\mathrm{Tc}^{\mathrm{R}} ; \mathrm{P}_{a m p c 171}$ fused to the lacZ of mini-CTX-lacZ & This study \\
\hline pOC4 & $\mathrm{Tc}^{\mathrm{R}} ; \mathrm{P}_{a m p c 151}$ fused to the lacZ of mini-CTX-lacZ & This study \\
\hline pOC5 & $\mathrm{Tc}^{\mathrm{R}} ; \mathrm{P}_{a m p c 130}$ fused to the lacZ of mini-CTX-lacZ & This study \\
\hline p0C6 & $\mathrm{Tc}^{\mathrm{R}} ; \mathrm{P}_{a m p c 111}$ fused to the lacZ of mini-CTX-lacZ & This study \\
\hline pOC7 & $\mathrm{Tc}^{\mathrm{R}} ; \mathrm{P}_{\text {amp } 90}$ fused to the $l a c Z$ of mini-CTX-lacZ & This study \\
\hline pOC8 & $\mathrm{Tc}^{\mathrm{R}} ; \mathrm{P}_{\text {атрс70 }}$ fused to the $l a c Z$ of mini-CTX-lacZ & This study \\
\hline pOC9 & $\mathrm{Tc}^{\mathrm{R}} ; \mathrm{P}_{\text {ampc51 }}$ fused to the $l a c Z$ of mini-CTX-lacZ & This study \\
\hline pOC10 & $\mathrm{Tc}^{\mathrm{R}} ; \mathrm{P}_{\text {ampR193 }}$ fused to the lacZ of mini-CTX-lacZ & This study \\
\hline p0C11 & $\begin{array}{l}\mathrm{Ap}^{\mathrm{R}} ; \mathrm{pBSK}-\mathrm{AmpR}-\mathrm{His}_{6} \text { (933-bp EcoRI-BamHI } \\
\text { fragment containing AmpR His-tagged at C- } \\
\text { terminus) }\end{array}$ & This study \\
\hline p0C12 & $\mathrm{Ap}^{\mathrm{R}} ; \mathrm{p} 0 \mathrm{C} 11-\mathrm{AmpR}_{\text {Ser38Ala }}$ & This study \\
\hline p0C13 & $\mathrm{Ap}^{\mathrm{R}} ; \mathrm{pOC} 11-\mathrm{AmpR}_{\mathrm{His} 39 \mathrm{Ala}}$ & This study \\
\hline p0C14 & $\mathrm{Ap}^{\mathrm{R}} ; \mathrm{p} 0 \mathrm{C} 11-\mathrm{AmpR}_{\mathrm{Lys} 42 \mathrm{Ala}}$ & This study \\
\hline pOC15 & $\mathrm{Ap}^{\mathrm{R}} ; \mathrm{p} 0 \mathrm{C} 11-\mathrm{AmpR}_{\mathrm{Ser} 43 \mathrm{Ala}}$ & This study \\
\hline p0C16 & $\mathrm{Ap}^{\mathrm{R}} ; \mathrm{p} 0 \mathrm{C} 11-\mathrm{AmpR}_{\mathrm{Glu} 46 \mathrm{Ala}}$ & This study \\
\hline p0C17 & $\mathrm{Ap}^{\mathrm{R}} ; \mathrm{pOC} 11-\mathrm{AmpR}_{\mathrm{Gly} 102 \mathrm{Glu}}$ & This study \\
\hline p0C18 & $\mathrm{Ap}^{\mathrm{R}} ; \mathrm{p} 0 \mathrm{C} 11-\mathrm{AmpR}_{\mathrm{Asp} 135 \mathrm{Asn}}$ & This study \\
\hline p0C19 & $\begin{array}{l}\mathrm{Gm}^{\mathrm{R}} ; \mathrm{pMMB67EHGM} \text { containing 933-bp EcoRI- } \\
\text { BamHI } a m p R \text {-His } 6 \text { fragment from p0C11 }\end{array}$ & $\begin{array}{l}\text { pAmpR- } \\
\text { His }_{6} \text {, This } \\
\text { study }\end{array}$ \\
\hline pOC20 & $\begin{array}{l}\mathrm{Gm}^{\mathrm{R}} \text {; pMMB67EHGM with EcoRI-BamHI fragment } \\
\text { containing AmpR-His } 6 \text {-Ser38Ala from pOC12 }\end{array}$ & This study \\
\hline p0C21 & $\begin{array}{l}\mathrm{Gm}^{\mathrm{R}} \text {; pMMB67EHGM with EcoRI-BamHI fragment } \\
\text { containing AmpR-His } 6 \text { - His39Ala from p0C13 }\end{array}$ & This study \\
\hline pOC22 & $\begin{array}{l}\mathrm{Gm}^{\mathrm{R}} \text {; pMMB67EHGM with EcoRI-BamHI fragment } \\
\text { containing AmpR-His } 6 \text {-Lys42Ala from p0C14 }\end{array}$ & This study \\
\hline p0C23 & $\begin{array}{l}\mathrm{Gm}^{\mathrm{R}} \text {; pMMB67EHGM with EcoRI-BamHI fragment } \\
\text { containing AmpR-His } 6 \text {-Ser43Ala from pOC15 }\end{array}$ & This study \\
\hline pOC24 & $\begin{array}{l}\mathrm{Gm}^{\mathrm{R}} \text {; pMMB67EHGM with EcoRI-BamHI fragment } \\
\text { containing AmpR-His } 6 \text {-Glu46Ala from pOC16 }\end{array}$ & This study \\
\hline p0C25 & $\begin{array}{l}\mathrm{Gm}^{\mathrm{R}} \text {; pMMB67EHGM with EcoRI-BamHI fragment } \\
\text { containing AmpR-His } 6 \text {-Gly102Glu from pOC17 }\end{array}$ & This study \\
\hline p0C26 & $\begin{array}{l}\mathrm{Gm}^{\mathrm{R}} \text {; pMMB67EHGM with EcoRI-BamHI fragment } \\
\text { containing AmpR-His }{ }_{6-\text { Asp } 135 \text { Asn }} \text { from p0C18 }\end{array}$ & This study \\
\hline pKK862 & $\begin{array}{l}\text { Ap }{ }^{\mathrm{R}} \text {, pTrclacZ derivative with a } 1220 \text {-bp fragment } \\
\text { containing ampR and the lacZ gene at HindIII site }\end{array}$ & This study \\
\hline pKK863 & $\begin{array}{l}\text { Ap }{ }^{\mathrm{R}}, \mathrm{p} \operatorname{Trcpho} A \text { derivative with a } 1220-\mathrm{bp} \text { fragment } \\
\text { containing } a m p R \text { and the } p h o A \text { gene at HindIII site }\end{array}$ & This study \\
\hline pKK864 & $\begin{array}{l}\text { Ap }{ }^{\mathrm{R}}, \mathrm{p} \text { TrclacZ derivative with a } 1220 \text {-bp fragment } \\
\text { containing } a m p R \text { and the lacZ gene at HincII site }\end{array}$ & This study \\
\hline
\end{tabular}




\begin{tabular}{|c|c|c|}
\hline pKK865 & $\begin{array}{l}\text { Ap }{ }^{\mathrm{R}}, \mathrm{p} \operatorname{Trc} p h o A \text { derivative with a } 1220 \text {-bp fragment } \\
\text { containing } a m p R \text { and the } p h o A \text { gene at HincII site }\end{array}$ & This study \\
\hline pKK866 & $\begin{array}{l}\mathrm{Ap}^{\mathrm{R}}, \mathrm{p} \text { TrclacZ derivative with a } 1220 \text {-bp fragment } \\
\text { containing ampR and the lacZ gene at PstI site }\end{array}$ & This study \\
\hline pKK867 & $\begin{array}{l}A p^{\mathrm{R}}, \mathrm{pTrcphoA} \text { derivative with a } 1220 \text {-bp fragment } \\
\text { containing } a m p R \text { and the } p h o A \text { gene at PstI site }\end{array}$ & This study \\
\hline \multicolumn{3}{|l|}{ Primers } \\
\hline DZampCUF1 & 5'-GGAATTCAAGACGATGCTCCGGGTCAGTG-3' & $(760)$ \\
\hline DZampCUR1 & $\begin{array}{l}\text { 5'-GATACCAGATTCCCCTGCCTGTCTAGCTAGCTAGA } \\
\text { ATGCTC-3' }\end{array}$ & $(760)$ \\
\hline DZampCDF2 & $\begin{array}{l}\text { 5'-CTAGCTAGCTAGAATGCTCAAGCGCGCTCGCGAGG } \\
\text { GCGA CGGA-3' }\end{array}$ & $(760)$ \\
\hline DZampCDR2 & 5'-CGGGATCCGACCCTGCATACCATCAAGG-3' & $(760)$ \\
\hline KMampRUF1 & $\begin{array}{l}\text { 5'-GGAATTCAGCCTGGAACTTCCGCAATCTCGACG } \\
\text { AGA-3' }\end{array}$ & $(843)$ \\
\hline KMampRUR1 & $\begin{array}{l}\text { 5'GAGCATTCTAGCTAGCTAGGTTCGCCACCGAAGT } \\
\text { GAGTACC-3' }\end{array}$ & $(843)$ \\
\hline KMampRDF2 & $\begin{array}{l}\text { 5'-CTAGCTAGCTAGAATGCTCAATGGGGTCGAACCAA } \\
\text { TCTCT GCTCC-3' }\end{array}$ & $(843)$ \\
\hline KMampRDR2 & 5’ CGGGATCCGATGCTCGGGTTGGAATAGA-3' & $(843)$ \\
\hline OCAmpR-His-For & $\begin{array}{l}\text { 5'ACAGAATTCGAAGGAGATATACCTTGGTTCGACCC } \\
\text { CATTT GCC-3' }\end{array}$ & This study \\
\hline OCAmpR-His-Rev & $\begin{array}{l}\text { 5'-GAGGATCCTTAGTGATGATGATGATGATGTCTCCC } \\
\text { CCGCGCCTCAACGGCAGCC-3' }\end{array}$ & This study \\
\hline SBJ03ampCRFor & $\begin{array}{l}\text { 5'-GGAATTCTGAGGCCGCGCGGCAGACGCTTGAACA- } \\
\text { 3' }\end{array}$ & This study \\
\hline OCP $_{a m p c}$ For193 & 5'-CGGGAATTCGCGGCAAATGGGGTCGAACC-3' & This study \\
\hline OCP $_{a m p c}$ For173 & 5'-CGGGAATTCTCTCTGCTCCAAATTTTTCT-3' & This study \\
\hline OCP $_{a m p c}$ For151 & 5'-CGGGAATTCAATGGCTGCCGCGAGTATTCG-3' & This study \\
\hline OCP $_{a m p c}$ For131 & 5'-CGGGAATTCTCGTTTGCCGCAAATCCTGC-3' & This study \\
\hline OCP $_{a m p c}$ For111 & 5'-CGGGAATTCGCAAGCCTAGATTTTCCCCG-3' & This study \\
\hline OCP $_{a m p c}$ For91 & 5'-CGGGAATTCCCCGCCGATCAAGGAGCGCT-3' & This study \\
\hline OCP $_{a m p c}$ For71 & 5'-CGGGAATTCCCCGGGGCGGTTTCTCATGC-3' & This study \\
\hline OCР ${ }_{a m p c}$ For51 & 5'-CGGGAATTCAGCCAACGACAAAGGACGC-3' & This study \\
\hline $\mathrm{OCP}_{a m p c} \operatorname{RevBc}$ & 5'-CGGGGATCCTCAGGGGAATCTGGTATCGCG-3'C & This study \\
\hline $\mathrm{OCP}_{a m p R} \operatorname{Rev193}$ & 5'-CGGGGATCCGCGGCAAATGGGGTCGAACC-3' & This study \\
\hline $\mathrm{OCP}_{a m p R}$ ForEc & 5'-CGGGAATTCCAGGGGAATCTGGTATCGCGC-3' & This study \\
\hline $\operatorname{alg} 44$ For & 5'-CGAAGCCCAGCGCCAGTTCG-3' & This study \\
\hline $\operatorname{alg} 44 \mathrm{Rev}$ & 5'-GGTCCACCGAGCGCACCTGG-3' & This study \\
\hline AmpRSer38AlaFor & 5'-ACCCAGGCGGCGGTCGCCCACCAGGTGAAGAGC-3' & This study \\
\hline AmpRSer38AlaRev & 5'-GCTCTTCACCTGGTGGGCGACCGCCGCCTGGGT-3' & This study \\
\hline AmpRHis39AlaFor & 5'-CAGGCGGCGGTCAGCGCCCAGGTGAAGAGCCTC-3' & This study \\
\hline AmpRHis39AlaRev & 5'-GAGGCTCTTCACCTGGGCGCTGACCGCCGCCTG-3' & This study \\
\hline AmpRLys42AlaFor & 5'-GTCAGCCACCAGGTGGCGAGCCTCGAGGAGCGT-3' & This study \\
\hline
\end{tabular}




\begin{tabular}{|c|c|c|}
\hline AmpRLys42AlaRev & 5'-ACGCTCCTCGAGGCTCGCCACCTGGTGGCTGAC-3' & This study \\
\hline AmpRSer43AlaFor & 5'-AGCCACCAGGTGAAGGCCCTCGAGGAGCGTCTC-3' & This study \\
\hline AmpRSer43AlaRev & 5'-GAGACGCTCCTCGAGGGCCTTCACCTGGTGGCT-3' & This study \\
\hline AmpRGlu46AlaFor & 5'-GTGAAGAGCCTCGAGGCGCGTCTCGGCGTGGCC-3' & This study \\
\hline AmpRGlu46AlaRev & 5'-GGCCACGCCGAGACGCGCCTCGAGGCTCTTCAC-3' & This study \\
\hline AmpRGly102GluFor & $\begin{array}{l}\text { 5'- } \\
\text { CACCGTCGGCGCGGTCGAAACCTTCACGGTCGGTT-3' }\end{array}$ & This study \\
\hline AmpRGly102GluRev & $\begin{array}{l}\text { 5'- } \\
\text { AACCGACCGTGAAGGTTTCGACCGCGCCGACGGTG-3' }\end{array}$ & This study \\
\hline $\begin{array}{l}\text { AmpRAsp135Asn } \\
\text { For }\end{array}$ & $\begin{array}{l}\text { 5'-CCCACAACAACCGCGTCAACATCGCCGCCGAGGG- } \\
\text { 3' }\end{array}$ & This study \\
\hline $\begin{array}{l}\text { AmpRAsp135Asn } \\
\text { Rev }\end{array}$ & $\begin{array}{l}\text { 5'-CCCTCGGCGGCGATGTTGACGCGGTTGTTGTGGG- } \\
3^{\prime}\end{array}$ & This study \\
\hline 5RA- $\mathrm{P}_{a m p c} 233$ & 5'-GGGTCTCCGGCGTCACCCGG-3' & This study \\
\hline 5RA-P ${ }_{a m p c} 154$ & 5'-GGCTACGGCCAGGCCCGG-3' & This study \\
\hline 5RA-P ${ }_{a m p c} 113$ & 5'-CCGGTTGTACGGCGGCGTCG-3' & This study \\
\hline 5RA-P ${ }_{a m p R} 229$ & 5'-GCAGGCCGGCGATGCGGTCG-3' & This study \\
\hline 5RA-P ${ }_{a m p R} 169$ & 5'-GGTCAGCATGAGGCCGCGCG-3' & This study \\
\hline 5RA-P $\mathrm{P}_{a m p R} 99$ & 5'-CCTGGTGGCTGACCGCCGCC-3' & This study \\
\hline $\mathrm{Q}_{\mathrm{t}}$ & $\begin{array}{l}\text { 5'- } \\
\text { GCCAGGTGAGCAGGAGTGACGGGAATTCAGCTGGAT } \\
\text { CCTGCACCCTTTTTTTTTTTTTTTTTT-3' }\end{array}$ & $(764)$ \\
\hline $\mathrm{Q}_{0}$ & 5'- GCCAGGTGAGCAGGAGTGACG-3' & $(764)$ \\
\hline$Q_{i}$ & 5'-GGAATTCAGCTGGATCCTGCACCC-3' & $(764)$ \\
\hline DBS_ChIP_ampCF & 5'-GGTCGAACCAATCTCTGCTCCAAA-3' & $(841)$ \\
\hline DBS_ChIP_ampCR & 5'-TTGTCGTTGGCTGCATGAGAAACC-3' & $(841)$ \\
\hline DBS_qRT_clpXF & 5'-TGCGATTACGATGTGGAGA-3' & $(843)$ \\
\hline DBS_qRT_clpXR & 5'-CCCTCGATGAGCTTCAGCA-3' & $(843)$ \\
\hline DBS_QRTAmpRFwd & 5'-CATTGGCCTTCATCACCGGTTGTA-3' & $(760)$ \\
\hline DBS_QRTAmpRRev & 5'-GGTTTCTCATGCAGCCAACGACAA-3' & $(760)$ \\
\hline qRT_ampCF & 5'-CGCCGTACAACCGGTGAT-3' & $(971)$ \\
\hline qRT_ampCR & 5'-CGGCCGTCCTCTTTCGA-3' & $(971)$ \\
\hline
\end{tabular}


His-tagged AmpR is functional. PA0 $\triangle a m p R$ was complemented with the vector, pMMB67EH-Gm, or with the His-tagged AmpR. $\beta$-lactamase assays show that in the absence of AmpR there is low constitutive ampC expression (Table S2). However, overexpression of AmpR-His 6 in PA0 $\triangle a m p R$ in the presence of $\beta$-lactams restores the $\beta$ lactamase activity to that of the wild-type induced strain. Additionally, the inherently resistant PA01 strain shows a significant increase in sensitivity to both amoxicillin and ampicillin when $a m p R$ is deleted, as determined by E-test (Table S2). The resistance is restored upon complementation with AmpR-His 6 . The AmpR-His 6 construct thus complements the $a m p R$ deletion and retains its native function.

Table S2: Complementing assays showing His-tagged AmpR is functional.

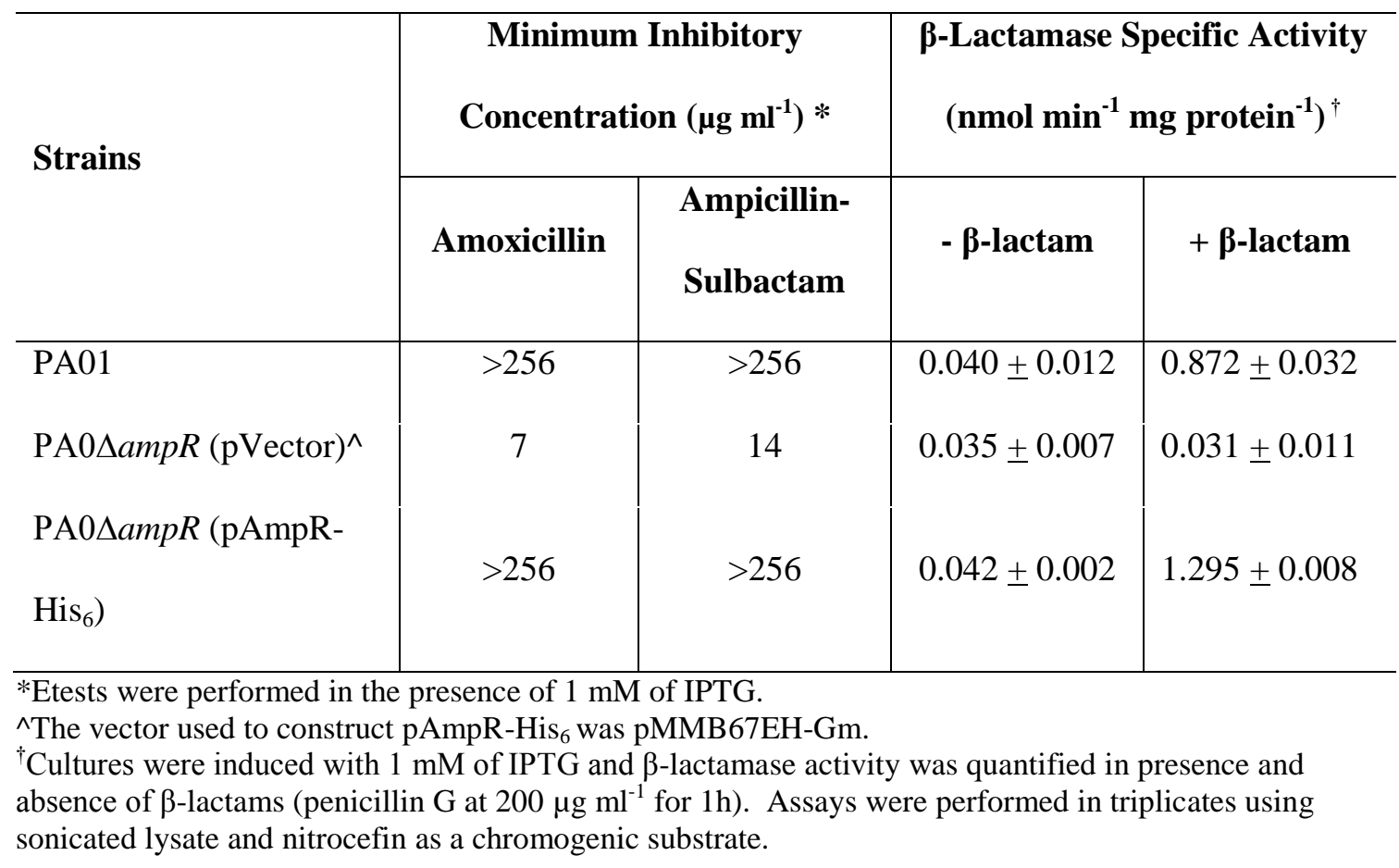


VSV-G-tagged AmpR is functional. The VSV-G-tagged AmpR was introduced into PA0 $\triangle a m p R$. The inherently resistant PA01 strain shows a significant increase in sensitivity to both amoxicillin and ampicillin-sublactam when $a m p R$ is deleted, as determined by E-test (Table S3). The resistance is restored upon integration of ampRVSV-G. The AmpR-VSV-G complements the $a m p R$ deletion and retains its native function.

Table S3: Complementing assays showing VSV-G-tagged AmpR is functional.

\begin{tabular}{l|c|c}
\hline \multirow{2}{*}{ Strains } & \multicolumn{2}{|c}{ Minimum Inhibitory Concentration $\left(\mu \mathrm{g} \mathrm{ml}^{-1}\right) *$} \\
\cline { 2 - 3 } & Amoxicillin & Ampicillin-Sulbactam \\
\hline PA01 & $>256$ & $>256$ \\
PA0 $\triangle a m p R$ & 8 & $>256$ \\
PAO $\triangle a m p R:: a m p R$-VSV-G & $>256$ & \\
\hline
\end{tabular}

*MIC was determined by Etest. 


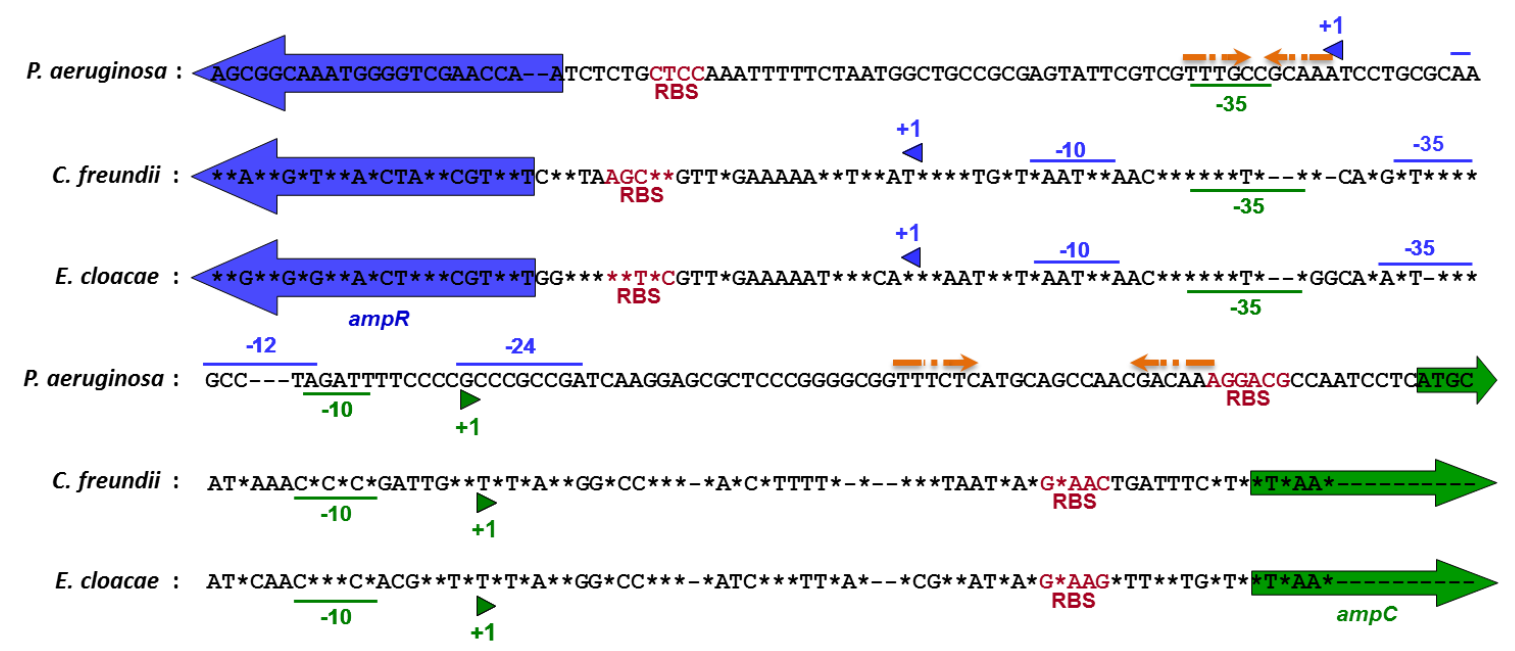

Figure S1. Multiple alignment of the $a m p R$ - $a m p C$ intergenic region. The $C$. freundii, $E$. cloacae and $P$. aeruginosa ampR-ampC intergenic regions were aligned using ClustalW2. The $a m p R$ and $a m p C$ ORFs (arrows), TSSs (triangles), and sigma sequences (underlined) are depicted in blue and green, respectively. A downstream shift in the $P$. aeruginosa $a m p R$ TSS is likely responsible for the change in sigma factor control observed here. Orange arrows denote palindromic sequences.

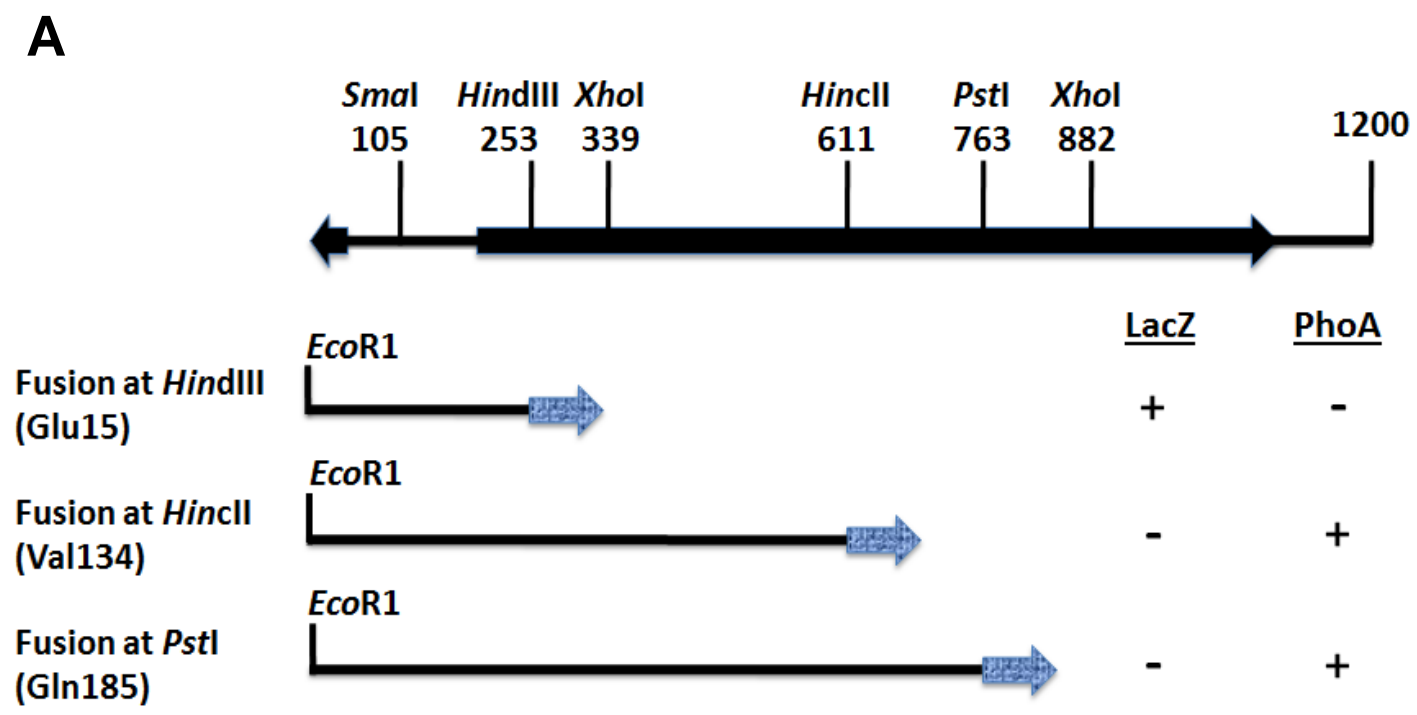




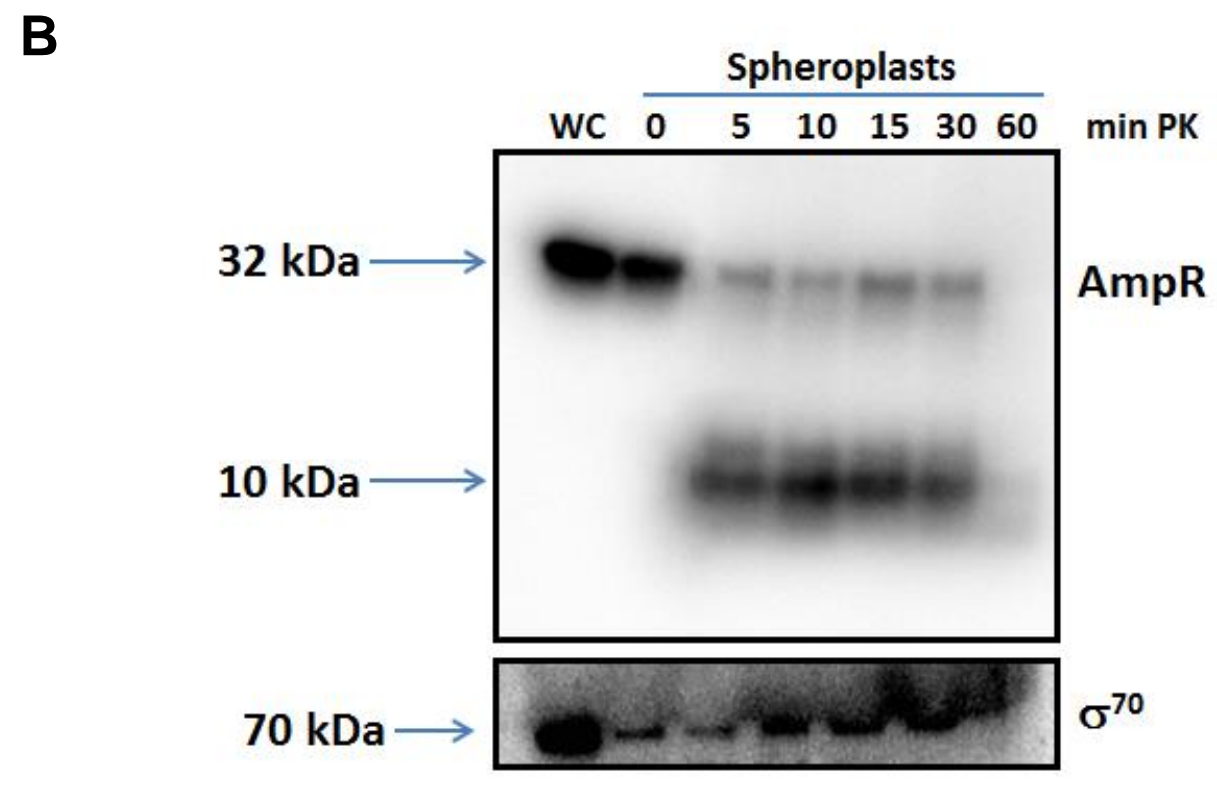

Figure S2. Localization studies of $P$. aeruginosa AmpR. (A) Restriction map and topology of AmpR. The topology of AmpR was investigated by introducing phoA and lacZ fusions at the restriction sites HindIII, HincII and PstI restriction sites corresponding to amino acid positions Glu15, Val134 and Gln185, respectively. Fusions at Glu15 were LacZ-positive but PhoA-negative, whereas fusions at Val134 and Gln185 were PhoApositive and LacZ-negative. (B) Spheroplasts of PA0 $\triangle a m p R$ (pAmpR-His ${ }_{6}$ ) were obtained and treated with $10 \mu \mathrm{g} / \mathrm{ml}$ of Proteinase $\mathrm{K}$ for varying lengths of time. AntiAmpR antibodies were used to monitor the presence of the full-length AmpR (32 kDA) and the shaved product $(10 \mathrm{kDa})$ by Western blotting. The membranes were stripped and re-probed with anti- $\sigma^{70}$ antibodies. 


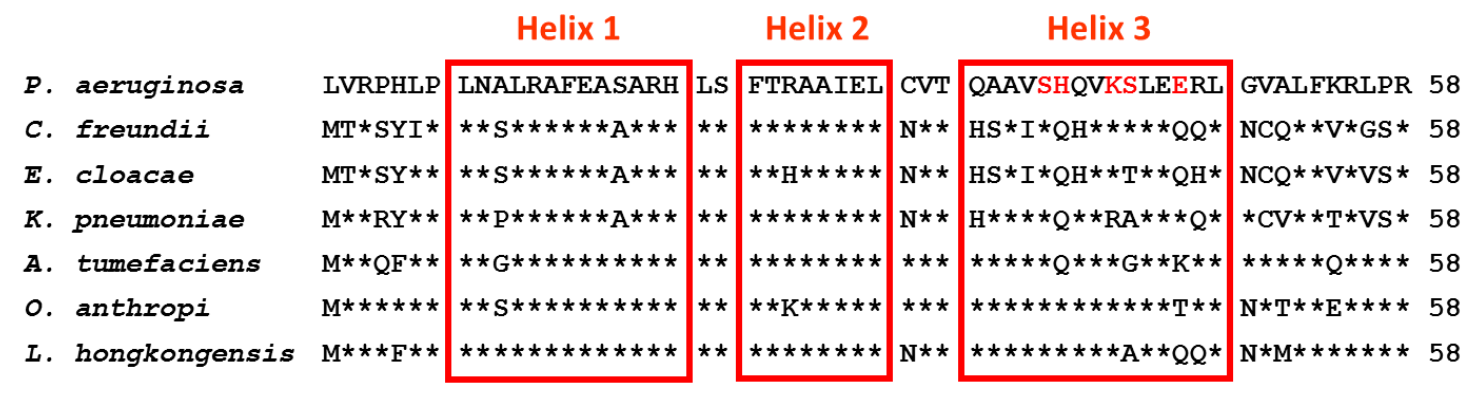

Figure S3. Multiple alignment of the AmpR HTH motif. N-terminal sequences from $P$. aeruginosa, C. freundii, E. cloacae, K. pneumonia, A. tumefaciens, $O$. anthropic, and $L$. hongkongensis AmpR were aligned using ClustalW2 (http://www.ebi.ac.uk/Tools/msa/clustalw2/). Asterisks denote conserved residues that do not deviate from those in the reference sequence of $P$. aeruginosa. Residues denoted in red were replaced with alanine in P. aeruginosa AmpR-His 6 by site-directed mutagenesis.

\section{SUPPLEMENTAL MATERIALS AND METHODS}

$\boldsymbol{\beta}$-lactamase assay. The $\beta$-lactamase assay was modified from a previously published protocol (765). Briefly, stationary-phase cultures were diluted in $30 \mathrm{ml}$ of LB broth to an $\mathrm{OD}_{600}$ of 0.02 and incubated with shaking at $37^{\circ} \mathrm{C}$. At an $\mathrm{OD}_{600}$ of 0.2 , cells containing the expression plasmid were induced with $1 \mathrm{mM}$ IPTG for an hour. At an $\mathrm{OD}_{600}$ of 0.6 , the remaining cultures were induced with $200 \mu \mathrm{g} \mathrm{ml}^{-1}$ of penicillin $\mathrm{G}$ for an hour before harvesting. Cells were harvested by centrifugation, resuspended in $1 \mathrm{ml}$ of $50 \mathrm{mM}$ of sodium phosphate buffer and sonicated on ice for $1 \mathrm{~min} 20 \mathrm{~s}(20$-sec pulse ON, 20-sec pulse OFF, amplitude $30 \%$ ). The sonicated cells were centrifuged at $10,000 \mathrm{X}$ g at $4^{\circ} \mathrm{C}$ for 30 minutes to collect the $\beta$-lactamase-containing supernatant. A $2-\mu 1$ aliquot of cell lysate is added to nitrocefin (final concentration, $100 \mu \mathrm{M}$ ) in $1 \mathrm{ml}$ of assay buffer. The reaction was incubated at $37^{\circ} \mathrm{C}$ for 20 minutes and the hydrolysis of nitrocefin was 
measured spectrophotometrically at $482 \mathrm{~nm}$. Total protein concentration was determined with Bradford on the same supernatant. The activity was expressed as nmol of nitrocefin degraded per minute per milligram of total protein.

Minimum Inhibitory concentration (MIC). The MIC was determined by the E-test system according to the manufacturer's instructions (bioMérieux, Marcy l'Etoile). 


\section{BIBLIOGRAPHY}

1. Kapatral V, A. Zago, S. Kamath, and S. Chugani. 2000. Pseudomonas. Encyclopedia of Microbiology, 2nd Edition ed. Academic Press, New York, NY.

2. Cox CD, Parker J. 1979. Use of 2-aminoacetophenone production in identification of Pseudomonas aeruginosa. J Clin Microbiol 9:479-484.

3. Palleroni NJ. 2001. Pseudomonadaceae. Bergey's Manual of Systemic Bacteriology, 6th Edition ed. William \& Wilkins, Baltimore, MD.

4. Vander Wauven C, Pierard A, Kley-Raymann M, Haas D. 1984. Pseudomonas aeruginosa mutants affected in anaerobic growth on arginine: evidence for a four-gene cluster encoding the arginine deiminase pathway. $\mathrm{J}$ Bacteriol 160:928-934.

5. Livermore DM. 2002. Multiple mechanisms of antimicrobial resistance in Pseudomonas aeruginosa: our worst nightmare? Clin Infect Dis 34:634-640.

6. Hassett DJ, Charniga L, Bean K, Ohman DE, Cohen MS. 1992. Response of Pseudomonas aeruginosa to pyocyanin: mechanisms of resistance, antioxidant defenses, and demonstration of a manganese-cofactored superoxide dismutase. Infect Immun 60:328-336.

7. Wilson R, Pitt T, Taylor G, Watson D, MacDermot J, Sykes D, Roberts D, Cole P. 1987. Pyocyanin and 1-hydroxyphenazine produced by Pseudomonas aeruginosa inhibit the beating of human respiratory cilia in vitro. J Clin Invest 79:221-229.

8. Stewart-Tull DE, Armstrong AV. 1972. The effect of 1-hydroxyphenazine and pyocyanin from Pseudomonas aeruginosa on mammalian cell respiration. J Med Microbiol 5:67-73.

9. Sorensen RU, Klinger JD, Cash HA, Chase PA, Dearborn DG. 1983. In vitro inhibition of lymphocyte proliferation by Pseudomonas aeruginosa phenazine pigments. Infect Immun 41:321-330.

10. Cruickshank CN, Lowbury EJ. 1953. The effect of pyocyanin on human skin cells and leucocytes. Br J Exp Pathol 34:583-587.

11. Cox CD, Adams P. 1985. Siderophore activity of pyoverdin for Pseudomonas aeruginosa. Infect Immun 48:130-138. 
12. Vasil ML. 1986. Pseudomonas aeruginosa: biology, mechanisms of virulence, epidemiology. J Pediatr 108:800-805.

13. Kanthakumar K, Taylor GW, Cundell DR, Dowling RB, Johnson M, Cole PJ, Wilson R. 1996. The effect of bacterial toxins on levels of intracellular adenosine nucleotides and human ciliary beat frequency. Pulm Pharmacol 9:223230.

14. McClure CD, Schiller NL. 1996. Inhibition of macrophage phagocytosis by Pseudomonas aeruginosa rhamnolipids in vitro and in vivo. Curr Microbiol 33:109-117.

15. Jensen PO, Bjarnsholt T, Phipps R, Rasmussen TB, Calum H, Christoffersen L, Moser C, Williams P, Pressler T, Givskov M, Hoiby N. 2007. Rapid necrotic killing of polymorphonuclear leukocytes is caused by quorum-sensingcontrolled production of rhamnolipid by Pseudomonas aeruginosa. Microbiology 153:1329-1338.

16. Zulianello L, Canard C, Kohler T, Caille D, Lacroix JS, Meda P. 2006. Rhamnolipids are virulence factors that promote early infiltration of primary human airway epithelia by Pseudomonas aeruginosa. Infect Immun 74:31343147.

17. Ito S, Honda H, Tomita F, Suzuki T. 1971. Rhamnolipids produced by Pseudomonas aeruginosa grown on n-paraffin (mixture of C 12, C 13 and C 14 fractions). J Antibiot (Tokyo) 24:855-859.

18. Haba E, Pinazo A, Jauregui O, Espuny MJ, Infante MR, Manresa A. 2003. Physicochemical characterization and antimicrobial properties of rhamnolipids produced by Pseudomonas aeruginosa 47T2 NCBIM 40044. Biotechnol Bioeng 81:316-322.

19. Gilligan PH. 1995. Pseudomonas and Burkholderia. Manual of Clinical Microbiology, 6th Edition ed. American Society for Microbiology, Washintgon, D.C.

20. Cogen AL, Nizet V, Gallo RL. 2008. Skin microbiota: a source of disease or defence? Br J Dermatol 158:442-455.

21. Gao Z, Tseng CH, Pei Z, Blaser MJ. 2007. Molecular analysis of human forearm superficial skin bacterial biota. Proc Natl Acad Sci U S A 104:29272932. 
22. Grice EA, Kong HH, Renaud G, Young AC, Program NCS, Bouffard GG, Blakesley RW, Wolfsberg TG, Turner ML, Segre JA. 2008. A diversity profile of the human skin microbiota. Genome Res 18:1043-1050.

23. Dekio I, Hayashi H, Sakamoto M, Kitahara M, Nishikawa T, Suematsu M, Benno Y. 2005. Detection of potentially novel bacterial components of the human skin microbiota using culture-independent molecular profiling. J Med Microbiol 54:1231-1238.

24. Stoodley BJ, Thom BT. 1970. Observations on the intestinal carriage of Pseudomonas aeruginosa. J Med Microbiol 3:367-375.

25. Hall JH, Callaway JL, Tindall JP, Smith JG, Jr. 1968. Pseudomonas aeruginosa in dermatology. Arch Dermatol 97:312-324.

26. Jacobson JA. 1985. Pool-associated Pseudomonas aeruginosa dermatitis and other bathing-associated infections. Infect Control 6:398-401.

27. Anaissie EJ, Penzak SR, Dignani MC. 2002. The hospital water supply as a source of nosocomial infections: a plea for action. Arch Intern Med 162:14831492.

28. Favero MS, Carson LA, Bond WW, Petersen NJ. 1971. Pseudomonas aeruginosa: growth in distilled water from hospitals. Science 173:836-838.

29. Bert F, Maubec E, Bruneau B, Berry P, Lambert-Zechovsky N. 1998. Multiresistant Pseudomonas aeruginosa outbreak associated with contaminated tap water in a neurosurgery intensive care unit. J Hosp Infect 39:53-62.

30. Grundmann H, Kropec A, Hartung D, Berner R, Daschner F. 1993. Pseudomonas aeruginosa in a neonatal intensive care unit: reservoirs and ecology of the nosocomial pathogen. J Infect Dis 168:943-947.

31. Ferroni A, Nguyen L, Pron B, Quesne G, Brusset MC, Berche P. 1998. Outbreak of nosocomial urinary tract infections due to Pseudomonas aeruginosa in a paediatric surgical unit associated with tap-water contamination. J Hosp Infect 39:301-307.

32. Trautmann M, Lepper PM, Haller M. 2005. Ecology of Pseudomonas aeruginosa in the intensive care unit and the evolving role of water outlets as a reservoir of the organism. Am J Infect Control 33:S41-49.

33. Rogues AM, Boulestreau H, Lasheras A, Boyer A, Gruson D, Merle C, Castaing Y, Bebear CM, Gachie JP. 2007. Contribution of tap water to patient 
colonisation with Pseudomonas aeruginosa in a medical intensive care unit. J Hosp Infect 67:72-78.

34. Doring G, Ulrich M, Muller W, Bitzer J, Schmidt-Koenig L, Munst L, Grupp H, Wolz C, Stern M, Botzenhart K. 1991. Generation of Pseudomonas aeruginosa aerosols during handwashing from contaminated sink drains, transmission to hands of hospital personnel, and its prevention by use of a new heating device. Zentralbl Hyg Umweltmed 191:494-505.

35. Ayliffe GA, Babb JR, Collins BJ, Lowbury EJ, Newsom SW. 1974. Pseudomonas aeruginosa in hospital sinks. Lancet 2:578-581.

36. Doring G, Jansen S, Noll H, Grupp H, Frank F, Botzenhart K, Magdorf K, Wahn U. 1996. Distribution and transmission of Pseudomonas aeruginosa and Burkholderia cepacia in a hospital ward. Pediatr Pulmonol 21:90-100.

37. Doring G, Horz M, Ortelt J, Grupp H, Wolz C. 1993. Molecular epidemiology of Pseudomonas aeruginosa in an intensive care unit. Epidemiol Infect 110:427436.

38. Fazeli H, Akbari R, Moghim S, Narimani T, Arabestani MR, Ghoddousi AR. 2012. Pseudomonas aeruginosa infections in patients, hospital means, and personnel's specimens. J Res Med Sci 17:332-337.

39. Kirschner AK, Atteneder M, Schmidhuber A, Knetsch S, Farnleitner AH, Sommer R. 2012. Holy springs and holy water: underestimated sources of illness? J Water Health 10:349-357.

40. Rees JC, Allen KD. 1996. Holy water-a risk factor for hospital-acquired infection. J Hosp Infect 32:51-55.

41. Kush BJ, Hoadley AW. 1980. A preliminary survey of the association of Pseudomonas aeruginosa with commercial whirlpool bath waters. Am J Public Health 70:279-281.

42. Hollyoak V, Boyd P, Freeman R. 1995. Whirlpool baths in nursing homes: use, maintenance, and contamination with Pseudomonas aeruginosa. Commun Dis Rep CDR Rev 5:R102-104.

43. Ratnam S, Hogan K, March SB, Butler RW. 1986. Whirlpool-associated folliculitis caused by Pseudomonas aeruginosa: report of an outbreak and review. J Clin Microbiol 23:655-659. 
44. Thomas P, Moore M, Bell E, Friedman S, Decker J, Shayegani M, Martin K. 1985. Pseudomonas dermatitis associated with a swimming pool. JAMA 253:1156-1159.

45. Hopkins RS, Abbott DO, Wallace LE. 1981. Follicular dermatitis outbreak caused by Pseudomonas aeruginosa associated with a motel's indoor swimming pool. Public Health Rep 96:246-249.

46. Jacobs RF, McCarthy RE, Elser JM. 1989. Pseudomonas osteochondritis complicating puncture wounds of the foot in children: a 10-year evaluation. $\mathbf{J}$ Infect Dis 160:657-661.

47. Fox AB, Hambrick GW, Jr. 1984. Recreationally associated Pseudomonas aeruginosa folliculitis. Report of an epidemic. Arch Dermatol 120:1304-1307.

48. Tate D, Mawer S, Newton A. 2003. Outbreak of Pseudomonas aeruginosa folliculitis associated with a swimming pool inflatable. Epidemiol Infect 130:187192.

49. Havelaar AH, Bosman M, Borst J. 1983. Otitis externa by Pseudomonas aeruginosa associated with whirlpools. J Hyg (Lond) 90:489-498.

50. Reid TM, Porter IA. 1981. An outbreak of otitis externa in competitive swimmers due to Pseudomonas aeruginosa. J Hyg (Lond) 86:357-362.

51. van Asperen IA, de Rover CM, Schijven JF, Oetomo SB, Schellekens JF, van Leeuwen NJ, Colle C, Havelaar AH, Kromhout D, Sprenger MW. 1995. Risk of otitis externa after swimming in recreational fresh water lakes containing Pseudomonas aeruginosa. BMJ 311:1407-1410.

52. Seyfried PL, Fraser DJ. 1978. Pseudomonas aeruginosa in swimming pools related to the incidence of otitis externa infection. Health Lab Sci 15:50-57.

53. Ramphal R, McNiece MT, Polack FM. 1981. Adherence of Pseudomonas aeruginosa to the injured cornea: a step in the pathogenesis of corneal infections. Ann Ophthalmol 13:421-425.

54. Stern GA. 1990. Pseudomonas keratitis and contact lens wear: the lens/eye is at fault. Cornea 9 Suppl 1:S36-38; discussion S39-40.

55. Klotz SA, Misra RP, Butrus SI. 1990. Contact lens wear enhances adherence of Pseudomonas aeruginosa and binding of lectins to the cornea. Cornea 9:266-270. 
56. Fletcher EL, Fleiszig SM, Brennan NA. 1993. Lipopolysaccharide in adherence of Pseudomonas aeruginosa to the cornea and contact lenses. Invest Ophthalmol Vis Sci 34:1930-1936.

57. Fleiszig SM, Efron N, Pier GB. 1992. Extended contact lens wear enhances Pseudomonas aeruginosa adherence to human corneal epithelium. Invest Ophthalmol Vis Sci 33:2908-2916.

58. Fisher MC, Goldsmith JF, Gilligan PH. 1985. Sneakers as a source of Pseudomonas aeruginosa in children with osteomyelitis following puncture wounds. J Pediatr 106:607-609.

59. Sapico FL, Montgomerie JZ. 1980. Vertebral osteomyelitis in intravenous drug abusers: report of three cases and review of the literature. Rev Infect Dis 2:196206.

60. Wiesseman GJ, Wood VE, Kroll LL, Linda L. 1973. Pseudomonas vertebral osteomyelitis in heroin addicts. Report of five cases. J Bone Joint Surg Am 55:1416-1424.

61. Shekar R, Rice TW, Zierdt CH, Kallick CA. 1985. Outbreak of endocarditis caused by Pseudomonas aeruginosa serotype O11 among pentazocine and tripelennamine abusers in Chicago. J Infect Dis 151:203-208.

62. Saroff AL, Armstrong D, Johnson WD. 1973. Pseudomonas endocarditis. Am J Cardiol 32:234-237.

63. Archer G, Fekety FR, Supena R. 1974. Pseudomonas aeruginosa endocarditis in drug addicts. Am Heart J 88:570-578.

64. Laguno M, Miro O, Font C, de la Sierra A. 1998. Pacemaker-related endocarditis. Report of 7 cases and review of the literature. Cardiology 90:244248.

65. Regules JA, Glasser JS, Wolf SE, Hospenthal DR, Murray CK. 2008. Endocarditis in burn patients: clinical and diagnostic considerations. Burns 34:610-616.

66. Molina DN, Colon M, Bermudez RH, Ramirez-Ronda CH. 1991. Unusual presentation of Pseudomonas aeruginosa infections: a review. Bol Asoc Med P R 83:160-163. 
67. Pittet D, Harbarth S, Ruef C, Francioli P, Sudre P, Petignat C, Trampuz A, Widmer A. 1999. Prevalence and risk factors for nosocomial infections in four university hospitals in Switzerland. Infect Control Hosp Epidemiol 20:37-42.

68. Kim JM, Park ES, Jeong JS, Kim KM, Kim JM, Oh HS, Yoon SW, Chang HS, Chang KH, Lee SI, Lee MS, Song JH, Kang MW, Park SC, Choe KW, Pai CH. 2000. Multicenter surveillance study for nosocomial infections in major hospitals in Korea. Nosocomial Infection Surveillance Committee of the Korean Society for Nosocomial Infection Control. Am J Infect Control 28:454-458.

69. Lizioli A, Privitera G, Alliata E, Antonietta Banfi EM, Boselli L, Panceri ML, Perna MC, Porretta AD, Santini MG, Carreri V. 2003. Prevalence of nosocomial infections in Italy: result from the Lombardy survey in 2000. J Hosp Infect 54:141-148.

70. $\quad$ Erbay H, Yalcin AN, Serin S, Turgut H, Tomatir E, Cetin B, Zencir M. 2003. Nosocomial infections in intensive care unit in a Turkish university hospital: a 2year survey. Intensive Care Med 29:1482-1488.

71. Richards MJ, Edwards JR, Culver DH, Gaynes RP. 1999. Nosocomial infections in medical intensive care units in the United States. National Nosocomial Infections Surveillance System. Crit Care Med 27:887-892.

72. Magill SS, Edwards JR, Bamberg W, Beldavs ZG, Dumyati G, Kainer MA, Lynfield R, Maloney M, McAllister-Hollod L, Nadle J, Ray SM, Thompson DL, Wilson LE, Fridkin SK, Emerging Infections Program HealthcareAssociated I, Antimicrobial Use Prevalence Survey T. 2014. Multistate pointprevalence survey of health care-associated infections. N Engl J Med 370:11981208.

73. Sievert DM, Ricks P, Edwards JR, Schneider A, Patel J, Srinivasan A, Kallen A, Limbago B, Fridkin S, National Healthcare Safety Network T, Participating NF. 2013. Antimicrobial-resistant pathogens associated with healthcare-associated infections: summary of data reported to the National Healthcare Safety Network at the Centers for Disease Control and Prevention, 2009-2010. Infect Control Hosp Epidemiol 34:1-14.

74. Lynch JP, 3rd. 2001. Hospital-acquired pneumonia: risk factors, microbiology, and treatment. Chest 119:373S-384S.

75. Kollef MH, Shorr A, Tabak YP, Gupta V, Liu LZ, Johannes RS. 2005. Epidemiology and outcomes of health-care-associated pneumonia: results from a large US database of culture-positive pneumonia. Chest 128:3854-3862. 
76. Chastre J, Fagon JY. 2002. Ventilator-associated pneumonia. Am J Respir Crit Care Med 165:867-903.

77. Fagon JY, Chastre J, Domart Y, Trouillet JL, Gibert C. 1996. Mortality due to ventilator-associated pneumonia or colonization with Pseudomonas or Acinetobacter species: assessment by quantitative culture of samples obtained by a protected specimen brush. Clin Infect Dis 23:538-542.

78. Kollef MH, Silver P, Murphy DM, Trovillion E. 1995. The effect of late-onset ventilator-associated pneumonia in determining patient mortality. Chest 108:1655-1662.

79. Shigemura K, Arakawa S, Sakai Y, Kinoshita S, Tanaka K, Fujisawa M. 2006. Complicated urinary tract infection caused by Pseudomonas aeruginosa in a single institution (1999-2003). Int J Urol 13:538-542.

80. Askarian M, Hosseini RS, Kheirandish P, Assadian O. 2004. Incidence and outcome of nosocomial infections in female burn patients in Shiraz, Iran. Am J Infect Control 32:23-26.

81. Geyik MF, Aldemir M, Hosoglu S, Tacyildiz HI. 2003. Epidemiology of burn unit infections in children. Am J Infect Control 31:342-346.

82. Lari AR, Alaghehbandan R. 2000. Nosocomial infections in an Iranian burn care center. Burns 26:737-740.

83. Yildirim S, Nursal TZ, Tarim A, Torer N, Noyan T, Demiroglu YZ, Moray G, Haberal M. 2005. Bacteriological profile and antibiotic resistance: comparison of findings in a burn intensive care unit, other intensive care units, and the hospital services unit of a single center. J Burn Care Rehabil 26:488-492.

84. Song W, Lee KM, Kang HJ, Shin DH, Kim DK. 2001. Microbiologic aspects of predominant bacteria isolated from the burn patients in Korea. Burns 27:136139.

85. de Macedo JL, Santos JB. 2005. Bacterial and fungal colonization of burn wounds. Mem Inst Oswaldo Cruz 100:535-539.

86. Erol S, Altoparlak U, Akcay MN, Celebi F, Parlak M. 2004. Changes of microbial flora and wound colonization in burned patients. Burns 30:357-361.

87. McManus AT, Mason AD, Jr., McManus WF, Pruitt BA, Jr. 1985. Twentyfive year review of Pseudomonas aeruginosa bacteremia in a burn center. Eur $\mathbf{J}$ Clin Microbiol 4:219-223. 
88. Osmon S, Ward S, Fraser VJ, Kollef MH. 2004. Hospital mortality for patients with bacteremia due to Staphylococcus aureus or Pseudomonas aeruginosa. Chest 125:607-616.

89. Bodey GP, Bolivar R, Fainstein V, Jadeja L. 1983. Infections caused by Pseudomonas aeruginosa. Rev Infect Dis 5:279-313.

90. Lee WI, Kuo ML, Huang JL, Lin SJ, Wu CJ. 2005. Distribution and clinical aspects of primary immunodeficiencies in a Taiwan pediatric tertiary hospital during a 20-year period. J Clin Immunol 25:162-173.

91. Lee WI, Jaing TH, Hsieh MY, Kuo ML, Lin SJ, Huang JL. 2006. Distribution, infections, treatments and molecular analysis in a large cohort of patients with primary immunodeficiency diseases (PIDs) in Taiwan. J Clin Immunol 26:274283.

92. Chatzinikolaou I, Abi-Said D, Bodey GP, Rolston KV, Tarrand JJ, Samonis G. 2000. Recent experience with Pseudomonas aeruginosa bacteremia in patients with cancer: Retrospective analysis of 245 episodes. Arch Intern Med 160:501509.

93. Funada H, Matsuda T. 1998. Changes in the incidence and etiological patterns of bacteremia associated with acute leukemia over a 25-year period. Intern Med 37:1014-1018.

94. Vidal F, Mensa J, Martinez JA, Almela M, Marco F, Gatell JM, Richart C, Soriano E, Jimenez de Anta MT. 1999. Pseudomonas aeruginosa bacteremia in patients infected with human immunodeficiency virus type 1. Eur J Clin Microbiol Infect Dis 18:473-477.

95. Kiehn TE. 1989. Bacteremia and fungemia in the immunocompromised patient. Eur J Clin Microbiol Infect Dis 8:832-837.

96. Kielhofner M, Atmar RL, Hamill RJ, Musher DM. 1992. Life-threatening Pseudomonas aeruginosa infections in patients with human immunodeficiency virus infection. Clin Infect Dis 14:403-411.

97. Kramer MR, Marshall SE, Starnes VA, Gamberg P, Amitai Z, Theodore J. 1993. Infectious complications in heart-lung transplantation. Analysis of 200 episodes. Arch Intern Med 153:2010-2016.

98. Bodey GP, Jadeja L, Elting L. 1985. Pseudomonas bacteremia. Retrospective analysis of 410 episodes. Arch Intern Med 145:1621-1629. 
99. Rolston KV, Bodey GP. 1992. Pseudomonas aeruginosa infection in cancer patients. Cancer Invest 10:43-59.

100. Mendelson MH, Gurtman A, Szabo S, Neibart E, Meyers BR, Policar M, Cheung TW, Lillienfeld D, Hammer G, Reddy S, et al. 1994. Pseudomonas aeruginosa bacteremia in patients with AIDS. Clin Infect Dis 18:886-895.

101. Flores G, Stavola JJ, Noel GJ. 1993. Bacteremia due to Pseudomonas aeruginosa in children with AIDS. Clin Infect Dis 16:706-708.

102. Eng RH, Bishburg E, Smith SM, Geller H, Kapila R. 1986. Bacteremia and fungemia in patients with acquired immune deficiency syndrome. Am J Clin Pathol 86:105-107.

103. Afessa B, Green B. 2000. Bacterial pneumonia in hospitalized patients with HIV infection: the Pulmonary Complications, ICU Support, and Prognostic Factors of Hospitalized Patients with HIV (PIP) Study. Chest 117:1017-1022.

104. Afessa B, Green W, Chiao J, Frederick W. 1998. Pulmonary complications of HIV infection: autopsy findings. Chest 113:1225-1229.

105. Taylor IK, Coker RJ, Clarke J, Moss FM, Nieman R, Evans DJ, Veale D, Shaw RJ, Robinson DS, Mitchell DM. 1995. Pulmonary complications of HIV disease: 10 year retrospective evaluation of yields from bronchoalveolar lavage, 1983-93. Thorax 50:1240-1245.

106. Franzetti F, Grassini A, Piazza M, Degl'innocenti M, Bandera A, Gazzola L, Marchetti G, Gori A. 2006. Nosocomial bacterial pneumonia in HIV-infected patients: risk factors for adverse outcome and implications for rational empiric antibiotic therapy. Infection 34:9-16.

107. Traill ZC, Miller RF, Ali N, Shaw PJ. 1996. Pseudomonas aeruginosa bronchopulmonary infection in patients with advanced human immunodeficiency virus disease. Br J Radiol 69:1099-1103.

108. Busi Rizzi E, Schinina V, Bordi E, Buontempo G, Narciso P, Bibbolino C. 2006. HIV-related bronchopulmonary infection by Pseudomonas aeruginosa in the HAART era: radiological findings. Acta Radiol 47:793-797.

109. Asboe D, Gant V, Aucken HM, Moore DA, Umasankar S, Bingham JS, Kaufmann ME, Pitt TL. 1998. Persistence of Pseudomonas aeruginosa strains in respiratory infection in AIDS patients. AIDS 12:1771-1775. 
110. Domingo P, Ferre A, Baraldes MA, Ris J, Sanchez F. 1998. Pseudomonas aeruginosa bronchopulmonary infection in patients with AIDS, with emphasis on relapsing infection. Eur Respir J 12:107-112.

111. Ali NJ, Kessel D, Miller RF. 1995. Bronchopulmonary infection with Pseudomonas aeruginosa in patients infected with human immunodeficiency virus. Genitourin Med 71:73-77.

112. Hersh EM, Bodey GP, Nies BA, Freireich E. 1965. Causes of death in acute leukemia: A ten-year study of 414 patients from 1954-1963. JAMA 193:105-109.

113. Curtin JA, Petersdorf RG, Bennett JIL. 1961. Pseudomonas bacteremia: Review of ninety-one cases. Ann Intern Med 54:1077-1107.

114. Forkner Jr CE, Frei Iii E, Edgcomb JH, Utz JP. 1958. Pseudomonas septicemia: Observations on twenty-three cases. Am J Med 25:877-889.

115. Chang HY, Rodriguez V, Narboni G, Bodey GP, Luna MA, Freireich EJ. 1976. Causes of death in adults with acute leukemia. Medicine (Baltimore) 55:259-268.

116. Schimpff SC, Greene WH, Young VM, Wiernik PH. 1974. Significance of Pseudomonas aeruginosa in the patient with leukemia or lymphoma. J Infect Dis 130 Suppl:S24-31.

117. Tapper ML, Armstrong D. 1974. Bacteremia due to Pseudomonas aeruginosa complicating neoplastic disease: a progress report. J Infect Dis 130 Suppl:S14-23.

118. Maschmeyer G, Braveny I. 2000. Review of the incidence and prognosis of Pseudomonas aeruginosa infections in cancer patients in the 1990s. Eur J Clin Microbiol Infect Dis 19:915-925.

119. Varaiya A, Kulkarni M, Bhalekar P, Dogra J. 2008. Incidence of carbapenemresistant Pseudomonas aeruginosa in diabetes and cancer patients. Indian J Med Microbiol 26:238-240.

120. Marin M, Gudiol C, Ardanuy C, Garcia-Vidal C, Calvo M, Arnan M, Carratala J. 2014. Bloodstream infections in neutropenic patients with cancer: Differences between patients with haematological malignancies and solid tumours. J Infect 69:417-423.

121. Saiman L, and J. Siegel. 2004. Infection control in cystic fibrosis. Clin Microbiol Rev 17:51-71. 
122. Foundation CF. 2012. Cystic Fibrosis Foundation patient registry 2012. Cystic Fibrosis Foundation, Bethesda, Maryland.

123. Lyczak JB, Cannon CL, Pier GB. 2002. Lung infections associated with cystic fibrosis. Clin Microbiol Rev 15:194-222.

124. Emerson J, Rosenfeld M, McNamara S, Ramsey B, Gibson RL. 2002. Pseudomonas aeruginosa and other predictors of mortality and morbidity in young children with cystic fibrosis. Pediatr Pulmonol 34:91-100.

125. Kosorok MR, Zeng L, West SE, Rock MJ, Splaingard ML, Laxova A, Green CG, Collins J, Farrell PM. 2001. Acceleration of lung disease in children with cystic fibrosis after Pseudomonas aeruginosa acquisition. Pediatr Pulmonol 32:277-287.

126. Gaspar MC, Couet W, Olivier JC, Pais AA, Sousa JJ. 2013. Pseudomonas aeruginosa infection in cystic fibrosis lung disease and new perspectives of treatment: a review. Eur J Clin Microbiol Infect Dis 32:1231-1252.

127. Kerem B, Rommens JM, Buchanan JA, Markiewicz D, Cox TK, Chakravarti A, Buchwald M, Tsui LC. 1989. Identification of the cystic fibrosis gene: genetic analysis. Science 245:1073-1080.

128. Riordan JR, Rommens JM, Kerem B, Alon N, Rozmahel R, Grzelczak Z, Zielenski J, Lok S, Plavsic N, Chou JL, et al. 1989. Identification of the cystic fibrosis gene: cloning and characterization of complementary DNA. Science 245:1066-1073.

129. Rommens JM, Iannuzzi MC, Kerem B, Drumm ML, Melmer G, Dean M, Rozmahel R, Cole JL, Kennedy D, Hidaka N, et al. 1989. Identification of the cystic fibrosis gene: chromosome walking and jumping. Science 245:1059-1065.

130. Anderson MP, Gregory RJ, Thompson S, Souza DW, Paul S, Mulligan RC, Smith AE, Welsh MJ. 1991. Demonstration that CFTR is a chloride channel by alteration of its anion selectivity. Science 253:202-205.

131. Bear CE, Li CH, Kartner N, Bridges RJ, Jensen TJ, Ramjeesingh M, Riordan JR. 1992. Purification and functional reconstitution of the cystic fibrosis transmembrane conductance regulator (CFTR). Cell 68:809-818.

132. Howell LD, Borchardt R, Kole J, Kaz AM, Randak C, Cohn JA. 2004. Protein kinase A regulates ATP hydrolysis and dimerization by a CFTR (cystic fibrosis transmembrane conductance regulator) domain. Biochem J 378:151-159. 
133. Berger HA, Anderson MP, Gregory RJ, Thompson S, Howard PW, Maurer RA, Mulligan R, Smith AE, Welsh MJ. 1991. Identification and regulation of the cystic fibrosis transmembrane conductance regulator-generated chloride channel. J Clin Invest 88:1422-1431.

134. Cheng SH, Rich DP, Marshall J, Gregory RJ, Welsh MJ, Smith AE. 1991. Phosphorylation of the R domain by cAMP-dependent protein kinase regulates the CFTR chloride channel. Cell 66:1027-1036.

135. Denning GM, Ostedgaard LS, Cheng SH, Smith AE, Welsh MJ. 1992. Localization of cystic fibrosis transmembrane conductance regulator in chloride secretory epithelia. J Clin Invest 89:339-349.

136. Anderson MP, Berger HA, Rich DP, Gregory RJ, Smith AE, Welsh MJ. 1991. Nucleoside triphosphates are required to open the CFTR chloride channel. Cell 67:775-784.

137. Jovov B, Ismailov, II, Benos DJ. 1995. Cystic fibrosis transmembrane conductance regulator is required for protein kinase A activation of an outwardly rectified anion channel purified from bovine tracheal epithelia. J Biol Chem 270:1521-1528.

138. Jovov B, Ismailov, II, Berdiev BK, Fuller CM, Sorscher EJ, Dedman JR, Kaetzel MA, Benos DJ. 1995. Interaction between cystic fibrosis transmembrane conductance regulator and outwardly rectified chloride channels. J Biol Chem 270:29194-29200.

139. Schwiebert EM, Egan ME, Hwang TH, Fulmer SB, Allen SS, Cutting GR, Guggino WB. 1995. CFTR regulates outwardly rectifying chloride channels through an autocrine mechanism involving ATP. Cell 81:1063-1073.

140. Reddy MM, Light MJ, Quinton PM. 1999. Activation of the epithelial $\mathrm{Na}^{+}$ channel $(\mathrm{ENaC})$ requires $\mathrm{CFTR} \mathrm{Cl}^{-}$channel function. Nature 402:301-304.

141. Reddy MM, Quinton PM. 2005. ENaC activity requires CFTR channel function independently of phosphorylation in sweat duct. J Membr Biol 207:23-33.

142. Stutts MJ, Canessa CM, Olsen JC, Hamrick M, Cohn JA, Rossier BC, Boucher RC. 1995. CFTR as a cAMP-dependent regulator of sodium channels. Science 269:847-850.

143. Reddy MM, Quinton PM. 2003. Functional interaction of CFTR and ENaC in sweat glands. Pflugers Arch 445:499-503. 
144. Bobadilla JL, Macek M, Jr., Fine JP, Farrell PM. 2002. Cystic fibrosis: a worldwide analysis of CFTR mutations--correlation with incidence data and application to screening. Hum Mutat 19:575-606.

145. McKone EF, Emerson SS, Edwards KL, Aitken ML. 2003. Effect of genotype on phenotype and mortality in cystic fibrosis: a retrospective cohort study. Lancet 361:1671-1676.

146. McKone EF, Goss CH, Aitken ML. 2006. CFTR genotype as a predictor of prognosis in cystic fibrosis. Chest 130:1441-1447.

147. Kerem E, Corey M, Kerem BS, Rommens J, Markiewicz D, Levison H, Tsui LC, Durie P. 1990. The relation between genotype and phenotype in cystic fibrosis- analysis of the most common mutation $\left(\Delta \mathrm{F}_{508}\right)$. N Engl J Med 323:15171522.

148. Gan KH, Veeze HJ, van den Ouweland AM, Halley DJ, Scheffer H, van der Hout A, Overbeek SE, de Jongste JC, Bakker W, Heijerman HG. 1995. A cystic fibrosis mutation associated with mild lung disease. N Engl J Med 333:9599.

149. Gaskin K, Gurwitz D, Durie P, Corey M, Levison H, Forstner G. 1982. Improved respiratory prognosis in patients with cystic fibrosis with normal fat absorption. J Pediatr 100:857-862.

150. Wilschanski M, Zielenski J, Markiewicz D, Tsui LC, Corey M, Levison H, Durie PR. 1995. Correlation of sweat chloride concentration with classes of the cystic fibrosis transmembrane conductance regulator gene mutations. J Pediatr 127:705-710.

151. Welsh MJ, Smith AE. 1993. Molecular mechanisms of CFTR chloride channel dysfunction in cystic fibrosis. Cell 73:1251-1254.

152. Haardt M, Benharouga M, Lechardeur D, Kartner N, Lukacs GL. 1999. Cterminal truncations destabilize the cystic fibrosis transmembrane conductance regulator without impairing its biogenesis. A novel class of mutation. J Biol Chem 274:21873-21877.

153. Rowe SM, Miller S, Sorscher EJ. 2005. Mechanisms underlying cystic fibrosis. N Engl J Med 352:1992-2001.

154. Robinson M, Bye PT. 2002. Mucociliary clearance in cystic fibrosis. Pediatr Pulmonol 33:293-306. 
155. Robinson M, Eberl S, Tomlinson C, Daviskas E, Regnis JA, Bailey DL, Torzillo PJ, Menache M, Bye PT. 2000. Regional mucociliary clearance in patients with cystic fibrosis. J Aerosol Med 13:73-86.

156. Mall MA. 2008. Role of cilia, mucus, and airway surface liquid in mucociliary dysfunction: lessons from mouse models. J Aerosol Med Pulm Drug Deliv 21:1324.

157. Newhouse MT, Rossman CM, Dolovich J, Dolovich MB, Wilson WM. 1976. Impairment of mucociliary transport in cystic fibrosis. Mod Probl Paediatr 19:190-198.

158. Regnis JA, Robinson M, Bailey DL, Cook P, Hooper P, Chan HK, Gonda I, Bautovich G, Bye PT. 1994. Mucociliary clearance in patients with cystic fibrosis and in normal subjects. Am J Respir Crit Care Med 150:66-71.

159. Rutland J, Cole PJ. 1981. Nasal mucociliary clearance and ciliary beat frequency in cystic fibrosis compared with sinusitis and bronchiectasis. Thorax 36:654-658.

160. Knowles MR, Boucher RC. 2002. Mucus clearance as a primary innate defense mechanism for mammalian airways. J Clin Invest 109:571-577.

161. Wanner A, Salathe M, O'Riordan TG. 1996. Mucociliary clearance in the airways. Am J Respir Crit Care Med 154:1868-1902.

162. Chilvers MA, O'Callaghan C. 2000. Local mucociliary defence mechanisms. Paediatr Respir Rev 1:27-34.

163. Serafini SM, Michaelson ED. 1977. Length and distribution of cilia in human and canine airways. Bull Eur Physiopathol Respir 13:551-559.

164. Button B, Cai LH, Ehre C, Kesimer M, Hill DB, Sheehan JK, Boucher RC, Rubinstein M. 2012. A periciliary brush promotes the lung health by separating the mucus layer from airway epithelia. Science 337:937-941.

165. Widdicombe JH. 2002. Regulation of the depth and composition of airway surface liquid. J Anat 201:313-318.

166. Stannard W, O'Callaghan C. 2006. Ciliary function and the role of cilia in clearance. J Aerosol Med 19:110-115.

167. Fahy JV, Dickey BF. 2010. Airway mucus function and dysfunction. N Engl J Med 363:2233-2247. 
168. Matsui H, Randell SH, Peretti SW, Davis CW, Boucher RC. 1998. Coordinated clearance of periciliary liquid and mucus from airway surfaces. J Clin Invest 102:1125-1131.

169. Sleigh MA, Blake JR, Liron N. 1988. The propulsion of mucus by cilia. Am Rev Respir Dis 137:726-741.

170. Boucher RC. 2007. Cystic fibrosis: a disease of vulnerability to airway surface dehydration. Trends Mol Med 13:231-240.

171. Mall M, Grubb BR, Harkema JR, O'Neal WK, Boucher RC. 2004. Increased airway epithelial $\mathrm{Na}+$ absorption produces cystic fibrosis-like lung disease in mice. Nat Med 10:487-493.

172. Livraghi-Butrico A, Kelly EJ, Wilkinson KJ, Rogers TD, Gilmore RC, Harkema JR, Randell SH, Boucher RC, O'Neal WK, Grubb BR. 2013. Loss of CFTR function exacerbates the phenotype of $\mathrm{Na}(+)$ hyperabsorption in murine airways. Am J Physiol Lung Cell Mol Physiol 304:L469-480.

173. Zhou Z, Duerr J, Johannesson B, Schubert SC, Treis D, Harm M, Graeber SY, Dalpke A, Schultz C, Mall MA. 2011. The ENaC-overexpressing mouse as a model of cystic fibrosis lung disease. J Cyst Fibros 10 Suppl 2:S172-182.

174. Matsui H, Grubb BR, Tarran R, Randell SH, Gatzy JT, Davis CW, Boucher RC. 1998. Evidence for periciliary liquid layer depletion, not abnormal ion composition, in the pathogenesis of cystic fibrosis airways disease. Cell 95:10051015.

175. Tarran R, Grubb BR, Parsons D, Picher M, Hirsh AJ, Davis CW, Boucher RC. 2001. The CF salt controversy: in vivo observations and therapeutic approaches. Mol Cell 8:149-158.

176. Mall MA, Graeber SY, Stahl M, Zhou-Suckow Z. 2014. Early cystic fibrosis lung disease: Role of airway surface dehydration and lessons from preventive rehydration therapies in mice. Int J Biochem Cell Biol.

177. Treacy K, Tunney M, Elborn JS, Bradley JM. 2011. Mucociliary clearance in cystic fibrosis: physiology and pharmacological treatments. Paediatr Child Health 21:425-430.

178. Andersen DH. 1938. Cystic fibrosis of the pancreas and its relation to celiac disease: a clinical and pathologic study. Am J Dis Child 56:344-399. 
179. Lowe CU, May CD, Reed SC. 1949. Fibrosis of the pancreas in infants and children; a statistical study of clinical and hereditary features. Am J Dis Child 78:349-374.

180. Williams SG, Westaby D, Tanner MS, Mowat AP. 1992. Liver and biliary problems in cystic fibrosis. Br Med Bull 48:877-892.

181. Feranchak AP. 2004. Hepatobiliary complications of cystic fibrosis. Curr Gastroenterol Rep 6:231-239.

182. Gross K, Desanto A, Grosfeld JL, West KW, Eigen H. 1985. Intra-abdominal complications of cystic fibrosis. J Pediatr Surg 20:431-435.

183. Chillon M, Casals T, Mercier B, Bassas L, Lissens W, Silber S, Romey MC, Ruiz-Romero J, Verlingue C, Claustres M, et al. 1995. Mutations in the cystic fibrosis gene in patients with congenital absence of the vas deferens. N Engl $\mathbf{J}$ Med 332:1475-1480.

184. De Braekeleer M, Ferec C. 1996. Mutations in the cystic fibrosis gene in men with congenital bilateral absence of the vas deferens. Mol Hum Reprod 2:669677.

185. Mushtaq I, Wright VM, Drake DP, Mearns MB, Wood CB. 1998. Meconium ileus secondary to cystic fibrosis. The East London experience. Pediatr Surg Int 13:365-369.

186. Lissens W, Mercier B, Tournaye H, Bonduelle M, Ferec C, Seneca S, Devroey P, Silber S, Van Steirteghem A, Liebaers I. 1996. Cystic fibrosis and infertility caused by congenital bilateral absence of the vas deferens and related clinical entities. Hum Reprod 11 Suppl 4:55-78; discussion 79-80.

187. Phillipson G. 1998. Cystic fibrosis and reproduction. Reprod Fertil Dev 10:113119.

188. Kaplan E, Shwachman H, Perlmutter AD, Rule A, Khaw KT, Holsclaw DS. 1968. Reproductive failure in males with cystic fibrosis. N Engl J Med 279:65-69.

189. Gibson RL, Burns JL, Ramsey BW. 2003. Pathophysiology and management of pulmonary infections in cystic fibrosis. Am J Respir Crit Care Med 168:918-951.

190. Doud MS. 2010. A multi-faceted diagnostic approach to lung infections in patients with cystic fibrosis. Doctoral dissertation. Florida International University, Miami, Fl. 
191. Bakare N, Rickerts V, Bargon J, Just-Nubling G. 2003. Prevalence of Aspergillus fumigatus and other fungal species in the sputum of adult patients with cystic fibrosis. Mycoses 46:19-23.

192. Horre R, Marklein G, Siekmeier R, Nidermajer S, Reiffert SM. 2009. Selective isolation of Pseudallescheria and Scedosporium species from respiratory tract specimens of cystic fibrosis patients. Respiration 77:320-324.

193. Haase G, Skopnik H, Groten T, Kusenbach G, Posselt HG. 1991. Long-term fungal cultures from sputum of patients with cystic fibrosis. Mycoses 34:373-376.

194. Burns JL, Gibson RL, McNamara S, Yim D, Emerson J, Rosenfeld M, Hiatt P, McCoy K, Castile R, Smith AL, Ramsey BW. 2001. Longitudinal assessment of Pseudomonas aeruginosa in young children with cystic fibrosis. J Infect Dis 183:444-452.

195. Li Z, Kosorok MR, Farrell PM, Laxova A, West SE, Green CG, Collins J, Rock MJ, Splaingard ML. 2005. Longitudinal development of mucoid Pseudomonas aeruginosa infection and lung disease progression in children with cystic fibrosis. JAMA 293:581-588.

196. Mathee K, Ciofu O, Sternberg C, Lindum PW, Campbell JI, Jensen P, Johnsen AH, Givskov M, Ohman DE, Molin S, Hoiby N, Kharazmi A. 1999. Mucoid conversion of Pseudomonas aeruginosa by hydrogen peroxide: a mechanism for virulence activation in the cystic fibrosis lung. Microbiology 145 ( Pt 6):1349-1357.

197. Parad RB, Gerard CJ, Zurakowski D, Nichols DP, Pier GB. 1999. Pulmonary outcome in cystic fibrosis is influenced primarily by mucoid Pseudomonas aeruginosa infection and immune status and only modestly by genotype. Infect Immun 67:4744-4750.

198. Pedersen SS. 1992. Lung infection with alginate-producing, mucoid Pseudomonas aeruginosa in cystic fibrosis. APMIS Suppl 28:1-79.

199. Pedersen SS, Hoiby N, Espersen F, Koch C. 1992. Role of alginate in infection with mucoid Pseudomonas aeruginosa in cystic fibrosis. Thorax 47:6-13.

200. Donlan RM, Costerton JW. 2002. Biofilms: survival mechanisms of clinically relevant microorganisms. Clin Microbiol Rev 15:167-193.

201. Kanj SS, Kanafani ZA. 2011. Current concepts in antimicrobial therapy against resistant Gram-negative organisms: extended-spectrum $\beta$-lactamase-producing 
Enterobacteriaceae, carbapenem-resistant Enterobacteriaceae, and multidrugresistant Pseudomonas aeruginosa. Mayo Clin Proc 86:250-259.

202. Driscoll JA, Brody SL, Kollef MH. 2007. The epidemiology, pathogenesis and treatment of Pseudomonas aeruginosa infections. Drugs 67:351-368.

203. Nys PS, Kurochkina VB, Skliarenko AV, Veinberg GA. 2000. $\beta$-Lactams. Structure and biological activity relationship. Antibiot Khimioter 45:36-42.

204. Manhas MS, Bose AK. 1971. $\beta$-lactams: natural and synthetic. John Wiley \& Sons, Inc. New York, N.Y.

205. Hamilton-Miller JM. 1999. $\beta$-lactams: variations on a chemical theme, with some surprising biological results. J Antimicrob Chemother 44:729-734.

206. Demain AL, Elander RP. 1999. The $\beta$-lactam antibiotics: past, present, and future. Antonie Van Leeuwenhoek 75:5-19.

207. Breilh D, Texier-Maugein J, Allaouchiche B, Saux MC, Boselli E. 2013. Carbapenems. J Chemother 25:1-17.

208. Zhanel GG, Wiebe R, Dilay L, Thomson K, Rubinstein E, Hoban DJ, Noreddin AM, Karlowsky JA. 2007. Comparative review of the carbapenems. Drugs 67:1027-1052.

209. Johnson DH, Cunha BA. 1995. Aztreonam. Med Clin North Am 79:733-743.

210. Westley-Horton E, Koestner JA. 1991. Aztreonam: a review of the first monobactam. Am J Med Sci 302:46-49.

211. Gonzalez LS, 3rd, Spencer JP. 1998. Aminoglycosides: a practical review. Am Fam Physician 58:1811-1820.

212. Kothari U, Krilov LR. 2012. Aminoglycosides. Pediatr Rev 33:531-532; discussion 533.

213. Weinstein MJ, Luedemann GM, Oden EM, Wagman GH. 1963. Gentamicin, a new broad-spectrum antibiotic complex. Antimicrob Agents Chemother 161:17.

214. Jackson J, Chen C, Buising K. 2013. Aminoglycosides: how should we use them in the 21st century? Curr Opin Infect Dis 26:516-525. 
215. Tran Ba Huy P, Deffrennes D. 1988. Aminoglycoside ototoxicity: influence of dosage regimen on drug uptake and correlation between membrane binding and some clinical features. Acta Otolaryngol 105:511-515.

216. Chamot E, Boffi El Amari E, Rohner P, Van Delden C. 2003. Effectiveness of combination antimicrobial therapy for Pseudomonas aeruginosa bacteremia. Antimicrob Agents Chemother 47:2756-2764.

217. Haller I. 1985. Comprehensive evaluation of ciprofloxacin-aminoglycoside combinations against Enterobacteriaceae and Pseudomonas aeruginosa strains. Antimicrob Agents Chemother 28:663-666.

218. Wolfson JS, Hooper DC. 1989. Fluoroquinolone antimicrobial agents. Clin Microbiol Rev 2:378-424.

219. Jensen T, Pedersen SS, Hoiby N, Koch C. 1987. Efficacy of oral fluoroquinolones versus conventional intravenous antipseudomonal chemotherapy in treatment of cystic fibrosis. Eur J Clin Microbiol 6:618-622.

220. Davis R, Markham A, Balfour JA. 1996. Ciprofloxacin. An updated review of its pharmacology, therapeutic efficacy and tolerability. Drugs 51:1019-1074.

221. Klinger JD, Aronoff SC. 1985. In-vitro activity of ciprofloxacin and other antibacterial agents against Pseudomonas aeruginosa and Pseudomonas cepacia from cystic fibrosis patients. J Antimicrob Chemother 15:679-684.

222. Hodson ME, Roberts CM, Butland RJ, Smith MJ, Batten JC. 1987. Oral ciprofloxacin compared with conventional intravenous treatment for Pseudomonas aeruginosa infection in adults with cystic fibrosis. Lancet 1:235237.

223. Ball P. 1990. Emergent resistance to ciprofloxacin amongst Pseudomonas aeruginosa and Staphylococcus aureus: clinical significance and therapeutic approaches. J Antimicrob Chemother 26 Suppl F:165-179.

224. Shalit I, Stutman HR, Marks MI, Chartrand SA, Hilman BC. 1987. Randomized study of two dosage regimens of ciprofloxacin for treating chronic bronchopulmonary infection in patients with cystic fibrosis. Am J Med 82:189195.

225. Norrby SR. 1991. Side-effects of quinolones: comparisons between quinolones and other antibiotics. Eur J Clin Microbiol Infect Dis 10:378-383.

226. Stahlmann R, Lode H. 1999. Toxicity of quinolones. Drugs 58 Suppl 2:37-42. 
227. Lang BJ, Aaron SD, Ferris W, Hebert PC, MacDonald NE. 2000. Multiple combination bactericidal antibiotic testing for patients with cystic fibrosis infected with multiresistant strains of Pseudomonas aeruginosa. Am J Respir Crit Care Med 162:2241-2245.

228. Graber CD, Tumbusch WT, Vogel EH, Jr. 1959. In vitro sensitivity of pseudomonads from burned patients to colistin sulfate. Antibiot Annu 7:77-79.

229. Gordon DM, Mc LJ. 1960. Colistin in Pseudomonas infection. Report of a successfully treated case. Am J Ophthalmol 50:33-35.

230. McMeel JW. 1961. Colistin treatment of experimentally produced Pseudomonas corneal infection. Am J Ophthalmol 51:391-394.

231. Hoeprich PD. 1970. The polymyxins. Med Clin North Am 54:1257-1265.

232. Wolinsky E, Hines JD. 1962. Neurotoxic and nephrotoxic effects of colistin patients with renal disease. N Engl J Med 266:759-762.

233. Holmes KK. 1964. Toxicity of colistin and polymixin B. N Engl J Med 271:633634.

234. Linden PK, Kusne S, Coley K, Fontes P, Kramer DJ, Paterson D. 2003. Use of parenteral colistin for the treatment of serious infection due to antimicrobialresistant Pseudomonas aeruginosa. Clin Infect Dis 37:e154-160.

235. Mastoraki A, Douka E, Kriaras I, Stravopodis G, Manoli H, Geroulanos S. 2008. Pseudomonas aeruginosa susceptible only to colistin in intensive care unit patients. Surg Infect (Larchmt) 9:153-160.

236. Kallel H, Hergafi L, Bahloul M, Hakim A, Dammak H, Chelly H, Hamida CB, Chaari A, Rekik N, Bouaziz M. 2007. Safety and efficacy of colistin compared with imipenem in the treatment of ventilator-associated pneumonia: a matched case-control study. Intensive Care Med 33:1162-1167.

237. Falagas ME, Kasiakou SK. 2006. Toxicity of polymyxins: a systematic review of the evidence from old and recent studies. Crit Care 10:R27.

238. Kallel H, Bahloul M, Hergafi L, Akrout M, Ketata W, Chelly H, Hamida CB, Rekik N, Hammami A, Bouaziz M. 2006. Colistin as a salvage therapy for nosocomial infections caused by multidrug-resistant bacteria in the ICU. Int $\mathbf{J}$ Antimicrob Agents 28:366-369. 
239. Falagas ME, Kasiakou SK. 2005. Colistin: the revival of polymyxins for the management of multidrug-resistant Gram-negative bacterial infections. Clin Infect Dis 40:1333-1341.

240. Michalopoulos A, Kasiakou SK, Mastora Z, Rellos K, Kapaskelis AM, Falagas ME. 2005. Aerosolized colistin for the treatment of nosocomial pneumonia due to multidrug-resistant Gram-negative bacteria in patients without cystic fibrosis. Crit Care 9:R53-59.

241. Bauldoff GS, Nunley DR, Manzetti JD, Dauber JH, Keenan RJ. 1997. Use of aerosolized colistin sodium in cystic fibrosis patients awaiting lung transplantation. Transplantation 64:748-752.

242. Doring G, Conway SP, Heijerman HG, Hodson ME, Hoiby N, Smyth A, Touw DJ. 2000. Antibiotic therapy against Pseudomonas aeruginosa in cystic fibrosis: a European consensus. Eur Respir J 16:749-767.

243. Haagensen JA, Klausen M, Ernst RK, Miller SI, Folkesson A, Tolker-Nielsen T, Molin S. 2007. Differentiation and distribution of colistin- and sodium dodecyl sulfate-tolerant cells in Pseudomonas aeruginosa biofilms. J Bacteriol 189:28-37.

244. Ohmori H, Azuma A, Suzuki Y, Hashimoto Y. 1977. Factors involved in $\beta$ lactam antibiotic resistance in Pseudomonas aeruginosa. Antimicrob Agents Chemother 12:537-539.

245. Ogawara H. 1981. Antibiotic resistance in pathogenic and producing bacteria, with special reference to $\beta$-lactam antibiotics. Microbiol Rev 45:591-619.

246. Lambert PA. 2002. Mechanisms of antibiotic resistance in Pseudomonas aeruginosa. J R Soc Med 95 Suppl 41:22-26.

247. Hancock RE, Speert DP. 2000. Antibiotic resistance in Pseudomonas aeruginosa: mechanisms and impact on treatment. Drug Resist Updat 3:247-255.

248. Nordmann P, Dortet L, Poirel L. 2012. Carbapenem resistance in Enterobacteriaceae: here is the storm! Trends Mol Med 18:263-272.

249. Glauert AM, Thornley MJ. 1969. The topography of the bacterial cell wall. Annu Rev Microbiol 23:159-198.

250. Hancock RE. 1997. The bacterial outer membrane as a drug barrier. Trends Microbiol 5:37-42. 
251. Clarke K, Gray GW, Reaveley DA. 1967. The cell walls of Pseudomonas aeruginosa. General composition. Biochem J 105:749-754.

252. Silhavy TJ, Kahne D, Walker S. 2010. The bacterial cell envelope. Cold Spring Harb Perspect Biol 2:a000414.

253. Esko JD, Doering TL, Raetz CR. 2009. Eubacteria and Archaea. In: Varki A, Cummings RD, Esko JD, et al., editors. Essentials of Glycobiology. 2nd edition. Cold Spring Harbor Laboratory Press, Cold Spring Harbor , NY.

254. Nikaido H, Vaara M. 1985. Molecular basis of bacterial outer membrane permeability. Microbiol Rev 49:1-32.

255. Hancock RE, Bell A. 1988. Antibiotic uptake into Gram-negative bacteria. Eur J Clin Microbiol Infect Dis 7:713-720.

256. Hancock RE. 1981. Aminoglycoside uptake and mode of action-with special reference to streptomycin and gentamicin. II. Effects of aminoglycosides on cells. J Antimicrob Chemother 8:429-445.

257. Bennett PM, Chopra I. 1993. Molecular basis of $\beta$-lactamase induction in bacteria. Antimicrob Agents Chemother 37:153-158.

258. Sherbet GV, Lakshmi MS. 1973. Characterisation of Escherichia coli cell surface by isoelectric equilibrium analysis. Biochim Biophys Acta 298:50-58.

259. Wilkinson SG, Galbrath L. 1975. Studies of lipopolysaccharides from Pseudomonas aeruginosa. Eur J Biochem 52:331-343.

260. Schindler M, Osborn MJ. 1979. Interaction of divalent cations and polymyxin B with lipopolysaccharide. Biochemistry 18:4425-4430.

261. Eagon RG. 1969. Cell wall-associated inorganic substances from Pseudomonas aeruginosa. Can J Microbiol 15:235-236.

262. Galanos C, Luderitz O. 1975. Electrodialysis of lipopolysaccharides and their conversion to uniform salt forms. Eur J Biochem 54:603-610.

263. Burton AJ, Carter HE. 1964. Purification and characterization of the lipid a component of the lipopolysaccharides from Escherichia coli. Biochemistry 3:411418.

264. Vincent JM, Humphrey BA. 1963. Partition of divalent cations between bacterial wall and cell contents. Nature 199:149-151. 
265. Coughlin RT, Caldwell CR, Haug A, McGroarty EJ. 1981. A cationic electron spin resonance probe used to analyze cation interactions with lipopolysaccharide. Biochem Biophys Res Commun 100:1137-1142.

266. Eagon RG, Carson KJ. 1965. Lysis of cell walls and intact cells of Pseudomonas aeruginosa by ethylenediamine tetraacetic acid and by lysozyme. Can J Microbiol 11:193-201.

267. Asbell MA, Eagon RG. 1966. The role of multivalent cations in the organization and structure of bacterial cell walls. Biochem Biophys Res Commun 22:664-671.

268. Asbell MA, Eagon RG. 1966. Role of multivalent cations in the organization, structure, and assembly of the cell wall of Pseudomonas aeruginosa. J Bacteriol 92:380-387.

269. Delcour AH. 2009. Outer membrane permeability and antibiotic resistance. Biochim Biophys Acta 1794:808-816.

270. Nikaido H, Hancock RE. 1985. Outer membrane permeability of Pseudomonas aeruginosa, p. 145-193, In: Sokatch, J.R. (ed.): The bacteria: a treatise on structue and function, vol. 10. Academic Press, New York.

271. Hancock RE. 1984. Alterations in outer membrane permeability. Annu Rev Microbiol 38:237-264.

272. Muschel LH, Gustafson L. 1968. Antibiotic, detergent, and complement sensitivity of Salmonella typhi after ethylenediaminetetraacetic acid treatment. J Bacteriol 95:2010-2013.

273. Plesiat P, Nikaido H. 1992. Outer membranes of gram-negative bacteria are permeable to steroid probes. Mol Microbiol 6:1323-1333.

274. Vaara M, Vaara T. 1983. Polycations sensitize enteric bacteria to antibiotics. Antimicrob Agents Chemother 24:107-113.

275. Vaara M, Vaara T. 1983. Polycations as outer membrane-disorganizing agents. Antimicrob Agents Chemother 24:114-122.

276. Vaara M. 1992. Agents that increase the permeability of the outer membrane. Microbiol Rev 56:395-411.

277. Loh B, Grant C, Hancock RE. 1984. Use of the fluorescent probe 1-Nphenylnaphthylamine to study the interactions of aminoglycoside antibiotics with 
the outer membrane of Pseudomonas aeruginosa. Antimicrob Agents Chemother 26:546-551.

278. Nicas TI, Hancock RE. 1983. Alteration of susceptibility to EDTA, polymyxin B and gentamicin in Pseudomonas aeruginosa by divalent cation regulation of outer membrane protein H1. J Gen Microbiol 129:509-517.

279. Vaara M. 1991. The outer membrane permeability-increasing action of linear analogues of polymyxin B nonapeptide. Drugs Exp Clin Res 17:437-443.

280. Kimura Y, Matsunaga H, Vaara M. 1992. Polymyxin B octapeptide and polymyxin B heptapeptide are potent outer membrane permeability-increasing agents. J Antibiot (Tokyo) 45:742-749.

281. Bradshaw J. 2003. Cationic antimicrobial peptides: issues for potential clinical use. BioDrugs 17:233-240.

282. Hancock RE, Raffle VJ, Nicas TI. 1981. Involvement of the outer membrane in gentamicin and streptomycin uptake and killing in Pseudomonas aeruginosa. Antimicrob Agents Chemother 19:777-785.

283. Warren GH, Gray J, Yurchenco JA. 1957. Effect of polymyxin on the lysis of Neisseria catarrhalis by lysozyme. J Bacteriol 74:788-793.

284. Hancock RE, Wong PG. 1984. Compounds which increase the permeability of the Pseudomonas aeruginosa outer membrane. Antimicrob Agents Chemother 26:48-52.

285. Leive L. 1974. The barrier function of the gram-negative envelope. Ann N Y Acad Sci 235:109-129.

286. Hancock RE, Decad GM, Nikaido H. 1979. Identification of the protein producing transmembrane diffusion pores in the outer membrane of Pseudomonas aeruginosa PA01. Biochim Biophys Acta 554:323-331.

287. Nikaido H, Nikaido K, Harayama S. 1991. Identification and characterization of porins in Pseudomonas aeruginosa. J Biol Chem 266:770-779.

288. Bellido F, Martin NL, Siehnel RJ, Hancock RE. 1992. Reevaluation, using intact cells, of the exclusion limit and role of porin OprF in Pseudomonas aeruginosa outer membrane permeability. J Bacteriol 174:5196-5203. 
289. Yoshihara E, Nakae T. 1989. Identification of porins in the outer membrane of Pseudomonas aeruginosa that form small diffusion pores. J Biol Chem 264:62976301.

290. Nestorovich EM, Sugawara E, Nikaido H, Bezrukov SM. 2006. Pseudomonas aeruginosa porin OprF: properties of the channel. J Biol Chem 281:16230-16237.

291. Yoshimura F, Zalman LS, Nikaido H. 1983. Purification and properties of Pseudomonas aeruginosa porin. J Biol Chem 258:2308-2314.

292. Yoshimura F, Nikaido H. 1982. Permeability of Pseudomonas aeruginosa outer membrane to hydrophilic solutes. J Bacteriol 152:636-642.

293. Sugawara E, Nestorovich EM, Bezrukov SM, Nikaido H. 2006. Pseudomonas aeruginosa porin OprF exists in two different conformations. J Biol Chem 281:16220-16229.

294. Sugawara E, Steiert M, Rouhani S, Nikaido H. 1996. Secondary structure of the outer membrane proteins OmpA of Escherichia coli and $\mathrm{OprF}$ of Pseudomonas aeruginosa. J Bacteriol 178:6067-6069.

295. Sugawara E, Nagano K, Nikaido H. 2010. Factors affecting the folding of Pseudomonas aeruginosa OprF porin into the one-domain open conformer. MBio 1:4e00228-00210.

296. Koebnik R. 1995. Proposal for a peptidoglycan-associating alpha-helical motif in the $\mathrm{C}$-terminal regions of some bacterial cell-surface proteins. Mol Microbiol 16: $1269-1270$.

297. Woodruff WA, Hancock RE. 1989. Pseudomonas aeruginosa outer membrane protein F: structural role and relationship to the Escherichia coli OmpA protein. J Bacteriol 171:3304-3309.

298. Hancock RE, Irvin RT, Costerton JW, Carey AM. 1981. Pseudomonas aeruginosa outer membrane: peptidoglycan-associated proteins. J Bacteriol 145:628-631.

299. Rawling EG, Brinkman FS, Hancock RE. 1998. Roles of the carboxy-terminal half of Pseudomonas aeruginosa major outer membrane protein OprF in cell shape, growth in low-osmolarity medium, and peptidoglycan association. J Bacteriol 180:3556-3562. 
300. Gotoh N, Wakebe H, Nishino T. 1989. Ultrastructural aspects of fragility of Pseudomonas aeruginosa outer membrane devoid of protein F. FEMS Microbiol Lett 50:51-53.

301. Gotoh N, Wakebe H, Yoshihara E, Nakae T, Nishino T. 1989. Role of protein $\mathrm{F}$ in maintaining structural integrity of the Pseudomonas aeruginosa outer membrane. J Bacteriol 171:983-990.

302. Nicas TI, Hancock RE. 1983. Pseudomonas aeruginosa outer membrane permeability: isolation of a porin protein F-deficient mutant. J Bacteriol 153:281285 .

303. Livermore DM, Yang YJ. 1989. Comparative activity of meropenem against Pseudomonas aeruginosa strains with well-characterized resistance mechanisms. J Antimicrob Chemother 24 Suppl A:149-159.

304. Lee JY, Ko KS. 2012. OprD mutations and inactivation, expression of efflux pumps and AmpC, and metallo- $\beta$-lactamases in carbapenem-resistant Pseudomonas aeruginosa isolates from South Korea. Int J Antimicrob Agents 40:168-172.

305. Livermore DM. 1992. Interplay of impermeability and chromosomal $\beta$-lactamase activity in imipenem-resistant Pseudomonas aeruginosa. Antimicrob Agents Chemother 36:2046-2048.

306. Perez FJ, Gimeno C, Navarro D, Garcia-de-Lomas J. 1996. Meropenem permeation through the outer membrane of Pseudomonas aeruginosa can involve pathways other than the OprD porin channel. Chemotherapy 42:210-214.

307. Fung-Tomc JC, Huczko E, Banville J, Menard M, Kolek B, Gradelski E, Kessler RE, Bonner DP. 1995. Structure-activity relationships of carbapenems that determine their dependence on porin protein D2 for activity against Pseudomonas aeruginosa. Antimicrob Agents Chemother 39:394-399.

308. El Amin N, Giske CG, Jalal S, Keijser B, Kronvall G, Wretlind B. 2005. Carbapenem resistance mechanisms in Pseudomonas aeruginosa: alterations of porin OprD and efflux proteins do not fully explain resistance patterns observed in clinical isolates. APMIS 113:187-196.

309. Kohler T, Michea-Hamzehpour M, Epp SF, Pechere JC. 1999. Carbapenem activities against Pseudomonas aeruginosa: respective contributions of OprD and efflux systems. Antimicrob Agents Chemother 43:424-427. 
310. Masuda N, Ohya S. 1992. Cross-resistance to meropenem, cephems, and quinolones in Pseudomonas aeruginosa. Antimicrob Agents Chemother 36:18471851.

311. Mushtaq S, Ge Y, Livermore DM. 2004. Doripenem versus Pseudomonas aeruginosa in vitro: activity against characterized isolates, mutants, and transconjugants and resistance selection potential. Antimicrob Agents Chemother 48:3086-3092.

312. Masuda N, Sakagawa E, Ohya S, Gotoh N, Tsujimoto H, Nishino T. 2000. Substrate specificities of MexAB-OprM, MexCD-OprJ, and MexXY-oprM efflux pumps in Pseudomonas aeruginosa. Antimicrob Agents Chemother 44:33223327.

313. Wolter DJ, Hanson ND, Lister PD. 2004. Insertional inactivation of oprD in clinical isolates of Pseudomonas aeruginosa leading to carbapenem resistance. FEMS Microbiol Lett 236:137-143.

314. Fang ZL, Zhang LY, Huang YM, Qing Y, Cao KY, Tian GB, Huang X. 2014. OprD mutations and inactivation in imipenem-resistant Pseudomonas aeruginosa isolates from China. Infect Genet Evol 21:124-128.

315. Yoneyama H, Nakae T. 1993. Mechanism of efficient elimination of protein D2 in outer membrane of imipenem-resistant Pseudomonas aeruginosa. Antimicrob Agents Chemother 37:2385-2390.

316. Pai H, Kim J, Kim J, Lee JH, Choe KW, Gotoh N. 2001. Carbapenem resistance mechanisms in Pseudomonas aeruginosa clinical isolates. Antimicrob Agents Chemother 45:480-484.

317. Li XZ, Nikaido H, Poole K. 1995. Role of MexA-MexB-OprM in antibiotic efflux in Pseudomonas aeruginosa. Antimicrob Agents Chemother 39:19481953.

318. Hancock RE, Brinkman FS. 2002. Function of Pseudomonas porins in uptake and efflux. Annu Rev Microbiol 56:17-38.

319. Piddock LJ. 2006. Clinically relevant chromosomally encoded multidrug resistance efflux pumps in bacteria. Clin Microbiol Rev 19:382-402.

320. Grkovic S, Brown MH, Skurray RA. 2002. Regulation of bacterial drug export systems. Microbiol Mol Biol Rev 66:671-701. 
321. Van Bambeke F, Balzi E, Tulkens PM. 2000. Antibiotic efflux pumps. Biochem Pharmacol 60:457-470.

322. Schweizer HP. 2003. Efflux as a mechanism of resistance to antimicrobials in Pseudomonas aeruginosa and related bacteria: unanswered questions. Genet Mol Res 2:48-62.

323. Poole K. 2004. Efflux-mediated multiresistance in Gram-negative bacteria. Clin Microbiol Infect 10:12-26.

324. Piddock LJ. 2006. Multidrug-resistance efflux pumps - not just for resistance. Nat Rev Microbiol 4:629-636.

325. Prouty AM, Brodsky IE, Falkow S, Gunn JS. 2004. Bile-salt-mediated induction of antimicrobial and bile resistance in Salmonella typhimurium. Microbiology 150:775-783.

326. Lin J, Sahin O, Michel LO, Zhang Q. 2003. Critical role of multidrug efflux pump CmeABC in bile resistance and in vivo colonization of Campylobacter jejuni. Infect Immun 71:4250-4259.

327. Shafer WM, Qu X, Waring AJ, Lehrer RI. 1998. Modulation of Neisseria gonorrhoeae susceptibility to vertebrate antibacterial peptides due to a member of the resistance/nodulation/division efflux pump family. Proc Natl Acad Sci U S A 95:1829-1833.

328. Elkins CA, Mullis LB. 2006. Mammalian steroid hormones are substrates for the major RND- and MFS-type tripartite multidrug efflux pumps of Escherichia coli. J Bacteriol 188:1191-1195.

329. Blair JM, Piddock LJ. 2009. Structure, function and inhibition of RND efflux pumps in Gram-negative bacteria: an update. Curr Opin Microbiol 12:512-519.

330. Zgurskaya HI, Nikaido H. 1999. Bypassing the periplasm: reconstitution of the AcrAB multidrug efflux pump of Escherichia coli. Proc Natl Acad Sci U S A 96:7190-7195.

331. Li XZ, Ma D, Livermore DM, Nikaido H. 1994. Role of efflux pump(s) in intrinsic resistance of Pseudomonas aeruginosa: active efflux as a contributing factor to $\beta$-lactam resistance. Antimicrob Agents Chemother 38:1742-1752.

332. Aires JR, Nikaido H. 2005. Aminoglycosides are captured from both periplasm and cytoplasm by the AcrD multidrug efflux transporter of Escherichia coli. J Bacteriol 187:1923-1929. 
333. Nikaido H, Basina M, Nguyen V, Rosenberg EY. 1998. Multidrug efflux pump AcrAB of Salmonella typhimurium excretes only those $\beta$-lactam antibiotics containing lipophilic side chains. J Bacteriol 180:4686-4692.

334. Poole K, Heinrichs DE, Neshat S. 1993. Cloning and sequence analysis of an EnvCD homologue in Pseudomonas aeruginosa: regulation by iron and possible involvement in the secretion of the siderophore pyoverdine. Mol Microbiol 10:529-544.

335. Poole K, Krebes K, McNally C, Neshat S. 1993. Multiple antibiotic resistance in Pseudomonas aeruginosa: evidence for involvement of an efflux operon. $\mathbf{J}$ Bacteriol 175:7363-7372.

336. Poole K, Gotoh N, Tsujimoto H, Zhao Q, Wada A, Yamasaki T, Neshat S, Yamagishi J, Li XZ, Nishino T. 1996. Overexpression of the mexC-mexD-oprJ efflux operon in $n f x B$-type multidrug-resistant strains of Pseudomonas aeruginosa. Mol Microbiol 21:713-724.

337. Gotoh N, Tsujimoto H, Tsuda M, Okamoto K, Nomura A, Wada T, Nakahashi M, Nishino T. 1998. Characterization of the MexC-MexD-OprJ multidrug efflux system in $\Delta$ mexA-mexB-oprM mutants of Pseudomonas aeruginosa. Antimicrob Agents Chemother 42:1938-1943.

338. Kohler T, Michea-Hamzehpour M, Henze U, Gotoh N, Curty LK, Pechere JC. 1997. Characterization of MexE-MexF-OprN, a positively regulated multidrug efflux system of Pseudomonas aeruginosa. Mol Microbiol 23:345-354.

339. Aendekerk S, Ghysels B, Cornelis P, Baysse C. 2002. Characterization of a new efflux pump, MexGHI-OpmD, from Pseudomonas aeruginosa that confers resistance to vanadium. Microbiology 148:2371-2381.

340. Sekiya H, Mima T, Morita Y, Kuroda T, Mizushima T, Tsuchiya T. 2003. Functional cloning and characterization of a multidrug efflux pump, MexHIOpmD, from a Pseudomonas aeruginosa mutant. Antimicrob Agents Chemother 47:2990-2992.

341. Chuanchuen R, Narasaki CT, Schweizer HP. 2002. The MexJK efflux pump of Pseudomonas aeruginosa requires OprM for antibiotic efflux but not for efflux of triclosan. J Bacteriol 184:5036-5044.

342. Mima T, Sekiya H, Mizushima T, Kuroda T, Tsuchiya T. 2005. Gene cloning and properties of the RND-type multidrug efflux pumps MexPQ-OpmE and MexMN-OprM from Pseudomonas aeruginosa. Microbiol Immunol 49:9991002 . 
343. Li Y, Mima T, Komori Y, Morita Y, Kuroda T, Mizushima T, Tsuchiya T. 2003. A new member of the tripartite multidrug efflux pumps, MexVW-OprM, in Pseudomonas aeruginosa. J Antimicrob Chemother 52:572-575.

344. Westbrock-Wadman S, Sherman DR, Hickey MJ, Coulter SN, Zhu YQ, Warrener P, Nguyen LY, Shawar RM, Folger KR, Stover CK. 1999. Characterization of a Pseudomonas aeruginosa efflux pump contributing to aminoglycoside impermeability. Antimicrob Agents Chemother 43:2975-2983.

345. Mine T, Morita Y, Kataoka A, Mizushima T, Tsuchiya T. 1999. Expression in Escherichia coli of a new multidrug efflux pump, MexXY, from Pseudomonas aeruginosa. Antimicrob Agents Chemother 43:415-417.

346. Mima T, Joshi S, Gomez-Escalada M, Schweizer HP. 2007. Identification and characterization of TriABC-OpmH, a triclosan efflux pump of Pseudomonas aeruginosa requiring two membrane fusion proteins. J Bacteriol 189:7600-7609.

347. Mima T, Kohira N, Li Y, Sekiya H, Ogawa W, Kuroda T, Tsuchiya T. 2009. Gene cloning and characteristics of the RND-type multidrug efflux pump MuxABC-OpmB possessing two RND components in Pseudomonas aeruginosa. Microbiology 155:3509-3517.

348. Hassan MT, van der Lelie D, Springael D, Romling U, Ahmed N, Mergeay M. 1999. Identification of a gene cluster, $c z r$, involved in cadmium and zinc resistance in Pseudomonas aeruginosa. Gene 238:417-425.

349. Lister PD, Wolter DJ, Hanson ND. 2009. Antibacterial-resistant Pseudomonas aeruginosa: clinical impact and complex regulation of chromosomally encoded resistance mechanisms. Clin Microbiol Rev 22:582-610.

350. Poole K, Srikumar R. 2001. Multidrug efflux in Pseudomonas aeruginosa: components, mechanisms and clinical significance. Curr Top Med Chem 1:59-71.

351. Yang L, Chen L, Shen L, Surette M, Duan K. 2011. Inactivation of MuxABCOpmB transporter system in Pseudomonas aeruginosa leads to increased ampicillin and carbenicillin resistance and decreased virulence. J Microbiol 49:107-114.

352. Morita Y, Komori Y, Mima T, Kuroda T, Mizushima T, Tsuchiya T. 2001. Construction of a series of mutants lacking all of the four major mex operons for multidrug efflux pumps or possessing each one of the operons from Pseudomonas aeruginosa PAO1: MexCD-OprJ is an inducible pump. FEMS Microbiol Lett 202:139-143. 
353. Masuda N, Sakagawa E, Ohya S, Gotoh N, Tsujimoto H, Nishino T. 2000. Contribution of the MexX-MexY-OprM efflux system to intrinsic resistance in Pseudomonas aeruginosa. Antimicrob Agents Chemother 44:2242-2246.

354. Masuda N, Gotoh N, Ohya S, Nishino T. 1996. Quantitative correlation between susceptibility and $\mathrm{OprJ}$ production in $\mathrm{NfxB}$ mutants of Pseudomonas aeruginosa. Antimicrob Agents Chemother 40:909-913.

355. Heilmann HD. 1974. On the peptidoglycan of the cell walls of Pseudomonas aeruginosa. Structure of the peptide side chains. Eur J Biochem 43:35-38.

356. Heilmann HD. 1972. On the peptidoglycan of the cell walls of Pseudomonas aeruginosa. Eur J Biochem 31:456-463.

357. Mirelman D, Nuchamowitz Y. 1979. Biosynthesis of peptidoglycan in Pseudomonas aeruginosa. 1. The incorporation of peptidoglycan into the cell wall. Eur J Biochem 94:541-548.

358. van Heijenoort J. 2001. Formation of the glycan chains in the synthesis of bacterial peptidoglycan. Glycobiology 11:25R-36R.

359. Vollmer W, Blanot D, de Pedro MA. 2008. Peptidoglycan structure and architecture. FEMS Microbiol Rev 32:149-167.

360. Sauvage E, Kerff F, Terrak M, Ayala JA, Charlier P. 2008. The penicillinbinding proteins: structure and role in peptidoglycan biosynthesis. FEMS Microbiol Rev 32:234-258.

361. Georgopapadakou NH, Liu FY. 1980. Penicillin-binding proteins in bacteria. Antimicrob Agents Chemother 18:148-157.

362. Pratt RF. 2008. Substrate specificity of bacterial DD-peptidases (penicillinbinding proteins). Cell Mol Life Sci 65:2138-2155.

363. Goffin C, Ghuysen JM. 2002. Biochemistry and comparative genomics of SxxK superfamily acyltransferases offer a clue to the mycobacterial paradox: presence of penicillin-susceptible target proteins versus lack of efficiency of penicillin as therapeutic agent. Microbiol Mol Biol Rev 66:702-738.

364. Ghuysen JM. 1991. Serine $\beta$-lactamases and penicillin-binding proteins. Annu Rev Microbiol 45:37-67.

365. Zapun A, Contreras-Martel C, Vernet T. 2008. Penicillin-binding proteins and $\beta$-lactam resistance. FEMS Microbiol Rev 32:361-385. 
366. Zeng X, Lin J. 2013. $\beta$-lactamase induction and cell wall metabolism in Gramnegative bacteria. Front Microbiol 4:128.

367. Godfrey AJ, Bryan LE, Rabin HR. 1981. $\beta$-Lactam-resistant Pseudomonas aeruginosa with modified penicillin-binding proteins emerging during cystic fibrosis treatment. Antimicrob Agents Chemother 19:705-711.

368. Gotoh N, Nunomura K, Nishino T. 1990. Resistance of Pseudomonas aeruginosa to cefsulodin: modification of penicillin-binding protein 3 and mapping of its chromosomal gene. J Antimicrob Chemother 25:513-523.

369. Moya B, Dotsch A, Juan C, Blazquez J, Zamorano L, Haussler S, Oliver A. 2009. $\beta$-lactam resistance response triggered by inactivation of a nonessential penicillin-binding protein. PLoS Pathog 5:e1000353.

370. Zamorano L, Moya B, Juan C, Oliver A. 2010. Differential $\beta$-lactam resistance response driven by $a m p D$ or $d a c B$ (PBP4) inactivation in genetically diverse Pseudomonas aeruginosa strains. J Antimicrob Chemother 65:1540-1542.

371. Georgopapadakou NH. 1993. Penicillin-binding proteins and bacterial resistance to $\beta$-lactams. Antimicrob Agents Chemother 37:2045-2053.

372. Chambers HF. 1999. Penicillin-binding protein-mediated resistance in pneumococci and staphylococci. J Infect Dis 179 Suppl 2:S353-359.

373. Livermore DM. 1995. $\beta$-Lactamases in laboratory and clinical resistance. Clin Microbiol Rev 8:557-584.

374. Livermore DM. 2009. $\beta$-Lactamases- The threat renews. Curr Protein Pept Sci 10:397-400.

375. Hanson ND, Sanders CC. 1999. Regulation of inducible AmpC $\beta$-lactamase expression among Enterobacteriaceae. Curr Pharm Des 5:881-894.

376. Normark S, Lindquist S, Lindberg F. 1986. Chromosomal $\beta$-lactam resistance in enterobacteria. Scand J Infect Dis Suppl 49:38-45.

377. Wiedemann B, Peter K. 1989. Induction of $\beta$-lactamase in Gram-negative bacteria. Diagn Microbiol Infect Dis 12:131S-137S.

378. Bergstrom S, Lindberg FP, Olsson O, Normark S. 1983. Comparison of the overlapping frd and ampC operons of Escherichia coli with the corresponding DNA sequences in other Gram-negative bacteria. J Bacteriol 155:1297-1305. 
379. Juan C, Macia MD, Gutierrez O, Vidal C, Perez JL, Oliver A. 2005. Molecular mechanisms of $\beta$-lactam resistance mediated by $\mathrm{AmpC}$ hyperproduction in Pseudomonas aeruginosa clinical strains. Antimicrob Agents Chemother 49:4733-4738.

380. Giwercman B, Lambert PA, Rosdahl VT, Shand GH, Hoiby N. 1990. Rapid emergence of resistance in Pseudomonas aeruginosa in cystic fibrosis patients due to in-vivo selection of stable partially derepressed $\beta$-lactamase producing strains. J Antimicrob Chemother 26:247-259.

381. Giwercman B, Meyer C, Lambert PA, Reinert C, Hoiby N. 1992. High-level $\beta$-lactamase activity in sputum samples from cystic fibrosis patients during antipseudomonal treatment. Antimicrob Agents Chemother 36:71-76.

382. Strateva T, Yordanov D. 2009. Pseudomonas aeruginosa - a phenomenon of bacterial resistance. J Med Microbiol 58:1133-1148.

383. Smith H. 1977. Penicillin and cephalosporins, p. 25-55. 3rd edn. Antibiotics in clinical practice. University Park Press, Baltimore, M.D.

384. Fleming A. 1929. On the antibacterial action of cultures of a penicillium, with special reference to their use in the isolation of B. influenzae. Br J Exp Pathol 10:226-236.

385. Chain E, Florey HW, Gardner AD, Heatley NG, Jennings MA, Orr-Ewing J, Sanders AG. 1940. Penicillin as a chemotherapeutic agent. The Lancet 236:226228.

386. Abraham EP, Chain E, Fletcher CM, Gardner AD, Heatley NG, Jennings MA, Florey HW. 1941. Further observations on penicillin. The Lancet 238:177189.

387. Hodgkin DC. 1949. The X-ray analysis of the structure of penicillin. Adv Sci 6:85-89.

388. Newton GG, Abraham EP. 1955. Cephalosporin C, a new antibiotic containing sulphur and D-alpha-aminoadipic acid. Nature 175:548.

389. Newton GG, Abraham EP. 1956. Isolation of cephalosporin C, a penicillin-like antibiotic containing D-alpha-aminoadipic acid. Biochem J 62:651-658.

390. Abraham EP, Newton GG. 1961. The structure of cephalesporin C. Biochem J 79:377-393. 
391. Batchelor FR, Doyle FP, Nayler JH, Rolinson GN. 1959. Synthesis of penicillin: 6-aminopenicillanic acid in penicillin fermentations. Nature 183:257258.

392. Kato K. 1953. Occurrence of penicillin-nucleus in culture broths. J Antibiot (Tokyo) 6:130-136.

393. Loder B, Newton GG, Abraham EP. 1961. The cephalosporin C nucleus (7aminocephalosporanic acid) and some of its derivatives. Biochem J 79:408-416.

394. Doyle FP, Nayler JH, Smith H, Stove ER. 1961. Some novel acid-stable penicillins. Nature 191:1091-1092.

395. Doyle FP, Long AA, Nayler JH, Stove ER. 1961. New penicillins stable towards both acid and penicillinase. Nature 192:1183-1184.

396. Gottstein WJ, Babel RB, Crast LB, Essery JM, Fraser RR, Godfrey JC, Holdrege CT, Minor WF, Neubert ME, Panetta CA, Cheney LC. 1965. Derivatives of 6-aminopenicillanic acid. VI. Synthesis of some derivatives of 6aminothiopenicillanic acid. J Med Chem 8:794-797.

397. Long AA, Nayler JH, Smith H, Taylor T, Ward N. 1971. Derivatives of 6aminopenicillanic acid. XI. Alpha-amino-p-hydroxybenzylpenicillin. J Chem Soc Perkin 1 10:1920-1922.

398. Naito T, Nakagawa S, Okumura J, Konishi M, Kawaguchi H. 1965. Synthesis of 6-aminopenicillanic acid derivatives. I. 6-(Acylureido) penicillanates and some related compounds. J Antibiot (Tokyo) 18:145-157.

399. Basoglu S, Demirbas A, Ulker S, Alpay-Karaoglu S, Demirbas N. 2013. Design, synthesis and biological activities of some 7-aminocephalosporanic acid derivatives. Eur J Med Chem 69:622-631.

400. Singh J, Arrieta AC. 1999. New cephalosporins. Semin Pediatr Infect Dis 10:1422.

401. Rolinson GN. 1979. 6-APA and the development of the $\beta$-lactam antibiotics. J Antimicrob Chemother 5:7-14.

402. Sader HS, Jones RN. 1992. Historical overview of the cephalosporin spectrum: Four generations of structural evolution. Antimicrobic Newsletter 8:75-82.

403. Kirby WM, Bulger RJ. 1964. The new penicillins and cephalosporins. Annu Rev Med 15:393-412. 
404. Kahan JS, Kahan FM, Goegelman R, Currie SA, Jackson M, Stapley EO, Miller TW, Miller AK, Hendlin D, Mochales S, Hernandez S, Woodruff HB, Birnbaum J. 1979. Thienamycin, a new $\beta$-lactam antibiotic. I. Discovery, taxonomy, isolation and physical properties. J Antibiot (Tokyo) 32:1-12.

405. Akagawa H, Okanishi M, Umezawa H. 1975. A plasmid involved in chloramphenicol production in Streptomyces venezuelae: evidence from genetic mapping. J Gen Microbiol 90:336-346.

406. Distler J, Ebert A, Mansouri K, Pissowotzki K, Stockmann M, Piepersberg W. 1987. Gene cluster for streptomycin biosynthesis in Streptomyces griseus: nucleotide sequence of three genes and analysis of transcriptional activity. Nucleic Acids Res 15:8041-8056.

407. Dulmage HT. 1953. The production of neomycin by Streptomyces fradiae in synthetic media. Appl Microbiol 1:103-106.

408. Nagarajan R, Boeck LD, Gorman M, Hamill RL, Higgens CE, Hoehn MM, Stark WM, Whitney JG. 1971. $\beta$-Lactam antibiotics from Streptomyces. J Am Chem Soc 93:2308-2310.

409. Procopio RE, Silva IR, Martins MK, Azevedo JL, Araujo JM. 2012. Antibiotics produced by Streptomyces. Braz J Infect Dis 16:466-471.

410. Aoki H, Sakai H, Kohsaka M, Konomi T, Hosoda J. 1976. Nocardicin A, a new monocyclic $\beta$-lactam antibiotic. I. Discovery, isolation and characterization. J Antibiot (Tokyo) 29:492-500.

411. Nisbet LJ, Mehta RJ, Oh Y, Pan CH, Phelen CG, Polansky MJ, Shearer MC, Giovenella AJ, Grappel SF. 1985. Chlorocardicin, a monocyclic $\beta$-lactam from a Streptomyces sp. I. Discovery, production and biological activities. J Antibiot (Tokyo) 38:133-138.

412. Imada A, Kitano K, Kintaka K, Muroi M, Asai M. 1981. Sulfazecin and isosulfazecin, novel $\beta$-lactam antibiotics of bacterial origin. Nature 289:590-591.

413. Sykes RB, Cimarusti CM, Bonner DP, Bush K, Floyd DM, Georgopapadakou NH, Koster WM, Liu WC, Parker WL, Principe PA, Rathnum ML, Slusarchyk WA, Trejo WH, Wells JS. 1981. Monocyclic $\beta$ lactam antibiotics produced by bacteria. Nature 291:489-491.

414. Wells JS, Trejo WH, Principe PA, Bush K, Georgopapadakou N, Bonner DP, Sykes RB. 1982. EM5400, a family of monobactam antibiotics produced by 
Agrobacterium radiobacter. I. Taxonomy, fermentation and biological properties. J Antibiot (Tokyo) 35:295-299.

415. Sykes RB, Bonner DP. 1985. Discovery and development of the monobactams. Rev Infect Dis 7 Suppl 4:S579-593.

416. Sykes RB, Wells JS, Parker WL, Koster WH, Cimarusti CM. 1986. Aztreonam: discovery and development of the monobactams. N J Med Spec No:8-15.

417. Sykes RB, Bonner DP, Bush K, Georgopapadakou NH. 1982. Azthreonam (SQ 26,776), a synthetic monobactam specifically active against aerobic Gramnegative bacteria. Antimicrob Agents Chemother 21:85-92.

418. Percival A, Thomas E, Hart CA, Karayiannis P. 1981. In-vitro activity of monobactam, SQ 26,776, against Gram-negative bacteria. J Antimicrob Chemother 8 Suppl E:49-55.

419. Livermore DM, Williams JD. 1981. In vitro activity of the monobactam, SQ 26,776, against Gram-negative bacteria and its stability to their $\beta$-lactamases. J Antimicrob Chemother 8 Suppl E:29-37.

420. Phillips I, King A, Shannon K, Warren C. 1981. SQ 26,776: In vitro antibacterial activity and susceptibility to $\beta$-lactamases. J Antimicrob Chemother 8 Suppl E:103-110.

421. Sakurai Y, Yoshida Y, Saitoh K, Nemoto M, Yamaguchi A, Sawai T. 1990. Characteristics of aztreonam as a substrate, inhibitor and inducer for $\beta$-lactamases. J Antibiot (Tokyo) 43:403-410.

422. Bush K, Freudenberger JS, Sykes RB. 1982. Interaction of azthreonam and related monobactams with $\beta$-lactamases from Gram-negative bacteria. Antimicrob Agents Chemother 22:414-420.

423. Bush K. 1988. $\beta$-lactamase inhibitors from laboratory to clinic. Clin Microbiol Rev 1:109-123.

424. Neu HC. 1988. Aztreonam: the first monobactam. Med Clin North Am 72:555566.

425. Lister PD, Sanders WE, Jr., Sanders CC. 1998. Cefepime-aztreonam: a unique double $\beta$-lactam combination for Pseudomonas aeruginosa. Antimicrob Agents Chemother 42:1610-1619. 
426. English AR, Retsema JA, Girard AE, Lynch JE, Barth WE. 1978. CP-45,899, a $\beta$-lactamase inhibitor that extends the antibacterial spectrum of $\beta$-lactams: initial bacteriological characterization. Antimicrob Agents Chemother 14:414-419.

427. Reading C, Cole M. 1977. Clavulanic acid: a $\beta$-lactamase-inhiting $\beta$-lactam from Streptomyces clavuligerus. Antimicrob Agents Chemother 11:852-857.

428. Drawz SM, Bonomo RA. 2010. Three decades of $\beta$-lactamase inhibitors. Clin Microbiol Rev 23:160-201.

429. Bush K, Macalintal C, Rasmussen BA, Lee VJ, Yang Y. 1993. Kinetic interactions of tazobactam with $\beta$-lactamases from all major structural classes. Antimicrob Agents Chemother 37:851-858.

430. Monnaie D, Frere JM. 1993. Interaction of clavulanate with class C $\beta$ lactamases. FEBS Lett 334:269-271.

431. Noguchi JK, Gill MA. 1988. Sulbactam: a $\beta$-lactamase inhibitor. Clin Pharm 7:37-51.

432. Kazmierczak A, Cordin X, Duez JM, Siebor E, Pechinot A, Sirot J. 1990. Differences between clavulanic acid and sulbactam in induction and inhibition of cephalosporinases in enterobacteria. J Int Med Res 18 Suppl 4:67D-77D.

433. Akova M, Yang Y, Livermore DM. 1990. Interactions of tazobactam and clavulanate with inducibly- and constitutively-expressed Class I $\beta$-lactamases. J Antimicrob Chemother 25:199-208.

434. Fass RJ, Prior RB. 1989. Comparative in vitro activities of piperacillintazobactam and ticarcillin-clavulanate. Antimicrob Agents Chemother 33:12681274.

435. Sader HS, Tosin I, Sejas L, Miranda E. 2000. Comparative evaluation of the in vitro activity of three combinations of $\beta$-lactams with $\beta$-lactamase inhibitors: piperacillin/tazobactam, ticarcillin/clavulanic acid and ampicillin/sulbactam. Braz J Infect Dis 4:22-28.

436. Stover CK, Pham XQ, Erwin AL, Mizoguchi SD, Warrener P, Hickey MJ, Brinkman FS, Hufnagle WO, Kowalik DJ, Lagrou M, Garber RL, Goltry L, Tolentino E, Westbrock-Wadman S, Yuan Y, Brody LL, Coulter SN, Folger KR, Kas A, Larbig K, Lim R, Smith K, Spencer D, Wong GK, Wu Z, Paulsen IT, Reizer J, Saier MH, Hancock RE, Lory S, Olson MV. 2000. Complete genome sequence of Pseudomonas aeruginosa PA01, an opportunistic pathogen. Nature 406:959-964. 
437. Abraham EP, Chain E. 1940. An enzyme from bacteria able to destroy penicillin. Nature (London) 146:837.

438. Kirby WM. 1944. Extraction of a highly potent penicillin inactivator from penicillin resistant staphylococci. Science 99:452-453.

439. Barber M, Rozwadowska-Dowzenko M. 1948. Infection by penicillin-resistant staphylococci. Lancet 2:641-644.

440. Bondi A, Jr., Dietz CC. 1945. Penicillin resistant staphylococci. Proc Soc Exp Biol Med 60:55-58.

441. Forbes GB. 1949. Infection with penicillin-resistant staphylococci in hospital and general practice. Br Med J 2:569-571.

442. Williams RE. 1959. Epidemic staphylococci. Lancet 1:190-195.

443. Shooter RA, Smith MA, Griffiths JD, Brown ME, Williams RE, Rippon JE, Jevons MP. 1958. Spread of staphylococci in a surgical ward. Br Med J 1:607613.

444. Buhr AJ, Scott JC. 1959. Penicillin-resistant staphylococci; incidence in outpatients with hand infections. Lancet 1:1019-1021.

445. Frieden EH, Whiteley HR, Frazier CN. 1947. Some characteristics of penicillin-resistant staphylococci. Tex Rep Biol Med 5:74-91.

446. Sanders CC, Sanders WE, Jr. 1992. $\beta$-lactam resistance in Gram-negative bacteria: global trends and clinical impact. Clin Infect Dis 15:824-839.

447. Anderson ES, Datta N. 1965. Resistance to penicillins and its transfer in Enterobacteriaceae. Lancet 1:407-409.

448. Matthew M. 1979. Plasmid-mediated $\beta$-lactamases of Gram-negative bacteria: properties and distribution. J Antimicrob Chemother 5:349-358.

449. Medeiros AA. 1997. Evolution and dissemination of $\beta$-lactamases accelerated by generations of $\beta$-lactam antibiotics. Clin Infect Dis 24 Suppl 1:S19-45.

450. Bush K. 2001. New $\beta$-lactamases in Gram-negative bacteria: diversity and impact on the selection of antimicrobial therapy. Clin Infect Dis 32:1085-1089.

451. Thomson JM, Bonomo RA. 2005. The threat of antibiotic resistance in Gramnegative pathogenic bacteria: $\beta$-lactams in peril! Curr Opin Microbiol 8:518-524. 
452. Ambler RP. 1980. The structure of $\beta$-lactamases. Philos Trans R Soc Lond B Biol Sci 289:321-331.

453. Ambler RP, Coulson AF, Frere JM, Ghuysen JM, Joris B, Forsman M, Levesque RC, Tiraby G, Waley SG. 1991. A standard numbering scheme for the class A $\beta$-lactamases. Biochem J 276 ( Pt 1):269-270.

454. Galleni M, Lamotte-Brasseur J, Raquet X, Dubus A, Monnaie D, Knox JR, Frere JM. 1995. The enigmatic catalytic mechanism of active-site serine $\beta$ lactamases. Biochem Pharmacol 49:1171-1178.

455. Lamotte-Brasseur J, Knox J, Kelly JA, Charlier P, Fonze E, Dideberg O, Frere JM. 1994. The structures and catalytic mechanisms of active-site serine $\beta$ lactamases. Biotechnol Genet Eng Rev 12:189-230.

456. Palzkill T. 2013. Metallo- $\beta$-lactamase structure and function. Ann N Y Acad Sci 1277:91-104.

457. Walsh TR, Toleman MA, Poirel L, Nordmann P. 2005. Metallo- $\beta$-lactamases: the quiet before the storm? Clin Microbiol Rev 18:306-325.

458. Jack GW, Richmond MH. 1970. A comparative study of eight distinct $\beta$ lactamases synthesized by gram-negative bacteria. J Gen Microbiol 61:43-61.

459. Richmond MH, Sykes RB. 1973. The $\beta$-lactamases of gram-negative bacteria and their possible physiological role. Adv Microb Physiol 9:31-88.

460. Bush K. 1989. Characterization of $\beta$-lactamases. Antimicrob Agents Chemother 33:259-263.

461. Bush K, Jacoby GA, Medeiros AA. 1995. A functional classification scheme for $\beta$-lactamases and its correlation with molecular structure. Antimicrob Agents Chemother 39:1211-1233.

462. Bush K. 1989. Classification of $\beta$-lactamases: groups $1,2 a, 2 b$, and $2 b^{\prime}$. Antimicrob Agents Chemother 33:264-270.

463. Bush K. 1989. Classification of $\beta$-lactamases: groups 2c, 2d, 2e, 3, and 4. Antimicrob Agents Chemother 33:271-276.

464. Philippon A, Labia R, Jacoby G. 1989. Extended-spectrum $\beta$-lactamases. Antimicrob Agents Chemother 33:1131-1136. 
465. Liu PY, Gur D, Hall LM, Livermore DM. 1992. Survey of the prevalence of $\beta$ lactamases amongst 1000 Gram-negative bacilli isolated consecutively at the Royal London Hospital. J Antimicrob Chemother 30:429-447.

466. Paterson DL, Bonomo RA. 2005. Extended-spectrum $\beta$-lactamases: a clinical update. Clin Microbiol Rev 18:657-686.

467. Payne DJ, Cramp R, Winstanley DJ, Knowles DJ. 1994. Comparative activities of clavulanic acid, sulbactam, and tazobactam against clinically important $\beta$-lactamases. Antimicrob Agents Chemother 38:767-772.

468. Cheung TK, Ho PL, Woo PC, Yuen KY, Chau PY. 2002. Cloning and expression of class A $\beta$-lactamase gene blaA $A_{\mathrm{BPS}}$ in Burkholderia pseudomallei. Antimicrob Agents Chemother 46:1132-1135.

469. Castanheira M, Mendes RE, Walsh TR, Gales AC, Jones RN. 2004. Emergence of the extended-spectrum $\beta$-lactamase GES-1 in a Pseudomonas aeruginosa strain from Brazil: report from the SENTRY antimicrobial surveillance program. Antimicrob Agents Chemother 48:2344-2345.

470. Poirel L, Le Thomas I, Naas T, Karim A, Nordmann P. 2000. Biochemical sequence analyses of GES-1, a novel class A extended-spectrum $\beta$-lactamase, and the class 1 integron In52 from Klebsiella pneumoniae. Antimicrob Agents Chemother 44:622-632.

471. Aubert D, Girlich D, Naas T, Nagarajan S, Nordmann P. 2004. Functional and structural characterization of the genetic environment of an extended-spectrum $\beta$ lactamase bla $a_{\mathrm{VEB}}$ gene from a Pseudomonas aeruginosa isolate obtained in India. Antimicrob Agents Chemother 48:3284-3290.

472. Medeiros AA. 1984. $\beta$-lactamases. Br Med Bull 40:18-27.

473. Datta N, Kontomichalou P. 1965. Penicillinase synthesis controlled by infectious $R$ factors in Enterobacteriaceae. Nature 208:239-241.

474. Cooksey R, Swenson J, Clark N, Gay E, Thornsberry C. 1990. Patterns and mechanisms of $\beta$-lactam resistance among isolates of Escherichia coli from hospitals in the United States. Antimicrob Agents Chemother 34:739-745.

475. Matthew M, Hedges RW. 1976. Analytical isoelectric focusing of R factordetermined $\beta$-lactamases: correlation with plasmid compatibility. J Bacteriol 125:713-718. 
476. Ambler RP, Scott GK. 1978. Partial amino acid sequence of penicillinase coded by Escherichia coli plasmid R6K. Proc Natl Acad Sci U S A 75:3732-3736.

477. Matthew M, Hedges RW, Smith JT. 1979. Types of $\beta$-lactamase determined by plasmids in Gram-negative bacteria. J Bacteriol 138:657-662.

478. Tzouvelekis LS, Bonomo RA. 1999. SHV-type $\beta$-lactamases. Curr Pharm Des 5:847-864.

479. Chaves J, Coira A, Segura C, Reig R. 1995. Identification and location of the SHV-1 gene in Klebsiella pneumoniae strains. J Chemother 7 Suppl 4:49-51.

480. Levy SB, Hedges RW, Sullivan F, Medeiros AA, Sosroseputro H. 1985. Multiple antibiotic resistance plasmids in Enterobacteriaceae isolated from diarrhoeal specimens of hospitalized children in Indonesia. $\mathrm{J}$ Antimicrob Chemother 16:7-16.

481. Kuzin AP, Nukaga M, Nukaga Y, Hujer AM, Bonomo RA, Knox JR. 1999. Structure of the SHV-1 $\beta$-lactamase. Biochemistry 38:5720-5727.

482. Weldhagen GF, Poirel L, Nordmann P. 2003. Ambler class A extendedspectrum $\beta$-lactamases in Pseudomonas aeruginosa: novel developments and clinical impact. Antimicrob Agents Chemother 47:2385-2392.

483. Bradford PA. 2001. Extended-spectrum $\beta$-lactamases in the 21st century: characterization, epidemiology, and detection of this important resistance threat. Clin Microbiol Rev 14:933-951.

484. Bauernfeind A, Grimm H, Schweighart S. 1990. A new plasmidic cefotaximase in a clinical isolate of Escherichia coli. Infection 18:294-298.

485. Matsumoto Y, Ikeda F, Kamimura T, Yokota Y, Mine Y. 1988. Novel plasmid-mediated $\beta$-lactamase from Escherichia coli that inactivates oxyiminocephalosporins. Antimicrob Agents Chemother 32:1243-1246.

486. Bonnet R. 2004. Growing group of extended-spectrum $\beta$-lactamases: the CTX-M enzymes. Antimicrob Agents Chemother 48:1-14.

487. Bernard H, Tancrede C, Livrelli V, Morand A, Barthelemy M, Labia R. 1992. A novel plasmid-mediated extended-spectrum $\beta$-lactamase not derived from TEM- or SHV-type enzymes. J Antimicrob Chemother 29:590-592. 
488. Cao V, Lambert T, Courvalin P. 2002. ColE1-like plasmid pIP843 of Klebsiella pneumoniae encoding extended-spectrum $\beta$-lactamase CTX-M-17. Antimicrob Agents Chemother 46:1212-1217.

489. Kariuki S, Corkill JE, Revathi G, Musoke R, Hart CA. 2001. Molecular characterization of a novel plasmid-encoded cefotaximase (CTX-M-12) found in clinical Klebsiella pneumoniae isolates from Kenya. Antimicrob Agents Chemother 45:2141-2143.

490. Karim A, Poirel L, Nagarajan S, Nordmann P. 2001. Plasmid-mediated extended-spectrum $\beta$-lactamase (CTX-M-3 like) from India and gene association with insertion sequence ISEcp1. FEMS Microbiol Lett 201:237-241.

491. Sabate M, Tarrago R, Navarro F, Miro E, Verges C, Barbe J, Prats G. 2000. Cloning and sequence of the gene encoding a novel cefotaxime-hydrolyzing $\beta$ lactamase (CTX-M-9) from Escherichia coli in Spain. Antimicrob Agents Chemother 44:1970-1973.

492. Rossi A, Lopardo H, Woloj M, Picandet AM, Marino M, Galds M, Radice M, Gutkind G. 1995. Non-typhoid Salmonella spp. resistant to cefotaxime. J Antimicrob Chemother 36:697-702.

493. Gazouli M, Tzelepi E, Markogiannakis A, Legakis NJ, Tzouvelekis LS. 1998. Two novel plasmid-mediated cefotaxime-hydrolyzing $\beta$-lactamases (CTX-M-5 and CTX-M-6) from Salmonella typhimurium. FEMS Microbiol Lett 165:289293.

494. Gazouli M, Sidorenko SV, Tzelepi E, Kozlova NS, Gladin DP, Tzouvelekis LS. 1998. A plasmid-mediated $\beta$-lactamase conferring resistance to cefotaxime in a Salmonella typhimurium clone found in St Petersburg, Russia. J Antimicrob Chemother 41:119-121.

495. Gniadkowski M, Schneider I, Palucha A, Jungwirth R, Mikiewicz B, Bauernfeind A. 1998. Cefotaxime-resistant Enterobacteriaceae isolates from a hospital in Warsaw, Poland: identification of a new CTX-M-3 cefotaximehydrolyzing $\beta$-lactamase that is closely related to the CTX-M-1/MEN-1 enzyme. Antimicrob Agents Chemother 42:827-832.

496. Palucha A, Mikiewicz B, Hryniewicz W, Gniadkowski M. 1999. Concurrent outbreaks of extended-spectrum $\beta$-lactamase-producing organisms of the family Enterobacteriaceae in a Warsaw hospital. J Antimicrob Chemother 44:489-499.

497. Pai H, Choi EH, Lee HJ, Hong JY, Jacoby GA. 2001. Identification of CTXM-14 extended-spectrum $\beta$-lactamase in clinical isolates of Shigella sonnei, 
Escherichia coli, and Klebsiella pneumoniae in Korea. J Clin Microbiol 39:37473749.

498. Bonnet R, Sampaio JL, Labia R, De Champs C, Sirot D, Chanal C, Sirot J. 2000. A novel CTX-M $\beta$-lactamase (CTX-M-8) in cefotaxime-resistant Enterobacteriaceae isolated in Brazil. Antimicrob Agents Chemother 44:19361942.

499. Doucet-Populaire F, Ghnassia JC, Bonnet R, Sirot J. 2000. First isolation of a CTX-M-3-producing Enterobacter cloacae in France. Antimicrob Agents Chemother 44:3239-3240.

500. Saladin M, Cao VT, Lambert T, Donay JL, Herrmann JL, Ould-Hocine Z, Verdet C, Delisle F, Philippon A, Arlet G. 2002. Diversity of CTX-M $\beta$ lactamases and their promoter regions from Enterobacteriaceae isolated in three Parisian hospitals. FEMS Microbiol Lett 209:161-168.

501. Petroni A, Corso A, Melano R, Cacace ML, Bru AM, Rossi A, Galas M. 2002. Plasmidic extended-spectrum $\beta$-lactamases in Vibrio cholerae O1 El Tor isolates in Argentina. Antimicrob Agents Chemother 46:1462-1468.

502. Gomez-Garces JL, Saez D, Almagro M, Fernandez-Romero S, Merino F, Campos J, Oteo J. 2011. Osteomyelitis associated to CTX-M-15-producing Aeromonas hydrophila: first description in the literature. Diagn Microbiol Infect Dis 70:420-422.

503. Yamasaki K, Komatsu M, Yamashita T, Shimakawa K, Ura T, Nishio H, Satoh K, Washidu R, Kinoshita S, Aihara M. 2003. Production of CTX-M-3 extended-spectrum $\beta$-lactamase and IMP-1 metallo $\beta$-lactamase by five Gramnegative bacilli: survey of clinical isolates from seven laboratories collected in 1998 and 2000, in the Kinki region of Japan. J Antimicrob Chemother 51:631638.

504. Humeniuk C, Arlet G, Gautier V, Grimont P, Labia R, Philippon A. 2002. $\beta$ lactamases of Kluyvera ascorbata, probable progenitors of some plasmid-encoded CTX-M types. Antimicrob Agents Chemother 46:3045-3049.

505. Rodriguez MM, Power P, Radice M, Vay C, Famiglietti A, Galleni M, Ayala JA, Gutkind G. 2004. Chromosome-encoded CTX-M-3 from Kluyvera ascorbata: a possible origin of plasmid-borne CTX-M-1-derived cefotaximases. Antimicrob Agents Chemother 48:4895-4897. 
506. Decousser JW, Poirel L, Nordmann P. 2001. Characterization of a chromosomally encoded extended-spectrum class A $\beta$-lactamase from Kluyvera cryocrescens. Antimicrob Agents Chemother 45:3595-3598.

507. Poirel L, Kampfer P, Nordmann P. 2002. Chromosome-encoded Ambler class A $\beta$-lactamase of Kluyvera georgiana, a probable progenitor of a subgroup of CTX-M extended-spectrum $\beta$-lactamases. Antimicrob Agents Chemother 46:4038-4040.

508. Arduino SM, Roy PH, Jacoby GA, Orman BE, Pineiro SA, Centron D. 2002. bla $_{\text {CTX-M-2 }}$ is located in an unusual class 1 integron (In35) which includes Orf513. Antimicrob Agents Chemother 46:2303-2306.

509. Di Conza J, Ayala JA, Power P, Mollerach M, Gutkind G. 2002. Novel class 1 integron (InS21) carrying bla $a_{\mathrm{CTX}-\mathrm{M}-2}$ in Salmonella enterica serovar infantis. Antimicrob Agents Chemother 46:2257-2261.

510. Brizio A, Vasco S, Goncalves AR, Lito LM, Cristino JM, Salgado MJ, Duarte A. 2006. Survey of extended-spectrum $\beta$-lactamases in Escherichia coli isolates from a Portuguese hospital and characterisation of a novel class 1 integron (In60A) carrying the $b l a_{\text {CTX-M-9 }}$ gene. Int J Antimicrob Agents 28:320-324.

511. Chanawong A, M'Zali FH, Heritage J, Xiong JH, Hawkey PM. 2002. Three cefotaximases, CTX-M-9, CTX-M-13, and CTX-M-14, among Enterobacteriaceae in the People's Republic of China. Antimicrob Agents Chemother 46:630-637.

512. Sabate M, Navarro F, Miro E, Campoy S, Mirelis B, Barbe J, Prats G. 2002. Novel complex sull-type integron in Escherichia coli carrying bla $a_{\mathrm{CTX}-\mathrm{M}-9}$. Antimicrob Agents Chemother 46:2656-2661.

513. Rasmussen BA, Bush K, Keeney D, Yang Y, Hare R, O'Gara C, Medeiros AA. 1996. Characterization of IMI-1 $\beta$-lactamase, a class A carbapenemhydrolyzing enzyme from Enterobacter cloacae. Antimicrob Agents Chemother 40:2080-2086.

514. Naas T, Cattoen C, Bernusset S, Cuzon G, Nordmann P. 2012. First identification of bla $\mathrm{IMI}_{\mathrm{I}-1}$ in an Enterobacter cloacae clinical isolate from France. Antimicrob Agents Chemother 56:1664-1665.

515. Blanco VM, Rojas LJ, De La Cadena E, Maya JJ, Camargo RD, Correa A, Quinn JP, Villegas MV. 2013. First report of a nonmetallocarbapenemase class A carbapenemase in an Enterobacter cloacae isolate from Colombia. Antimicrob Agents Chemother 57:3457. 
516. Carrer A, Poirel L, Pitout JD, Church D, Nordmann P. 2008. Occurrence of an SME-2-producing Serratia marcescens isolate in Canada. Int J Antimicrob Agents 31:181-182.

517. Fairfax MR, Queenan AM, Lephart PR, Kaye KS, Dror M, Arnous N, Salimnia TT, Mitchell RA, Alangaden G, Salimnia H. 2011. Detection of 2 SME-1 carbapenemase-producing Serratia marcescens in Detroit. Diagn Microbiol Infect Dis 71:325-326.

518. Mataseje LF, Boyd DA, Delport J, Hoang L, Imperial M, Lefebvre B, Kuhn M, Van Caeseele P, Willey BM, Mulvey MR. 2014. Serratia marcescens harbouring SME-type class A carbapenemases in Canada and the presence of $b l a_{\mathrm{SME}}$ on a novel genomic island, SmarGI1-1. J Antimicrob Chemother 69:18251829.

519. Queenan AM, Bush K. 2007. Carbapenemases: the versatile $\beta$-lactamases. Clin Microbiol Rev 20:440-458.

520. Walther-Rasmussen J, Hoiby N. 2007. Class A carbapenemases. J Antimicrob Chemother 60:470-482.

521. Yigit H, Queenan AM, Anderson GJ, Domenech-Sanchez A, Biddle JW, Steward CD, Alberti S, Bush K, Tenover FC. 2001. Novel carbapenemhydrolyzing $\beta$-lactamase, KPC-1, from a carbapenem-resistant strain of Klebsiella pneumoniae. Antimicrob Agents Chemother 45:1151-1161.

522. Bratu S, Landman D, Alam M, Tolentino E, Quale J. 2005. Detection of KPC carbapenem-hydrolyzing enzymes in Enterobacter spp. from Brooklyn, New York. Antimicrob Agents Chemother 49:776-778.

523. Smith Moland E, Hanson ND, Herrera VL, Black JA, Lockhart TJ, Hossain A, Johnson JA, Goering RV, Thomson KS. 2003. Plasmid-mediated, carbapenem-hydrolysing $\beta$-lactamase, KPC-2, in Klebsiella pneumoniae isolates. J Antimicrob Chemother 51:711-714.

524. Miriagou V, Tzouvelekis LS, Rossiter S, Tzelepi E, Angulo FJ, Whichard JM. 2003. Imipenem resistance in a Salmonella clinical strain due to plasmidmediated class A carbapenemase KPC-2. Antimicrob Agents Chemother 47:1297-1300.

525. Bratu S, Mooty M, Nichani S, Landman D, Gullans C, Pettinato B, Karumudi U, Tolaney P, Quale J. 2005. Emergence of KPC-possessing Klebsiella pneumoniae in Brooklyn, New York: epidemiology and recommendations for detection. Antimicrob Agents Chemother 49:3018-3020. 
526. Bratu S, Landman D, Haag R, Recco R, Eramo A, Alam M, Quale J. 2005. Rapid spread of carbapenem-resistant Klebsiella pneumoniae in New York City: a new threat to our antibiotic armamentarium. Arch Intern Med 165:1430-1435.

527. Meyer KS, Urban C, Eagan JA, Berger BJ, Rahal JJ. 1993. Nosocomial outbreak of Klebsiella infection resistant to late-generation cephalosporins. Ann Intern Med 119:353-358.

528. Quale JM, Landman D, Bradford PA, Visalli M, Ravishankar J, Flores C, Mayorga D, Vangala K, Adedeji A. 2002. Molecular epidemiology of a citywide outbreak of extended-spectrum $\beta$-lactamase-producing Klebsiella pneumoniae infection. Clin Infect Dis 35:834-841.

529. Woodford N, Tierno PM, Jr., Young K, Tysall L, Palepou MF, Ward E, Painter RE, Suber DF, Shungu D, Silver LL, Inglima K, Kornblum J, Livermore DM. 2004. Outbreak of Klebsiella pneumoniae producing a new carbapenem-hydrolyzing class A $\beta$-lactamase, KPC-3, in a New York Medical Center. Antimicrob Agents Chemother 48:4793-4799.

530. Gomez SA, Pasteran FG, Faccone D, Tijet N, Rapoport M, Lucero C, Lastovetska O, Albornoz E, Galas M, Group KPC, Melano RG, Corso A, Petroni A. 2011. Clonal dissemination of Klebsiella pneumoniae ST258 harbouring KPC-2 in Argentina. Clin Microbiol Infect 17:1520-1524.

531. Villegas MV, Lolans K, Correa A, Suarez CJ, Lopez JA, Vallejo M, Quinn JP, Colombian Nosocomial Resistance Study G. 2006. First detection of the plasmid-mediated class A carbapenemase KPC-2 in clinical isolates of Klebsiella pneumoniae from South America. Antimicrob Agents Chemother 50:2880-2882.

532. Curiao T, Morosini MI, Ruiz-Garbajosa P, Robustillo A, Baquero F, Coque TM, Canton R. 2010. Emergence of $b l a_{\mathrm{KPC}-3}-\mathrm{Tn} 4401 \mathrm{a}$ associated with a pKPN3/4-like plasmid within ST384 and ST388 Klebsiella pneumoniae clones in Spain. J Antimicrob Chemother 65:1608-1614.

533. Cuzon G, Naas T, Demachy MC, Nordmann P. 2008. Plasmid-mediated carbapenem-hydrolyzing $\beta$-lactamase KPC-2 in Klebsiella pneumoniae isolate from Greece. Antimicrob Agents Chemother 52:796-797.

534. Naas T, Nordmann P, Vedel G, Poyart C. 2005. Plasmid-mediated carbapenem-hydrolyzing $\beta$-lactamase KPC in a Klebsiella pneumoniae isolate from France. Antimicrob Agents Chemother 49:4423-4424.

535. Pournaras S, Protonotariou E, Voulgari E, Kristo I, Dimitroulia E, Vitti D, Tsalidou M, Maniatis AN, Tsakris A, Sofianou D. 2009. Clonal spread of KPC- 
2 carbapenemase-producing Klebsiella pneumoniae strains in Greece. J Antimicrob Chemother 64:348-352.

536. Samuelsen O, Naseer U, Tofteland S, Skutlaberg DH, Onken A, Hjetland R, Sundsfjord A, Giske CG. 2009. Emergence of clonally related Klebsiella pneumoniae isolates of sequence type 258 producing plasmid-mediated KPC carbapenemase in Norway and Sweden. J Antimicrob Chemother 63:654-658.

537. Wendt C, Schutt S, Dalpke AH, Konrad M, Mieth M, Trierweiler-Hauke B, Weigand MA, Zimmermann S, Biehler K, Jonas D. 2010. First outbreak of Klebsiella pneumoniae carbapenemase (KPC)-producing $K$. pneumoniae in Germany. Eur J Clin Microbiol Infect Dis 29:563-570.

538. Woodford N, Zhang J, Warner M, Kaufmann ME, Matos J, Macdonald A, Brudney D, Sompolinsky D, Navon-Venezia S, Livermore DM. 2008. Arrival of Klebsiella pneumoniae producing KPC carbapenemase in the United Kingdom. J Antimicrob Chemother 62:1261-1264.

539. Leavitt A, Navon-Venezia S, Chmelnitsky I, Schwaber MJ, Carmeli Y. 2007. Emergence of KPC-2 and KPC-3 in carbapenem-resistant Klebsiella pneumoniae strains in an Israeli hospital. Antimicrob Agents Chemother 51:3026-3029.

540. Wei ZQ, Du XX, Yu YS, Shen P, Chen YG, Li LJ. 2007. Plasmid-mediated KPC-2 in a Klebsiella pneumoniae isolate from China. Antimicrob Agents Chemother 51:763-765.

541. Ma L, Siu LK, Lin JC, Wu TL, Fung CP, Wang JT, Lu PL, Chuang YC. 2013. Updated molecular epidemiology of carbapenem-non-susceptible Escherichia coli in Taiwan: first identification of KPC-2 or NDM-1-producing $E$. coli in Taiwan. BMC Infect Dis 13:599.

542. Gootz TD, Lescoe MK, Dib-Hajj F, Dougherty BA, He W, Della-Latta P, Huard RC. 2009. Genetic organization of transposase regions surrounding bla $a_{\mathrm{KPC}}$ carbapenemase genes on plasmids from Klebsiella strains isolated in a New York City hospital. Antimicrob Agents Chemother 53:1998-2004.

543. Naas T, Cuzon G, Villegas MV, Lartigue MF, Quinn JP, Nordmann P. 2008. Genetic structures at the origin of acquisition of the $\beta$-lactamase bla $_{\mathrm{KPC}}$ gene. Antimicrob Agents Chemother 52:1257-1263.

544. Arnold RS, Thom KA, Sharma S, Phillips M, Kristie Johnson J, Morgan DJ. 2011. Emergence of Klebsiella pneumoniae carbapenemase-producing bacteria. South Med J 104:40-45. 
545. da Silva RM, Traebert J, Galato D. 2012. Klebsiella pneumoniae carbapenemase (KPC)-producing Klebsiella pneumoniae: a review of epidemiological and clinical aspects. Expert Opin Biol Ther 12:663-671.

546. Villegas MV, Lolans K, Correa A, Kattan JN, Lopez JA, Quinn JP, Colombian Nosocomial Resistance Study G. 2007. First identification of Pseudomonas aeruginosa isolates producing a KPC-type carbapenemhydrolyzing $\beta$-lactamase. Antimicrob Agents Chemother 51:1553-1555.

547. Hossain A, Ferraro MJ, Pino RM, Dew RB, 3rd, Moland ES, Lockhart TJ, Thomson KS, Goering RV, Hanson ND. 2004. Plasmid-mediated carbapenemhydrolyzing enzyme KPC-2 in an Enterobacter sp. Antimicrob Agents Chemother 48:4438-4440.

548. Sacha P, Ostas A, Jaworowska J, Wieczorek P, Ojdana D, Ratajczak J, Tryniszewska E. 2009. The KPC type $\beta$-lactamases: new enzymes that confer resistance to carbapenems in Gram-negative bacilli. Folia Histochem Cytobiol 47:537-543.

549. Zhao WH, Hu ZQ. 2010. $\beta$-lactamases identified in clinical isolates of Pseudomonas aeruginosa. Crit Rev Microbiol 36:245-258.

550. Poirel L, Weldhagen GF, De Champs C, Nordmann P. 2002. A nosocomial outbreak of Pseudomonas aeruginosa isolates expressing the extended-spectrum $\beta$-lactamase GES-2 in South Africa. J Antimicrob Chemother 49:561-565.

551. Labuschagne Cde J, Weldhagen GF, Ehlers MM, Dove MG. 2008. Emergence of class 1 integron-associated GES-5 and GES-5-like extended-spectrum $\beta$ lactamases in clinical isolates of Pseudomonas aeruginosa in South Africa. Int J Antimicrob Agents 31:527-530.

552. Dubois V, Poirel L, Marie C, Arpin C, Nordmann P, Quentin C. 2002. Molecular characterization of a novel class 1 integron containing $b l a_{\mathrm{GES}-1}$ and a fused product of aac3-Ib/aac6'-Ib' gene cassettes in Pseudomonas aeruginosa. Antimicrob Agents Chemother 46:638-645.

553. Jeong SH, Bae IK, Kim D, Hong SG, Song JS, Lee JH, Lee SH. 2005. First outbreak of Klebsiella pneumoniae clinical isolates producing GES-5 and SHV12 extended-spectrum $\beta$-lactamases in Korea. Antimicrob Agents Chemother 49:4809-4810.

554. Duarte A, Boavida F, Grosso F, Correia M, Lito LM, Cristino JM, Salgado MJ. 2003. Outbreak of GES-1 $\beta$-lactamase-producing multidrug-resistant 
Klebsiella pneumoniae in a university hospital in Lisbon, Portugal. Antimicrob Agents Chemother 47:1481-1482.

555. Sykes RB, Matthew M. 1976. The $\beta$-lactamases of Gram-negative bacteria and their role in resistance to $\beta$-lactam antibiotics. J Antimicrob Chemother 2:115157.

556. Pitout JD, Laupland KB. 2008. Extended-spectrum $\beta$-lactamase-producing Enterobacteriaceae: an emerging public-health concern. Lancet Infect Dis 8:159166.

557. Perez-Llarena FJ, Bou G. 2009. $\beta$-lactamase inhibitors: the story so far. Curr Med Chem 16:3740-3765.

558. Massidda O, Rossolini GM, Satta G. 1991. The Aeromonas hydrophila cphA gene: molecular heterogeneity among class B metallo- $\beta$-lactamases. J Bacteriol 173:4611-4617.

559. Hussain M, Carlino A, Madonna MJ, Lampen JO. 1985. Cloning and sequencing of the metallothioprotein $\beta$-lactamase II gene of Bacillus cereus $569 / \mathrm{H}$ in Escherichia coli. J Bacteriol 164:223-229.

560. Chen Y, Succi J, Tenover FC, Koehler TM. 2003. $\beta$-lactamase genes of the penicillin-susceptible Bacillus anthracis Sterne strain. J Bacteriol 185:823-830.

561. Saavedra MJ, Peixe L, Sousa JC, Henriques I, Alves A, Correia A. 2003. SfhI, a subclass B2 metallo- $\beta$-lactamase from a Serratia fonticola environmental isolate. Antimicrob Agents Chemother 47:2330-2333.

562. Boschi L, Mercuri PS, Riccio ML, Amicosante G, Galleni M, Frere JM, Rossolini GM. 2000. The Legionella (Fluoribacter) gormanii metallo- $\beta$ lactamase: a new member of the highly divergent lineage of molecular-subclass B3 $\beta$-lactamases. Antimicrob Agents Chemother 44:1538-1543.

563. Mammeri H, Bellais S, Nordmann P. 2002. Chromosome-encoded $\beta$-lactamases TUS-1 and MUS-1 from Myroides odoratus and Myroides odoratimimus (formerly Flavobacterium odoratum), new members of the lineage of molecular subclass B1 metalloenzymes. Antimicrob Agents Chemother 46:3561-3567.

564. Sanschagrin F, Dufresne J, Levesque RC. 1998. Molecular heterogeneity of the L-1 metallo- $\beta$-lactamase family from Stenotrophomonas maltophilia. Antimicrob Agents Chemother 42:1245-1248. 
565. Avison MB, Niumsup P, Walsh TR, Bennett PM. 2000. Aeromonas hydrophila $\mathrm{AmpH}$ and $\mathrm{CepH} \beta$-lactamases: derepressed expression in mutants of Escherichia coli lacking creB. J Antimicrob Chemother 46:695-702.

566. Walsh TR, Stunt RA, Nabi JA, MacGowan AP, Bennett PM. 1997. Distribution and expression of $\beta$-lactamase genes among Aeromonas spp. J Antimicrob Chemother 40:171-178.

567. Alksne LE, Rasmussen BA. 1997. Expression of the AsbA1, OXA-12, and AsbM1 $\beta$-lactamases in Aeromonas jandaei AER 14 is coordinated by a twocomponent regulon. J Bacteriol 179:2006-2013.

568. Hayes MV, Thomson CJ, Amyes SG. 1994. Three $\beta$-lactamases isolated from Aeromonas salmonicida, including a carbapenemase not detectable by conventional methods. Eur J Clin Microbiol Infect Dis 13:805-811.

569. Avison MB, Higgins CS, Ford PJ, von Heldreich CJ, Walsh TR, Bennett PM. 2002. Differential regulation of L1 and L2 $\beta$-lactamase expression in Stenotrophomonas maltophilia. J Antimicrob Chemother 49:387-389.

570. Hu RM, Huang KJ, Wu LT, Hsiao YJ, Yang TC. 2008. Induction of L1 and L2 $\beta$-lactamases of Stenotrophomonas maltophilia. Antimicrob Agents Chemother 52:1198-1200.

571. Okazaki A, Avison MB. 2008. Induction of L1 and L2 $\beta$-lactamase production in Stenotrophomonas maltophilia is dependent on an AmpR-type regulator. Antimicrob Agents Chemother 52:1525-1528.

572. Lin CW, Huang YW, Hu RM, Chiang KH, Yang TC. 2009. The role of AmpR in regulation of L1 and L2 $\beta$-lactamases in Stenotrophomonas maltophilia. Res Microbiol 160:152-158.

573. Watanabe M, Iyobe S, Inoue M, Mitsuhashi S. 1991. Transferable imipenem resistance in Pseudomonas aeruginosa. Antimicrob Agents Chemother 35:147151.

574. Osano E, Arakawa Y, Wacharotayankun R, Ohta M, Horii T, Ito H, Yoshimura F, Kato N. 1994. Molecular characterization of an enterobacterial metallo $\beta$-lactamase found in a clinical isolate of Serratia marcescens that shows imipenem resistance. Antimicrob Agents Chemother 38:71-78.

575. Ito H, Arakawa Y, Ohsuka S, Wacharotayankun R, Kato N, Ohta M. 1995. Plasmid-mediated dissemination of the metallo- $\beta$-lactamase gene bla $a_{\mathrm{IMP}}$ among 
clinically isolated strains of Serratia marcescens. Antimicrob Agents Chemother 39:824-829.

576. Arakawa Y, Murakami M, Suzuki K, Ito H, Wacharotayankun R, Ohsuka S, Kato N, Ohta M. 1995. A novel integron-like element carrying the metallo- $\beta$ lactamase gene $b l a_{\mathrm{IMP}}$. Antimicrob Agents Chemother 39:1612-1615.

577. Senda K, Arakawa Y, Nakashima K, Ito H, Ichiyama S, Shimokata K, Kato N, Ohta M. 1996. Multifocal outbreaks of metallo- $\beta$-lactamase-producing Pseudomonas aeruginosa resistant to broad-spectrum $\beta$-lactams, including carbapenems. Antimicrob Agents Chemother 40:349-353.

578. Yano H, Kuga A, Okamoto R, Kitasato H, Kobayashi T, Inoue M. 2001. Plasmid-encoded metallo- $\beta$-lactamase (IMP-6) conferring resistance to carbapenems, especially meropenem. Antimicrob Agents Chemother 45:13431348 .

579. Senda K, Arakawa Y, Ichiyama S, Nakashima K, Ito H, Ohsuka S, Shimokata K, Kato N, Ohta M. 1996. PCR detection of metallo- $\beta$-lactamase gene $\left(\right.$ bla $\left._{\mathrm{IMP}}\right)$ in gram-negative rods resistant to broad-spectrum $\beta$-lactams. J Clin Microbiol 34:2909-2913.

580. Shibata N, Doi Y, Yamane K, Yagi T, Kurokawa H, Shibayama K, Kato H, Kai K, Arakawa Y. 2003. PCR typing of genetic determinants for metallo- $\beta$ lactamases and integrases carried by gram-negative bacteria isolated in Japan, with focus on the class 3 integron. J Clin Microbiol 41:5407-5413.

581. Arakawa Y, Shibata N, Shibayama K, Kurokawa H, Yagi T, Fujiwara H, Goto M. 2000. Convenient test for screening metallo- $\beta$-lactamase-producing gram-negative bacteria by using thiol compounds. J Clin Microbiol 38:40-43.

582. Hirakata Y, Izumikawa K, Yamaguchi T, Takemura H, Tanaka H, Yoshida R, Matsuda J, Nakano M, Tomono K, Maesaki S, Kaku M, Yamada Y, Kamihira S, Kohno S. 1998. Rapid detection and evaluation of clinical characteristics of emerging multiple-drug-resistant gram-negative rods carrying the metallo- $\beta$-lactamase gene $b a_{\mathrm{IMP}}$. Antimicrob Agents Chemother 42:20062011.

583. Yomoda S, Okubo T, Takahashi A, Murakami M, Iyobe S. 2003. Presence of Pseudomonas putida strains harboring plasmids bearing the metallo- $\beta$-lactamase gene $b l a_{\mathrm{IMP}}$ in a hospital in Japan. J Clin Microbiol 41:4246-4251. 
584. Da Silva GJ, Leitao GJ, Peixe L. 1999. Emergence of carbapenem-hydrolyzing enzymes in Acinetobacter baumannii clinical isolates. J Clin Microbiol 37:21092110 .

585. Riccio ML, Franceschini N, Boschi L, Caravelli B, Cornaglia G, Fontana R, Amicosante G, Rossolini GM. 2000. Characterization of the metallo- $\beta$-lactamase determinant of Acinetobacter baumannii AC-54/97 reveals the existence of bla $a_{\mathrm{IMP}}$ allelic variants carried by gene cassettes of different phylogeny. Antimicrob Agents Chemother 44:1229-1235.

586. Gibb AP, Tribuddharat C, Moore RA, Louie TJ, Krulicki W, Livermore DM, Palepou MF, Woodford N. 2002. Nosocomial outbreak of carbapenemresistant Pseudomonas aeruginosa with a new bla $a_{\mathrm{IMP}}$ allele, bla $_{\mathrm{IMP}-7}$. Antimicrob Agents Chemother 46:255-258.

587. Chu YW, Afzal-Shah M, Houang ET, Palepou MI, Lyon DJ, Woodford N, Livermore DM. 2001. IMP-4, a novel metallo- $\beta$-lactamase from nosocomial Acinetobacter spp. collected in Hong Kong between 1994 and 1998. Antimicrob Agents Chemother 45:710-714.

588. Hawkey PM, Xiong J, Ye H, Li H, M'Zali FH. 2001. Occurrence of a new metallo- $\beta$-lactamase IMP-4 carried on a conjugative plasmid in Citrobacter youngae from the People's Republic of China. FEMS Microbiol Lett 194:53-57.

589. Peleg AY, Franklin C, Bell J, Spelman DW. 2004. Emergence of IMP-4 metallo- $\beta$-lactamase in a clinical isolate from Australia. J Antimicrob Chemother 54:699-700.

590. Poirel L, Pham JN, Cabanne L, Gatus BJ, Bell SM, Nordmann P. 2004. Carbapenem-hydrolysing metallo- $\beta$-lactamases from Klebsiella pneumoniae and Escherichia coli isolated in Australia. Pathology 36:366-367.

591. Tysall L, Stockdale MW, Chadwick PR, Palepou MF, Towner KJ, Livermore DM, Woodford N. 2002. IMP-1 carbapenemase detected in an Acinetobacter clinical isolate from the UK. J Antimicrob Chemother 49:217-218.

592. Oh EJ, Lee S, Park YJ, Park JJ, Park K, Kim SI, Kang MW, Kim BK. 2003. Prevalence of metallo- $\beta$-lactamase among Pseudomonas aeruginosa and Acinetobacter baumannii in a Korean university hospital and comparison of screening methods for detecting metallo- $\beta$-lactamase. J Microbiol Methods 54:411-418.

593. Lee K, Lee WG, Uh Y, Ha GY, Cho J, Chong Y, Korean Nationwide Surveillance of Antimicrobial Resistance G. 2003. VIM- and IMP-type 
metallo-beta-lactamase-producing Pseudomonas spp. and Acinetobacter spp. in Korean hospitals. Emerg Infect Dis 9:868-871.

594. Galan JC, Gonzalez-Candelas F, Rolain JM, Canton R. 2013. Antibiotics as selectors and accelerators of diversity in the mechanisms of resistance: from the resistome to genetic plasticity in the $\beta$-lactamases world. Front Microbiol 4:9.

595. Lauretti L, Riccio ML, Mazzariol A, Cornaglia G, Amicosante G, Fontana R, Rossolini GM. 1999. Cloning and characterization of bla $a_{\mathrm{VIM}}$, a new integronborne metallo- $\beta$-lactamase gene from a Pseudomonas aeruginosa clinical isolate. Antimicrob Agents Chemother 43:1584-1590.

596. Riccio ML, Pallecchi L, Fontana R, Rossolini GM. 2001. In70 of plasmid pAX22, a bla $a_{\mathrm{VIM}-1}$-containing integron carrying a new aminoglycoside phosphotransferase gene cassette. Antimicrob Agents Chemother 45:1249-1253.

597. Poirel L, Collet L, Nordmann P. 2000. Carbapenem-hydrolyzing metallo- $\beta$ lactamase from a nosocomial isolate of Pseudomonas aeruginosa in France. Emerg Infect Dis 6:84-85.

598. Poirel L, Lambert T, Turkoglu S, Ronco E, Gaillard J, Nordmann P. 2001. Characterization of Class 1 integrons from Pseudomonas aeruginosa that contain the $b l a_{\mathrm{VIM}-2}$ carbapenem-hydrolyzing $\beta$-lactamase gene and of two novel aminoglycoside resistance gene cassettes. Antimicrob Agents Chemother 45:546552.

599. Pournaras S, Maniati M, Petinaki E, Tzouvelekis LS, Tsakris A, Legakis NJ, Maniatis AN. 2003. Hospital outbreak of multiple clones of Pseudomonas aeruginosa carrying the unrelated metallo- $\beta$-lactamase gene variants bla $a_{\mathrm{VIM}-2}$ and bla $_{\mathrm{VIM}-4 .}$ J Antimicrob Chemother 51:1409-1414.

600. Yatsuyanagi J, Saito S, Harata S, Suzuki N, Ito Y, Amano K, Enomoto K. 2004. Class 1 integron containing metallo- $\beta$-lactamase gene bla $a_{\mathrm{VIM}-2}$ in Pseudomonas aeruginosa clinical strains isolated in Japan. Antimicrob Agents Chemother 48:626-628.

601. Lagatolla C, Tonin EA, Monti-Bragadin C, Dolzani L, Gombac F, Bearzi C, Edalucci E, Gionechetti F, Rossolini GM. 2004. Endemic carbapenem-resistant Pseudomonas aeruginosa with acquired metallo- $\beta$-lactamase determinants in European hospital. Emerg Infect Dis 10:535-538.

602. Cardoso O, Leitao R, Figueiredo A, Sousa JC, Duarte A, Peixe LV. 2002. Metallo- $\beta$-lactamase VIM-2 in clinical isolates of Pseudomonas aeruginosa from Portugal. Microb Drug Resist 8:93-97. 
603. Mendes RE, Castanheira M, Garcia P, Guzman M, Toleman MA, Walsh TR, Jones RN, Program SAS. 2004. First isolation of bla $a_{\mathrm{VIM}-2}$ in Latin America: report from the SENTRY Antimicrobial Surveillance Program. Antimicrob Agents Chemother 48:1433-1434.

604. Yum JH, Yong D, Lee K, Kim HS, Chong Y. 2002. A new integron carrying VIM-2 metallo- $\beta$-lactamase gene cassette in a Serratia marcescens isolate. Diagn Microbiol Infect Dis 42:217-219.

605. Toleman MA, Simm AM, Murphy TA, Gales AC, Biedenbach DJ, Jones RN, Walsh TR. 2002. Molecular characterization of SPM-1, a novel metallo- $\beta$ lactamase isolated in Latin America: report from the SENTRY antimicrobial surveillance programme. J Antimicrob Chemother 50:673-679.

606. Poirel L, Magalhaes M, Lopes M, Nordmann P. 2004. Molecular analysis of metallo- $\beta$-lactamase gene $b l a_{\mathrm{SPM}-1}$-surrounding sequences from disseminated Pseudomonas aeruginosa isolates in Recife, Brazil. Antimicrob Agents Chemother 48: 1406-1409.

607. Gales AC, Menezes LC, Silbert S, Sader HS. 2003. Dissemination in distinct Brazilian regions of an epidemic carbapenem-resistant Pseudomonas aeruginosa producing SPM metallo- $\beta$-lactamase. J Antimicrob Chemother 52:699-702.

608. Martins AF, Zavascki AP, Gaspareto PB, Barth AL. 2007. Dissemination of Pseudomonas aeruginosa producing SPM-1-like and IMP-1-like metallo- $\beta$ lactamases in hospitals from southern Brazil. Infection 35:457-460.

609. Fontes LC, Neves PR, Oliveira S, Silva KC, Hachich EM, Sato MI, Lincopan N. 2011. Isolation of Pseudomonas aeruginosa coproducing metallo- $\beta$-lactamase SPM-1 and 16S rRNA methylase RmtD1 in an urban river. Antimicrob Agents Chemother 55:3063-3064.

610. Fuentefria DB, Ferreira AE, Graf T, Corcao G. 2009. Spread of metallo- $\beta$ lactamases: screening reveals the presence of a $b a_{\mathrm{SPM}-1}$ gene in hospital sewage in southern Brazil. Braz J Microbiol 40:82-85.

611. Shahcheraghi F, Abbasalipour M, Feizabadi M, Ebrahimipour G, Akbari N. 2011. Isolation and genetic characterization of metallo- $\beta$-lactamase and carbapenamase producing strains of Acinetobacter baumannii from patients at Tehran hospitals. Iran J Microbiol 3:68-74.

612. Salabi AE, Toleman MA, Weeks J, Bruderer T, Frei R, Walsh TR. 2010. First report of the metallo- $\beta$-lactamase SPM-1 in Europe. Antimicrob Agents Chemother 54:582. 
613. Castanheira M, Toleman MA, Jones RN, Schmidt FJ, Walsh TR. 2004. Molecular characterization of a $\beta$-lactamase gene, $b l a_{\mathrm{GIM}-1}$, encoding a new subclass of metallo- $\beta$-lactamase. Antimicrob Agents Chemother 48:4654-4661.

614. Rieber H, Frontzek A, von Baum H, Pfeifer Y. 2012. Emergence of metallo- $\beta$ lactamases GIM-1 and VIM in multidrug-resistant Pseudomonas aeruginosa in North Rhine-Westphalia, Germany. J Antimicrob Chemother 67:1043-1045.

615. Wendel AF, Brodner AH, Wydra S, Ressina S, Henrich B, Pfeffer K, Toleman MA, Mackenzie CR. 2013. Genetic characterization and emergence of the metallo- $\beta$-lactamase GIM-1 in Pseudomonas spp. and Enterobacteriaceae during a long-term outbreak. Antimicrob Agents Chemother 57:5162-5165.

616. Hamprecht A, Poirel L, Gottig S, Seifert H, Kaase M, Nordmann P. 2013. Detection of the carbapenemase GIM-1 in Enterobacter cloacae in Germany. J Antimicrob Chemother 68:558-561.

617. Rieber H, Frontzek A, Pfeifer Y. 2012. Emergence of metallo- $\beta$-lactamase GIM-1 in a clinical isolate of Serratia marcescens. Antimicrob Agents Chemother 56:4945-4947.

618. Kaase M, Szabados F, Pfennigwerth N, Anders A, Geis G, Pranada AB, Rossler S, Lang U, Gatermann SG. 2014. Description of the metallo- $\beta$ lactamase GIM-1 in Acinetobacter pittii. J Antimicrob Chemother 69:81-84.

619. Lee K, Yum JH, Yong D, Lee HM, Kim HD, Docquier JD, Rossolini GM, Chong Y. 2005. Novel acquired metallo- $\beta$-lactamase gene, bla $a_{\text {SIM-1 }}$, in a class 1 integron from Acinetobacter baumannii clinical isolates from Korea. Antimicrob Agents Chemother 49:4485-4491.

620. Lee K, Kim CK, Hong SG, Choi J, Song S, Koh E, Yong D, Jeong SH, Yum JH, Docquier JD, Rossolini GM, Chong Y. 2010. Characteristics of clinical isolates of Acinetobacter genomospecies 10 carrying two different metallo- $\beta$ lactamases. Int J Antimicrob Agents 36:259-263.

621. Zhou Z, Du X, Wang L, Yang Q, Fu Y, Yu Y. 2011. Clinical carbapenemresistant Acinetobacter baylyi strain coharboring blaSIM-1 and blaOXA-23 from China. Antimicrob Agents Chemother 55:5347-5349.

622. Lin MF, Lan CY. 2014. Antimicrobial resistance in Acinetobacter baumannii: From bench to bedside. World J Clin Cases 2:787-814.

623. Jacoby GA. 2009. AmpC $\beta$-lactamases. Clin Microbiol Rev 22:161-182. 
624. Walther-Rasmussen J, Hoiby N. 2002. Plasmid-borne AmpC $\beta$-lactamases. Can J Microbiol 48:479-493.

625. Galleni M, Amicosante G, Frere JM. 1988. A survey of the kinetic parameters of class $C \beta$-lactamases. Cephalosporins and other $\beta$-lactam compounds. Biochem J 255:123-129.

626. Galleni M, Frere JM. 1988. A survey of the kinetic parameters of class C $\beta$ lactamases. Penicillins. Biochem J 255:119-122.

627. Sanders CC. 1987. Chromosomal cephalosporinases responsible for multiple resistance to newer $\beta$-lactam antibiotics. Annu Rev Microbiol 41:573-593.

628. Lindberg F, Lindquist S, Normark S. 1986. Induction of chromosomal $\beta$ lactamase expression in enterobacteria. J Antimicrob Chemother 18 Suppl C:4350.

629. Bergstrom S, Olsson O, Normark S. 1982. Common evolutionary origin of chromosomal $\beta$-lactamase genes in enterobacteria. J Bacteriol 150:528-534.

630. Lindberg F, Normark S. 1986. Contribution of chromosomal $\beta$-lactamases to $\beta$ lactam resistance in enterobacteria. Rev Infect Dis 8 Suppl 3:S292-304.

631. Grundstrom T, Jaurin B. 1982. Overlap between ampC and frd operons on the Escherichia coli chromosome. Proc Natl Acad Sci U S A 79:1111-1115.

632. Jaurin B, Grundstrom T. 1981. ampC cephalosporinase of Escherichia coli K12 has a different evolutionary origin from that of $\beta$-lactamases of the penicillinase type. Proc Natl Acad Sci U S A 78:4897-4901.

633. Jaurin B, Grundstrom T, Edlund T, Normark S. 1981. The E. coli $\beta$-lactamase attenuator mediates growth rate-dependent regulation. Nature 290:221-225.

634. Olsson O, Bergstrom S, Lindberg FP, Normark S. 1983. аmpC $\beta$-lactamase hyperproduction in Escherichia coli: natural ampicillin resistance generated by horizontal chromosomal DNA transfer from Shigella. Proc Natl Acad Sci U S A 80:7556-7560.

635. Jaurin B, Grundstrom T, Normark S. 1982. Sequence elements determining ampC promoter strength in E. coli. EMBO J 1:875-881.

636. Siu LK, Lu PL, Chen JY, Lin FM, Chang SC. 2003. High-level expression of атр $C \beta$-lactamase due to insertion of nucleotides between -10 and -35 promoter 
sequences in Escherichia coli clinical isolates: cases not responsive to extendedspectrum-cephalosporin treatment. Antimicrob Agents Chemother 47:2138-2144.

637. Caroff N, Espaze E, Berard I, Richet H, Reynaud A. 1999. Mutations in the ampC promoter of Escherichia coli isolates resistant to oxyiminocephalosporins without extended spectrum $\beta$-lactamase production. FEMS Microbiol Lett 173:459-465.

638. Jaurin B, Normark S. 1983. Insertion of IS2 creates a novel ampC promoter in Escherichia coli. Cell 32:809-816.

639. Nelson EC, Elisha BG. 1999. Molecular basis of AmpC hyperproduction in clinical isolates of Escherichia coli. Antimicrob Agents Chemother 43:957-959.

640. Shayan S, Bokaeian M, Shahraki S. 2014. Prevalence and molecular characterization of AmpC-producing clinical isolates of Escherichia coli from southeastern Iran. Microb Drug Resist 20:104-107.

641. Tzelepi E, Tzouvelekis LS, Vatopoulos AC, Mentis AF, Tsakris A, Legakis NJ. 1992. High prevalence of stably derepressed class-I $\beta$-lactamase expression in multiresistant clinical isolates of Enterobacter cloacae from Greek hospitals. J Med Microbiol 37:91-95.

642. Livermore DM. 1987. Clinical significance of $\beta$-lactamase induction and stable derepression in Gram-negative rods. Eur J Clin Microbiol 6:439-445.

643. Juan C, Moya B, Perez JL, Oliver A. 2006. Stepwise upregulation of the Pseudomonas aeruginosa chromosomal cephalosporinase conferring high-level $\beta$ lactam resistance involves three AmpD homologues. Antimicrob Agents Chemother 50:1780-1787.

644. Tam VH, Schilling AN, LaRocco MT, Gentry LO, Lolans K, Quinn JP, Garey KW. 2007. Prevalence of AmpC over-expression in bloodstream isolates of Pseudomonas aeruginosa. Clin Microbiol Infect 13:413-418.

645. Drissi M, Ahmed ZB, Dehecq B, Bakour R, Plesiat P, Hocquet D. 2008. Antibiotic susceptibility and mechanisms of $\beta$-lactam resistance among clinical strains of Pseudomonas aeruginosa: first report in Algeria. Med Mal Infect 38:187-191.

646. Bauernfeind A, Chong Y, Schweighart S. 1989. Extended broad spectrum $\beta$ lactamase in Klebsiella pneumoniae including resistance to cephamycins. Infection 17:316-321. 
647. Bauernfeind A, Stemplinger I, Jungwirth R, Giamarellou H. 1996. Characterization of the plasmidic $\beta$-lactamase CMY-2, which is responsible for cephamycin resistance. Antimicrob Agents Chemother 40:221-224.

648. Papanicolaou GA, Medeiros AA, Jacoby GA. 1990. Novel plasmid-mediated $\beta$ lactamase (MIR-1) conferring resistance to oxyimino- and alpha-methoxy $\beta$ lactams in clinical isolates of Klebsiella pneumoniae. Antimicrob Agents Chemother 34:2200-2209.

649. Philippon A, Arlet G, Jacoby GA. 2002. Plasmid-determined AmpC-type $\beta$ lactamases. Antimicrob Agents Chemother 46:1-11.

650. Horii T, Arakawa Y, Ohta M, Ichiyama S, Wacharotayankun R, Kato N. 1993. Plasmid-mediated AmpC-type $\beta$-lactamase isolated from Klebsiella pneumoniae confers resistance to broad-spectrum $\beta$-lactams, including moxalactam. Antimicrob Agents Chemother 37:984-990.

651. Nakano R, Okamoto R, Nakano Y, Kaneko K, Okitsu N, Hosaka Y, Inoue M. 2004. CFE-1, a novel plasmid-encoded AmpC $\beta$-lactamase with an ampR gene originating from Citrobacter freundii. Antimicrob Agents Chemother 48:11511158 .

652. Barlow M, Hall BG. 2002. Origin and evolution of the AmpC $\beta$-lactamases of Citrobacter freundii. Antimicrob Agents Chemother 46:1190-1198.

653. Bauernfeind A, Stemplinger I, Jungwirth R, Wilhelm R, Chong Y. 1996. Comparative characterization of the cephamycinase $b l a_{\mathrm{CMY}-1}$ gene and its relationship with other $\beta$-lactamase genes. Antimicrob Agents Chemother 40:1926-1930.

654. Alvarez M, Tran JH, Chow N, Jacoby GA. 2004. Epidemiology of conjugative plasmid-mediated AmpC $\beta$-lactamases in the United States. Antimicrob Agents Chemother 48:533-537.

655. Chen YT, Lauderdale TL, Liao TL, Shiau YR, Shu HY, Wu KM, Yan JJ, Su IJ, Tsai SF. 2007. Sequencing and comparative genomic analysis of pK29, a 269kilobase conjugative plasmid encoding CMY-8 and CTX-M-3 $\beta$-lactamases in Klebsiella pneumoniae. Antimicrob Agents Chemother 51:3004-3007.

656. Hanson ND, Thomson KS, Moland ES, Sanders CC, Berthold G, Penn RG. 1999. Molecular characterization of a multiply resistant Klebsiella pneumoniae encoding ESBLs and a plasmid-mediated AmpC. J Antimicrob Chemother 44:377-380. 
657. Miriagou V, Tzouvelekis LS, Villa L, Lebessi E, Vatopoulos AC, Carattoli A, Tzelepi E. 2004. CMY-13, a novel inducible cephalosporinase encoded by an Escherichia coli plasmid. Antimicrob Agents Chemother 48:3172-3174.

658. Bauernfeind A, Wagner S, Jungwirth R, Schneider I, Meyer D. 1997. A novel class C $\beta$-lactamase (FOX-2) in Escherichia coli conferring resistance to cephamycins. Antimicrob Agents Chemother 41:2041-2046.

659. M'Zali FH, Heritage J, Gascoyne-Binzi DM, Denton M, Todd NJ, Hawkey PM. 1997. Transcontinental importation into the UK of Escherichia coli expressing a plasmid-mediated AmpC-type $\beta$-lactamase exposed during an outbreak of SHV-5 extended-spectrum $\beta$-lactamase in a Leeds hospital. J Antimicrob Chemother 40:823-831.

660. Gazouli M, Kaufmann ME, Tzelepi E, Dimopoulou H, Paniara O, Tzouvelekis LS. 1997. Study of an outbreak of cefoxitin-resistant Klebsiella pneumoniae in a general hospital. J Clin Microbiol 35:508-510.

661. Pai H, Kang CI, Byeon JH, Lee KD, Park WB, Kim HB, Kim EC, Oh MD, Choe KW. 2004. Epidemiology and clinical features of bloodstream infections caused by AmpC-type- $\beta$-lactamase-producing Klebsiella pneumoniae. Antimicrob Agents Chemother 48:3720-3728.

662. Yan JJ, Ko WC, Wu JJ, Tsai SH, Chuang CL. 2004. Epidemiological investigation of bloodstream infections by extended spectrum cephalosporinresistant Escherichia coli in a Taiwanese teaching hospital. J Clin Microbiol 42:3329-3332.

663. Mitsuhashi S, Harada K, Hashimoto H, Kameda M, Suzuki M. 1962. Combination of two types of transmissible drug-resistance factors in a host bacterium. J Bacteriol 84:9-16.

664. Harada K, Kameda M, Suzuki M, Mitsuhashi S. 1963. Drug resistance of enteric bacteria. II. Transduction of transmissible drug-resistance (R) factors with phage Epsilon. J Bacteriol 86:1332-1338.

665. Harada K, Kameda M, Suzuki M, Mitsuhashi S. 1964. Drug resistance of enteric bacteria. 3. Acquisition of transferability of nontransmissible R(TC) factor in cooperation with F factor and formation of FR(TC). J Bacteriol 88:1257-1265.

666. Lederberg J. 1952. Cell genetics and hereditary symbiosis. Physiol Rev 32:403430. 
667. Meynell E, Datta N. 1966. The relation of resistance transfer factors to the Ffactor (sex-factor) of Escherichia coli K12. Genet Res 7:134-140.

668. Dale JW, Smith JT. 1971. The purification and properties of the $\beta$-lactamase specified by the resistance factor R-1818 in Escherichia coli and Proteus mirabilis. Biochem J 123:493-500.

669. Dale JW. 1971. Characterization of the $\beta$-lactamase specified by the resistance factor R-1818 in E. coli $\mathrm{K} 12$ and other Gram-negative bacteria. Biochem J 123:501-505.

670. Dale JW, Smith JT. 1972. A direct comparison of two unusual R-factormediated $\beta$-lactamases. Biochem J 128:173-174.

671. Hedges RW, Datta N, Kontomichalou P, Smith JT. 1974. Molecular specificities of $\mathrm{R}$ factor-determined $\beta$-lactamases: correlation with plasmid compatibility. J Bacteriol 117:56-62.

672. Smith JT. 1969. R-factor gene expression Gram-negative bacteria. J Gen Microbiol 55:109-120.

673. Egawa R, Sawai T, Mitsuhashi S. 1967. Drug resistance of enteric bacteria. XII. Unique substrate specificity of penicillinase produced by R-Factor. Japanese Journal of Microbiology 11:173-178.

674. Dale JW, Smith JT. 1974. R-factor-mediated $\beta$-lactamases that hydrolyze oxacillin: evidence for two distinct groups. J Bacteriol 119:351-356.

675. Yamagishi S, O'Hara K, Sawai T, Mitsuhashi S. 1969. The purification and properties of penicillin $\beta$-lactamases mediated by transmissible $\mathrm{R}$ factors in Escherichia coli. J Biochem 66:11-20.

676. Ouellette M, Bissonnette L, Roy PH. 1987. Precise insertion of antibiotic resistance determinants into Tn21-like transposons: nucleotide sequence of the OXA-1 $\beta$-lactamase gene. Proc Natl Acad Sci U S A 84:7378-7382.

677. Jenkins PH, Drabble WT. 1971. $\beta$-lactamases of R factors derived from Shigella and Salmonella strains. J Bacteriol 108:159-165.

678. Witchitz JL, Chabbert YA. 1971. High level transferable resistance to gentamicin. J Antibiot (Tokyo) 24:137-139. 
679. Datta N, Hedges RW. 1972. R factors identified in Paris, some conferring gentamicin resistance, constitute a new compatibility group. Ann Inst Pasteur (Paris) 123:849-852.

680. Poirel L, Naas T, Nordmann P. 2010. Diversity, epidemiology, and genetics of class D $\beta$-lactamases. Antimicrob Agents Chemother 54:24-38.

681. Aubert D, Poirel L, Chevalier J, Leotard S, Pages JM, Nordmann P. 2001. Oxacillinase-mediated resistance to cefepime and susceptibility to ceftazidime in Pseudomonas aeruginosa. Antimicrob Agents Chemother 45:1615-1620.

682. Medeiros AA, Cohenford M, Jacoby GA. 1985. Five novel plasmid-determined $\beta$-lactamases. Antimicrob Agents Chemother 27:715-719.

683. Huovinen P, Huovinen S, Jacoby GA. 1988. Sequence of PSE-2 $\beta$-lactamase. Antimicrob Agents Chemother 32:134-136.

684. Yang Y, Bush K. 1995. Oxacillin hydrolysis by the LCR-1 $\beta$-lactamase. Antimicrob Agents Chemother 39:1209.

685. Evans BA, Amyes SG. 2014. OXA $\beta$-lactamases. Clin Microbiol Rev 27:241263.

686. Hall LM, Livermore DM, Gur D, Akova M, Akalin HE. 1993. OXA-11, an extended-spectrum variant of OXA-10 (PSE-2) $\beta$-lactamase from Pseudomonas aeruginosa. Antimicrob Agents Chemother 37:1637-1644.

687. Danel F, Hall LM, Gur D, Livermore DM. 1995. OXA-14, another extendedspectrum variant of OXA-10 (PSE-2) $\beta$-lactamase from Pseudomonas aeruginosa. Antimicrob Agents Chemother 39:1881-1884.

688. Danel F, Hall LM, Gur D, Livermore DM. 1998. OXA-16, a further extendedspectrum variant of OXA-10 $\beta$-lactamase, from two Pseudomonas aeruginosa isolates. Antimicrob Agents Chemother 42:3117-3122.

689. Danel F, Hall LM, Duke B, Gur D, Livermore DM. 1999. OXA-17, a further extended-spectrum variant of OXA-10 $\beta$-lactamase, isolated from Pseudomonas aeruginosa. Antimicrob Agents Chemother 43:1362-1366.

690. Mugnier P, Casin I, Bouthors AT, Collatz E. 1998. Novel OXA-10-derived extended-spectrum $\beta$-lactamases selected in vivo or in vitro. Antimicrob Agents Chemother 42:3113-3116. 
691. Danel F, Hall LM, Gur D, Livermore DM. 1997. OXA-15, an extendedspectrum variant of OXA-2 $\beta$-lactamase, isolated from a Pseudomonas aeruginosa strain. Antimicrob Agents Chemother 41:785-790.

692. Brown S, Amyes S. 2006. OXA $\beta$-lactamases in Acinetobacter: the story so far. J Antimicrob Chemother 57:1-3.

693. Walther-Rasmussen J, Hoiby N. 2006. OXA-type carbapenemases. J Antimicrob Chemother 57:373-383.

694. Kaitany KC, Klinger NV, June CM, Ramey ME, Bonomo RA, Powers RA, Leonard DA. 2013. Structures of the class D carbapenemases OXA-23 and OXA-146: mechanistic basis of activity against carbapenems, extended-spectrum cephalosporins, and aztreonam. Antimicrob Agents Chemother 57:4848-4855.

695. Scaife W, Young HK, Paton RH, Amyes SG. 1995. Transferable imipenemresistance in Acinetobacter species from a clinical source. J Antimicrob Chemother 36:585-586.

696. Heritier C, Poirel L, Fournier PE, Claverie JM, Raoult D, Nordmann P. 2005. Characterization of the naturally occurring oxacillinase of Acinetobacter baumannii. Antimicrob Agents Chemother 49:4174-4179.

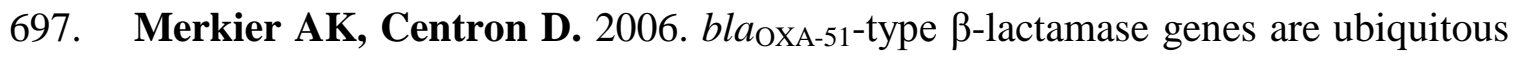
and vary within a strain in Acinetobacter baumannii. Int J Antimicrob Agents 28:110-113.

698. Figueiredo S, Poirel L, Croize J, Recule C, Nordmann P. 2009. In vivo selection of reduced susceptibility to carbapenems in Acinetobacter baumannii related to ISAbal-mediated overexpression of the natural bla OXA-66 Oxacillinase gene. Antimicrob Agents Chemother 53:2657-2659.

699. Figueiredo S, Poirel L, Papa A, Koulourida V, Nordmann P. 2009. Overexpression of the naturally occurring $b_{\text {OXA-51 }}$ gene in Acinetobacter baumannii mediated by novel insertion sequence ISAba9. Antimicrob Agents Chemother 53:4045-4047.

700. Turton JF, Ward ME, Woodford N, Kaufmann ME, Pike R, Livermore DM, Pitt TL. 2006. The role of ISAbal in expression of OXA carbapenemase genes in Acinetobacter baumannii. FEMS Microbiol Lett 258:72-77.

701. Bou G, Oliver A, Martinez-Beltran J. 2000. OXA-24, a novel class D $\beta$ lactamase with carbapenemase activity in an Acinetobacter baumannii clinical strain. Antimicrob Agents Chemother 44:1556-1561. 
702. Poirel L, Heritier C, Tolun V, Nordmann P. 2004. Emergence of oxacillinasemediated resistance to imipenem in Klebsiella pneumoniae. Antimicrob Agents Chemother 48:15-22.

703. Poirel L, Marque S, Heritier C, Segonds C, Chabanon G, Nordmann P. 2005. OXA-58, a novel class D $\beta$-lactamase involved in resistance to carbapenems in Acinetobacter baumannii. Antimicrob Agents Chemother 49:202-208.

704. Sari AN, Bicmen M, Gulay Z. 2013. The first report on the outbreak of OXA24/40-like carbapenemase-producing Acinetobacter baumannii in Turkey. Jpn J Infect Dis 66:439-442.

705. Manageiro V, Ferreira E, Pinto M, Canica M. 2014. First description of OXA48 carbapenemase harbored by Escherichia coli and Enterobacter cloacae from a single patient in Portugal. Antimicrob Agents Chemother 58:7613-7614.

706. Hammoudi D, Moubareck CA, Aires J, Adaime A, Barakat A, Fayad N, Hakime N, Houmani M, Itani T, Najjar Z, Suleiman M, Sarraf R, Sarkis DK. 2014. Countrywide spread of OXA-48 carbapenemase in Lebanon: surveillance and genetic characterization of carbapenem-non-susceptible Enterobacteriaceae in 10 hospitals over a one-year period. Int J Infect Dis 29:139-144.

707. Bonnin RA, Poirel L, Benoit-Cattin T, Nordmann P. 2013. Ceftazidimesusceptible and imipenem-non-susceptible OXA-58-producing Acinetobacter baumannii from the Comoros archipelago. Int J Antimicrob Agents 41:297-298.

708. Amudhan SM, Sekar U, Arunagiri K, Sekar B. 2011. OXA $\beta$-lactamasemediated carbapenem resistance in Acinetobacter baumannii. Indian $\mathrm{J}$ Med Microbiol 29:269-274.

709. Poirel L, Nordmann P. 2006. Carbapenem resistance in Acinetobacter baumannii: mechanisms and epidemiology. Clin Microbiol Infect 12:826-836.

710. Kong K-F, Jayawardena SR, Indulkar SD, del Puerto A, Koh C-L, Hoiby N, Mathee K. 2005. Pseudomonas aeruginosa AmpR is a global transcriptional factor that regulates expression of AmpC and PoxB $\beta$-Lactamases, proteases, quorum sensing, and other virulence factors. Antimicrob Agents Chemother 49:4567-4575.

711. Lodge JM, Minchin SD, Piddock LJ, Busby JW. 1990. Cloning, sequencing and analysis of the structural gene and regulatory region of the Pseudomonas aeruginosa chromosomal ampC $\beta$-lactamase. Biochem J 272:627-631. 
712. Lindberg F, Normark S. 1987. Common mechanism of ampC $\beta$-lactamase induction in enterobacteria: regulation of the cloned Enterobacter cloacae P99 $\beta$ lactamase gene. J Bacteriol 169:758-763.

713. Normark S. 1995. $\beta$-lactamase induction in Gram-negative bacteria is intimately linked to peptidoglycan recycling. Microb Drug Resist 1:111-114.

714. Dietz H, Pfeifle D, Wiedemann B. 1997. The signal molecule for $\beta$-lactamase induction in Enterobacter cloacae is the anhydromuramyl-pentapeptide. Antimicrob Agents Chemother 41:2113-2120.

715. Chahboune A, Decaffmeyer M, Brasseur R, Joris B. 2005. Membrane topology of the Escherichia coli AmpG permease required for recycling of cell wall anhydromuropeptides and $\mathrm{AmpC} \beta$-lactamase induction. Antimicrob Agents Chemother 49:1145-1149.

716. Jacobs C, Huang LJ, Bartowsky E, Normark S, Park JT. 1994. Bacterial cell wall recycling provides cytosolic muropeptides as effectors for $\beta$-lactamase induction. EMBO J 13:4684-4694.

717. Jacobs C, Joris B, Jamin M, Klarsov K, Van Beeumen J, Mengin-Lecreulx D, van Heijenoort J, Park JT, Normark S, Frere JM. 1995. AmpD, essential for both $\beta$-lactamase regulation and cell wall recycling, is a novel cytosolic Nacetylmuramyl-L-alanine amidase. Mol Microbiol 15:553-559.

718. Jacobs C, Frere JM, Normark S. 1997. Cytosolic intermediates for cell wall biosynthesis and degradation control inducible $\beta$-lactam resistance in Gramnegative bacteria. Cell 88:823-832.

719. Holtje JV, Kopp U, Ursinus A, Wiedemann B. 1994. The negative regulator of $\beta$-lactamase induction AmpD is a $\mathrm{N}$-acetyl-anhydromuramyl-L-alanine amidase. FEMS Microbiol Lett 122:159-164.

720. Jacobs C. 1997. Pharmacia Biotech \& Science prize. 1997 grand prize winner. Life in the balance: cell walls and antibiotic resistance. Science 278:1731-1732.

721. Tuomanen E, Lindquist S, Sande S, Galleni M, Light K, Gage D, Normark S. 1991. Coordinate regulation of $\beta$-lactamase induction and peptidoglycan composition by the amp operon. Science 251:201-204.

722. Bagge N, Ciofu O, Hentzer M, Campbell JI, Givskov M, Hoiby N. 2002. Constitutive high expression of chromosomal $\beta$-lactamase in Pseudomonas aeruginosa caused by a new insertion sequence (IS1669) located in ampD. Antimicrob Agents Chemother 46:3406-3411. 
723. Lindberg F, Lindquist S, Normark S. 1987. Inactivation of the ampD gene causes semiconstitutive overproduction of the inducible Citrobacter freundii $\beta$ lactamase. J Bacteriol 169:1923-1928.

724. Heritier C, Poirel L, Nordmann P. 2006. Cephalosporinase over-expression resulting from insertion of ISAbal in Acinetobacter baumannii. Clin Microbiol Infect 12:123-130.

725. Zhou H, Pi BR, Yang Q, Yu YS, Chen YG, Li LJ, Zheng SS. 2007. Dissemination of imipenem-resistant Acinetobacter baumannii strains carrying the ISAbal bla ${ }_{\text {OXA-23 }}$ genes in a Chinese hospital. J Med Microbiol 56:1076-1080.

726. Lu PL, Huang LY, Lian ST, Chang K, Lin CL, Hwang IJ, Chiang WG, Chen YH, Lin SF, Siu LK. 2008. How carbapenem-resistant Acinetobacter spp. established in a newly constructed hospital. Int J Antimicrob Agents 31:463-466.

727. Martinez P, Mattar S. 2012. Imipenem-resistant Acinetobacter baumannii carrying the ISAbal-bla $a_{\mathrm{OXA}-23,51}$ and ISAbal-bla $\mathrm{ADC}_{\mathrm{AD}}$ genes in Monteria, Colombia. Braz J Microbiol 43:1274-1280.

728. Poirel L, Cabanne L, Vahaboglu H, Nordmann P. 2005. Genetic environment and expression of the extended-spectrum $\beta$-lactamase $b a_{\mathrm{PER}-1}$ gene in Gramnegative bacteria. Antimicrob Agents Chemother 49:1708-1713.

729. Niumsup P, Simm AM, Nurmahomed K, Walsh TR, Bennett PM, Avison MB. 2003. Genetic linkage of the penicillinase gene, amp, and blrAB, encoding the regulator of $\beta$-lactamase expression in Aeromonas spp. J Antimicrob Chemother 51:1351-1358.

730. Girlich D, Naas T, Nordmann P. 2006. Regulation of class D $\beta$-lactamase gene expression in Ralstonia pickettii. Microbiology 152:2661-2672.

731. Girlich D, Naas T, Nordmann P. 2004. OXA-60, a chromosomal, inducible, and imipenem-hydrolyzing class D $\beta$-lactamase from Ralstonia pickettii. Antimicrob Agents Chemother 48:4217-4225.

732. Nordmann P, Poirel L, Kubina M, Casetta A, Naas T. 2000. Biochemicalgenetic characterization and distribution of OXA-22, a chromosomal and inducible class D $\beta$-lactamase from Ralstonia (Pseudomonas) pickettii. Antimicrob Agents Chemother 44:2201-2204.

733. Girlich D, Kolb A, Naas T, Nordmann P. 2009. Characterization of regulatory element Rp3 of regulation of $\beta$-lactamases from Ralstonia pickettii. FEMS Microbiol Lett 301:50-56. 
734. Iaconis JP, Sanders CC. 1990. Purification and characterization of inducible $\beta$ lactamases in Aeromonas spp. Antimicrob Agents Chemother 34:44-51.

735. Rasmussen BA, Keeney D, Yang Y, Bush K. 1994. Cloning and expression of a cloxacillin-hydrolyzing enzyme and a cephalosporinase from Aeromonas sobria AER 14M in Escherichia coli: requirement for an E. coli chromosomal mutation for efficient expression of the class D enzyme. Antimicrob Agents Chemother 38:2078-2085.

736. Yang Y, Bush K. 1996. Biochemical characterization of the carbapenemhydrolyzing $\beta$-lactamase AsbM1 from Aeromonas sobria AER 14M: a member of a novel subgroup of metallo- $\beta$-lactamases. FEMS Microbiol Lett 137:193-200.

737. Walsh TR, Payne DJ, MacGowan AP, Bennett PM. 1995. A clinical isolate of Aeromonas sobria with three chromosomally mediated inducible $\beta$-lactamases: a cephalosporinase, a penicillinase and a third enzyme, displaying carbapenemase activity. J Antimicrob Chemother 35:271-279.

738. Walsh TR, Hall L, MacGowan AP, Bennett PM. 1995. Sequence analysis of two chromosomally mediated inducible $\beta$-lactamases from Aeromonas sobria, strain 163a, one a class D penicillinase, the other an AmpC cephalosporinase. J Antimicrob Chemother 36:41-52.

739. Niumsup P, Simm AM, Nurmahomed K, Walsh TR, Bennett PM, Avison MB. 2003. Genetic linkage of the penicillinase gene, amp, and blrAB, encoding the regulator of $\beta$-lactamase expression in Aeromonas spp. J Antimicrob Chemother 51:1351-1358.

740. Kong KF, Jayawardena SR, Del Puerto A, Wiehlmann L, Laabs U, Tummler B, Mathee K. 2005. Characterization of poxB, a chromosomal-encoded Pseudomonas aeruginosa oxacillinase. Gene 358:82-92.

741. Girlich D, Naas T, Nordmann P. 2004. Biochemical characterization of the naturally occurring oxacillinase OXA-50 of Pseudomonas aeruginosa. Antimicrob Agents Chemother 48:2043-2048.

742. Koch C, Hoiby N. 1993. Pathogenesis of cystic fibrosis. Lancet 341:1065-1069.

743. Wolter DJ, Lister PD. 2013. Mechanisms of $\beta$-lactam resistance among Pseudomonas aeruginosa. Curr Pharm Des 19:209-222.

744. Tsakris A, Pournaras S, Woodford N, Palepou MF, Babini GS, Douboyas J, Livermore DM. 2000. Outbreak of infections caused by Pseudomonas 
aeruginosa producing VIM-1 carbapenemase in Greece. J Clin Microbiol 38:1290-1292.

745. Neyestanaki DK, Mirsalehian A, Rezagholizadeh F, Jabalameli F, Taherikalani M, Emaneini M. 2014. Determination of extended spectrum $\beta$ lactamases, metallo- $\beta$-lactamases and AmpC- $\beta$-lactamases among carbapenem resistant Pseudomonas aeruginosa isolated from burn patients. Burns 40:15561561.

746. Najar Peerayeh S, Pirhajati Mahabadi R, Pakbaten Toupkanlou S, Siadat SD. 2014. Diversity of $\beta$-lactamases produced by imipenem resistant, Pseudomonas aeruginosa isolates from the bloodstream. Burns 40:1360-1364.

747. Tawfik AF, Shibl AM, Aljohi MA, Altammami MA, Al-Agamy MH. 2012. Distribution of Ambler class A, B and D $\beta$-lactamases among Pseudomonas aeruginosa isolates. Burns 38:855-860.

748. Livermore DM. 1991. $\beta$-lactamases of Pseudomonas aeruginosa. Antibiot Chemother (1971) 44:215-220.

749. Cabot G, Ocampo-Sosa AA, Tubau F, Macia MD, Rodriguez C, Moya B, Zamorano L, Suarez C, Pena C, Martinez-Martinez L, Oliver A, Spanish Network for Research in Infectious D. 2011. Overexpression of AmpC and efflux pumps in Pseudomonas aeruginosa isolates from bloodstream infections: prevalence and impact on resistance in a Spanish multicenter study. Antimicrob Agents Chemother 55:1906-1911.

750. Dale JW, Godwin D, Mossakowska D, Stephenson P, Wall S. 1985. Sequence of the OXA-2 $\beta$-lactamase: comparison with other penicillin-reactive enzymes. FEBS Lett 191:39-44.

751. Matthew M. 1978. Properties of the $\beta$-lactamase specified by the Pseudomonas plasmid R151. FEMS Microbiol Lett 4:241 -244.

752. Nakazawa H, Mitsuhashi S. 1983. Tn2011, a new transposon encoding oxacillin-hydrolyzing $\beta$-lactamase. Antimicrob Agents Chemother 23:407-412.

753. Couture F, Lachapelle J, Levesque RC. 1992. Phylogeny of LCR-1 and OXA-5 with class A and class D $\beta$-lactamases. Mol Microbiol 6:1693-1705.

754. Joris B, Ledent P, Dideberg O, Fonze E, Lamotte-Brasseur J, Kelly JA, Ghuysen JM, Frere JM. 1991. Comparison of the sequences of class A $\beta$ lactamases and of the secondary structure elements of penicillin-recognizing proteins. Antimicrob Agents Chemother 35:2294-2301. 
755. Schneider I, Queenan AM, Bauernfeind A. 2006. Novel carbapenemhydrolyzing oxacillinase OXA-62 from Pandoraea pnomenusa. Antimicrob Agents Chemother 50:1330-1335.

756. Arora SK, Ritchings BW, Almira EC, Lory S, Ramphal R. 1997. A transcriptional activator, FleQ, regulates mucin adhesion and flagellar gene expression in Pseudomonas aeruginosa in a cascade manner. J Bacteriol 179:5574-5581.

757. Trias J, Nikaido H. 1990. Protein D2 channel of the Pseudomonas aeruginosa outer membrane has a binding site for basic amino acids and peptides. J Biol Chem 265:15680-15684.

758. Horton RM, Cai ZL, Ho SN, Pease LR. 1990. Gene splicing by overlap extension: tailor-made genes using the polymerase chain reaction. Biotechniques 8:528-535.

759. Rietsch A, Vallet-Gely I, Dove SL, Mekalanos JJ. 2005. ExsE, a secreted regulator of type III secretion genes in Pseudomonas aeruginosa. Proc Natl Acad Sci U S A 102:8006-8011.

760. Kumari H, Balasubramanian D, Zincke D, Mathee K. 2014. Role of Pseudomonas aeruginosa AmpR on $\beta$-lactam and non- $\beta$-lactam transient crossresistance upon pre-exposure to subinhibitory concentrations of antibiotics. J Med Microbiol 63:544-555.

761. Studier FW, Moffatt BA. 1986. Use of bacteriophage T7 RNA polymerase to direct selective high-level expression of cloned genes. J Mol Biol 189:113-130.

762. Holloway BW, Krishnapillai V, Morgan AF. 1979. Chromosomal genetics of Pseudomonas. Microbiol Rev 43:73-102.

763. Furste JP, Pansegrau W, Frank R, Blocker H, Scholz P, Bagdasarian M, Lanka E. 1986. Molecular cloning of the plasmid RP4 primase region in a multihost-range tacP expression vector. Gene 48:119-131.

764. Brencic A, Lory S. 2009. Determination of the regulon and identification of novel mRNA targets of Pseudomonas aeruginosa RsmA. Mol Microbiol 72:612632.

765. O'Callaghan CH, Morris A, Kirby SM, Shingler AH. 1972. Novel method for detection of $\beta$-lactamases by using a chromogenic cephalosporin substrate. Antimicrob Agents Chemother 1:283-288. 
766. Jorgensen JH. 1993. Antimicrobial susceptibility testing of bacteria that grow aerobically. Infect Dis Clin North Am 7:393-409.

767. Institute CaLS. 2012. Methods for dilution antimicrobial susceptibility tests for bacteria that grow aerobically. Approved Standard 9th Ed. CLSI document M07A9., Clinical and Laboratory Standards Institute. CLSI, Wayne, PA.

768. Friedman L, Kolter R. 2004. Two genetic loci produce distinct carbohydraterich structural components of the Pseudomonas aeruginosa biofilm matrix. J Bacteriol 186:4457-4465.

769. Ma L, Jackson KD, Landry RM, Parsek MR, Wozniak DJ. 2006. Analysis of Pseudomonas aeruginosa conditional psl variants reveals roles for the Psl polysaccharide in adhesion and maintaining biofilm structure postattachment. J Bacteriol 188:8213-8221.

770. Ghafoor A, Hay ID, Rehm BH. 2011. Role of exopolysaccharides in Pseudomonas aeruginosa biofilm formation and architecture. Appl Environ Microbiol 77:5238-5246.

771. Pechere JC, Kohler T. 1999. Patterns and modes of $\beta$-lactam resistance in Pseudomonas aeruginosa. Clin Microbiol Infect 5 Suppl 1:S15-S18.

772. Llanes C, Pourcel C, Richardot C, Plesiat P, Fichant G, Cavallo JD, Merens A, Group GS. 2013. Diversity of $\beta$-lactam resistance mechanisms in cystic fibrosis isolates of Pseudomonas aeruginosa: a French multicentre study. J Antimicrob Chemother 68:1763-1771.

773. Fehlberg LC, Xavier DE, Peraro PP, Marra AR, Edmond MB, Gales AC. 2012. $\beta$-lactam resistance mechanisms in Pseudomonas aeruginosa strains causing bloodstream infections: comparative results between Brazilian and American isolates. Microb Drug Resist 18:402-407.

774. Hakemi Vala M, Hallajzadeh M, Hashemi A, Goudarzi H, Tarhani M, Sattarzadeh Tabrizi M, Bazmi F. 2014. Detection of Ambler class A, B and D $\beta$-lactamases among Pseudomonas aeruginosa and Acinetobacter baumannii clinical isolates from burn patients. Ann Burns Fire Disasters 27:8-13.

775. Wirth FW, Picoli SU, Cantarelli VV, Goncalves AL, Brust FR, Santos LM, Barreto MF. 2009. Metallo- $\beta$-lactamase-producing Pseudomonas aeruginosa in two hospitals from southern Brazil. Braz J Infect Dis 13:170-172. 
776. Lodge J, Busby S, Piddock L. 1993. Investigation of the Pseudomonas aeruginosa ampR gene and its role at the chromosomal ampC $\beta$-lactamase promoter. FEMS Microbiol Lett 111:315-320.

777. Ollis DL, Cheah E, Cygler M, Dijkstra B, Frolow F, Franken SM, Harel M, Remington SJ, Silman I, Schrag J, et al. 1992. The $\alpha / \beta$ hydrolase fold. Protein Eng 5:197-211.

778. Nardini M, Dijkstra BW. 1999. $\alpha / \beta$ hydrolase fold enzymes: the family keeps growing. Curr Opin Struct Biol 9:732-737.

779. Gates ML, Sanders CC, Goering RV, Sanders WE, Jr. 1986. Evidence for multiple forms of type I chromosomal $\beta$-lactamase in Pseudomonas aeruginosa. Antimicrob Agents Chemother 30:453-457.

780. Murata T, Minami S, Yasuda K, Iyobe S, Inoue M, Mitsuhashi S. 1981. Purification and properties of cephalosporinase from Pseudomonas aeruginosa. $\mathbf{J}$ Antibiot (Tokyo) 34:1164-1170.

781. Chen HY, Yuan M, Livermore DM. 1995. Mechanisms of resistance to $\beta$ lactam antibiotics amongst Pseudomonas aeruginosa isolates collected in the UK in 1993. J Med Microbiol 43:300-309.

782. Langaee TY, Gagnon L, Huletsky A. 2000. Inactivation of the ampD gene in Pseudomonas aeruginosa leads to moderate-basal-level and hyperinducible AmpC $\beta$-lactamase expression. Antimicrob Agents Chemother 44:583-589.

783. Schmidtke AJ, Hanson ND. 2008. Role of $a m p D$ homologs in overproduction of AmpC in clinical isolates of Pseudomonas aeruginosa. Antimicrob Agents Chemother 52:3922-3927.

784. Trias J, Nikaido H. 1990. Outer membrane protein D2 catalyzes facilitated diffusion of carbapenems and penems through the outer membrane of Pseudomonas aeruginosa. Antimicrob Agents Chemother 34:52-57.

785. Yoneyama H, Nakae T. 1991. Cloning of the protein D2 gene of Pseudomonas aeruginosa and its functional expression in the imipenem-resistant host. FEBS Lett 283:177-179.

786. Lynch MJ, Drusano GL, Mobley HL. 1987. Emergence of resistance to imipenem in Pseudomonas aeruginosa. Antimicrob Agents Chemother 31:18921896. 
787. Okamoto K, Gotoh N, Nishino T. 2002. Alterations of susceptibility of Pseudomonas aeruginosa by overproduction of multidrug efflux systems, MexAB-OprM, MexCD-OprJ, and MexXY/OprM to carbapenems: substrate specificities of the efflux systems. J Infect Chemother 8:371-373.

788. Masuda N, Gotoh N, Ishii C, Sakagawa E, Ohya S, Nishino T. 1999. Interplay between chromosomal $\beta$-lactamase and the MexAB-OprM efflux system in intrinsic resistance to $\beta$-lactams in Pseudomonas aeruginosa. Antimicrob Agents Chemother 43:400-402.

789. Spratt BG, Jobanputra V, Zimmermann W. 1977. Binding of thienamycin and clavulanic acid to the penicillin-binding proteins of Escherichia coli K-12. Antimicrob Agents Chemother 12:406-409.

790. Yokota T. 1989. Inactivation of $\beta$-lactamases by sulbactam and enhanced clinical activity due to target-site binding of the combination of sulbactam and ampicillin. APMIS Suppl 5:9-16.

791. Labia R, Morand A, Lelievre V, Mattioni D, Kazmierczak A. 1986. Sulbactam: biochemical factors involved in its synergy with ampicillin. Rev Infect Dis 8 Suppl 5:S496-502.

792. Urban C, Rahal JJ, Luft B. 1991. Effect of a $\beta$-lactamase inhibitor, tazobactam, on growth and penicillin-binding proteins of Borrelia burgdorferi. FEMS Microbiol Lett 66:113-116.

793. Adediran SA, Pratt RF. 2008. Inhibition of serine $\beta$-lactamases by vanadatecatechol complexes. Biochemistry 47:9467-9474.

794. Bush K. 1986. Evaluation of enzyme inhibition data in screening for new drugs. Drugs Exp Clin Res 12:565-576.

795. Brenner DG, Knowles JR. 1984. Penicillanic acid sulfone: nature of irreversible inactivation of RTEM $\beta$-lactamase from Escherichia coli. Biochemistry 23:58335839 .

796. Sulton D, Pagan-Rodriguez D, Zhou X, Liu Y, Hujer AM, Bethel CR, Helfand MS, Thomson JM, Anderson VE, Buynak JD, Ng LM, Bonomo RA. 2005. Clavulanic acid inactivation of SHV-1 and the inhibitor-resistant S130G SHV-1 $\beta$-lactamase. Insights into the mechanism of inhibition. J Biol Chem 280:35528-35536.

797. Brown AG. 1986. Clavulanic acid, a novel $\beta$-lactamase inhibitor: a case study in drug discovery and development. Drug Des Deliv 1:1-21. 
798. Brown AG, Butterworth D, Cole M, Hanscomb G, Hood JD, Reading C, Rolinson GN. 1976. Naturally-occurring $\beta$-lactamase inhibitors with antibacterial activity. J Antibiot (Tokyo) 29:668-669.

799. Retsema JA, English AR, Girard A, Lynch JE, Anderson M, Brennan L, Cimochowski C, Faiella J, Norcia W, Sawyer P. 1986. Sulbactam/ampicillin: in vitro spectrum, potency, and activity in models of acute infection. Rev Infect Dis 8 Suppl 5:S528-534.

800. Wexler HM, Harris B, Carter WT, Finegold SM. 1985. In vitro efficacy of sulbactam combined with ampicillin against anaerobic bacteria. Antimicrob Agents Chemother 27:876-878.

801. Gin A, Dilay L, Karlowsky JA, Walkty A, Rubinstein E, Zhanel GG. 2007. Piperacillin-tazobactam: a $\beta$-lactam/ $\beta$-lactamase inhibitor combination. Expert Rev Anti Infect Ther 5:365-383.

802. Daniel KP, Krop LC. 1996. Piperacillin-Tazobactam: a new $\beta$-lactam- $\beta$ lactamase inhibitor combination. Pharmacotherapy 16:149-162.

803. Zhanel GG, Lawson CD, Adam H, Schweizer F, Zelenitsky S, Lagace-Wiens PR, Denisuik A, Rubinstein E, Gin AS, Hoban DJ, Lynch JP, 3rd, Karlowsky JA. 2013. Ceftazidime-avibactam: a novel cephalosporin/ $\beta$-lactamase inhibitor combination. Drugs 73:159-177.

804. Ehmann DE, Jahic H, Ross PL, Gu RF, Hu J, Kern G, Walkup GK, Fisher SL. 2012. Avibactam is a covalent, reversible, non- $\beta$-lactam $\beta$-lactamase inhibitor. Proc Natl Acad Sci U S A 109:11663-11668.

805. Ehmann DE, Jahic H, Ross PL, Gu RF, Hu J, Durand-Reville TF, Lahiri S, Thresher J, Livchak S, Gao N, Palmer T, Walkup GK, Fisher SL. 2013. Kinetics of avibactam inhibition against Class A, C, and D $\beta$-lactamases. J Biol Chem 288:27960-27971.

806. Livermore DM, Mushtaq S, Warner M, Miossec C, Woodford N. 2008. NXL104 combinations versus Enterobacteriaceae with CTX-M extendedspectrum $\beta$-lactamases and carbapenemases. J Antimicrob Chemother 62:10531056.

807. Lahiri SD, Mangani S, Durand-Reville T, Benvenuti M, De Luca F, Sanyal G, Docquier JD. 2013. Structural insight into potent broad-spectrum inhibition with reversible recyclization mechanism: avibactam in complex with CTX-M-15 and Pseudomonas aeruginosa AmpC $\beta$-lactamases. Antimicrob Agents Chemother 57:2496-2505. 
808. Xu H, Hazra S, Blanchard JS. 2012. NXL104 irreversibly inhibits the $\beta$ lactamase from Mycobacterium tuberculosis. Biochemistry 51:4551-4557.

809. Flamm RK, Farrell DJ, Sader HS, Jones RN. 2014. Ceftazidime/avibactam activity tested against Gram-negative bacteria isolated from bloodstream, pneumonia, intra-abdominal and urinary tract infections in US medical centres (2012). J Antimicrob Chemother 69:1589-1598.

810. Sader HS, Castanheira M, Flamm RK, Farrell DJ, Jones RN. 2014. Antimicrobial activity of ceftazidime-avibactam against Gram-negative organisms collected from U.S. medical centers in 2012. Antimicrob Agents Chemother 58:1684-1692.

811. Wang X, Zhang F, Zhao C, Wang Z, Nichols WW, Testa R, Li H, Chen H, He W, Wang Q, Wang H. 2014. In vitro activities of ceftazidime-avibactam and aztreonam-avibactam against 372 Gram-negative bacilli collected in 2011 and 2012 from 11 teaching hospitals in China. Antimicrob Agents Chemother 58:1774-1778.

812. Stachyra T, Pechereau MC, Bruneau JM, Claudon M, Frere JM, Miossec C, Coleman K, Black MT. 2010. Mechanistic studies of the inactivation of TEM-1 and P99 by NXL104, a novel non- $\beta$-lactam $\beta$-lactamase inhibitor. Antimicrob Agents Chemother 54:5132-5138.

813. Porres-Osante N, Dupont $\mathbf{H}$, Torres $\mathbf{C}$, Ammenouche $\mathbf{N}$, de Champs $\mathbf{C}$, Mammeri H. 2014. Avibactam activity against extended-spectrum AmpC $\beta$ lactamases. J Antimicrob Chemother.

814. Stachyra T, Levasseur P, Pechereau MC, Girard AM, Claudon M, Miossec C, Black MT. 2009. In vitro activity of the $\beta$-lactamase inhibitor NXL104 against KPC-2 carbapenemase and Enterobacteriaceae expressing KPC carbapenemases. J Antimicrob Chemother 64:326-329.

815. Endimiani A, Hujer KM, Hujer AM, Pulse ME, Weiss WJ, Bonomo RA. 2011. Evaluation of ceftazidime and NXL104 in two murine models of infection due to KPC-producing Klebsiella pneumoniae. Antimicrob Agents Chemother 55:82-85.

816. Aktas Z, Kayacan C, Oncul O. 2012. In vitro activity of avibactam (NXL104) in combination with $\beta$-lactams against Gram-negative bacteria, including OXA-48 $\beta$-lactamase-producing Klebsiella pneumoniae. Int J Antimicrob Agents 39:86-89.

817. Levasseur P, Girard AM, Claudon M, Goossens H, Black MT, Coleman K, Miossec C. 2012. In vitro antibacterial activity of the ceftazidime-avibactam 
(NXL104) combination against Pseudomonas aeruginosa clinical isolates. Antimicrob Agents Chemother 56:1606-1608.

818. Livermore DM, Mushtaq S, Warner M, Zhang J, Maharjan S, Doumith M, Woodford N. 2011. Activities of NXL104 combinations with ceftazidime and aztreonam against carbapenemase-producing Enterobacteriaceae. Antimicrob Agents Chemother 55:390-394.

819. Heritier C, Poirel L, Aubert D, Nordmann P. 2003. Genetic and functional analysis of the chromosome-encoded carbapenem-hydrolyzing oxacillinase OXA40 of Acinetobacter baumannii. Antimicrob Agents Chemother 47:268-273.

820. Pournaras S, Markogiannakis A, Ikonomidis A, Kondyli L, Bethimouti K, Maniatis AN, Legakis NJ, Tsakris A. 2006. Outbreak of multiple clones of imipenem-resistant Acinetobacter baumannii isolates expressing OXA-58 carbapenemase in an intensive care unit. J Antimicrob Chemother 57:557-561.

821. Zahm JM, Baconnais S, Davidson DJ, Webb S, Dorin J, Bonnet N, Balossier G, Puchelle E. 2001. X-ray microanalysis of airway surface liquid collected in cystic fibrosis mice. Am J Physiol Lung Cell Mol Physiol 281:L309-313.

822. Jayaraman S, Joo NS, Reitz B, Wine JJ, Verkman AS. 2001. Submucosal gland secretions in airways from cystic fibrosis patients have normal $[\mathrm{Na}(+)]$ and pH but elevated viscosity. Proc Natl Acad Sci U S A 98:8119-8123.

823. Jayaraman S, Song Y, Vetrivel L, Shankar L, Verkman AS. 2001. Noninvasive in vivo fluorescence measurement of airway-surface liquid depth, salt concentration, and pH. J Clin Invest 107:317-324.

824. Pier GB, Ramphal R. 2010. Pseudomonas aeruginosa, p. 2835-2860. In Mandell GL, Bennett JE, Dolin R (ed.), Mandell, Douglas, and Bennett's Principles and Practice of Infectious Diseases, 7th ed, vol. 2. Churchill Livingstone, Philadelphia, PA.

825. Friedman L, Kolter R. 2004. Genes involved in matrix formation in Pseudomonas aeruginosa PA14 biofilms. Mol Microbiol 51:675-690.

826. Colvin KM, Gordon VD, Murakami K, Borlee BR, Wozniak DJ, Wong GC, Parsek MR. 2011. The Pel polysaccharide can serve a structural and protective role in the biofilm matrix of Pseudomonas aeruginosa. PLoS Pathog 7:e1001264.

827. Jackson KD, Starkey M, Kremer S, Parsek MR, Wozniak DJ. 2004. Identification of $p s l$, a locus encoding a potential exopolysaccharide that is 
essential for Pseudomonas aeruginosa PAO1 biofilm formation. J Bacteriol 186:4466-4475.

828. Yang L, Hu Y, Liu Y, Zhang J, Ulstrup J, Molin S. 2011. Distinct roles of extracellular polymeric substances in Pseudomonas aeruginosa biofilm development. Environ Microbiol 13:1705-1717.

829. Colvin KM, Irie Y, Tart CS, Urbano R, Whitney JC, Ryder C, Howell PL, Wozniak DJ, Parsek MR. 2012. The Pel and Psl polysaccharides provide Pseudomonas aeruginosa structural redundancy within the biofilm matrix. Environ Microbiol 14:1913-1928.

830. Weiner R, Seagren E, Arnosti C, Quintero E. 1999. Bacterial survival in biofilms: probes for exopolysaccharide and its hydrolysis, and measurements of intra- and interphase mass fluxes. Methods Enzymol 310:403-426.

831. Solano C, Garcia B, Valle J, Berasain C, Ghigo JM, Gamazo C, Lasa I. 2002. Genetic analysis of Salmonella enteritidis biofilm formation: critical role of cellulose. Mol Microbiol 43:793-808.

832. Zogaj X, Nimtz M, Rohde M, Bokranz W, Romling U. 2001. The multicellular morphotypes of Salmonella typhimurium and Escherichia coli produce cellulose as the second component of the extracellular matrix. Mol Microbiol 39:14521463 .

833. Spiers AJ, Bohannon J, Gehrig SM, Rainey PB. 2003. Biofilm formation at the air-liquid interface by the Pseudomonas fluorescens SBW25 wrinkly spreader requires an acetylated form of cellulose. Mol Microbiol 50:15-27.

834. Hammar M, Arnqvist A, Bian Z, Olsen A, Normark S. 1995. Expression of two $\operatorname{cs} g$ operons is required for production of fibronectin- and congo red-binding curli polymers in Escherichia coli K-12. Mol Microbiol 18:661-670.

835. Collinson SK, Doig PC, Doran JL, Clouthier S, Trust TJ, Kay WW. 1993. Thin, aggregative fimbriae mediate binding of Salmonella enteritidis to fibronectin. J Bacteriol 175:12-18.

836. Romling U, Sierralta WD, Eriksson K, Normark S. 1998. Multicellular and aggregative behaviour of Salmonella typhimurium strains is controlled by mutations in the agfD promoter. Mol Microbiol 28:249-264.

837. Kong KF, Aguila A, Schneper L, Mathee K. 2010. Pseudomonas aeruginosa $\beta$ lactamase induction requires two permeases, AmpG and AmpP. BMC Microbiol 10:328. 
838. Petrova OE, Sauer K. 2009. A novel signaling network essential for regulating Pseudomonas aeruginosa biofilm development. PLoS Pathog 5:e1000668.

839. Bartowsky E, Normark S. 1993. Interactions of wild-type and mutant AmpR of Citrobacter freundii with target DNA. Mol Microbiol 10:555-565.

840. Lindquist S, Lindberg F, Normark S. 1989. Binding of the Citrobacter freundii AmpR regulator to a single DNA site provides both autoregulation and activation of the inducible ampC $\beta$-lactamase gene. J Bacteriol 171:3746-3753.

841. Balasubramanian D, Kumari H, Jaric M, Fernandez M, Turner KH, Dove SL, Narasimhan G, Lory S, Mathee K. 2014. Deep sequencing analyses expands the Pseudomonas aeruginosa AmpR regulon to include small RNAmediated regulation of iron acquisition, heat shock and oxidative stress response. Nucleic Acids Res 42:979-998.

842. Balasubramanian D, Schneper L, Kumari H, Mathee K. 2013. A dynamic and intricate regulatory network determines Pseudomonas aeruginosa virulence. Nucleic Acids Res 41:1-20.

843. Balasubramanian D, Schneper L, Merighi M, Smith R, Narasimhan G, Lory S, Mathee K. 2012. The regulatory repertoire of Pseudomonas aeruginosa AmpC $\beta$-lactamase regulator AmpR includes virulence genes. PLoS One 7:e34067.

844. Kumari H, Murugapiran SK, Balasubramanian D, Schneper L, Merighi M, Sarracino D, Lory S, Mathee K. 2014. LTQ-XL mass spectrometry proteome analysis expands the Pseudomonas aeruginosa AmpR regulon to include cyclic di-GMP phosphodiesterases and phosphoproteins, and identifies novel open reading frames. J Proteomics 96:328-342. doi:10.1016/j.jprot.2013.11.018.

845. Tabor CW, Tabor H. 1984. Polyamines. Annu Rev Biochem 53:749-790.

846. Wortham BW, Patel CN, Oliveira MA. 2007. Polyamines in bacteria: pleiotropic effects yet specific mechanisms. Adv Exp Med Biol 603:106-115.

847. Tabor CW, Tabor H. 1985. Polyamines in microorganisms. Microbiol Rev 49:81-99.

848. Burton DR, Forsen S, Reimarsson P. 1981. The interaction of polyamines with DNA: a 23Na NMR study. Nucleic Acids Res 9:1219-1228.

849. Matthews HR. 1993. Polyamines, chromatin structure and transcription. Bioessays 15:561-566. 
850. Wang JY. 2007. Polyamines and mRNA stability in regulation of intestinal mucosal growth. Amino Acids 33:241-252.

851. Zou T, Rao JN, Liu L, Xiao L, Yu TX, Jiang P, Gorospe M, Wang JY. 2010. Polyamines regulate the stability of JunD mRNA by modulating the competitive binding of its 3' untranslated region to HuR and AUF1. Mol Cell Biol 30:50215032.

852. Caille O, Zincke D, Merighi M, Balasubramanian D, Kumari H, Kong KF, Silva-Herzog E, Narasimhan G, Schneper L, Lory S, Mathee K. 2014. Structural and functional characterization of Pseudomonas aeruginosa global regulator AmpR. J Bacteriol 196:3890-3902.

853. Hoang TT, Kutchma AJ, Becher A, Schweizer HP. 2000. Integration-proficient plasmids for Pseudomonas aeruginosa: site-specific integration and use for engineering of reporter and expression strains. Plasmid 43:59-72.

854. Mathee K, McPherson CJ, Ohman DE. 1997. Posttranslational control of the $\operatorname{alg} T(\operatorname{alg} U)$-encoded $\sigma^{22}$ for expression of the alginate regulon in Pseudomonas aeruginosa and localization of its antagonist proteins MucA and MucB (AlgN). J Bacteriol 179:3711-3720.

855. Goodman AL, Kulasekara B, Rietsch A, Boyd D, Smith RS, Lory S. 2004. A signaling network reciprocally regulates genes associated with acute infection and chronic persistence in Pseudomonas aeruginosa. Dev Cell 7:745-754.

856. Schell MA. 1993. Molecular biology of the LysR family of transcriptional regulators. Annu Rev Microbiol 47:597-626.

857. Maloy S, Stewart V. 1993. Autogenous regulation of gene expression. J Bacteriol 175:307-316.

858. Thieffry D, Huerta AM, Perez-Rueda E, Collado-Vides J. 1998. From specific gene regulation to genomic networks: a global analysis of transcriptional regulation in Escherichia coli. Bioessays 20:433-440.

859. Shen-Orr SS, Milo R, Mangan S, Alon U. 2002. Network motifs in the transcriptional regulation network of Escherichia coli. Nat Genet 31:64-68.

860. Goldberger RF. 1974. Autogenous regulation of gene expression. Science 183:810-816. 
861. Kulasekara HD, Ventre I, Kulasekara BR, Lazdunski A, Filloux A, Lory S. 2005. A novel two-component system controls the expression of Pseudomonas aeruginosa fimbrial cup genes. Mol Microbiol 55:368-380.

862. Lampe DJ, Churchill ME, Robertson HM. 1996. A purified mariner transposase is sufficient to mediate transposition in vitro. EMBO J 15:5470-5479.

863. Robertson HM, Lampe DJ. 1995. Recent horizontal transfer of a mariner transposable element among and between Diptera and Neuroptera. Mol Biol Evol 12:850-862.

864. Lu CD, Itoh Y, Nakada Y, Jiang Y. 2002. Functional analysis and regulation of the divergent spuABCDEFGH-spuI operons for polyamine uptake and utilization in Pseudomonas aeruginosa PAO1. J Bacteriol 184:3765-3773.

865. Agostinelli E, Marques MP, Calheiros R, Gil FP, Tempera G, Viceconte N, Battaglia V, Grancara S, Toninello A. 2010. Polyamines: fundamental characters in chemistry and biology. Amino Acids 38:393-403.

866. Andreasson B, Nordenskiold L, Schultz J. 1996. Interactions of spermidine and methylspermidine with DNA studied by nuclear magnetic resonance selfdiffusion measurements. Biophys J 70:2847-2856.

867. Choe WS, Middelberg AP. 2001. Selective precipitation of DNA by spermine during the chemical extraction of insoluble cytoplasmic protein. Biotechnol Prog 17:1107-1113.

868. Hoopes BC, McClure WR. 1981. Studies on the selectivity of DNA precipitation by spermine. Nucleic Acids Res 9:5493-5504.

869. Tabor H. 1962. The protective effect of spermine and other polyamines against heat denaturation of deoxyribonucleic acid. Biochemistry 1:496-501.

870. Bloomfield VA. 1997. DNA condensation by multivalent cations. Biopolymers 44:269-282.

871. Kaiser D, Tabor H, Tabor CW. 1963. Spermine protection of coliphage lambda DNA against breakage by hydrodynamic shear. J Mol Biol 6:141-147.

872. Cochet C, Chambaz EM. 1983. Polyamine-mediated protein phosphorylations: a possible target for intracellular polyamine action. Mol Cell Endocrinol 30:247266. 
873. Atmar VJ, Kuehn GD. 1983. Polyamine-dependent protein kinase and phosphorylation of ornithine decarboxylase in Physarum polycephalum. Methods Enzymol 99:366-372.

874. Pegg AE, Casero RA, Jr. 2011. Current status of the polyamine research field. Methods Mol Biol 720:3-35.

875. Dela Vega AL, Delcour AH. 1996. Polyamines decrease Escherichia coli outer membrane permeability. J Bacteriol 178:3715-3721.

876. Dela Vega AL, Delcour AH. 1995. Cadaverine induces closing of E. coli porins. EMBO J 14:6058-6065.

877. Samartzidou H, Delcour AH. 1999. Excretion of endogenous cadaverine leads to a decrease in porin-mediated outer membrane permeability. J Bacteriol 181:791-798.

878. Igarashi K, Kashiwagi K. 1999. Polyamine transport in bacteria and yeast. Biochem J 344 Pt 3:633-642.

879. Nishijyo T, Haas D, Itoh Y. 2001. The CbrA-CbrB two-component regulatory system controls the utilization of multiple carbon and nitrogen sources in Pseudomonas aeruginosa. Mol Microbiol 40:917-931.

880. Furuchi T, Kashiwagi K, Kobayashi H, Igarashi K. 1991. Characteristics of the gene for a spermidine and putrescine transport system that maps at 15 min on the Escherichia coli chromosome. J Biol Chem 266:20928-20933.

881. Pistocchi R, Kashiwagi K, Miyamoto S, Nukui E, Sadakata Y, Kobayashi H, Igarashi K. 1993. Characteristics of the operon for a putrescine transport system that maps at 19 minutes on the Escherichia coli chromosome. J Biol Chem 268:146-152.

882. Kashiwagi K, Hosokawa N, Furuchi T, Kobayashi H, Sasakawa C, Yoshikawa M, Igarashi K. 1990. Isolation of polyamine transport-deficient mutants of Escherichia coli and cloning of the genes for polyamine transport proteins. J Biol Chem 265:20893-20897.

883. Wu D, Lim SC, Dong Y, Wu J, Tao F, Zhou L, Zhang LH, Song H. 2012. Structural basis of substrate binding specificity revealed by the crystal structures of polyamine receptors $\mathrm{SpuD}$ and SpuE from Pseudomonas aeruginosa. J Mol Biol 416:697-712. 
884. Zhou L, Wang J, Zhang LH. 2007. Modulation of bacterial Type III secretion system by a spermidine transporter dependent signaling pathway. PLoS One 2:e1291.

885. Kwon DH, Lu CD. 2006. Polyamines induce resistance to cationic peptide, aminoglycoside, and quinolone antibiotics in Pseudomonas aeruginosa PAO1. Antimicrob Agents Chemother 50:1615-1622.

886. Kwon DH, Lu CD. 2006. Polyamines increase antibiotic susceptibility in Pseudomonas aeruginosa. Antimicrob Agents Chemother 50:1623-1627.

887. Malone L, Kwon DH. 2013. Carbapenem-associated multidrug-resistant Acinetobacter baumannii are sensitised by aztreonam in combination with polyamines. Int J Antimicrob Agents 41:70-74.

888. Terui Y, Saroj SD, Sakamoto A, Yoshida T, Higashi K, Kurihara S, Suzuki H, Toida T, Kashiwagi K, Igarashi K. 2014. Properties of putrescine uptake by PotFGHI and PuuP and their physiological significance in Escherichia coli. Amino Acids 46:661-670.

889. Kurihara S, Tsuboi Y, Oda S, Kim HG, Kumagai H, Suzuki H. 2009. The putrescine importer PuuP of Escherichia coli K-12. J Bacteriol 191:2776-2782.

890. Kurihara S, Oda S, Kato K, Kim HG, Koyanagi T, Kumagai H, Suzuki H. 2005. A novel putrescine utilization pathway involves $\gamma$-glutamylated intermediates of Escherichia coli K-12. J Biol Chem 280:4602-4608.

891. Sakurada K, Ohta T, Fujishiro K, Hasegawa M, Aisaka K. 1996. Acetylpolyamine amidohydrolase from Mycoplana ramosa: gene cloning and characterization of the metal-substituted enzyme. J Bacteriol 178:5781-5786.

892. Tkachenko AG, Pozhidaeva ON, Shumkov MS. 2006. Role of polyamines in formation of multiple antibiotic resistance of Escherichia coli under stress conditions. Biochemistry (Mosc) 71:1042-1049.

893. Kwon DH, Lu CD. 2007. Polyamine effects on antibiotic susceptibility in bacteria. Antimicrob Agents Chemother 51:2070-2077.

894. Hennessey TD. 1967. Inducible $\beta$-lactamase in Enterobacter. J Gen Microbiol 49:277-285.

895. Honore N, Nicolas MH, Cole ST. 1986. Inducible cephalosporinase production in clinical isolates of Enterobacter cloacae is controlled by a regulatory gene that has been deleted from Escherichia coli. EMBO J 5:3709-3714. 
896. Lindberg F, Westman L, Normark S. 1985. Regulatory components in Citrobacter freundii ampC $\beta$-lactamase induction. Proc Natl Acad Sci U S A 82:4620-4624.

897. Maddocks SE, Oyston PC. 2008. Structure and function of the LysR-type transcriptional regulator (LTTR) family proteins. Microbiology 154:3609-3623.

898. Cheng Q, Park JT. 2002. Substrate specificity of the AmpG permease required for recycling of cell wall anhydro-muropeptides. J Bacteriol 184:6434-6436.

899. Cheng Q, Li H, Merdek K, Park JT. 2000. Molecular characterization of the $\beta$ $\mathrm{N}$-acetylglucosaminidase of Escherichia coli and its role in cell wall recycling. J Bacteriol 182:4836-4840.

900. Votsch W, Templin MF. 2000. Characterization of a $\beta-\mathrm{N}$-acetylglucosaminidase of Escherichia coli and elucidation of its role in muropeptide recycling and $\beta$ lactamase induction. J Biol Chem 275:39032-39038.

901. Dietz H, Wiedemann B. 1996. The role of N-actylglucosaminyl-1,6 anhydro Nacetylmuramyl-L-alanyl-D-glutamyl-meso-diaminopimelic acid-D-alanine for the induction of $\beta$-lactamase in Enterobacter cloacae. Zentralbl Bakteriol 284:207217.

902. Mengin-Lecreulx D, Flouret B, van Heijenoort J. 1982. Cytoplasmic steps of peptidoglycan synthesis in Escherichia coli. J Bacteriol 151:1109-1117.

903. Dietz H, Pfeifle D, Wiedemann B. 1996. Location of N-acetylmuramyl-Lalanyl-D-glutamylmesodiaminopimelic acid, presumed signal molecule for $\beta$ lactamase induction, in the bacterial cell. Antimicrob Agents Chemother 40:21732177.

904. Langaee TY, Dargis M, Huletsky A. 1998. An ampD gene in Pseudomonas aeruginosa encodes a negative regulator of AmpC $\beta$-Lactamase expression. Antimicrob Agents Chemother 42:3296-3300.

905. Zamorano L, Reeve TM, Juan C, Moya B, Cabot G, Vocadlo DJ, Mark BL, Oliver A. 2011. AmpG inactivation restores susceptibility of pan- $\beta$-lactamresistant Pseudomonas aeruginosa clinical strains. Antimicrob Agents Chemother 55:1990-1996.

906. Zhang Y, Bao Q, Gagnon LA, Huletsky A, Oliver A, Jin S, Langaee T. 2010. ampG gene of Pseudomonas aeruginosa and its role in $\beta$-lactamase expression. Antimicrob Agents Chemother 54:4772-4779. 
907. Scotto-Lavino E, Du G, Frohman MA. 2006. 5' end cDNA amplification using classic RACE. Nat Protoc 1:2555-2562.

908. Castang S, McManus HR, Turner KH, Dove SL. 2008. H-NS family members function coordinately in an opportunistic pathogen. Proc Natl Acad Sci U S A 105:18947-18952.

909. Campo N, Rudner DZ. 2006. A branched pathway governing the activation of a developmental transcription factor by regulated intramembrane proteolysis. Mol Cell 23:25-35.

910. Smith PK, Krohn RI, Hermanson GT, Mallia AK, Gartner FH, Provenzano MD, Fujimoto EK, Goeke NM, Olson BJ, Klenk DC. 1985. Measurement of protein using bicinchoninic acid. Anal Biochem 150:76-85.

911. Blank TE, Donnenberg MS. 2001. Novel topology of BfpE, a cytoplasmic membrane protein required for type IV fimbrial biogenesis in enteropathogenic Escherichia coli. J Bacteriol 183:4435-4450.

912. Boucher JC, Schurr MJ, Deretic V. 2000. Dual regulation of mucoidy in Pseudomonas aeruginosa and sigma factor antagonism. Mol Microbiol 36:341351.

913. Reitzer LJ, Bueno R, Cheng WD, Abrams SA, Rothstein DM, Hunt TP, Tyler B, Magasanik B. 1987. Mutations that create new promoters suppress the $\sigma^{54}$ dependence of $g \ln A$ transcription in Escherichia coli. J Bacteriol 169:42794284.

914. Huffman JL, Brennan RG. 2002. Prokaryotic transcription regulators: more than just the helix-turn-helix motif. Curr Opin Struct Biol 12:98-106.

915. Aravind L, Anantharaman V, Balaji S, Babu MM, Iyer LM. 2005. The many faces of the helix-turn-helix domain: transcription regulation and beyond. FEMS Microbiol Rev 29:231-262.

916. Bushman FD, Ptashne M. 1988. Turning $\lambda$ Cro into a transcriptional activator. Cell 54:191-197.

917. Hochschild A, Irwin N, Ptashne M. 1983. Repressor structure and the mechanism of positive control. Cell 32:319-325.

918. Whipple FW, Ptashne M, Hochschild A. 1997. The activation defect of a $\lambda \mathrm{cI}$ positive control mutant. J Mol Biol 265:261-265. 
919. Bushman FD, Shang C, Ptashne M. 1989. A single glutamic acid residue plays a key role in the transcriptional activation function of $\lambda$ repressor. Cell 58:11631171.

920. Gosink KK, Gaal T, Bokal AJ, Gourse RL. 1996. A positive control mutant of the transcription activator protein FIS. J Bacteriol 178:5182-5187.

921. Guarente L, Nye JS, Hochschild A, Ptashne M. 1982. Mutant $\lambda$ phage repressor with a specific defect in its positive control function. Proc Natl Acad Sci U S A 79:2236-2239.

922. Zhou Y, Zhang X, Ebright RH. 1993. Identification of the activating region of catabolite gene activator protein (CAP): isolation and characterization of mutants of CAP specifically defective in transcription activation. Proc Natl Acad Sci U S A 90:6081-6085.

923. Bartowsky E, Normark S. 1991. Purification and mutant analysis of Citrobacter freundii AmpR, the regulator for chromosomal AmpC $\beta$-lactamase. Mol Microbiol 5:1715-1725.

924. Kuga A, Okamoto R, Inoue M. 2000. ampR gene mutations that greatly increase class C $\beta$-lactamase activity in Enterobacter cloacae. Antimicrob Agents Chemother 44:561-567.

925. McFall SM, Chugani SA, Chakrabarty AM. 1998. Transcriptional activation of the catechol and chlorocatechol operons: variations on a theme. Gene 223:257267.

926. Miller BE, Kredich NM. 1987. Purification of the CysB protein from Salmonella typhimurium. J Biol Chem 262:6006-6009.

927. Muraoka S, Okumura R, Ogawa N, Nonaka T, Miyashita K, Senda T. 2003. Crystal structure of a full-length LysR-type transcriptional regulator, CbnR: unusual combination of two subunit forms and molecular bases for causing and changing DNA bend. J Mol Biol 328:555-566.

928. Parsek MR, Shinabarger DL, Rothmel RK, Chakrabarty AM. 1992. Roles of CatR and cis,cis-muconate in activation of the catBC operon, which is involved in benzoate degradation in Pseudomonas putida. J Bacteriol 174:7798-7806.

929. Schell MA, Brown PH, Raju S. 1990. Use of saturation mutagenesis to localize probable functional domains in the NahR protein, a LysR-type transcription activator. J Biol Chem 265:3844-3850. 
930. Balcewich MD, Reeve TM, Orlikow EA, Donald LJ, Vocadlo DJ, Mark BL. 2010. Crystal structure of the AmpR effector binding domain provides insight into the molecular regulation of inducible Ampc $\beta$-lactamase. J Mol Biol 400:9981010 .

931. Bishop RE, Weiner JH. 1993. Overproduction, solubilization, purification and DNA-binding properties of AmpR from Citrobacter freundii. Eur $\mathrm{J}$ Biochem 213:405-412.

932. Kyte J, Doolittle RF. 1982. A simple method for displaying the hydropathic character of a protein. J Mol Biol 157:105-132.

933. von Heijne G. 1992. Membrane protein structure prediction. Hydrophobicity analysis and the positive-inside rule. J Mol Biol 225:487-494.

934. Cserzo M, Wallin E, Simon I, von Heijne G, Elofsson A. 1997. Prediction of transmembrane alpha-helices in prokaryotic membrane proteins: the dense alignment surface method. Protein Eng 10:673-676.

935. Jones DT, Taylor WR, Thornton JM. 1994. A model recognition approach to the prediction of all-helical membrane protein structure and topology. Biochemistry 33:3038-3049.

936. K. H, W. S. 1993. A database of membrane spanning protein segments. Biol Chem Hoppe-Seyler 374:166.

937. Bernsel A, Viklund H, Falk J, Lindahl E, von Heijne G, Elofsson A. 2008. Prediction of membrane-protein topology from first principles. Proc Natl Acad Sci U S A 105:7177-7181.

938. Lee M, Artola-Recolons C, Carrasco-Lopez C, Martinez-Caballero S, Hesek D, Spink E, Lastochkin E, Zhang W, Hellman LM, Boggess B, Hermoso JA, Mobashery S. 2013. Cell-wall remodeling by the zinc-protease AmpDh3 from Pseudomonas aeruginosa. J Am Chem Soc 135:12604-12607.

939. Zhang W, Lee M, Hesek D, Lastochkin E, Boggess B, Mobashery S. 2013. Reactions of the three AmpD enzymes of Pseudomonas aeruginosa. J Am Chem Soc 135:4950-4953.

940. Schlaman HR, Spaink HP, Okker RJ, Lugtenberg BJ. 1989. Subcellular localization of the nodD gene product in Rhizobium leguminosarum. J Bacteriol 171:4686-4693. 
941. Dell CL, Neely MN, Olson ER. 1994. Altered pH and lysine signalling mutants of $c a d C$, a gene encoding a membrane-bound transcriptional activator of the Escherichia coli cadBA operon. Mol Microbiol 14:7-16.

942. Hanson BR, Lowe BA, Neely MN. 2011. Membrane topology and DNA-binding ability of the Streptococcal CpsA protein. J Bacteriol 193:411-420.

943. Miller VL, Taylor RK, Mekalanos JJ. 1987. Cholera toxin transcriptional activator ToxR is a transmembrane DNA binding protein. Cell 48:271-279.

944. Pendleton JN, Gorman SP, Gilmore BF. 2013. Clinical relevance of the ESKAPE pathogens. Expert Rev Anti Infect Ther 11:297-308.

945. Rice LB. 2008. Federal funding for the study of antimicrobial resistance in nosocomial pathogens: no ESKAPE. J Infect Dis 197:1079-1081.

946. Nikaido H. 2003. Molecular basis of bacterial outer membrane permeability revisited. Microbiol Mol Biol Rev 67:593-656.

947. Ocampo-Sosa AA, Cabot G, Rodriguez C, Roman E, Tubau F, Macia MD, Moya B, Zamorano L, Suarez C, Pena C, Dominguez MA, Moncalian G, Oliver A, Martinez-Martinez L, Spanish Network for Research in Infectious D. 2012. Alterations of OprD in carbapenem-intermediate and -susceptible strains of Pseudomonas aeruginosa isolated from patients with bacteremia in a Spanish multicenter study. Antimicrob Agents Chemother 56:1703-1713.

948. Boutoille D, Corvec S, Caroff N, Giraudeau C, Espaze E, Caillon J, Plesiat P, Reynaud A. 2004. Detection of an IS21 insertion sequence in the mexR gene of Pseudomonas aeruginosa increasing $\beta$-lactam resistance. FEMS Microbiol Lett 230:143-146.

949. Adewoye L, Sutherland A, Srikumar R, Poole K. 2002. The MexR repressor of the mexAB-oprM multidrug efflux operon in Pseudomonas aeruginosa: characterization of mutations compromising activity. J Bacteriol 184:4308-4312.

950. Mathee K, Narasimhan G, Valdes C, Qiu X, Matewish JM, Koehrsen M, Rokas A, Yandava CN, Engels R, Zeng E, Olavarietta R, Doud M, Smith RS, Montgomery P, White JR, Godfrey PA, Kodira C, Birren B, Galagan JE, Lory S. 2008. Dynamics of Pseudomonas aeruginosa genome evolution. Proc Natl Acad Sci U S A 105:3100-3105.

951. Sevillano E, Gallego L, Garcia-Lobo JM. 2009. First detection of the OXA-40 carbapenemase in $P$. aeruginosa isolates, located on a plasmid also found in $A$. baumannii. Pathol Biol (Paris) 57:493-495. 
952. El Garch F, Bogaerts P, Bebrone C, Galleni M, Glupczynski Y. 2011. OXA198 , an acquired carbapenem-hydrolyzing class $\mathrm{D} \beta$-lactamase from Pseudomonas aeruginosa. Antimicrob Agents Chemother 55:4828-4833.

953. Sevillano E, Valderrey C, Canduela MJ, Umaran A, Calvo F, Gallego L. 2006. Resistance to antibiotics in clinical isolates of Pseudomonas aeruginosa. Pathol Biol (Paris) 54:493-497.

954. Lopez-Otsoa F, Gallego L, Towner KJ, Tysall L, Woodford N, Livermore DM. 2002. Endemic carbapenem resistance associated with OXA-40 carbapenemase among Acinetobacter baumannii isolates from a hospital in northern Spain. J Clin Microbiol 40:4741-4743.

955. Fowler RC, Hanson ND. 2014. Emergence of carbapenem resistance due to the novel insertion sequence ISPa8 in Pseudomonas aeruginosa. PLoS One 9:e91299.

956. Mulvey MR, Boyd DA, Baker L, Mykytczuk O, Reis EM, Asensi MD, Rodrigues DP, Ng LK. 2004. Characterization of a Salmonella enterica serovar Agona strain harbouring a class 1 integron containing novel OXA-type $\beta$ lactamase $\left(\right.$ bla $\left._{\text {OXA-53 }}\right)$ and 6'-N-aminoglycoside acetyltransferase genes [aac $\left(6^{\prime}\right)$ I30]. J Antimicrob Chemother 54:354-359.

957. Philippon LN, Naas T, Bouthors AT, Barakett V, Nordmann P. 1997. OXA18 , a class D clavulanic acid-inhibited extended-spectrum $\beta$-lactamase from Pseudomonas aeruginosa. Antimicrob Agents Chemother 41:2188-2195.

958. Toleman MA, Rolston K, Jones RN, Walsh TR. 2003. Molecular and biochemical characterization of OXA-45, an extended-spectrum class $2 \mathrm{~d}^{\prime} \beta$ lactamase in Pseudomonas aeruginosa. Antimicrob Agents Chemother 47:28592863.

959. Poirel L, Gerome P, De Champs C, Stephanazzi J, Naas T, Nordmann P. 2002. Integron-located oxa-32 gene cassette encoding an extended-spectrum variant of OXA-2 $\beta$-lactamase from Pseudomonas aeruginosa. Antimicrob Agents Chemother 46:566-569.

960. Rasmussen BA, Bush K. 1997. Carbapenem-hydrolyzing $\beta$-lactamases. Antimicrob Agents Chemother 41:223-232.

961. Bou G, Martinez-Beltran J. 2000. Cloning, nucleotide sequencing, and analysis of the gene encoding an AmpC $\beta$-lactamase in Acinetobacter baumannii. Antimicrob Agents Chemother 44:428-432. 
962. Ben RJ, Yang MC, Hsueh JC, Shiang JC, Chien ST. 2011. Molecular characterisation of multiple drug-resistant Acinetobacter baumannii isolates in southern Taiwan. Int J Antimicrob Agents 38:403-408.

963. Lin MF, Chang KC, Lan CY, Chou J, Kuo JW, Chang CK, Liou ML. 2011. Molecular epidemiology and antimicrobial resistance determinants of multidrugresistant Acinetobacter baumannii in five proximal hospitals in Taiwan. Jpn J Infect Dis 64:222-227.

964. Segal H, Nelson EC, Elisha BG. 2004. Genetic environment and transcription of aтpC in an Acinetobacter baumannii clinical isolate. Antimicrob Agents Chemother 48:612-614.

965. Corvec S, Caroff N, Espaze E, Giraudeau C, Drugeon H, Reynaud A. 2003. AmpC cephalosporinase hyperproduction in Acinetobacter baumannii clinical strains. J Antimicrob Chemother 52:629-635.

966. Aubert D, Naas T, Nordmann P. 2003. IS 1999 increases expression of the extended-spectrum $\beta$-lactamase VEB-1 in Pseudomonas aeruginosa. J Bacteriol 185:5314-5319.

967. Wang J, Zhou JY, Qu TT, Shen P, Wei ZQ, Yu YS, Li LJ. 2010. Molecular epidemiology and mechanisms of carbapenem resistance in Pseudomonas aeruginosa isolates from Chinese hospitals. Int J Antimicrob Agents 35:486-491.

968. Takeda S, Nakai T, Ikeda F, Hatano K. 2008. Overproduction of a metallo- $\beta$ lactamase by a strong promoter causes high-level imipenem resistance in a clinical isolate of Pseudomonas aeruginosa. Chemotherapy 54:181-187.

969. Walsh F, Amyes SG. 2007. Carbapenem resistance in clinical isolates of Pseudomonas aeruginosa. J Chemother 19:376-381.

970. Heurlier K, Denervaud V, Pessi G, Reimmann C, Haas D. 2003. Negative control of quorum sensing by RpoN $\left(\sigma^{54}\right)$ in Pseudomonas aeruginosa PA01. J Bacteriol 185:2227-2235.

971. Quale J, Bratu S, Gupta J, Landman D. 2006. Interplay of efflux system, aтpC, and oprD expression in carbapenem resistance of Pseudomonas aeruginosa clinical isolates. Antimicrob Agents Chemother 50:1633-1641. 
VITA

\section{DIANSY ZINCKE}

2000-2005

B. Sc., Biological Sciences, Cum Laude

Florida International University, Miami, FL

2004-2005 Undergraduate Research Assistant

Department of Biological Sciences, Florida International University, Miami, FL

Advisor: Professor Evelyn Gaiser

Fall $2006 \quad$ Post Baccalaureate Research Assistant

Department of Biological Sciences, Florida International University, Miami, FL

Advisor: Professor Kalai Mathee

American Society for Microbiology Travel Award [\$500]

2008

$\mathrm{NIH}$ - Access to Biomedical Research Summer Award [\$2,500]

2008

Cystic Fibrosis Foundation Traineeship Award [\$1,500]

2008-13 Research Initiative for Scientific Enhancement (RISE) Fellowship

2009

$\mathrm{NIH}$ - Access to Biomedical Research Summer Award [\$3,000]

2010-2015

Doctoral Candidate in Biological Sciences

Florida International University,

Miami, FL

Teaching Assistant

Florida International University,

Miami, FL

2011

American Society for Microbiology Travel Award [\$500] 


\section{PUBLICATIONS AND PRESENTATIONS}

H. Kumari, D. Balasubramanian, D. Zincke, and K. Mathee (2014). Role of Pseudomonas aeruginosa AmpR on $\beta$-lactam and non- $\beta$-lactam transient cross-resistance upon pre-exposure to subinhibitory concentrations of antibiotics. J Med Microbiol. 63(Pt 4):544-55.

Caille O, Zincke D, Merighi M, Balasubramanian D, Kumari H, Kong KF, Silva-Herzog E, Narasimhan G, Schneper L, Lory S, Mathee K. 2014. Structural and functional characterization of Pseudomonas aeruginosa global regulator AmpR. J Bacteriol 196:3890-3902.

$10^{\text {th }}$ Annual Biology Research Symposium, Feb $16^{\text {th }}, 2008$, Kampong Botanical Gardens, Miami, FL. D. Zincke, D. Balasubramanian, L. Schneper, K. Mathee. Characterization of the poxAB operon in Pseudomonas aeruginosa.

$107^{\text {th }}$ Annual Meeting of the American Society for Microbiology in Boston, MA. June $1^{\text {st }}$ - $5^{\text {th }}$, 2008. D. Zincke, D. Balasubramanian, K-F.Kong, L. Schneper, K. Mathee. Characterization of the poxAB operon in Pseudomonas aeruginosa. Abstract \#A-083

$111^{\text {th }}$ Annual Meeting of the American Society for Microbiology, New Orleans, Louisiana. May $21^{\text {st }}-24^{\text {th }}, 2011$, D. Zincke, D. Balasubramanian, L. Schneper, K. Mathee. Investigating the contribution of the poxAB operon to $\beta$-lactam resistance in Pseudomonas aeruginosa. Abstract \#A-3144

Annual RISE Symposium, Oct $10^{\text {th }}$, 2008, Florida International University, Miami, FL. D. Zincke, D. Balasubramanian, K-F.Kong, L. Schneper, K. Mathee. Function of the poxAB operon in Pseudomonas aeruginosa.

$12^{\text {th }}$ Annual Biology Research Symposium, Feb $6^{\text {th }}$, 2010, BBC Campus, Florida International University, Miami, FL. D. Zincke, D. Balasubramanian, L. Schneper, K. Mathee. Investigating the contribution of the poxAB operon to $\beta$-lactam resistance in Pseudomonas aeruginosa.

Annual RISE Symposium, Oct $8^{\text {th }}$, 2010, Florida International University, Miami, FL. D. Zincke, D. Balasubramanian, K-F.Kong, L. Schneper, K. Mathee. Function of the poxAB operon in Pseudomonas aeruginosa.

Annual RISE Symposium, Oct $7^{\text {th }}$, 2011, Florida International University, Miami, FL. D. Zincke, D. Balasubramanian, L. Schneper, K. Mathee. Investigating $\beta$-lactam resistance in Pseudomonas aeruginosa.

Annual RISE Symposium, Oct $9^{\text {th }}$, 2012, Florida International University, Miami, FL. D. Zincke, Camille de Rimonteil de Lombares, Olivier Caille, K. Mathee. Characterization of the poxAB operon in Pseudomonas aeruginosa. 